Tل Book Series on Vibroengineering ISSN 2351-5260 Volume 1 ISBN 978-609-95549-2-1

\title{
Rafał Burdzik
}

Identification of sources, propagation and structure of vibrations affecting men in means of transport based on the example of automotive vehicles
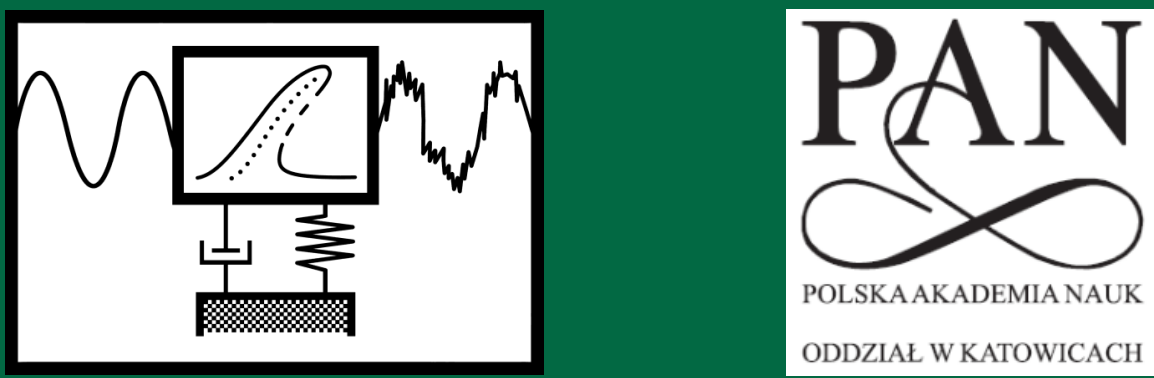
Identification of sources, propagation and structure of vibrations affecting men in means of transport based on the example of automotive vehicles 


\section{JVE Book Series on Vibroengineering}

\section{Aims and Scope}

JVE Book Series on Vibroengineering covers a wide area of topics describing modern developments in vibroengineering of dynamical systems (macro-, micro-, nano- mechanical, mechatronic and biomechanical systems).

The following subjects are principal topics:

Vibration and wave processes; Vibration and wave technologies;

Nonlinear vibrations; Vibroshock systems; Generation of vibrations and waves;

Vibrostabilization; Transformation of motion by vibrations and waves;

Dynamics of intelligent mechanical systems;

Vibration control, identification, diagnostics and monitoring. 


\section{Rafał Burdzik}

Identification of sources, propagation and structure of vibrations affecting men in means of transport based on the example of automotive vehicles
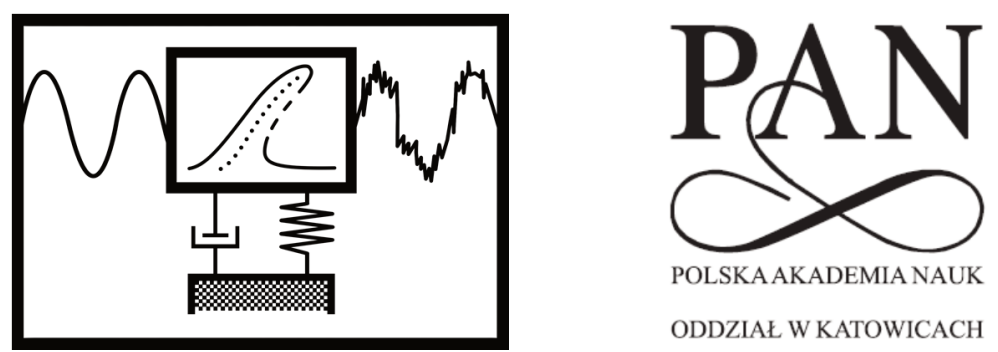


\section{Published by}

JVE International Ltd.

Gèlių ratas 15A, LT-50282, Kaunas, Lithuania

http://www.jve.lt

\section{Official Reviewers}

Prof. Kazimieras Ragulskis, Lithuanian Academy of Sciences, Lithuania

Prof. Bogusław Łazarz, Silesian University of Technology, Poland

\section{Patronage}

The Katowice Branch of the Polish Academy of Sciences

The Committee on Transport, The Katowice Branch of the Polish Academy of Sciences

\section{JVE Book Series on Vibroengineering}

IDENTIFICATION OF SOURCES, PROPAGATION AND STRUCTURE OF VIBRATIONS AFFECTING MEN IN MEANS OF TRANSPORT BASED ON THE EXAMPLE OF AUTOMOTIVE VEHICLES - Vol. 1

\section{Copyright $\odot 2014$ by JVE International Ltd.}

All rights reserved. This book, or parts thereof, may not be reproduced in any form or by any means, electronic or mechanical, including photocopying, recording or any information storage and retrieval system now know or to be invented, without written permission from the Publisher.

ISSN 2351-5260

ISBN 978-609-95549-2-1

Printed in Lithuania by Design and Publishing JSC KOPA 


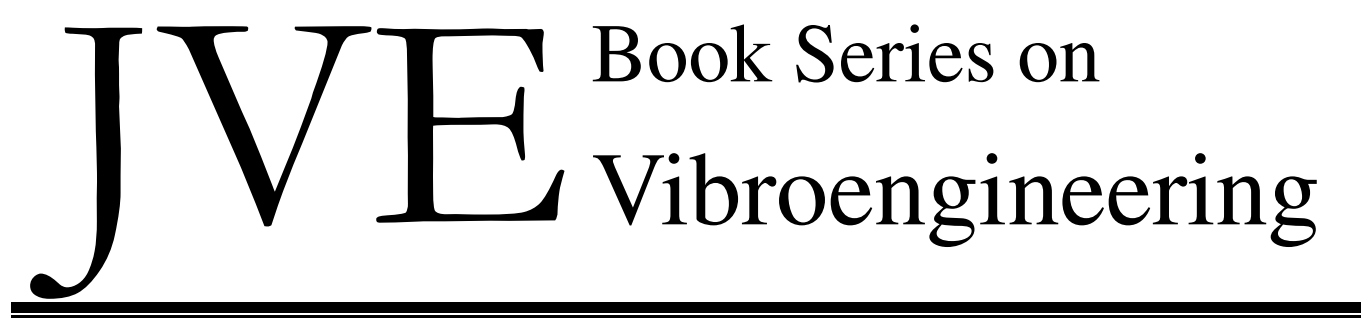

ISSN 2351-5260. APRIL 2014. VOLUME 1, ISBN 978-609-95549-2-1

\section{Contents}

SUMMARY

NOMENCLATURE

1. INTRODUCTION $\quad 7$

2. VIBRATION EFFECTS ON THE TRANSPORT SYSTEM 9

2.1. GENERAL INFORMATION ON VEHICLE VIBRATION 9

2.2. VIBRATION EFFECTS ON THE HUMAN 10

2.3. PERCEPTION OF VIBRATIONS IN AUTOMOTIVE VEHICLES

2.4. ENVIRONMENTAL IMPACT OF TRANSPORT 16

3. ANALYSIS OF VIBRATION PROPAGATION INTO VEHICLE`S STRUCTURE 19

3.1. PROPAGATION OF VIBRATION IN TRANSPORT SYSTEM 19

3.2. VIBRATION - STOCHASTIC WAVE PROPAGATION 20

3.3. PROPAGATION OF VIBRATIONS AND VIBROACOUSTIC DIAGNOSTICS

3.4. ANALYSIS AND COMPARISON OF THE VIBRATION DYNAMICS FOR THE CHOSEN 22

POINTS IN THE VEHICLE STRUCTURE

4. ADVANCED SIGNAL PROCESSING METHODS IN RESEARCH ON VIBRATION 27

PROPAGATION IN VEHICLE STRUCTURE

4.1. VEHICLE VIBRATION SIGNAL PROCESSING METHODS 27

5. IDENTIFICATION OF FLOOR PANEL VIBRATION CHARACTERISTICS FOR A MOTOR 4

VEHICLE, EMPIRICAL EVALUATION OF DISTRIBUTION OF NATURAL VIBRATIONS

5.1. MATERIAL VIBRATION SIGNAL PROCESSING METHODS 41

5.2. EXPERIMENTAL RESEARCH METHOD

5.3. PROPAGATION AND STRUCTURE OF THE FLOOR PANEL'S VIBRATION 46

5.4. DISCUSSION, ESTIMATES OF THE FLOOR PANEL'S NATURAL VIBRATION SURFACE 49

6. THE INFLUENCE OF THE TECHNICAL CONDITION OF SUSPENSION ELEMENTS AND 53

VEHICLE OPERATING PARAMETERS ON THE VIBRATION DISTRIBUTION IN A VEHICLE

CONSTRUCTION GENERATED BY THE FORCES ACTING ON THE WHEEL

6.1. RESEARCH ON THE INFLUENCE OF CHANGES IN THE TECHNICAL CONDITION OF 53

SUSPENSION ELEMENTS AND OF THE VEHICLE OPERATING PARAMETERS ON THE

VIBRATION DISTRIBUTION IN A VEHICLE STRUCTURE

6.2. DISTRIBUTION OF VIBRATION IN VEHICLE STRUCTURE FOR DIFFERENT TECHNICAL

CONDITIONS OF SUSPENSION ELEMENTS AND OPERATING PARAMETERS OF THE CAR 
6.3. DISCUSSION ON IMPACT OF SUSPENSION COMPONENTS AND OPERATING

PARAMETERS OF A VEHICLE ON THE PROPAGATION OF VIBRATIONS

7. THE INFLUENCE OF THE TECHNICAL CONDITION OF SUSPENSION ELEMENTS AND VEHICLE OPERATING PARAMETERS ON THE DOMINANT FREQUENCY COMPONENTS OF THE VIBRATION

7.1. FOURIER TRANSFORM

7.2. DYNAMICS OF VIBRATION IN VEHICLE STRUCTURE FOR DIFFERENT TECHNICAL

CONDITIONS OF SUSPENSION ELEMENTS AND OPERATING PARAMETERS OF THE CAR, IDENTIFICATION OF DOMINANT FREQUENCY COMPONENTS

7.3. FREQUENCY BASED ESTIMATORS

7.4. RESULT ANALYSIS AND DISCUSSION

8. IDENTIFICATION OF VEHICLE VIBRATION'S STRUCTURE IN RESEARCH ON THE

INFLUENCE OF CHANGES IN THE TECHNICAL CONDITION OF SUSPENSION ELEMENTS AND OF THE VEHICLE OPERATING PARAMETERS ON THE VIBRATION DISTRIBUTION IN A VEHICLE STRUCTURE

8.1. APPLICATION OF TIME-FREQUENCY REPRESENTATION METHODS FOR

DETERMINATION OF VIBRATION STRUCTURE

8.2. ANALYSIS OF TIME STRUCTURE OF VIBRATION IN DETERMINED FREQUENCY BANDS

9. FUNCTION OF EXPOSURE TIME TO THE VIBRATION OF DEFINED FREQUENCY BANDS IN

RESEARCH ON THE INFLUENCE OF THE TECHNICAL CONDITION OF SUSPENSION

ELEMENTS AND OF THE VEHICLE OPERATING PARAMETERS ON THE VIBRATION

9.1. DISTRIBUTION OF THE VIBRATION OF DEFINED FREQUENCY BANDS

9.2. FUNCTION OF EXPOSURE TIME TO THE VIBRATION OF DEFINED FREQUENCY BANDS

10. RESONANCE SPECTRUM IN RESEARCH ON THE INFLUENCE OF THE TECHNICAL

CONDITION OF SUSPENSION ELEMENTS AND OF THE VEHICLE OPERATING PARAMETERS

ON THE VIBRATION

10.1. SPECTRUM OF THE VIBRATION OCCURRING DURING RESONANCE

10.2. RESONANCE SPECTRUM - VIBRATION FUNCTION

11. ENGINE AS THE VIBRATION SOURCE IN VEHICLE

11.1. MOTOR-ENGINE AS THE SOURCE OF VIBRATION

11.2. IDENTIFICATION OF THE VIBRATION GENERATED BY THE ENGINE

11.3. INFLUENCE OF ENGINE ROTATIONAL SPEED ON THE VIBRATION GENERATION

12. IDENTIFICATION OF VIBRATION PROPAGATION PATHS FROM ENGINE INTO THE

12.1. RESEARCH ON VIBRATION PROPAGATION PATHS FROM ENGINE TO CAR BODY IN

LOCATIONS OF PENETRATE INTO HUMAN BODY

12.2. PROPAGATION AND STRUCTURE OF VIBRATION TRANSFERRED FROM ENGINE TO CAR BODY

13. INFLUENCE OF ENGINE ROTATIONAL SPEED ON FLOOR PANEL VIBRATION

13.1. RESEARCH ON VIBRATION PROPAGATION PATHS FROM ENGINE TO CAR BODY IN LOCATIONS OF PENETRATE INTO HUMAN BODY

13.2. RESEARCH RESULTS

13.3. ANALYSIS OF ENGINE ROTATIONAL SPEED IMPACT ON FLOOR PANEL VIBRATION

13.4. RESULT AND FUNCTIONS ANALYSIS

14. INFLUENCE OF GEAR POSITION IN TRANSMISSION GEARBOX ON VIBRATION 171

14.1. GEARBOX VIBRATION

14.2. RESEARCH METHOD

14.3. RESEARCH RESULT

14.4. IDENTIFICATION OF VIBRATION COMPONENTS CAUSED BY THE GEARBOX 
15. IDENTIFICATION OF VIBRATION COMPONENTS OF RIDE VEHICLE CAUSED BY ROAD

ROUGHNESS

15.1. RIDE VEHICLE VIBRATION GENERATED BY THE GROUND UNEVENNESS

15.2. METHOD OF IDENTIFICATION OF VIBRATION COMPONENTS OF RIDING VEHICLE $\quad 184$

CAUSED BY THE ROAD ROUGHNESS

15.3. IDENTIFICATION OF VIBRATION COMPONENTS CAUSED BY THE POWERTRAIN

185

SYSTEM AND ROAD ROUGHNESS

16. INFLUENCE OF VEHICLE TECHNICAL CONDITION AND POWER TRANSMISSION

SYSTEM ON EVALUATION OF HUMAN EXPOSURE TO WHOLE BODY VIBRATION

PENETRATED VIA FEET

16.1. HUMAN EXPOSURE TO WHOLE-BODY VIBRATION

192

16.2. THE STANDARDS OF WBV FOR MEANS OF TRANSPORT

192

16.3. THE MODEL OF VIBRATION SPINE RESPONSE - ISO 2631

193

16.4. WBV EVALUATION

194

16.5. INVESTIGATION ON INFLUENCE OF VEHICLE TECHNICAL CONDITION AND POWER TRANSMISSION SYSTEM ON EVALUATION OF HUMAN EXPOSURE TO WHOLE BODY VIBRATION VIA FEET

17. THE MEASURES OF DISPERSION OF VIBRATION ENERGY OF VEHICLE CONSTRUCTION

17.1. METHODS OF ANALYSIS OF DISPERSION OF VIBRATION ENERGY

17.2. THE MEASURES OF DISPERSION OF VIBRATION ENERGY OF VEHICLE CONSTRUCTION

206

17.3. DISPERSION OF FLOOR PANEL VIBRATION GENERATED BY THE ENGINE AND

POWER TRANSMISSION SYSTEM

18. APPLICATION OF VIBROACOUSTIC METHODS FOR MONITORING OF VIBRATION PROPAGATION AND COMFORT IN VEHICLES

18.1. IMPACT OF VIBRATION ON VEHICLE'S COMFORT AND SAFETY

18.2. METHODS OF VIBRATION SIGNAL ANALYSIS

18.3. METHOD OF MULTIDIMENSIONAL IDENTIFICATION OF SIGNAL CHARACTERISTICS

IN THE ANALYSIS OF VIBRATION PROPERTIES

18.4. PROTOTYPE OF SYSTEMS OF MONITORING OF VIBRATION PROPERTIES AND

220

PROPAGATION IN VEHICLES

18.5. CONCEPT OF ON-BOARD COMFORT AND PROPAGATION OF VIBRATION

223

MONITORING SYSTEM FOR VEHICLES

18.6. THE POTENTIAL PRACTICAL APPLICATION OF THE WSA 



\section{Summary}

Vibration problems are very important for vehicle dynamics, especially due to safety and comfort. The range of impacts vibrations exert on a vehicle driver is very broad, starting from the feeling of discomfort to safety hazards caused by vibrations at resonant frequencies of specific organs, thus affecting the driver's responses. Therefore, it is important to study the paths of vibration propagation from their sources into the human organism and to assess the vibration exposure for different input function conditions.

In a decided majority of cases, modelling of the vehicle vibration dynamics is based on the assumed input functions reflecting sources of vibrations, e.g. the random ones caused by road irregularities, and they are identified at testing stations featuring vibration inductors characterised by sinusoidal or random input functions. The range of applications of models thus developed is very broad, however, the selection of publications addressing the impact on men is definitely more limited, the reason for which is the difficulty in reflecting biodynamic phenomena taking place in the human-vehicle-road (HVR) system as well we the fact that input functions are brought down to a single source, namely the road irregularity.

This book provides a discussion on the results of studies the author was conducting for several years, addressing the impact exerted by operating parameters of the engine and the power transmission system as well as the vehicle's technical condition and service factors of the suspension system components on the vibrations being generated. The author proposed specific methods dedicated to identification of the vibration structure in the function of time, frequency and time-frequency distributions, compared characteristics of the vibration structure of wave propagation in three orthogonal axes as well as identified the vibration distribution in a vehicle structure. Ultimately, such a wide range of experimental studies and analyses enables accurate assessment of the human exposure to vibrations in means of transport based on multidimensional analysis of the vibration exposure time for successive frequency bands. It may also initiate a new approach to experimental modelling and identification of the oscillatory wave propagation in a vehicle structure.

In terms of the vibration propagation analysis from the perspective of safety aspects, the publication addresses methods of testing oscillatory phenomena taking place in the vehicle suspension system, being responsible for the contact between the wheel and the road pavement. Oscillatory comfort was studied based on vibration signals in points of the impact exerted on the human organism by general vibrations propagating through the feet and the lumbosacral spine section. However, the objective nature of the feeling of comfort and discomfort may lead to disturbances in the human organism, and consequently to safety hazards related connected with vehicle driving. It is exactly for this reason that studies and collective analyses should be conducted in the fields of safety and comfort.

Results of the studies discussed in the book were obtained in the course of numerous experiments conducted with actual vehicles by application of three types of vibration input functions: kinematic, at a harmonic station of programmable frequency and amplitude structure, input functions caused by road irregularities and aerodynamic phenomena as well we those isolated from the road irregularity-induced and aerodynamic input functions caused by the engine and the power transmission system. The quantities recorded each time were the vibration accelerations measured simultaneously at several points of the vehicle structure in three 
orthogonal axes ( $X$ - in parallel to the vehicle's longitudinal axis, $Y$ - horizontally and perpendicularly to the vehicle's longitudinal axis, $Z$ - vertically and perpendicularly to the vehicle's longitudinal axis).

In the first part of the book, the author has discussed the results obtained while studying the impact of the vehicle operating parameters as well as technical condition of the suspension system components on vibrations, and special emphasis has been put on describing how passengers are affects through feet. Studies of the impact exerted by the technical condition of the suspension system components and the chosen operating parameters of a vehicle on the propagation of vibrations generated by a road wheel vertical motion to the vehicle structure are particularly important in cognitive terms for the analysis and identification of the vibration sources and propagation in vehicles. The purpose of the said studies was to identify factors affecting the propagation of vibrations caused by the dynamic impact of road irregularities on wheels of a moving vehicle. The aspects analysed included the accelerations of vibrations of the suspension system components and of the vehicle floor panel generated by the kinematic input function and affecting the vehicle wheel. For the sake of accurate analysis of both the stationary and non-stationary parts of signals, an algorithm for automatic identification of the signal's stationary characteristic and a division into successive time windows were developed. The research methodology proposed and a dedicated analysis of results enable comparison of the vibration dynamics between specific frequency bands. Also mathematical and programming algorithms were developed, allowing for in-depth analysis of automotive vehicle structure vibrations. The influence exerted by selected factors on vibrations was assessed based on energy and frequency measures. Furthermore, the author developed time and frequency measures for the vibration structure as a function of time of exposure to vibration for defined frequency bands and a frequency resonance function for the sake of studying the influence of technical condition of suspension elements and of the vehicle operating parameters on vibrations. These measures demonstrated a considerable susceptibility to changes in the technical condition of suspension elements and in the vehicle operating parameters with regard to the assessment of human exposure to vibrations.

The second part of the book addresses studies of the engine and the power transmission system considered as sources of vibrations in automotive vehicles. With regard to differences in the human perception of vibrations depending on the action direction, the engine was analysed as a directional vibration source. Having assumed such an approach, one could assess the energy fraction of vibrations propagating in 3 directions as well as analyse their dynamics and determine the predominant direction and frequency components of the vibration source. The author has also described propagation paths for whole-body vibrations (WBV) starting from the source (i.e. the engine) towards the driver and passengers, at points where lower limbs as well as the lumbosacral spine section are affected.

What is required in order to consider the engine as a source of vibrations in a moving vehicle is an analysis of the impact exerted by other mechanisms of the power transmission system on the vibration propagation from the source. And bearing the vibroacoustic emission in mind, one of the most crucial mechanisms is the transmission.

This part of the book provides results obtained in the studies of the influence exerted by basic operating parameters of the engine, namely rotational speed, and of the transmission, i.e. gear ratio at successive gears, on the propagation and impact of vibrations in a vehicle-human system. For this purpose, a series of measures was compared and some additional new measures were developed as energy estimators of the vibration spectrum and energy estimators of the vibration TFR, which represent frequency as well as time-frequency distributions of vibrations.

For the sake of verification of the methods developed for the vibration source identification in vehicles, results of vibration studies conducted in a moving vehicle have been discussed. The method proposed for identification of components of road irregularity induced vibrations for a moving vehicle may be brought down to comprehensive laboratory and road tests of the same vehicle while maintaining identical engine and power transmission operating parameters. It can 
be achieved by isolating components correlated with the signals obtained in the course of laboratory tests of a motionless car, excited to vibrate by an engine operating at a constant rotational speed and with the given transmission gear ratio, from the recorded signals of directional vibration propagation of a moving vehicle's load bearing structure. The foregoing is most evident in the difference spectrum function defined, one which represents the frequency distribution of absolute values based on the difference of vibration spectra obtained in road and laboratory tests.

The book also addresses an experimental approach to exposure to WBV penetrating the human organism through the floor panel via feet. Different evaluation methods have been compared for the assessment of the influence exerted by technical condition of the vehicle and the power transmission system on the human exposure to whole body vibration. Attempts to use the acceleration dose and equivalent static compressive stress for the assessment of health effects of WBV transferred from the floor panel, according to methods described in ISO 2631-5, have been discussed as well.

Based on an analysis of such a vast selection of study results, bearing in mind that the research purpose was to identify sources, propagation and structure of vibrations affecting men in means of transport based on the example of automotive vehicles, a decision has been made to extend the studies with an analysis of the vibration energy dissipation across the vehicle structure. It will enable assessment of the damping properties of the vehicle structure and its equipment, and may prove to be a valid source of information to be applied while designing eliminators and dampers of vibrations propagating into the human organism. The notion of vibration energy dissipation is inextricably linked with the wave propagation phenomenon, being most commonly described by means of wave propagation velocity derivatives. What matters more in terms of the vibration related phenomena and their impact on men is the energy-oriented approach. Having established a correlation between energy measures and dynamic properties of vibrations or their TFR distribution, one can develop dedicated measures of propagation and dissipation of vibrations in a vehicle structure from the perspective of their impact on the vehicle passengers. Based on satisfactory results of the efficiency assessment for the measures proposed in the book with reference to the studies of propagation of vibrations and how they are affected by technical parameters of the suspension system, the engine and the transmission, specific measures of the vibration energy dissipation at structural points of a vehicle have been proposed.

The measures developed enable observation and assessment of the energy and amplitude dissipation of vibrations in the domains of time, frequency and TFR. The dissipation measures are correlated with vibration signals of the vibration sources envisaged as int signals. In order to verify the efficiency of the measures proposed for the vibration dissipation in a vehicle structure, analytical experiments were conducted, comprising assessment of the susceptibility of the said measures to changes of the input function parameters. The source of vibrations was assumed to be the engine and the transmission. The measures of vibration dissipation in automotive vehicles have been defined and verified. They enable the vibration propagation and damping to be analysed and assessed by application of simple energy measures in the domains of time and frequency. The measures envisaged can also be used in experimental studies, which has been confirmed by the results of validation experiments. Directional distributions of the said coefficients make it possible to determine the vibration damping characteristics and the directional propagation, whereas by comparing the values obtained at the selected structural points in the propagation path, one can assess the dynamics of vibration damping or suppression as well as determine the most exposed locations.

The study results discussed in the book as well as the methods developed for the sake of analysis and assessment of vibrations have displayed a considerable application potential. The author has proposed several concepts of vibration monitoring systems to be used in vehicles for diagnostic purposes, for monitoring the state of comfort and safety as well as for vibration "management" by means of active insulation and damping systems.

The overall body of problems discussed in the book is also crucial in cognitive and utilitarian terms, which stems from an increasing share of forced stops while travelling with means of 
transport due to larger traffic volume and growing congestion. These factors are responsible for intensification and growth of the fraction of exposure to vehicle vibrations at stops, experienced by all vehicle users, the sources of which should not be sought in road irregularities and aerodynamic phenomena but exactly in the operation of the engine and the power transmission system. 


\section{Nomenclature}

\begin{tabular}{|c|c|}
\hline$A_{\text {wmax }}$ & Maximum transient vibration value \\
\hline$D_{\text {TabsTFR }}, D_{S_{\text {TabsTFR }}}$ & TFR directional dispersion factor for $i$-direction, total TFR dispersion factor \\
\hline$D_{T a b s_{i}}, D_{S_{T a b s}}$ & $\begin{array}{l}\text { Frequency directional dispersion factor for } i \text {-direction, total frequency dispersion } \\
\text { factor }\end{array}$ \\
\hline$D_{R M S i}, D_{S R M S}$ & $R M S$ directional dispersion factor for $i$-direction, total $R M S$ dispersion factor \\
\hline$D_{k}, D_{k d}$ & Acceleration dose, average daily acceleration dose \\
\hline$F_{d}(\omega)$ & Differential spectrum \\
\hline$F_{f}$ & Coulomb friction \\
\hline$R_{x y}$ & Cross-corelation function \\
\hline$S_{R M S}$ & Total energy estimator (sum of $R M S$ for $X, Y, Z$ axes) \\
\hline$S_{\text {Tabs }}$ & Total energy of vibration spectrum estimator (sum of $T_{a b s}$ for $X, Y, Z$ axes) \\
\hline$S_{\text {TabsTFR }}$ & Total energy of TFR of vibration estimator (sum of $t_{a b s t f r}$ for $X, Y, Z$ axes) \\
\hline$S_{\text {Tavr }}$ & Function of average value of time period vibration in frequency domain \\
\hline$S_{\text {avr }}$ & Function of average values of frequency bands vibration in time domain \\
\hline$S_{e}, S_{e d}$ & Equivalent static compressive stress and its daily equivalent dose \\
\hline$S_{f}$ & Time function of separate frequency bands \\
\hline$S_{t}$ & Function of frequency distribution for determined time period \\
\hline$T_{a b s}$ & Estimator of energy of vibration spectrum \\
\hline$T_{a b s T F R}$ & Energy of TFR of vibration estimator \\
\hline$X_{R M S}, R M S$ & Root-mean-square \\
\hline$a_{l}$ & Acceleration of vibration of lumbar response (lumbar spine acceleration) \\
\hline$H[s(t)]$ & Hilbert transform of the signal $s(t)$ \\
\hline$A(8)$ & Daily exposure to vibration \\
\hline$E M A$ & Experimental modal analysis \\
\hline$F(\omega)$ & Fourier transform \\
\hline$F F T, D F T$ & Fast fourier transform, discrete fourier transform \\
\hline$F R F$ & Frequency response function \\
\hline I & Vibration intensity \\
\hline IRI & International roughness index (metric to describe road roughness) \\
\hline$M, C, K$ & Mass, damping, stiffness matrix \\
\hline$N D N$ & Vibration limit value \\
\hline$Q$ & Quantity of the energy transferred \\
\hline$R$ & Risk factor \\
\hline STFT & Short time fourier transform \\
\hline$T F R$ & Time-frequency representation \\
\hline$V D V$ & Vibration dose value \\
\hline$W B V, H A V, H V R$ & Whole-body vibration, hand-arm vibration, human-vehicle-road \\
\hline$W S A$ & Vibroacoustic signals analyzer \\
\hline$W T, \Psi(t)$ & Wavelet transformation, wavelet family \\
\hline WVD & Wigner-ville distribution \\
\hline$X, Y, Z$ & $X$ - longitudinal, $Y$ - lateral, $Z$ - vertical (direction, axes) \\
\hline$n-D O F$ & The $n$ degrees of freedom \\
\hline rpm & Revolutions per minute \\
\hline$\lambda$ & Wave length \\
\hline$\omega, f$ & Frequency \\
\hline
\end{tabular}





\section{Introduction}

The complex distribution of oscillatory motion and force within the body during whole-body vibration produces complex sensations. The location and character of the sensations vary greatly according to the vibration frequency, axis and other factors. The term "iscomfort" is applied to the sensations arising directly from the vibration. A wider term is sometimes used in transportation systems to include reactions to other aspects of the environment (e.g. vibration, noise) and the effects of motion on common activities. Whether a degree of discomfort is acceptable will depend on many factors. Vibration is the mechanical phenomena caused by machines in operation. Generally the vibration is undesirable, wasting energy and creating unwanted effects. The vehicle vibration are one of the most important unwanted effects. It causes decrease of safety and comfort factors and increase of fuel consumption. Vibration transmission to human has a large influence on comfort, performance and health. Comfortable ride is essential for a vehicle in order to obtain passenger satisfaction.

Ride quality and comfort increasingly begins refer to vibration and noise in terms of sensation or feel of the passengers. Lower range of vibration frequencies mostly are correlated to vibration comfort and higher (more than $80 \mathrm{~Hz}$ ) to noise $[137,155]$. Vibration and noise are perceived differently by humans, so there is a need to adopt methods that help quantify and control them. Independent of method it is important to define the sources of these phenomena.

Group of interesting problems are relating to the understanding of vibration in complex mechanical structures such as means of transport. One of the usual reason to seek such understanding is to control the vibrations. The book presents research on identification of sources, propagation and structure of vibration in complex mechanical structure of passenger car. The vehicle vibration are results from many kind of dynamic interactions. The proper identification of the vibration is very difficult research and scientific problem. It requires good knowledge fundament and correct measurement tools and signal processing. An automotive vehicle, being a complex mechanical system, includes a set of specific natural vibrations frequencies depending on the direction of the oscillatory wave propagation.

Model representation of an automotive vehicle as a complex mechanical object requires that various aspects should be taken into consideration from the perspective of statics and dynamics, bearing the random phenomena in mind as well. In the study discussed in the monograph, the author focused on non-linear vibration related phenomena occurring in vehicles $[34,35,63,70-72,74,80,114,115,133]$. What is characteristic about dynamic vehicle models is that they entail a set of simplifications, applied across quartile models, several point masses, to finally mention full vehicle models of numerous degrees of freedom and non-linear characteristics.

The main areas of the author's interest under the past studies undertaken included an assessment of vibration damping from the perspective of safety and comfort based on vibration signals recorded in a direct vicinity of shock absorbers, at points of shock absorber mounting to both unsprung and sprung masses [56]. Furthermore, the author conducted a series of studies pertaining to identification of other vibration sources occurring in vehicles, such as the engine and the power transmission systems. The range of impacts vibrations exert on a vehicle driver is very broad, starting from the feeling of discomfort to safety hazards caused by vibrations at resonant frequencies of specific organs, thus affecting the driver's responses. Therefore, it is important to study the routes of vibration propagation from their sources to the human organism and to assess 
the vibration exposure for different input function conditions. The studies discussed in papers $[39,47]$ illustrate the outcomes of the influence of input parameters on the distribution of the vibrations being generated as well as their propagation.

The scope of studies of vehicle vibration dynamics, identification of sources and propagation paths have to be extended by stationary empirical tests of oscillatory wave propagation in a vehicle structure as well as identification of natural vibrations. One must also bear in mind that a detailed analysis of vibration related phenomena requires that other properties and mechanical phenomena taking place in the course of degradation as well as the impact of external factors should be taken into consideration.

During operating of vehicles there are many vibration generated by the different sources. Motor engine should be considered as the vibration generator as well. This kind of machine generate a disturbing force of one sort or another, but the frequency of the disturbing force should not be at, or near, a natural frequency of the structure otherwise resonance will occur, with the resulting high amplitudes of vibration and dynamic stresses, and noise and fatigue problems. There are two basic types of structural vibration: steady-state vibration caused by continually running machines such as engines, air-conditioning plants and generators either within the structure or situated in a neighbouring structure, and transient vibration caused by a short-duration disturbance such as a lorry or train passing over an expansion joint in a road or over a bridge. Vibration from engines can agitate the body to the point of causing micro fractures in the vertebrae, disc protrusion, nerve damage and acute lower back pain.

Ride comfort is extremely difficult to determine because of the variations in individual sensitivity to vibration. Therefore many researchers have concentrated their efforts on reducing the amount of vibration from vehicles. Some interesting researches were conducted for the low frequency discomfort for human analysed [88, 89]. Ride vibrations are transmitted to the driver buttocks and back by the seat. The floor panel, pedal and steering wheel transmit additional vibrations to the feet and hands of the driver. These vibrations are producing a level of discomfort for driver and passengers.

With advancements in transportation, we often travel longer distances at faster speeds. Whole-Body vibration research is now focusing on determining the vibration and exposure levels that initiate physical and mechanical changes in the body. The results of current research could provide helpful guidelines for vehicle manufacturers. To reach this purpose it is extremely important to define all sources of vibration in driving vehicle and paths of propagation into the occupants.

The book address the results of large scope research on identification of sources, propagation paths and structure of vibration affecting on human in means of transport. According to scope of the research others authors and the current state of art the book is focused on consideration on combustion engine as source of vibration affecting on occupants. For fulfilment of the engine working condition during drive the investigation were conducted on vibration generated by working engine and powertrain system with different gear ratio. For the identification of vibration propagation paths there were developed some estimators of vibration energy dissipation. As the second main source of vehicle vibration the impact of vertical movement of wheels on vibration propagation into car-body were investigated. The influence of technical condition of suspension system and operating parameters of the vehicle on vibration caused by forcing a wheel were described in the book. The author discussed the influence of vibration sources on exposure to whole-body vibration.

Basing on the conclusions some mathematical and software application for vehicle vibration comfort and safety were developed. Thus it allowed to describe the systems for monitoring and control of vibration propagation in means of transport in terms of comfort and safety. 


\section{Vibration effects on the transport system}

\subsection{General information on vehicle vibration}

The effects caused by transport should be examined in a breakdown into desirable and undesirable as well as external and internal ones. The desirable effects of transport are related to its function of purpose, namely moving and increasing of mobility. The undesirable ones involve a negative impact on the surrounding. The division into external and internal effects stems from the influence exerted on the components of a system which do not take part in the transport process as well as those directly related to the process. The internal effects are of a feedback nature and they influence the efficiency of transport processes. An example of undesirable transport effects of both external and internal relevance is vibration. Representing phenomena generated and propagated into the environment, vibrations may cause damage to the transport infrastructure and other surrounding elements. Vibrations perceived as internal phenomena reduce the efficiency of transport processes as well as transport safety and comfort $[67,197]$. Sudden or intensifying vibration phenomena of local nature may compromise safety. A man operating a mean of transport subjected to local vibrations may lose control over the vehicle. Also general vibrations are very significant in this respect, as they influence the feeling of discomfort to a considerable extent. It is more and more common that vibrations, being undesirable residual phenomena, are used in machinery monitoring and diagnostics $[37,61,72,94,126,130,148-151,160,192,193,201]$.

Studying vibration phenomena in automotive vehicles should primarily pertain to their effects on men and the chosen structural elements of a vehicle. In the scope of the impact on structural elements, one should perceive vibrations in terms of destructive factors. Consequences of the human exposure to vibrations include various harmful changes occurring in the organism. They have a direct or indirect influence on comfort and safety [2, 33, 83, 84, 121, 134, 143, 152]. The scope and the procedure of handling them depend on a considerable extent on the location where they penetrate the organism. Mechanical vibrations occurring in a working environment may be classified as general vibrations, i.e. those affecting the human organism via lower extremities, the pelvis and the back. The second category comprises local vibrations affecting the human organism through upper limbs. It allows to describe the vibration affecting on the human as Hand-Arm Vibration (HAV) and Whole-Body Vibration (WBV) (Fig. 2.1). For the HAV the standards of measurement and evaluation have been collected in ISO 5349:2001 - Measurement and evaluation of Human Exposure to Hand Transmitted Vibration. For the WBV the main standards are described in ISO 2631 - Mechanical vibration and shock - Evaluation of human exposure to whole-body vibration [103-105]. Directives and guidelines based on measurement standards define allowable exposure limits for HAV and WBV.

Vehicle vibrations are classified into two general types of the rigid body vibration of sprung and unsprung mass and stiffness vibration. Stiffness vibration is determined by mass, stiffness and damping forces which are contained in suspension component parts. Some interesting investigation on vibration energy according to type and structure of the tire have been presented in [131]. In many papers the main goal of investigation on vehicle tires are noise. Interaction between tire and road is recognised as the main source of interior and exterior noise for velocities over the $40 \mathrm{~km} / \mathrm{h}$. In the paper [166] a three-dimensional elemental approach has been adopted to predict the stochastic tire vibration and hence the interior and exterior noise due to this kind of excitation. 
Drive comfort depends on many parameters, such as the mass of the vehicle, tire pressure $[147,174]$, shock absorber quality, the type of seat and the elasticity characteristics of the tires. The methods of investigation on the role of tires on the whole body vibration (WBV) of the operator have been presented in [69].

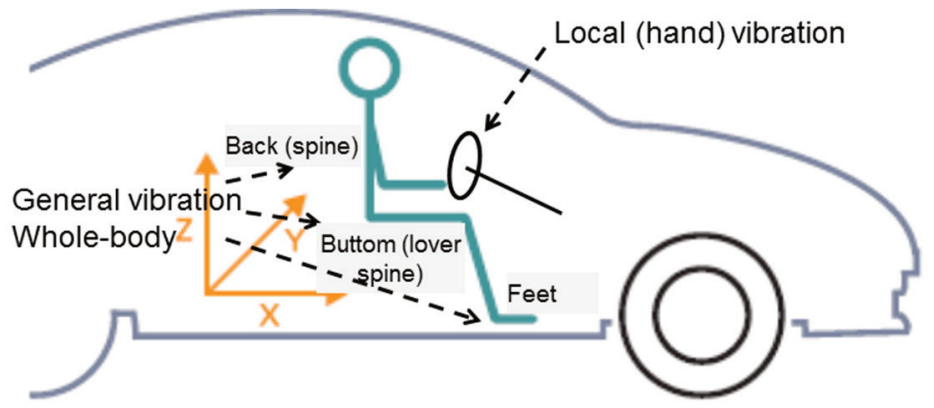

Fig. 2.1. Classification of vibration in term of exposure on human in passenger car

The characteristic of the spring and shock-absorber in the vehicle suspension system as well as other vehicle elastic elements are nonlinear. Therefore, vehicle nonlinear oscillatory models are used in oscillatory behavior analyses [25, 28]. The paper [173] presents results of the investigation on influence of air spring and hydraulic shock absorber of the bus driver's seat on driver comfort. Vibrations are transmitted from the bus floor via the driver seat suspension system to the driver's body. Effects of vibrations on the bus driver oscillatory comfort depend on the spring stiffness and the shock-absorber damping. The results presented show changes in the driver's vertical acceleration for various values of the spring stiffness and various values of the shock-absorber damping of the driver's seat.

The procedure as regulated by the international ISO 2631-1 standard [102] prescribes the vibration total value of weighted root mean square accelerations as a reference quantity for the assessment of vibration effects on the comfort. Many more estimators for the assessment of vibration effects on the comfort have been presented in [88, 89, 198], especially for the child seat.

The state of the art review indicate that main stream of investigation on vehicle vibration comfort is focused on research on vibration of the seat. Thus author decided to conduct the research on vibration of the floor panel in location when vibration are penetrate into the human organism via feet.

Vibrations are also a very ample source of information on the technical condition, and hence they are commonly used in diagnostic systems [49, 50, 52, 55, 60, 85, 108, 190-192]. Bearing in mind such extensive fields of influence and application of vibrations, it is highly recommendable that the impact exerted by selected parameters on the form of vibrations recorded as well as their distribution in the vehicle structure should be studied. One should also strive to verify the influence of material parameters and invasive repair methods on propagation of vibrations in structural elements $[27,36,119,196]$ with the many analysis on influence of some parameters on physical and chemical properties. Car manufacturers use more often lighter materials in the design of vehicles to reduce energy consumption. This loss in mass combined with the design of increasingly powerful vehicles lead to car frames more vulnerable to the propagation of vibration and the generation of noise [25].

\subsection{Vibration effects on the human}

A very large group of people exposed to general vibrations comprises car drivers, passengers, tram drivers or building and road machinery operators. Occupations that require driving long distances or operating heavy equipment expose workers daily to low-frequency vibrations generally less than $100 \mathrm{~Hz}$. Exposure to these vibrations can cause serious physical problems ranging from chronic back pain to nerve damage. Whole-body vibration is caused by twisted 
sitting postures combined with vibration. The combination increases stress and load on the neck, shoulder and lower back. Vibrations propagate into the human organism through vehicle seats via the pelvis, the back and lateral parts of the body as well as through the vehicle floor panel via feet. As vibration is transmitted to the body, the effect of the vibration can be amplified by factors such as body posture, type of seating and frequency of the vibration. Individual human body parts have their own resonant frequencies. The vibrations most harmful to people are those whose input frequency is close to the natural vibration pulsatance of human organs. For a vibration frequency lower than $2 \mathrm{~Hz}$, the human body behaves as a uniform mass. The first resonant frequency for a person remaining in a sitting position is $4 \mathrm{~Hz}$ or $6 \mathrm{~Hz}$. The human vibration perception depends on human body position, as standing, sitting and lying positions. The natural vibration frequency of an automotive vehicle's sprung masses is assumed to be contained within the range from 1 to $2.5[\mathrm{~Hz}]$. Such dynamics of vibration phenomena does not essentially exert any negative effects on passengers, since it corresponds to man's natural frequency of making steps (Fig. 2.2). Vibrations of the frequency below $1[\mathrm{~Hz}]$ cause effects similar to seasickness in people, whereas those of the frequency exceeding $2.5[\mathrm{~Hz}]$ bring prompt weariness and pain. The first resonant frequency for a man in a sitting position comes to ca. 4-6 [Hz] depending on individual body build features [14]. Input functions with the frequency of 3-4 [Hz] trigger strong vibrations in the abdominal cavity organs. The amplitude maximisation of the effects caused by these vibrations occurs at the frequency of 5-8 [Hz]. Close to these frequencies are those causing resonance in a human chest (i.e. $7-8[\mathrm{~Hz}]$ ). Organs of the head resonate in the band of 20-30 [Hz], whereas eyeballs at $60-90[\mathrm{~Hz}]$. However, it is the nervous as well as the cardiovascular system that are the most sensitive to the whole organism vibrations. The responses of these systems and their respective organs manifest themselves in their functions being disturbed, in poor physical and mental state, and even in certain forms of damage on higher amplitudes of effects and long exposure times. Health effects of excessive vibration for the HAV is the vascular and neuropathic effects and for the WBV are: low back pain, neck-shoulder disorders, digestive and circulatory disorders, cochleo-vestibular disorders and possible reproductive effects or vehicular safety hazards. The state of the art in scope of dynamics of the human vibration exposure on the sitting person via vehicle seats are published in numerous manuscripts [67, 92, 187, 200].

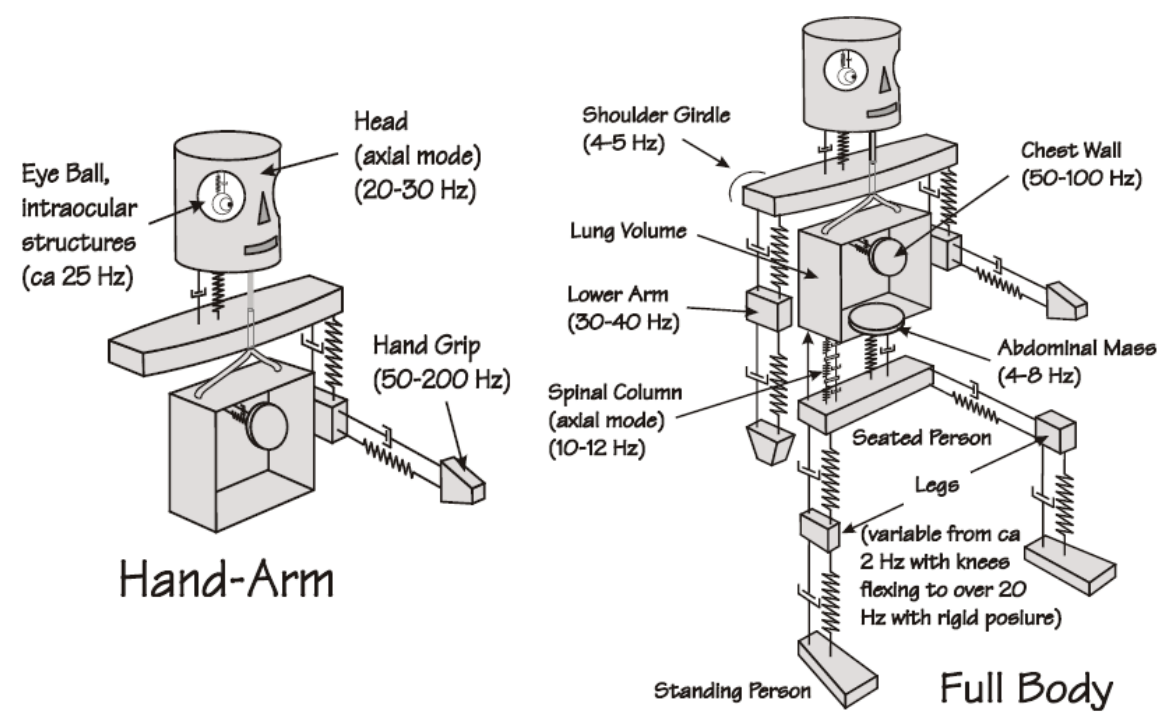

Fig. 2.2. Human body - resonant frequencies for the HAV and WBV [31]

Consequences of the human exposure to vibrations include various harmful changes occurring in the organism $[67,197,121,143,88,89,200]$. The scope and the procedure of handling them depend to a considerable extent on the location where they penetrate the organism. 
The human organism may exhibit various responses to vibrations:

- subjective responses,

- psychosomatic responses,

- responses being functional disorders.

Short-term exposure vibration causes only small physiological effects such as a slight degree of hyperventilation and increased heart rate. Vibration also causes increased muscle tension from voluntary and involuntary muscle contraction. Muscles become tense in order to dampen the vibration. The adverse health effects may also include reduced motion control, blurred vision, or impairment of the ability of free communication, memory processes, or perception. The issue of the influence of vibrations on adult people's bodies has been relatively well described, which is reflected in numerous normative acts. Low-frequency vibrations of moderate intensity can induce sleep. Higher frequencies have the opposite effect. Vision can also become blurred because of the movement of the image on the retina.

Intervertebral discs serve as shock absorbers and become susceptible to injury over prolonged periods. The research result [200] asserts that constant exposure to vibrations represents the ultimate cumulative trauma. The authors [197] also found that prolonged exposure to whole-body vibration can lead to bulging or herniated discs. The vibration sensitivity of organs has been depicted in Fig. 2.3.

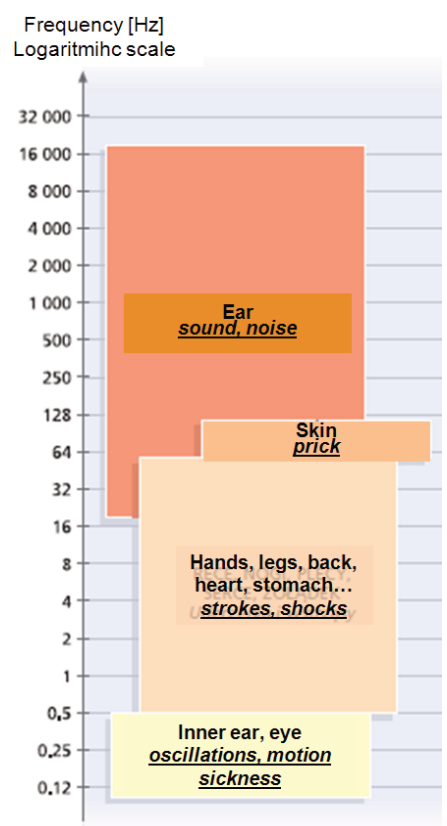

Fig. 2.3. Vibration sensitivity of chosen human organs

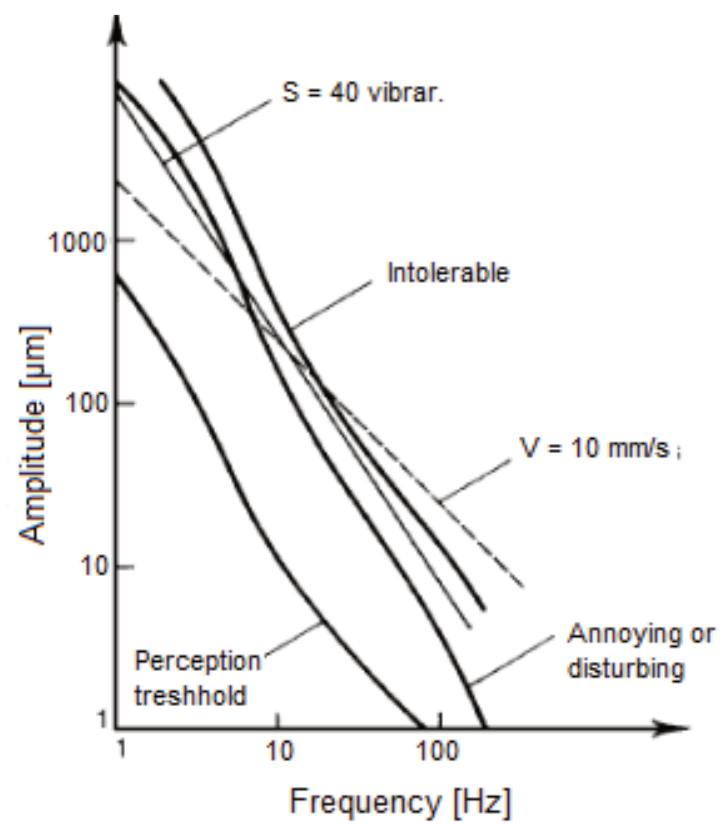

Fig. 2.4. Human response to vibration [23]

Human perception of vibration is very good. A human organism may then be perceived as a complex spring-mass system of a large number of degrees of freedom and diversified properties characterising elasticity, damping, masses, frequency of natural vibrations of individual components and human organs. The division of human perception of vibrations determines the subjective and psychosomatic responses as well as disturbances in the system functioning. As regards the location where vibrations penetrate the human organism, one may speak of general and local vibrations. General vibrations are transferred into the human organism via feet in a standing position or via the pelvis, the back and feet in a sitting or lying position. Local vibrations affect the human organism through upper limbs. Perception of the impact of wave related phenomena, such as noise and vibrations, on a human being is a complex matter, also in terms of 
the very nature of the said phenomena. The vibration energy affecting the human organism originates in the direct penetration area exclusively, therefore, what matters for the vibration perception is the energy affecting a unit of surface area in a unit of time. It is a real challenge in structural design to ensure that the perception threshold level is not exceeded. An indication of the likely human response to vertical vibration is shown in Fig. 2.4.

It shows harmonic vibration amplitude as a function of frequency. As it is presented the lines for constant velocity have smaller slopes than lines for constant vibration intensity. Therefore standards based on constant velocity give increased weight to lower frequency vibrations which are more likely to induce structural resonance and damage than frequencies above $50 \mathrm{~Hz}$.

\subsection{Perception of vibrations in automotive vehicles}

The scientific problems of vehicle's vibration in many aspects, especially in term of biodynamic response of the human body to the whole-body vibration, are main goal of many years investigation of Professor Michael Griffin from Southampton. Number of biodynamical models, vibration transmissibility concepts, and human biodynamic responses are considered in [91]. His research show the goal and way of investigation for many researchers $[29,67,111,186,127,128]$.

In order to examine vibration related phenomena occurring in a moving vehicle or a stationary one with its engine on, one should start with identification of vibration sources. Vibration sources in a vehicle are dynamic forces. The natural vibrations can be consider as well as forced, self-induced, parametrical, non-parametrical, random and stationary ones, all generated by the driving unit, the power transmission system and the road.

The vibrations experienced during vehicle ride may cause a variety of pathological symptoms arising in people such as alimentary system disorders, pains in the lumbosacral region and in the cervical spine section, or the occurrence of kyphosis and lordosis, joint and muscular pains, labyrinth disorders (travel sickness), or headaches [197].

The papers [88-92] present results of research on effects of vibration exposure on human organism. For this purpose are commonly used frequency analysis and spectrum's estimators. The transmission of vibration to the occupant of a car seat has been studied using the multiple vibration inputs to the seat $[92,158]$. The vibration experienced when riding in a vehicle may be greatly affected by the manner in which the vibration is attenuated or amplified by the suspension system and the seat $[67,120,202]$. The results discussed in [67] indicated that fore-aft vibration on the seat pan and the backrest were induced not only by fore-aft vibration at the car floor but also by vertical floor vibration, partly due to the inclination of the seat pan and backrest. Due to rotational motions and non-rigidity of the car floor, the vibration may not be identical at the four corners by which a car seat is normally secured to the car floor.

Due to the properties of vibration related phenomena, they may be analysed from the perspective of diagnostics, degradation and impact on men. A human organism may then be perceived as a complex spring-mass system of a large number of degrees of freedom and diversified properties characterising elasticity, damping, masses, frequency of natural vibrations of individual components and human organs. The division of human perception of vibrations determines the subjective and psychosomatic responses as well as disturbances in the system functioning. As regards the location where vibrations penetrate the human organism, one may speak of general and local vibrations. General vibrations are transferred into the human organism via feet in a standing position or via the pelvis, the back and feet in a sitting or lying position. Local vibrations affect the human organism through upper limbs. Perception of the impact of wave related phenomena, such as noise and vibrations, on a human being is a complex matter, also in terms of the very nature of the said phenomena.

The vibration energy affecting the human organism originates in the direct penetration area exclusively, therefore, what matters for the vibration perception is the energy affecting a unit of surface area in a unit of time. A quantity determined as wave power designated as $P$ divided by surface $S$ which the energy penetrates is referred to as wave intensity. It is defined according to 
the following dependence:

$I=\frac{P}{S}\left[\mathrm{~W} / \mathrm{m}^{2}\right]$.

In order to assess this perception, one applies multiple argument functions. An example in this respect may be a corrected measure of quantity of the energy transferred defined as follows:

$Q=I S t$

which entails vibration intensity $I$, vibration time $t$ and surface $S$ of the direct contact between a man and the vibrating object.

To recapitulate the above consideration, the perception of vibrations occurring in automotive vehicles depends on physical characteristics and the current state of the man, the location and the area of the vibration penetration into the organism, the time-frequency structure of the penetrating vibrations which enables assessment of the exposure time for vibrations of specific frequencies.

The perception of vibrations occurring in automotive vehicles depends on physical characteristics and the current state of the man, the location and the area of the vibration penetration into the organism, the time-frequency structure of the penetrating vibrations which enables assessment of the exposure time for vibrations of specific frequencies.

Vertical vibration limits from ride and vibration data manual (SAE) and "Janeway's comfort criterion" - based on sinusoidal vibration (single frequency) have been depicted in Fig. 2.5.

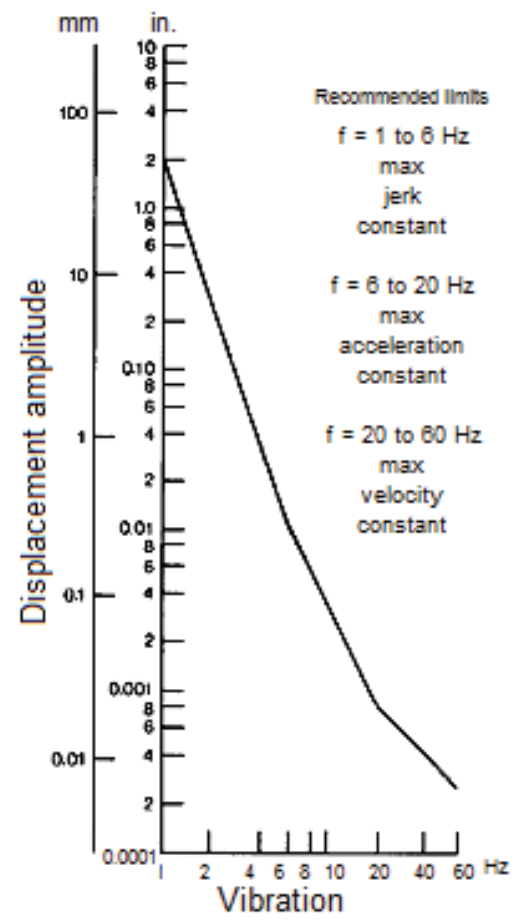

Fig. 2.5. Vertical vibration limits from ride and vibration [202]

The dynamics model of vehicle should permit for analysis of response function of the vehicle or human (occupant) on chosen excitation. The state of the art shows many publications on different approach to vehicle dynamics modeling. The paper [127] presents the three degrees of freedom (3-DOF) Human-Vehicle-Road (HVR) model, comprising a quarter-car and a biomechanical representation of the driver. The model used the Kelvin element as viscoelastic 
representation for modeling vehicle suspension systems and human muscular-skeletal structures. Differential equations are provided to describe the motions of various masses under the influence of a harmonic road excitation. The paper [127] formulates the optimization problem in terms of the requirements stipulated by ISO 2631 standards and utilizes a quarter-car model coupled with the biodynamical model of the driver. The model has been depicted in Fig. 2.6 in which $M_{3}$ denotes the driver's mass, $M_{2}$ stands for the mass of the vehicle body, and $M_{1}$ signifies the unsprung masses of the suspension. The model has been excited by a ground vertical motion, $u(t)=A e^{j \omega t}$, with an amplitude $A$ and a frequency $\omega$. The $z_{i}$ represents time-depending deflection. The $C_{i}$ are the viscous damping coefficients and $K_{i}$ are spring rates.

The differential equations of the motion for the $3-\mathrm{DOF}$ are given by:

$$
\begin{aligned}
& M_{1} \ddot{z}_{1}+C_{1} \dot{z}_{1}+K_{1} z_{1}+C_{2} \dot{z}_{1}+K_{2} z_{1}-C_{2} \dot{z}_{2}+K_{2} z_{2}=C_{1} \dot{u}(t)+K_{1} u(t), \\
& M_{2} \ddot{z}_{2}+C_{2} \dot{z}_{2}+K_{2} z_{2}-C_{2} \dot{z}_{1}-K_{2} z_{1}-C_{3} \dot{z}_{3}+C_{3} \dot{z}_{2}-K_{3} z_{3}+K_{3} z_{2}=0, \\
& M_{3} \ddot{z}_{3}+C_{3} \dot{z}_{3}+K_{3} z_{3}-C_{3} \dot{z}_{2}-K_{3} z_{2} .
\end{aligned}
$$

The equations can be expressed in a matrix form (detailed solution have been presented in [127]) it allows to obtain the expressions for the motions and accelerations of the masses $M_{1}, M_{2}$ and $M_{3}$ as equations as follows:

$$
\begin{aligned}
& a_{1}(t) \\
& =-\omega^{2} \frac{\left(K_{1}+j C_{1} \omega\right)\left(\omega^{2} K_{3} M_{2}-j \omega C_{2} K_{3}+\omega^{2} K_{3} M_{3}+\omega^{2} K_{2} M_{3}-j \omega K_{2} C_{3}-K_{2} K_{3}\right)}{\delta} A e^{j \omega t} \\
& -\omega^{2} \frac{\left(K_{1}+j C_{1} \omega\right)\left(-\omega^{4} M_{2} M_{3}+j \omega^{3} C_{3} M_{2}+j \omega^{3} C_{2} M_{3}+\omega^{2} C_{2} C_{3}+j \omega^{3} C_{3} M_{3}\right)}{\delta} A e^{j \omega t}, \\
& a_{2}(t)=-\omega^{2} \frac{\left(K_{1}+j C_{1} \omega\right)\left(K_{2}+j \omega C_{2}\right)\left(\omega^{2} M_{3}-j \omega C_{3}-K_{2}\right)}{\delta} A e^{j \omega t}, \\
& a_{3}(t)=\omega^{2} \frac{\left(K_{1}+j C_{1} \omega\right)\left(K_{2}+j \omega C_{2}\right)\left(K_{3}+j \omega C_{3}\right)}{\delta} A e^{j \omega t} .
\end{aligned}
$$

A generalized nonlinear two-degrees-of-freedom (2-DOF) model has been formulated in [29] for the dynamic analysis of suspension seats with passive, semi-active and active dampers. These model has been depicted in Fig. 2.7. The model incorporates Coulomb friction $F_{f}$ due to suspension linkages and bushings, forces arising from interactions with the elastic limit stops, a linear suspension spring and nonlinear damping force for passive, semi-active and active dampers, while the contribution due to biodynamics of the human operator is considered to be negligible. The model masses $m_{c}$ and $m_{s s}$ represent the masses due to occupant upon neglecting its biodynamic interactions and the seat, respectively. The cushion is characterized by linear stiffness $K_{c}$ and viscous damping coefficient $C_{c}$. The suspension is represented by its linear stiffness $K_{s S}$, a clearance spring $K_{s t}$, dry friction force (Columb) $F_{f}$ and a viscous damping coefficient $C_{s s}$ in the case of a passive suspension seat. The $z_{c}$ and $z_{s s}$ represent the vertical movement of the occupant mass $m_{c}$ and the suspension seat mass, respectively. The suspension force $F_{d}$ may be either $F_{a}$ for the active. The forces due to the passive components of the suspension are derived from the algorithm where $z_{s p}$ represents the displacement excitation at the base of the seat.

The equations of the motion for the 2-DOF suspension seat are given by:

$$
\begin{aligned}
& m_{c} \ddot{z}_{c}=-F_{c}, m_{s s} \ddot{z}_{s s}=F_{c}-F_{s p}, \\
& F_{c}=K_{c}\left(z_{c}-z_{s s}\right)+C_{c}\left(\dot{z}_{c}-\dot{z}_{s s}\right), \\
& F_{s p}=F_{s S}+F_{f}+F_{s t}+F_{d}, \\
& F_{s s}=K_{s s}\left(z_{s s}-z_{s p}\right)+C_{s s}\left(\dot{z}_{s s}-\dot{z}_{s p}\right), \\
& F_{f}=F_{f} \frac{\left\|\left(\dot{z}_{s s}-\dot{z}_{s p}\right)\right\|}{\left(\dot{z}_{s s}-\dot{z}_{s p}\right)}, \quad F_{a}=g \dot{z}_{s s},
\end{aligned}
$$


$F_{s t}=K_{s t}\left(\left(z_{s s}-z_{s p}\right)-\frac{d}{2}\right)$.

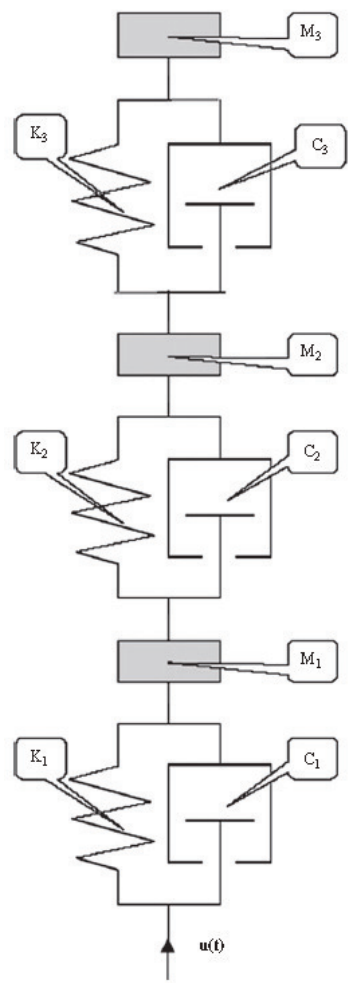

Fig. 2.6. 3-DOF HVR model of the motion [127]

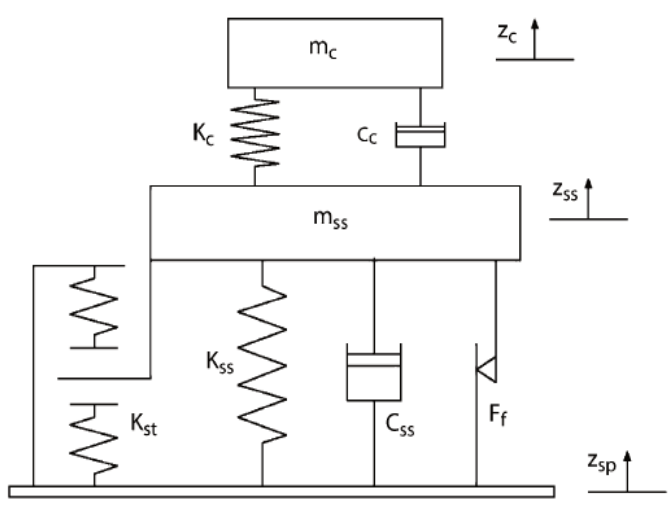

Fig. 2.7. Analytical 2-DOF model of the seat suspension of the motion [29]

New approach to system modeling based on possibilities of Finite Element or Neural Network methods allows to develop models dedicated to realize specific functions [154, 211].

\subsection{Environmental impact of transport}

Some documents indicate the cost to society caused by the vibration. Heavily regulated in EU ca. $\sim 25 \%$ of workforce is exposed to the vibration.

Traffic induced vibrations are a common source of environmental nuisance as they may cause malfunctioning of sensitive equipment, discomfort to people and damage to buildings and historical monuments $[2,66,129,195]$. During the last few years, the interaction problem between moving vehicles and buildings has drawn much attention. Noise and vibrations from road and rail traffic through residential areas are ones of major concern, because nearby buildings and residential areas need to be protected from them. Like most vibration problems, road and rail traffic vibrations can be characterized by a source-path-receiver scenario. Vehicle contact with irregularities of the road and rail surface induce dynamic loads that generate stress waves, which propagate in the soil, eventually reaching the foundations of adjacent buildings and causing them to vibrate. Road traffic vibrations are caused by heavy vehicles such as buses and trucks. Passenger cars and light trucks rarely induce vibrations that are perceptive in buildings. Road traffic tends to produce vibrations in the range from 5 to $25 \mathrm{~Hz}$. Rail traffic vibrations are produced in a similar way by vehicle contact with irregularities in the rail surface. The produced vibrations in this case are in the range from 60 to $100 \mathrm{~Hz}$, according with [98, 156]. 
The problem of vibration caused by underground traffic is discussed in [99, 207, 208]. Other aspects of the vibration analysis of bridges under moving vehicles and trains can be found in [9]. The environmental problems of vibrations, caused by train/traffic, construction activities and factory operation, and other man-made sources are investigated in [184].

The group of technical objects most considerably exposed to the impact of vibrations includes railway transport lines, vehicle transport roads, airfields and ports. The vibrations generated by means of transport, analysed from the perspective of their environmental impact, are to be classified as elastic foundation vibrations generating paraseismic waves. With regard to the impact surface, one can distinguish between surface waves and body waves. Surface waves propagate on boundaries of two media, e.g. on the ground surface. Body waves propagate in all directions $[26,179,180]$. Depending on the propagation direction, paraseismic waves can be divided into primary and secondary waves. The propagation velocity of longitudinal waves is about two times higher than that of transverse waves. Having reached the medium boundary, e.g. the ground surface, these waves keep propagating as surface waves of either the Rayleigh or the Love type. The energy dissipation of surface waves is far smaller than that of body waves. Moreover, surface waves are characterised by a lower frequency and a higher amplitude. For all these reasons, they constitute a greater hazard to the environment and the infrastructure [122].

What matters particularly from the scientific and engineering perspective of the analysis of environmental impacts of transport is the phenomena emerging at the point of contact between a running wheel and the road pavement. They constitute sources of kinematic input functions transferred to the road pavement, propagating as paraseismic vibrations to the surrounding infrastructure. The most crucial source of vibrations of a running car is the road irregularity, and the highest dynamic input functions occur as a consequence of running on considerable irregularities, such as a hole, a kerb, tracks, manholes etc [3, 4, 122, 123, 145, 179, 180]. All of these issues affects on human exposure to vibration in transport $[65,77,78,144]$.

An integrated approach to the analysis of vibration impacts in transport should entail both the vehicle-man and the vehicle-road-environment systems. 



\section{Analysis of vibration propagation into vehicle's structure}

\subsection{Propagation of vibration in transport system}

The analysis of the way in which the structure responds to the forcing, and in particular the way the vibration propagates from a source into distant parts of the system allow to research on vibration phenomena in means of transport. There is more than one approach to the theory of structural vibration, and it is useful at the outset to recognise a basic division into three general classes. These can be characterised as the 'waves', 'modes' and 'rays/wavepackets' approaches. All three have advantages for particular types of problem [11, 73, 97].

Transport system consists of infrastructure and suprastructure elements. As the sources of vibration the suprastructure elements, as means of transport, have to be considered. Means of transport generate the vibration which are propagating into the humans, buildings and environment via structure, air and combination of both (Fig. 3.1).

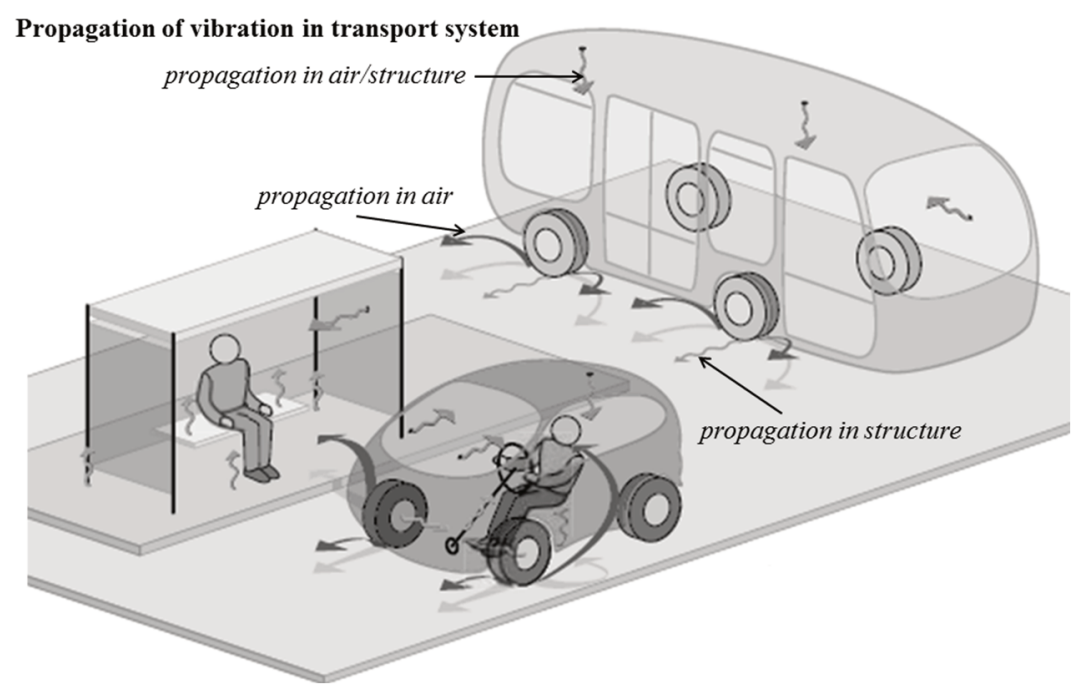

Fig. 3.1. Propagation of vibration in transport system

When the frequency of force usually acting on a vehicle matches or exceeds its natural oscillations, a vibration resonance is caused and the amplitude is increased, resulting frequent failures of the suspension and other elements of vehicle. During the drive it can cause wheels lose grip with the ground in effect vehicle becomes uncontrolled. Thus the vibration transfer from the road to the occupants can be illustrated as transmittance by multi-filter element. The excitation is given by the wheel movement caused by the road roughness. First filtering of vibration occurs as result of stiffness and damping properties of tire. Second and third will be results of stiffness and damping properties of suspension and car-body. The last filter is biodynamics properties of human body. These path of propagation determined the human exposure to vibration and feeling of discomfort (Fig. 3.2). 


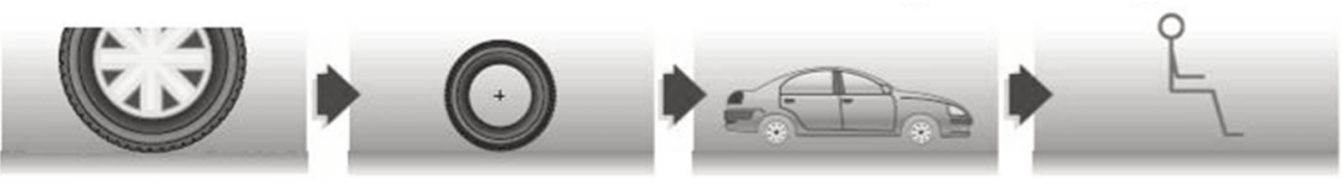

Fig. 3.2. Vibration transfer from road into the human in vehicle

\subsection{Vibration - stochastic wave propagation}

Vibration related phenomena, like all physical phenomena, should always be considered as random processes, even in deterministic models. Hence the identification and quantitative characterisation require that randomness should be taken into account each time. For the sake of proper analysis and description of random phenomena, one recognises the relevant regularities in order to complete a mathematical notation. In mechanics and material sciences, such an approach enables supplementation of imperfect equations and mathematical functions which then make it possible to model real phenomena. Direct outcomes of this methodology include such notions as entropy (defined by L. Boltzmann in 1866), statistical mechanics and Gibbs distributions (1903), a probabilistic model of Brownian motions (Einstein-Smoluchowski, years 1905-1906) and Langevin equations (formulated as early as in 1908) entailing random noise, constituting the origins of the entire contemporary stochastic dynamics. A true breakthrough in the analysis of random phenomena, regardless of the field of application, was Kolmogorov's theory of probability developed in 1933 to describe regularity in the scope of random phenomena. Numerous methods have been formulated for the sake of examination of random phenomena changing in time (theory of stochastic processes) and in space (random field theory and stochastic geometry), and recommendations have been provided with regard to the manner of extracting and analysing information contained in numerical data from observations and empirical tests (mathematical statistics) [177].

The necessity to complement real studies and modelling with stochastic methods in mechanics is particularly important in the analysis of dynamic processes. The foregoing is mainly due to the fact that a decided majority of external input functions affecting machines is characterised by an irregular and random course in time. An example of such input functions is the impact of road surface irregularities on structures of automotive vehicles. Bearing in mind other vibration sources affecting a moving vehicle, such as the engine, the power transmission system or aerodynamic phenomena, it occurs that correct analysis and modelling of vehicle vibrations is a problem of largely stochastic nature. It requires application of the theory of stochastic processes as well as the stochastic wave and vibration theory. In terms of modelling and optimisation of vehicle design, one must take random impacts of input functions into consideration. The mathematical and numerical methods applied in engineering practice should be based on stochastic differential equations describing the way a structure behaves.

An overall mathematical model of technical systems subject to random input functions changing in time is a system of stochastic equations which may be represented in the following vector form:

$\begin{aligned} \frac{d X(t)}{d t} & =F(X, t)+G(X, t) h(t, g), \\ X\left(t_{0}\right) & =X_{0}(g) .\end{aligned}$

In the Eq. 3.1 the $X(t)=\left[X_{1}(t), \ldots, X_{n}(t)\right]$ characterises the state of the system at instant $t$, $F(X, t)$ is a regular motion component, whereas $G(X, t) h(t, g)$ models random fluctuations, and $h(t, g)$ is a suitable stochastic process characterising a random external or internal impact. Symbol $g$ implies that the suitable quantity is random.

A stochastic dynamics problem thus defined consists in formulating equations of dynamics $d X(t) / d t$ relevant to the given phenomenon being studied, assigning appropriate stochastic 
process $h(t, g)$ characterising the random input functions and estimating its characteristics based on empirical data.

Modelling of vehicle structure vibrations requires that all linear and non-linear phenomena should be taken into consideration, both in the analysis of external impact and of internal relationships (e.g. suspension characteristics) [13, 15, 75, 113, 165, 182, 183].

The contemporary stochastic approach extends as far as to the observation of the structure and properties of various material media and structural materials. Assuming a homogeneous material continuum, providing grounds for classical material theories (e.g. theories of elasticity, plasticity etc.), does not reflect the complexity and inhomogeneity of real media and materials. Models and solutions based on classical theories are too simplified as regards the complexity of the deformation process for a number of real media. Depending on the thermomechanical working process, macroscopic mechanical properties may differ. A major scientific issue at the moment is an attempt to characterise and describe in mathematical terms complex and random material microstructures. In order to find the relevant solution, one needs both synthesis and integration in the spheres of random field theory, stochastic geometry and geometrical (spatial) statistics.

Since the loads affecting a vehicle structure essentially change in time, also stresses are of variable nature, which causes progressive changes in the material structure, i.e. degradation. Its effect is a system's decreasing capacity to transfer the loads assumed. As for vibrating systems, one mainly speaks of degradation in terms of rigidity of structural components caused by accumulation of fatigue failure. Therefore, a model should entail the system dynamics in the function of its internal degradation. Such a model will comprise a correlation between stochastic dynamics and the degradation occurring in time and caused by vibrations $[176,178]$.

A confirmation of how significant random dynamics of loads is from the perspective of technical degradation may be sought in the known case of buckling of an ideally rectilinear pole. For static load, the prerequisite of buckling is satisfied after Euler's critical load is exceeded. However, if the load contains components variable in time, then the buckling may occur even though the total load does not exceed Euler's static critical load at any time during the impact.

\subsection{Propagation of vibrations and vibroacoustic diagnostics}

Analysis of vibration related phenomena is a solution commonly applied in Structural Health Monitoring (SHM) systems. One may distinguish between two major approaches to detection and positioning of defects in SHM systems, i.e. global [191] and local [161] methods. The global methods rely on measurements of a structure and are mainly based on vibrations up to $1 \mathrm{kHz}$. The local ones consist in inducing phenomena sensitive to selected defects, primarily within small predefined areas (systems). The input functions used for the sake of these analyses are predominantly of high-frequency nature [190]. Among the methods mentioned, one may also speak of those based on elastic wave propagation. It makes it possible to analyse a broad band of ultrasonic frequencies, i.e. from 0.2 to $30 \mathrm{MHz}$ and higher. Standard ultrasonic techniques are based on application of two kinds of input functions: resonant and pulse ones $[5,19]$. The resonant techniques make use of narrow band input functions, whereas the pulse techniques are characterised by broad band inputs functions $[8,12,14]$. Grounds for this methodology are provided by the phenomenon of ultrasonic pulse propagation in the structure as well as its interactions with a potential defect. The ultrasonic wave frequency and length is determined as follows:

$\lambda=\frac{c}{f^{\prime}}$

where: $\lambda$ is the wave length and $c$ is the wave velocity in the material.

If wave velocity is constant, wave length will decrease as frequency increases, which implies that the capacity to detect a defect of small dimensions increases as the frequency increases. On the other hand, one of the phenomena occurring in materials is that wave amplitude changes along 
with the distance from the point of its generation. It declines in the course of propagation caused by damping. Ultrasonic wave damping is a function of frequency, i.e. if wave frequency increases, its propagation distance declines. On frequencies with wave lengths equalling the structure grain dimensions, there is an additional phenomenon of wave scattering occurring at the grain boundary. Contemporary techniques enable structure testing at frequencies exceeding $50 \mathrm{MHz}$. It makes it possible to detect defects of diameters smaller than $0.1 \mathrm{~mm}$. Unfortunately, in certain cases it is impossible to observe such high frequencies, just to mention an example of materials of high attenuation or those of large grain diameter (e.g. stainless steel) [6].

Two kinds of waves may propagate inside an elastic body, namely the transverse and longitudinal ones. Combining longitudinal and transverse vibrations make the vibrating point perform trajectories described by the Lissajous curves. Elastic waves propagating in solid bodies are channelled through boundaries of the medium in which they propagate. When applying the concept based on propagation of elastic waves, one strives to solve a wave equation for an elastic linear wave having assumed appropriate boundary conditions representing the geometry of the object studied. It is a classical problem of seeking eigenvalues.

While solving the wave equation for displacements in directions $x$ and $z$, one obtains the following [181]:

$\xi=A_{x} F_{x}(z) e^{i(t \omega-k x)}$,

$\xi=A_{z} F_{z x}(z) e^{i(t \omega-k x)}$.

The foregoing equations describe the phenomenon of wave propagation in direction $x$ for wave length of $2 \pi / k$ and frequency of $\omega / 2 \pi$. The displacement is a function of variables $x, z$ and $t$.

The main assumption made when taking advantage of the wave phenomenon to detect defects by means of surface waves is the fact that a wave introduced into a structure will change its parameters (e.g. velocity, amplitude) after it encounters an obstacle (diffraction).

Measures of defect detection in the function of time are the time of flight (TOF) and the energy dissipation factor. If it is not possible to measure a model signal (input function), one must apply reverse algorithms (time reversal) based on spectral transformations and the transfer function $[113,163,164]$.

One of examples of a low-frequency method is a modal filter. It is used to decompose a system's response signal into components connected with individual forms of natural vibrations.

Vibroacoustic diagnostics, based on the analysis of vibration or acoustic signals perceived as residual processes of non-invasive nature, is becoming more and more important in this respect. The scope of its application as well as the applicability of methods in numerous diagnostic systems also results from the capabilities of advanced methods of signal analysis and identification of numerous characteristics of technical condition [44, 57, 60, 62, 81, 86, 108, 118$]$.

\subsection{Analysis and comparison of the vibration dynamics for the chosen points in the vehicle structure}

As the first experimental investigation on vibration propagation into the vehicle's structure the research on dynamics of vibration distribution in different location in vehicle suspension, car-body and floor panel have been conducted. The first stage of the result analysis envisaged that dynamics of the vehicle vibration phenomena occurring in points of the structure where vibrations penetrated the human organism should be assessed. The section provides results of an analysis of general vibrations recorded on the floor panel in locations where passengers rest their feet. For the sake of the signal transformation, the FFT (Fast Fourier Transform) was applied, as it enabled determination of the Fourier spectra of signals. 


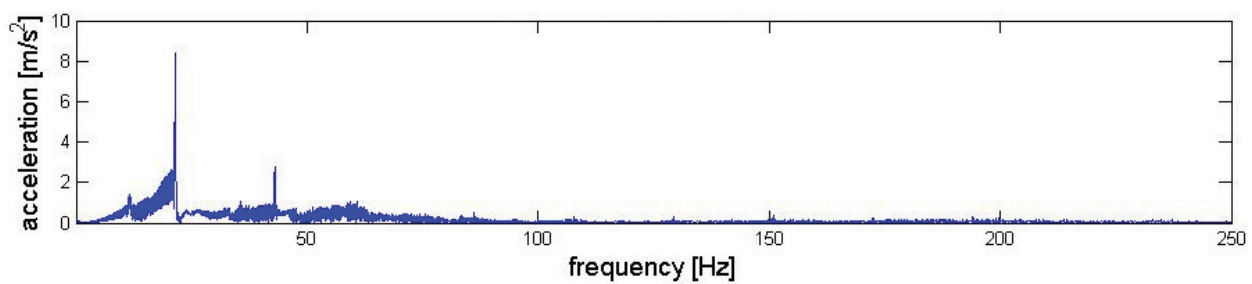

a) FFT - vibration exciter plate

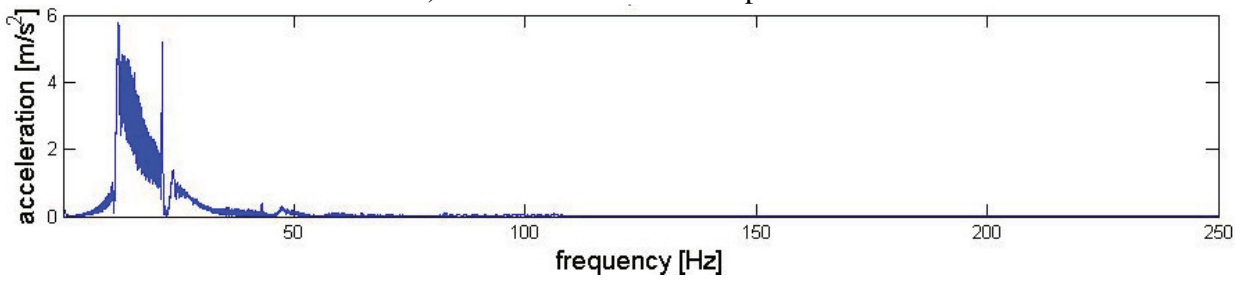

b) FFT - suspension arm

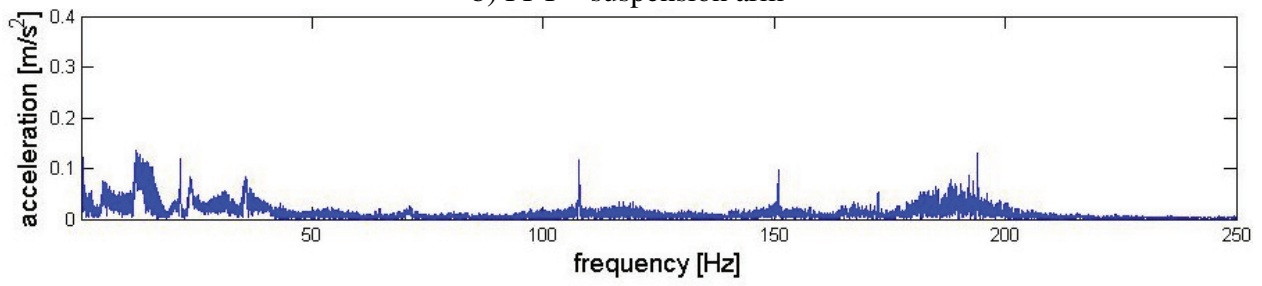

c) FFT - upper mounting of shock absorber

Fig. 3.3. Spectrums of the vibration signals recorded on exciter plate and suspension elements

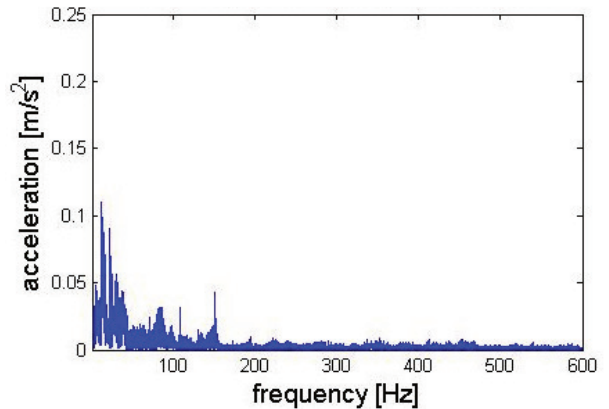

a) FFT - floor pan under the driver feet

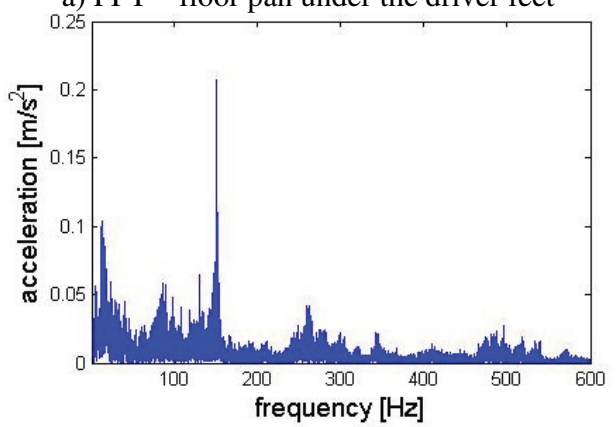

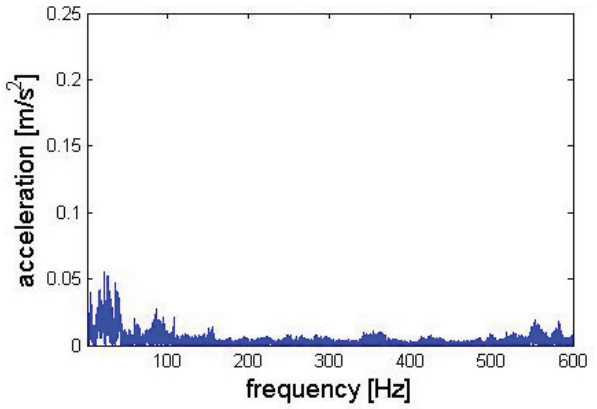

b) FFT - floor pan under the front passenger feet

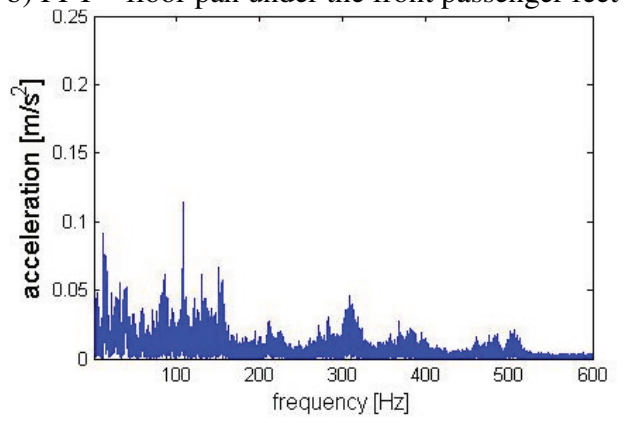

c) FFT - floor pan under the rear left passenger feet d) FFT - floor pan under the rear right passenger feet

Fig. 3.4. Spectrums of the vibration signals recorded on the floor panel

The comparison of the vibration dynamics for the chosen points in the vehicle structure enables evaluation of the dominant frequency components. It can be observed that for those locations in the vehicle structure there are different frequency components carrying most vibration energy 
values. Sample spectra of the signals recorded are presented in Figs. 3.3 and 3.4.

Basing on the vibration spectrum of the exciter plate, suspension arm and upper mounting of shock absorber analysis and comparison the evaluation of the damping properties can be done. The best results of the evaluation can be obtained by the analysis of the resonance of the unsprung masses (ca. 10-12 Hz). The presentation of the analysis is presented in upper chart in Fig. 3.5.

The evaluation of the differences in the vibration dynamics for chosen locations in the vehicle structure at the floor panel were conducted from an analysis of the signals spectrum for next frequency bands (Figs. 3.6-3.9).

This brief of result presentation shows the propagation of the vibration from unsprung masses excited by the wheel movement into sprung masses, i.e. car-body and floor panel. The comparison and propagation properties are illustrated in terms of vibration dynamics changes as spectrums of the signals recorded in chosen location of vehicle.

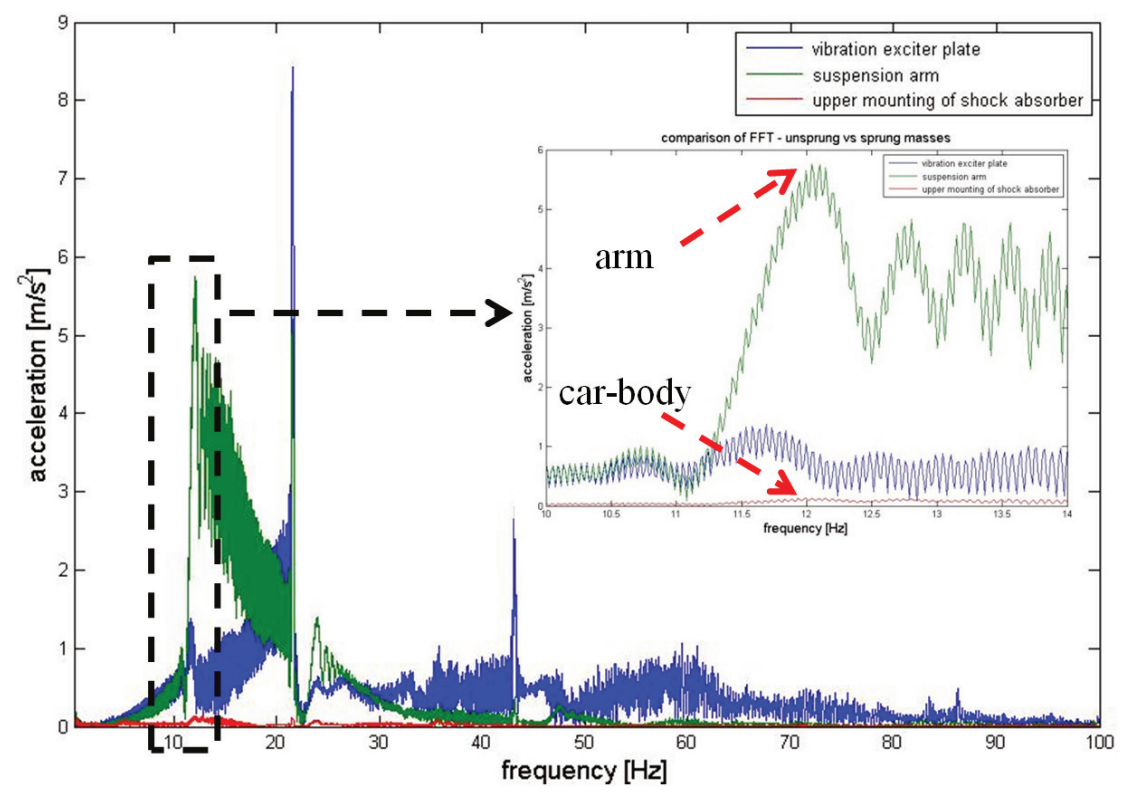

Fig. 3.5. Comparison of spectrums of unsprung and sprung masses (evaluation of the damping properties)

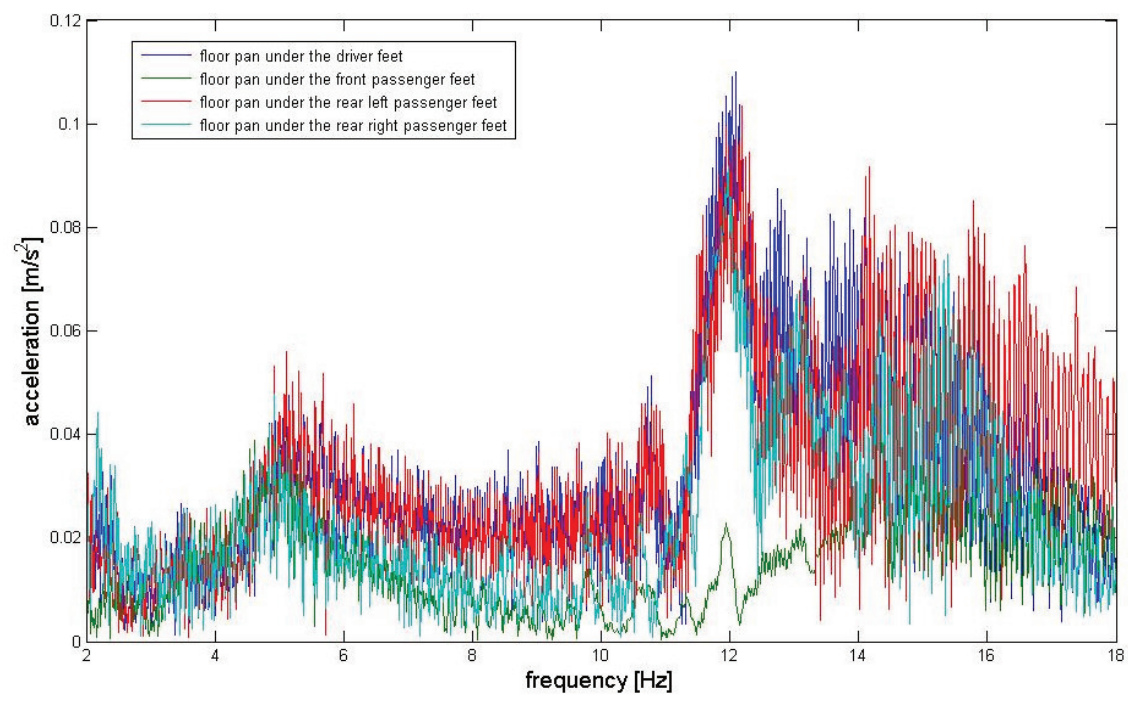

Fig. 3.6. Comparison of floor panel vibration dynamics, frequency band $2-18 \mathrm{~Hz}$ 


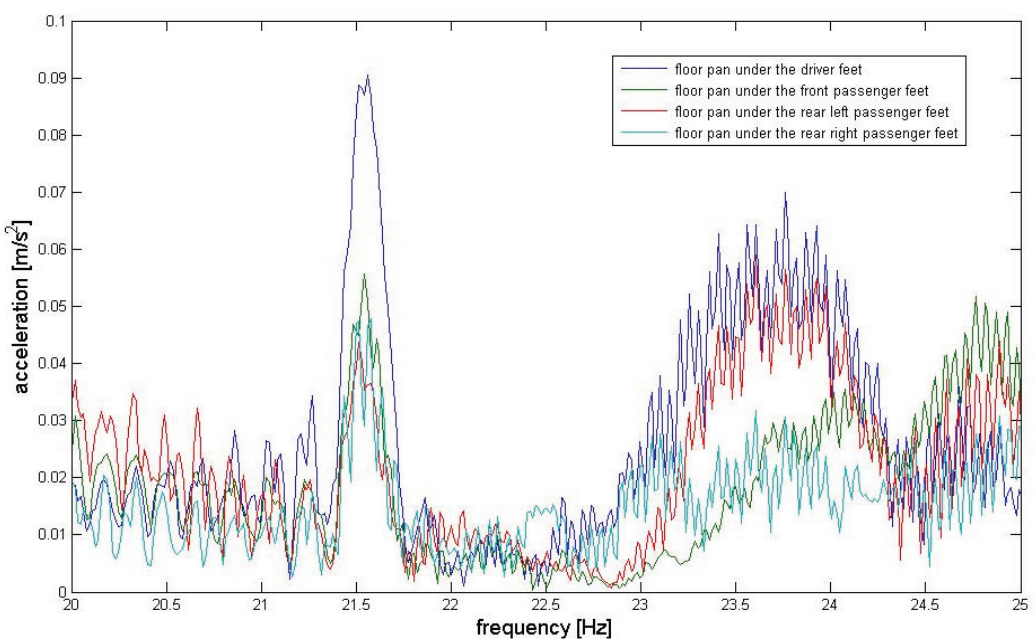

Fig. 3.7. Comparison of floor panel vibration dynamics, frequency band $20-25 \mathrm{~Hz}$

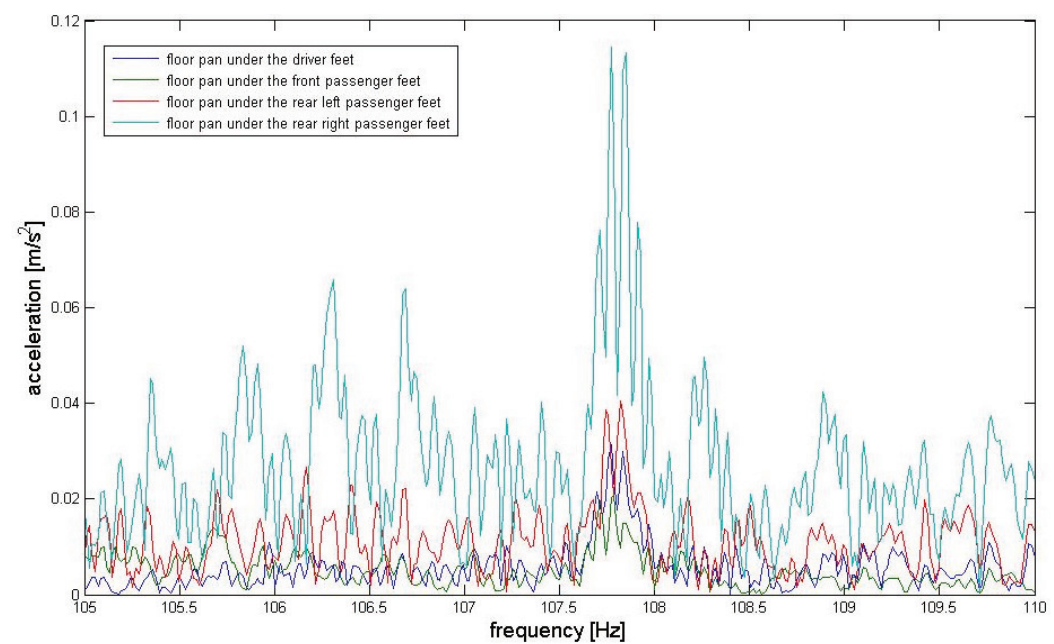

Fig. 3.8. Comparison of floor panel vibration dynamics, frequency band $105-110 \mathrm{~Hz}$

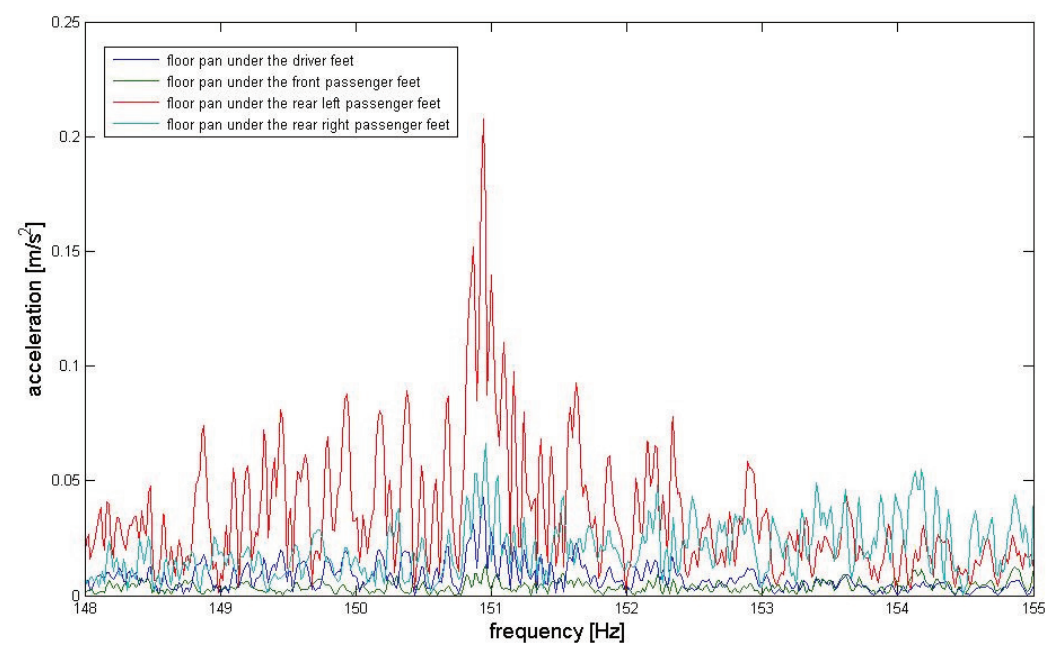

Fig. 3.9. Comparison of floor panel vibration dynamics, frequency band $148-155 \mathrm{~Hz}$ 



\section{Advanced signal processing methods in research on vibration propagation in vehicle structure}

The requirements to the passenger car become very broad. As the result we can observe constant development in automotive industries in numerous engineering directions. Designers and engineers are working on alternative engine, combustion, electrical, hybrid or light constructions and increase of passive and active safety. There are a lot of efforts put in environmental issues. Thus the safety and minimization of environmental impact become regular factors ensured by the homologation requirements. For the customers the most important issues are quality and comfort $[35,48,58]$.

Human vibration perception in transport is strongly correlated with comfort and safety. The chapter presents possibilities of advanced signal processing methods in research on vehicle vibration. Depending on the characteristics of the vibration signal or even its periods different mathematical transformations can be made. It enables elaborating of dedicated signal processing method for research on vibration in many terms.

The methodology was tested on results of research of vibration of passenger car forced to vibration by the special kinematic excitation machine. The signals were registered in chosen location of the vehicle for purpose of analysis path of propagation from source to the location when vibration penetrate to the driver and passengers via feet. The vibration sensors were placed in: exciter plate (source), suspension arm, upper mounting of shock absorber, floor panel in 4 locations for feet of driver and passengers. The location of the sensors are shown in Fig. 6.1.

\subsection{Vehicle vibration signal processing methods}

Analysis of the vibration propagation and influence on human body is difficult. It is important to observe the changes of the values and dynamics of the signal. The result obtained are signals with splot of stationary and non-stationary vibrations (Figs. 4.1-4.4).

The resonance is well noticed as the local amplitude of vibration increase, both in time and frequency domains. The quantity of vibration isolation by suspension system was presents as comparison of FFT spectrums between suspension arm (blue line) and upper mounting of shock absorber (green line) registered signals. As it can be calculated the energy of the vibration in unsprung masses resonance band (ca. 12-15 Hz) is more than 10 times lower in upper mounting than in suspension arm signal. The results are shown in Fig. 4.5.

The analysis of the dynamics of the vibration penetrating into the human body by the floor panel are presented in Fig. 4.6, as comparison of the spectrum of the vibration registered on floor panel. The analysis of the dominant frequency bands are shown in Fig. 4.7. 


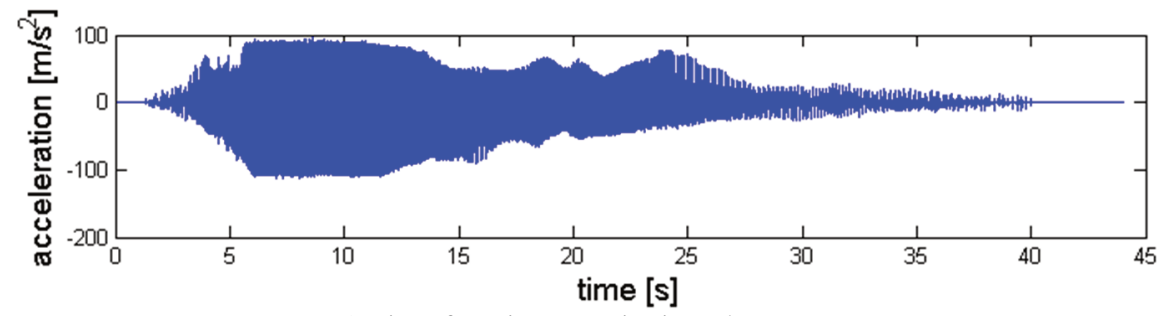

a) Time function - excitation plate

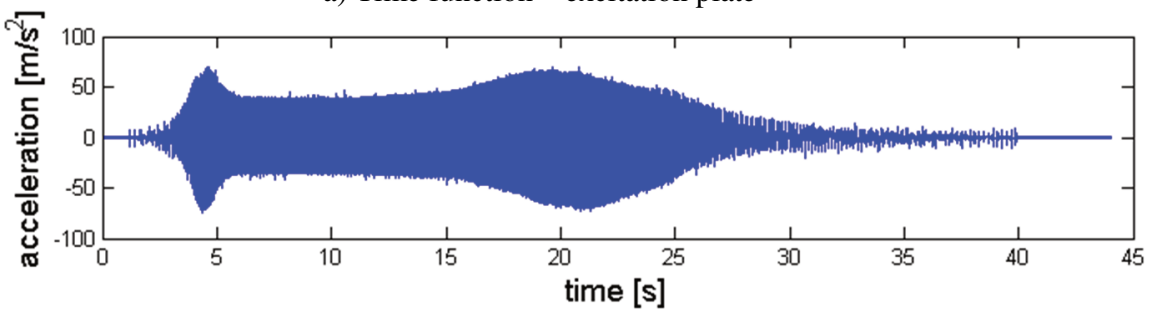

b) Time function - suspension arm

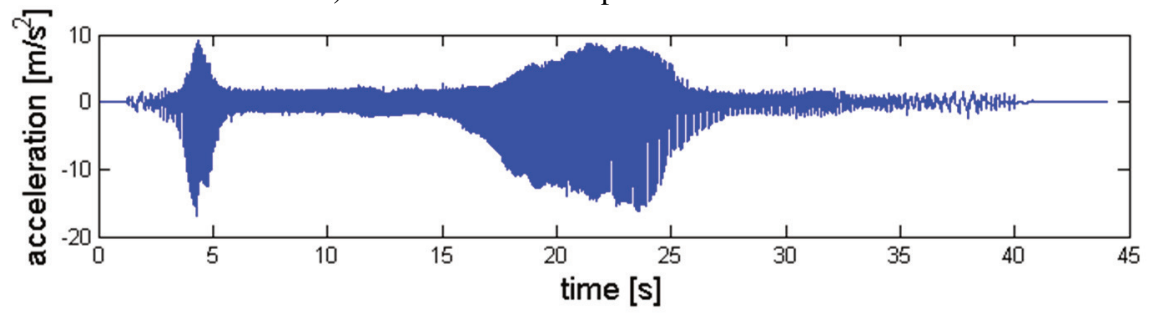

c) Time function - upper mounting of shock absorber

Fig. 4.1. Signals recorded

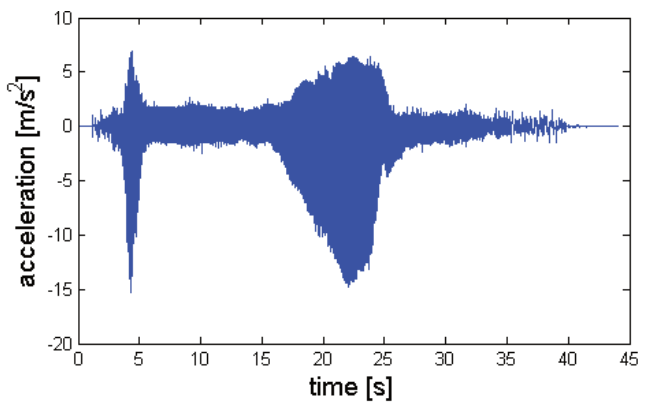

a) Time function - floor pan under the driver feet

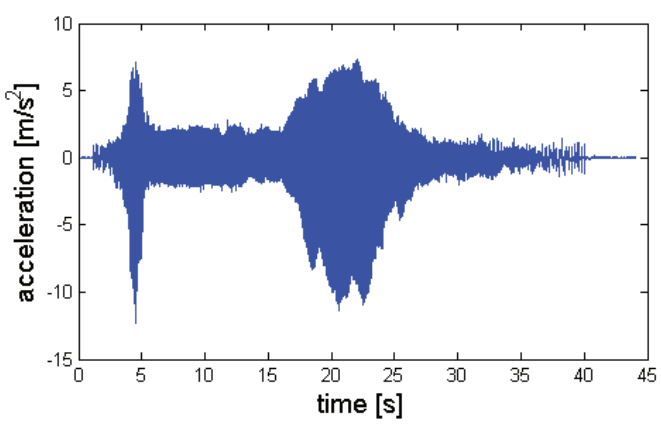

c) Time function - floor pan under the rear left passenger feet

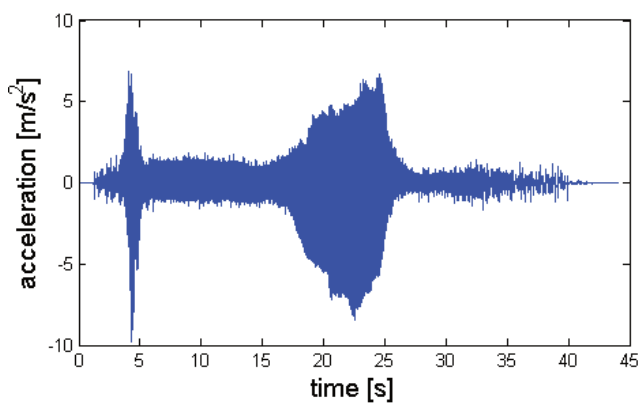

b) Time function - floor pan under the front passenger feet

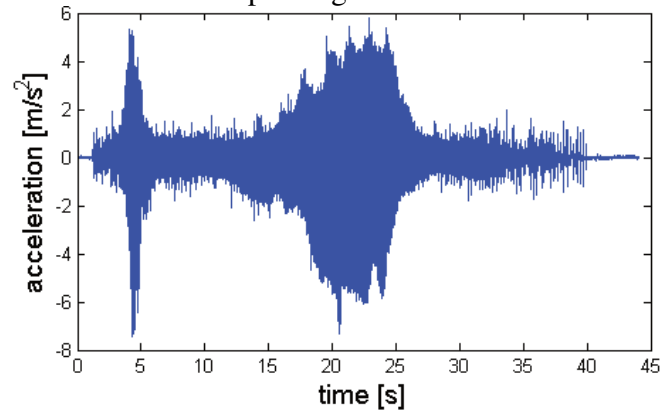

d) Time function - floor pan under the rear right passenger feet

Fig. 4.2. Signals recorded 


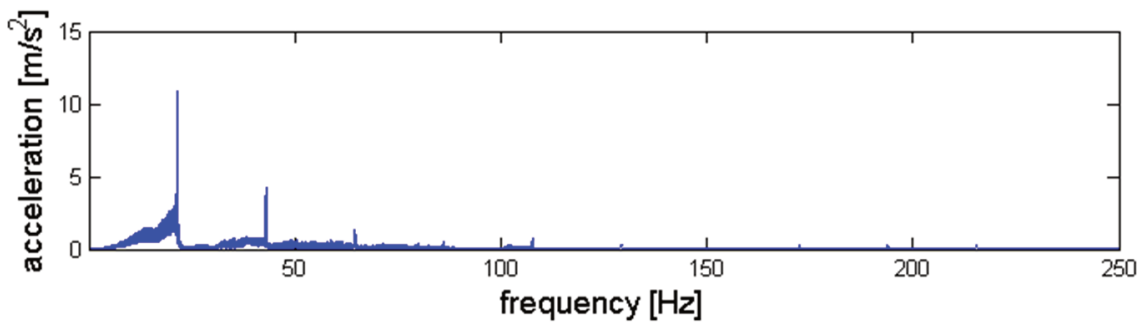

a) Time function - excitation plate

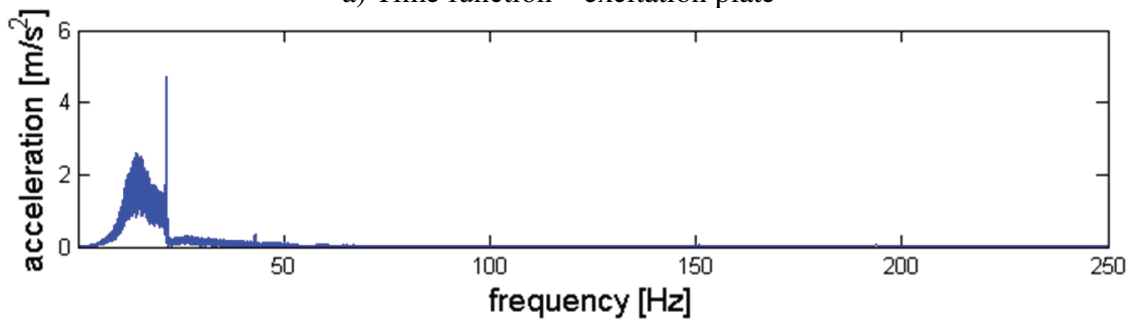

b) Time function - suspension arm

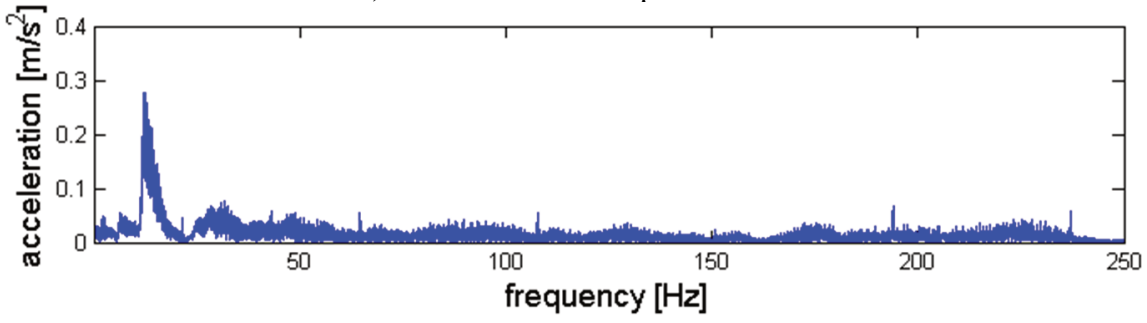

c) Time function - upper mounting of shock absorber

Fig. 4.3. Spectrum of the signals recorded - FFT
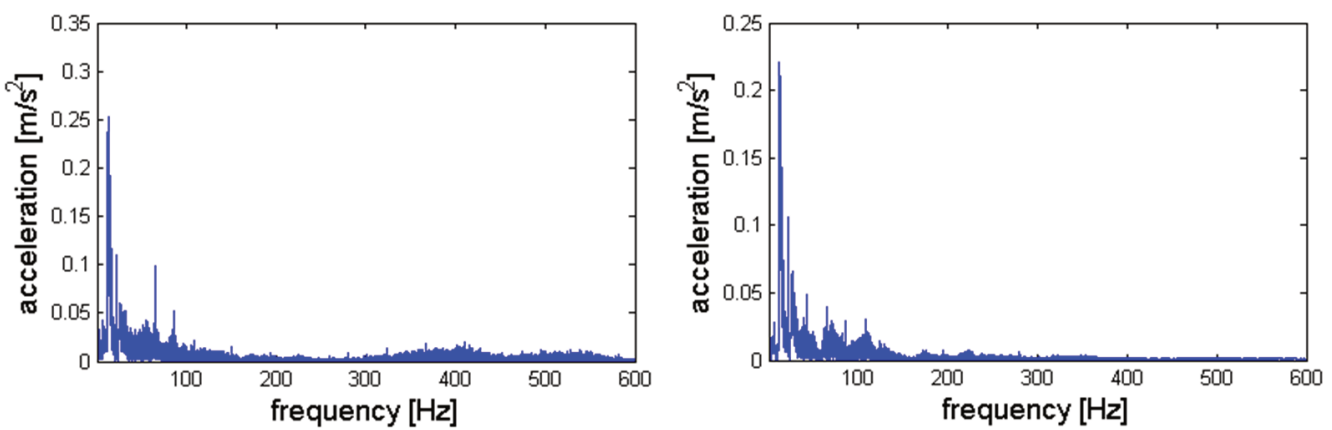

a) FFT - floor pan under the driver feet

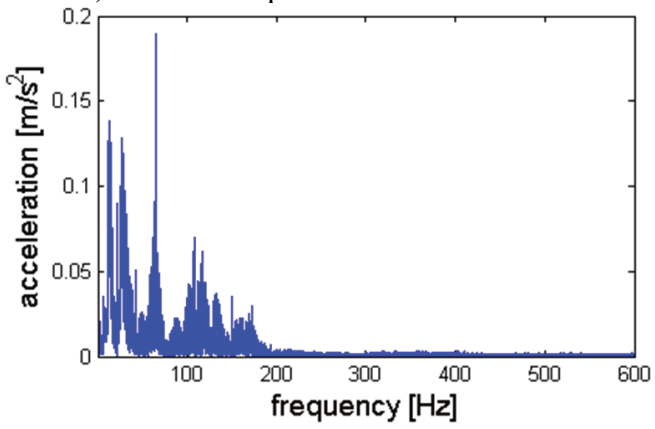

b) FFT - floor pan under the front passenger feet

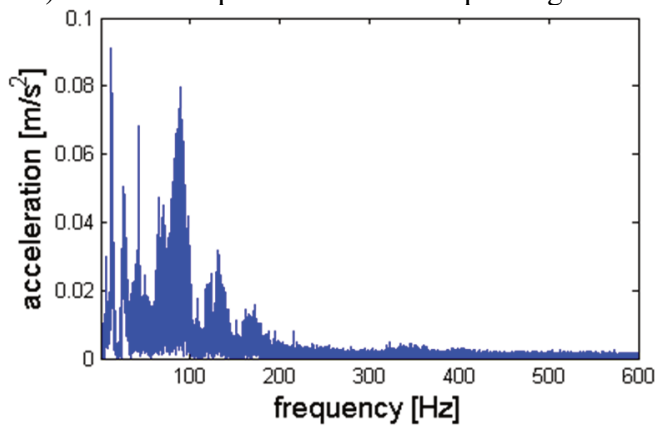

c) FFT - floor pan under the rear left passenger feet d) FFT - floor pan under the rear right passenger feet

Fig. 4.4. Spectrum of the signals recorded - FFT 


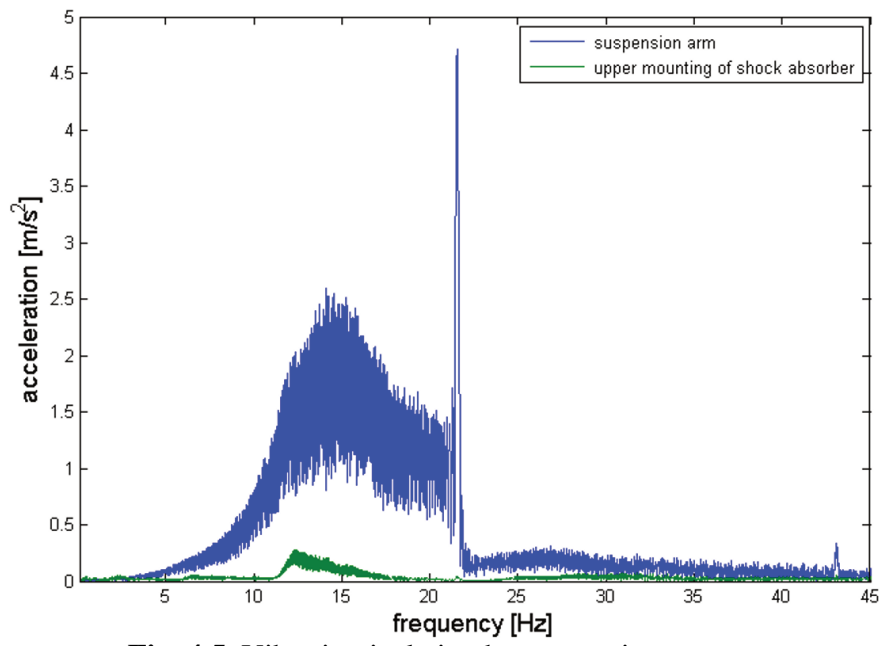

Fig. 4.5. Vibration isolation by suspension system

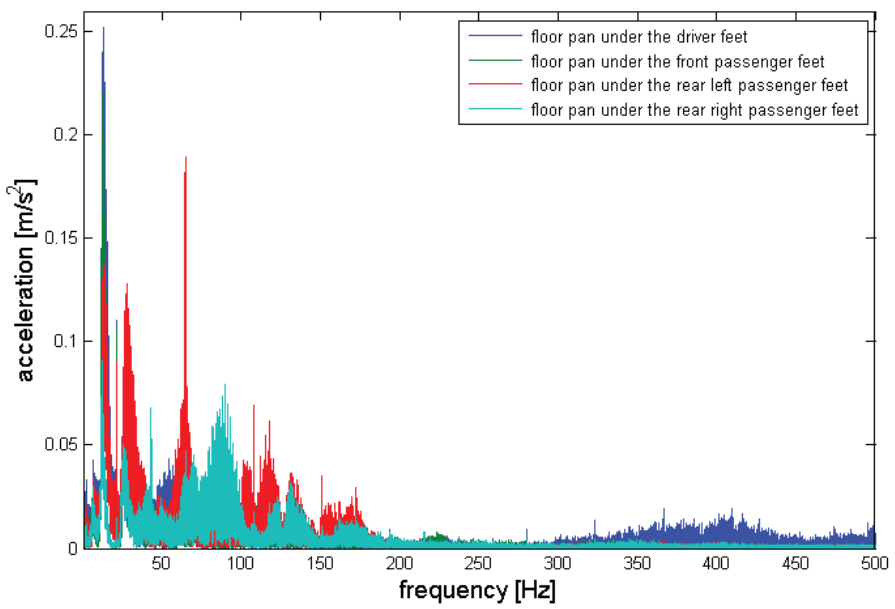

Fig. 4.6. Dynamics of the vibration penetrate into the human body by the floor panel

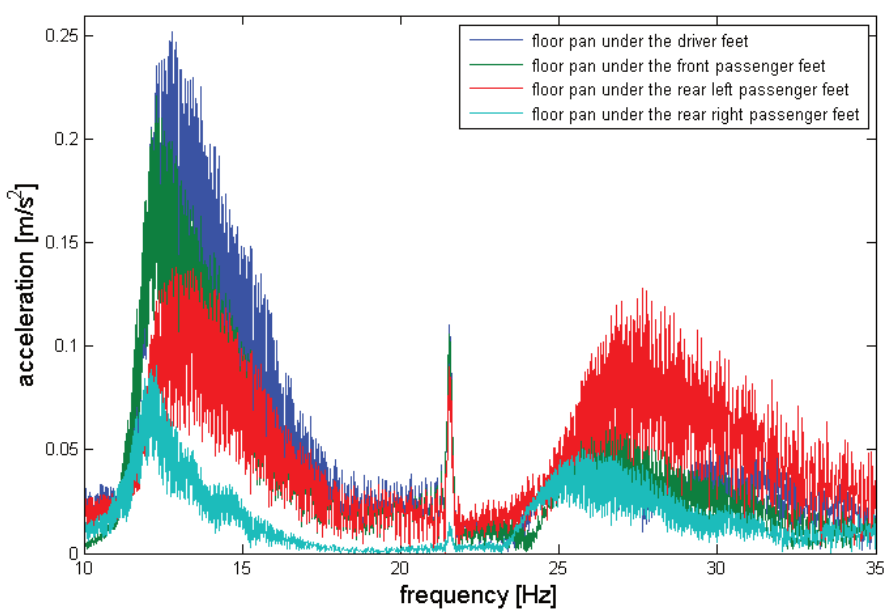

Fig. 4.7. Dominant frequency bands of the floor panel vibrations

Vehicles vibrations are random processes as results of vide range of possibilities forces and 
nonlinear characteristics of suspensions elements of the vehicles construction. The stationary and non-stationary signals require the use of different transformation for signal processing. For the proper analysis the distribution of the signal has to be made. The author developed the algorithm of the signal distribution by the stationary and non-stationary conditions. The mathematical algorithm prepared and programmed enables analysis and separation of the vibration signals in accordance with the stationary and non-stationary states. It is based on core of the frequency comparison to localize the signal with constant frequency as the main condition for stationary signal. Simultaneous the changes of the mean values of the signal in processed window are compared. Those operations allow dividing of the signal for windows with stationary and non-stationary part of the recorded signal. The markers of frequency comparison based on STFT transformation were used. The main reason of choosing this transformation was short realization time. The STFT transformation enables signal observation in time-frequency domains. It is possible to choose the constant frequency band and observe in time realization of the signal. Based on time function it can be identifying algorithm of frequency comparison. Elaborated algorithm is based on comparison of next value of analyzed frequency band ("analysis of edge") around set parameters. The divided signals are presented in Figs. 4.8 and 4.9.

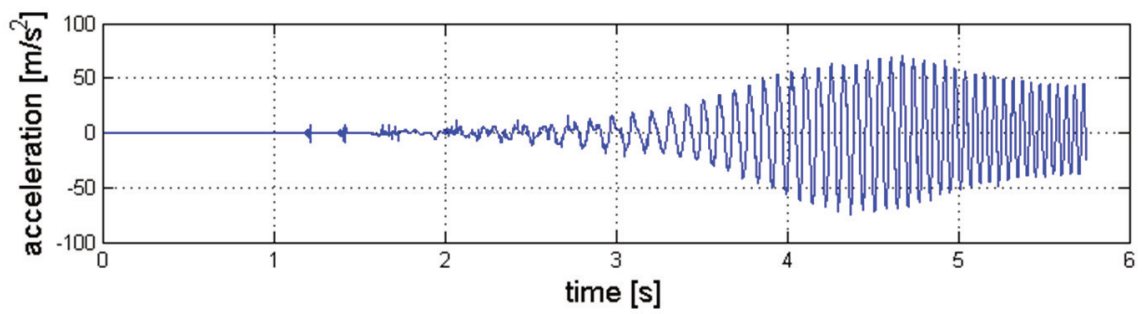

a) Time function of suspension arm - excitation frequency increase

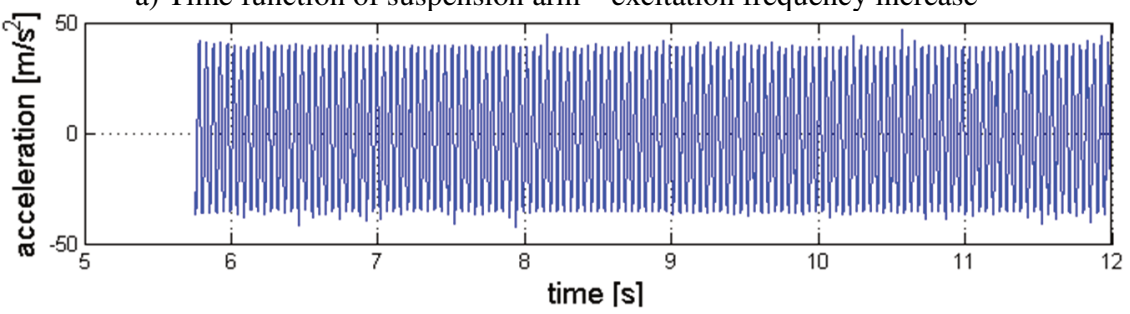

b) Time function of suspension arm - excitation with constatnt frequency

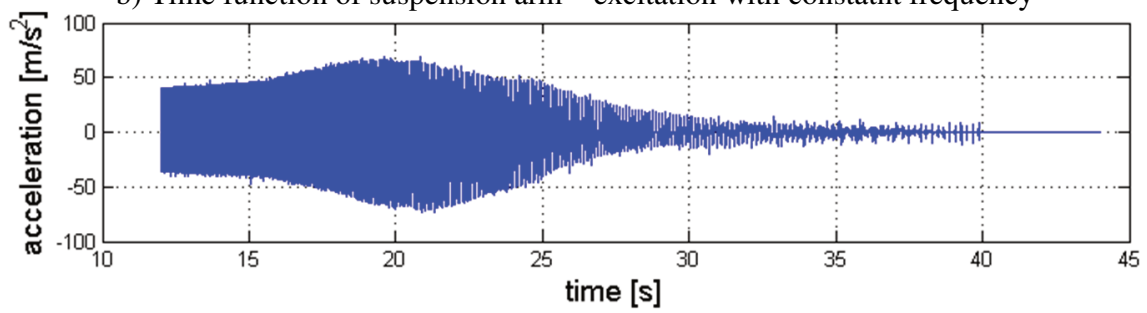

Fig. 4.8. Suspension arm vibration signal distribution by the stationary and non-stationary conditions

This methodology allows analysis of the signal with proper transformation. For the increase and decrease of the frequency the signal is non-stationary. The analysis spectrum is very difficult because frequency components are blurry. For the constant frequency the signal is stationary and frequency component are strongly isolated. Some of the results are shown in Figs. 4.10 and 4.11. The comparison of the spectrums of the stationary signals of vibration in suspension arm (unsprung masses), upper mounting of shock absorber, floor panel (sprung masses) are presented in Fig. 4.12. It can be observed that stationary signal of unsprung masses is typical harmonic signals without any polyharmonics components. The signals of sprung masses have got many polyharmonics components. 


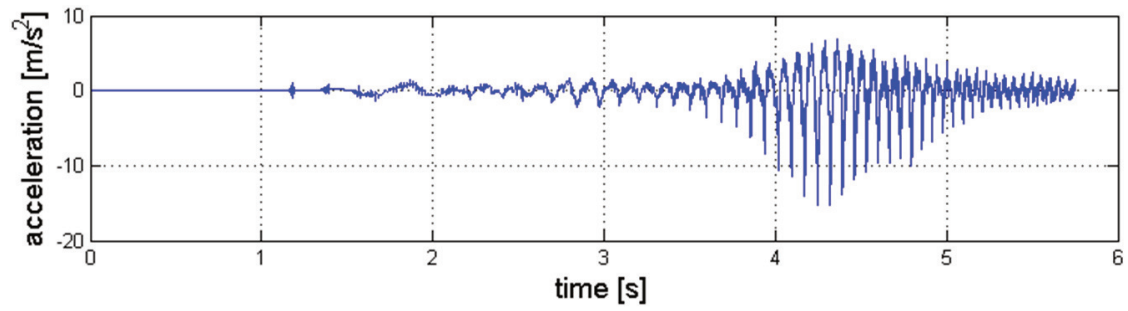

a) Time function of floor pan under the driver feet - excitation frequency increase

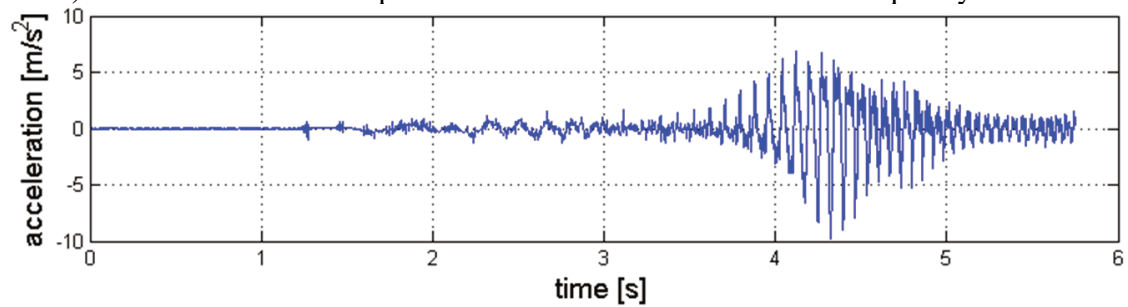

b) Time function of floor pan under the front passenger feet - excitation frequency increase

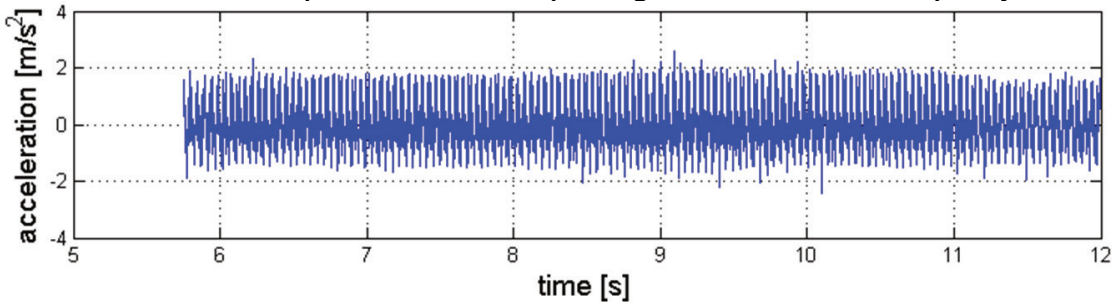

c) Time function of floor pan under the driver feet - excitation with constatnt frequency

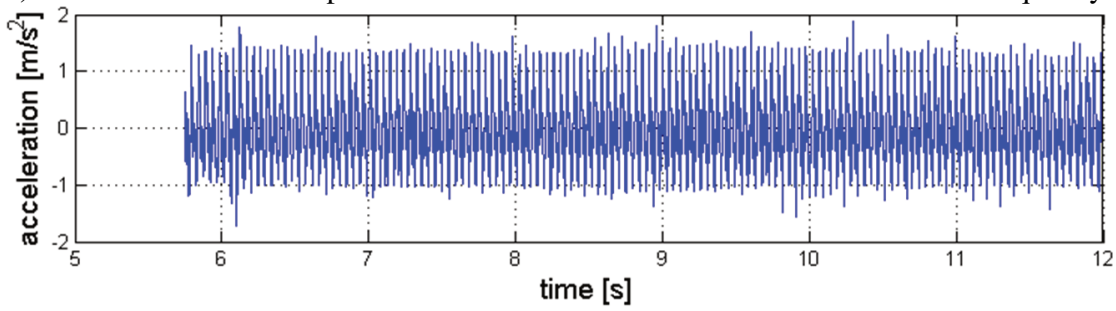

d) Time function of floor pan under the front passenger feet - excitation with constatnt frequency

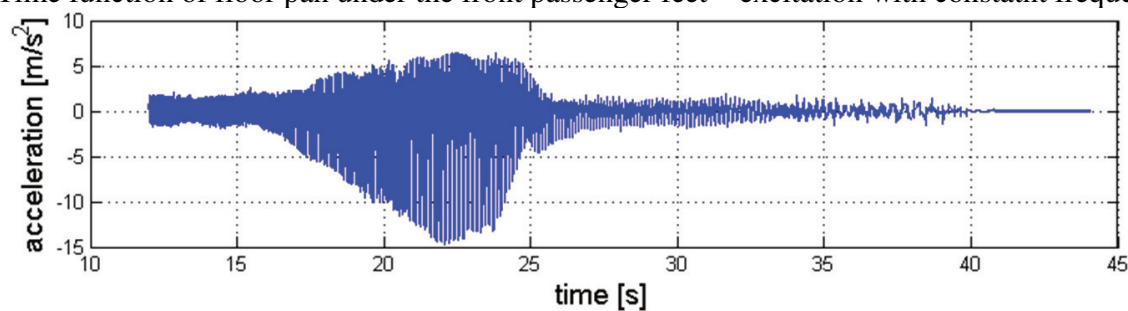

e) Time function of floor pan under the driver feet - excitation frequency decrease

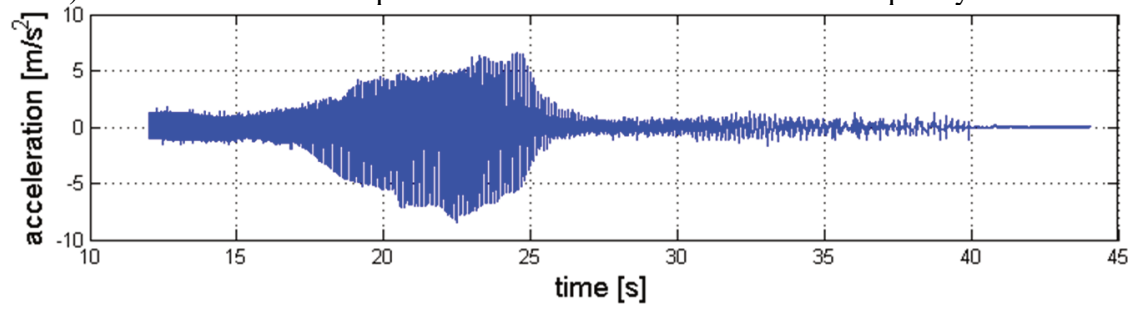

f) Time function of floor pan under the front passenger feet - excitation frequency decrease

Fig. 4.9. Vibration signal distribution by the stationary and non-stationary conditions 


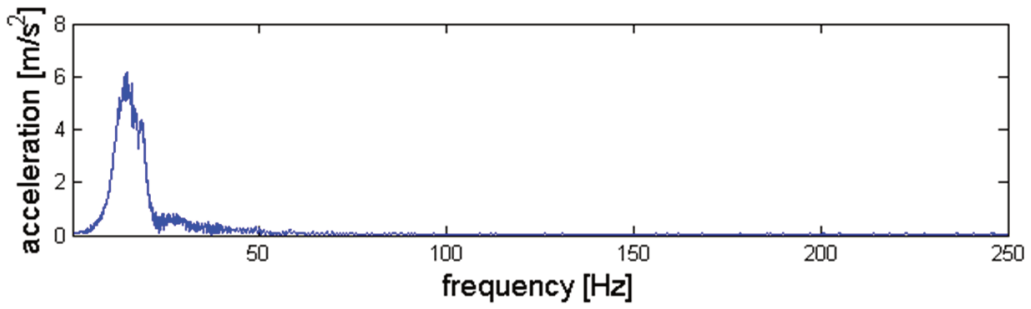

a) FFT excitation frequency increase - suspension arm

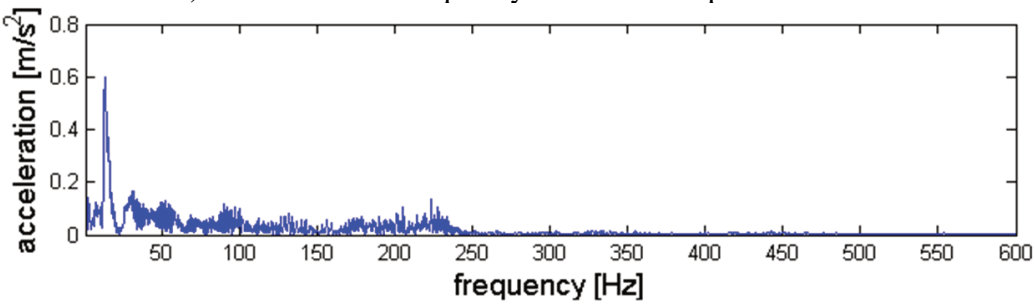

b) FFT excitation frequency increase - upper mounting of shock absorber

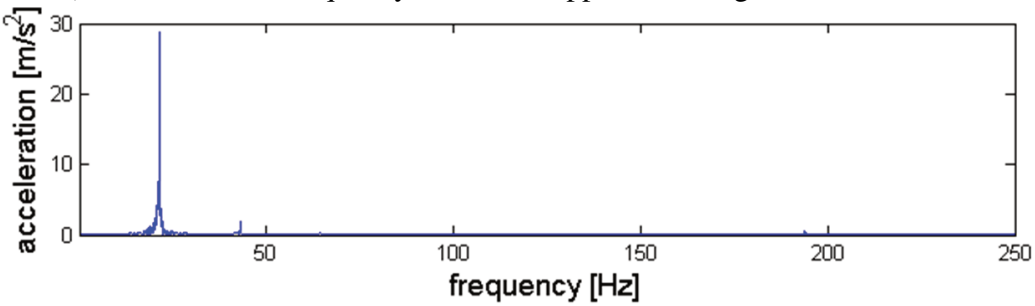

c) FFT excitation with constant frequency - suspension arm

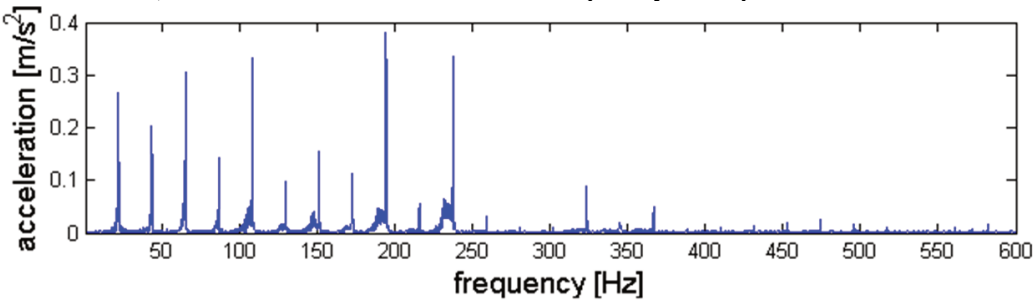

d) FFT excitation with constant frequency - upper mounting of shock absorber

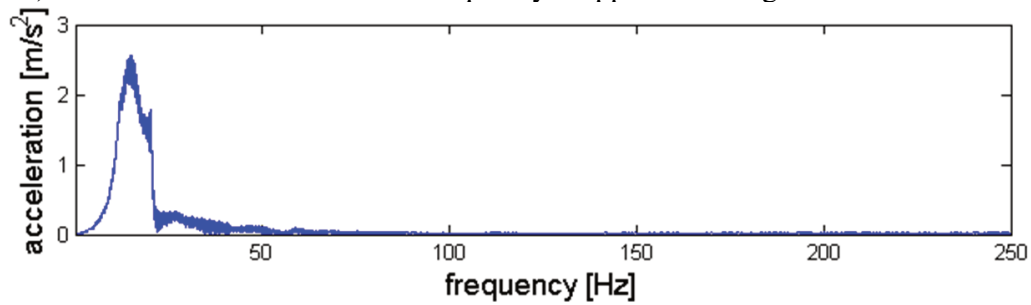

e) FFT excitation frequency decrease - suspension arm

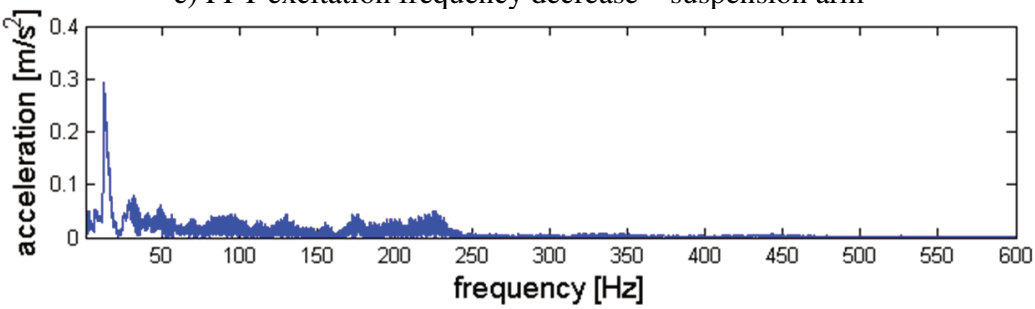

f) FFT excitation frequency decrease - upper mounting of shock absorber

Fig. 4.10. Spectrums of the divided signal by the stationary and non-stationary conditions 


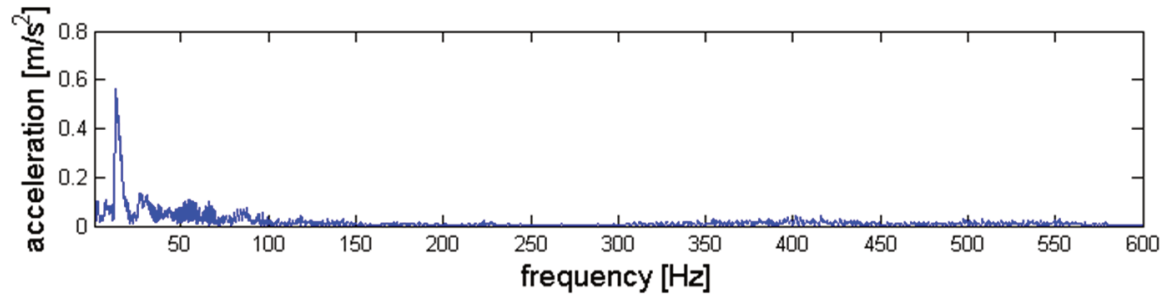

FFT excitation frequency increase

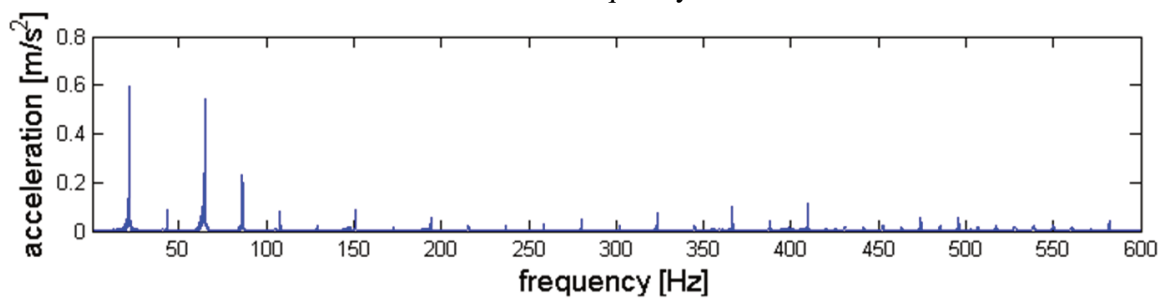

FFT excitation with constant frequency

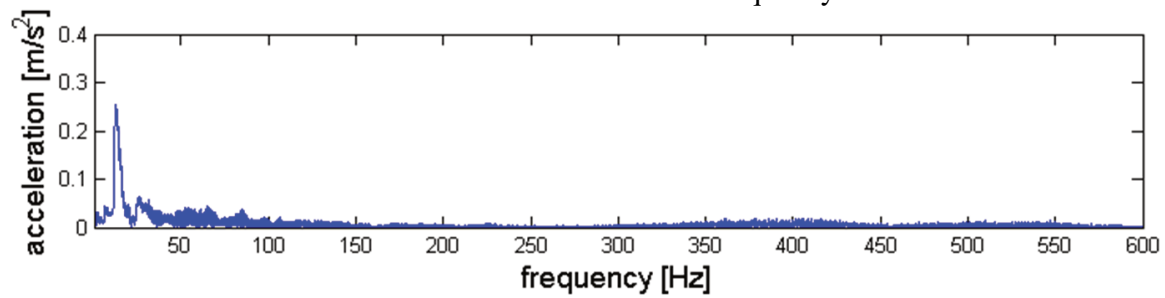

FFT excitation frequency decrease

a) Floor pan under the driver feet

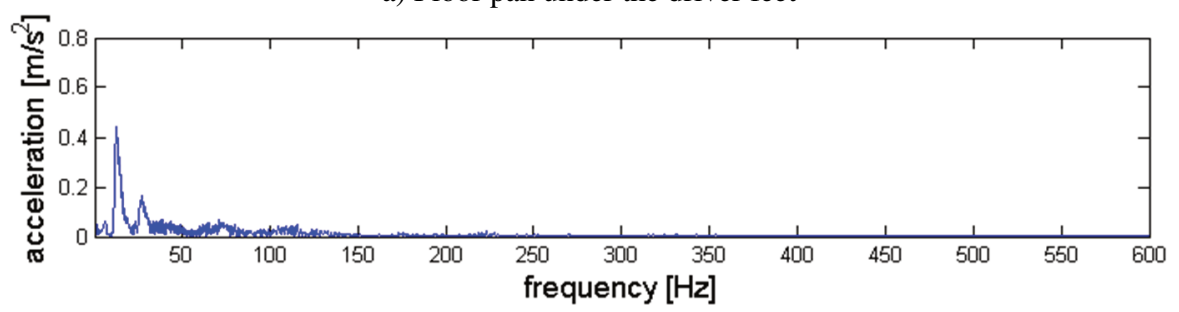

FFT excitation frequency increase

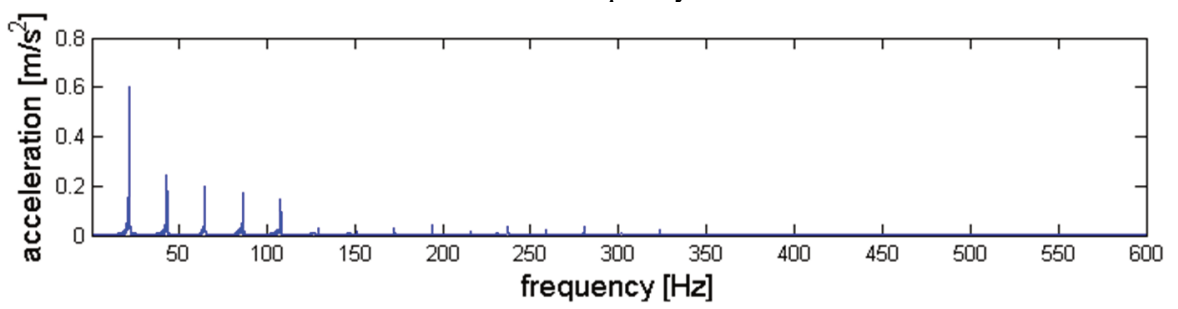

FFT excitation with constant frequency

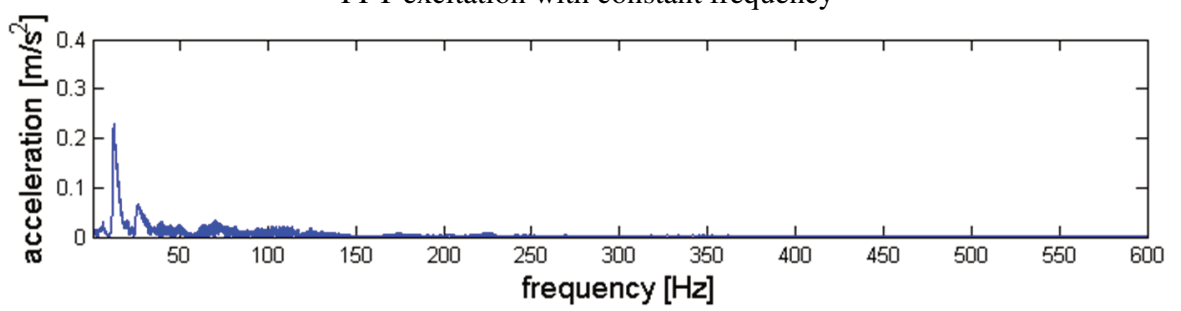

FFT excitation frequency decrease

b) Floor pan under the front passenger feet 


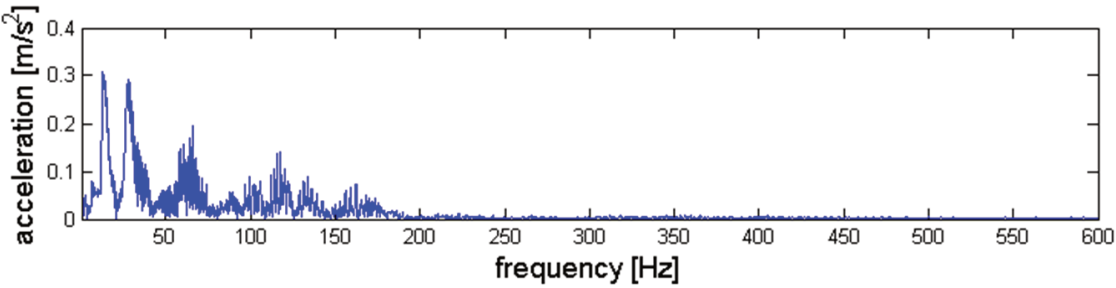

FFT excitation frequency increase

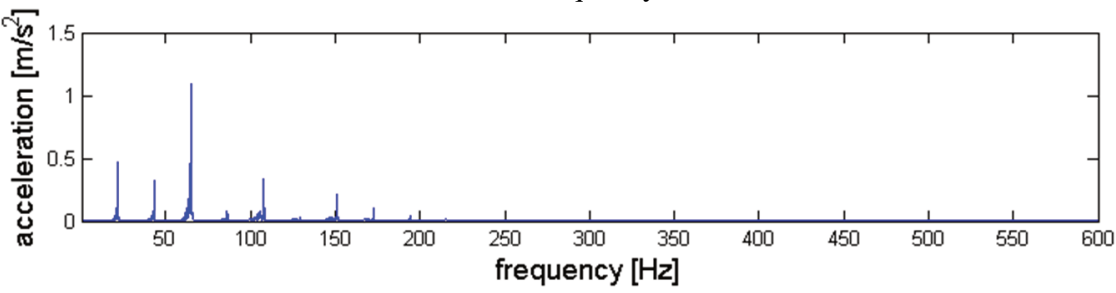

FFT excitation with constant frequency

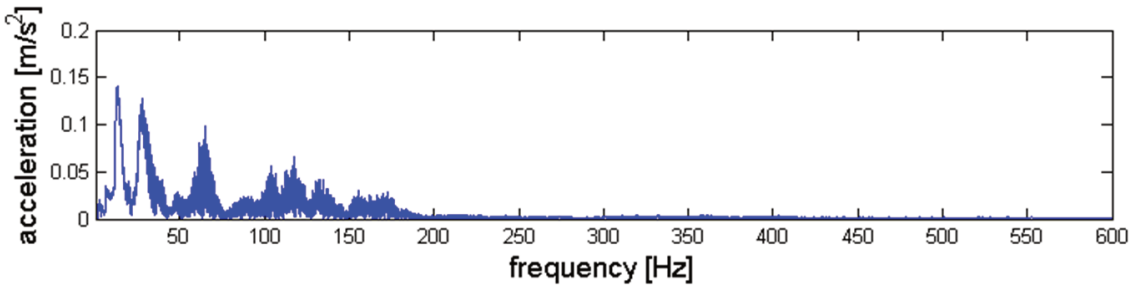

FFT excitation frequency decrease

c) Floor pan under the rear left passenger feet

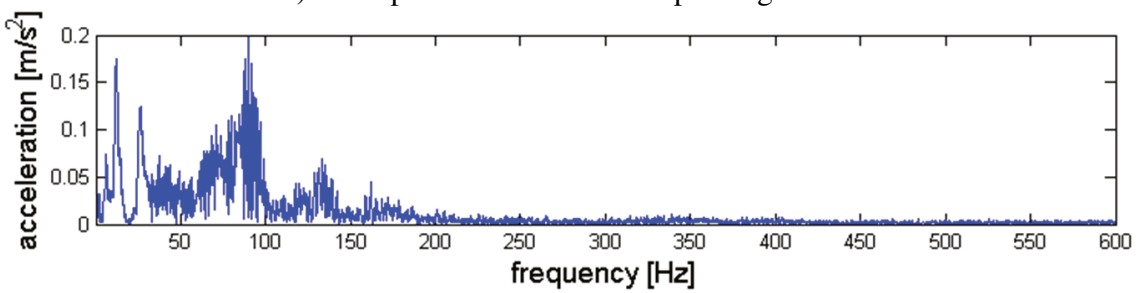

FFT excitation frequency increase

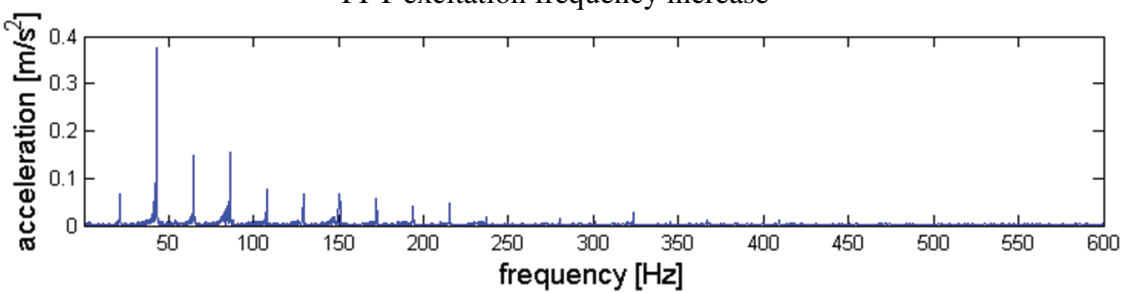

FFT excitation with constant frequency

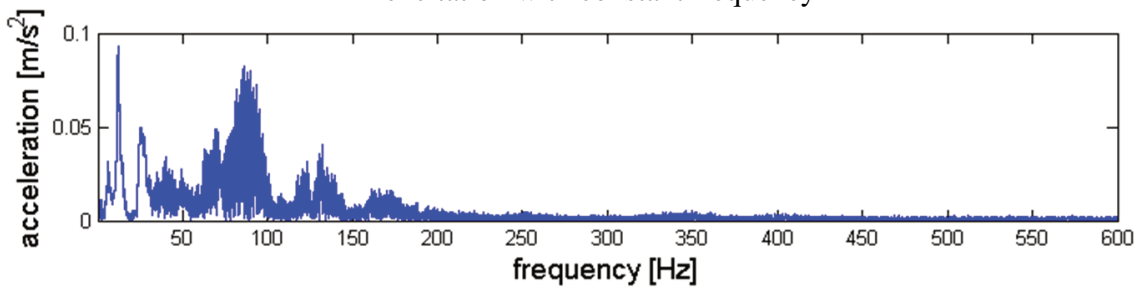

FFT excitation frequency decrease

d) Floor pan under the rear right passenger feet

Fig. 4.11. Spectrums of the divided floor panel vibration signal by the stationary and non-stationary conditions 


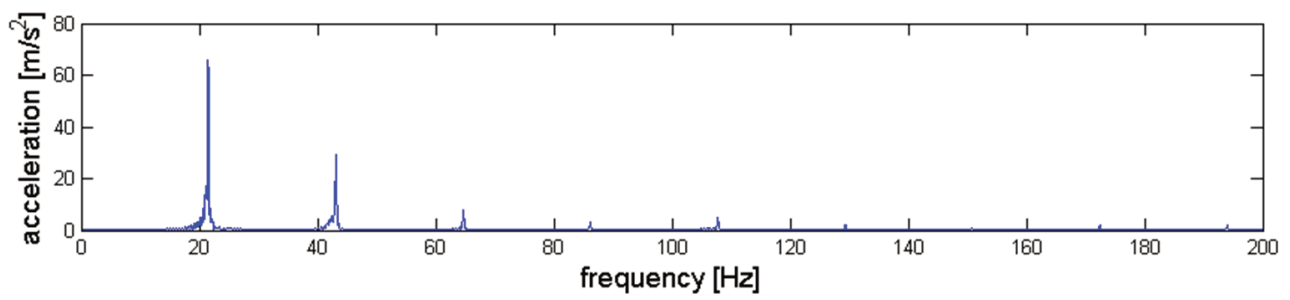

a) FFT excitation with constant frequency - excitation plate

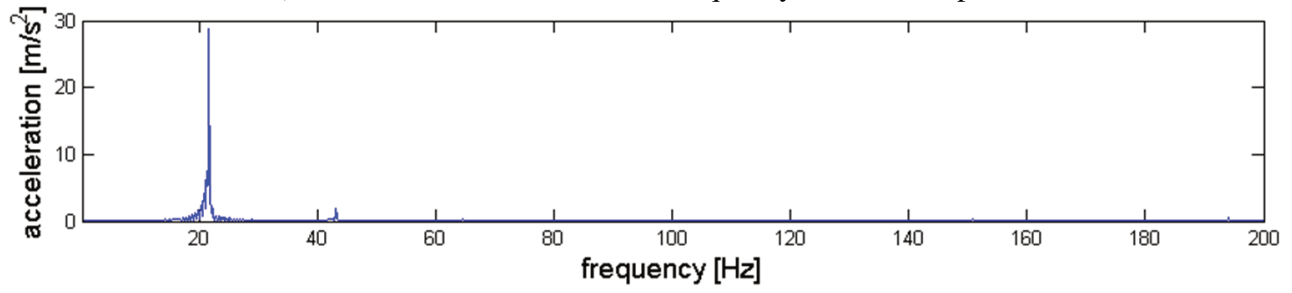

b) FFT excitation with constant frequency - suspension arm

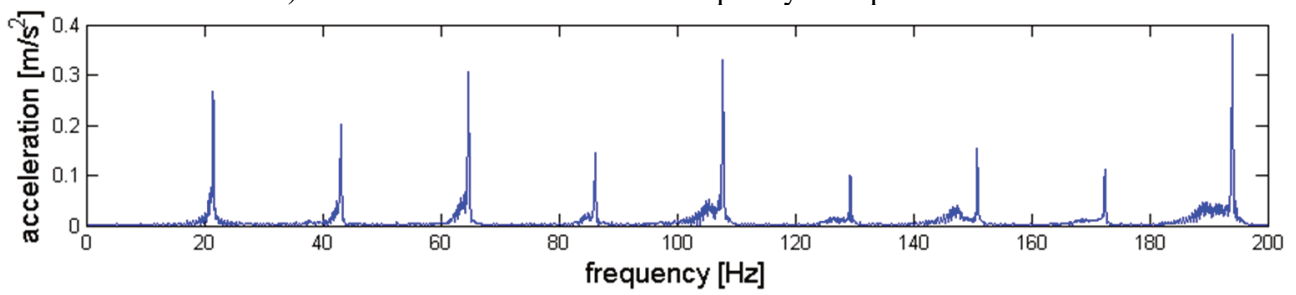

c) FFT excitation with constant frequency - upper mounting of shock absorber

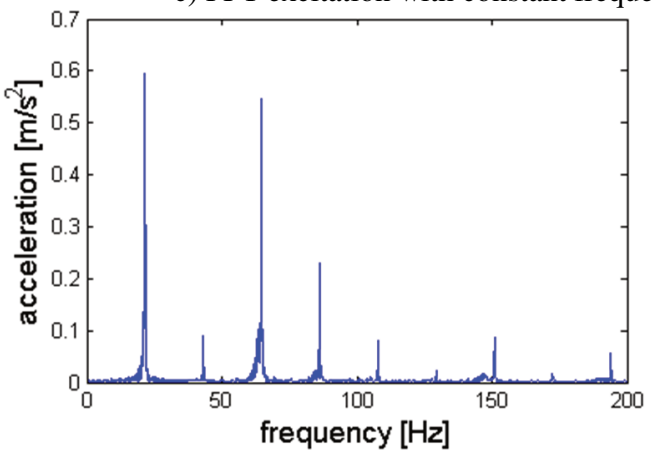

d) FFT excitation with constant frequency floor pan under the driver feet

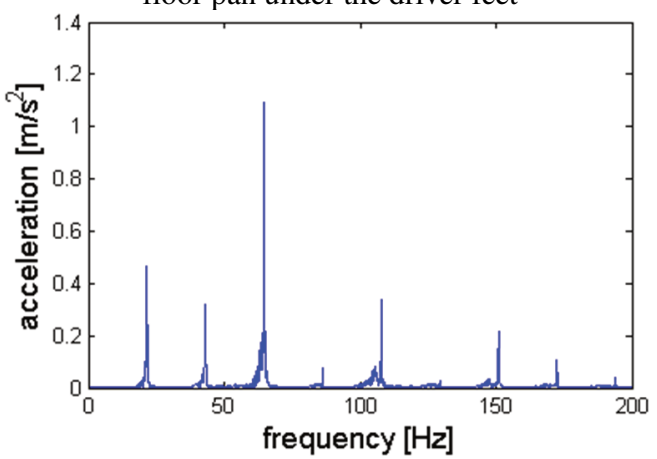

f) FFT excitation with constant frequency under the rear left passenger feet

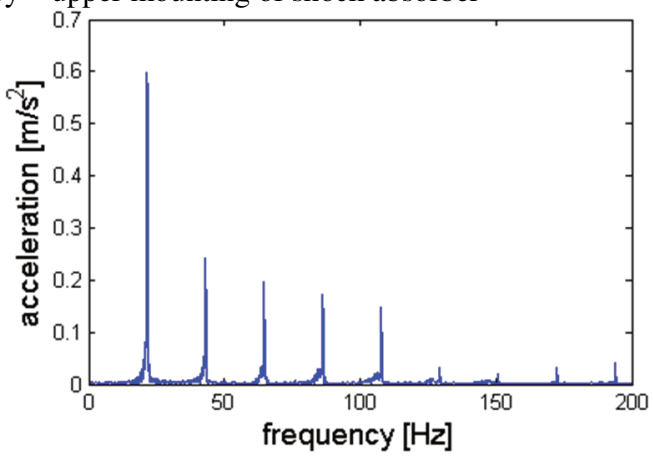

e) FFT excitation with constant frequency under the front passenger feet

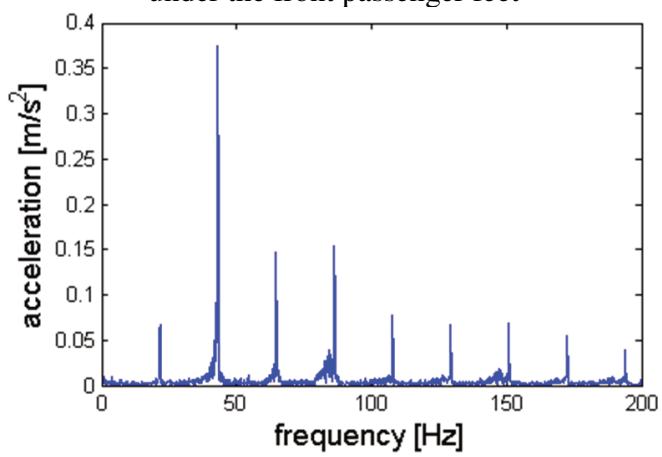

g) FFT excitation with constant frequency under the rear right passenger feet

Fig. 4.12. Comparison of the spectrums of the stationary periods of vibration signals 


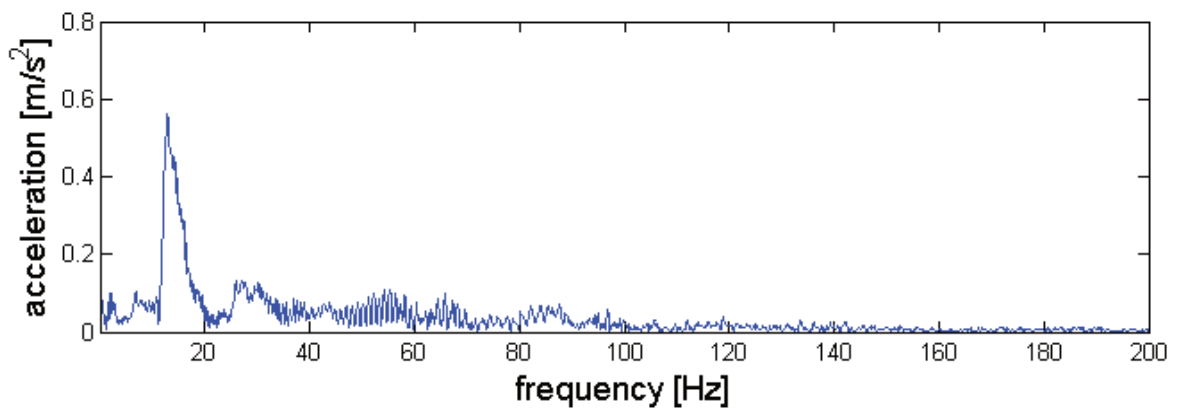

a) FFT excitation frequency increase - floor pan under the driver feet

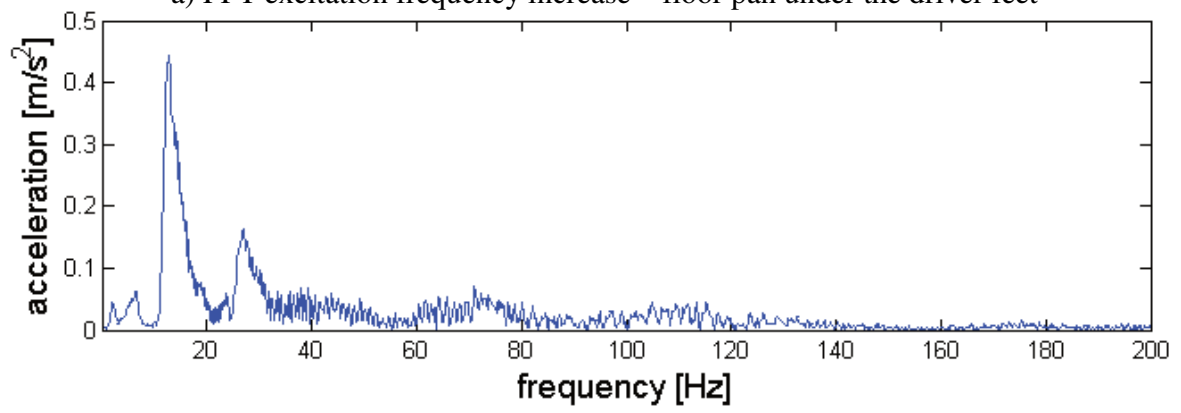

b) FFT excitation frequency increase - floor pan under the front passenger feet

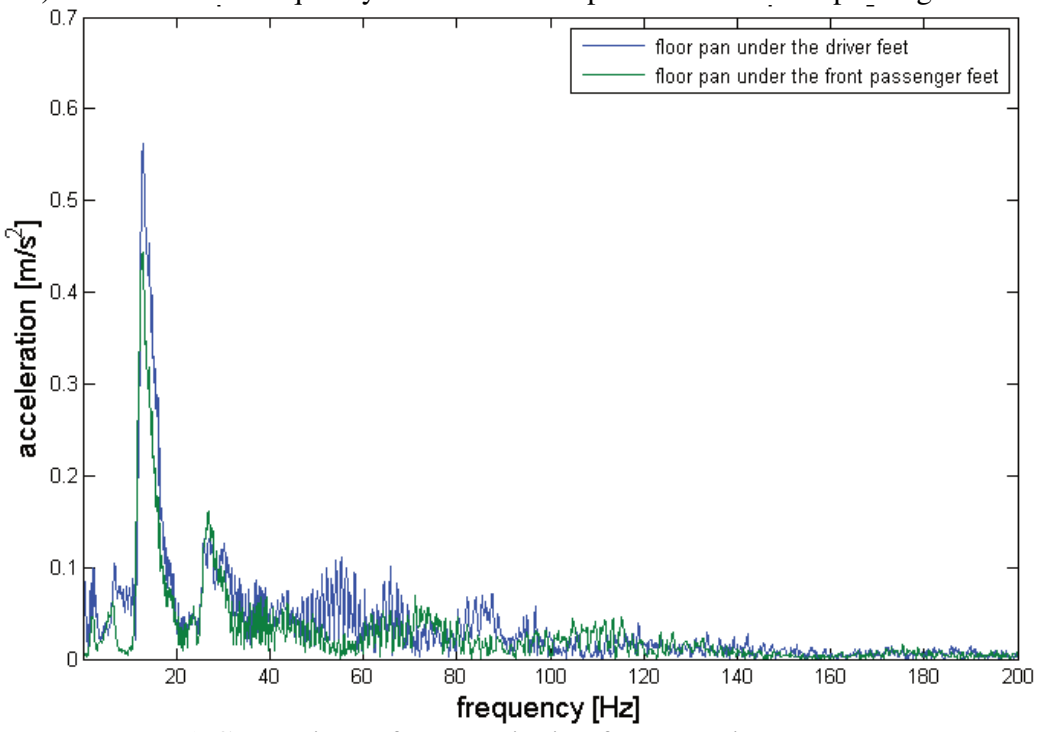

c) Comparison of FFT excitation frequency increase -

floor pan under the driver feet vs under the front passenger feet

Fig. 4.13. The correlation of the non-stationary periods of vibration signals dynamics of the front floor panel during the excitation frequency increase

The correlation of the non-stationary periods of vibration signals dynamics of the front floor panel are presented in Figures 4.13 and 4.14. The signals registered during increase and decrease of excitation frequencies were compared. It can be observed that vibration registered under the feet of driver and front passenger are strongly correlated, especially for unsprung masses resonance $(12-15 \mathrm{~Hz})$. Thorough analysis of the spectrums allow observing the resonance of sprung masses. The amplitudes in 1-2 Hz band are higher. Comparison of the sprung and unsprung masses resonances shows that energy of the signal for unsprung masses resonances is more than 5 times higher. 
For the proper analysis of non-stationary signals the time-frequency methods should be used. Simultaneous extraction of information concerning the time-frequency structure of a signal being analysed is possible owing to a Short Time Fourier Transform (STFT). Application of this method allows identifying with good precision the time of the resonance occurs. The Figs. 4.15 and 4.16 present some exemplary results of STFT transformation of the vibration registered during the increase frequencies of the excitation in whole vehicle structure.

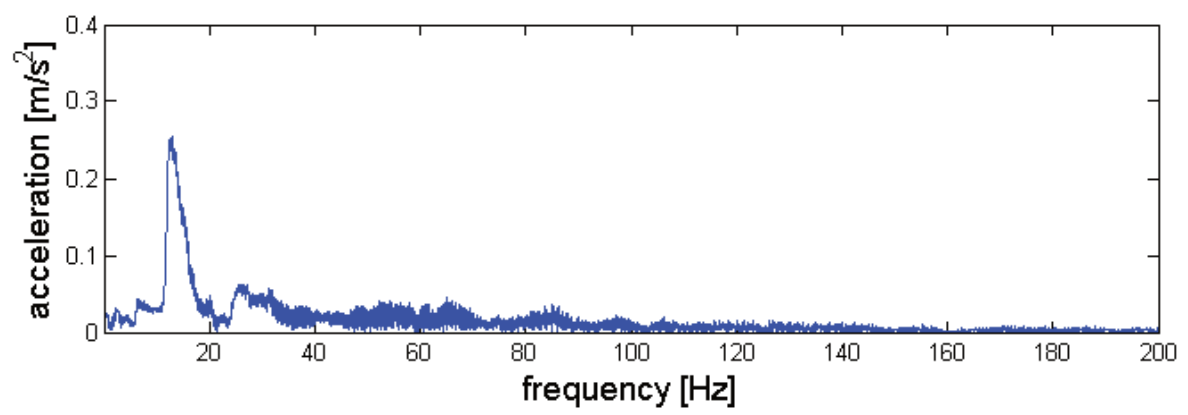

a) FFT excitation frequency decrease - floor pan under the driver feet

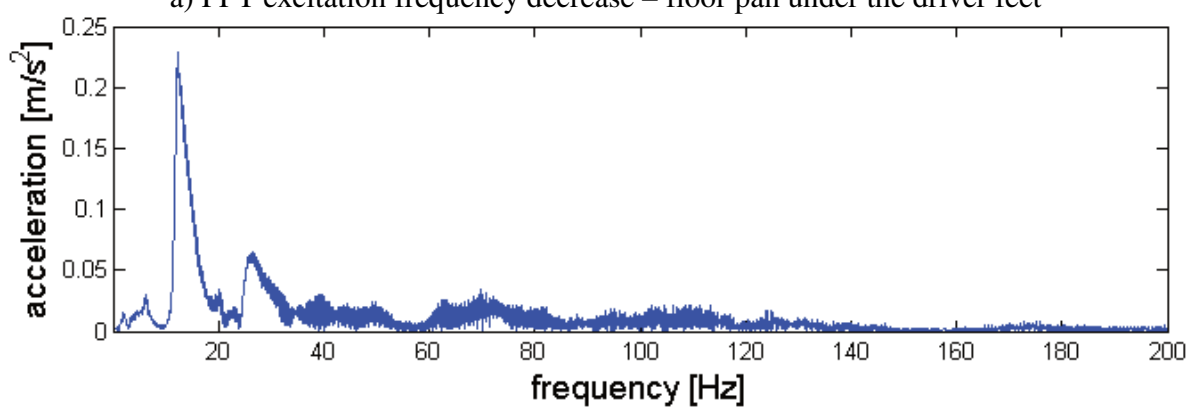

b) FFT excitation frequency decrease - floor pan under the front passenger feet

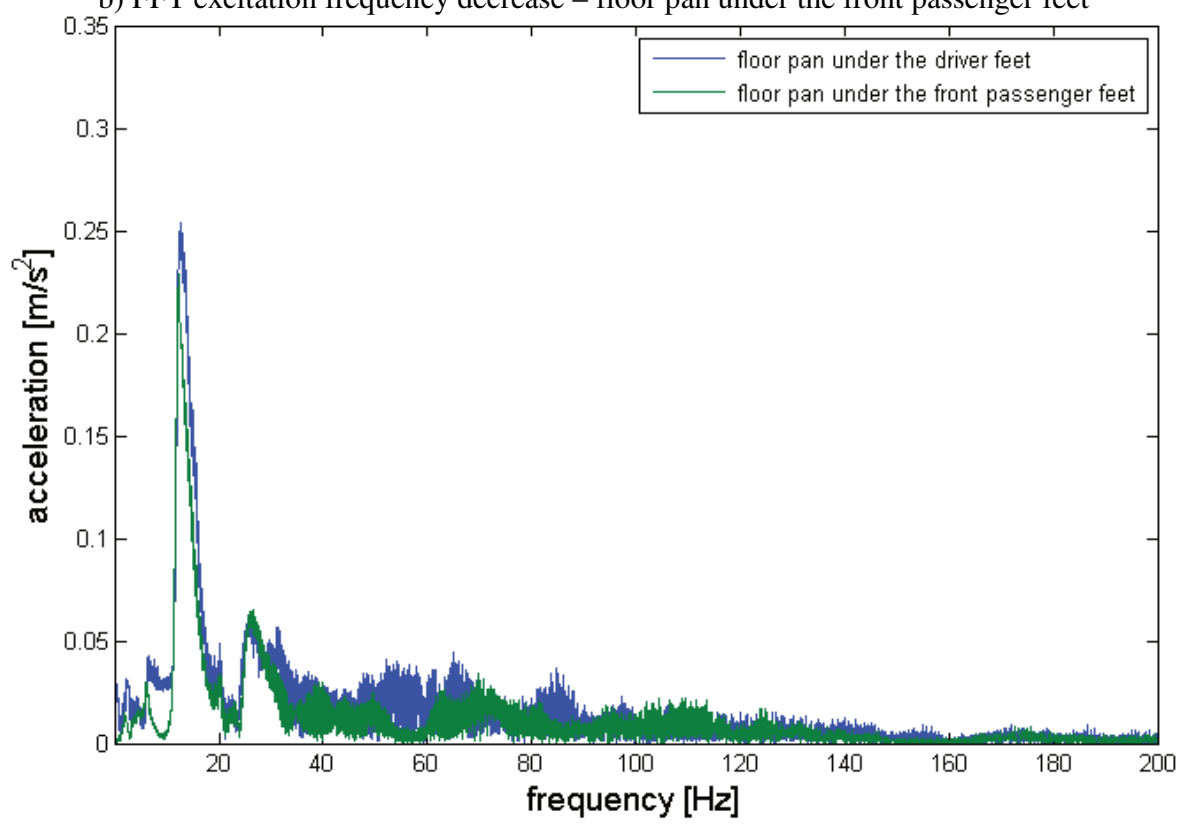

c) Comparison of FFT excitation frequency decrease -

floor pan under the driver feet vs under the front passenger feet

Fig. 4.14. The correlation of the non-stationary periods of vibration signals dynamics of the front floor panel during the excitation frequency decrease 


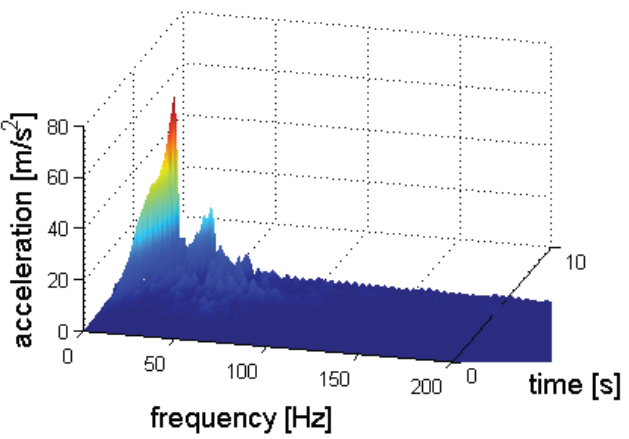

a) Excitation plate

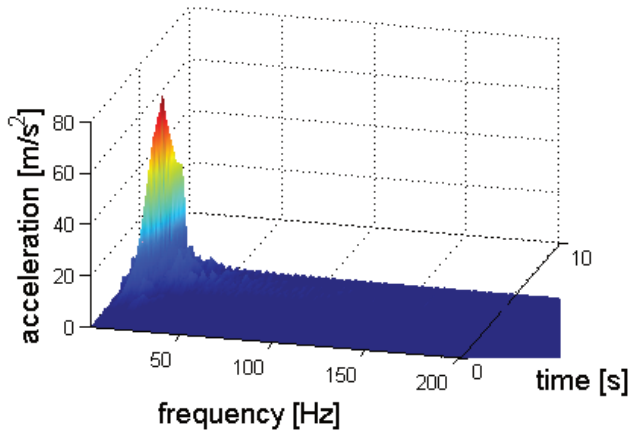

b) Suspension arm

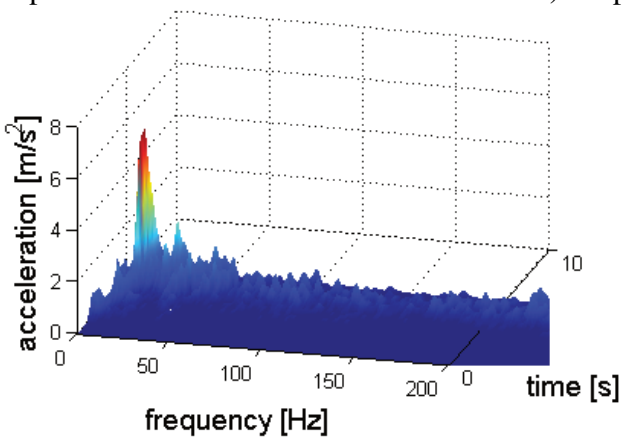

c) Upper mounting of shock absorber

Fig. 4.15. The STFT transformations of vibration registered during the increase frequencies of the excitation in vehicle suspension (time window $0.25 \mathrm{~s}$, resolution $0.4884 \mathrm{~Hz}$ )

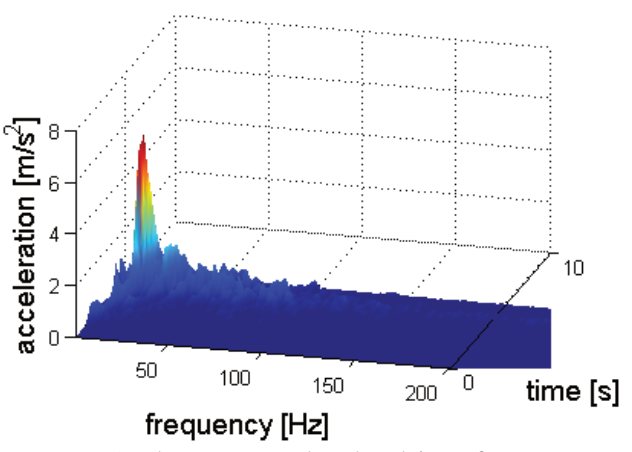

a) Floor pan under the driver feet

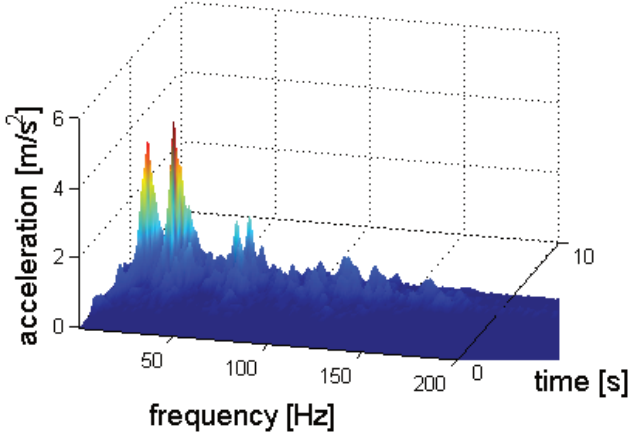

c) Under the rear left passenger feet

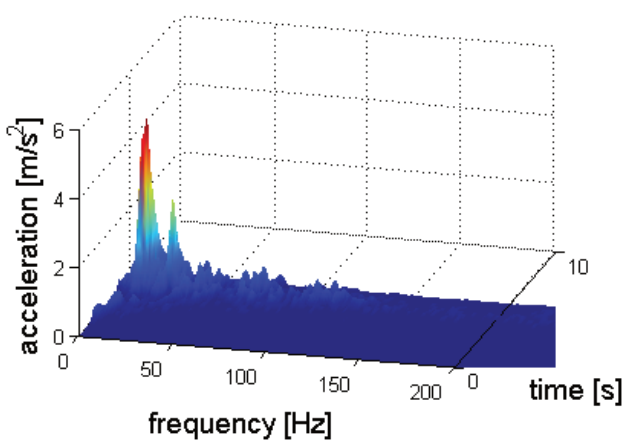

b) Under the front passenger feet

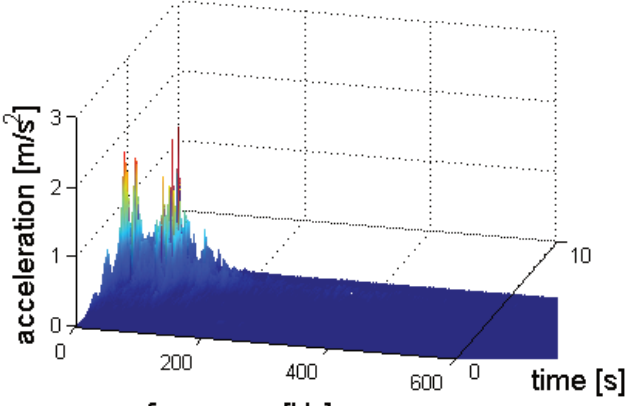

frequency $[\mathrm{Hz}]$

d) Under the right passenger feet

Fig. 4.16. The STFT transformations of vibration registered during the increase frequencies of the excitation on the floor panel (time window $0.25 \mathrm{~s}$, resolution $0.4884 \mathrm{~Hz}$ ) 
The chapter presents proposition of methodology on advanced signal processing for research on vibration propagation in vehicle construction. To evaluate the vibration research for the human vibration perception in vehicle it is necessary to analyse the structure of vibration signals in chosen elements of suspension and car body of the means of transport. For the human exposure to vibration the signals registered in places where vibrations are penetrating into the body can be analyzed. The scope of the research allows analysis of vibration isolation quantity by suspension system and distribution of the vibration penetrating into human body by feet. The algorithm of the signal distribution by the stationary and non-stationary conditions allows proper analysis of the signals separately as the stationary and non-stationary signal. 


\section{Identification of floor panel vibration characteristics for a motor vehicle, empirical evaluation of distribution of natural vibrations}

The ongoing development of the automotive industry is not only about improving vehicle subunits but also about using new structural materials manufactured by state-of-the-art metallurgical technologies and application of new technologies for joining vehicle components $[93,117]$. Therefore the scope of studies of vehicle vibration dynamics has been extended by stationary empirical tests of oscillatory wave propagation in a vehicle structure as well as identification of natural vibrations. A detailed analysis of vibration related phenomena requires that other properties and mechanical phenomena take place in the course of degradation as well as the impact of external factors should be taken into consideration [10, 51].

The purpose of this chapter is to propose a method of empirical studies for identification of characteristics of vehicle structure vibrations which enable modelling of vibration distribution in a broad range of frequencies. The chapter also contains a proposal as to the manner of modelling of floor panel vibration surface estimates based on global measures of propagation of random vibrations.

\subsection{Material vibration signal processing methods}

The vibration signal can be very useful for research on properties of the materials, diagnosing of the materials defects or even for monitoring and testing on quality of the metallurgical technologies in production processes. Large capacity of the information in vibration signals allows to create many research methods and results analysis. Vibroacoustics is the science discipline which describes the possibilities of vibration and acoustics signals useful for diagnosing and research purpose.

There are a lot of publications showing original methods of vibration signal analysis for many applications [1, 40, 53, 155]. The publication of Batko and Majkut presents the purposefulness in controlling the phase images of the vibration signals as a useful tool for fault development process identification in the object monitored. The presented diagnostics method based on phase trajectories analysis determines displacement and velocity in arbitrary chosen point [21, 22].

Interesting application of acoustic signal in structures defects research were presented in [9]. It shows application of the method of elastic wave propagation to detect defects in composite castings. It was proved that if a defect occurs, which is detected by the radiography, elastic wave propagation changes locally. Another example can be the photoacoustic transformation. In [141] the investigation of photoacoustic transformation in naturally-gyrotropic and magnetoactive crystals under internal stress by sound excitation in different modes by Bessel light beams was described.

Resonant is the main phenomenon useful in vibration research. Resonance vibration of materials is caused by an interaction between the inertial and elastic properties of the materials within a structure. Resonance vibration amplifies the vibration response more than the level of deflection, stress, and strain caused by static loading. Resonances are determined by the material properties, such as:

- mass, 
- stiffness,

- damping properties,

- boundary conditions of the structure.

The modal research allows the frequency response function (FRF) to be determined. This describes the input-output relationship between two points on a structure as a function of frequency. Nowadays the modal testing is mean effective for identifying and simulating dynamic behavior and responses of structures. A very useful group of this research is experimental modal analysis (EMA) because this is an example of non-destructive testing. Modal research is based on vibration responses of the structures. New methods of the signal processing allows the vibrational response of the structures to the impact excitation (instrumented hammer impact excitation), which are measured, transformed into frequency response functions using Fast Fourier Transformation (FFT) [95, 157]. In the practical applications the modal parameters are required to avoid resonance in structures affected by external periodic dynamic loads. Modal analysis is a process of describing a structure in terms of its natural characteristics which are the frequency, damping and mode shapes.

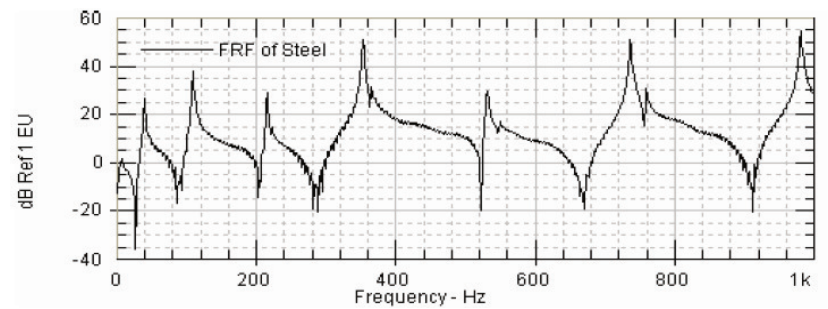

a) Steel

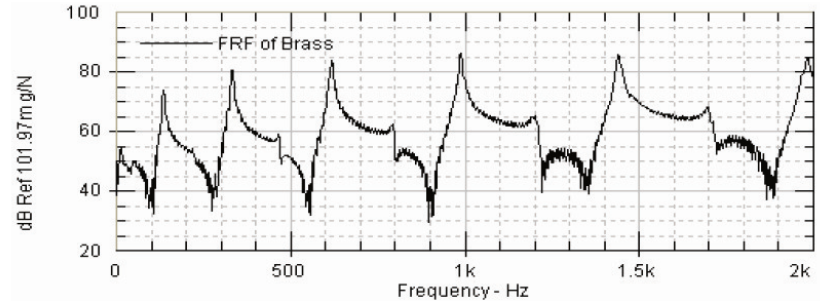

b) Brase

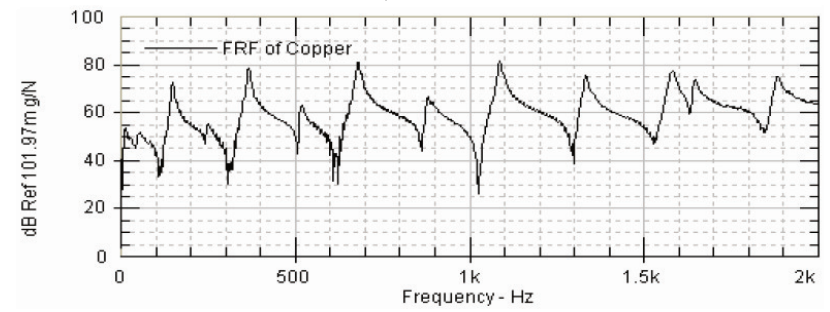

c) Copper

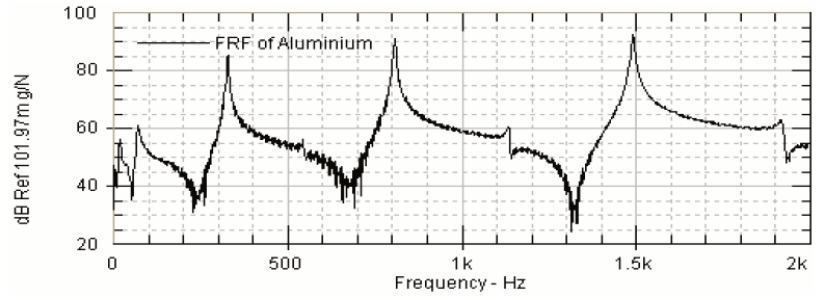

d) Aluminum

Fig. 5.1. Frequency response function [157]

D. J. Mead in [138] describes some methods developed at the University of Southampton to 
analyze and predict the free and forced wave motion in continuous periodic engineering structures. These kinds of methods are very commonly used in wide research. In the paper [112] the calculation of natural frequencies vibrational analysis of composite propeller shafts and decrease of weight was presented. The role of structural elements on the loss of total energy were investigated in [135].

The research on the nature of the wave dynamics in rod were presented in [109]. This investigation shows developed combination of finite element modeling and the transfer matrix method to solve the dynamics of the wave propagation of the periodic beam structures with defects.

Prasad and Seshu [157] investigated an experimental modal analysis of beams made with different materials such as Steel, Brass, Copper and Aluminum. The beams were excited using an impact hammer excitation technique. Some of their results are presented in Fig. 5.1.

By using SDOF (Single Degree of Freedom) and MDOF (Multiple Degree of Freedom) estimation algorithms, natural frequencies and damping ratio could be calculated. In SDOF and MDOF estimation the modal parameters are calculated using finite difference and quadrature methods. The research shows the differences between natural frequencies, damping ratio of the materials and shapes of modes (Tables 5.1-5.4).

Table 5.1. EMA natural frequencies calculated by SDOF estimation algorithm using finite difference method

\begin{tabular}{|c|c|c|c|c|}
\hline Mode No. & Steel [Hz] & Brass [Hz] & Copper [Hz] & Aluminum [Hz] \\
\hline 1 & 40.27 & 40.00 & 24.43 & 70.71 \\
\hline 2 & 110.34 & 105.52 & 45.21 & 170.29 \\
\hline 3 & 216.50 & 224.83 & 150.78 & 329.16 \\
\hline 4 & 353.72 & 333.61 & 250.108 & 548.42 \\
\hline 5 & 532.22 & 469.46 & 367.85 & 808.60 \\
\hline 6 & 533.75 & 619.46 & 518.46 & 1136.87 \\
\hline
\end{tabular}

Table 5.2. EMA damping ratio calculated by SDOF estimation algorithm using finite difference method

\begin{tabular}{|c|c|c|c|c|}
\hline Mode No. & Steel [\%] & Brass [\%] & Copper [\%] & Aluminum [\%] \\
\hline 1 & 2.02 & 14.43 & 39.21 & 6.12 \\
\hline 2 & 0.78 & 1.78 & 17.44 & 1.48 \\
\hline 3 & 0.33 & 1.02 & 1.96 & 0.51 \\
\hline 4 & 0.18 & 0.70 & 1.28 & 0.31 \\
\hline 5 & 0.13 & 0.56 & 1.01 & 0.22 \\
\hline 6 & 0.10 & 0.47 & 1.71 & 0.24 \\
\hline
\end{tabular}

Table 5.3. Modal shapes of steel beam

\begin{tabular}{|c|c|c|c|c|}
\hline Mode No. & 1 & 2 & 3 & 4 \\
\hline Mode shape & & & & \\
\hline Frequency & $40.27 \mathrm{~Hz}$ & $110.34 \mathrm{~Hz}$ & $216.50 \mathrm{~Hz}$ & $353.72 \mathrm{~Hz}$ \\
\hline
\end{tabular}

Table 5.4. Modal shapes of steel beam in SDOF (quadrature method)

\begin{tabular}{|c|c|c|c|c|}
\hline Mode No. & 1 & 2 & 3 & 4 \\
\hline Mode shape & & & & \\
\hline Frequency & $41.07 \mathrm{~Hz}$ & $110.00 \mathrm{~Hz}$ & $216.25 \mathrm{~Hz}$ & $353.75 \mathrm{~Hz}$ \\
\hline
\end{tabular}

The paper [60] presents some preliminary research on possibilities of vibration signal application in research on materials. During the research the vibration accelerations in a direction parallel to the symmetry axis of disks in two selected points were measured. The vibrations in the disk material structure were excited by impacts in specific points.

The qualitative impact assessment for the structural material the disk was made of was 
performed by establishing the correlograms of time sections of the wavelet coefficient matrices (Fig. 5.2). The time sections applied in the analysis were chosen bearing in mind the natural vibration frequencies of the components examined. The autocorrelation function for the chosen wavelet sections were used for the analysis.

A random signal autocorrelation function characterises the general dependency between the signal value at a certain instant and the signal value at another instant. For any chosen execution $x(t)$ of the process in question, the autocorrelation function value estimator, linking values $x(t)$ at instants $t$ and $t+\tau$ (where $\tau$ is the shift), can be obtained by calculating the product of these values and subsequently averaging it within the observation time range of $T$. When $T$ is approaching the infinity, a precise value of the autocorrelation function is calculated. This function is always real and even, and its maximum is at the point corresponding to a zero shift and may assume both positive and negative values. A cross-corelation function established for various random signals is a measure of their mutual dependency. It is defined as follows:

$R_{x y}(\tau)=\lim _{T \rightarrow \infty} \frac{1}{T} \int_{0}^{T} x(t) y(t+\tau) d t$.

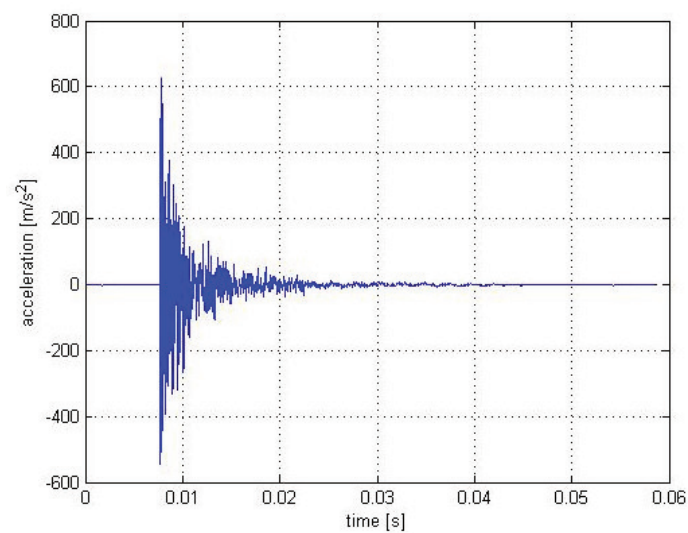

a)

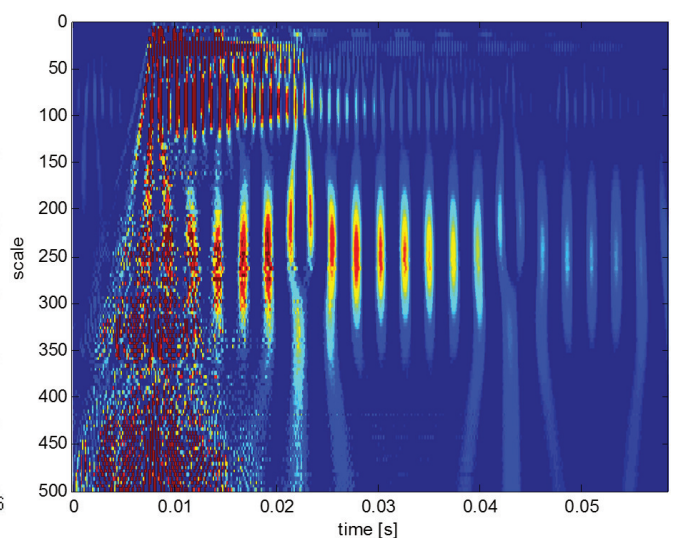

b)

Fig. 5.2. Results of the research: time course of vibration accelerations and distributions of wavelet coefficients [60]

This function constitutes an efficient tool for detection of predetermined processes (in the case of a random process, on high shift values, the autocorrelation function is approaching zero, whereas for a harmonic signal or other predetermined signals, the autocorrelation function does not fade as the shift value increases). When the aforementioned transformation is applied in the analysis of wavelet sections, the relevant function is as follows:

$R_{A, B}\left(t_{u}\right)=\lim _{T \rightarrow \infty} \frac{1}{T} \int_{0}^{T} W T_{A}(t) W T_{B}\left(t+t_{u}\right) d t$,

where: $A, B$ - indices of the measuring sensors mounting points, $W T_{x}(t)-$ time sections of matrices of wavelet coefficients, $t_{u}$ - time shift.

Fig. 5.3 presents a sample correlogram obtained for a steel disk excited to vibrate. 


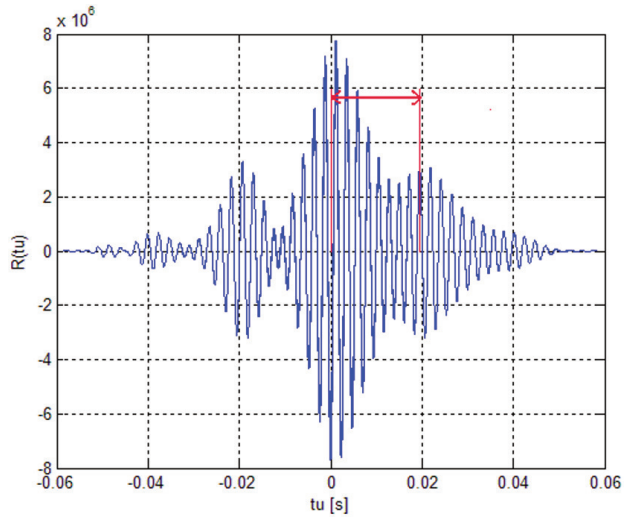

Fig. 5.3. Correlogram of wavelet sections with the time shift value marked on which the local maximum of the intercorrelation function occurs [60]

\subsection{Experimental research method}

Models of phenomena random in nature, defined on the grounds of the theory of probability, are also to be verified against the reality. For that purpose, one needs numerical data characterising the relevant properties of the phenomenon examined. Therefore, empirical data concerning the given phenomenon must be acquired and statistical conclusions drawn.

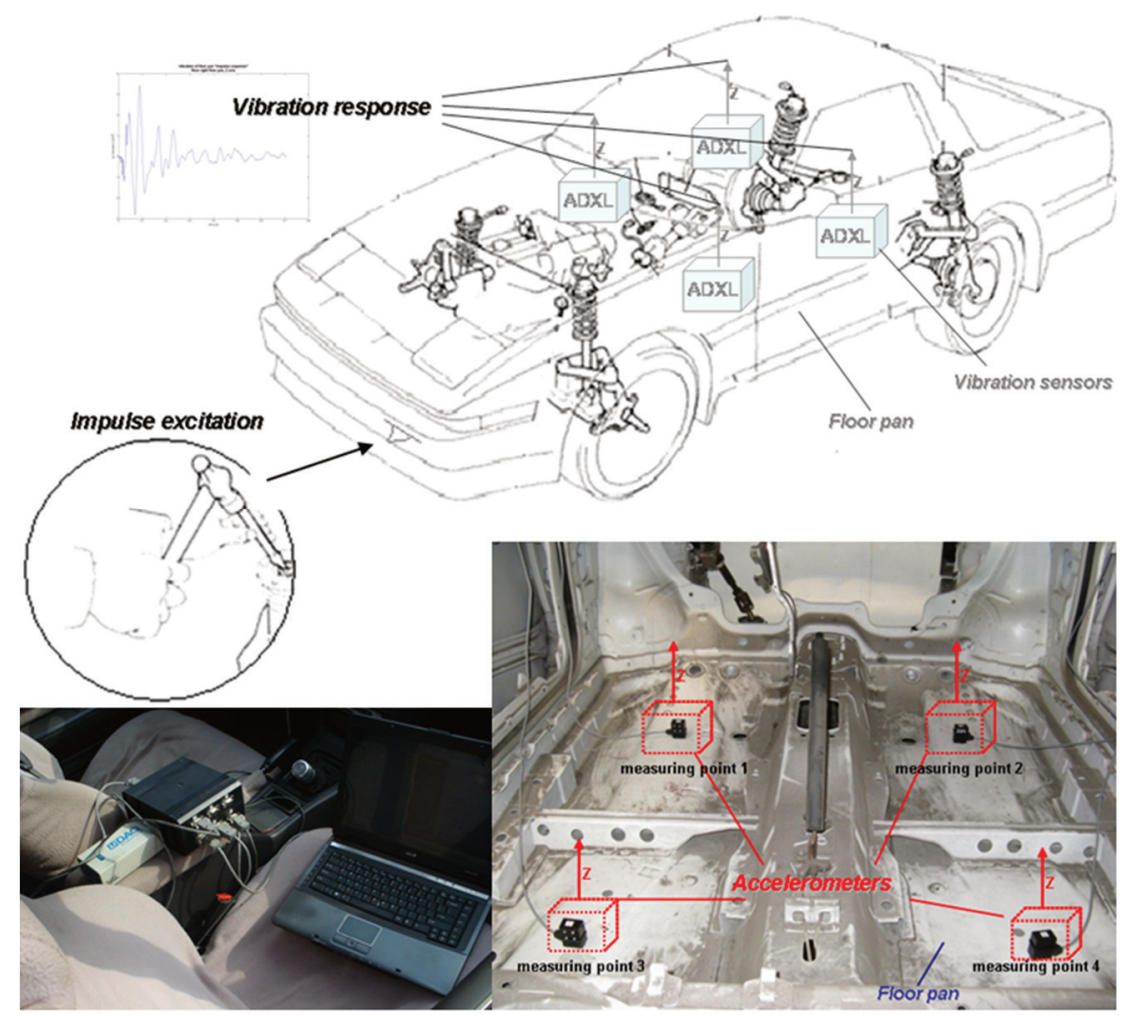

Fig. 5.4. Testing diagram and measurement chain

The experimental studies discussed in the chapter were conducted on an actual object, namely the Toyota Supra mk3 passenger car of the complete vehicle kerb weight of $1.540 \mathrm{~kg}$. The purpose assumed for the studies was to identify natural vibrations of the vehicle floor panel as well as to 
determine their structure in geometrically arranged measuring points. It was to enable determination of signal characteristics and a geometrical distribution of vibrations on the surface of the floor panel. Natural vibrations are those which nature depends on physical properties of the vibrating system (inertia, damping and elasticity), and not on the manner in which the vibrations are induced.

The nature of the studies conducted was that of an experimental modal analysis which does not require recording of the course of the vibration input function. An impulse response of the vehicle structure was examined at selected points by application of forced input function assuming the form of an impact pulse. Vibrations of the floor panel were recorded at 4 points. In order to refer the results obtained to the analysis of the passenger exposure to vibrations, the measuring points were arranged at locations where the vibrations were transferred into the human organism, i.e. where feet rested.

The measurement chain consisted of the piezoelectric sensors, a measuring unit with data acquisition card and a computer featuring the software. Fig. 5.4 presents the testing diagram and a view of the measurement chain.

\subsection{Propagation and structure of the floor panel's vibration}

As a result of the tests conducted, time courses of acceleration changes for vibrations recorded in 3 orthogonal axes: $X$ - horizontally along the vehicle axis, $Y$ - horizontally crosswise the vehicle axis, and $Z$ - vertically and perpendicularly towards plane $X Y$ were obtained. The chapter provides an analysis of the vertical vibrations that constitute the main cause of passenger exposure to vibrations. Courses of vertical vibration accelerations at structural points of the floor panel where passengers rest their feet are illustrated in Fig. 5.5.

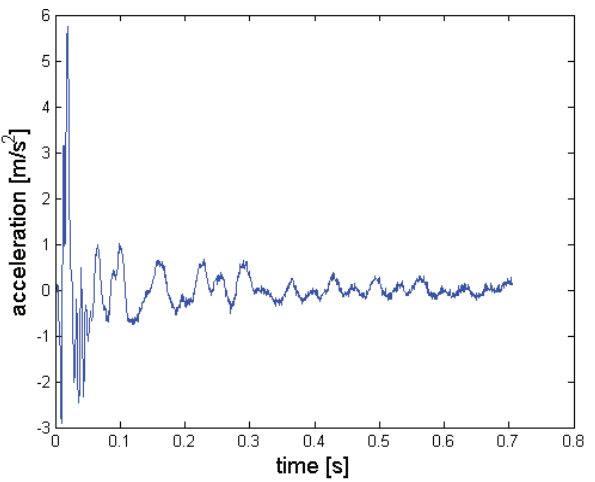

a) Driver

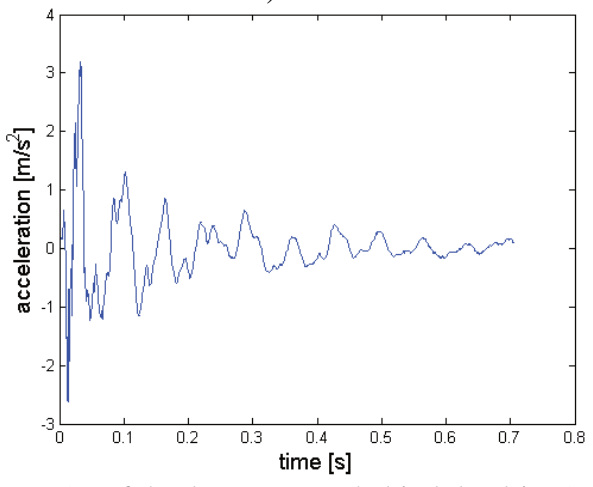

c) Left back passenger (behind the driver)

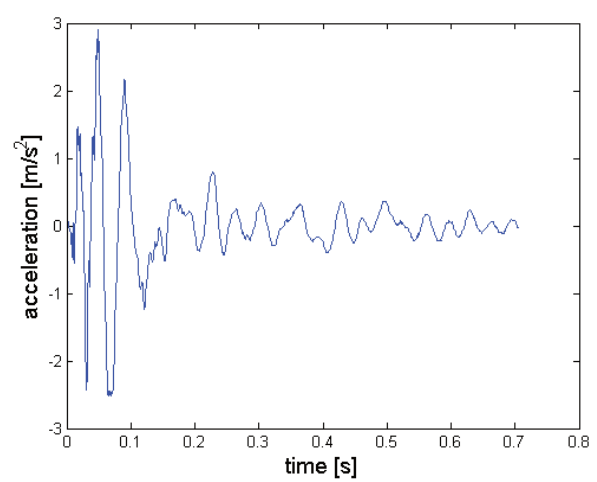

b) Front passenger

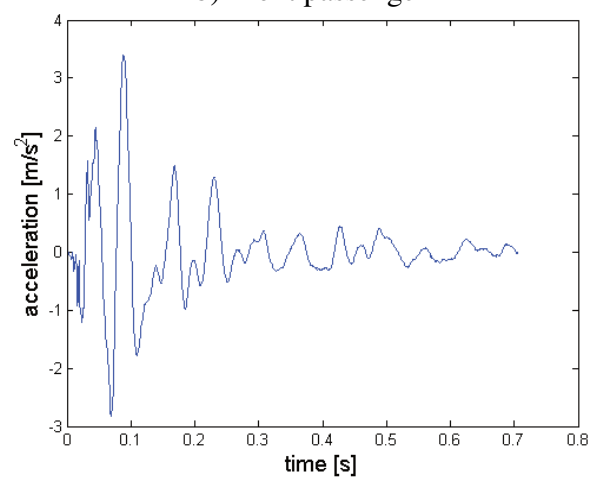

d) Right back passenger

Fig. 5.5. Recorded courses of vertical vibrations accelerations in the floor panel at points where passengers rest their feet 
In order to conduct a preliminary analysis of the frequency components of the signals recorded, their spectra with Fourier transforms application were established. Fig. 5.6 presents sample spectrum of floor panel vibrations.

Basing on the spectral analysis, it was found that the predominant energy content of the signals occurre in a band of up to $200 \mathrm{~Hz}$. Fig. 5.7 illustrates the vibration spectra of the signals recorded.

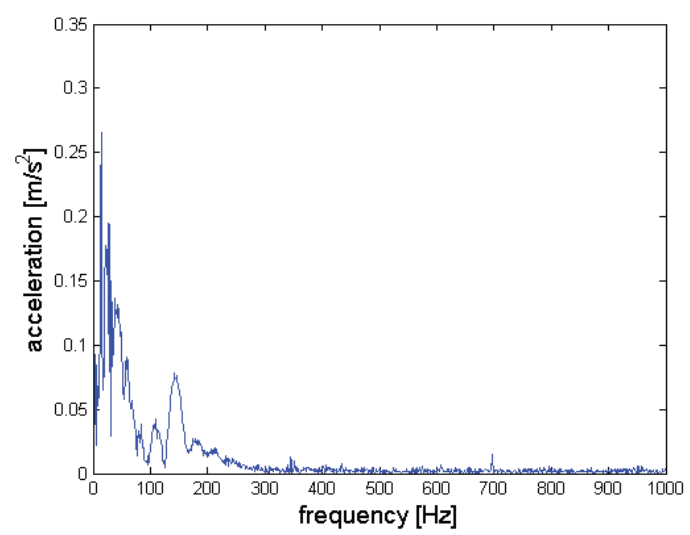

Fig. 5.6. Sample spectrum of floor panel vibrations

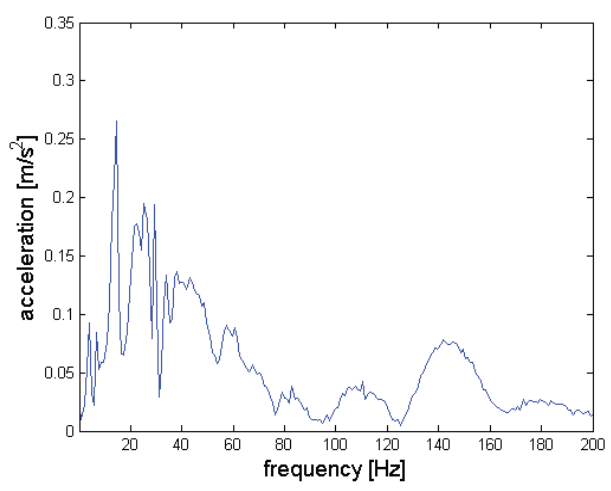

a) Driver

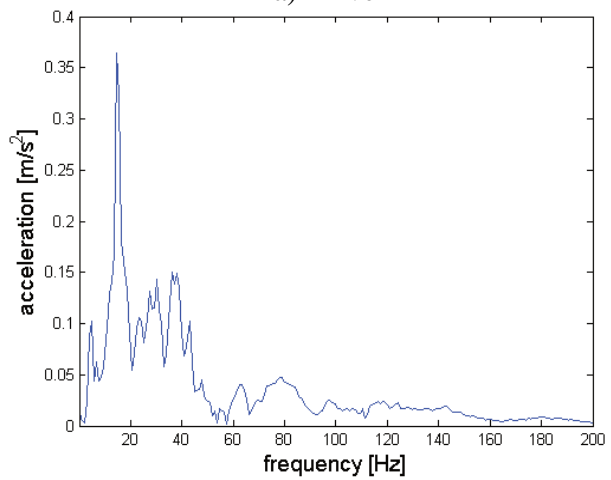

c) Left back passenger (behind the driver)

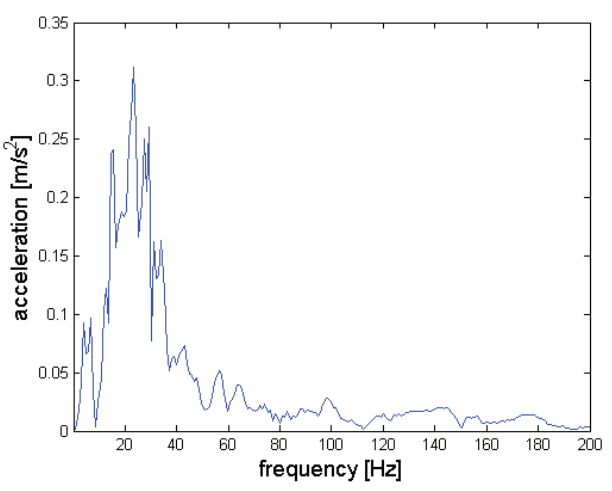

b) Front passenger

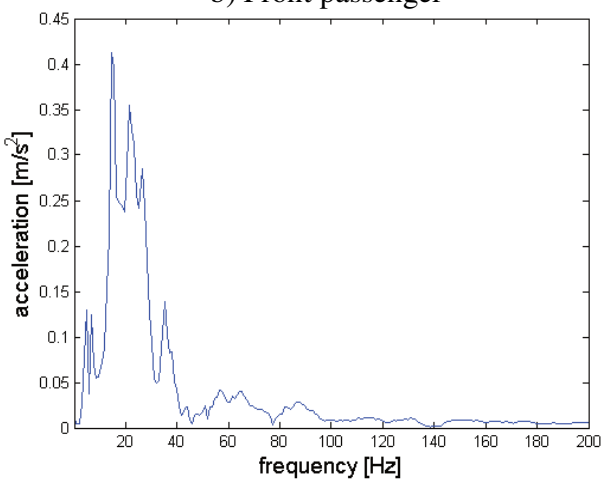

d) Right back passenger

Fig. 5.7. Spectrums of vertical vibrations accelerations in the floor panel at points where passengers rest their feet

Identification of distribution of natural vibration demands the relative phase of all measurement point determining. Fig. 5.8 show the phase shift of accelerations of vertical vibrations in the floor panel at points where passengers rest their feet. The data cursors indicated 
on left chart presents the time and amplitude of very first amplitude of response on impulse force. The Table 5.5 presents time of local maximum of vibration for measurement points and phase shift between next amplitudes and relative phase shift to the time of first maximum amplitude occurring.
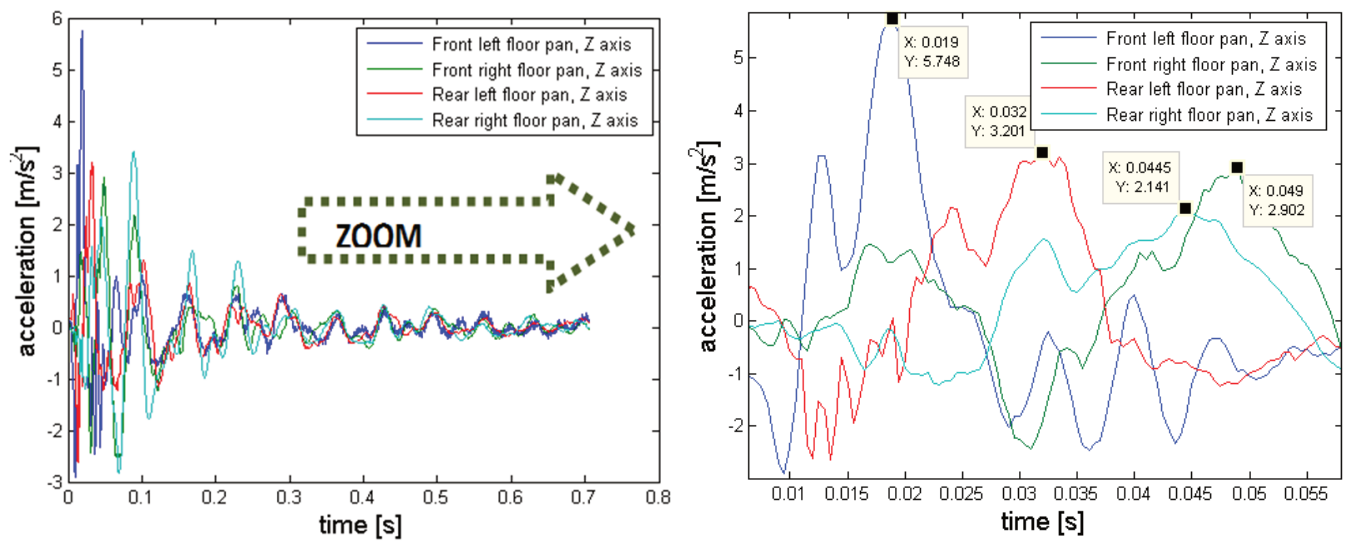

Fig. 5.8. Phase shift of accelerations of vertical vibrations in the floor panel

at points where passengers rest their feet

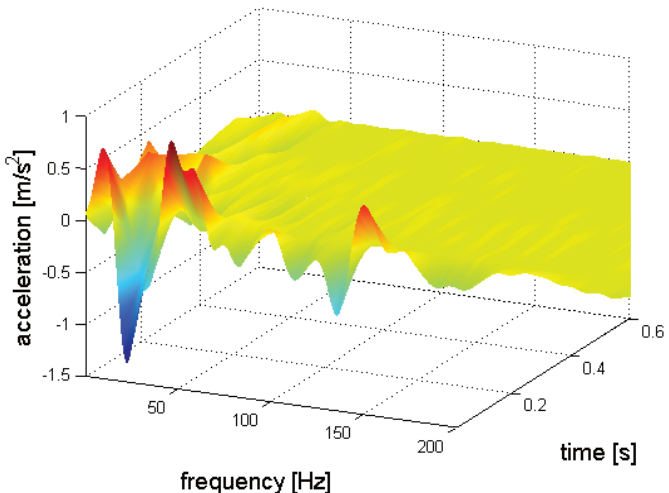

a) Driver

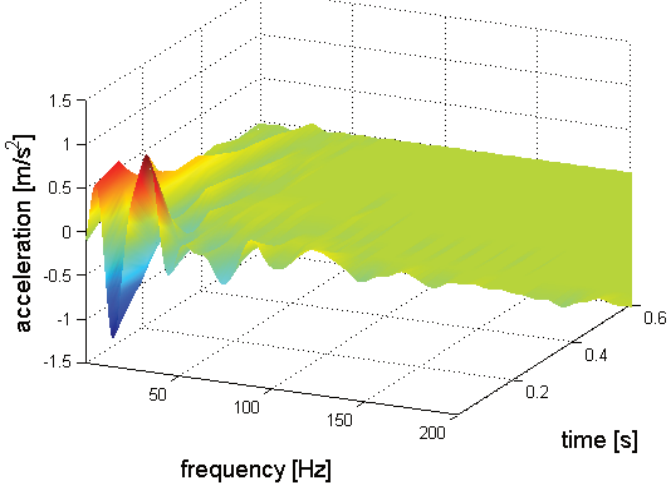

c) Left back passenger (behind the driver)

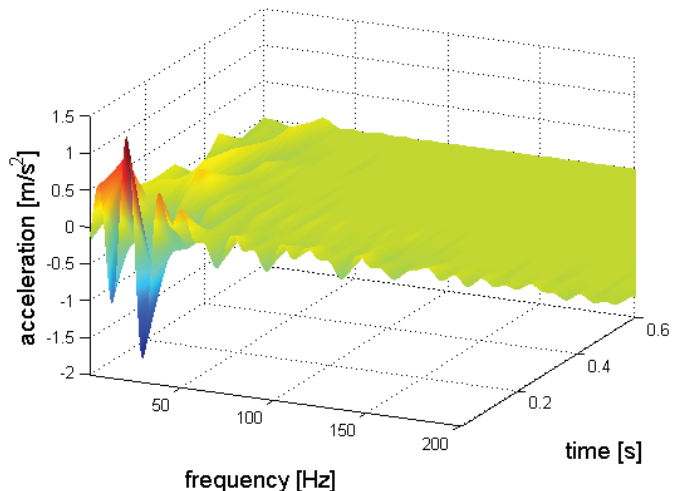

b) Front passenger

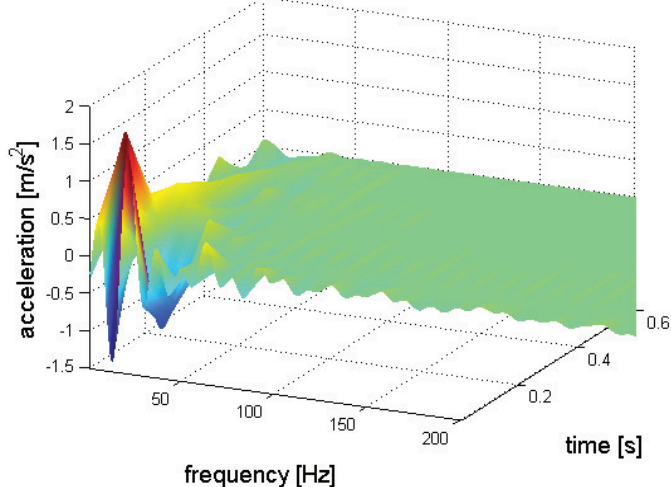

d) Right back passenger

Fig. 5.9. Time-frequency distribution of accelerations of vertical vibrations in the floor panel at points where passengers rest their feet

These vibrations are of non-stationary nature, and therefore, one should observe the distribution of component values of a signal in the domains of time and frequency simultaneously. 
For that purpose, the signals were transformed by application of the Short-Time Fourier Transform. The relevant results have been provided in the form of a time and frequency surface of distribution of the vibration signal components at the measuring points examined in Fig. 5.9.

Table 5.5. Phase shift of vibration measured on floor pan

\begin{tabular}{|l|c|c|c|c|}
\hline \multicolumn{1}{|c|}{ Measurement point } & Front left & Front right & Rear left & Rear right \\
\hline Time [s] & 0.019 & 0.032 & 0.0445 & 0.049 \\
\hline Phase shift [s] & & 0.013 & 0.0125 & 0.0045 \\
\hline Relative phase shift [s] & & 0.013 & 0.0255 & 0.03 \\
\hline
\end{tabular}

\subsection{Discussion, estimates of the floor panel's natural vibration surface}

The above transformations of vibration signals enable accurate analysis of the phenomena taking place. However, a representation of the said characteristics is a multi-element set assuming the form of vectors or matrices. It is for that fact that application of these measures in modelling becomes more difficult. Therefore, one should choose global measures of vibration signal characteristics. A measure commonly applied to determine the energy content of a signal is the root-mean-square value:

$X_{R M}=\left[\frac{1}{T} \int_{0}^{T} x(t) d t\right]^{1 / 2}$.

However, bearing the randomness and dynamism of vibrations in mind, it is still not an explicit representation of the signal characteristics. Therefore, it has been proposed for identification and evaluation of the surface of the vehicle floor panel vibrations should be developed basing on several statistical estimators of the vibration signal. It is assumed to enable representation of several signal characteristics in the form of a set of surfaces in a geometrical distribution of vibrations. The surface estimates provided were established basing on the following measures representing accordingly:

- measures of position - absolute mean value and peak-to-peak value,

- measures of dispersion - variance and standard deviation,

- measures of asymmetry and concentration - skewness and kurtosis,

- dimensionless measures - shape factor, peak factor, impulsivity factor and play factor.

Basing on the previous author's research those estimators show some sensitivity on changes of technical condition of suspension elements responding for vibration isolation and shock absorbing [40, 42, 56].

The methodology of determination of the surface estimates for characteristics of the vehicle floor panel vibrations entails determination of a set of global signal measures at specific geometrical points and determination of a surface approximating the distribution of these values on the floor panel. The vibration surfaces thus established are illustrated in Figs. 5.10-5.12.

Basing on the established analysis of the surfaces, one may conclude that assessment and identification of vibrations purely relying on the root-mean-square value, being the most uniform one, does not allow for defining the natural vibration surface in an appropriate manner. The changes observed in distributions of other values of the measures of position, dispersion and asymmetry imply considerable differences in the courses of vibrations at the measuring points analysed. The estimates of surfaces determined as functions of dimensionless coefficients show the changes occurring in the point distribution of floor panel vibrations. 


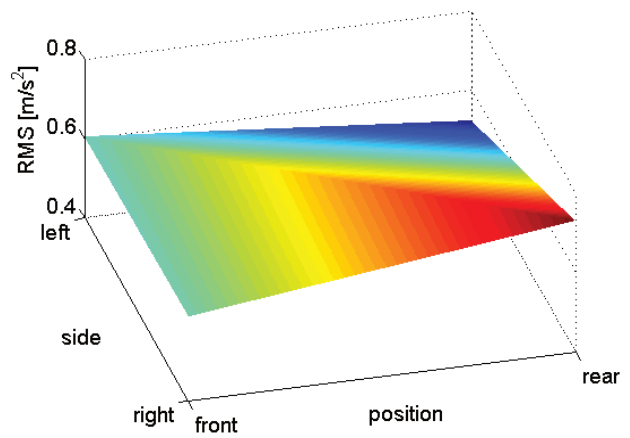

a)

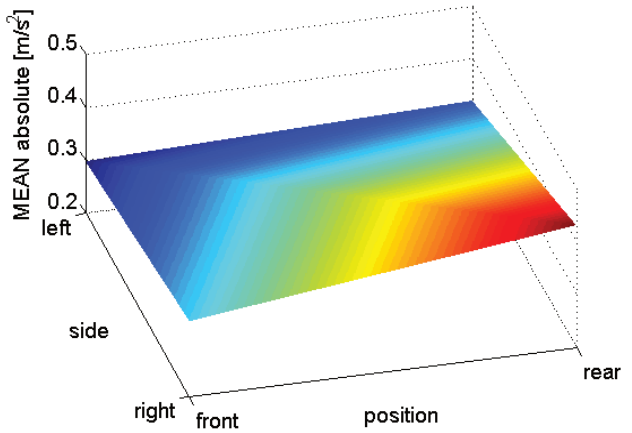

c)

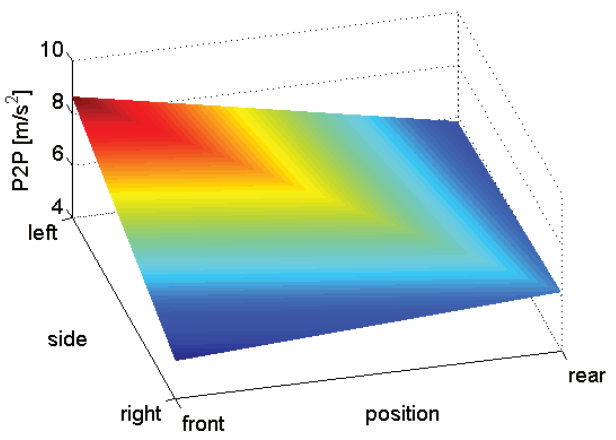

b)

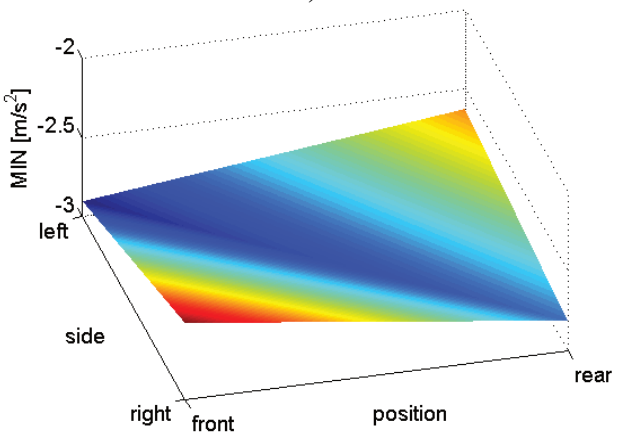

d)

Fig. 5.10. Global vibration surface estimates as functions:

a) value of $X_{R M S}$, b) peak-to-peak value, c) absolute mean value, d) minimal value

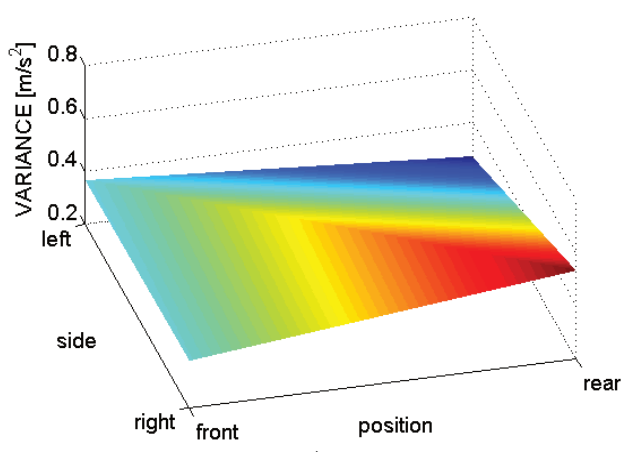

a)

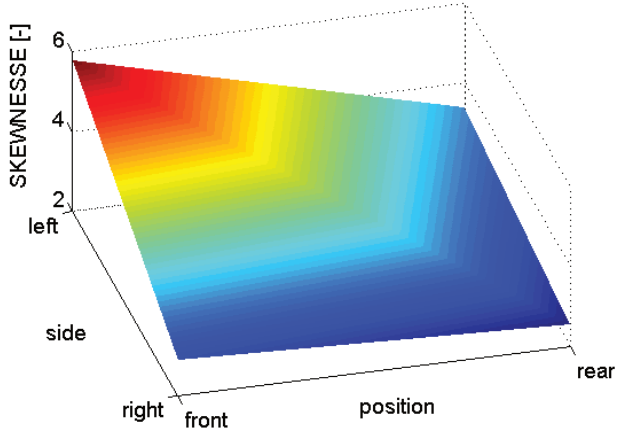

c)

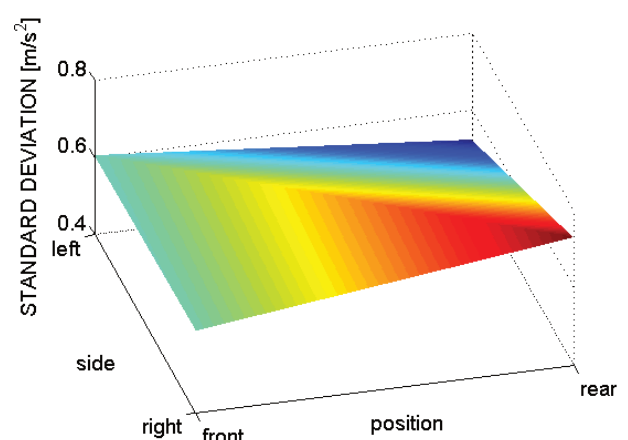

b)

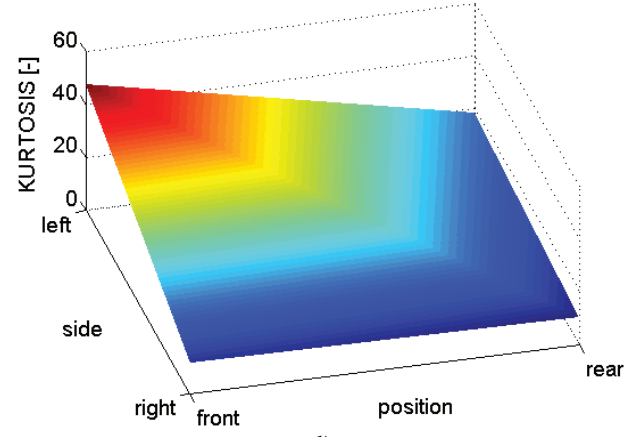

d)

Fig. 5.11. Dispersion and surface asymmetry estimates as functions: a) variance, b) standard deviation, c) skewness, d) kurtosis 


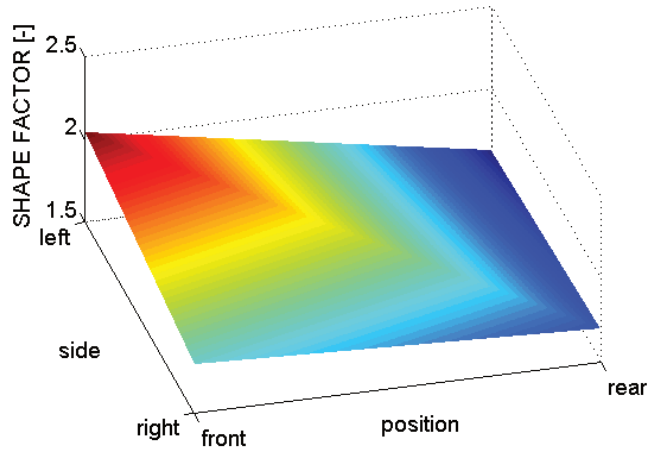

a)

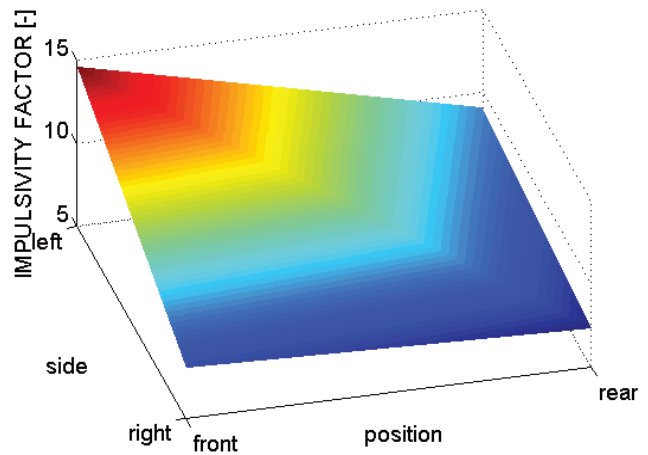

c)

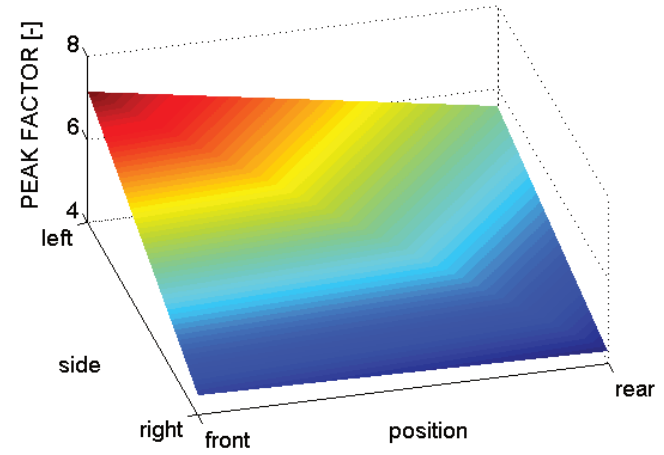

b)

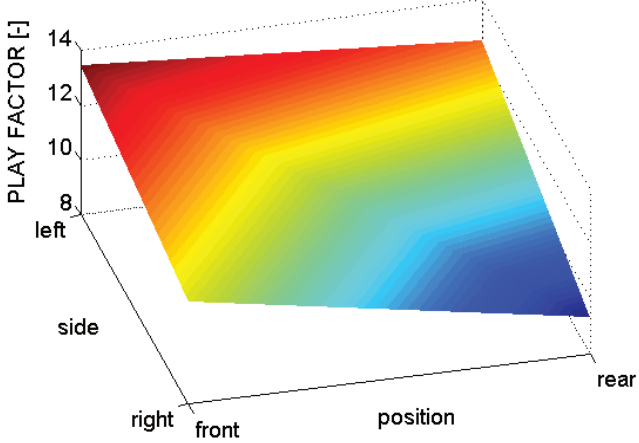

d)

Fig. 5.12. Estimates as functions: a) shape factor, b) peak factor, c) impulsivity factor, d) play factor

The presented methods, based on stochastic nature of signals, allows to observe the single vibration signal (time realization) as series of distribution of some estimators of the vibration. This kind of approach allows to define the vibration phenomena as vector of many statistical estimators described series of vibration values changes in time. Furthermore, a statistical analysis of the vibration courses being recorded was conducted as well, leading to determination of empirical surface estimates for natural vibrations, which enabled the vibrations to be assessed with regard to the geometrical position on the floor panel.

The studies performed are essentially of preliminary nature, and hence they require supplementation and further verification. 



\section{The influence of the technical condition of suspension elements and vehicle operating parameters on the vibration distribution in a vehicle construction generated by the forces acting on the wheel}

One of the main source of vehicle vibration is vertical movement of the wheel. It is caused by driving on road roughness. The excitation can be considered as time functions of the wheel strokes. It causes force transfer and dynamics response to the vehicle construction.

One may think of numerous systems and components of a vehicle which purpose is to absorb vibrations or minimise their impact. A vehicle suspension system, which consists of damping, springing and steering elements, are responsible to a considerable extent for damping of vibrations generated by road irregularities [28, 36, 38, 54, 55, 37, 134, 139]. Rubber components which provide mounting to such systems as engine, gearbox or exhaust system, also bear a fair share of responsibility for absorption of the vibrations generated by these assemblies. The purpose of every shock absorbing system and element is to minimise the impact exerted by vibrations on other systems as well as on the persons inside the vehicle. Oscillatory waves propagate and influence people via the vehicle structure, i.e. the frame or the body. Changes in the structure and composition of material may affect its capacity for the oscillatory wave propagation [153]. General vibrations are mainly transferred on men via lower extremities as well as the middle and lower section of the spine. The growing use of vehicles in urban areas causes that comfort in low-speed driving phase is an increasingly important consideration for manufacturers. The main sources of constraints in the occupant's environment are vibrations and sounds transmitted to the cabin via floor pan, dashboard, seats and steering wheels [7, 64].

\subsection{Research on the influence of changes in the technical condition of suspension elements and of the vehicle operating parameters on the vibration distribution in a vehicle structure}

The research comprised a series of active diagnostic experiments conducted in passenger cars. A car prepared to the tests, with the suspension components mounted having their technical condition known and their operating parameters preset, was subject to oscillatory input functions. For that purpose, a station enabling kinematic vibration excitation was used, making it possible to programme the dynamic frequency structure of input functions. The input frequency range envisaged comprised resonance frequency bands of both sprung and unsprung masses. Each time the test was conducted, a wheel of one vehicle axle was subject to the input function. The vibration accelerations were recorded at the chosen points of the structure (Fig. 6.1). For the sake of analysis of the suspension system damping parameters, vertical vibrations of unsprung elements (suspension arm) and the vehicle body (upper shock absorber mounting) were recorded coaxially. In order to analyse the distribution of vibrations being the sources of general vibration exposure of passengers, vibrations of the floor panel were recorded at locations where passengers rested their feet on the floor. These were also the points where general vibrations penetrated the human organism sitting inside the vehicle. 

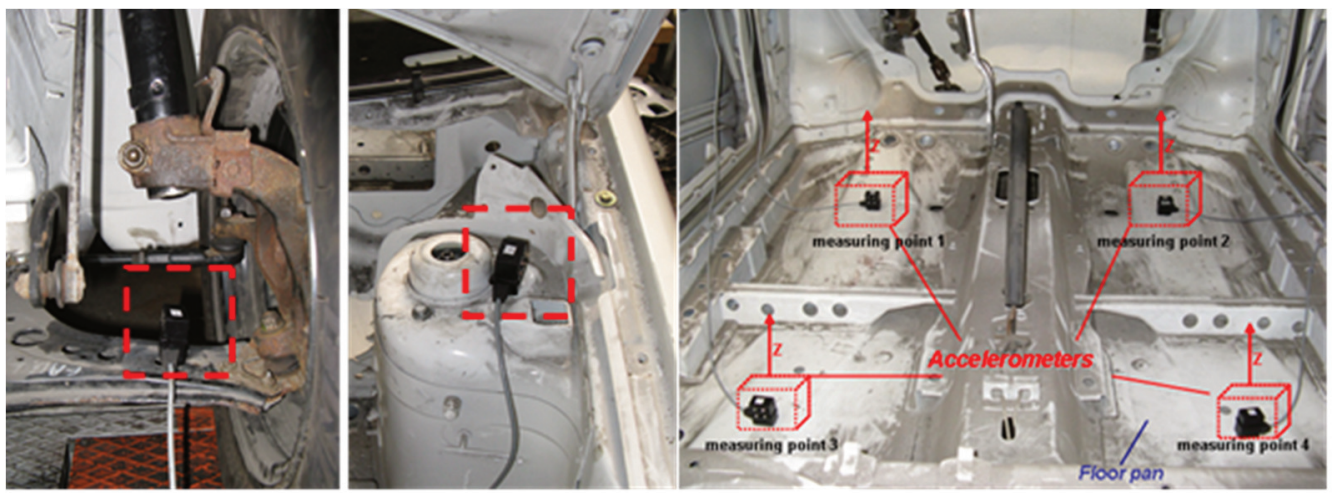

Fig. 6.1. Arrangement of vibration sensors across the structure of the vehicle tested

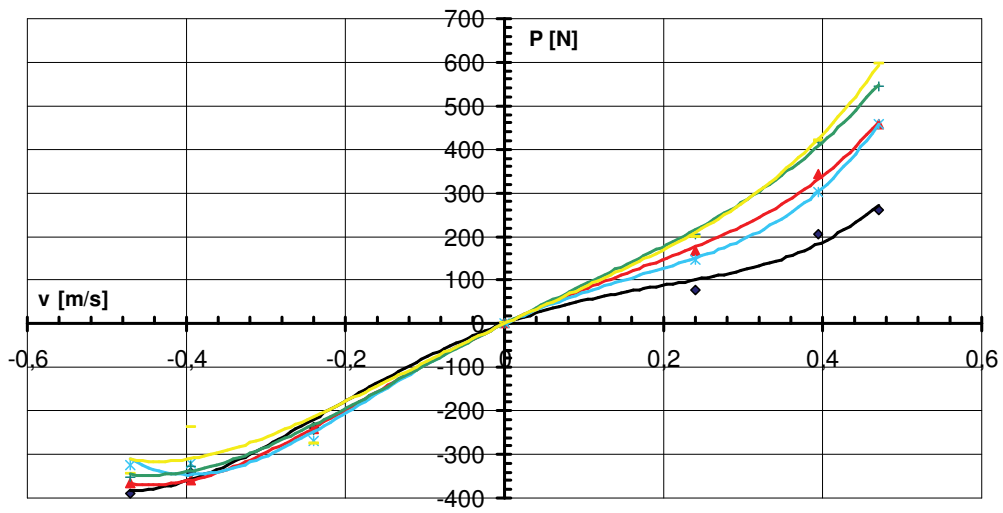

Fig. 6.2. Chosen applied damping characteristics of hydraulic shock-absorber: black - damping characteristics corresponding to the shock absorber with $60 \%$ of fluid volume, red $-70 \%$ of fluid volume, blue $-80 \%$ of fluid volume, green $-90 \%$ of fluid volume, yellow $-100 \%$ of fluid volume
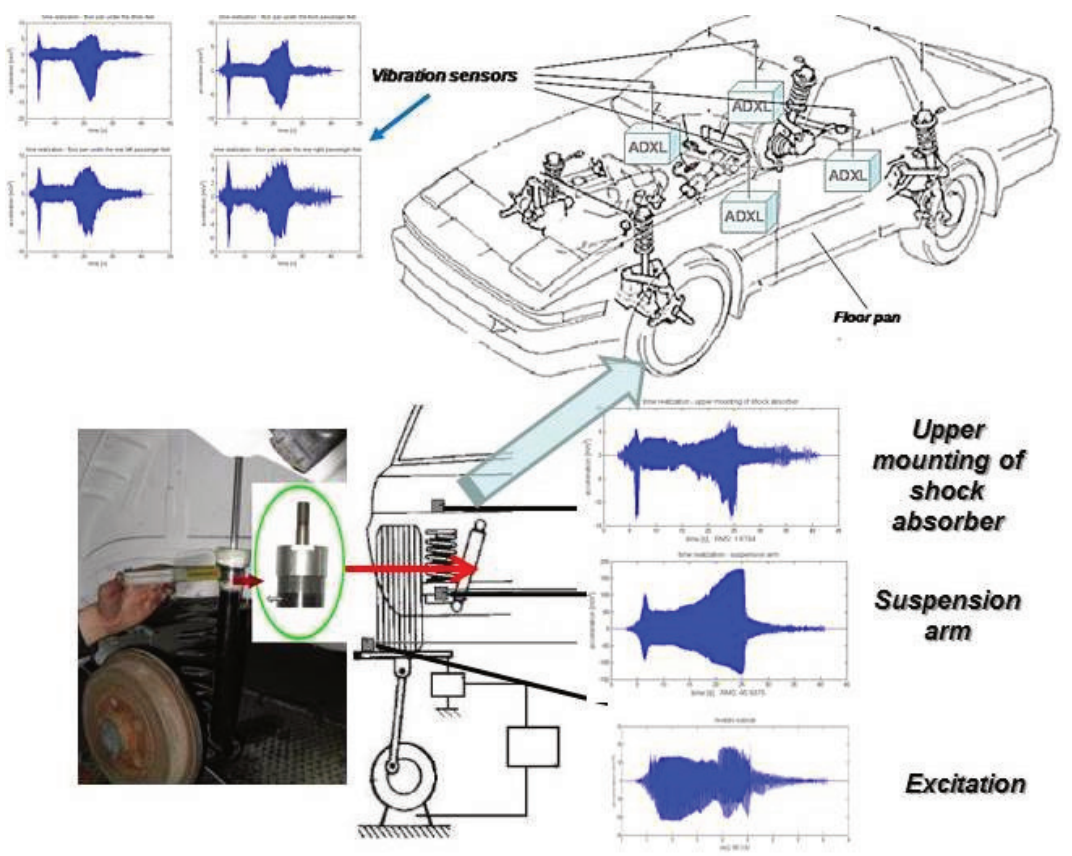

Fig. 6.3. Scheme of the experimental research 
For the sake of investigation the research on different technical condition of chosen suspension elements and operating parameters of vehicle were conducted. The analysis of the mechanical system dynamics requires conducting research with vibration excitation system. It enables forcing of the tested system with the excitation programmed for the chosen frequency bands. One of the assumption of the research method was guarantee of the force excitation recurrence with possibilities of changing of the technical parameters of the researched vehicle.

\subsubsection{Research on the impact of damping of shock absorbers on vehicle vibration}

The first investigation was conducted on main element of the suspension to minimize vibration transfer from wheel to car-body. This element is the shock absorber. It was assumed that the main factor of the vibration damping element's technical condition was the degree to which the shock absorber cylinder was filled with shock absorbing fluid. The author has conducted many of research on different damping parameters of vehicles as force-speed characteristics of shock absorbers [56]. Some of the results of damping characteristics of shock absorbers with different volume of fluid are shown in Fig. 6.2.

The scheme of the research and process of shock absorber filling with working medium is presented in Fig. 6.3.

\subsubsection{Research on the impact of the technical condition of springing elements on vehicle vibration}

The scope of the research contained the tests of the impact exerted by changes in the technical condition of a front suspension coil spring. A schematic representation of the suspension system of the vehicle tested with the subject of the research and the vibration recording points marked are presented in the Fig. 6.4.

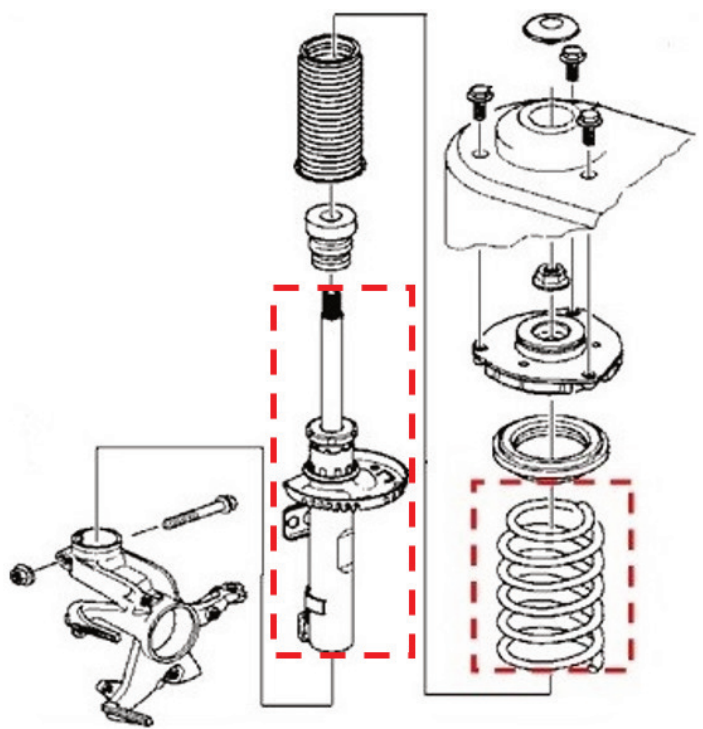

Fig. 6.4. Diagram of the front suspension system with marked objects of investigation

The identification of technical condition of suspension spring caused by defects (break) can be evaluated by the observation. The properties of coil springs are changing due to the operation period. It was assumed that the main factor of the vibration transfer related into spring is time and condition of operating in vehicle suspension. The experiments were conducted on passenger car with build in new and used (worn-out) suspension spring. The force vs. deflection characteristics of researching coil springs are presented in Fig. 6.5. 


\subsubsection{Research on the impact of the vehicle operating parameters on the vibration distribution}

The technical condition of suspension elements is extremely important for vehicle vibration. But there are some other factors impacted on vibration in car construction. Technical condition of the vehicle systems have to be tested periodically during the vehicle control in the service station. It has to be confirmed by the authorized car diagnostician. It doesn't relieve the vehicle's owners or users from obligatory car control and maintain the recommended values of operating parameters, as i.e. tire type and pressure. The scope of the research contained investigation on influence of pressure in tires and extra load, as operating parameters, on the vibration distribution in vehicle structure.

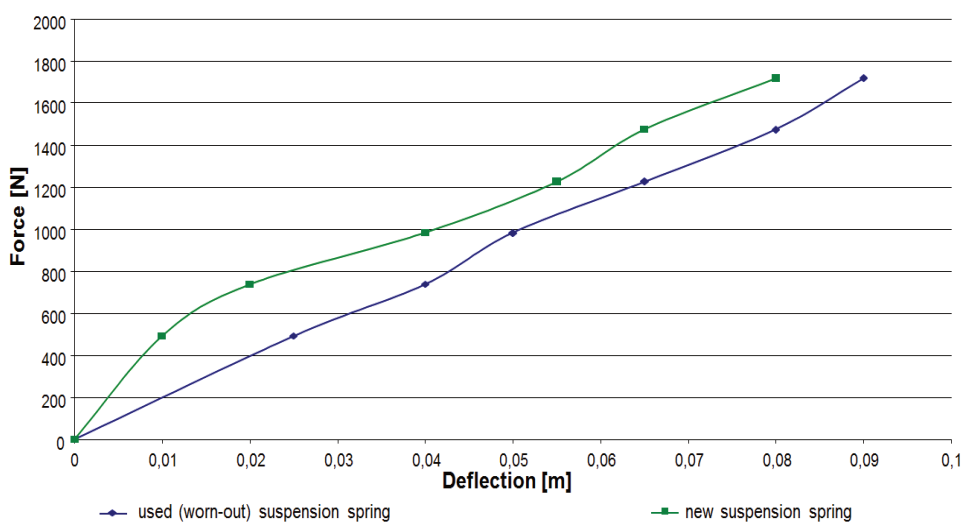

Fig. 6.5. Force vs. deflection characteristics of coil springs applied during research

\subsection{Distribution of vibration in vehicle structure for different technical conditions of suspension elements and operating parameters of the car}

Due to the scope of the research the vibration signals in chosen location in the vehicle structure were registered. Thus the analysis of vibration distribution in vehicle construction can be analysed. For the purpose of identification of the parameters are impacted on vibration transferred to car body from vertical movement of vehicle wheels some active diagnostics experiments were planned.

\subsubsection{Impact of the damping of shock absorber on distribution of vibration in vehicle structure}

Under study the volume of the shock absorber liquid have been changed and as the results the different damping characteristics of the suspension, presented in Fig. 6.2, were applied. The comparison of the vibration registered in chosen location in the vehicle structure with build in shock absorber with $100 \%$ and $50 \%$ of liquid volume are shown in Figs. 6.6 and 6.7.

For the sake of preliminary analysis of the influence of the investigated parameters on level of vibration occurring in vehicle structure the resonance windows were identified. According to mechanical vibration theory the impact of mechanical system properties on generation and propagation of vibration is significant during resonance frequencies passing. Therefore comparison of resonance window of vibration signal registered under studying of vehicle with shock absorbers with $50 \%$ and $100 \%$ of volume of liquid are shown in Fig. 6.8.

The preliminary evaluation of the influence can be conducted basing on simple global estimators, as RMS of total recorded signal and maximum value of the vibration acceleration in resonance window or maximum value of the absolute vibration acceleration in resonance window. The distribution of those estimators of 2 passenger cars vibration are presented in Figs. 6.9-6.11. 


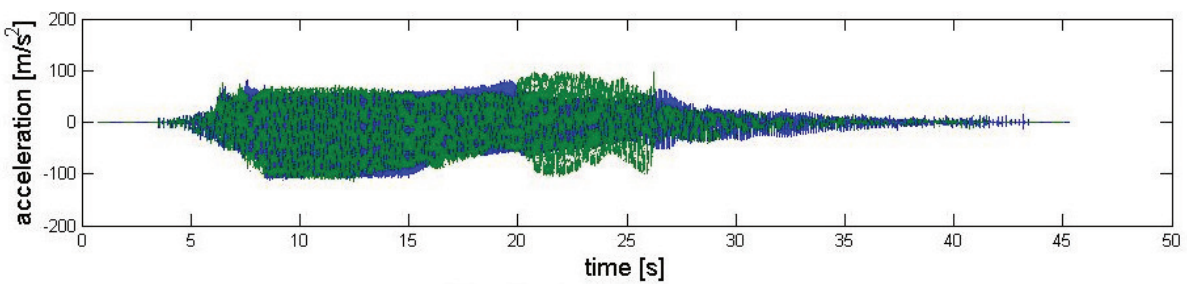

a) Time function - excitation plate

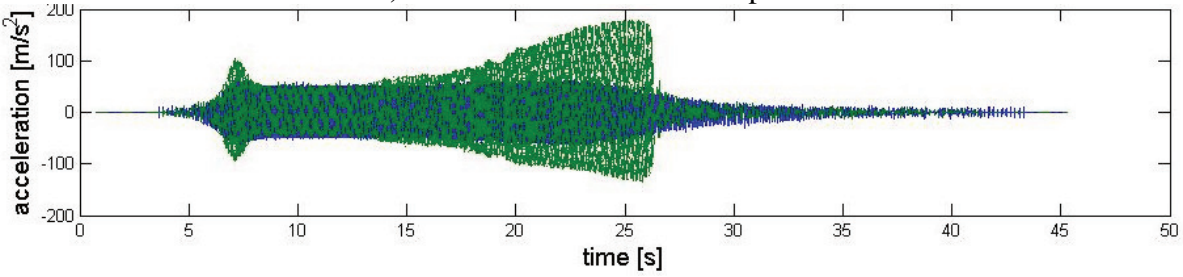

b) Time function - suspension arm

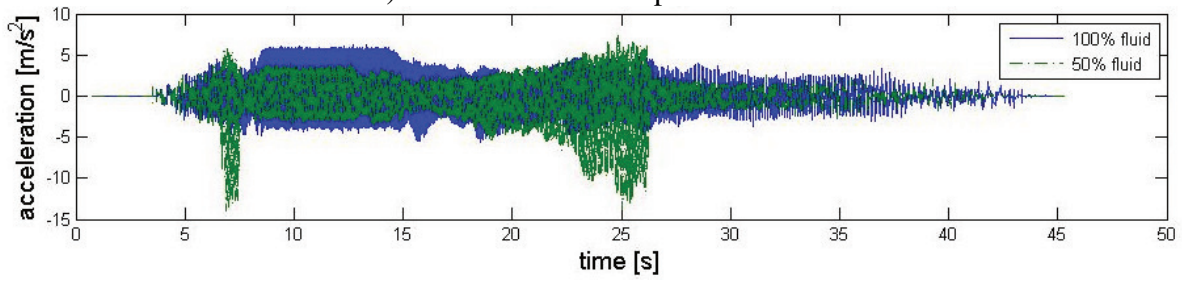

c) Time function - upper mounting of shock absorber

Fig. 6.6. Comparison in the vibration of exciter and suspension for the vehicles with build in shock absorbers with $100 \%$ (blue) and $50 \%$ (green) of liquid volume

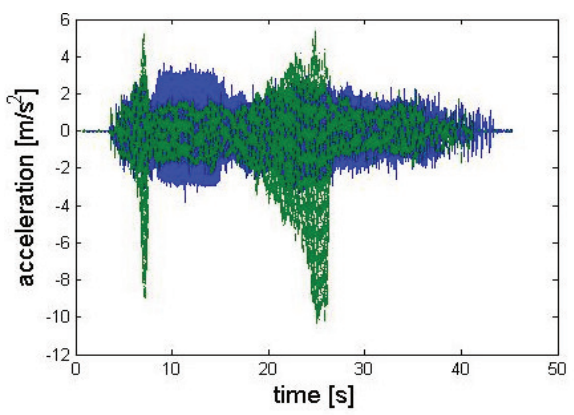

a) Time function - floor pan under the driver feet

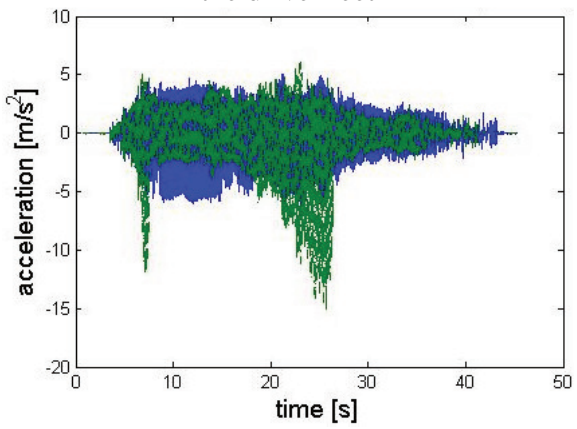

c) Time function - floor pan under the rear left passenger feet

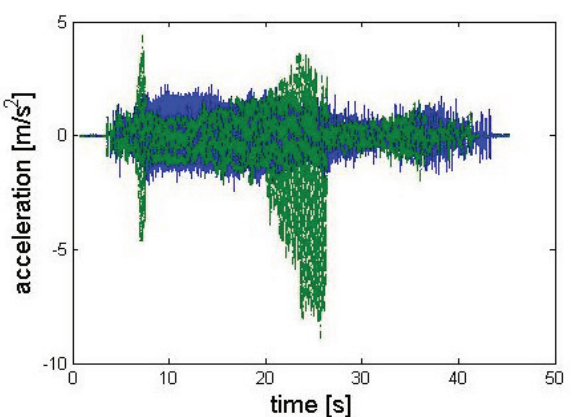

b) Time function - floor pan under the front passenger feet

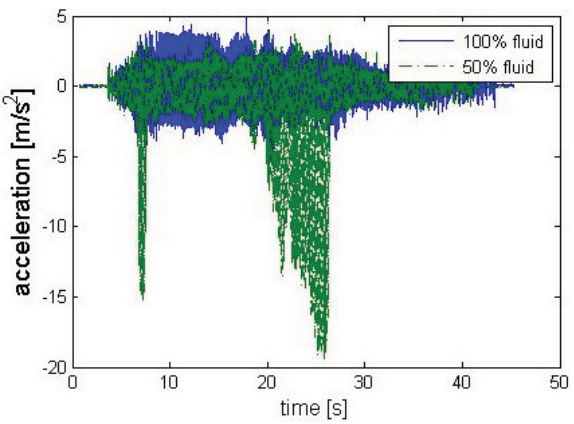

d) Time function - floor pan under the rear right passenger feet with build in shock absorbers with $100 \%$ (blue) and $50 \%$ (green) of liquid volume 


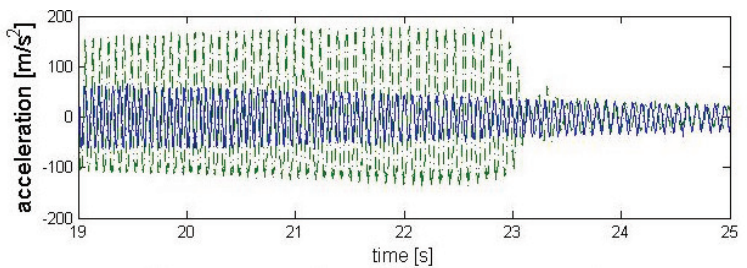

a) Suspension arm - resonance window

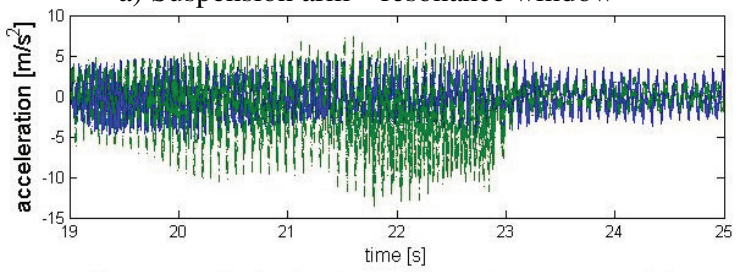

b) Upper mountig of shock absorber - resonance window

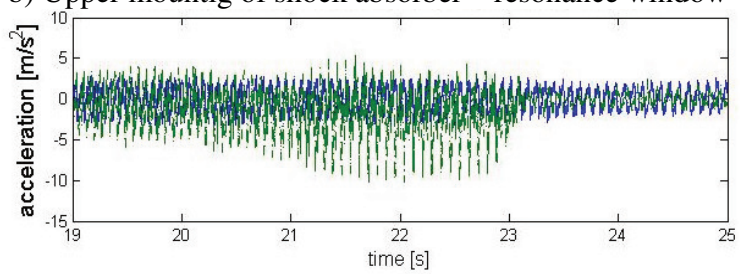

c) Floor pan under the driver feet - resonance window

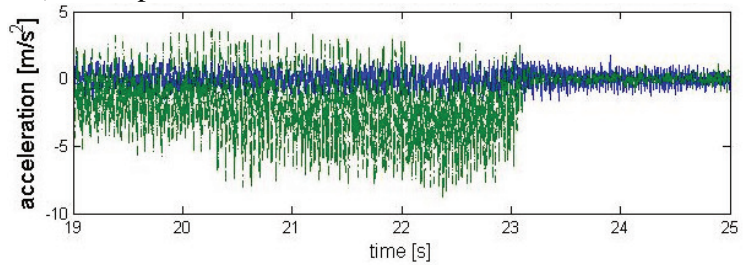

d) Floor pan under the front passenger feet

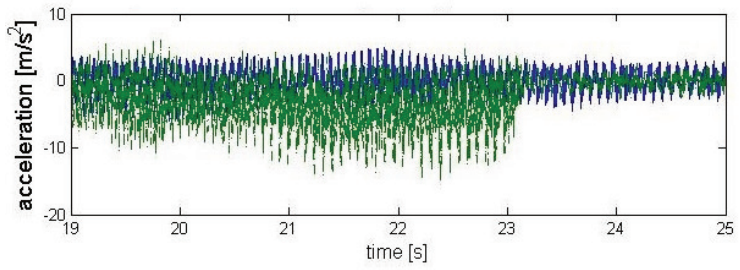

e) Floor pan under the rear left passenger feet - resonance window

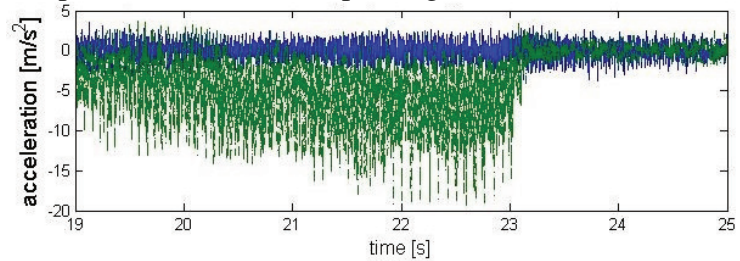

f) Floor pan under the rear right passenger feet - resonance window

Fig. 6.8. Comparison of resonance windows of vibration signal for the vehicles construction with build in shock absorbers with $100 \%$ (blue) and $50 \%$ (green) of liquid volume

The acceleration of vibration have been acquired by the channels: 2 - vibration exciter plate, 3 - suspension arm, 4 - upper mounting of shock absorber, 5 - floor under driver's feet, 6 - floor under front passenger's feet, 7 - floor under rear left passenger's feet, 8 - floor under rear right passenger's feet. 


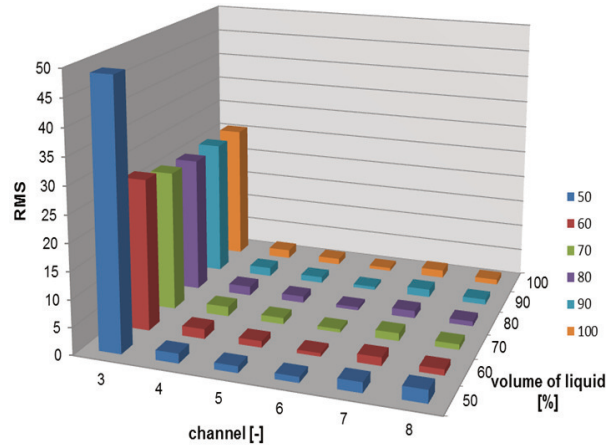

a)

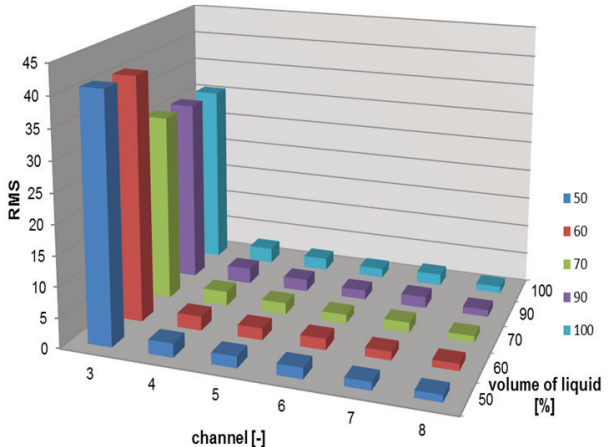

b)

Figs. 6.9. Distribution of changes in the RMS values of vibration acceleration signal for a variable damping properties of shock absorber: a) Skoda Fabia, b) Fiat Punto

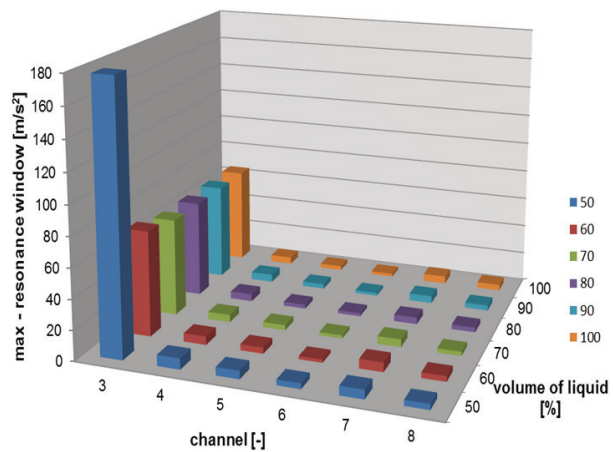

a)

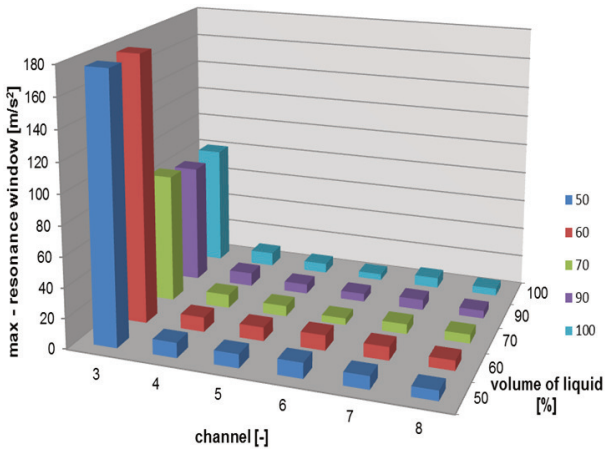

b)

Figs. 6.10. Distribution of changes in the maximum values of vibration accelerations in resonance window for a variable damping properties of shock absorber: a) Skoda Fabia, b) Fiat Punto

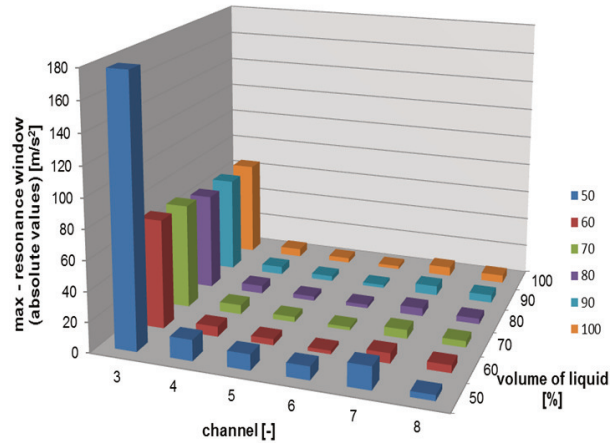

a)

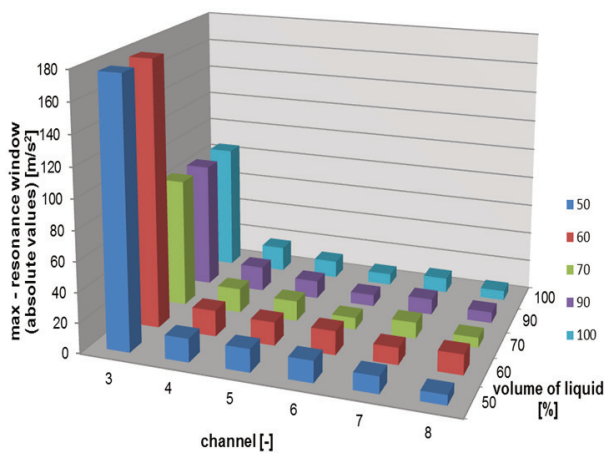

b)

Figs. 6.11. Distribution of changes in the maximum of absolute values of vibration accelerations in resonance window for a variable damping properties of shock absorber: a) Skoda Fabia, b) Fiat Punto

\subsubsection{Impact of the suspension spring properties on distribution of vibration in vehicle structure}

The coil springs with different characteristics, presented in Fig. 6.5, were applied into suspension of the vehicle. The comparison of the results obtained are shown in Figs. 6.12 and 6.13.

The properties of the spring is correlated to the suspension stiffness. It depends on many parameters and one of them is load of the vehicle. Thus the scope of the research contain investigation on different value of the vehicle's extra load. 


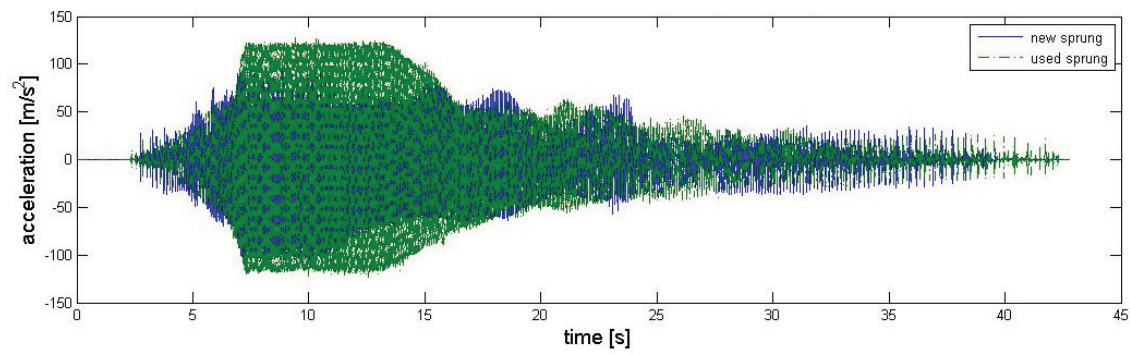

a) Time function - vibration exciter plate (channel 2)

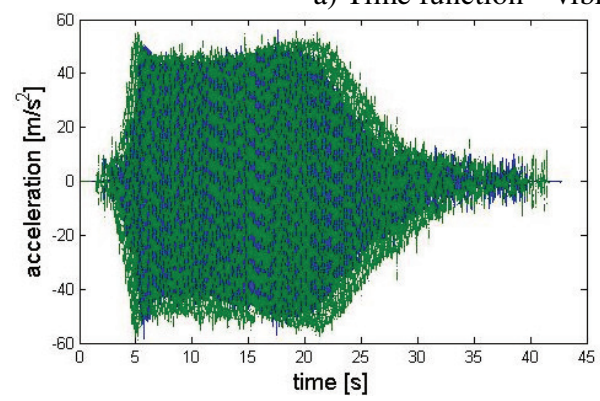

b) Time function - suspension arm (channel 3)

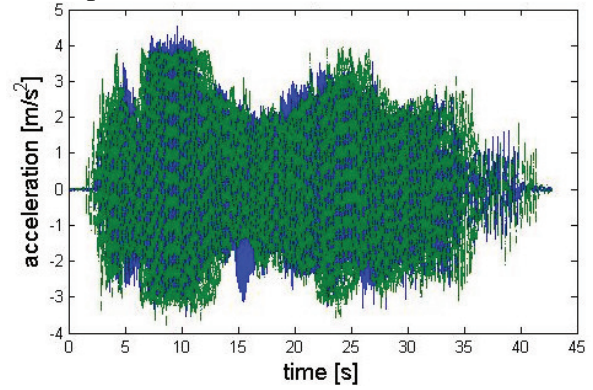

c) Time function - upper mounting of shock absorber (channel 4)

Fig. 6.12. Comparison in the vibration of exciter and suspension for the vehicles with build in new and used (worn-out) suspension spring

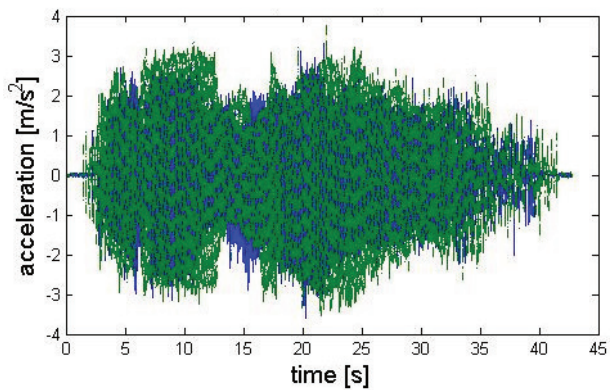

a) Time function - floor pan under the driver feet (channel 5)

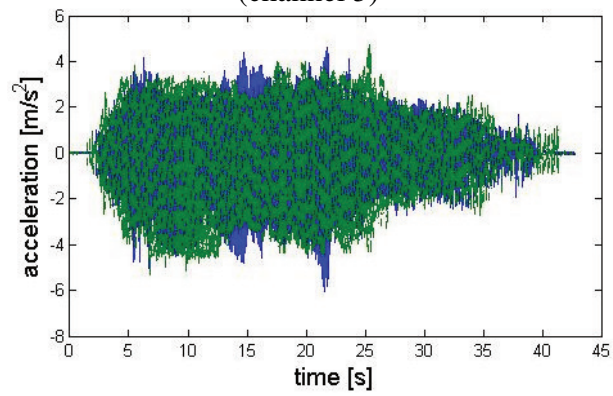

c) Time function - floor pan under the rear left passenger feet (channel 7)

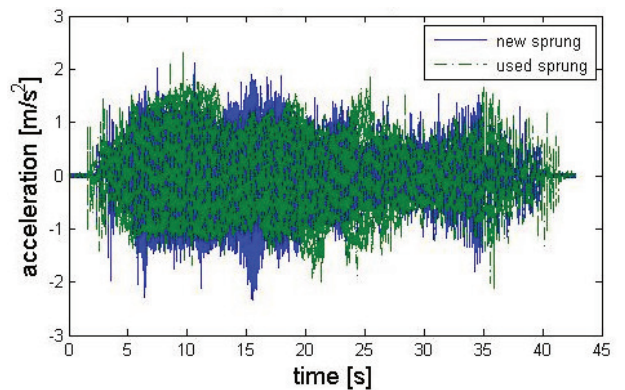

b) Time function - floor pan under the front passenger feet (channel 6)

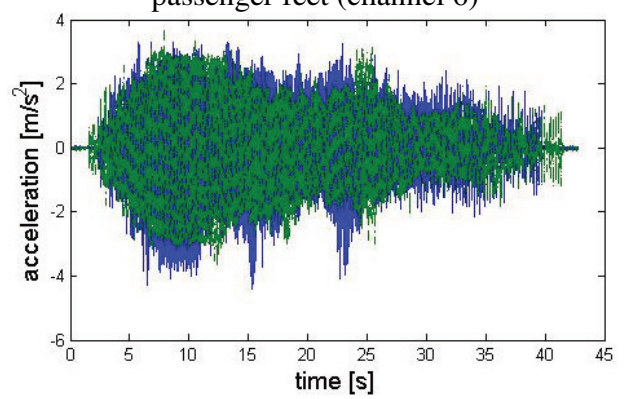

d) Time function - floor pan under the rear right passenger feet (channel 8)

Fig. 6.13. Comparison in the vibration of floor pan for the vehicles with build in new and used (worn-out) suspension spring

The distribution of the maximum value of the vibration acceleration in resonance window is presented. The distribution of those estimators of vehicle's vibration with build in new and used (worn-out) spring is presented in Fig. 6.14. 


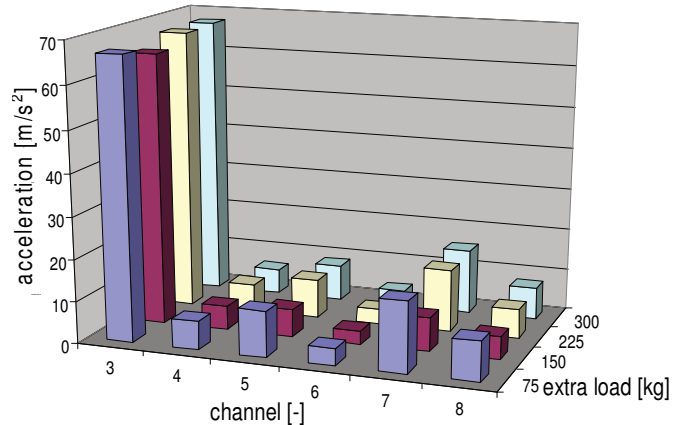

a)

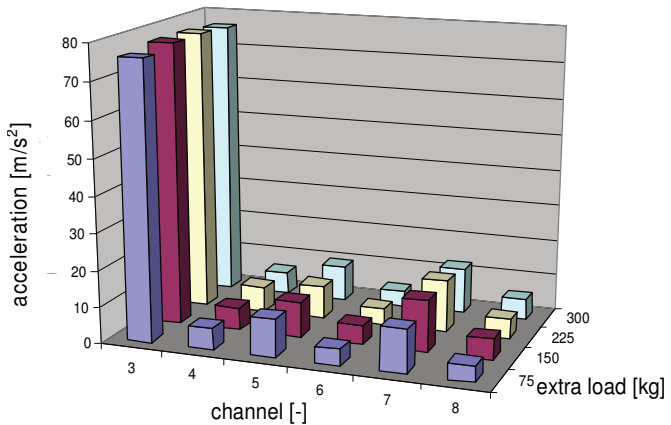

b)

Fig. 6.14. Distribution of changes in the maximum values of vibration accelerations in resonance window for a vehicle with build in a) new and b) used (worn-out) spring

\subsubsection{Impact of the pressure in tires on distribution of vibration in vehicle structure}

The scope of the research contain investigation of the vehicle operating parameters impact on the vibration distribution. During the research the pressure in tires and extra load were chosen as operating parameters. These parameters have significant influence on damping and stiffness properties of the vehicle suspension. It was assumed to conduct the investigation on complex factors of suspension stiffness properties, as combination of different values of tire pressure, extra load and coil spring properties. The comparison of the obtained results are shown in Figs. 6.15 and 6.16 .

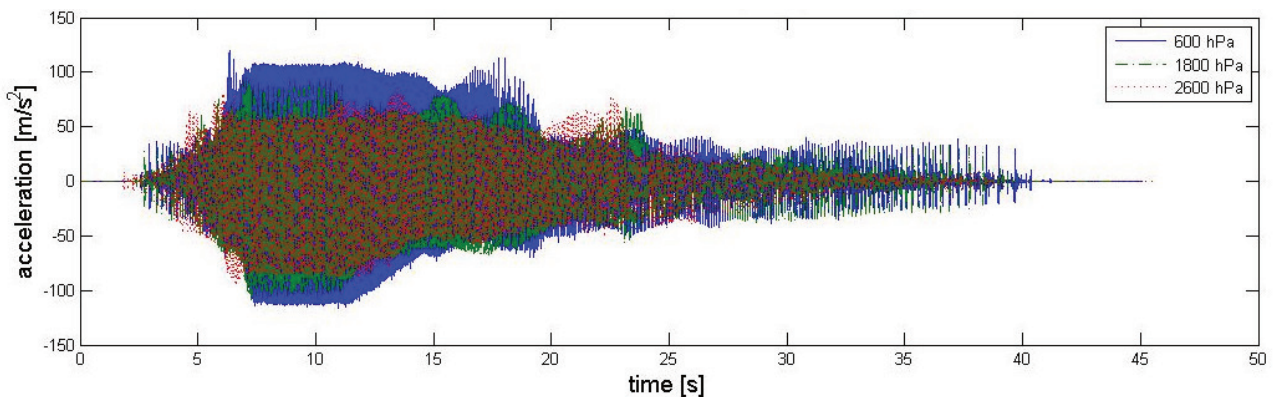

a) Time function - vibration exciter plate (channel 2)

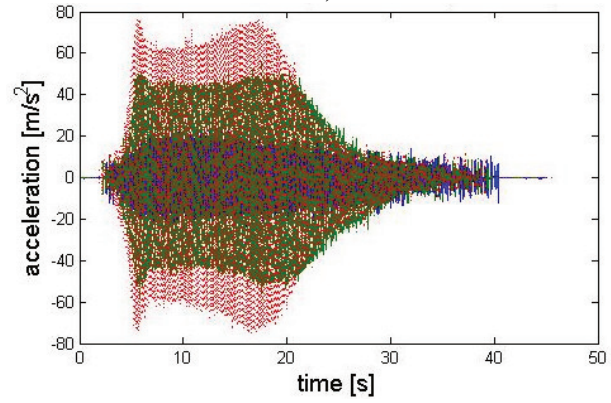

b) Time function - suspension arm (channel 3)

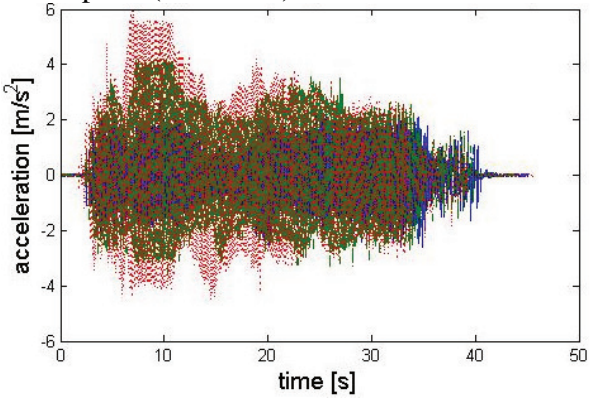

c) Time function - upper mounting of shock absorber (channel 4)

Fig. 6.15. Comparison in the vibration of exciter and suspension for the vehicles with different presure in tires $(600 \mathrm{hPA}, 1800 \mathrm{hPa}, 2600 \mathrm{hPA})$

The distribution of the vibration acceleration maximum values in resonance window is presented. Fig. 6.17 present the distribution of those estimators of vehicle's vibration with different pressure in tires with build in new and used (worn-out) spring. 


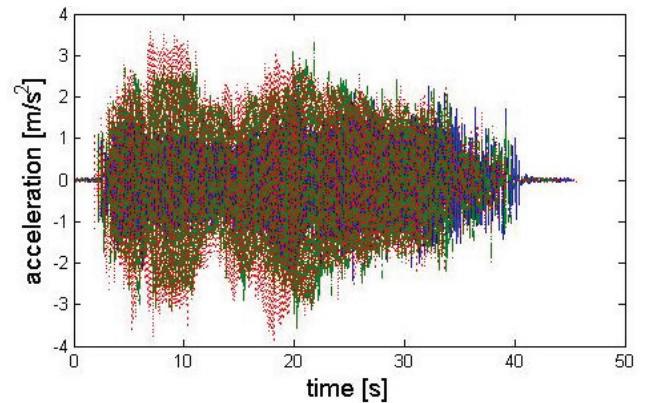

a) Time function - floor pan under the driver feet (channel 5)

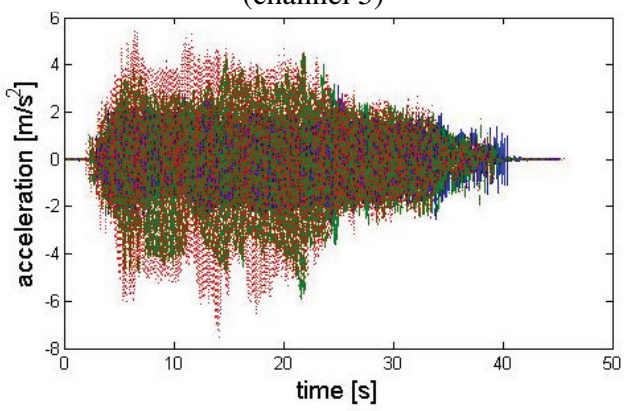

c) Time function - floor pan under the rear left passenger feet (channel 7)

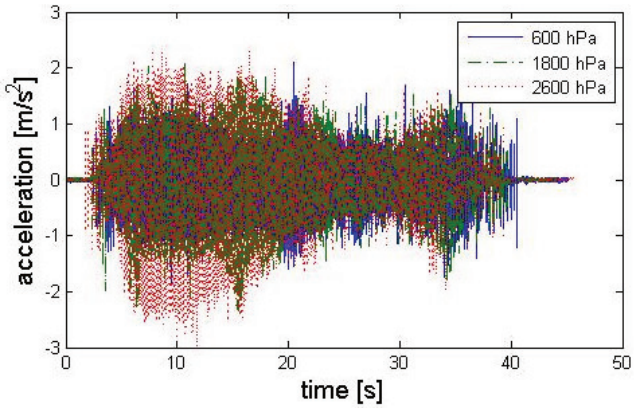

b) Time function - floor pan under the front passenger feet (channel 6)

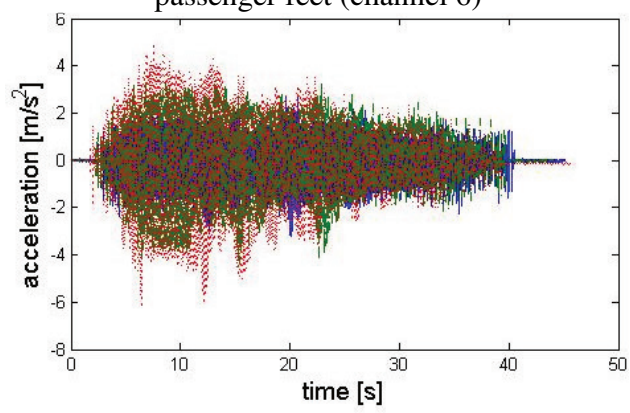

d) Time function - floor pan under the rear right passenger feet (channel 8)

Fig. 6.16. Comparison in the vibration of floor pan for the vehicles with different presure in tires (600 hPA, $1800 \mathrm{hPa}, 2600 \mathrm{hPA})$

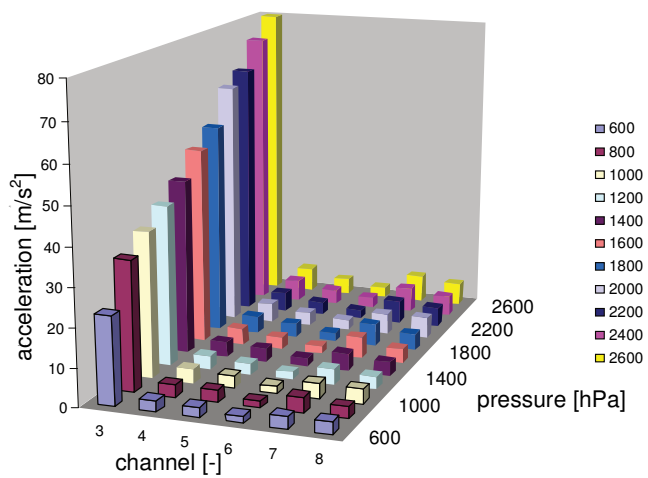

a)

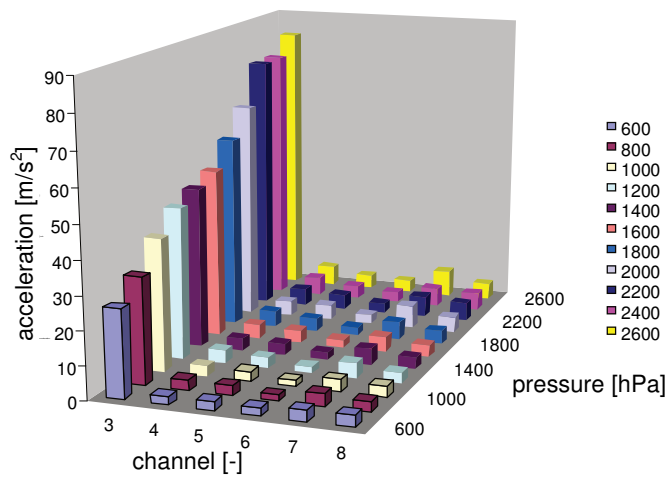

b)

Fig. 6.17. Distribution of changes in the maximum values of vibration accelerations in resonance window for a vehicle with different pressure in tires with build in a) new and b) used (worn-out) spring

\subsection{Discussion on impact of suspension components and operating parameters of a vehicle on the propagation of vibrations}

Studies of the impact exerted by the technical condition of suspension components and the chosen operating parameters of a vehicle on the propagation of vibrations generated by a road wheel vertical motion to the vehicle structure are particularly important in cognitive terms for the analysis and identification of the vibration sources and propagation in vehicles. The purpose of those studies was to identify factors affecting the propagation of vibrations caused by the dynamic impact of road irregularities on wheels of a moving vehicle.

Due to the fact that the technical condition of a decided majority of cars using roads is far from being normative, the vibration propagation studies were conducted for various technical 
conditions of suspension components as well as the vehicle operating parameters. For the sake of the preliminary assessment of the impact of the cases studied on the vibration propagation, courses of the vibration accelerations being recorded were compared and global measures of signals were determined and collated as maximum values, RMS values and maximum values in resonant windows.

For each case analysed, the impact of vibration propagation in a vehicle was established. The smallest impact was observed for the operating parameter of the additional vehicle load, which results from the car designers being well aware of the problem in question. As regards other factors, such as the vibration damping characteristics of shock absorbers (degree of filling with shock absorbing fluid), rigidity characteristics of a helical spring (determined by comparing a new and a used one) and tyre pressure, changes to these factors considerably affected the vibrations recorded at selected points of the vehicle structure. The recording results obtained proved the highest susceptibility to vibrations propagated through the vehicle structure for variable tire pressure, observed even on small changes. A significant impact was also exerted by a change in the vibration damping characteristics of a shock absorber, however, it was practically only experienced after exceeding the level of $60 \%$ corresponding to the absorber cylinder filling with shock absorbing fluid, and that is to be considered as considerable leakage. Furthermore, being analysed in such a manner, the results obtained have enabled assessment of the vehicle suspension damping properties by comparing the vibration values recorded at the suspension arm, being un unsprung element, and axially measured at the arm's upper mounting point, being a sprung element, as well as on the vehicle floor panel, comprising points of impact of the vehicle body induced vibrations on organisms of passengers. 



\section{The influence of the technical condition of suspension elements and vehicle operating parameters on the dominant frequency components of the vibration}

The evaluation of the technical condition of suspension elements and operating parameters of the motor vehicle based on time function of vibration signals or global estimators can be considered as preliminary overall assessment. The results presented in previous chapter confirm relations in founded case study and distribution of vibration in vehicle. The interpretation and conclusion based only on those results can be difficult. It enables comparison of total energy of vibration propagation in vehicle construction.

The human vibration perception has to be analysed in multi-criteria terms. It relies on amplitude and dynamics exposure to vibration. The evaluation of influence of technical condition of suspension elements and vehicle operating parameters on the vibration in terms of safety and comfort, should include analysis of the changes in dynamics of the phenomena. Thus the vibration signals have to be transformed to the frequency domain, because it enables analysis of dynamics. The most useful for that purpose is the Fourier transform.

\subsection{Fourier transform}

A signal is represented in the domain of frequency by application of the discrete Fourier transform. In the sphere of signal processing, it is mainly used to transform the $f(t)$ function, being continuous in the domain of time, into the $F(\omega)$ function, continuous in the domain of frequency. The discrete Fourier transform is based on an assumption that every signal may be obtained by adding sinusoid properties with appropriate phases and amplitudes. Therefore, a result of the discrete Fourier transform may be interpreted as a set of properties of the signal being examined in the function of frequency of component sinusoids. The Fourier transform based on Fourier series as periodic functions written as the sum of simple waves mathematically represented by sines and cosines. Due to the properties of sine and cosine, it is possible to recover the amplitude of each wave in a Fourier series using an integral. Thus the Fourier series, with period $T$, is an infinite sum of sinusoidal functions, each with a frequency that is an integer multiple of $1 / T$. The Fourier series also includes a constant, and hence can be written as:

$g(t)=a_{0}+\sum_{m=1}^{\infty} a_{m} \cos \left(\frac{2 \pi m t}{T}\right)+\sum_{n=1}^{\infty} b_{n} \sin \left(\frac{2 \pi n t}{T}\right)$,

where: $t$ - time, $T$ - period of fundamental function, $a_{0}-$ constant value, $a_{m}, b_{n}-$ constants coefficients of the Fourier series.

In many cases it is desirable to use complex exponential function (Euler's formula) to write Fourier series in terms of the basic waves $e^{-i 2 \pi \omega t}$, where Euler's equation can be derived by expanding the left side in a Taylor series (with variable theta or omega). Then expand the right side using the Taylor series expansions for cosine and sine and the results are identical:

$e^{i t}=\cos t+i \sin t$. 
Thus the Fourier transform can be express as formula:

$F(\omega)=\int_{-\infty}^{\infty} f(t) \cdot e^{-i 2 \pi \omega t} d t$.

Fig. 7.1 illustrates identification of Fourier series from fundamental function and transformation from time domain to frequency domain.

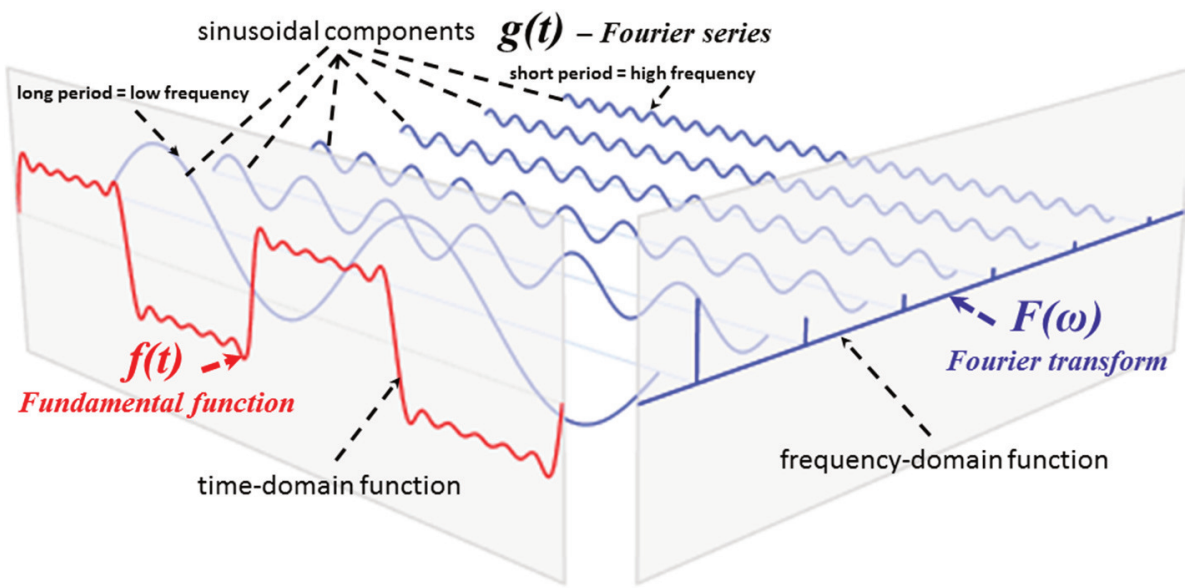

Fig. 7.1. Illustration of Fourier Transformation from time-domain function to frequency domain function

The continuous Fourier transform converts a time-domain function of infinite duration into a continuous spectrum composed of an infinite number of sinusoids. In engineering we deal with signals that are discretely sampled and of finite duration or periodic. For such data, only a finite number of sinusoids is needed and the Discrete Fourier Transform (DFT) is appropriate. The DFT of $N$ sampled data points $f_{j}$ are defined by:

$X_{k}=\sum_{j=0}^{N-1} f_{j} \cdot e^{-i 2 \pi j k / N}$.

The result of the DFT of an $N$-point input time series is an $N$-point frequency spectrum, with Fourier frequencies $k$ ranging from $-(N / 2-1)$, through the 0 -frequency and up to the highest Fourier frequency $N / 2$. The Discrete Fourier Transforms can be implemented with the Fast Fourier Transform (FFT) algorithm. FFTs are most efficient if the number of samples, $N$ is a power of 2. The advantage of the FFT over the DFT is that the operational complexity decreases from $O\left(N^{2}\right)$ for a DFT to $O\left(N \log _{2}(N)\right)$ for the FFT, so the speed of the algorithm is much higher.

It is important for a DFT or FFT to represent a function accurately, the original function must be sampled at a sufficiently high rate. The appropriate rate for a uniformly sampled time series is determined by the Nyquist-Shannon Theorem or Sampling Theorem. This theorem states that any continuous baseband signal may be identically reconstructed if the signal is bandwidth limited and the sampling frequency is at least twice the bandwidth of the signal. That critical sampling rate is known as the Nyquist rate, and it is a property of the time-domain signal based on its frequency content.

The chapter address results of analysis of influence of technical condition of suspension elements and vehicle operating parameters on the dominant frequency components of the vibration. For the purpose of identification of dominant frequency components of vibration dynamics it is sufficient to apply Fast Fourier Transformation (FFT). 
In order to analyse frequency components of vibration signals tests on vehicles excited to vibrate by means of an inductor controlled with a frequency converter were conducted. It enabled the system to be excited to vibrate within the chosen band of stabilised frequencies and, once the testing station had been switched off, made it possible to analyse the natural vibrations being damped. It is calculated for a car moving at a most popular speed about of $80 \mathrm{~km} / \mathrm{h}$, secondary roads, assuming an average wavelength of existing roads, the most energy of the vibration signal is contained in the band up to $20-30 \mathrm{~Hz}$. The active research experiments were conducted on real passenger car. The vehicle was excited to vibration by special kinematic excitation machine. The range of the frequency of the forced was set as dynamic linear increase up to ca. $21 \mathrm{~Hz}$, excitation with constant frequency (ca. $21 \mathrm{~Hz}$ ) for 5 seconds and excitation frequency decrease down to $0 \mathrm{~Hz}$ for 30 second period. This set up allows analysis bands of sprung and unsprung masses resonances.

\subsection{Dynamics of vibration in vehicle structure for different technical conditions of suspension elements and operating parameters of the car, identification of dominant frequency components}

For the purpose of analysis of the dynamics phenomena the transformation of the signal to the frequency domain has to be conducted. In the course of the results analysis the spectrums of the vibration were determined. The comparison of the vibration dynamics registered during research of the vehicle with build in shock absorbers with $100 \%$ and $50 \%$ of liquid volume and under research with different pressure level in the tires are presented in Figs. 7.2-7.5.

From the most general perspective of vibration phenomena that one may consider, what matters most is the natural vibration frequency bands for both sprung and unsprung masses, arranged in a vertical direction. For the proper identification of the dominant frequency component, which can determine dynamics of the vibration, analysis of the chosen frequency bands was conducted. Some of the exemplary results of the analysis are shown in Figs. 7.6-7.9.

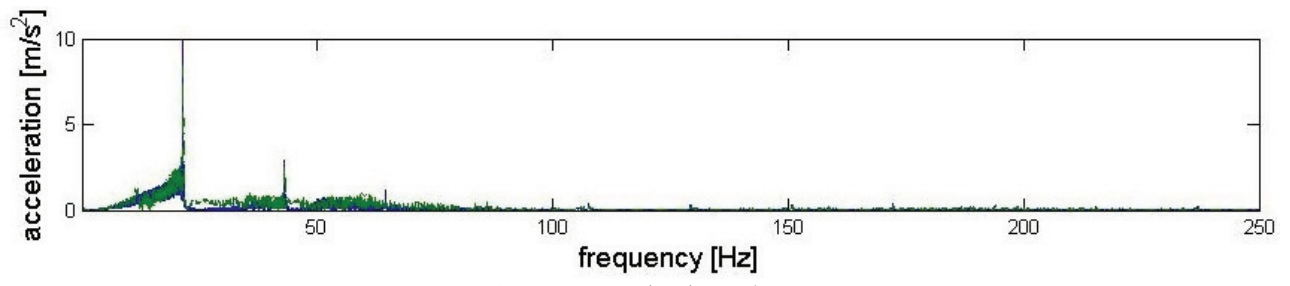

a) FFT - excitation plate

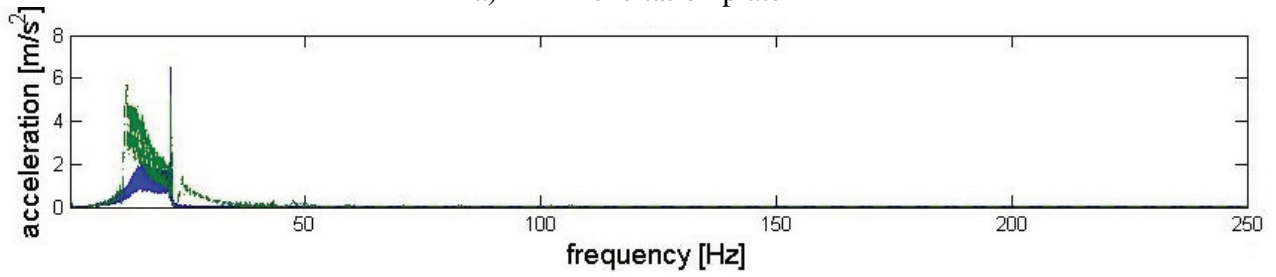

b) FFT - suspension arm

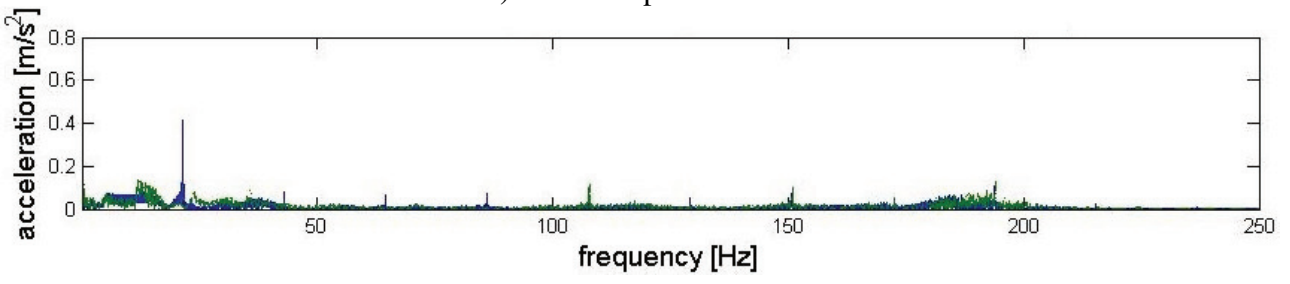

c) FFT- upper mounting of shock absorber

Fig. 7.2. Comparison in the vibration spectrums of exciter and suspension for the vehicles with build in shock absorbers with $100 \%$ (blue) and $50 \%$ (green) of liquid volume 


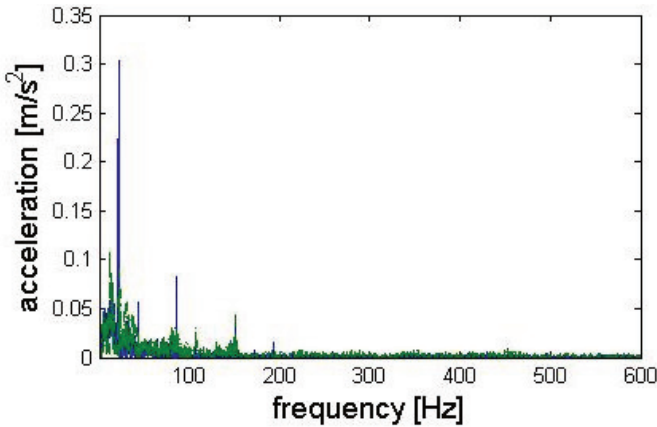

a) FFT - floor pan under the driver feet

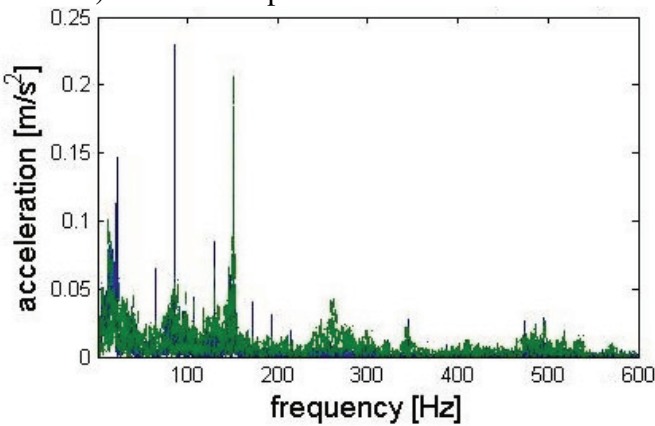

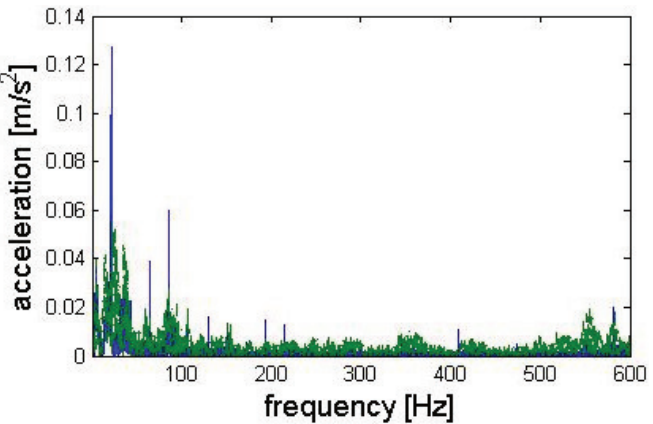

b) FFT - floor pan under the front passenger feet

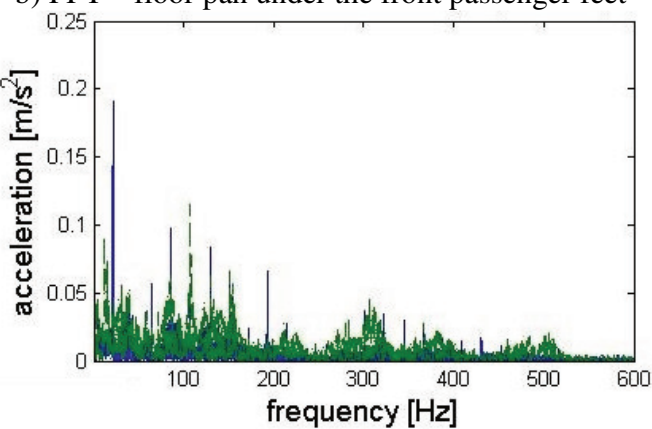

c) FFT - floor pan under the rear left passenger feet d) FFT - floor pan under the rear right passenger feet

Fig. 7.3. Comparison in the vibration spectrum of floor pan for the vehicles with build in shock absorbers with $100 \%$ (blue) and $50 \%$ (green) of liquid volume

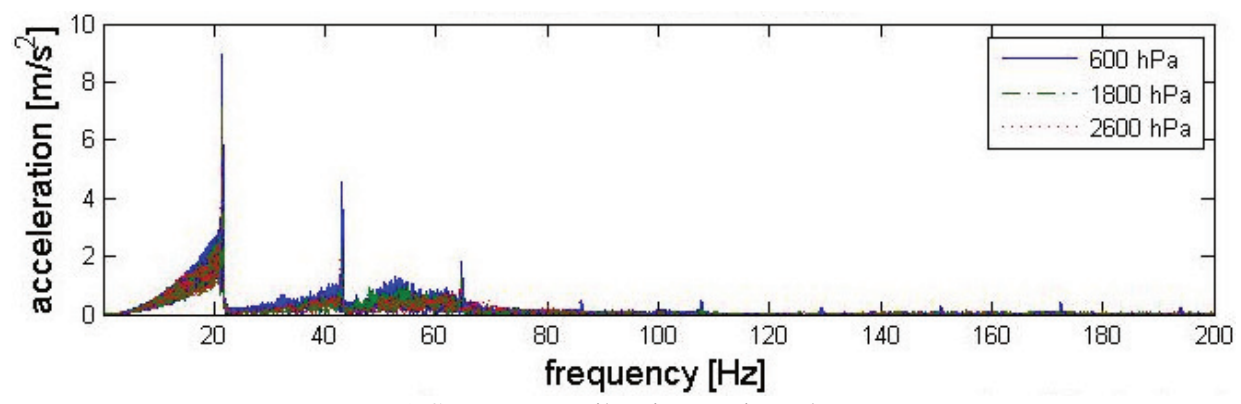

a) Spectrum - vibration exciter plate

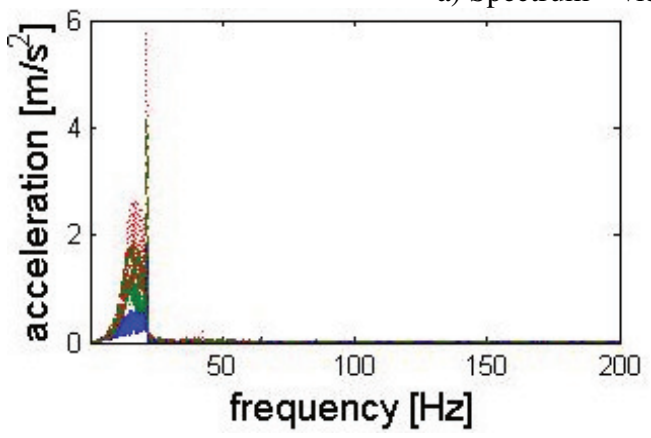

b) Spectrum - suspension arm

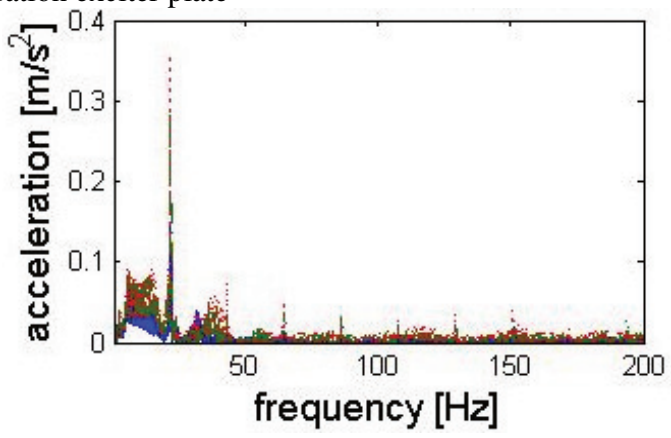

c) Spectrum - upper mounting of shock absorber

Fig. 7.4. Comparison in the vibration spectrums of exciter and suspension for the vehicles with different pressure level in tires (blue $-600 \mathrm{hPa}$, green $-1800 \mathrm{hPa}$, red $-2600 \mathrm{hPa}$ ) 


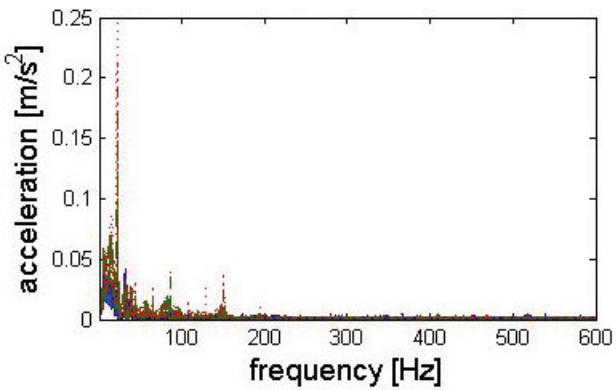

a) Spectrum - floor pan under the driver feet

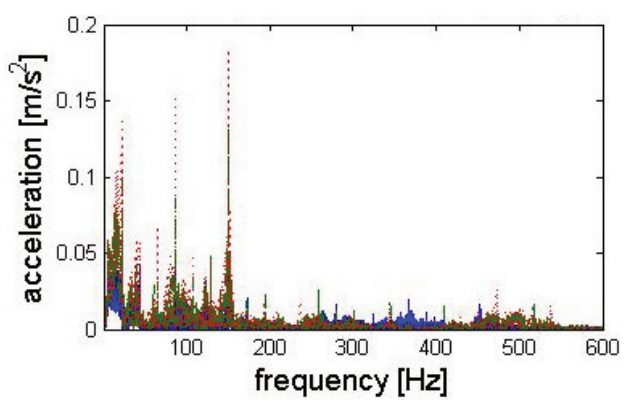

c) Spectrum - floor pan under the rear left passenger feet

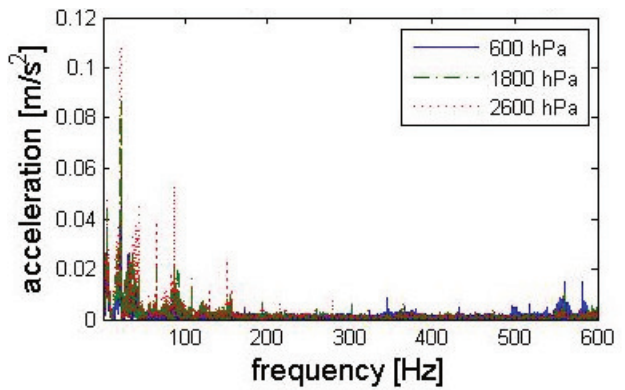

b) Spectrum - floor pan under the front passenger feet

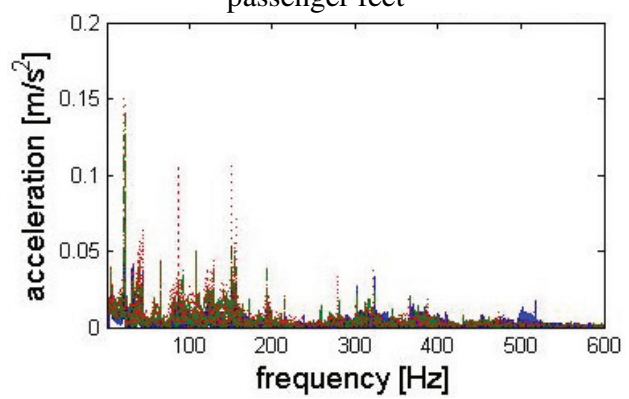

d) Spectrum - floor pan under the rear right passenger feet

Fig. 7.5. Comparison in the vibration spectrum of floor pan for the vehicles with different pressure level in tires (blue $-600 \mathrm{hPa}$, green $-1800 \mathrm{hPa}$, red $-2600 \mathrm{hPa}$ )

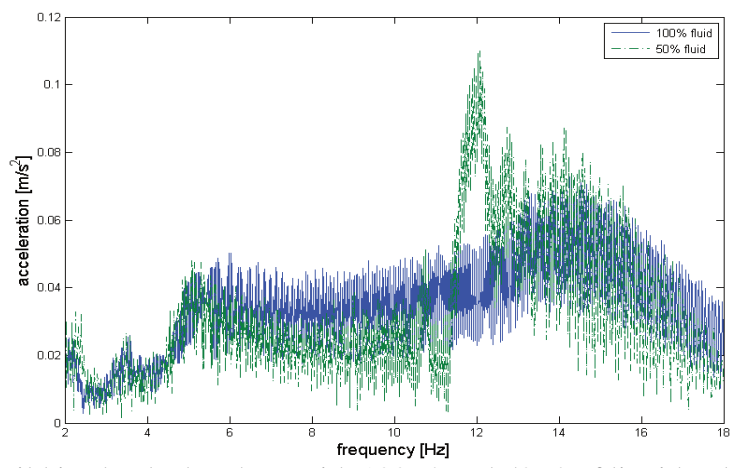

a) Build in shock absorbers with $100 \%$ and $50 \%$ of liquid volume

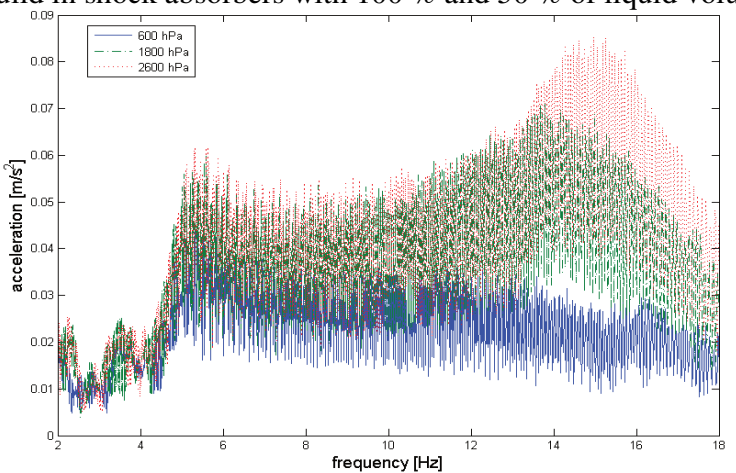

b) 600,1800 and $2600 \mathrm{hPa}$ pressure in tires

Fig. 7.6. Comparison of the spectrums of vibration under driver feet for the vehicles (2-18 Hz frequency band - resonances of sprung and unsprung masses) 


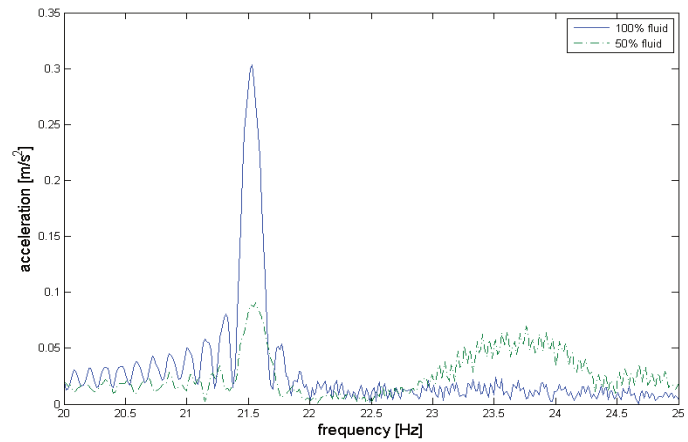

a) Build in shock absorbers with $100 \%$ and $50 \%$ of liquid volume

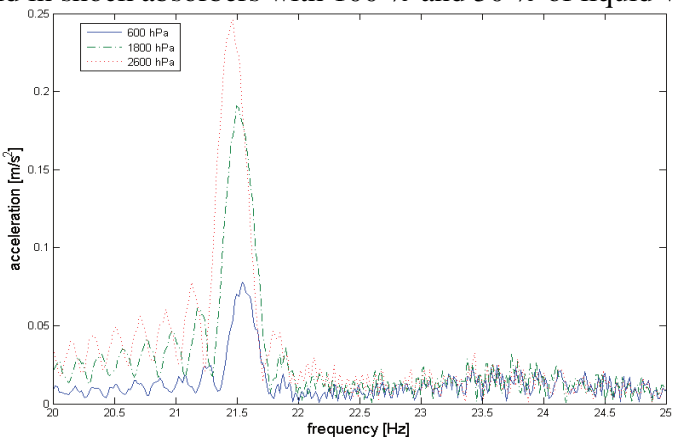

b) 600,1800 and $2600 \mathrm{hPa}$ pressure in tires

Fig. 7.7. Comparison of the spectrums of vibration under driver feet for the vehicles (20-25 Hz frequency band - frequency of constant period of excitation)

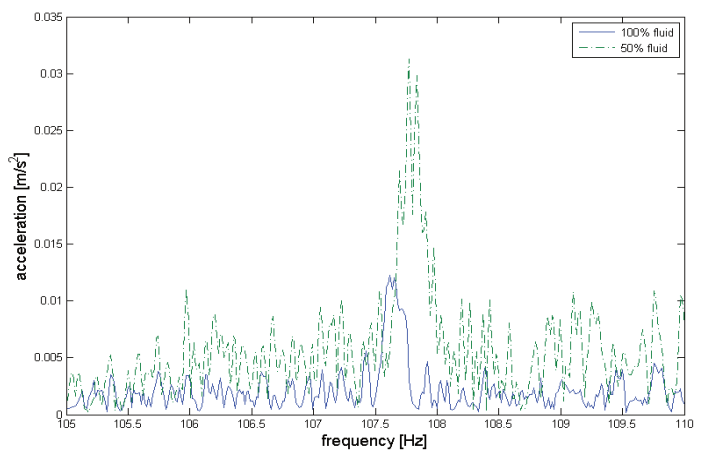

a) Build in shock absorbers with $100 \%$ and $50 \%$ of liquid volume

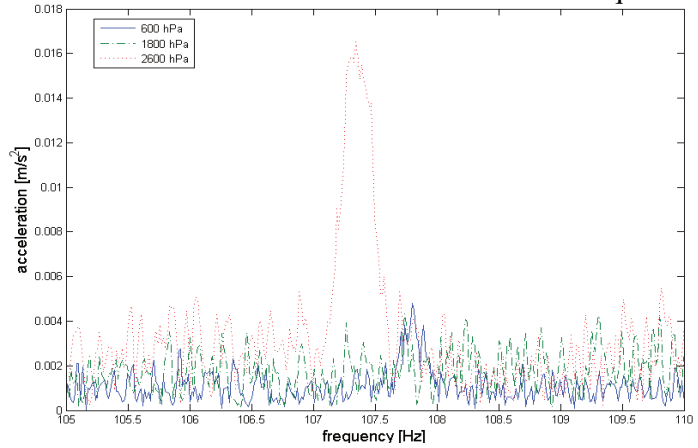

b) 600,1800 and $2600 \mathrm{hPa}$ pressure in tires

Fig. 7.8. Comparison of the spectrums of vibration under driver feet for the vehicles (105-110 Hz frequency band - frequency of 5th harmonics of constant period of excitation) 


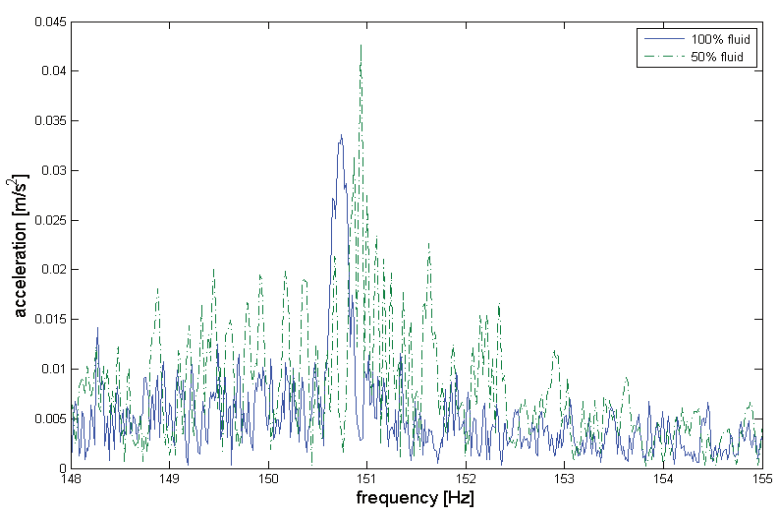

a) Build in shock absorbers with $100 \%$ and $50 \%$ of liquid volume

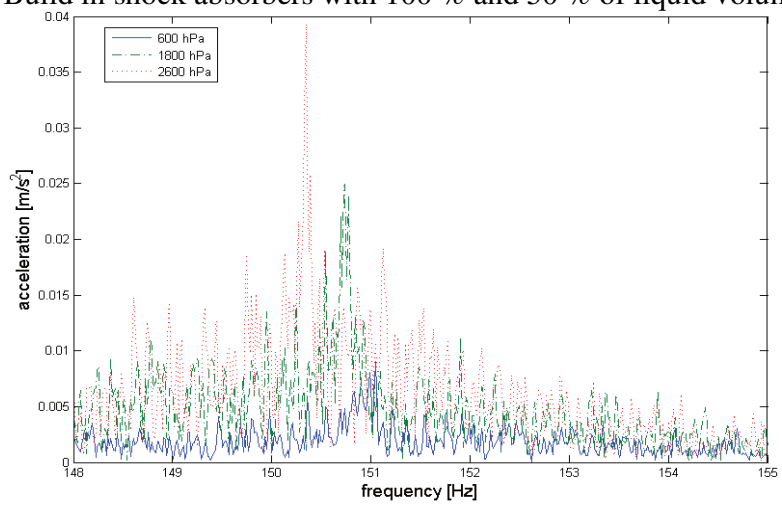

b) 600,1800 and $2600 \mathrm{hPa}$ pressure in tires

Fig. 7.9. Comparison of the spectrums of vibration under driver feet for the vehicles (148-155 Hz frequency band - frequency of 7 th harmonics of constant period of excitation)

\subsection{Frequency based estimators}

Frequency distribution of the signal enables analysis of the predominant dynamic components in the system. It also makes it possible to seek resonant frequencies and input frequencies. The detailed frequency analysis conducted consisted in a preliminary selection of characteristic frequency bands (with predominant values of the signal spectrum amplitudes) for individual sensor mounting points. The following frequency bands were selected for the analysis of the vehicle floor pan vibrations:

- from $2[\mathrm{~Hz}]$ to $4.5[\mathrm{~Hz}]$

- from $5[\mathrm{~Hz}]$ to $7[\mathrm{~Hz}]$

- from $12[\mathrm{~Hz}]$ to $17[\mathrm{~Hz}]$

- from $21[\mathrm{~Hz}]$ to $22[\mathrm{~Hz}]$

- from $33[\mathrm{~Hz}]$ to $38[\mathrm{~Hz}]$

- from $62[\mathrm{~Hz}]$ to $66[\mathrm{~Hz}]$

- from $85[\mathrm{~Hz}]$ to $88[\mathrm{~Hz}]$

- from $105[\mathrm{~Hz}]$ to $110[\mathrm{~Hz}]$

- from $150[\mathrm{~Hz}]$ to $155[\mathrm{~Hz}]$

- from $190[\mathrm{~Hz}]$ to $196[\mathrm{~Hz}]$

Fig. 7.10 illustrates the signal frequency bands assumed for the analysis of the vehicle floor panel vibrations.

The next stage of the analysis involved developing a collation of maximum values of the signal spectrum amplitudes in the chosen frequency bands. 


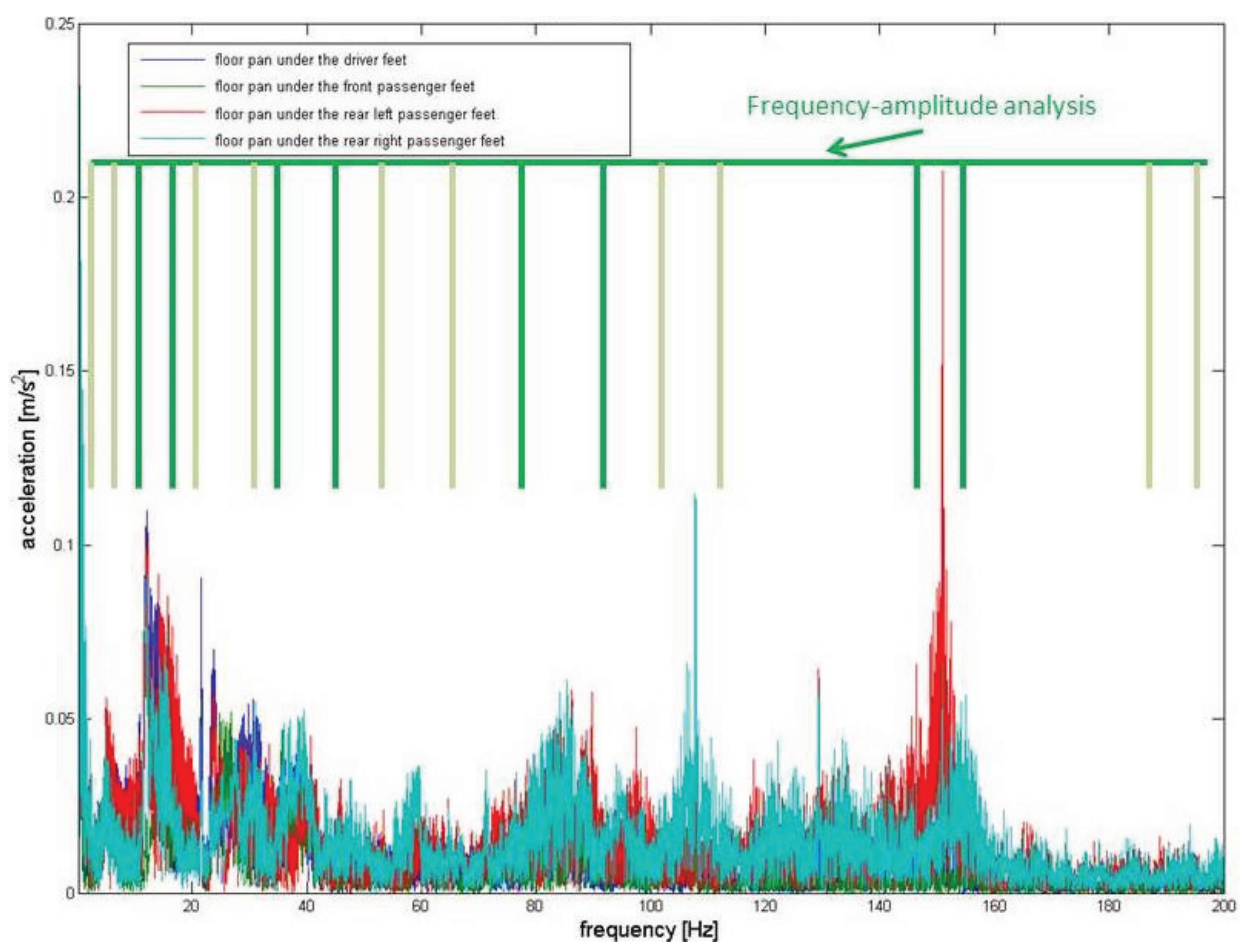

Fig. 7.10. Bands analyses for the vehicle floor panel vibration signals recorded

\subsubsection{Influence of the damping of shock absorber on dominant frequency components of vibration in vehicle structure on the floor pan}

The results of analysis of vibration for the chosen shock absorber technical condition parameters examined were collected in the database form. Figs. 7.11-7.12 graphically present chosen analysis results obtained.

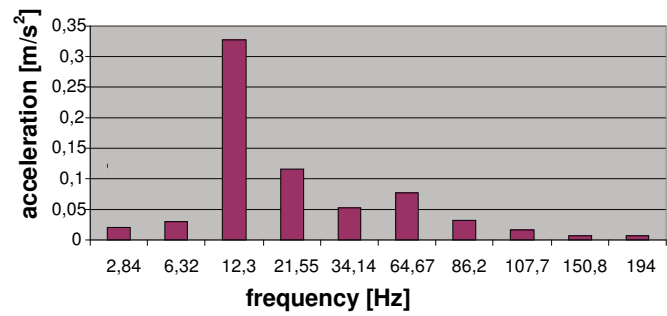

a) Driver's feet resting spot

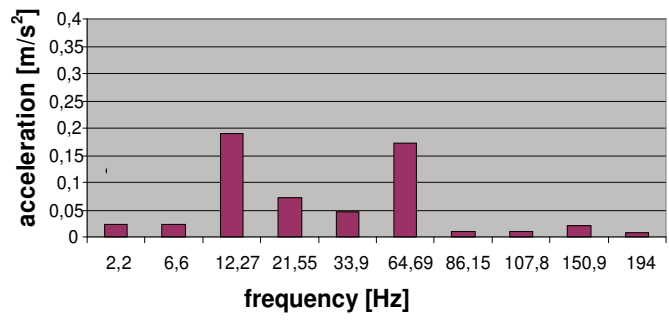

c) Rear left passenger's feet resting spot

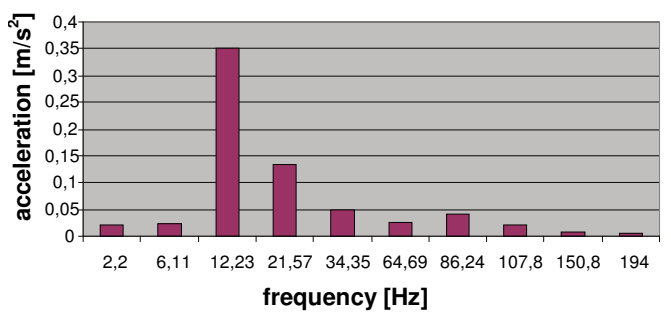

b) Front passenger's feet resting spot

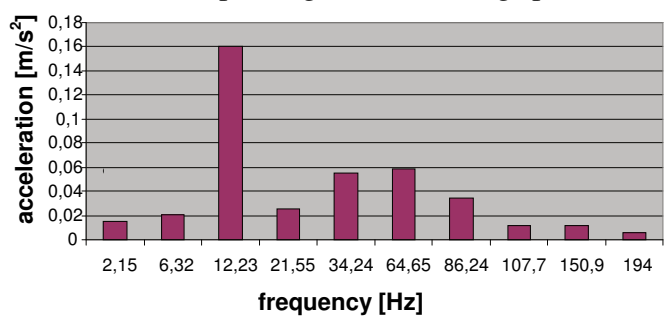

d) Rear right passenger's feet resting spot

Fig. 7.11. Distribution of maximum amplitudes of vibration signal spectra obtained for a floor panel of passenger car for the selected characteristic frequencies (shock absorber filled with working medium in $50 \%$ ) 


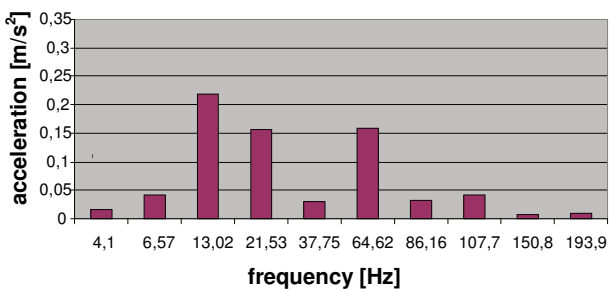

a) Driver's feet resting spot

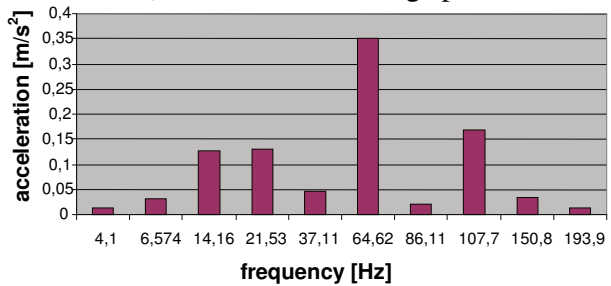

c) Rear left passenger's feet resting spot

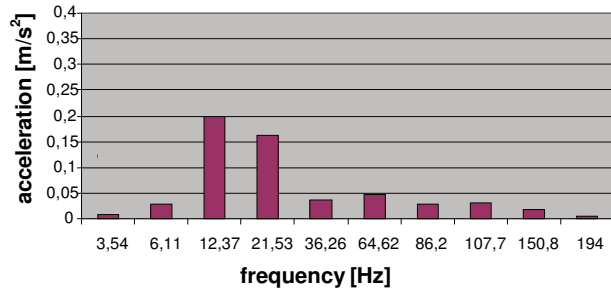

b) Front passenger's feet resting spot

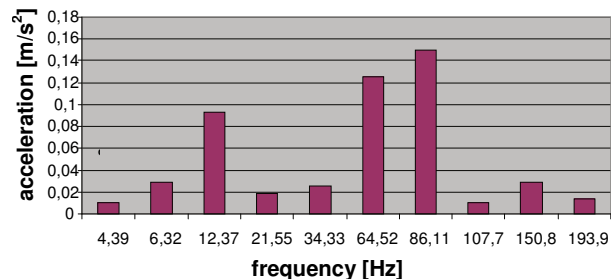

d) Rear right passenger's feet resting spot

Fig. 7.12. Distribution of maximum amplitudes of vibration signal spectra obtained for a floor panel of passenger car for the selected characteristic frequencies (shock absorber filled with working medium in $100 \%$ )

\subsubsection{Influence of the suspension spring properties on dominant frequency components of vibration in vehicle structure on the floor pan}

The results of analysis with build in new and used (worn-out) suspension spring vehicle's vibration were collected in the database form. The examined cases represent spring properties presented in Fig. 6.5, as the force vs. deflection characteristics of coil springs. Figs. 7.13-7.14 present chosen analysis results obtained.

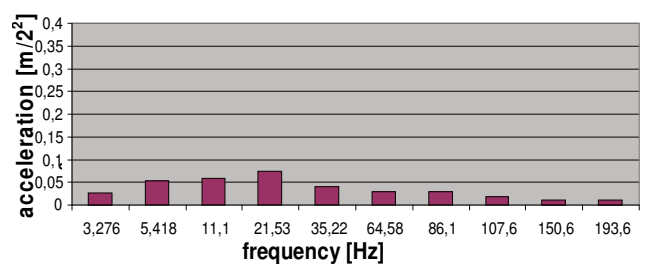

a) Driver's feet resting spot

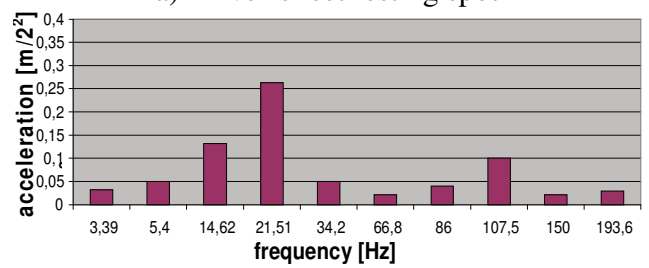

c) Rear left passenger's feet resting spot

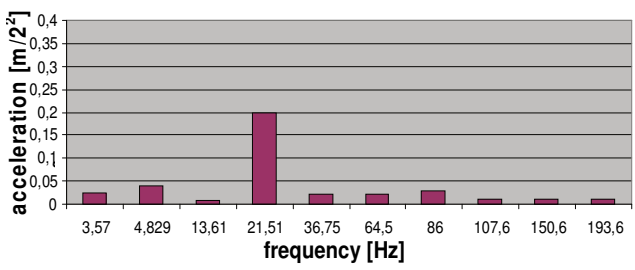

b) Front passenger's feet resting spot

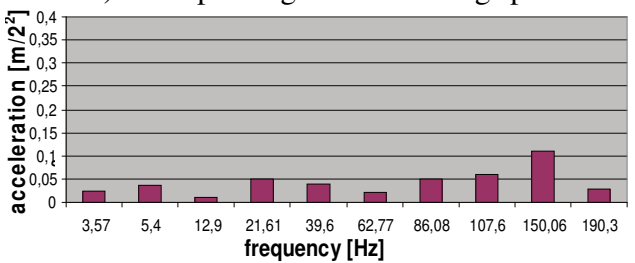

d) Rear right passenger's feet resting spot

Fig. 7.13. Distribution of maximum amplitudes of vibration signal spectra obtained for a floor panel of passenger car for the selected characteristic frequencies (the vehicles with build in new suspension spring)

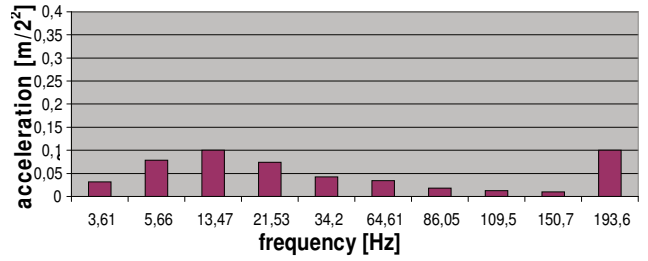

a) Driver's feet resting spot

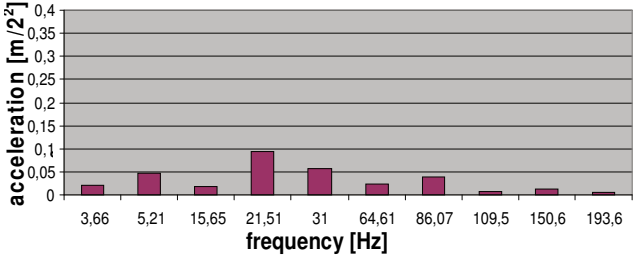

b) Front passenger's feet resting spot 


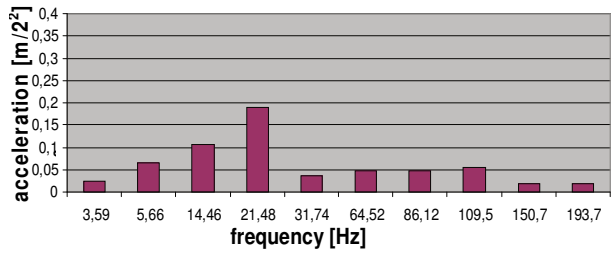

c) Rear left passenger's feet resting spot

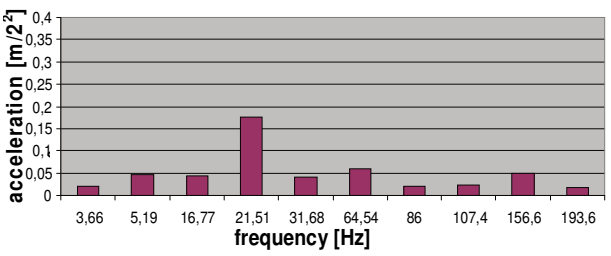

d) Rear right passenger's feet resting spot

Fig. 7.14. Distribution of maximum amplitudes of vibration signal spectra obtained for a floor panel of passenger car for the selected characteristic frequencies (the vehicles with build in used (worn-out) suspension spring)

\subsubsection{Influence of the pressure in tires on dominant frequency components of vibration in vehicle structure on the floor pan}

The analysis results of operation parameter's influence, as tire pressure, on vehicle vibration were collected in the database form. The examined cases represent tire pressure changes from 600 to $2600 \mathrm{hPa}$, with $200 \mathrm{hPa}$ steps. Fig. 7.15 present chosen analysis results obtained.

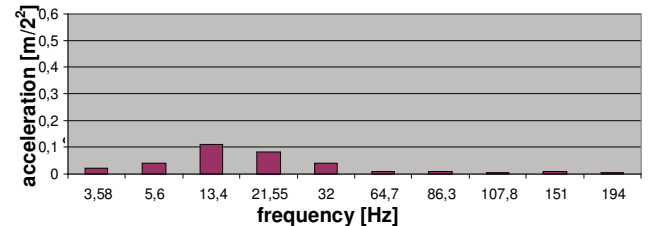

a) $600 \mathrm{hPa}$

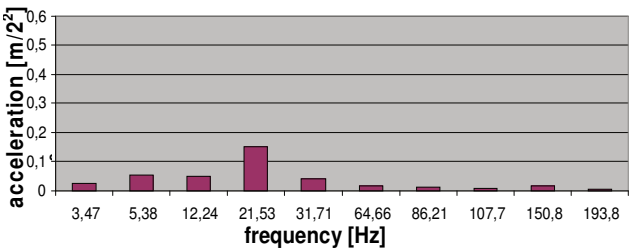

c) $1000 \mathrm{hPa}$

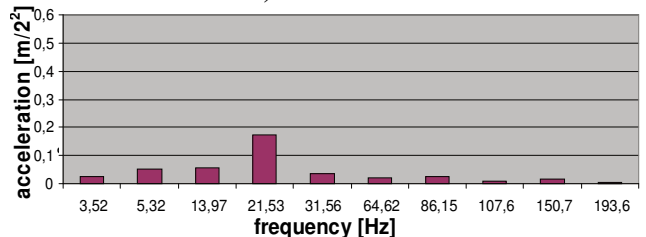

e) $1400 \mathrm{hPa}$

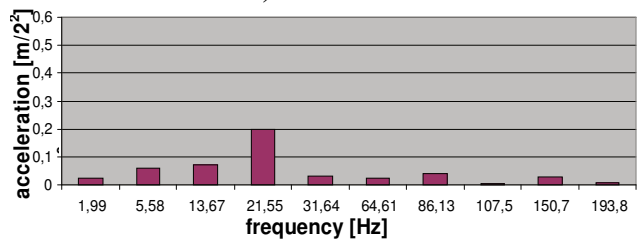

g) $1800 \mathrm{hPa}$

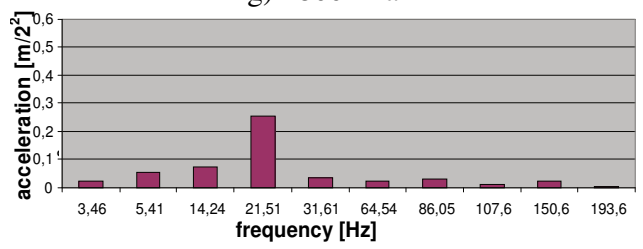

i) $2200 \mathrm{hPa}$

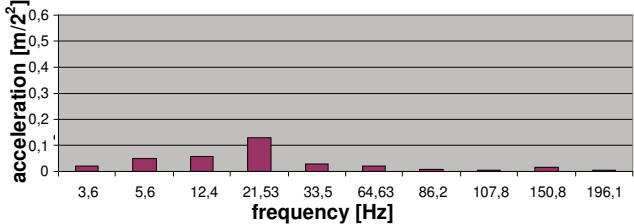

b) $800 \mathrm{hPa}$

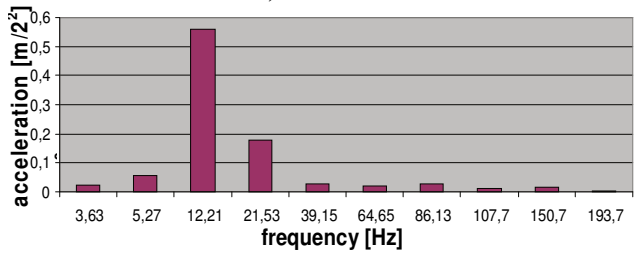

d) $1200 \mathrm{hPa}$

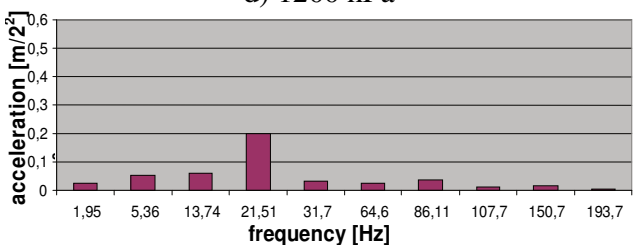

f) $1600 \mathrm{hPa}$

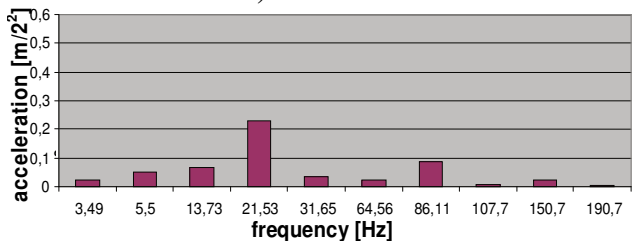

h) $2000 \mathrm{hPa}$

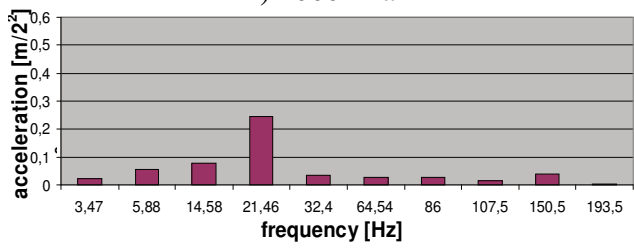

j) $2400 \mathrm{hPa}$ 


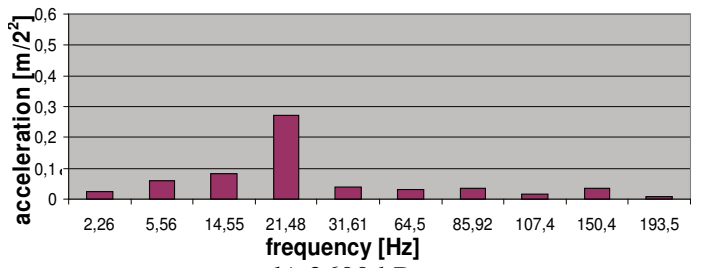

k) $2600 \mathrm{hPa}$

Fig. 7.15. Distribution of dominant frequencies of vibration of driver's feet resting spot for the pressure in tires

\subsubsection{Influence of the load of the passenger car on dominant frequency components of vibration in vehicle structure on the floor pan}

The influence of operation parameter, as extra load, on dynamics of vehicle vibration were collected in the database. The examined cases represent extra loads of 75, 150,225, $300 \mathrm{~kg}$, which can be correlated with 1-4 persons sitting in a car. Figs. 7.16-7.17 present chosen analysis results obtained.

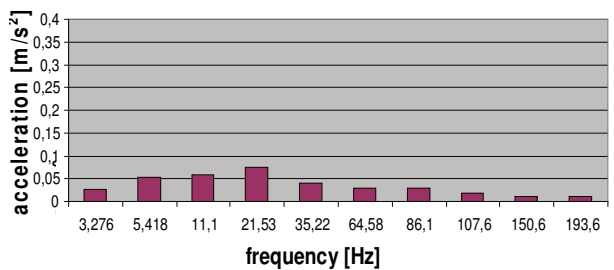

a) Driver's feet resting spot

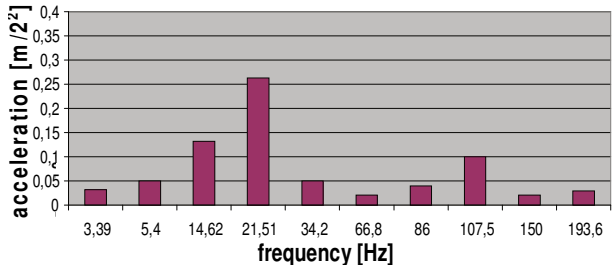

c) Rear left passenger's feet resting spot

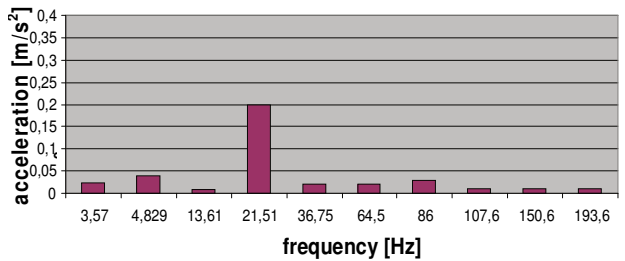

b) Front passenger's feet resting spot

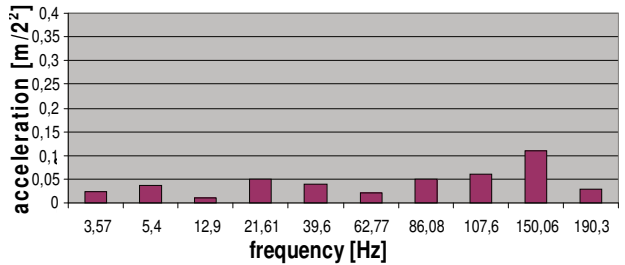

d) Rear right passenger's feet resting spot

Fig. 7.16. Distribution of maximum amplitudes of vibration signal spectra obtained for a floor panel of passenger car for the selected characteristic frequencies (the vehicles with $75 \mathrm{~kg}$ of extra load)

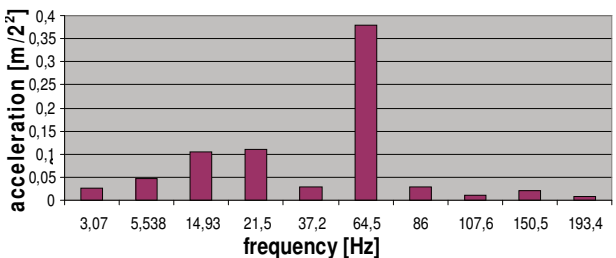

a) Driver's feet resting spot

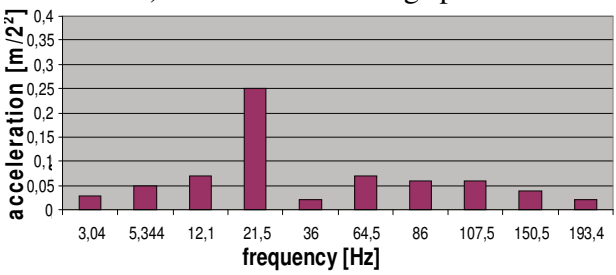

c) Rear left passenger's feet resting spot

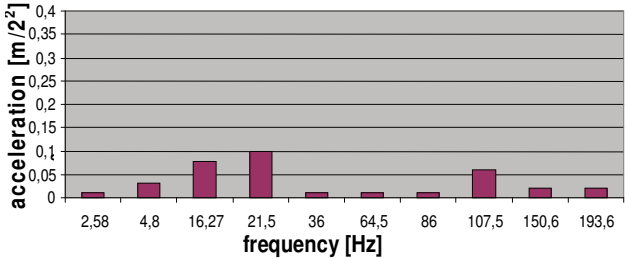

b) Front passenger's feet resting spot

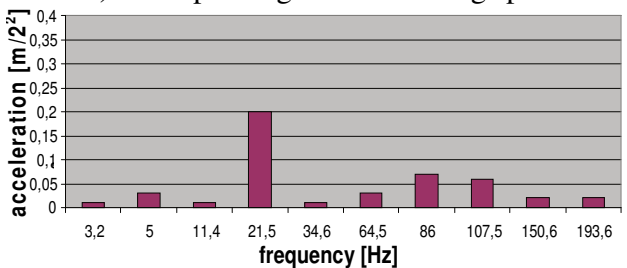

d) Rear right passenger's feet resting spot

Fig. 7.17. Distribution of maximum amplitudes of vibration signal spectra obtained for a floor panel of passenger car for the selected characteristic frequencies (the vehicles with $300 \mathrm{~kg}$ of extra load) 


\subsection{Result analysis and discussion}

This chapter addresses analyses results of frequency based vibration measures recorded on a vehicle floor panel and suspension elements in a function of technical condition of the suspension elements installed in automotive vehicles and its operation parameters. For the identification of the dominant frequency component, which can determined dynamics of the vibration, analysis of the chosen frequency bands have been conducted. The distribution of vibration in frequency bands: 2-18 Hz, 20-25 Hz, 105-110 Hz and 148-155 Hz show high sensitivity to changes of damping and stiffness (volume of liquid in shock absorber and tire pressure). The vibration are much higher for the vehicle with shock absorber filled with working medium in $50 \%$ and it increase due to level of pressure in tire.

The signal frequency bands assumed for the analysis of the vehicle floor panel vibrations enable involved developing a collation of maximum values of the signal spectrum amplitudes in the chosen frequency bands.

The highest sensitivity to vibrations was determined to be characteristic of a symptom of the shock absorber's technical condition, namely the maximum value of the vibration amplitude within the band of 12-17 Hz. It corresponds to a band of resonant frequency of unsprung masses. In the results of passenger car tests discussed in the chapter, this frequency equals ca. $12.5 \mathrm{~Hz}$. A drop in the amount of shock absorbing fluid causes a decrease of the vibration damping efficiency and an amplitude increase in the resonant band of unsprung masses. It is a very hazardous phenomenon, since it has a direct effect on the vehicle safety, and in extreme cases, it may even lead to a loss of road adhesion of a wheel. An interesting phenomenon was observed for a frequency of ca. $65 \mathrm{~Hz}$ which may be perceived as the 5th harmonic of resonance of unsprung masses. In this special case, one could observe a reverse dependence assuming the form of a decline in the value of maximum amplitudes as the amount of shock absorbing fluid decreased. Additionally, basing on the comparative analysis of the vibration distribution in the front and back of the floor panel, it was determined that the predominant frequency components, carrying the largest amounts of vibration energy, in locations where vibrations penetrated bodies of the sitting passengers were to be found within the band of $62-66 \mathrm{~Hz}$ when testing shock absorbers filled with the working medium in $100 \%$. One may consequently assume that correct technical condition of a shock absorber, damping efficiency aside, exerts an impact on shifting the dynamics of the system vibrations outside the band of hazardous frequencies, i.e. the resonance of unsprung masses.

The influence of the suspension spring properties on dominant frequency components of vibration in vehicle structure on the floor pan shows less sensitivity. The values of frequency based estimators are smaller for dynamics of vibration of vehicle with build in used (worn-out) suspension spring. The slight increase of the values can be observed in frequency components, as ca. $12 \mathrm{~Hz}$ and decrease in estimator of $21.5 \mathrm{~Hz}$ (correspond to the constant excitation).

The influence of operation parameters of the vehicle on the vibration dynamics were tested on two cases, tire pressure and extra load. The changes of the vibration dynamics due to the tire pressure level are observed for ca. 12 and $21.5 \mathrm{~Hz}$ frequency based estimators. This result are similar to influence of suspension spring properties, so it can be assumed that most sensitivity of suspension stiffness to vibrations dynamics is frequency component corresponded to the constant excitation (i.e. driving with constant speed on similar roughness road type). The distribution of maximum amplitudes of vibration signal spectra for the selected characteristic frequencies on different extra load show some influence. It is difficult to describe any clear conclusion for this case but one can be assumed that dominant frequency component is correlated to the constant excitation frequency.

Basing on empirical studies, resonant phenomena at higher frequencies, even exceeding 5 [Hz], have been identified, namely those which may cause considerable discomfort. In terms of unsprung masses, free vibration frequencies assume values within a range from several to more than a dozen hertz (i.e. 8-18 [Hz]). While an automotive vehicle is moving, free vibrations of sprung and unsprung masses occur simultaneously and overlap. 


\section{Identification of vehicle vibration's structure in research on the influence of changes in the technical condition of suspension elements and of the vehicle operating parameters on the vibration distribution in a vehicle structure}

The identification of sources and propagation's path of dynamics phenomena requires analysis in terms and conditions which are determining the course, modes and perception of the phenomena. The vibration has to be considered in terms of amplitude, time and frequency. The evaluation of impact of chosen factors on vibration occurring in motor vehicles requires wide scope of amplitude, time and frequency characteristics analysis. The previous chapter presents results of analysis of vibration distribution separately in time and frequency domains. It represents the amplitude-time and amplitude-frequency characteristics of vibration.

The properties of human vibration perception, discussed in Chapter 2, require observation of distribution of vibration in time and frequency domains for the analysis of vibration phenomena in mean of transport. Thus it is very helpful to identify structure of the vibration as correlated time-frequency distribution. Such an approach enables the analysis of exposure to vibration. This presentation of vibration's structure enables identification of time of exposure to chosen vibration dynamics. This approach allows precise evaluation of exposure to vibration correlated to natural vibration of human organs.

In the scope of engineering applications the presented approach allows designing and developing of elements and systems dedicated to absorb or isolate of vibration in chosen frequency bands.

\subsection{Application of time-frequency representation methods for determination of vibration structure}

Signal analysis addresses the problem of extracting information from registered signal and converting it into a recognizable form. The time courses recorded provide information on the phenomenon in question, however, in order to extract it, application of the appropriate mathematical method proving is necessary. The results obtained belong to a group of non-stationary signals the analysis of which enforces one to apply time-frequency methods. For the nonstationary signal, when the components of the signal changes with time, the representation of the signal in frequency domain become less useful. Simultaneous extraction of information concerning the time-frequency structure of a signal being analysed is possible owing to 2-dimensional transforms. To achieve frequency representation that includes dependence on time the double arguments function is needed, i.e. time-frequency representation (TFR).

The first attempt to develop such a function was carried out by Gabor in 1946 [87]. His approach can be considered as special case of the Short-Time Fourier Transform (STFT), expressed as: 
$S(\omega, b)=\int_{-\infty}^{+\infty} x(t) \omega_{a, b}(t) d t=\int_{-\infty}^{+\infty} x(t) g(t-b)^{-i \omega t} d t$

where: $b$ - window displacement, $\omega$ - analysing frequencies, $g(t-b)=$ const - constant width of successively analysed window.

The $g(t-b)$ window is typical chose as the Gaussian function or Hann function. The good properties of this window function occur from smoothly and symmetrically falls to zero around the time $t=0$ and is centered about $t=b$. The STFT segmenting the signal into narrow time intervals (i.e., narrow enough to be considered stationary) and takes the Fourier transform of each segment. Thus each Fourier transform provides the spectral information of a separate time-slice of the signal, providing simultaneous time and frequency information. Some disadvantage of STFT is a constant width of the window. If the window is too long transformation providing excellent frequency localization, but no time information. When the window is too short transformation gives the time signal back, with a phase factor, providing excellent time localization but no frequency information.

Simultaneous extraction of information concerning the time-frequency structure of a signal being analysed is possible owing to a wavelet transform. It is so because the wavelet function applied in this transformation can be extended or shortened. Narrow wavelets make it possible to analyse high-frequency components of a signal, and appropriately long base functions reveal its slowly-variable properties. The template role is performed by basic wavelet $\psi(t)$. The wavelet functions as a transformation kernel. A single wavelet is used in the given transformation, however, due to modification of scale coefficient $a$ and modification coefficient $b$, it forms what is referred to as a wavelet family. A wavelet transformation of signal $x(t)$ may be defined as follows:

$W T_{x}(a, b)=\frac{1}{\sqrt{a}} \int_{-\infty}^{\infty} x(t) \Psi\left(\frac{t-b}{a}\right) d t$,

where: $\Psi(t)$ - wavelet family, $\Psi((t-b) / a)$ - transformation kernel, $a$ - scaling parameter effecting frequency change $a \in R^{+} / 0 \wedge a \sim 1 / f, f$ - frequency, $1 / \sqrt{a}$ - wavelet standardized constant, $b$ - shift parameter locating the wavelet position against time axis $b \in R$.

The wavelet transform represents the correlation between the signal analysed and function $\Psi(t)$ having been appropriately scaled. The very idea of wavelet transformation is the decomposition of signal $x(t)$ into wavelet coefficients $W T_{x}(a, b)$ by application of the base function. As a result of such a transformation, one obtains coefficients being functions of scale and time. By altering the parameters of scale $a$ and time shift $b$, one may obtain the time-frequency distribution. The base function of the wavelet transformation is subject to the operations of scaling and shifting which makes it possible to obtain wavelets of various lengths of decay time and centre frequencies. The best results are obtained by this method when wavelets adjusted in shape to the relevant signal features are used.

The Wigner-Ville distribution approach is based on the use of the autocorrelation function for calculating the power spectrum. To construct the autocorrelation function, the signal is compared to itself for all possible relative shifts, or lags. The Wigner-Ville uses a variation of the autocorrelation function where time remains in the result, called instantaneous autocorrelation function. The Wigner-Ville Distribution (WVD) is defined as:

$W V D_{x x}(\theta, \tau)=\int x^{*}\left(t-\frac{\tau}{2}\right) x\left(t+\frac{\tau}{2}\right) e^{-j 2 \pi \theta t} d t$

where: $x^{*}$ - represents the complex conjugate of the signal $x, \tau$ - time shift, $\theta$ - frequency shift.

The analytic associate $x(t)$ of a signal $s(t)$ is defined such that $x(t) \equiv s(t)+i H[s(t)]$, 
where $H[s(t)]$ is the Hilbert Transform of the signal $s(t)$. In most cases, the TFR techniques are applied to the analytic associates of real signals unless noted otherwise - in particular, $x(t)$ is generally the complex-valued analytic associate of some real-valued time signal of interest. WVD is obtained by comparing the information of the signal with its own information at other times and frequencies. The WVD is a quadratic TFR, and most other TFR methods (including the spectrogram, i.e. STFT and the scalogram, i.e. WT) can be derived from the WVD, with a suitable choice of smoothing factors.

Wigner distribution was first introduced by E. Wigner in the context of quantum mechanics (Wigner, 1932), and later independently developed by J. Ville who applied the same transformation to signal processing and spectral analysis (Ville, 1948) [30, 194, 199].

\subsection{Analysis of time structure of vibration in determined frequency bands}

For the research results analysis on influence of suspension's technical condition and vehicle's operating parameters on structure of occurring vibration the STFT of the signals were calculated. The reason of choosing the STFT was time consumption of the analysis process, because utility and applicability requirements. The 3-D formats of absolute values of STFT transformation of vibration are shown in Figs. 8.1-8.10. The distribution in time of vibration energy for next frequencies can be observed.

\subsubsection{Influence of the damping of shock absorber on structure of vibration in vehicle construction}

The STFT transformation of vibration registered during research on vehicle with build in shock absorbers with $100 \%$ and $50 \%$ of liquid volume are shown in figures below. It shows that for the shock absorber with $50 \%$ of liquid the vibration energy become more centered and occurred in nonstationary parts of the signal.

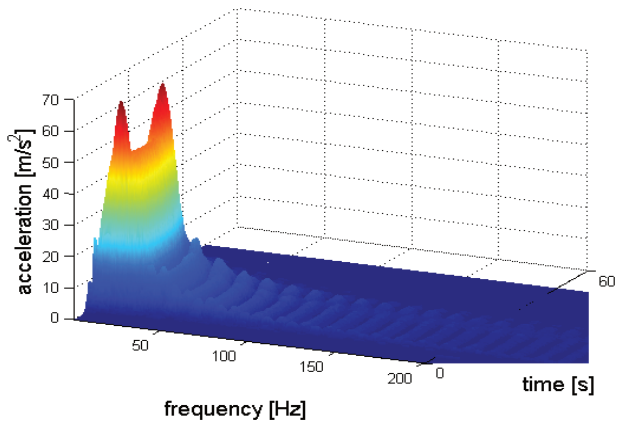

a) Shock absorber with $100 \%$ of liquid volume

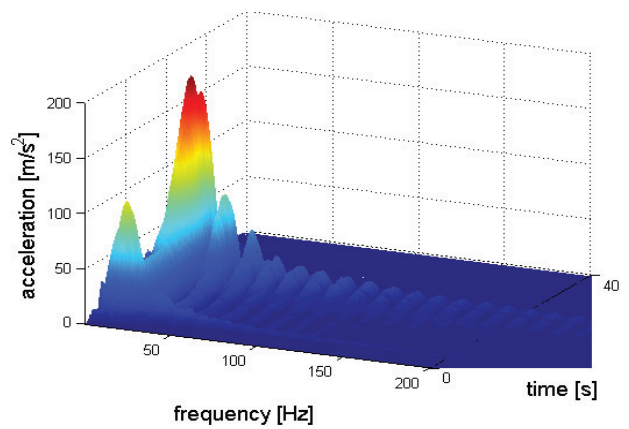

b) Shock absorber with $50 \%$ of liquid volume

Fig. 8.1. Time-frequency distribution of the vibration of suspension arm

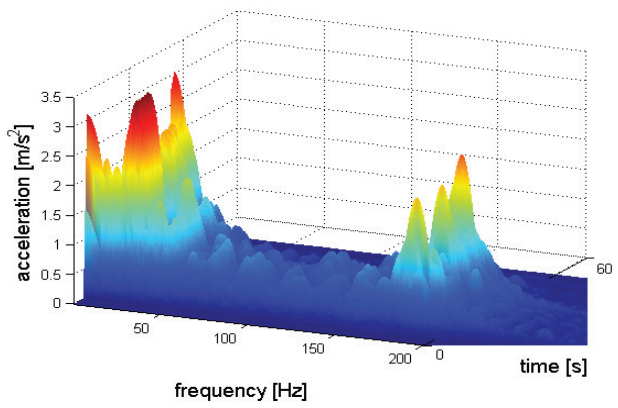

a) Shock absorber with $100 \%$ of liquid volume

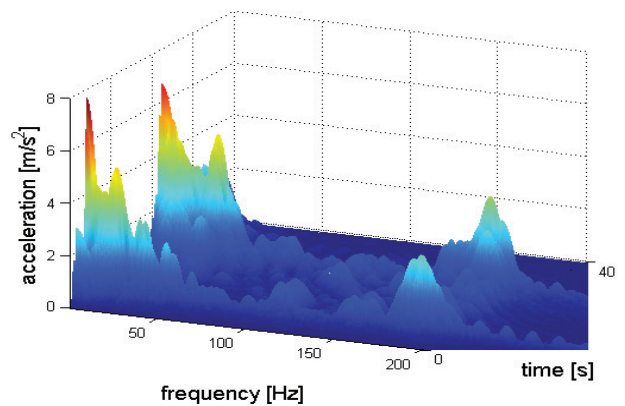

b) Shock absorber with $50 \%$ of liquid volume

Fig. 8.2. Time-frequency distribution of the vibration of upper mounting of shock absorber 


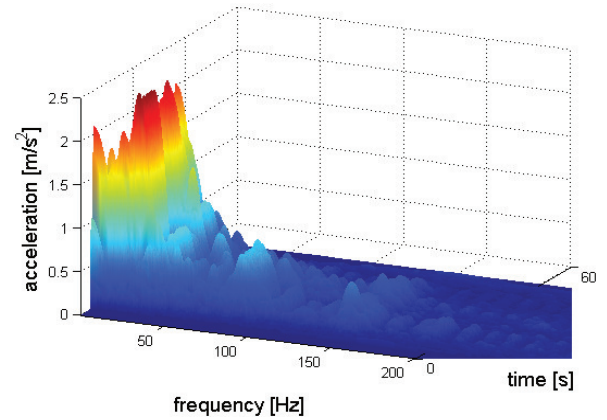

a) Shock absorber with $100 \%$ of liquid volume

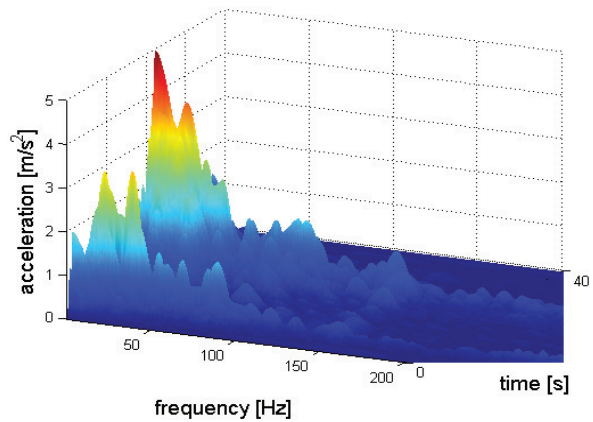

b) Shock absorber with $50 \%$ of liquid volume

Fig. 8.3. Time-frequency distribution of the vibration of floor under driver's feet

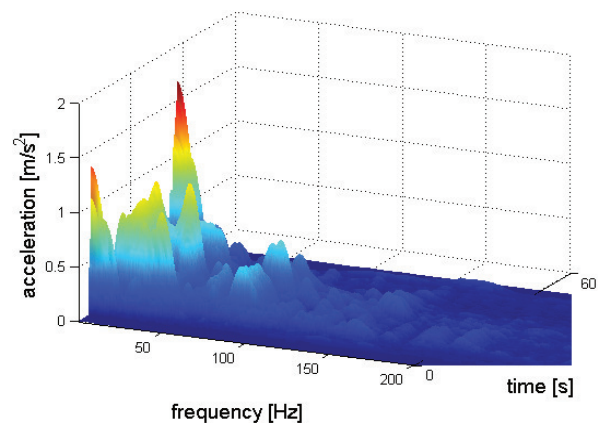

a) Shock absorber with $100 \%$ of liquid volume

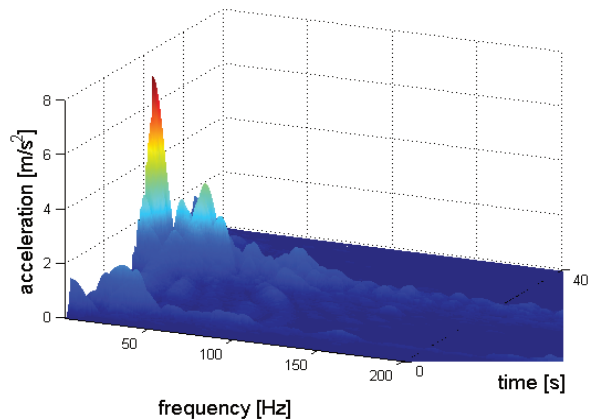

b) Shock absorber with $50 \%$ of liquid volume

Fig. 8.4. Time-frequency distribution of the vibration of floor under front passenger's feet

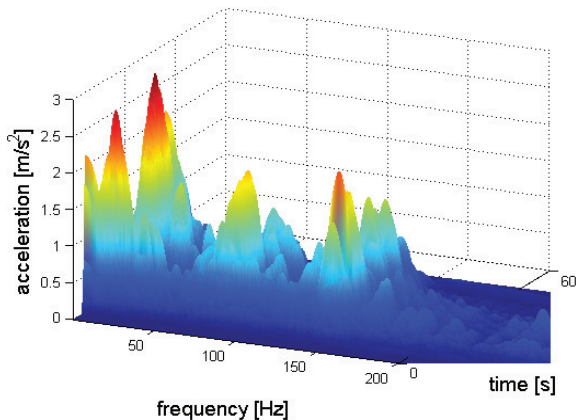

a) Shock absorber with $100 \%$ of liquid volume

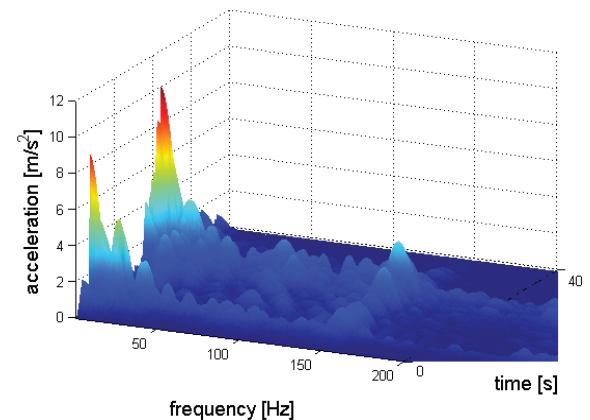

b) Shock absorber with $50 \%$ of liquid volume

Fig. 8.5. Time-frequency distribution of the vibration of floor under rear left passenger's feet

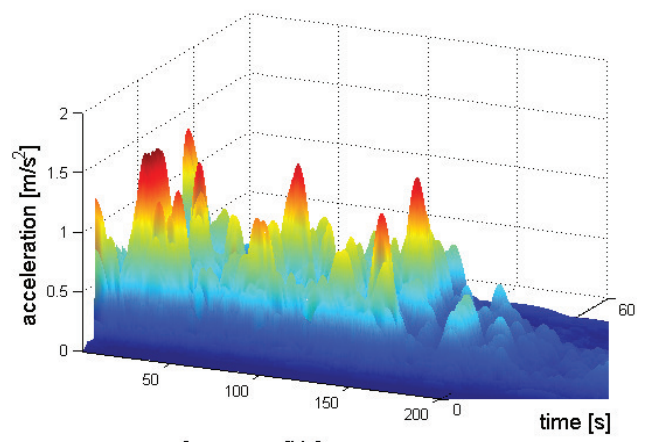

frequency $[\mathrm{Hz}]$

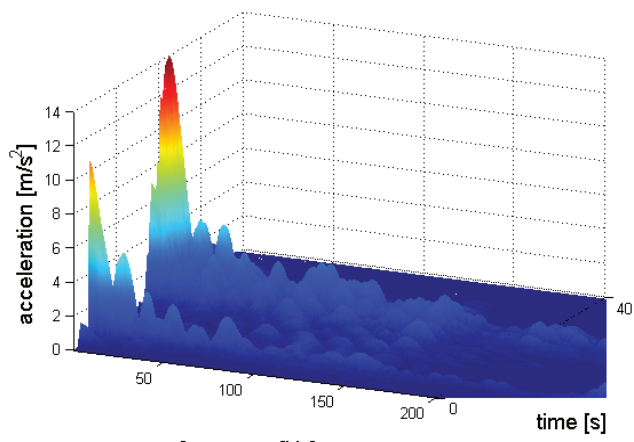

frequency $[\mathrm{Hz}]$

a) Shock absorber with $100 \%$ of liquid volume

b) Shock absorber with $50 \%$ of liquid volume

Fig. 8.6. Time-frequency distribution of the vibration of floor under rear right passenger's feet 
The Figs. 8.1 and 8.2 present time-frequency structure of the vibration of the suspension arm and upper mounting of shock absorber and the floor pan under driver's feet. The Figs. 8.3-8.6 show results of STFT of vibration registered on the floor panel under passenger's feet.

\subsubsection{Influence of the pressure in tires on structure of vibration in vehicle construction}

Figs. 8.7-8.12 present STFT transformation of vibration registered during research on vehicle with different pressure in tires. The results shows frequencies of the vibration occurring due to the increase of the pressure in tires. The number of time periods and frequencies of the vibration grows with the increase of the tire pressure.

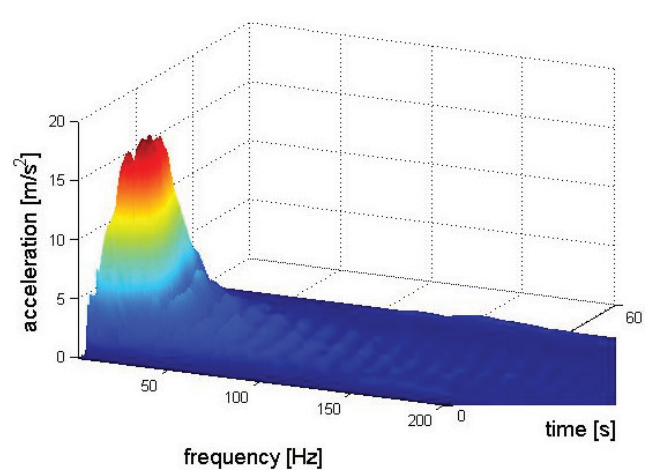

a) Tire pressure $600 \mathrm{hPa}$

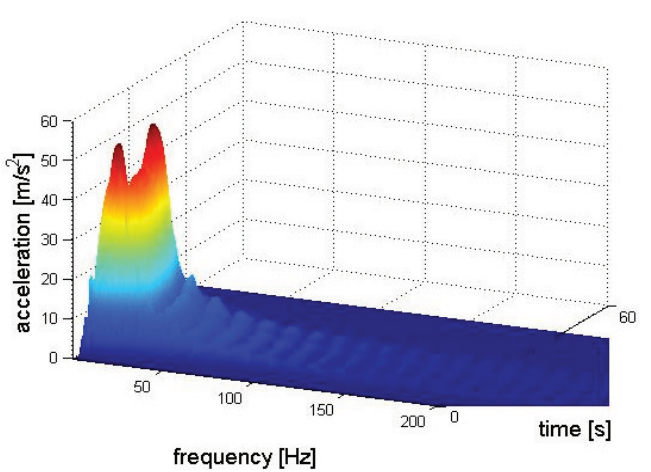

b) Tire pressure $1800 \mathrm{hPa}$

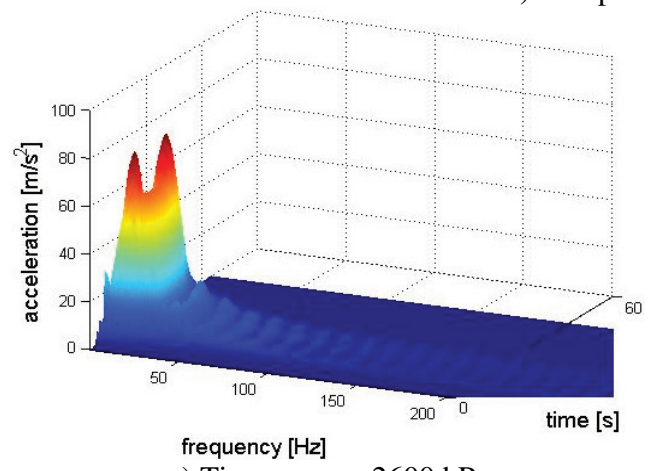

c) Tire pressure $2600 \mathrm{hPa}$

Fig. 8.7. Time-frequency distribution of the vibration of suspension arm

Exposure to vibration in means of transport depends on many factors. For the passengers it is important what type of vibration penetrates to the human body via vehicle construction or elements. For the perception properties as type of vibration we can consider the value, dynamics and exposure time of chosen frequency components of the vibration. The possibilities of simultaneous observation of distribution of signal in time and frequency domains are reached by the timefrequency representation (TFR) of the signal. There are many mathematical methods and techniques for transformation signal into TFR, mainly based on the transformation described in Chapter 8.2.

The chapter addresses results of analysis of application of one of the TFR techniques to the identification of structure of vibration. The possibilities of identification of characteristics components of vibration structure and sensitivity on the changes in mechanical system parameters have been investigated on the results of the research on influence of technical condition of suspension and operating parameters of the vehicle. The 3-D (amplitude, time, frequency) structure of the vibration obtained in result of STFT allow proper identification of the vibration and propagation into vehicle construction. 


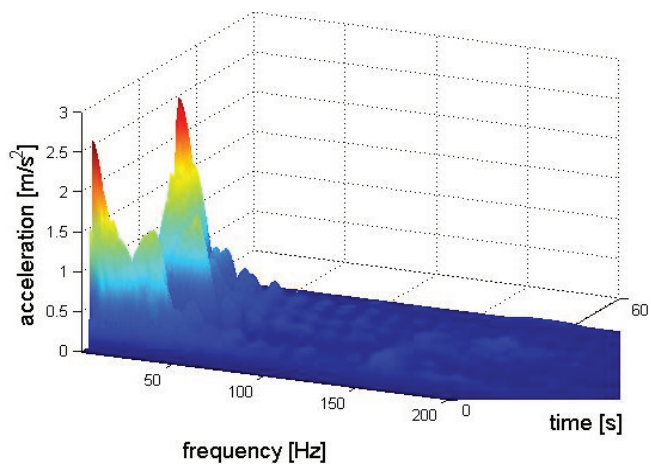

a) Tire pressure $600 \mathrm{hPa}$

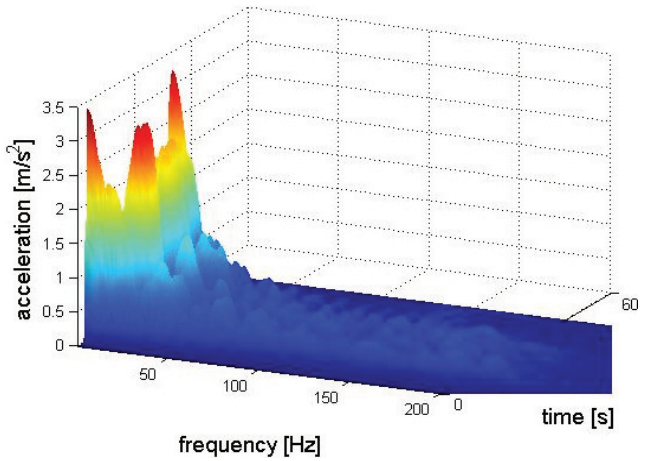

b) Tire pressure $1800 \mathrm{hPa}$

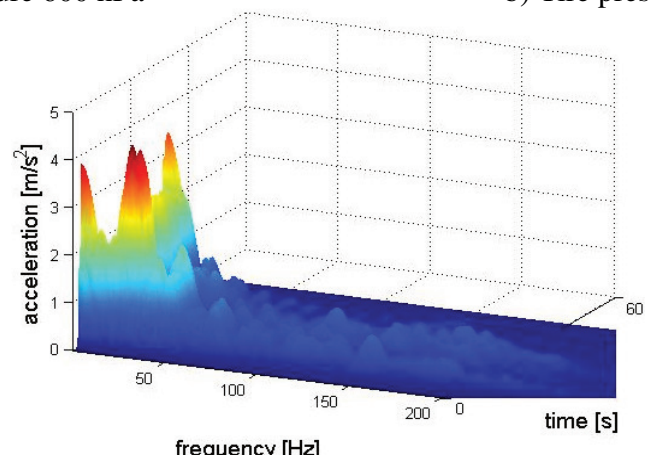

c) Tire pressure $2600 \mathrm{hPa}$

Fig. 8.8. Time-frequency distribution of the vibration of upper mounting of shock absorber

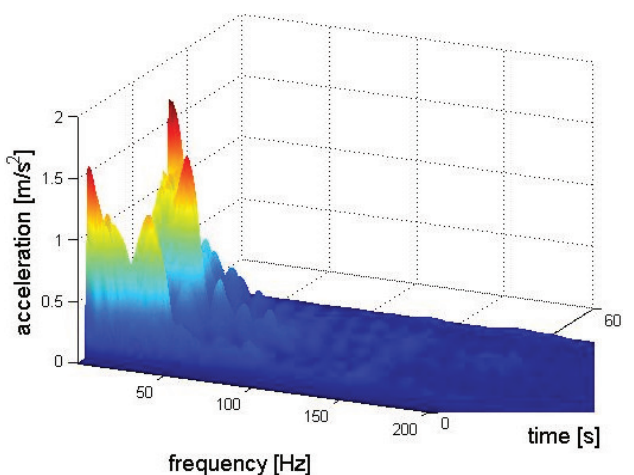

a) Tire pressure $600 \mathrm{hPa}$

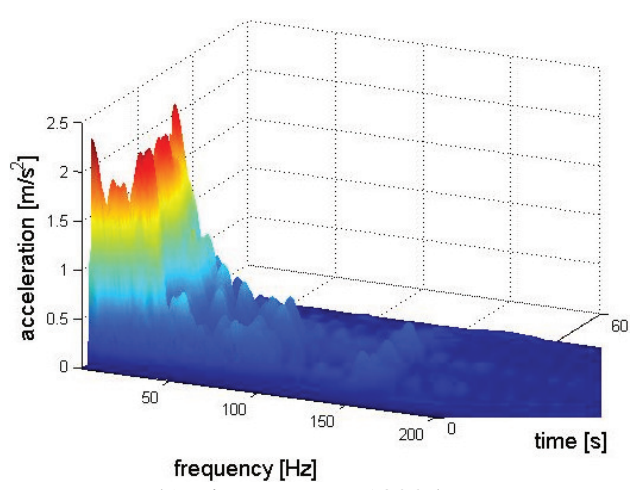

b) Tire pressure $1800 \mathrm{hPa}$

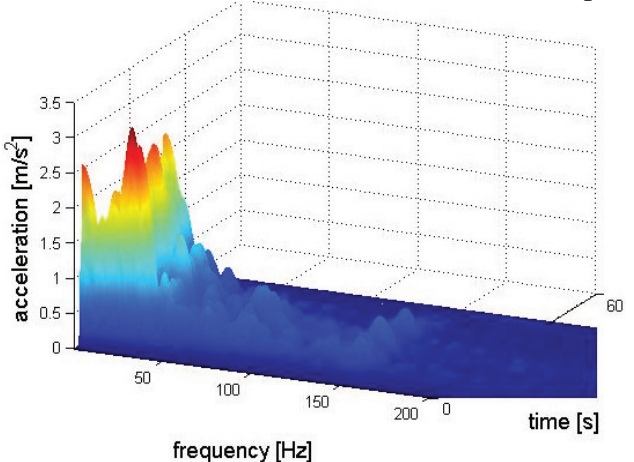

c) Tire pressure $2600 \mathrm{hPa}$

Fig. 8.9. Time-frequency distribution of the vibration of floor under driver's feet 


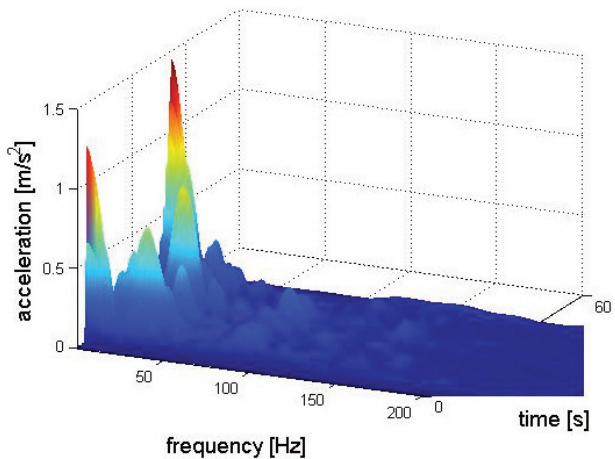

a) Tire pressure $600 \mathrm{hPa}$

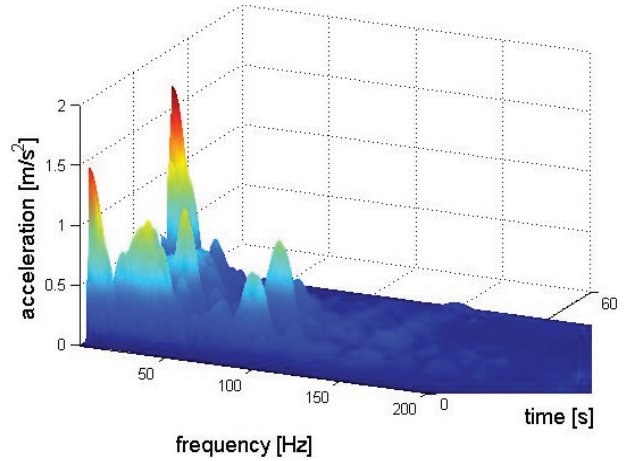

b) Tire pressure $1800 \mathrm{hPa}$

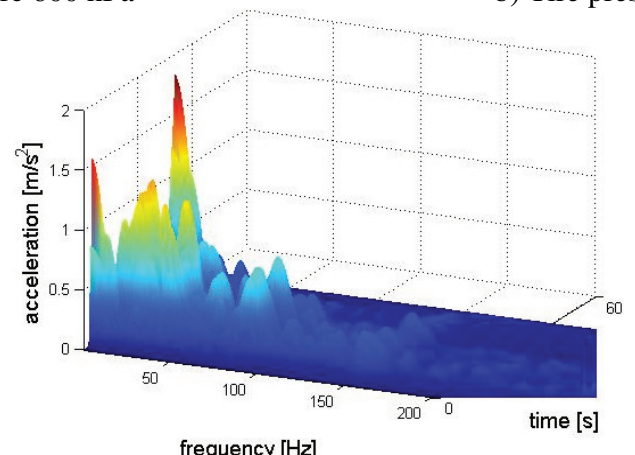

c) Tire pressure $2600 \mathrm{hPa}$

Fig. 8.10. Time-frequency distribution of the vibration of floor under front passenger's feet

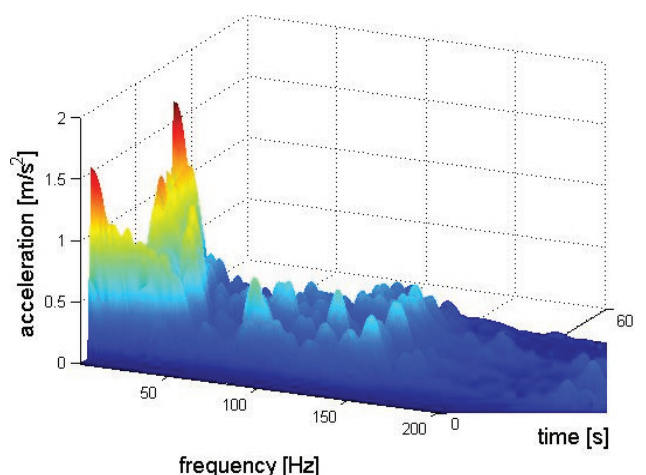

frequency $[\mathrm{Hz}]$

a) Tire pressure $600 \mathrm{hPa}$

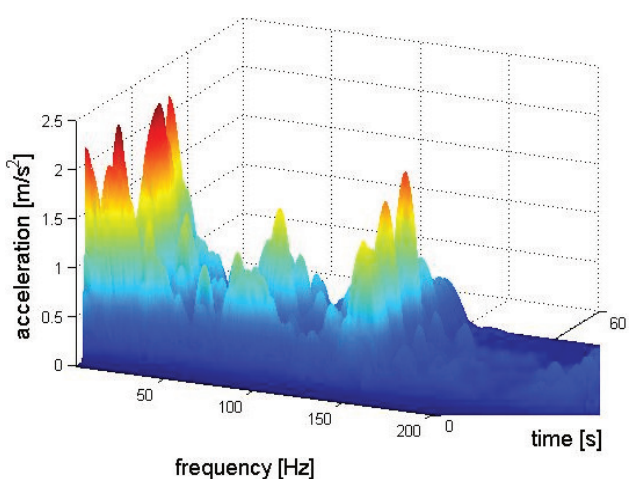

b) Tire pressure $1800 \mathrm{hPa}$

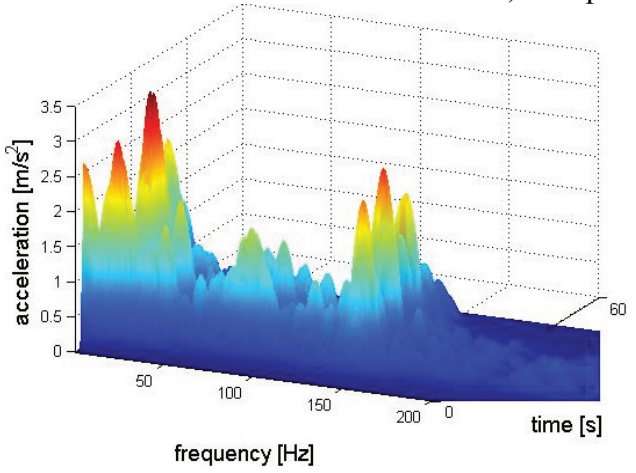

c) Tire pressure $2600 \mathrm{hPa}$

Fig. 8.11. Time-frequency distribution of the vibration of floor under rear left passenger's feet 


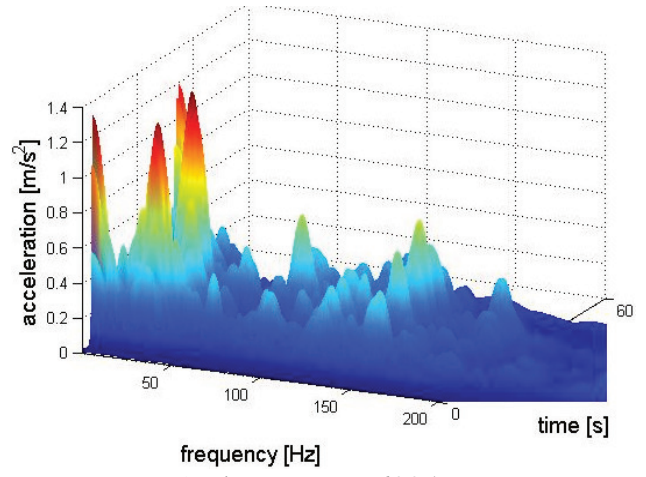

a) Tire pressure $600 \mathrm{hPa}$

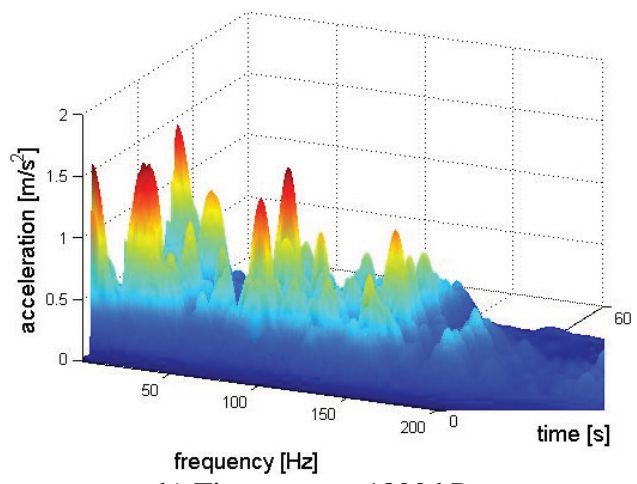

b) Tire pressure $1800 \mathrm{hPa}$

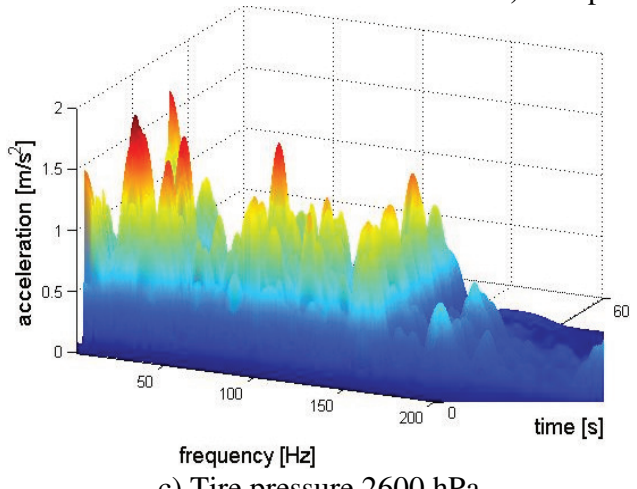

c) Tire pressure $2600 \mathrm{hPa}$

Fig. 8.12. Time-frequency distribution of the vibration of floor under rear right passenger's feet

The influence of the damping of shock absorber can be observed in vibration's structure, as an increase of the vibration energy. The time-frequency distribution of the vibration allows localization of window with highest increase of energy generated during passing by resonance frequency of unsprung masses in the time period of coasting of exciter machine. The structure of the vibration registered under study of influence of the pressure in tires shows increase of energy of the vibration due to increase of pressure in tires. The structures of the vibration are different than in results of damping influence. The total energy of the vibration is lower and there are many more higher frequencies components in the signals. The increase of the vibration can also be observed for time period of constant excitation (constant frequency). 


\section{Function of exposure time to the vibration of defined frequency bands in research on the influence of the technical condition of suspension elements and of the vehicle operating parameters on the vibration}

The time-frequency representations of the structure of the vibration presented in previous chapter enable selective evaluation of exposure to vibration in means of transport. For the purpose of analysis of the influence of the technical condition of suspension elements and of the vehicle operating parameters on the vibration propagation it is useful to identify the components of the signal which are best representation of those influences. Thus the symptoms of the technical conditions or value of the operating parameters can be determined.

The vibration occurring during research are results of forces acting on wheel by the exciter machine. The signals have non-stationary properties. Thus the evaluation of human vibration exposure based on global estimators is difficult. Distribution of the vibration in defined frequency bands enables observing of vibration exposition in time function.

\subsection{Distribution of the vibration of defined frequency bands}

Basing on the analysis of the results presented in Chapters 7 and 8 the main frequency bands were chosen for further observation. The obtained representation of the vibration as structure of STFT transformation allow to determine time function of separate frequency bands which represents the isolated vibration dynamics phenomena, expressed as:

$S_{f}=\sum_{f=a}^{b} \sum_{i=0}^{\infty} S\left(\omega_{f}, t_{i}\right)$,

where: $f$ - frequency band from $a$ to $b, t$ - time, $S\left(\omega_{f}, t_{i}\right)$ - Short-Time Fourier Transformation of the signal.

This function allows separation of the chosen dynamics structure of the vibration and analysis of energy changes by the time realization in isolated frequency bands.

This method of identification of vibration dynamics allows to observe time of increase of vibration activity of the vehicle and influence of the damping properties on time function of the chosen frequency bands of the signal. Results analysis of vibration structure, presented in previous chapter, occurred during research on different damping properties of shock absorbers it was assumed that the most sensitivity frequency components are ca. $12 \mathrm{~Hz}, 21.5 \mathrm{~Hz}$ and $64.5 \mathrm{~Hz}$. There were set as centers of the analysed frequency bands range ca. $2 \mathrm{~Hz}$ each. Figs. 9.1-9.6 present time distribution of the vibration in those frequencies.

The presented method has been tested too on results of research on influence of tire pressure on vibration propagation in vehicle construction. Results analysis of vibration structure, presented in previous chapter, it was assumed that the most sensitivity frequency components are ca. $5 \mathrm{~Hz}$, $15 \mathrm{~Hz}$ and $21.5 \mathrm{~Hz}$. Figs. 9.7-9.12 present time distribution of the vibration in those frequencies. 


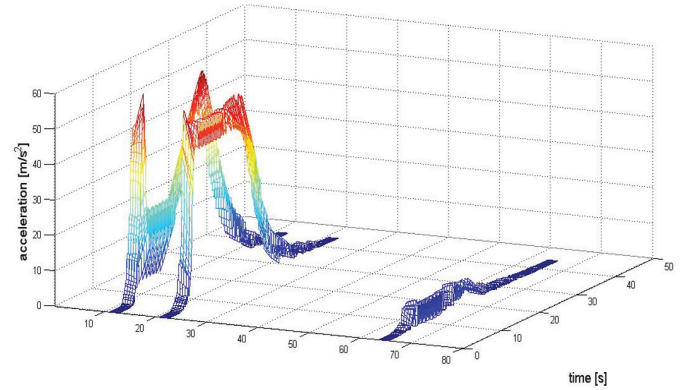

frequency $[\mathrm{Hz}]$

a) Shock absorber with $100 \%$ of liquid volume

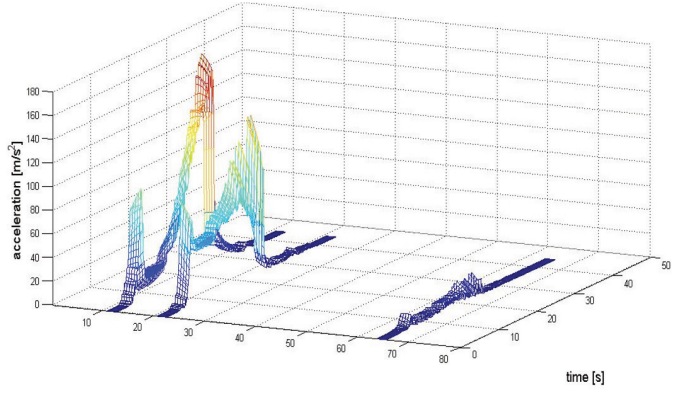

frequency $[\mathrm{Hz}]$

b) Shock absorber with $50 \%$ of liquid volume

Fig. 9.1. Time-frequency distribution of the vibration of suspension arm

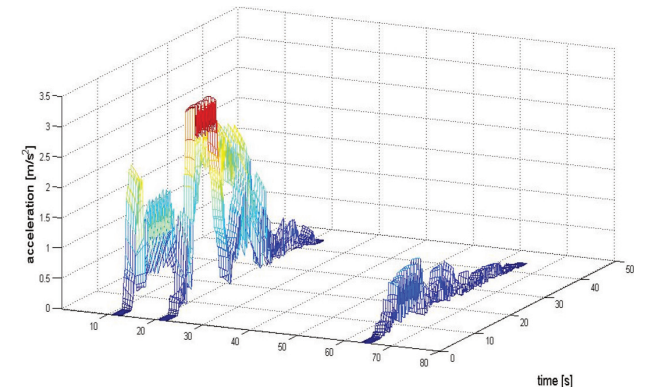

frequency $[\mathrm{Hz}]$

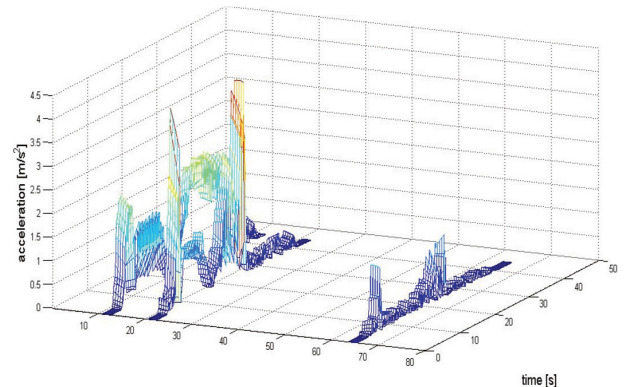

frequency $[\mathrm{Hz}]$

a) Shock absorber with $100 \%$ of liquid volume $\quad$ b) Shock absorber with $50 \%$ of liquid volume

Fig. 9.2. Time distribution of the vibration in chosen frequency bands of upper mounting of shock absorber

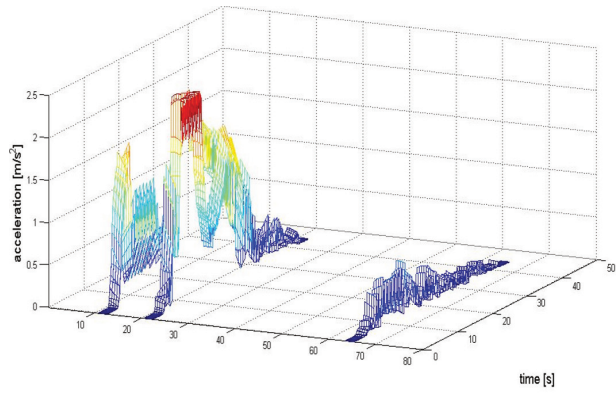

frequency $[\mathrm{Hz}]$

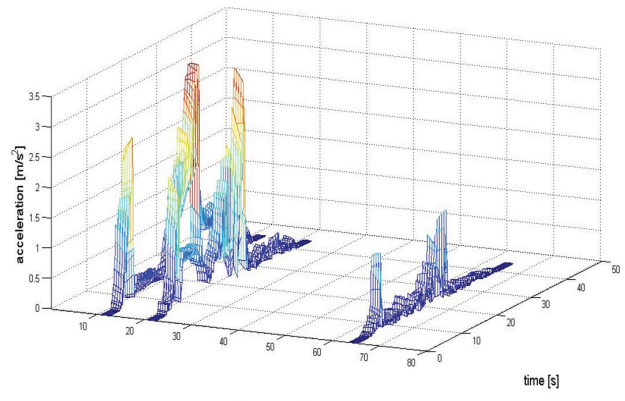

frequency $[\mathrm{Hz}]$

b) Shock absorber with $50 \%$ of liquid volume

a) Shock absorber with $100 \%$ of liquid volume

Fig. 9.3. Time distribution of the vibration in chosen frequency bands of floor under driver's feet

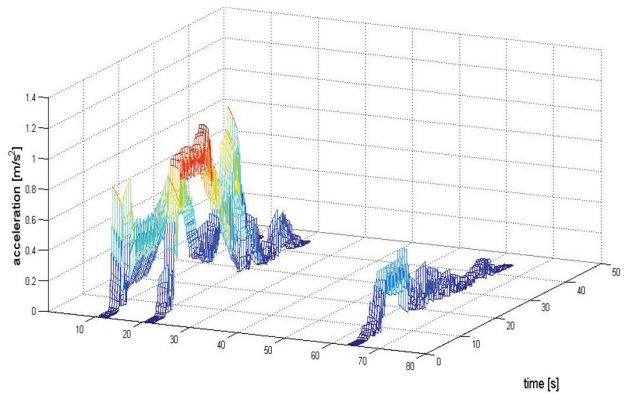

frequency $[\mathrm{Hz}]$

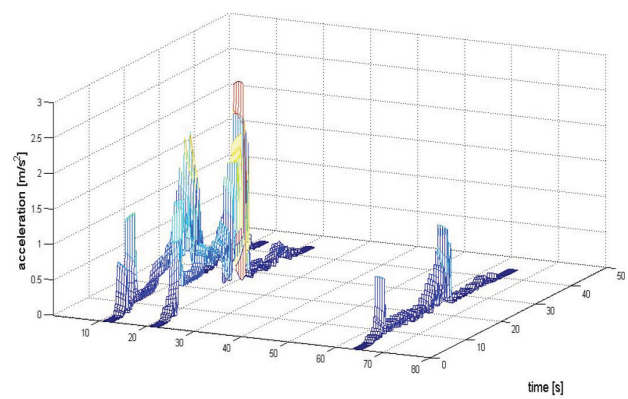

frequency $[\mathrm{Hz}]$
a) Shock absorber with $100 \%$ of liquid volume
b) Shock absorber with $50 \%$ of liquid volume

Fig. 9.4. Time distribution of the vibration in chosen frequency bands of floor under front passenger's feet 


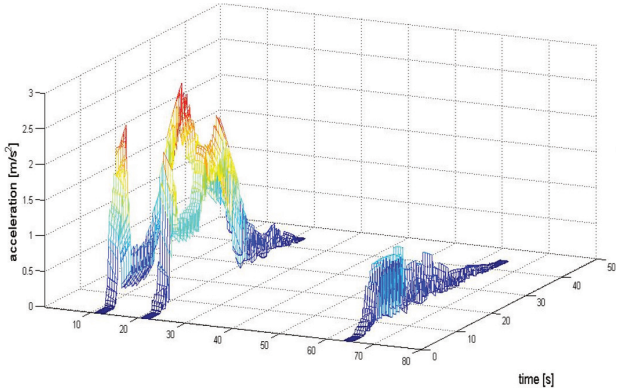

frequency $[\mathrm{Hz}]$

a) Shock absorber with $100 \%$ of liquid volume

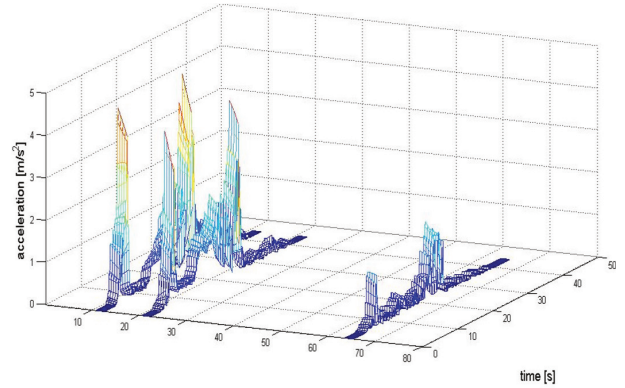

frequency [Hz]

b) Shock absorber with $50 \%$ of liquid volume

Fig. 9.5. Time distribution of the vibration in chosen frequency bands of floor under rear left passenger's feet

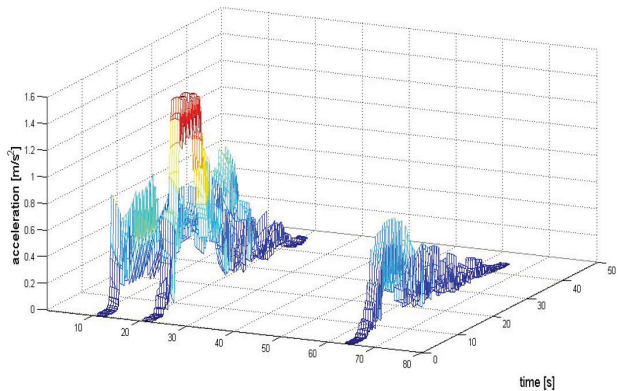

frequency $[\mathrm{Hz}]$

a) Shock absorber with $100 \%$ of liquid volume

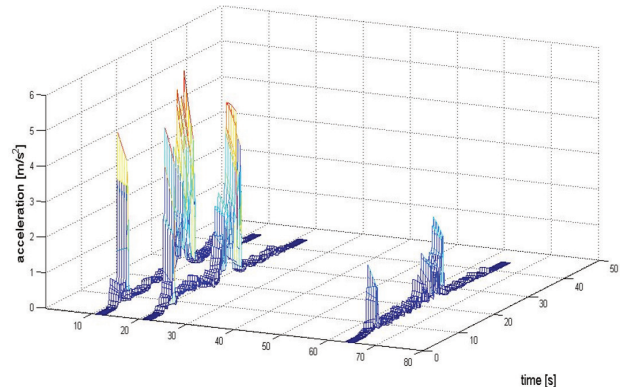

frequency [Hz]

b) Shock absorber with $50 \%$ of liquid volume

Fig. 9.6. Time distribution of the vibration in chosen frequency bands of floor under rear right passenger's feet

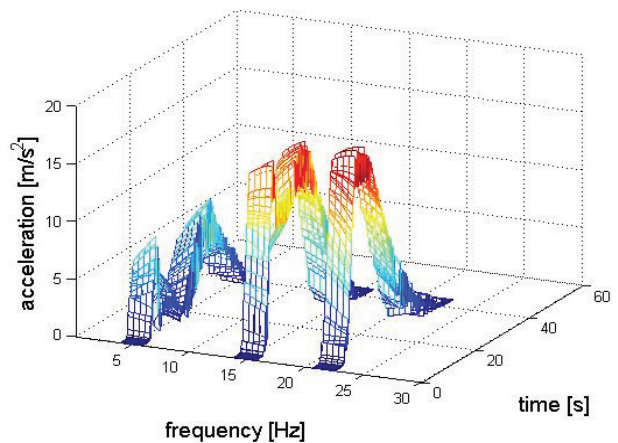

a) Tire pressure $600 \mathrm{hPa}$

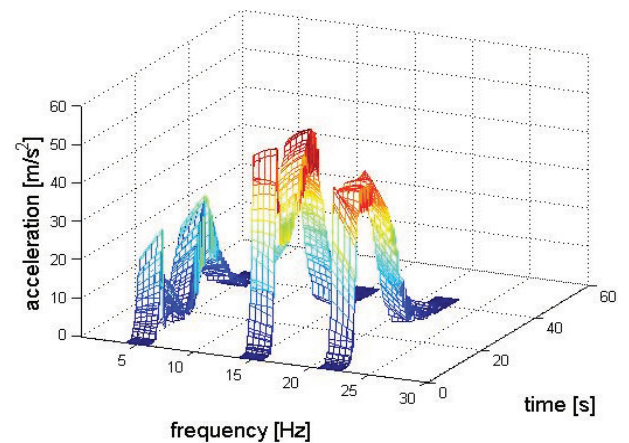

b) Tire pressure $1800 \mathrm{hPa}$

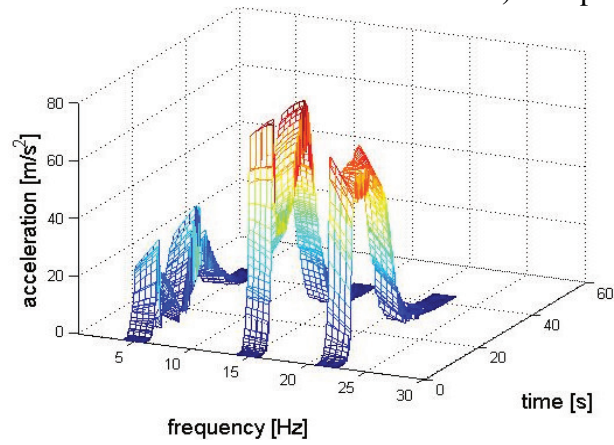

c) Tire pressure $2600 \mathrm{hPa}$

Fig. 9.7. Time distribution of the vibration in chosen frequency bands of suspension arm 


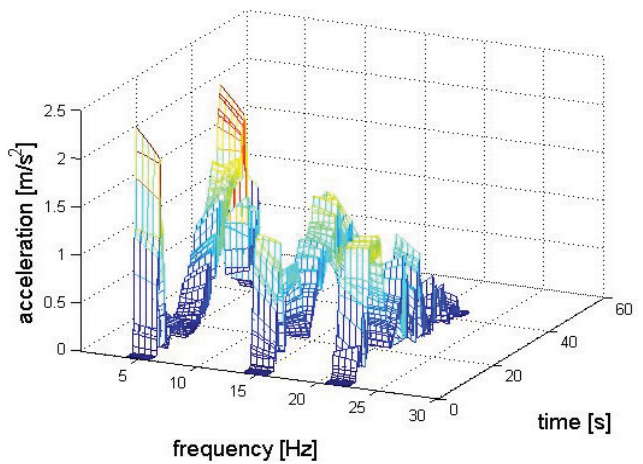

a) Tire pressure $600 \mathrm{hPa}$

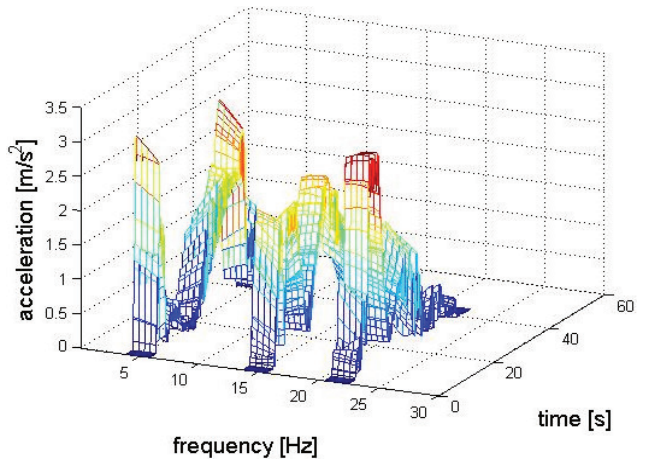

b) Tire pressure $1800 \mathrm{hPa}$

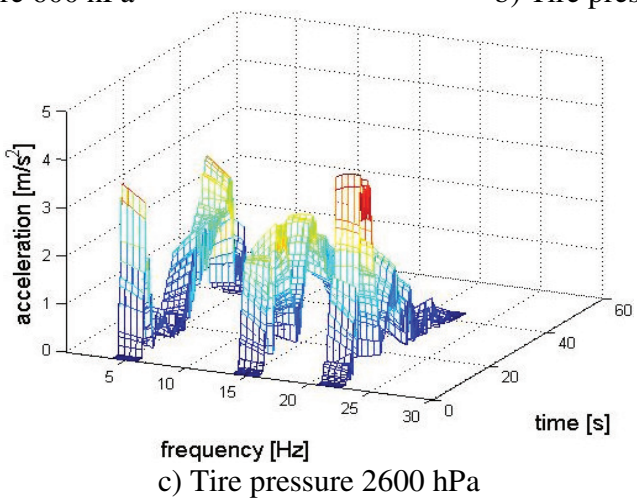

Fig. 9.8. Time distribution of the vibration in chosen frequency bands of upper mounting of shock absorber

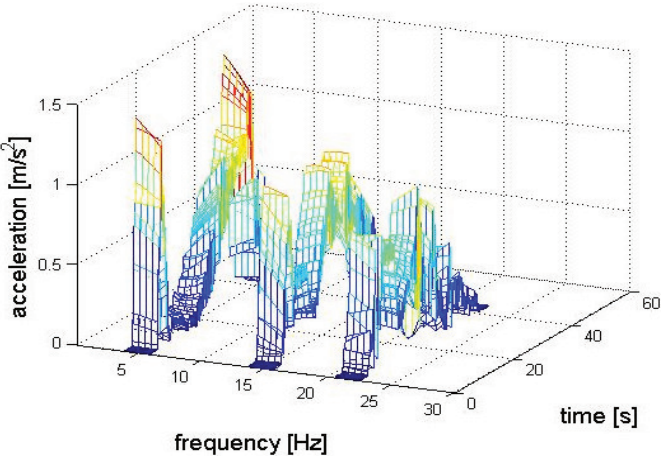

a) Tire pressure $600 \mathrm{hPa}$

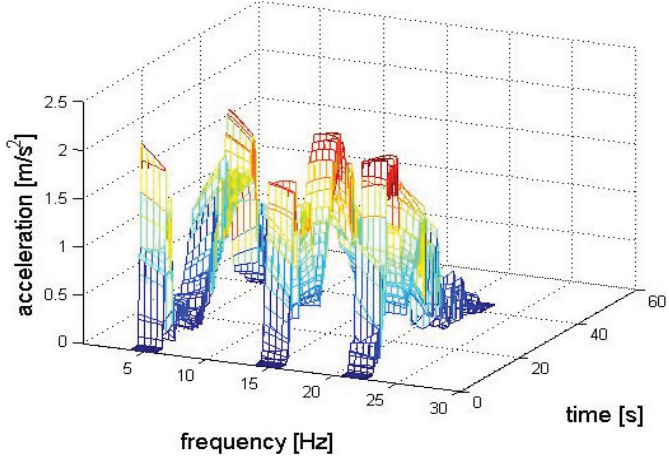

b) Tire pressure $1800 \mathrm{hPa}$

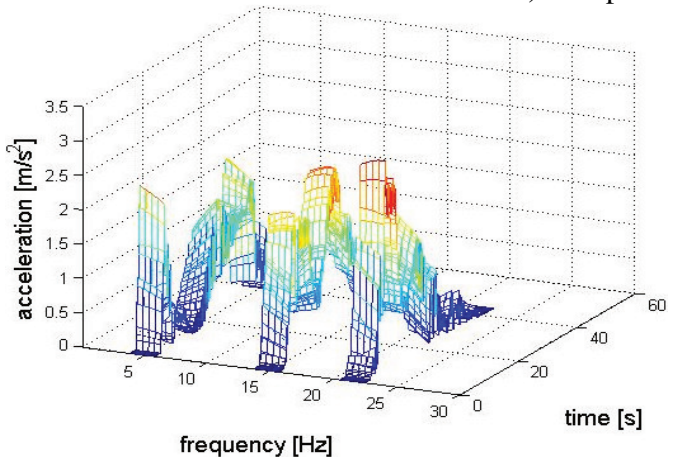

c) Tire pressure $2600 \mathrm{hPa}$

Fig. 9.9. Time distribution of the vibration in chosen frequency bands of floor under driver's feet 


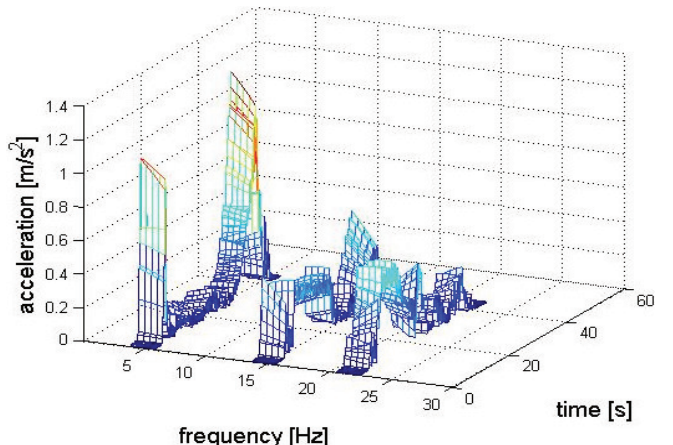

a) Tire pressure $600 \mathrm{hPa}$

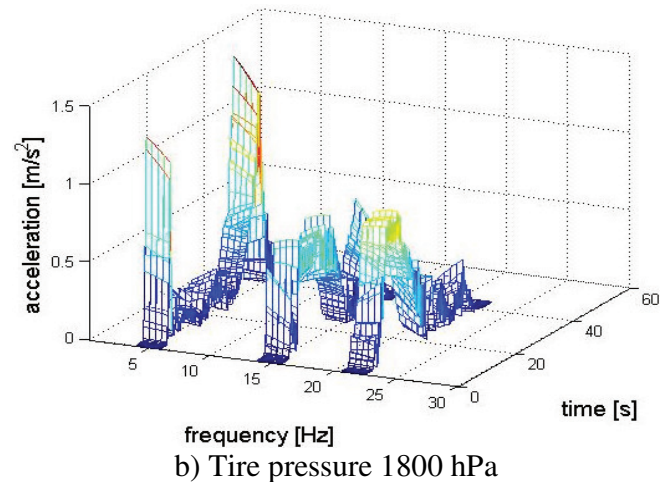

b) Tire pressure $1800 \mathrm{hPa}$

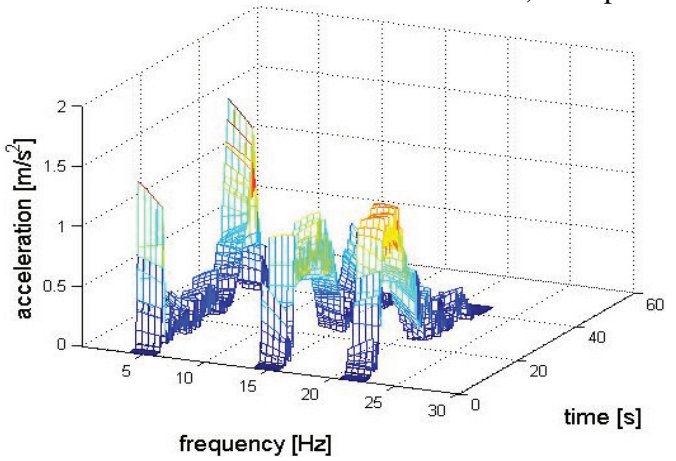

c) Tire pressure $2600 \mathrm{hPa}$

Fig. 9.10. Time distribution of the vibration in chosen frequency bands of floor under front passenger's feet

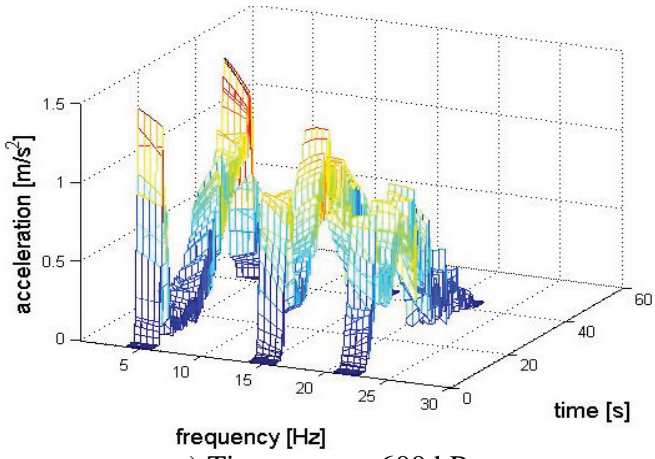

a) Tire pressure $600 \mathrm{hPa}$

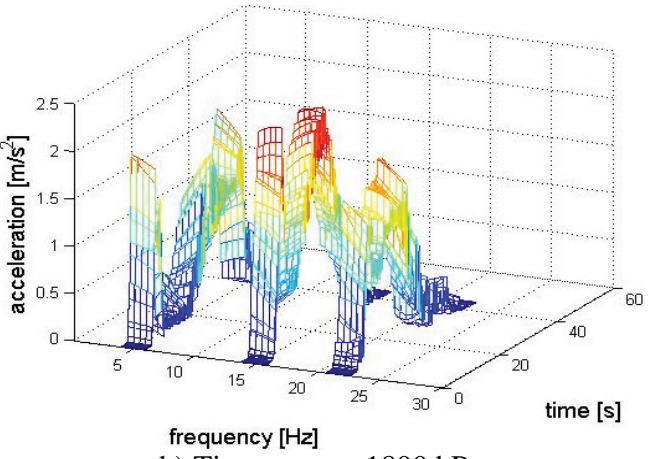

b) Tire pressure $1800 \mathrm{hPa}$

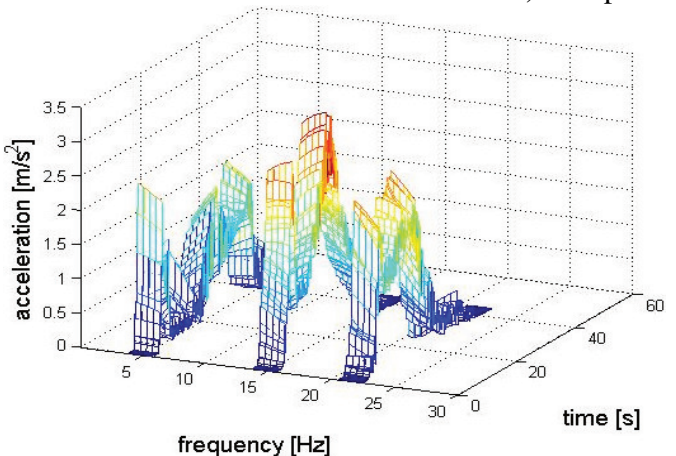

c) Tire pressure $2600 \mathrm{hPa}$

Fig. 9.11. Time distribution of the vibration in chosen frequency bands of floor under rear left passenger's feet 


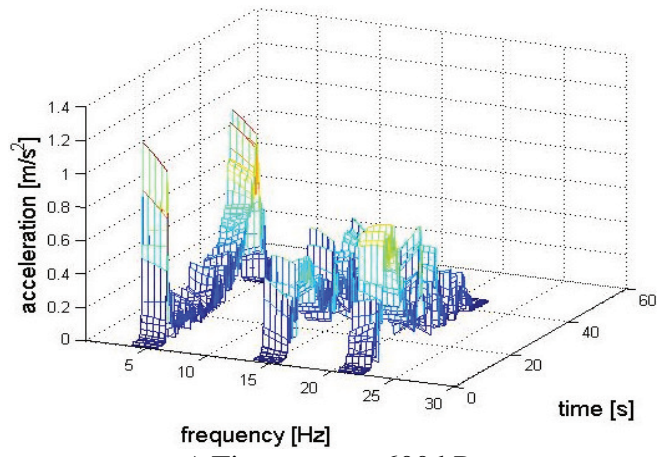

a) Tire pressure $600 \mathrm{hPa}$

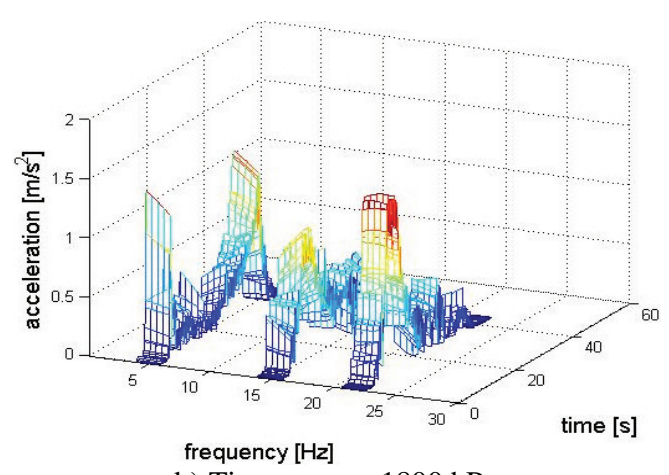

b) Tire pressure $1800 \mathrm{hPa}$

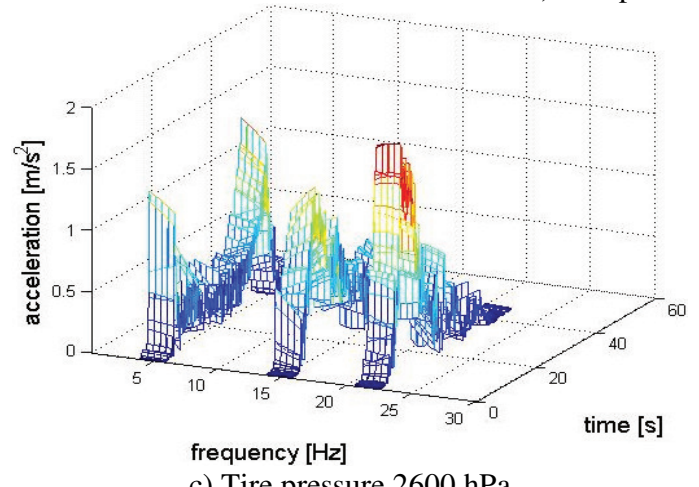

c) Tire pressure $2600 \mathrm{hPa}$

Fig. 9.12. Time distribution of the vibration in chosen frequency bands of floor under rear right passenger's feet

\subsection{Function of exposure time to the vibration of defined frequency bands}

Considering the variability of the time and frequency distribution of the vibration even in selected analyzed bands, the time function was developed as vibration exposure estimator. It is calculated of average value of frequency bands vibration in time domain, expressed as:

$S_{a v r}=\sum_{i=0}^{\infty}\left(\frac{1}{\frac{b-a}{d}} \sum_{f=a}^{b} S\left(\omega_{f}, t_{i}\right)\right)$,

where: $a$ - lover edge of the passband, $b$ - upper edge of the passband, $d$ - STFT frequency resolution (integer rounded value), $(b-a) / d$ - returns number of samples in frequency bands.

Fig. 9.13 presents process of the determination of the average value of frequency bands vibration $11-13 \mathrm{~Hz}$, in time domain.

Fig. 9.14 shows the comparison of the time function of average vibration in those frequency band for the car with shock absorber with $100 \%$ and $50 \%$ of liquid volume.

Figs. 9.15-9.20 present the comparison of time function of average vibration in chosen frequency bands for the vehicle with build in shock absorber with $100 \%$ and $50 \%$ of liquid volume. The chapter presents result obtained for the following frequencies bands: $11-13 \mathrm{~Hz}$ (Figs. 9.13 and 9.14), 20.5-22.5 Hz and 63.5-65.5 Hz.

The comparison of time function of average vibration in chosen frequency bands for the vehicle with tire pressure $600 \mathrm{hPa}, 1800 \mathrm{hPa}$ (nominal) and $2600 \mathrm{hPa}$ have been depicted in Figs. 9.21-9.26. The chapter presents result obtained for the frequencies bands: 4-6 Hz, 14-16 Hz and $20.5-22.5 \mathrm{~Hz}$. 


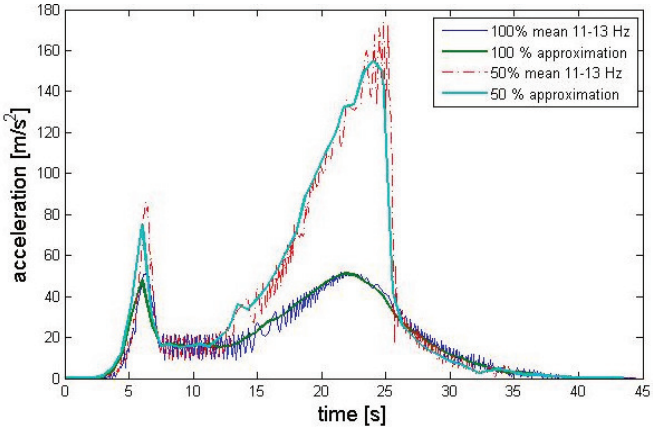

a) Suspension arm

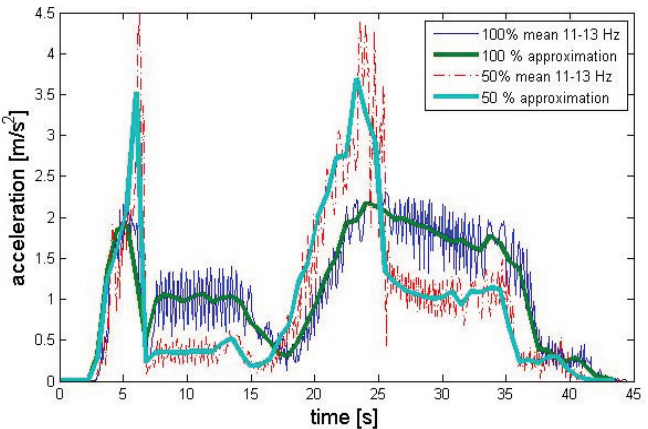

b) Upper mounting of shock absorber

Fig. 9.13. The process of the determination of the average value of frequency bands vibration $(11-13 \mathrm{~Hz})$ in time domain

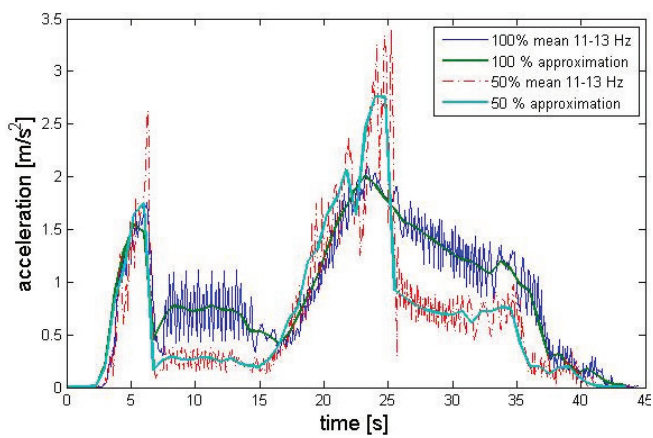

a) Floor under driver's feet

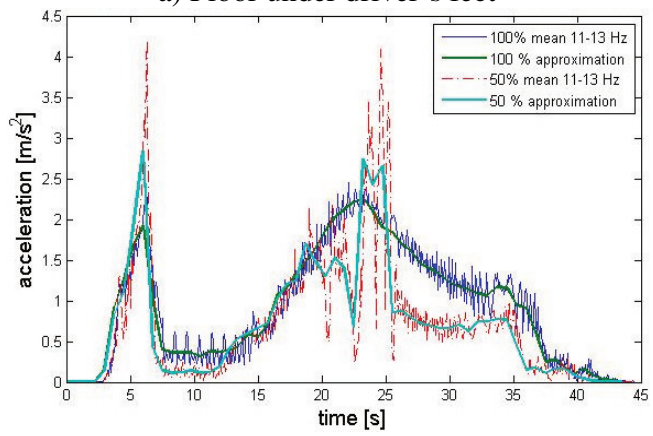

c) Under rear left passenger' feet

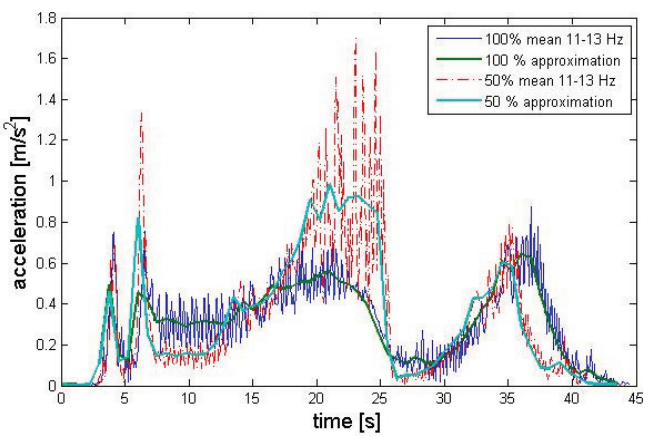

b) Under front passenger' feet

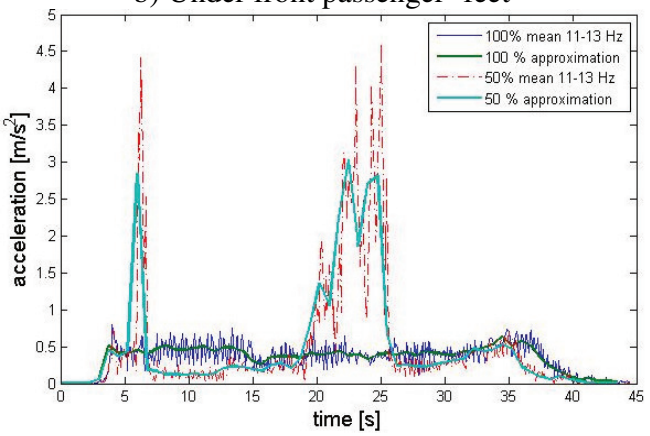

d) Under rear right passenger' feet

Fig. 9.14. The process of the determination of the average value of frequency bands vibration $(11-13 \mathrm{~Hz})$ in time domain

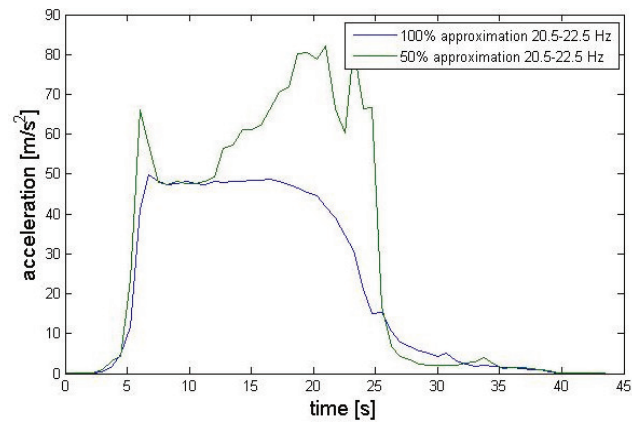

a) $20.5-22.5 \mathrm{~Hz}$

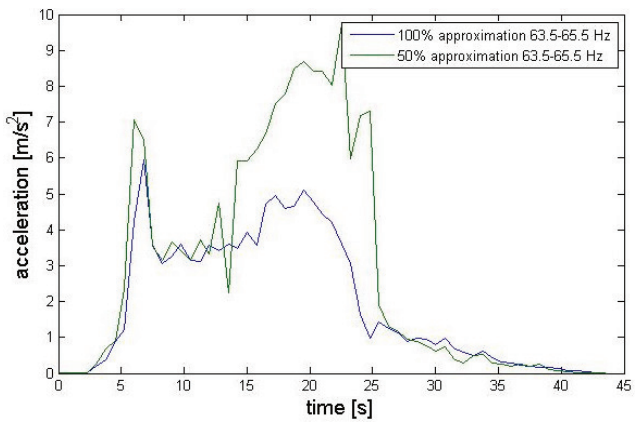

b) $63.5-65.5 \mathrm{~Hz}$

Fig. 9.15. Time function of average vibration in frequency bands: suspension arm of shock absorber 


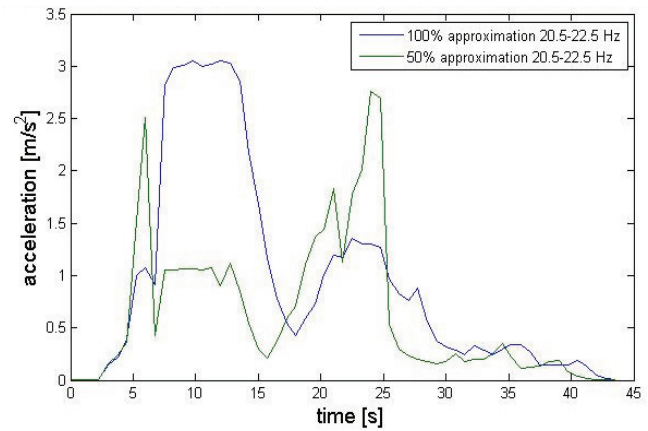

a) $20.5-22.5 \mathrm{~Hz}$

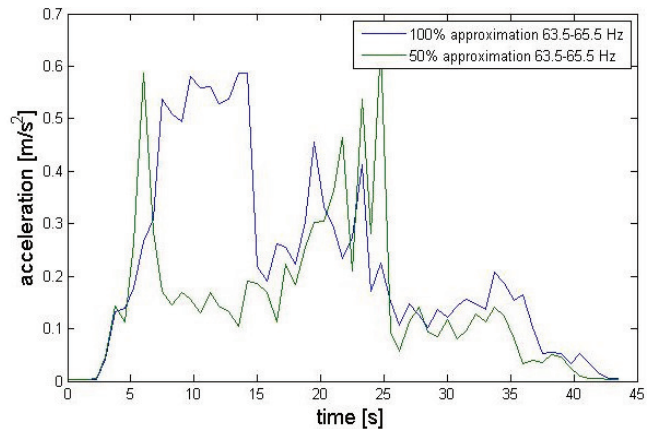

b) $63.5-65.5 \mathrm{~Hz}$

Fig. 9.16. Time function of average vibration in frequency bands: upper mounting of shock absorber

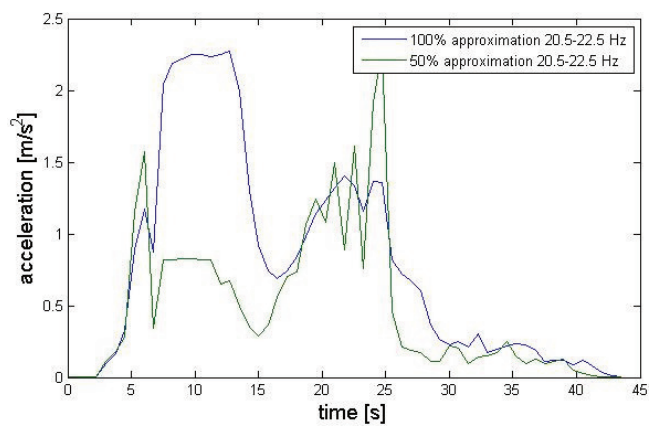

a) $20.5-22.5 \mathrm{~Hz}$

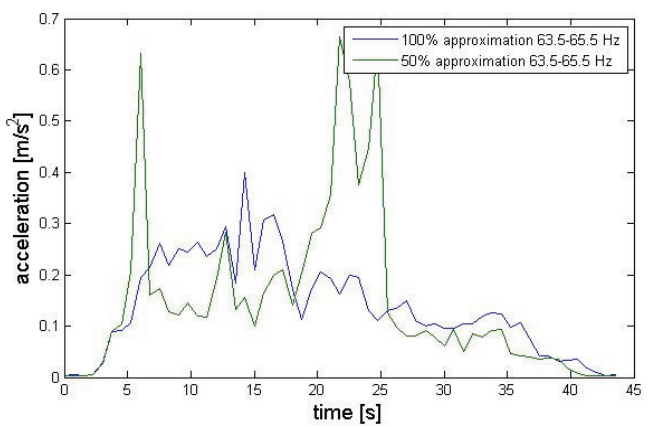

b) $63.5-65.5 \mathrm{~Hz}$

Fig. 9.17. Time function of average vibration in frequency bands: floor under driver's feet

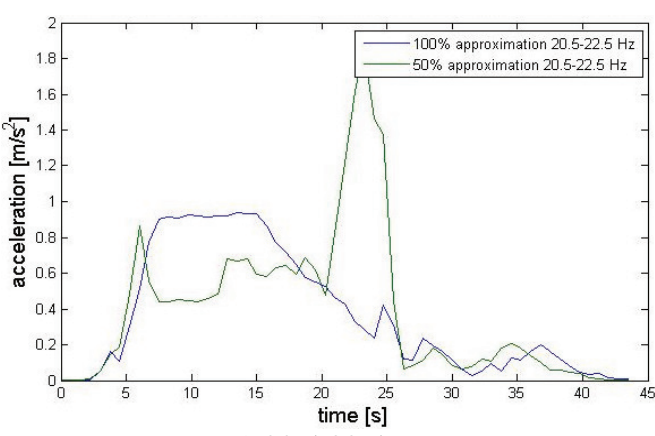

a) $20.5-22.5 \mathrm{~Hz}$

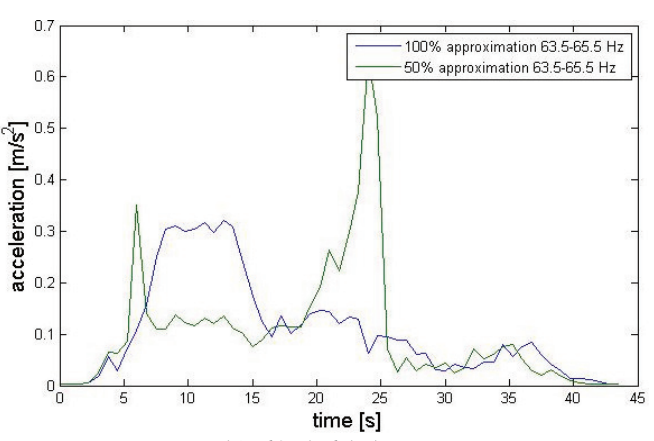

b) $63.5-65.5 \mathrm{~Hz}$

Fig. 9.18. Time function of average vibration in frequency bands: floor under front passenger's feet

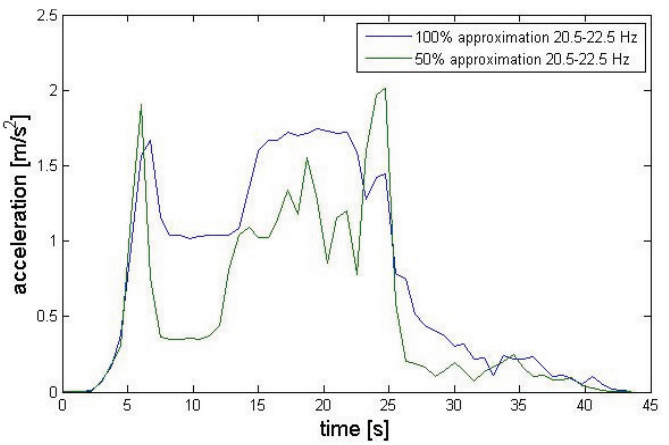

a) $20.5-22.5 \mathrm{~Hz}$

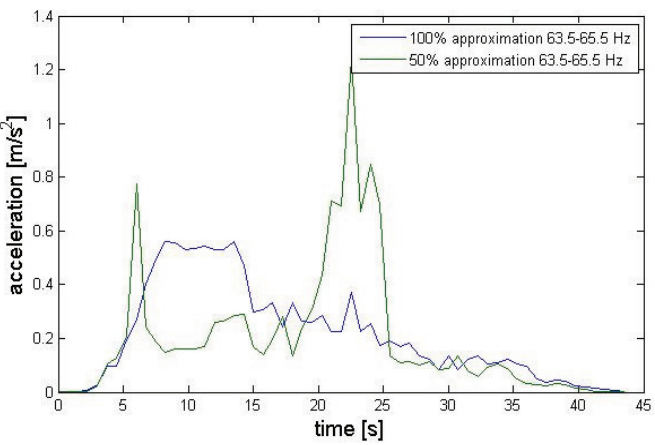

b) $63.5-65.5 \mathrm{~Hz}$

Fig. 9.19. Time function of average vibration in frequency bands: floor under rear left passenger's feet 


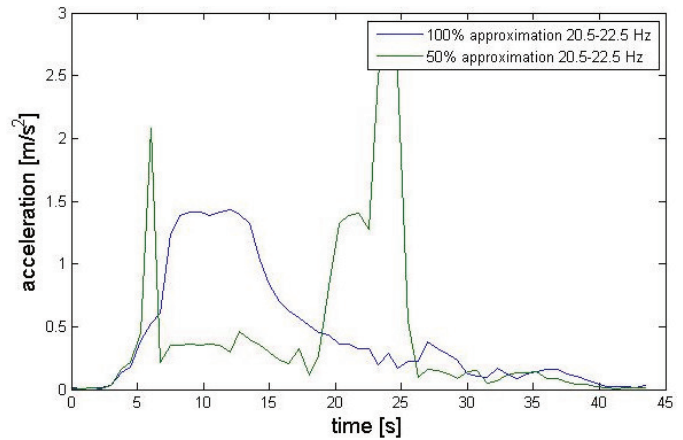

a) $20.5-22.5 \mathrm{~Hz}$

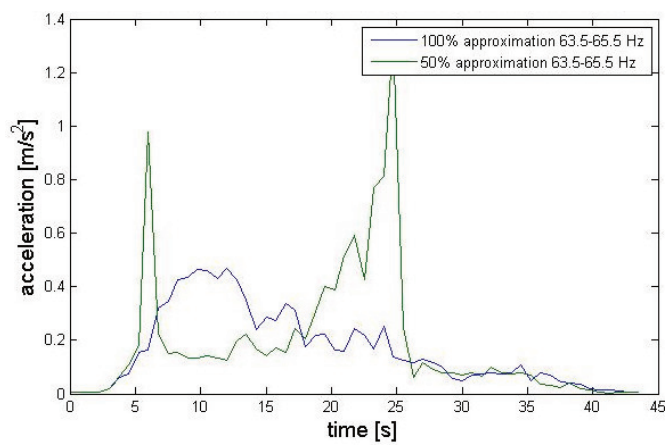

b) $63.5-65.5 \mathrm{~Hz}$

Fig. 9.20. Time function of average vibration in frequency bands: floor under rear right passenger's feet

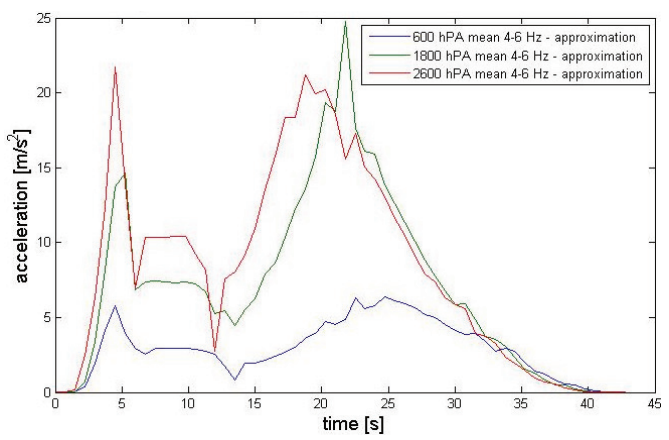

a) $4-6 \mathrm{~Hz}$

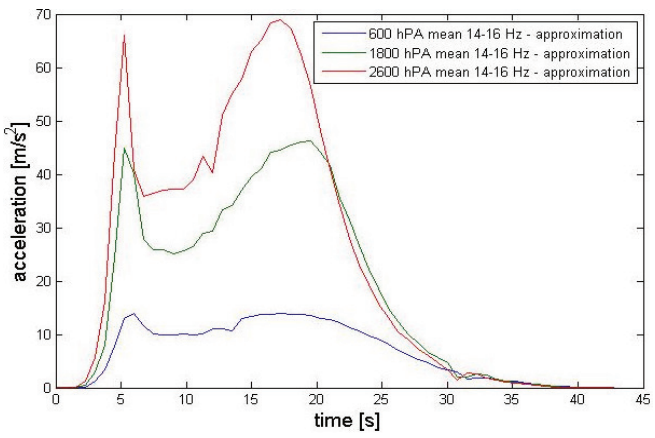

b) $14-16 \mathrm{~Hz}$

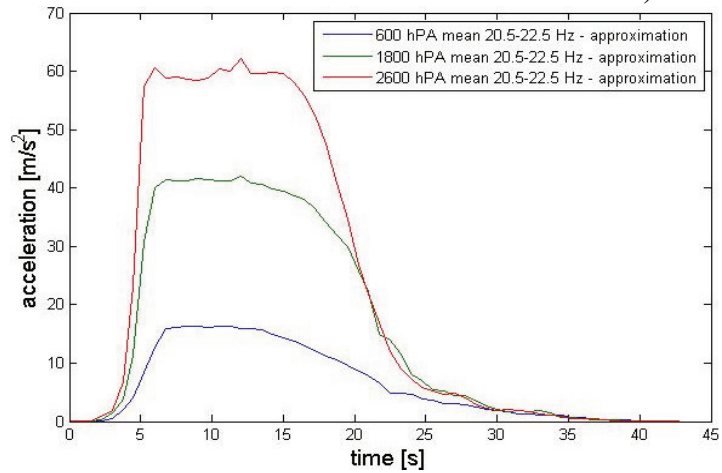

c) $20.5-22.5 \mathrm{~Hz}$

Fig. 9.21. Time function of average vibration of suspension arm in frequency bands

Previous chapter presents the application of STFT 3-D time-frequency distributions for proper observation of the vibration structure. The obtained representation of the vibration allow to determine time function of separate frequency bands represents the isolated vibration dynamics phenomena. The changes of the vibration energy in chosen frequency bands can be observed by the time function of average vibration in chosen frequency bands. The results of comparison of the vibration of the vehicle with build in shock absorber with $100 \%$ and $50 \%$ of liquid volume show the influence of damping properties on chosen frequency bands. The analysis of the results shows increase of the vibration energy for the vehicle with shock absorber with $50 \%$ of liquid volume but for determined time realization. For the rest of the time of excitation the vibration are even lower, especially during constant excitation (ca. between 9-14 second). The maximums values of the functions are in ca. 5th and 25 th seconds of the excitation. It is the effect of time of passing thru unsprung masses resonance, ca. $12 \mathrm{~Hz}$. 


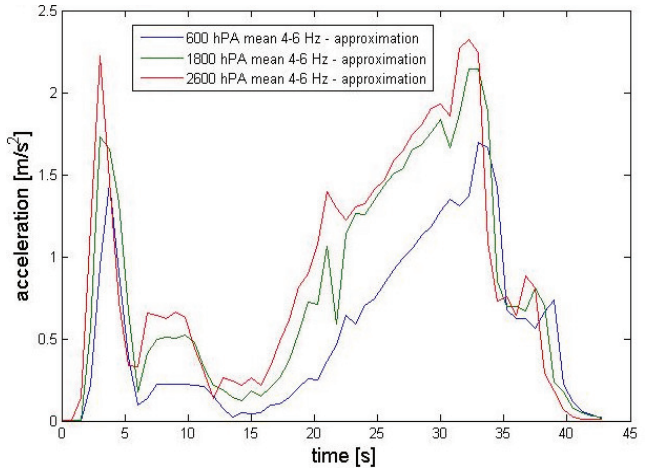

a) $4-6 \mathrm{~Hz}$

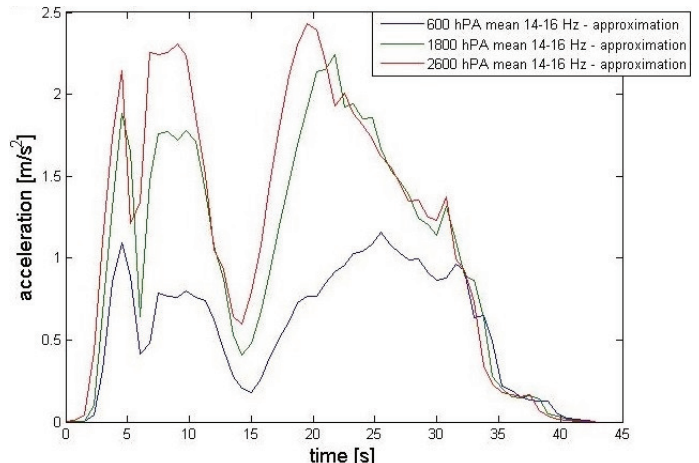

b) $14-16 \mathrm{~Hz}$

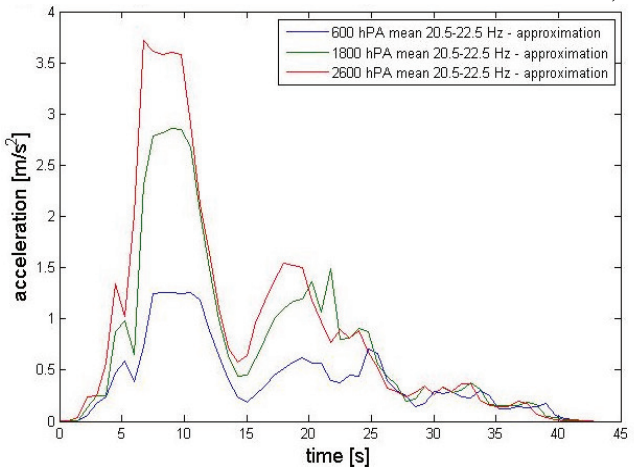

c) $20.5-22.5 \mathrm{~Hz}$

Fig. 9.22. Time function of average vibration of upper mounting of shock absorber in frequency bands

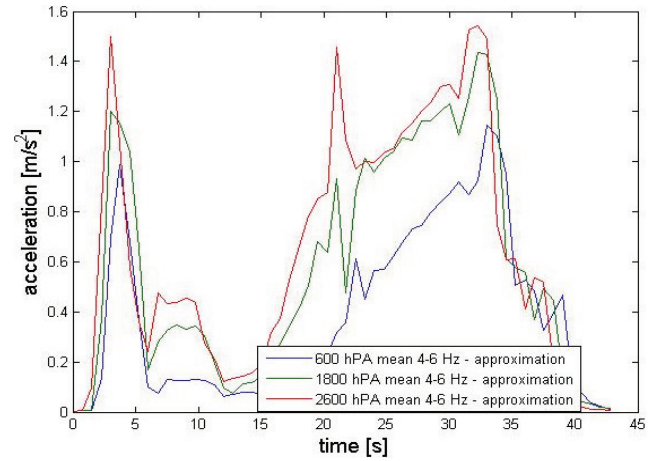

a) $4-6 \mathrm{~Hz}$

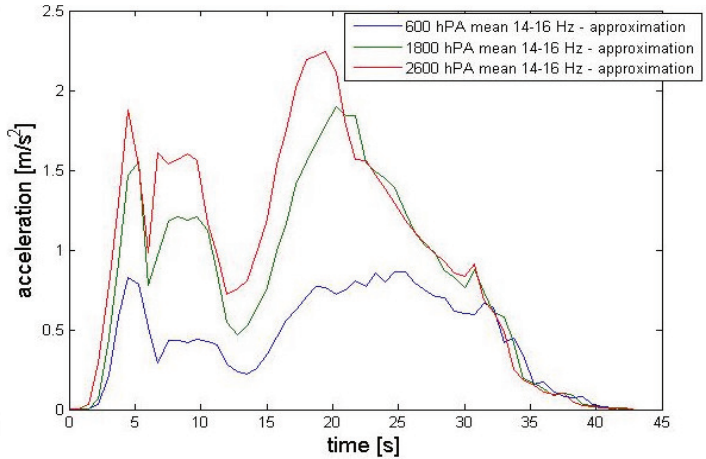

b) $14-16 \mathrm{~Hz}$

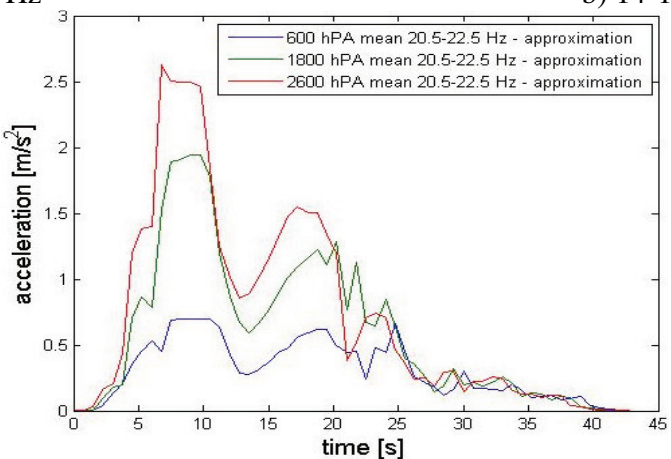

c) $20.5-22.5 \mathrm{~Hz}$

Fig. 9.23. Time function of average vibration of floor under driver's feet in frequency bands 


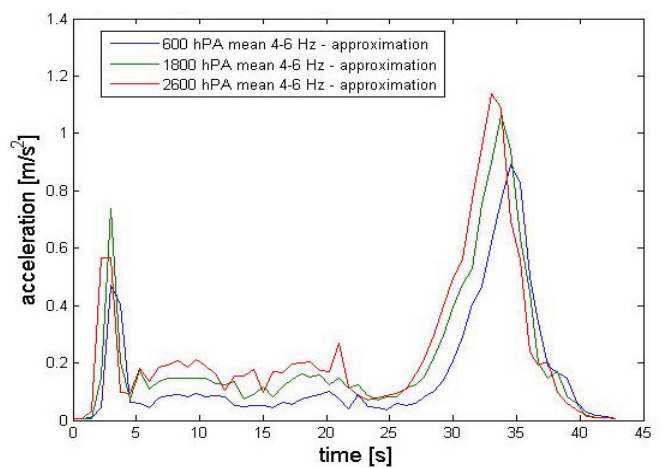

a) $4-6 \mathrm{~Hz}$

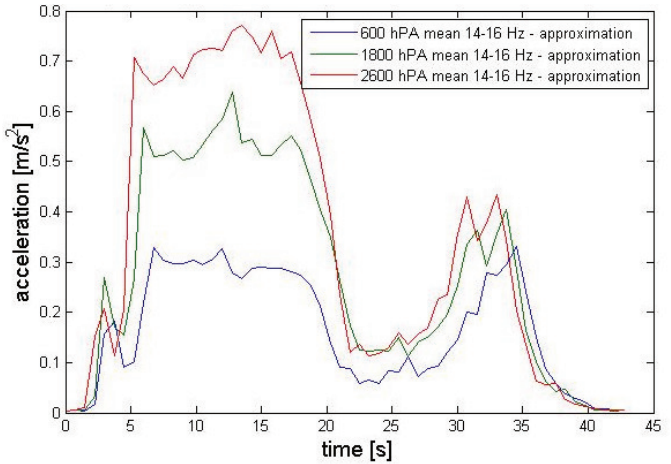

b) $14-16 \mathrm{~Hz}$

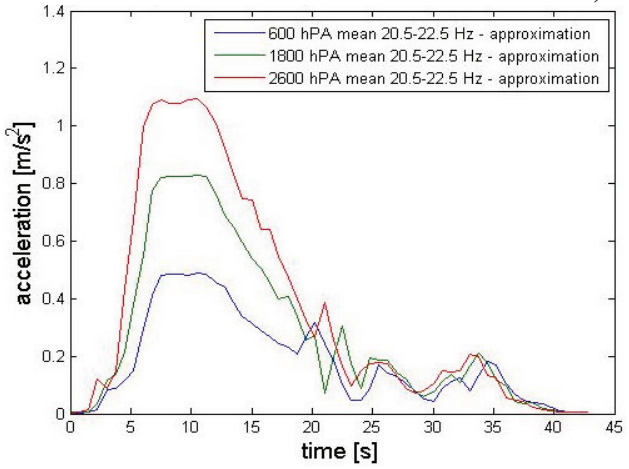

c) $20.5-22.5 \mathrm{~Hz}$

Fig. 9.24. Time function of average vibration of floor under front passenger's feet in frequency bands

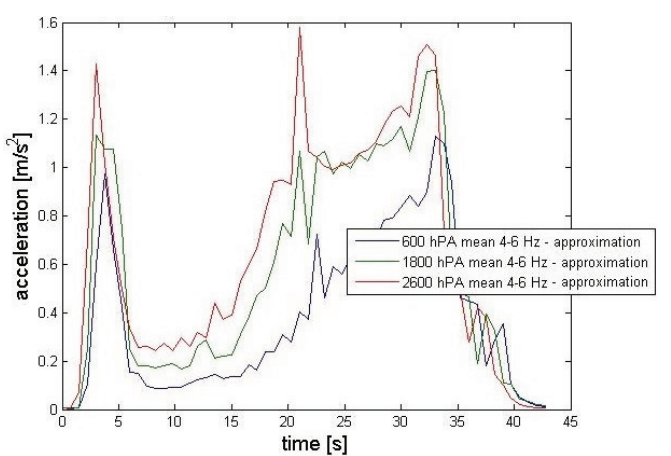

a) $4-6 \mathrm{~Hz}$

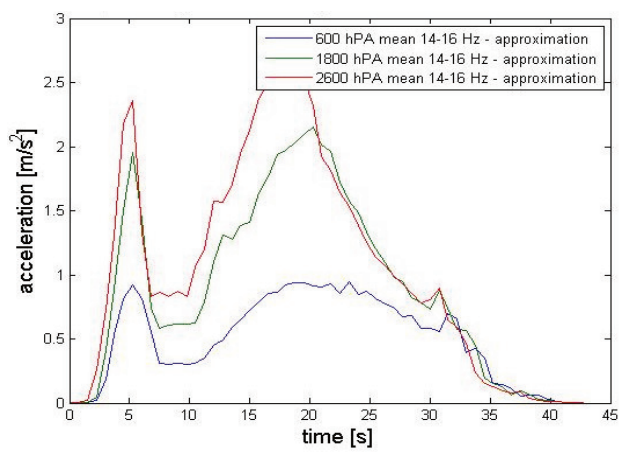

b) $14-16 \mathrm{~Hz}$

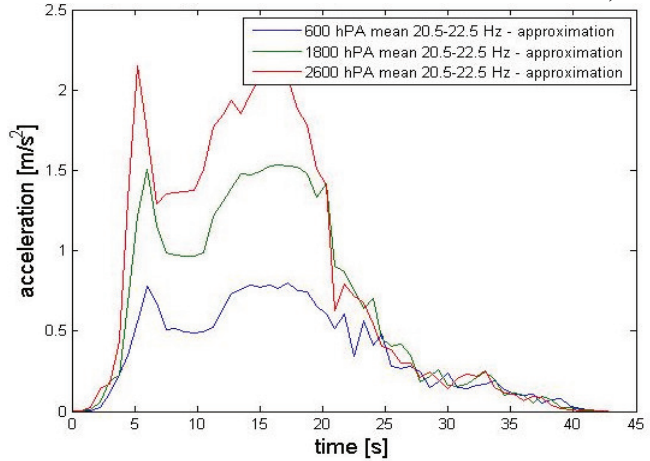

c) $20.5-22.5 \mathrm{~Hz}$

Fig. 9.25. Time function of average vibration of floor under rear left passenger's feet in frequency bands 


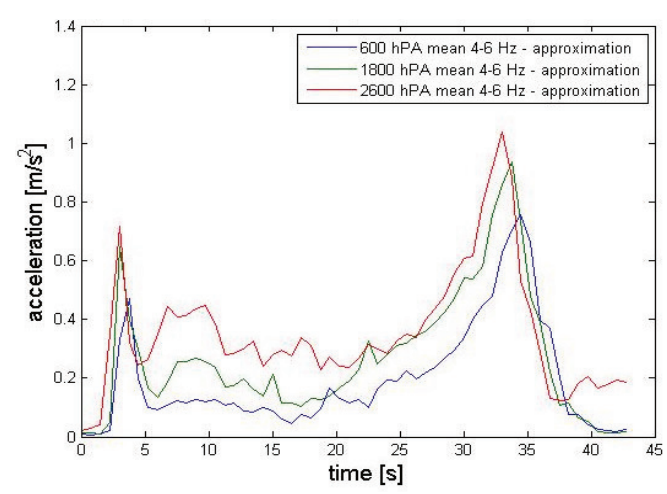

a) $4-6 \mathrm{~Hz}$

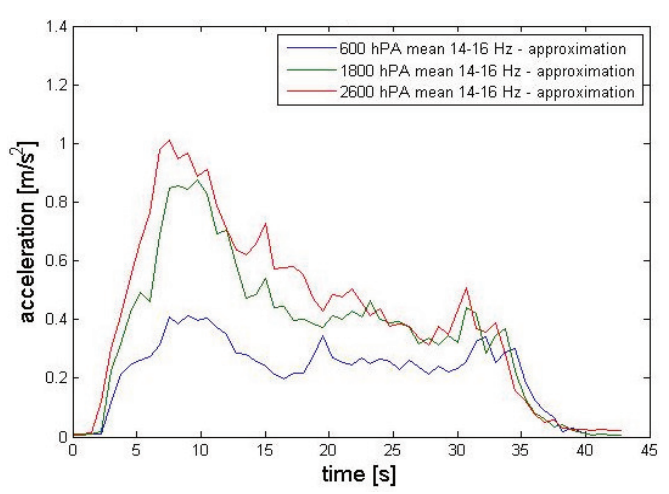

b) $14-16 \mathrm{~Hz}$

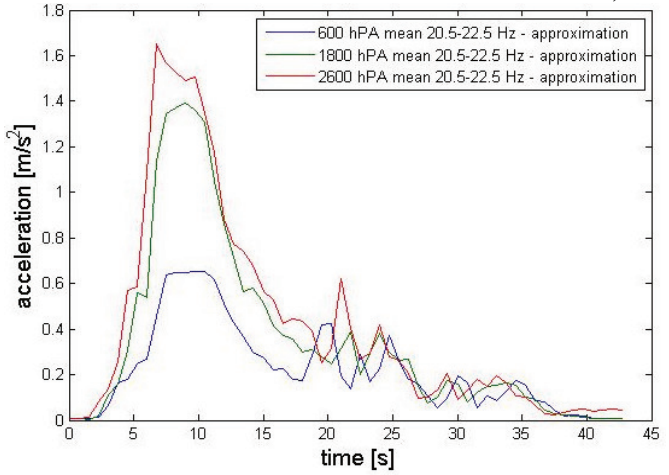

c) $20.5-22.5 \mathrm{~Hz}$

Fig. 9.26. Time function of average vibration of floor under rear right passenger's feet in frequency bands

The results of time function of average vibration in chosen frequency bands for the vehicle with tire pressure $600 \mathrm{hPa}, 1800 \mathrm{hPa}$ (nominal) and $2600 \mathrm{hPa}$ represents very sensitivity symptom estimator. Almost in every investigated case the functions were totally separable in whole time domain. The trend of increase of vibration due to increase of pressure in tire was noticeable for all results. The local maximum of the function can be localized ca. 5, 20 and 35 seconds.

Very important conclusion is that function proposed of exposure time to the vibration of defined frequency bands is sensitivity for vibration of the floor panel in location of driver and passengers feet. This is the place of vibration transfer from vehicle via feet to the humans in mean of transport. Thus formulated and developed function may be useful for exposure to vibration evaluation or monitoring. 


\section{Resonance spectrum in research on the influence of the technical condition of suspension elements and of the vehicle operating parameters on the vibration}

The functions of exposure time to the vibration of defined frequency bands $\left(S_{f}, S_{\text {avr }}\right)$ facilitate the identification and location of the time of increase of vibration activity of the mechanical system. The increase of vibration energy dissipation can be result of resonance occurring. For the mechanical system with the masses of the motor vehicle almost the total vibration energy can be related to the 2 types of resonances, of sprung and unsprung masses. For the purpose of evaluation of vibration in terms of human perception and exposure it is important to observe energy of the vibration in frequency bands correlated to the natural vibration of human organs. Thus the expanded methodology for identification of dominant frequency components forced during the resonance is developed. As result of the methodology the function estimator as resonance spectrum is determined.

\subsection{Spectrum of the vibration occurring during resonance}

Basing on the analysis of the time course of vibration in chosen frequencies bands, presented in previous chapter, the time of increased energy of vibration propagation can be clearly indicated. It can be result of passing by resonance frequency by this time. For observation of frequencies carrying most of the vibration energy the function of frequency distribution for determined time period was calculated and is expressed as:

$S_{t}=\sum_{t=a}^{b} \sum_{f=0}^{\infty} S\left(\omega_{f}, t_{i}\right)$,

where: $t$ - time period from $a$ to $b, f$-frequency, $S\left(\omega_{f}, t_{i}\right)$ - Short-Time Fourier Transformation of the signal.

The obtained results of research on influence of damping properties of shock absorbers on vehicle vibration, for all investigated cases, show that vibration occurs in ca. 5 and 25 seconds are higher. Figs. 10.1-10.6 present frequency distribution of the vibration for resonance passing.

The $S_{t}$ structure was calculated from results of research on influence of tire pressure on vibration propagation in vehicle construction. Results analysis of vibration structure, presented in previous chapter, confirms the increase of vibration occurring at ca. $4 \mathrm{~s}, 20 \mathrm{~s}$ and $34 \mathrm{~s}$. Figures below present frequency distribution of the vibration for those time passing. 


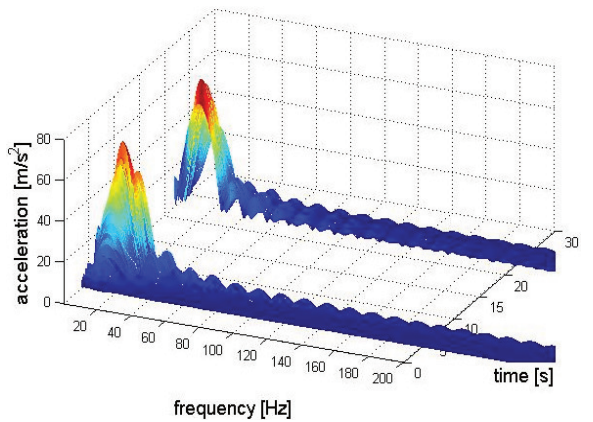

a) Shock absorber with $100 \%$ of liquid volume

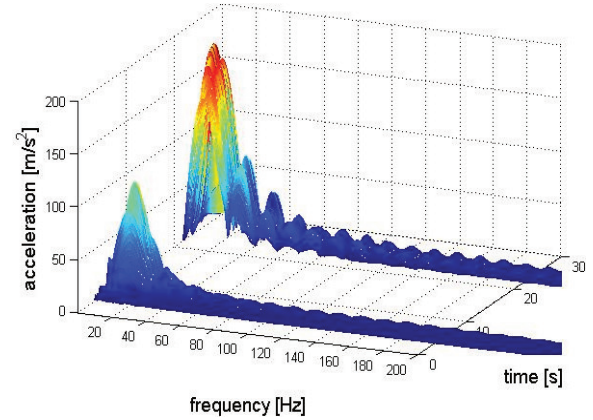

b) Shock absorber with $50 \%$ of liquid volume

Fig. 10.1. Frequency distribution of the vibration for resonance passing of suspension arm

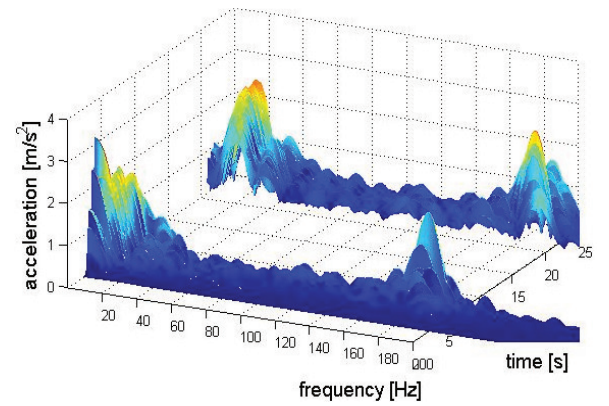

a) Shock absorber with $100 \%$ of liquid volume

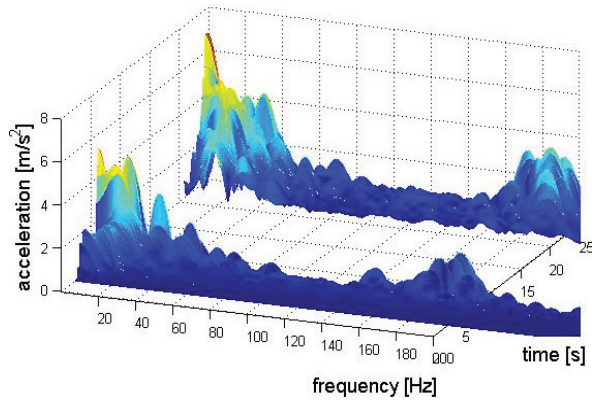

b) Shock absorber with $50 \%$ of liquid volume

Fig. 10.2. Frequency distribution of the vibration for resonance passing of upper mounting of shock absorber

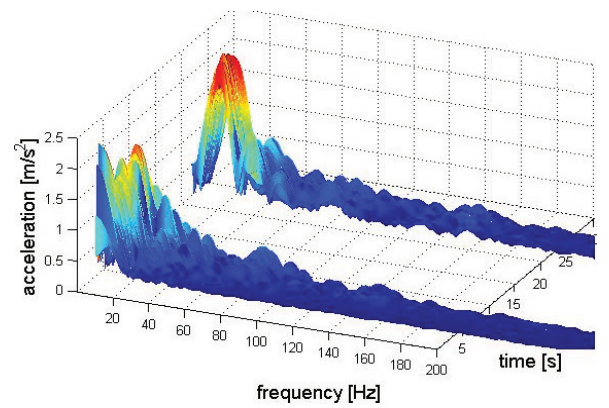

a) Shock absorber with $100 \%$ of liquid volume

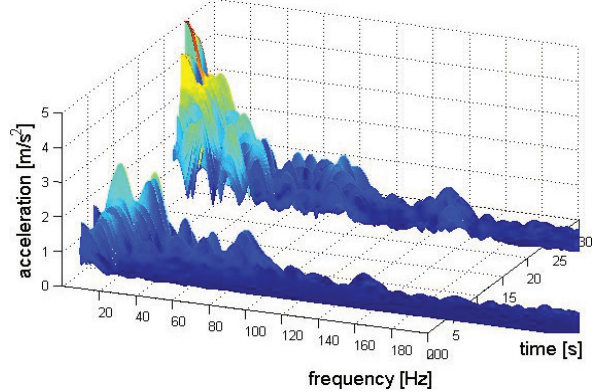

b) Shock absorber with $50 \%$ of liquid volume

Fig. 10.3. Frequency distribution of the vibration for resonance passing of floor under driver's feet

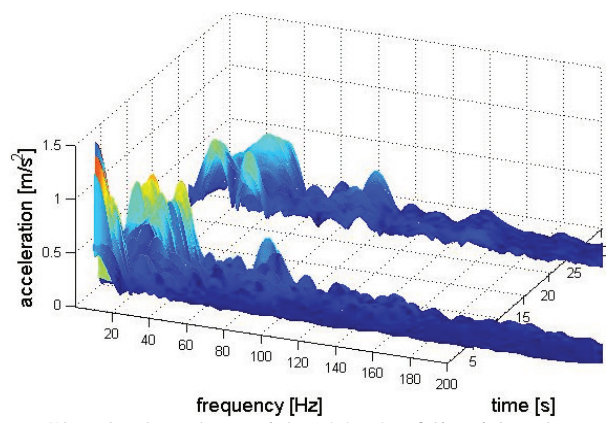

a) Shock absorber with $100 \%$ of liquid volume

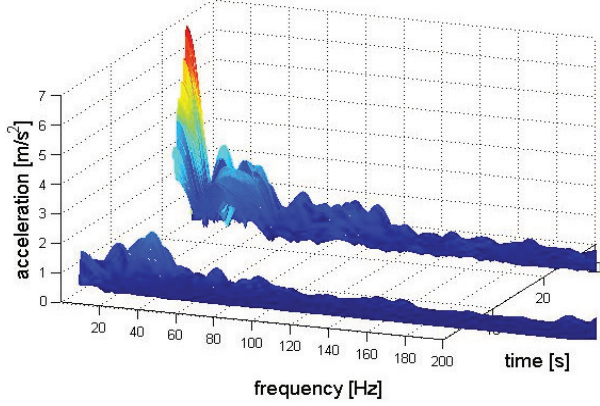

b) Shock absorber with $50 \%$ of liquid volume

Fig. 10.4. Frequency distribution of the vibration for resonance passing of floor under front passenger's feet 


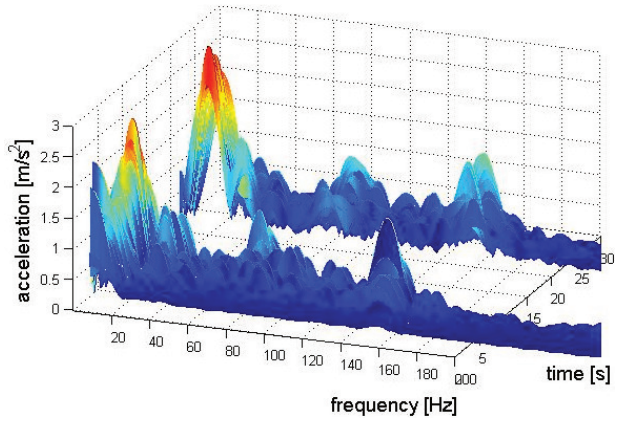

a) Shock absorber with $100 \%$ of liquid volume

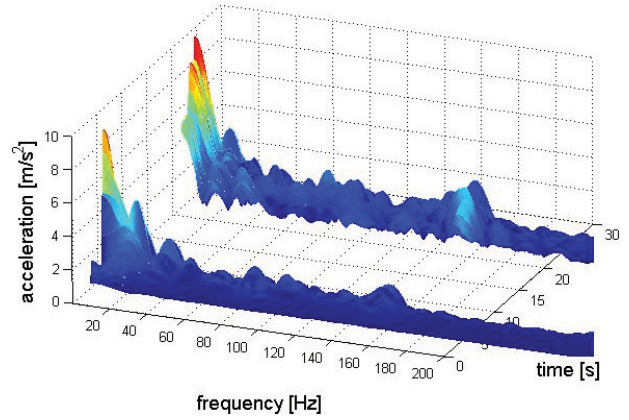

b) Shock absorber with $50 \%$ of liquid volume

Fig. 10.5. Frequency distribution of the vibration for resonance passing of floor under rear left passenger's feet

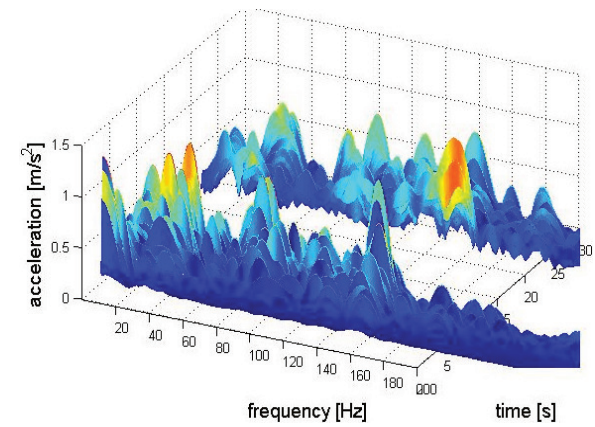

a) Shock absorber with $100 \%$ of liquid volume

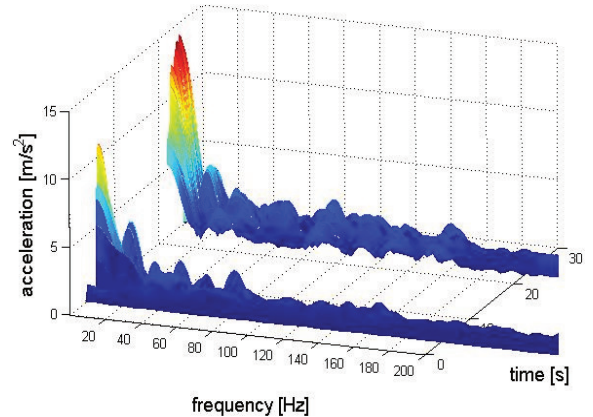

b) Shock absorber with $50 \%$ of liquid volume

Fig. 10.6. Frequency distribution of the vibration for resonance passing of floor under rear right passenger's feet

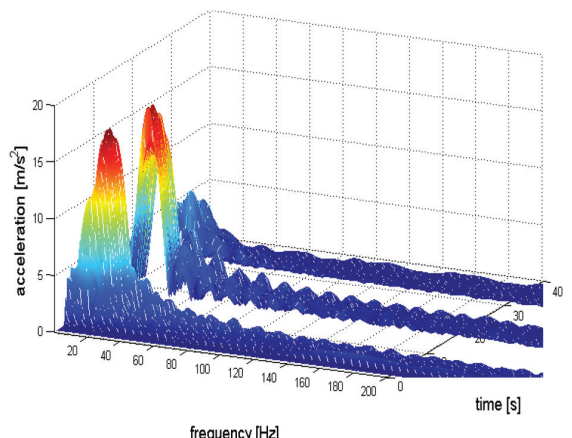

a) Tire pressure $600 \mathrm{hPa}$

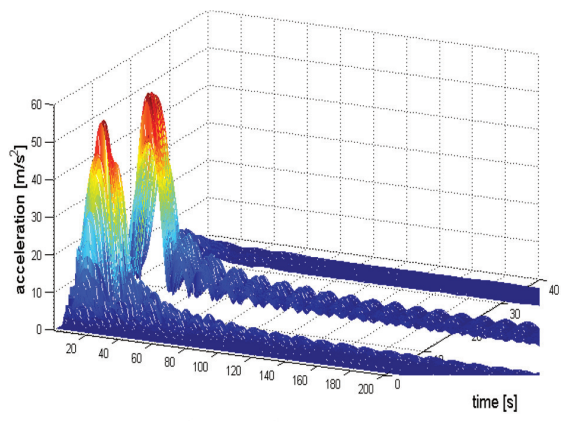

frequency $[\mathrm{Hz}]$

b) Tire pressure $1800 \mathrm{hPa}$

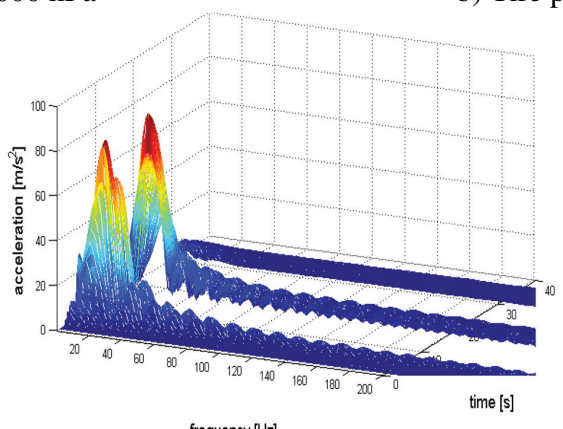

c) Tire pressure $2600 \mathrm{hPa}$

Fig. 10.7. Frequency distribution of the vibration for resonance passing of suspension arm 


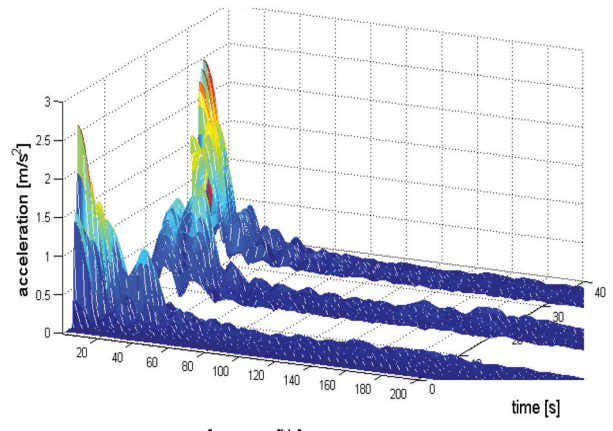

frequency [Hz]

a) Tire pressure $600 \mathrm{hPa}$

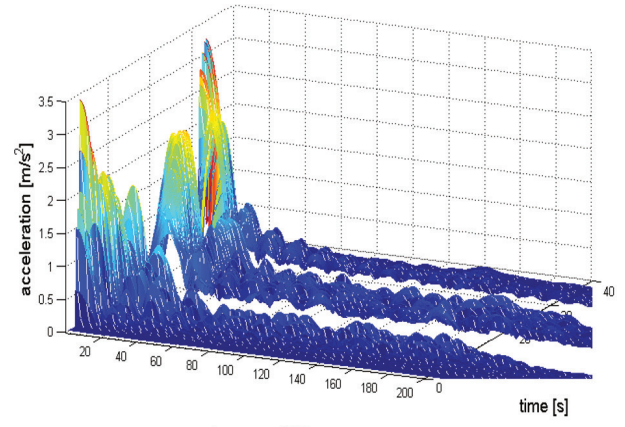

frequency $[\mathrm{Hz}]$

b) Tire pressure $1800 \mathrm{hPa}$

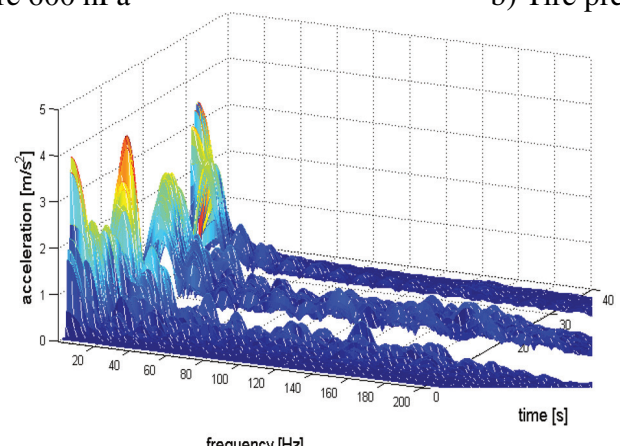

frequency $[\mathrm{Hz}]$

c) Tire pressure $2600 \mathrm{hPa}$

Fig. 10.8. Frequency distribution of the vibration for resonance passing of upper mounting of shock absorber

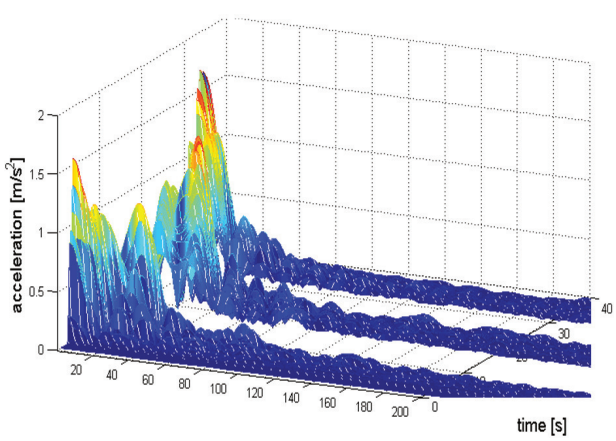

frequency $[\mathrm{Hz}]$

a) Tire pressure $600 \mathrm{hPa}$

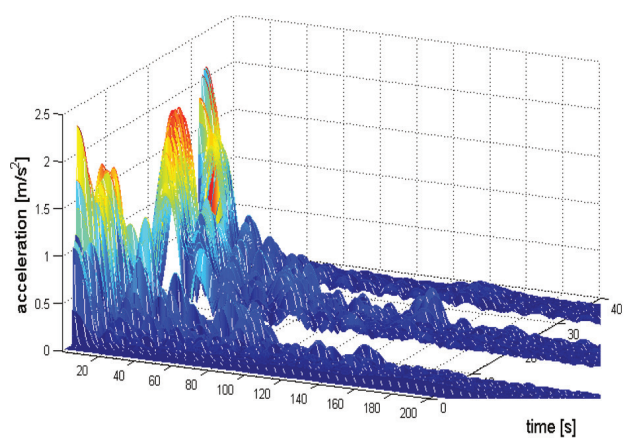

frequency $[\mathrm{Hz}]$

b) Tire pressure $1800 \mathrm{hPa}$

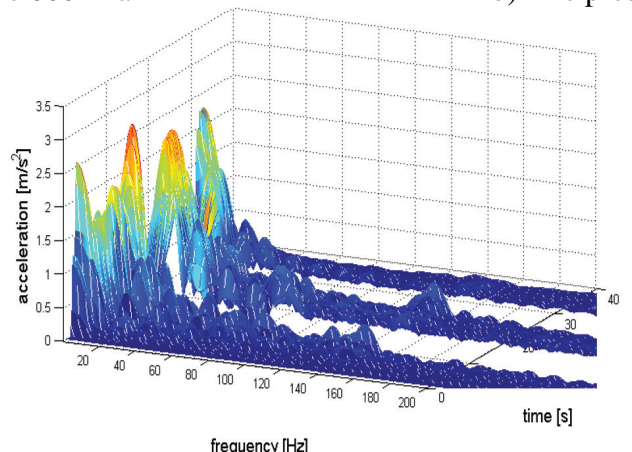

c) Tire pressure $2600 \mathrm{hPa}$

Fig. 10.9. Frequency distribution of the vibration for resonance passing of floor under driver's feet 


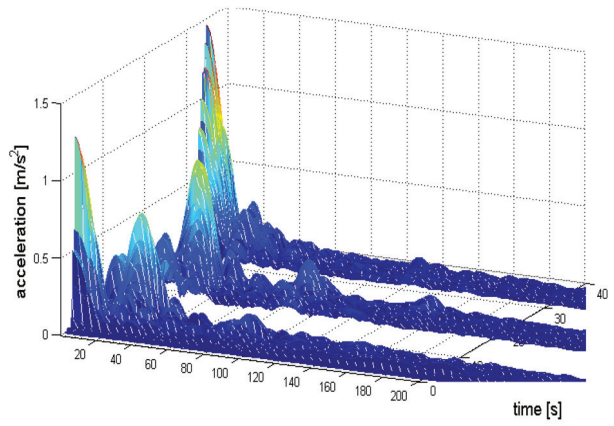

frequency $[\mathrm{Hz}]$

a) Tire pressure $600 \mathrm{hPa}$

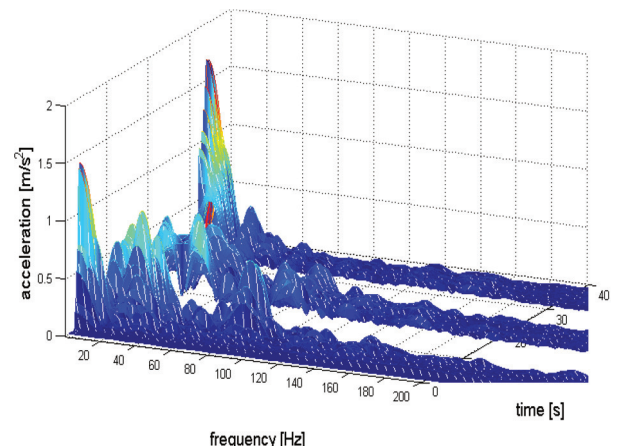

b) Tire pressure $1800 \mathrm{hPa}$

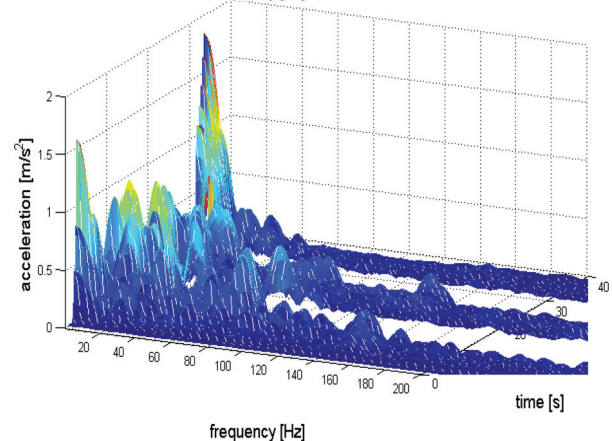

c) Tire pressure $2600 \mathrm{hPa}$

Fig. 10.10. Frequency distribution of the vibration for resonance passing of floor under front passenger's feet

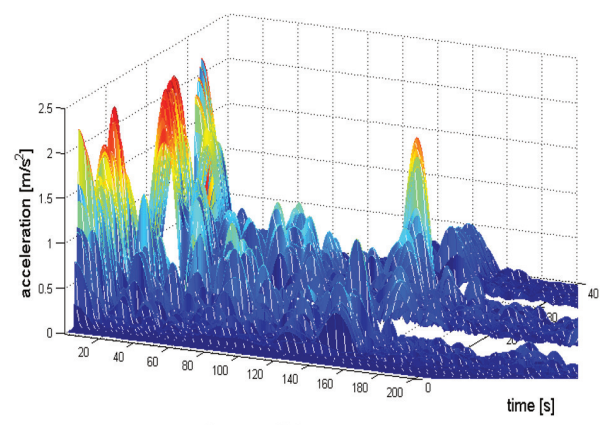

frequency $[\mathrm{Hz}]$

a) Tire pressure $600 \mathrm{hPa}$

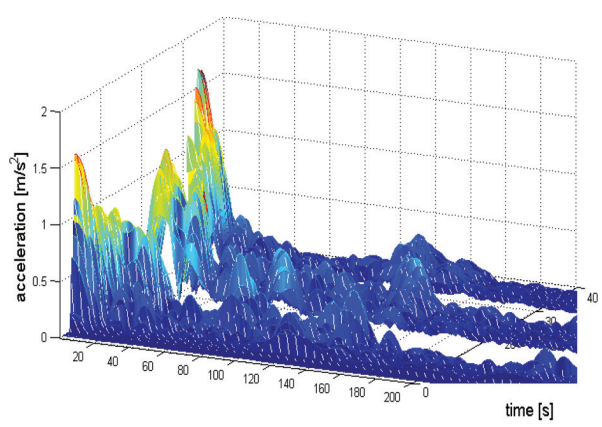

frequency $[\mathrm{Hz}]$

b) Tire pressure $1800 \mathrm{hPa}$

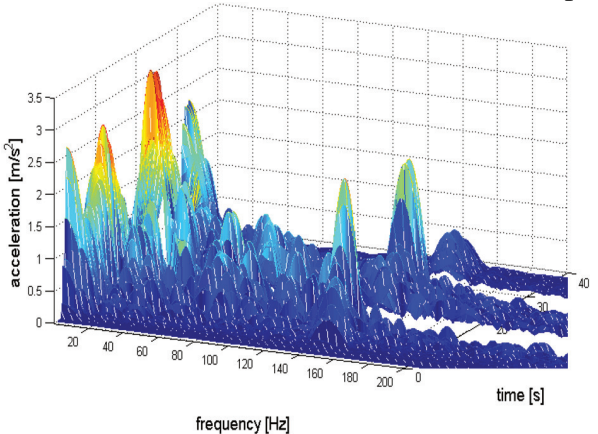

c) Tire pressure $2600 \mathrm{hPa}$

Fig. 10.11. Frequency distribution of the vibration for resonance passing of floor under rear left passenger's feet 


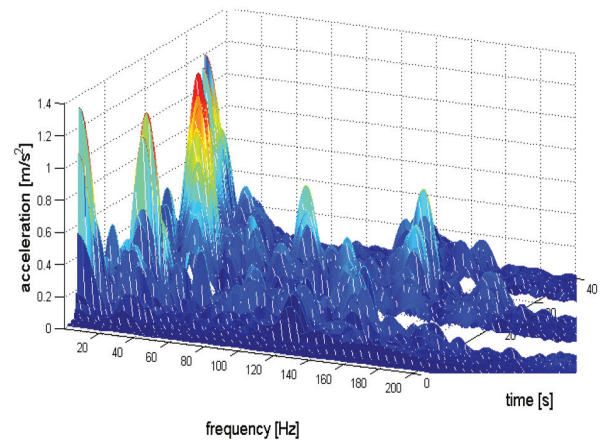

a) Tire pressure $600 \mathrm{hPa}$

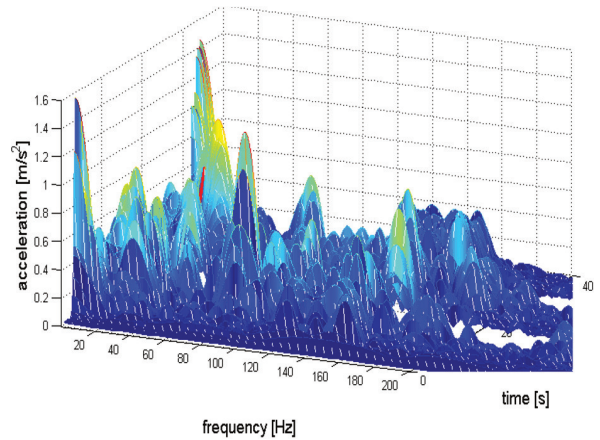

b) Tire pressure $1800 \mathrm{hPa}$

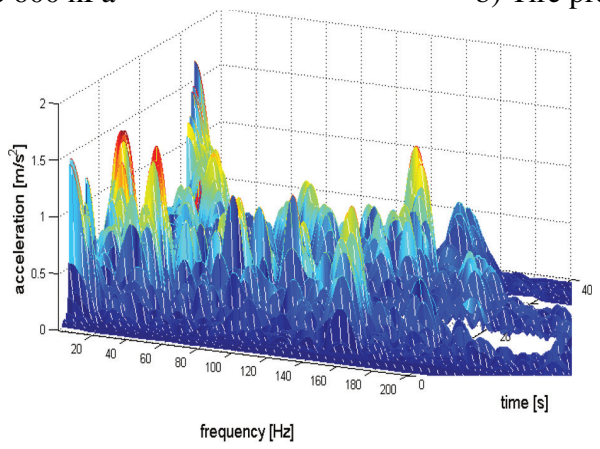

c) Tire pressure $2600 \mathrm{hPa}$

Fig. 10.12. Frequency distribution of the vibration for resonance passing of floor under rear right passenger's feet

\subsection{Resonance spectrum - vibration function}

The $S_{t}$ structures of vibration represent dominant dynamics components of vibration excited during most vibro-activity time of exposure. The matrix form of $S_{t}$ can make some difficulties in fast interpretation, especially for the purpose of the comparison. Thus the frequency function have been developed as vibration dynamics exposure estimator. It is calculated of average value of time period vibration in frequency domain, expressed as:

$S_{\text {Tavr }}=\sum_{f=0}^{\infty}\left(\frac{1}{(b-a) / d} \sum_{t=a}^{b} S\left(\omega_{f}, t_{i}\right)\right)$,

where: $a$ - start of the time period, $b$ - end of the time period, $d$-STFT time resolution (integer rounded value), $(b-a) / d-$ returns number of samples in time period.

The process of the determination of the average value of time period vibration in frequency domain, as result of $S_{\text {Tavr }}$ function, is similar to the process of determination of $S_{\text {avr }}$ function presented in Figs. 9.13 and 9.14. The difference is frequency domain instead time domain.

The comparison of frequency function of average vibration in chosen time period for the vehicle with build in shock absorber with $100 \%$ and $50 \%$ of liquid volume is presented in Figs. 10.13-10.18.

The comparison of frequency function of average vibration in chosen time period for the vehicle with tire pressure $600 \mathrm{hPa}, 1800 \mathrm{hPa}$ (nominal) and $2600 \mathrm{hPa}$ have been depicted in Figs. 10.19-10.24. The chapter presents result obtained for the time periods: 1-7 s, 17-23 s and 31-37 s. 


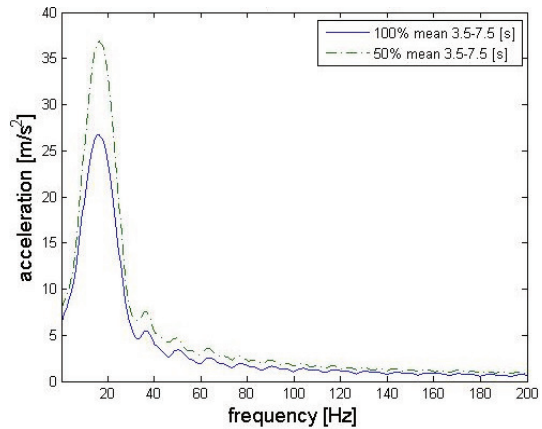

a) $3.5-7.5 \mathrm{~s}$

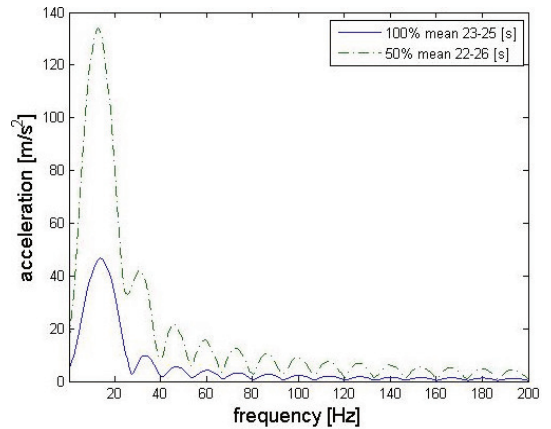

b) $22-26 \mathrm{~s}$

Fig. 10.13. Frequency function of average vibration in time periods: suspension arm

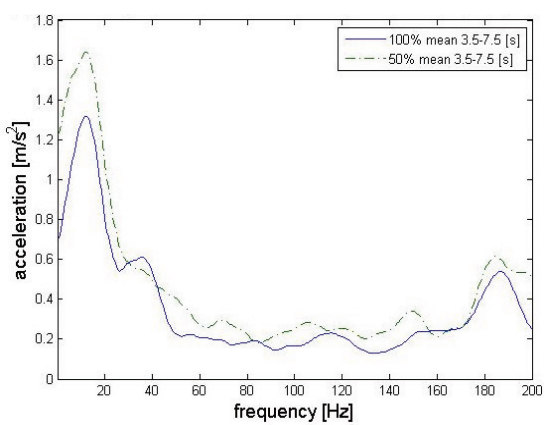

a) $3.5-7.5 \mathrm{~s}$

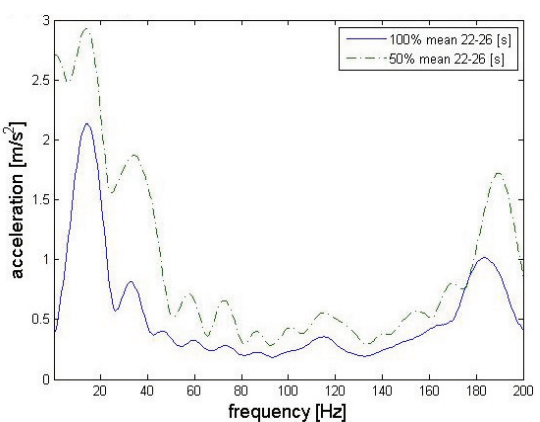

b) $22-26 \mathrm{~s}$

Fig. 10.14. Frequency function of average vibration in time periods: upper mounting of shock absorber

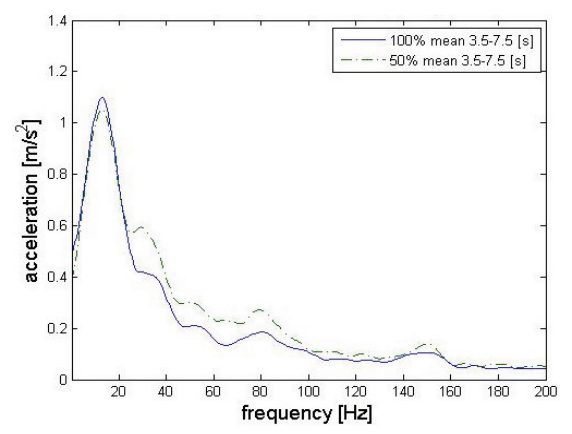

a) $3.5-7.5 \mathrm{~s}$

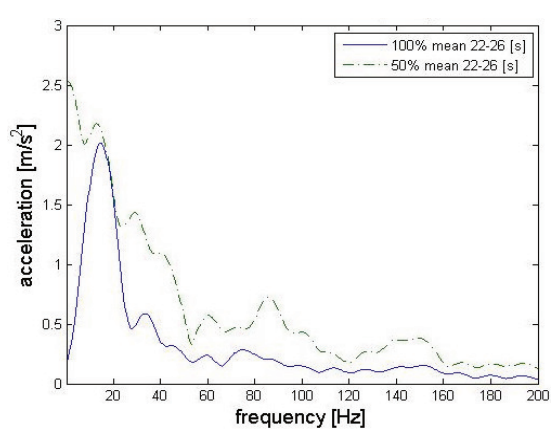

b) $22-26 \mathrm{~s}$

Fig. 10.15. Frequency function of average vibration in time periods: floor under driver's feet

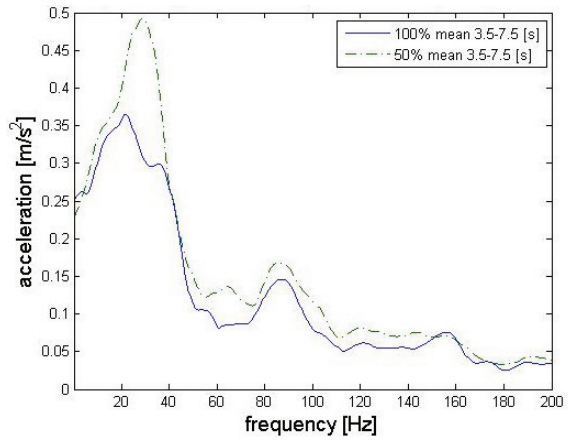

a) $3.5-7.5 \mathrm{~s}$

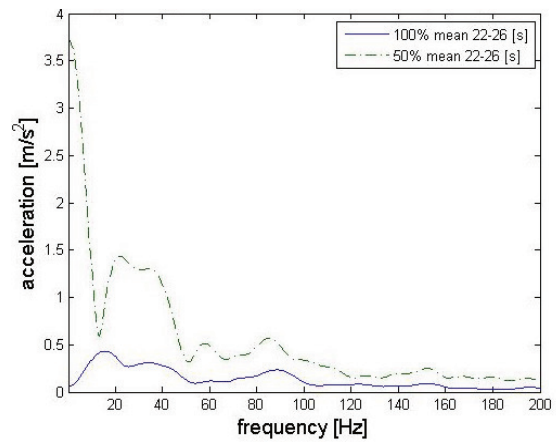

b) $22-26 \mathrm{~s}$

Fig. 10.16. Frequency function of average vibration in time periods: floor under front passenger's feet 


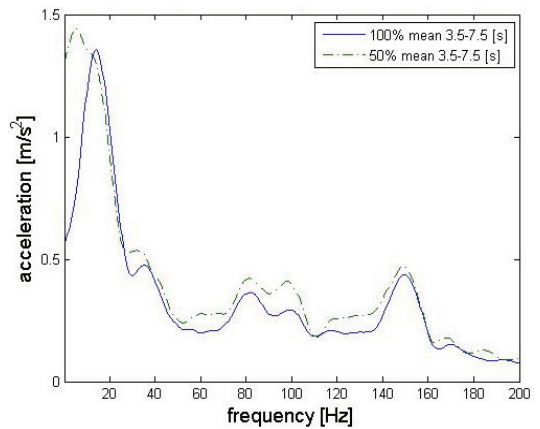

a) $3.5-7.5 \mathrm{~s}$

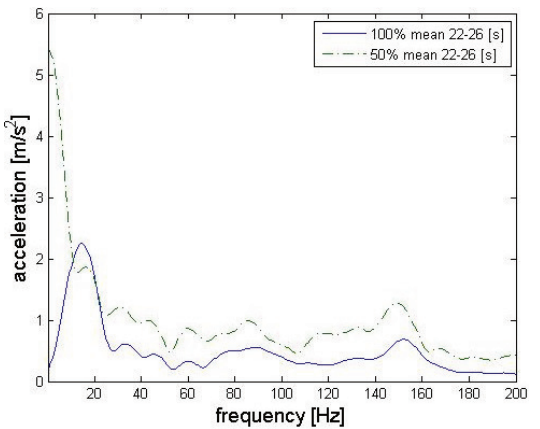

b) $22-26 \mathrm{~s}$

Fig. 10.17. Frequency function of average vibration in time periods: floor under rear left passenger's feet

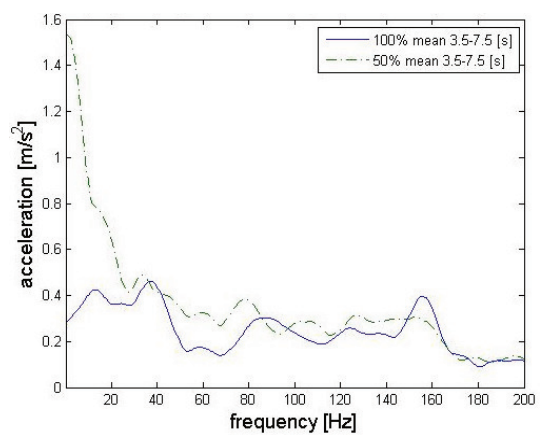

a) $3.5-7.5 \mathrm{~s}$

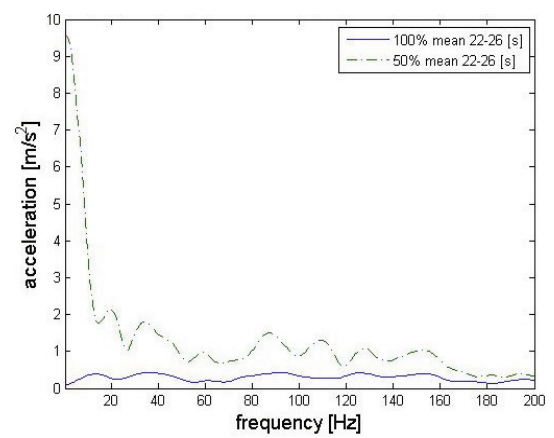

b) $22-26 \mathrm{~s}$

Fig. 10.18. Frequency function of average vibration in time periods: floor under rear right passenger's feet

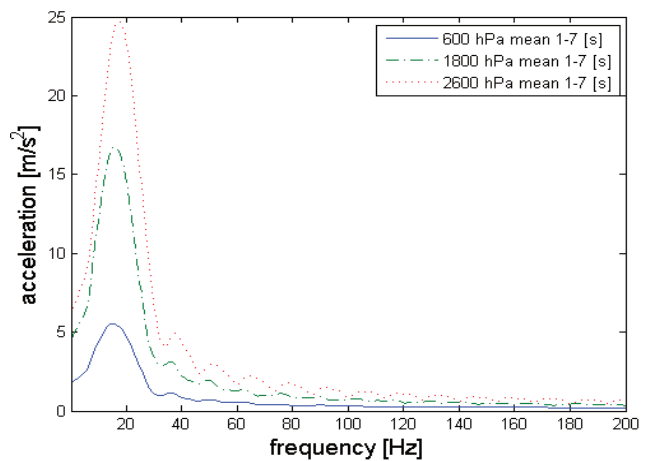

a) $1-7 \mathrm{~s}$

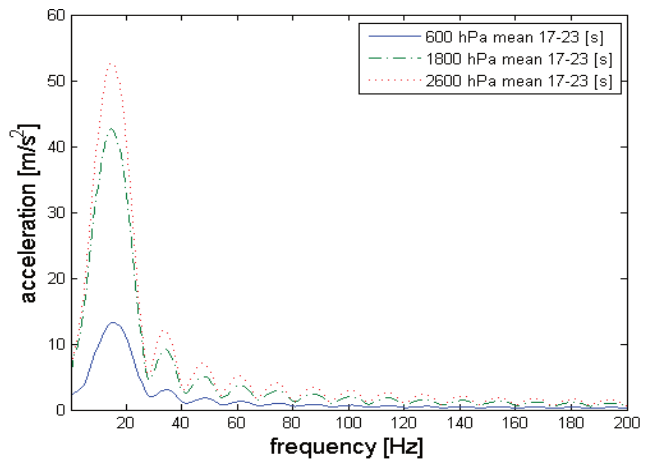

b) $17-23 \mathrm{~s}$

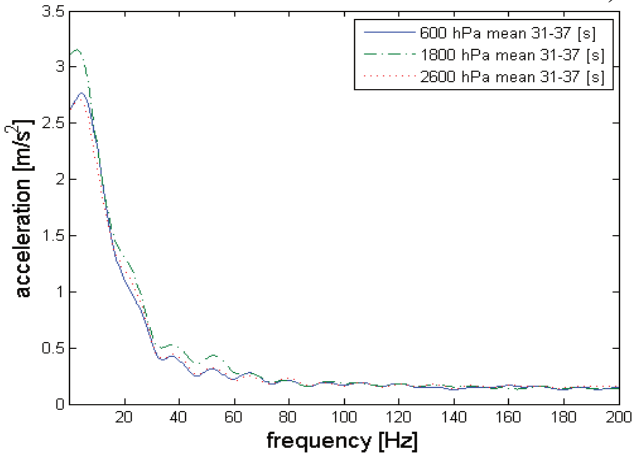

c) $31-37 \mathrm{~s}$

Fig. 10.19. Frequency function of average vibration in time periods: suspension arm 


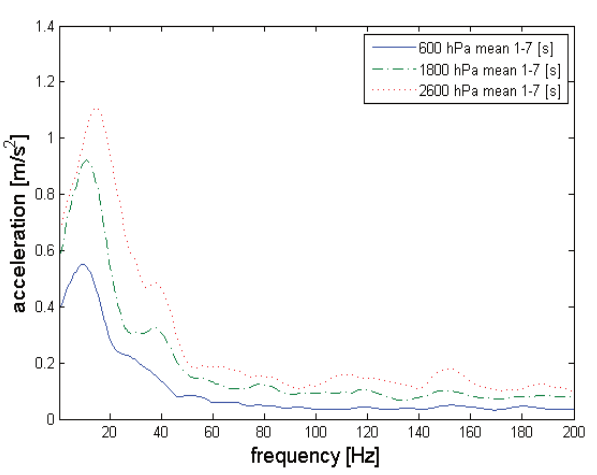

a) $1-7 \mathrm{~s}$

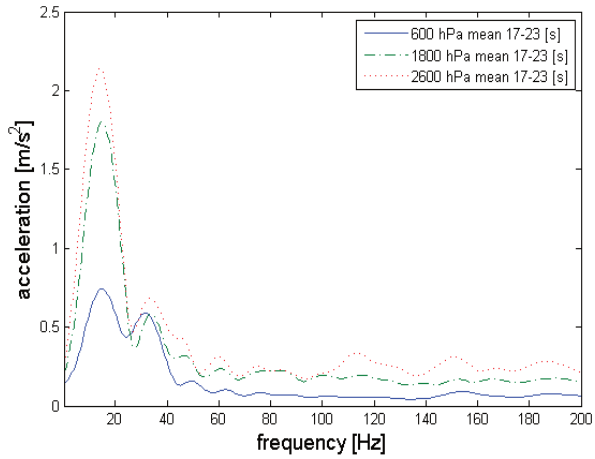

b) $17-23 \mathrm{~s}$

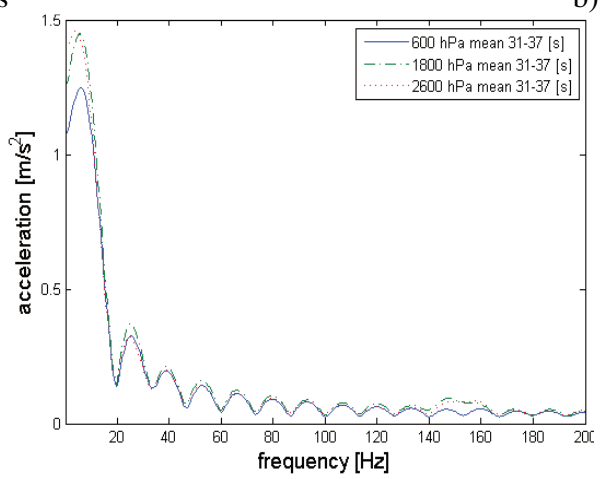

c) $31-37 \mathrm{~s}$

Fig. 10.20. Frequency function of average vibration in time periods: upper mounting of shock absorber

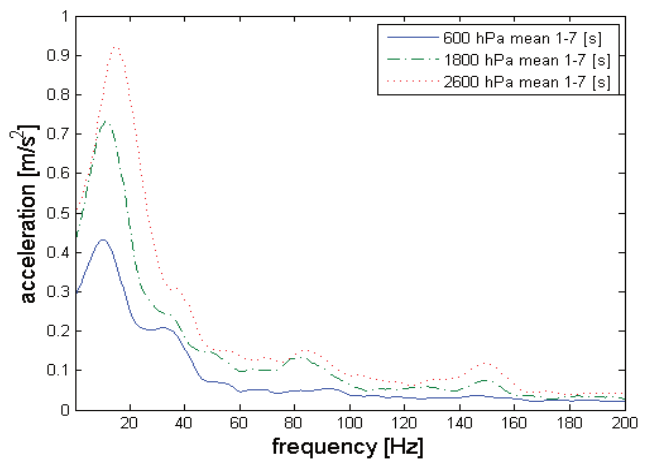

a) $1-7 \mathrm{~s}$

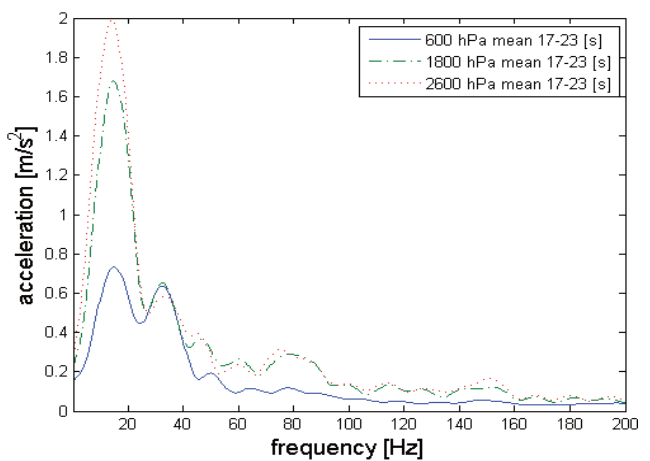

b) $17-23 \mathrm{~s}$

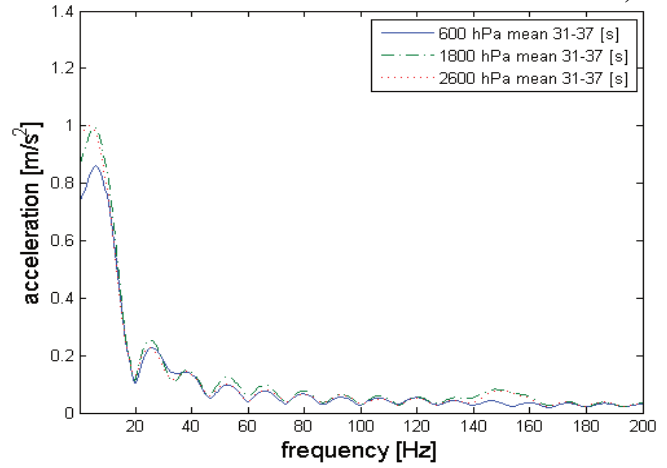

c) $31-37 \mathrm{~s}$

Fig. 10.21. Frequency function of average vibration in time periods: floor under driver's feet 


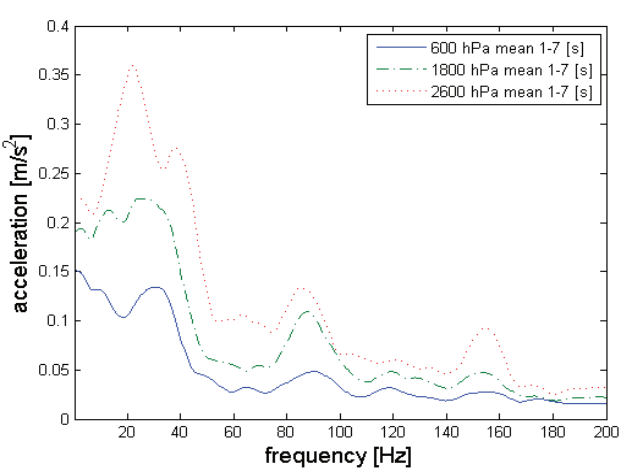

a) $1-7 \mathrm{~s}$

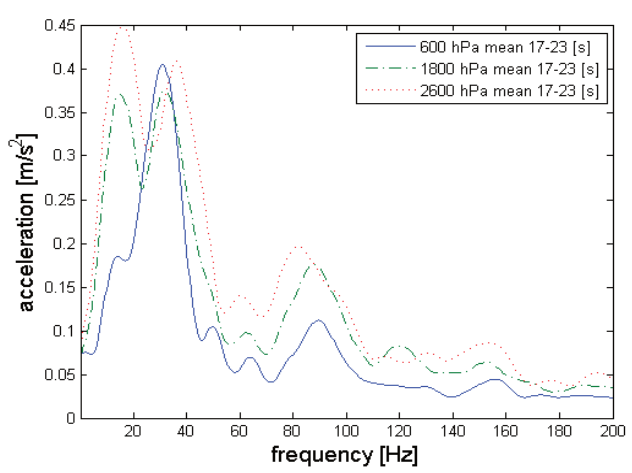

b) $17-23 \mathrm{~s}$

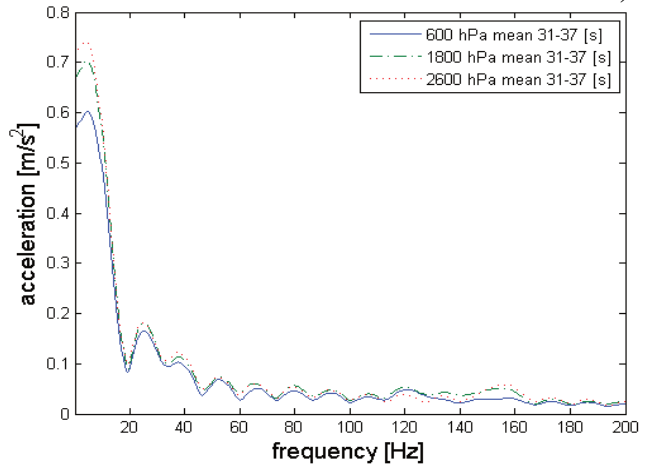

c) $31-37 \mathrm{~s}$

Fig. 10.22. Frequency function of average vibration in time periods: floor under front passenger's feet

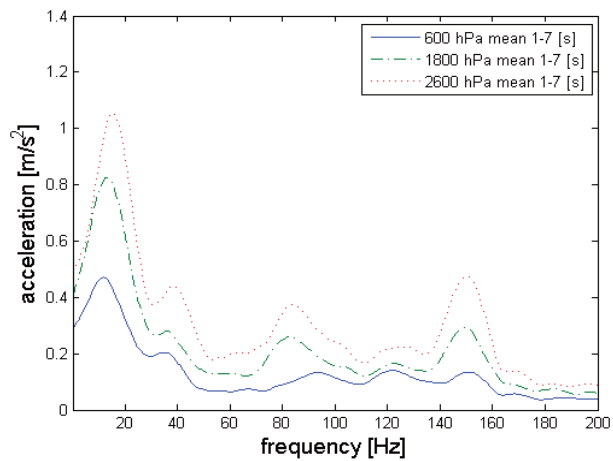

a) $1-7 \mathrm{~s}$

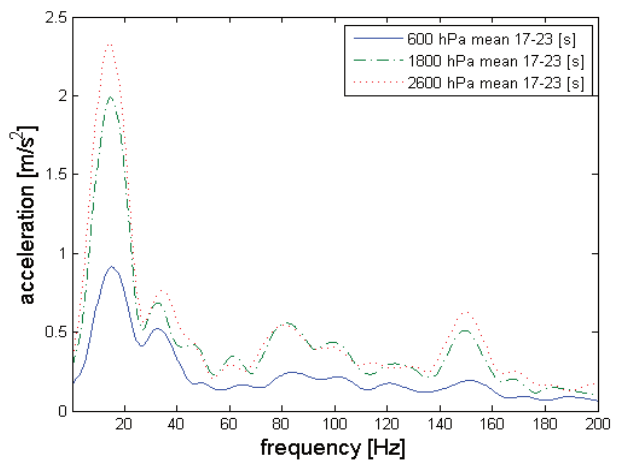

b) $17-23 \mathrm{~s}$

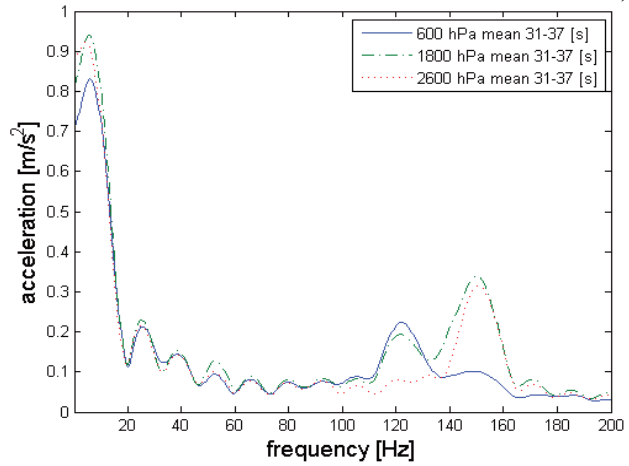

c) $31-37 \mathrm{~s}$

Fig. 10.23. Frequency function of average vibration in time periods: floor under rear left passenger's feet 


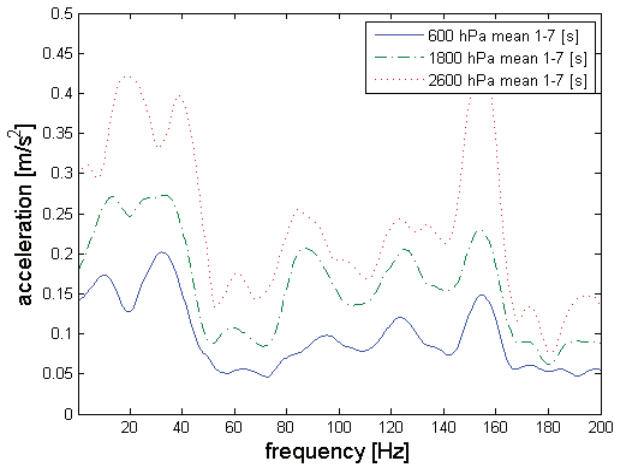

a) $1-7 \mathrm{~s}$

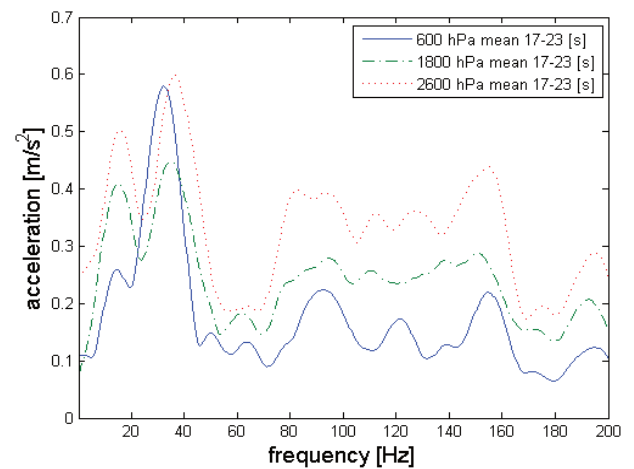

b) $17-23 \mathrm{~s}$

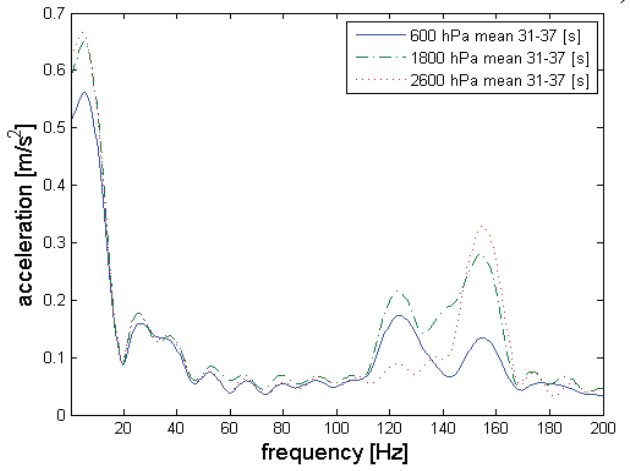

c) $31-37 \mathrm{~s}$

Fig. 10.24. Frequency function of average vibration in time periods: floor under rear right passenger's feet

The analysis of the technical condition of suspension elements and vehicle's operating parameters influence on the vibration in terms of human perception and exposure require identification of frequency component carrying most of the vibration energy. For the motor vehicle vibration the highest energy dissipation can be observed during time of passing thru resonance of unsprung masses. Thus the frequency distribution of the vibration in defined time period has to be analyzed. The developed functions $S_{t}, S_{\text {Tavr }}$ represent dominant dynamics components of vibration excited during most vibro-activity time of exposure and function of average value of time period vibration in frequency domain allow identify frequency components.

The results confirm good sensitivity of the function on the damping of shock absorber and pressure in tire. The course of the function allows to identify dominant frequency components. The most energy dissipation is result of the vibration in unsprung masses resonance $(12-18 \mathrm{~Hz})$ but some other frequencies represent dynamics of the vibration with high energy (i.e. $160 \mathrm{~Hz}$ ). 



\section{Engine as the vibration source in vehicle}

Road surface roughness often acts as a major source that excites the vibration of the vehicle running on the ground through the tire/wheel assembly and the suspension system [202, 205]. There are many different vibration sources in vehicles as well. The engine is primary vibration source along with external disturbance forces to the vehicle. Idle shake is one of the most important items to evaluate vehicle comfort [209]. A major comfort aspect is the transmission of engine-induced vibrations through powertrain mounts into the chassis.

The suspension system is very important for reduction of vibration transfer to car body. Before attempting to reduce the vibration levels in a machine or structure of the vehicles by increasing its damping, every effort should be made to reduce the vibration excitation at its source. Motor engine should be considered as one of the vibration generators. The vehicle engine mounting system, generally, consists of an engine, as vibration source and several mounts connected to the vehicle structure [210]. The engine mount is an efficient passive mean to isolate the car chassis structure from the engine vibration. The passive means for isolation is efficient only in the high frequency range. However the vibration disturbance generated by the engine occurs mainly in the low frequency range [206]. These vibrations result from fuel explosion in the cylinder and the rotation of the different parts of the engine.

\subsection{Motor-engine as the source of vibration}

The engine vibrations are strongly random processes because there are many different sources of vibration in engine. It has to be considered as dynamics responses which are most significantly dependent upon the nature of excitation forces and moments. They can have different mechanism of generation and different values and frequencies of vibration. The engine block mainly is characterised by the low coefficient of dumping. The vibrations of low and medium frequencies up to a few $\mathrm{kHz}$ are only slightly absorbed. Higher vibration absorbing effects appear only for frequencies of $10 \mathrm{kHz}$ or more. Those parameters are determined by materials used in engine construction. There are a lot of novel materials used in engine with modern production technologies. Thus the optimal material parameters can be obtained.

Motor engine can be considered as one of the vibration generators in vehicles (Fig. 11.1). Rotating machinery such as motors can generate disturbing forces at several different frequencies such as the rotational speed and blade passing frequency. Reciprocating machinery such as compressors and engines can rarely be perfectly balanced, and an exciting force is produced at the rotational speed and at harmonics. There are two basic types of structural vibration: steady-state vibration caused by continually running machines such as engines, air-conditioning plants and generators either within the structure or situated in a neighbouring structure, and transient vibration caused by a short-duration disturbance such as a lorry or train passing over an expansion joint in a road or over a bridge.

The engine exciting force mainly composed of the internal force generated by the rotational reciprocated motion of the piston-cranshaft system and of the fluctuation of torque generated by the fluctuation of gas pressure [209]. It can be assumed that vibration and forces excitations due to the engine is a complex function of the gas pressure, firing, unbalance and operating parameters, such as engine rotational speed. The mechanism of vibration generation is the firing cycle from each cylinder and the moving masses resulting from the firing cycle impact dynamic forces and 
moments to the internal combustion engine block which are transmitted into frame and car body.

Many publications reported that the engine vibration response are directly related to variations in the gas pressure. The chapter reports results of investigation on engine vibration response relation to the rotational speed of engine unbalanced masses.

If considering whole engine as body with six degrees of freedom it should be continued by using Lagrange's equation as shown in the following expression:

$\frac{d}{d_{t}}\left(\frac{\partial T}{\partial q_{j}}\right)-\frac{\partial T}{\partial q_{j}}+\frac{\partial V}{\partial q_{j}}=Q_{j}^{(n)}$,

where: $T$ - parameter of kinetic energy of the system, $V$ - parameter of potential energy of the system, $Q$ - is equal to both external forces and forces produced by damping objects.

Most models used to the engine vibration analysis, including the matrices with fixed components, i.e. mass matrix, $M$ stiffness matrix $K$ and damping matrix $C$. However, studying and optimizing such systems in terms of both free and forced vibrations require comprehensively accurate analysis as well as reliable input information [11]. It can be assumed that vibration amplitude of engine block is so small that structure is a linear vibration system. Thus the motion equation (vibration equation) which has a matrix form is expressed as:

$M(t) \ddot{q}+C(t) \dot{q}+K q=F(t)$

where: $M$ - global mass matrix, $C$ - global damping matrix, $K$ - global stiffness matrix, $\ddot{q}, \dot{q}, q$ are vectors of acceleration, velocity and displacement.

In equation $F(t)$ above both forces caused by combustion in the cylinder chamber and external forces exerted on the engine. To determine the natural frequencies of the whole engine system, homogeneous solution $(F(t)=0)$ is employed. Structural vibration responses depend on structural natural frequency, damping, stiffness and exciting condition [204].

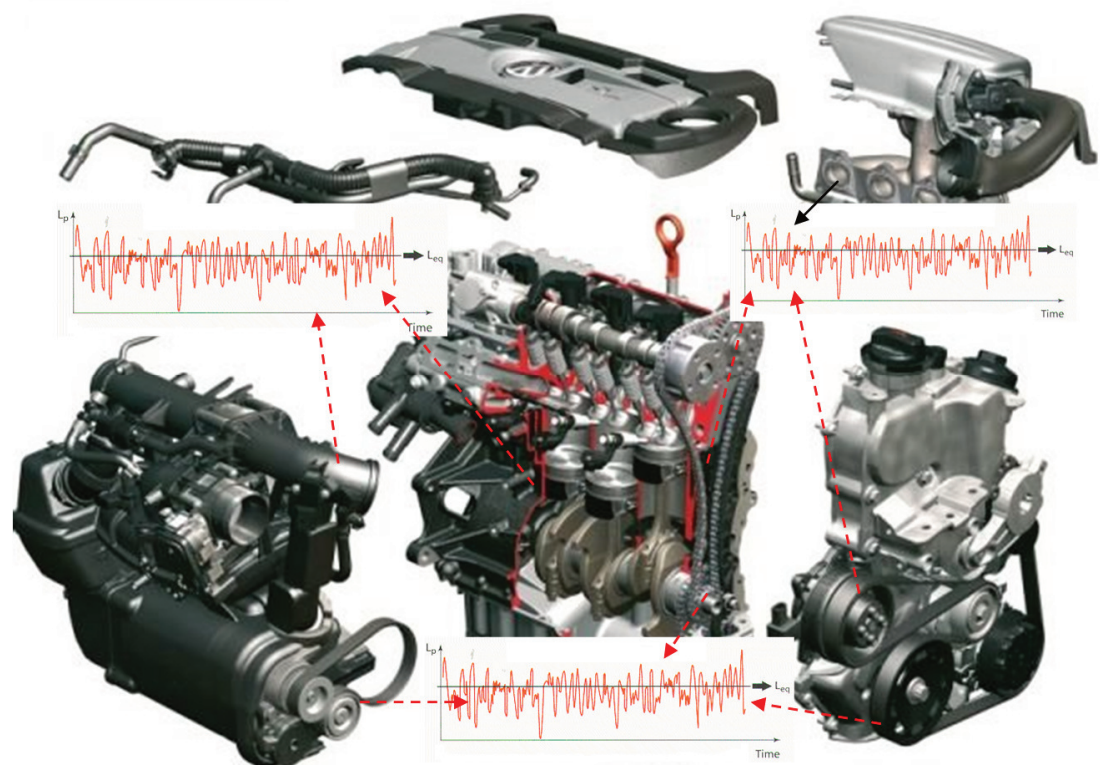

Fig. 11.1. Motor engine as the vibration generator

Considering the vibroacoustics analysis of an internal combustion engine it should be taken into account, that a high level of nominal vibrations is generated, resulting from the target function realisation. Internal combustion engine is an object under the influence of internal and external 
inputs. Among them there are mainly: burning pressure, the movement of the piston-crank mechanism, inputs from the timing gear system, inputs resulting from the work of the fittings of the engine, inputs transmitted from the motor-car body and the drive transmission system. One of the most important inputs during the work of the piston-crank mechanism are the impacts of the piston by the change of its work direction.

Vibration processes in combustion engine are unwanted effects but at the same time the can be very useful. This chapter take into account influence of the vibration as the detrimental effect on the safety and comfort in mean of transport. The human response to vibration depends on the values, frequencies and directions [46]. Perceptibility of the same frequencies and values of vibration in exposure in different directions can be diametrically different.

\subsection{Identification of the vibration generated by the engine}

The experimental research was conducted on directional distribution of vibrations generated by the combustion engine. The dual-axis accelerometer was used. Block of the combustion engine was studied. Active experiments were undertaken featuring measurements of vibration accelerations in three directions. The vibration in three orthogonal axes (X, $Y, Z-$ Fig. 11.2) have been measured. The position and directions of measurements is presented in Fig. 11.3.

As a result the time functions of acceleration of vibration generated in the three orthogonal axes by the combustion engine were determined. Fig. 11.2 presents some exemplary distribution of vibration signals.

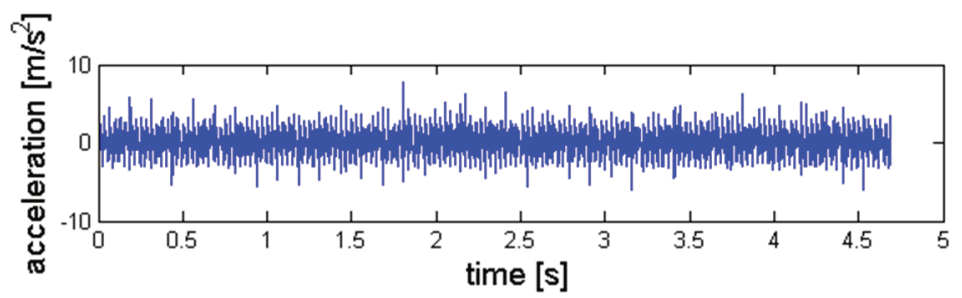

a) $X$ axis

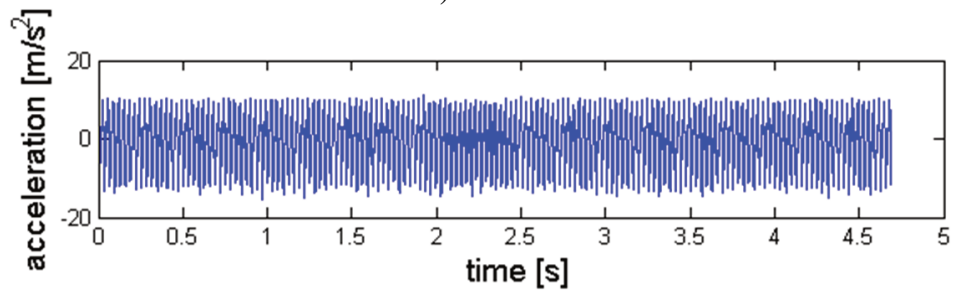

b) $Y$ axis

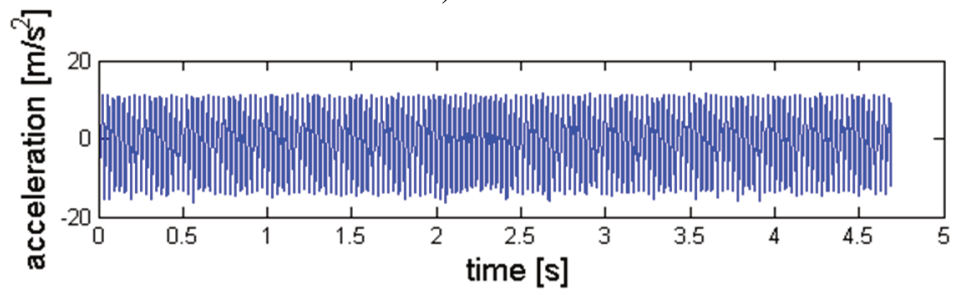

c) $Z$ axis

Fig. 11.2. Directional distribution of vibration signals of block of motor vehicle, $750 \mathrm{rpm}$

For the purpose of dynamics analysis of motor engine vibration the spectrums of the recorded signals in 3 axes were determined. For the identification of the frequency correlated to rpm (revolutions per minute) of the rotational speed of the engine the figures were analyzed separately with zoom window. 


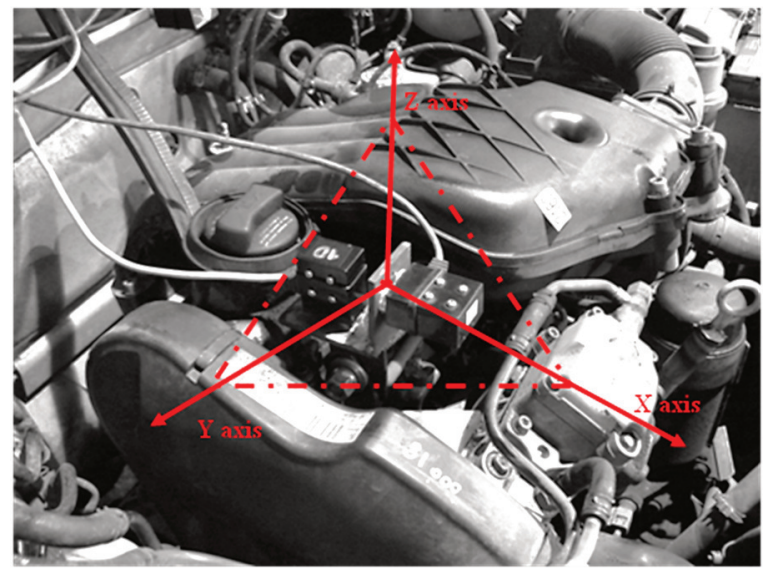

Fig. 11.3. The position and directions of measurement of the vibration of motor engine

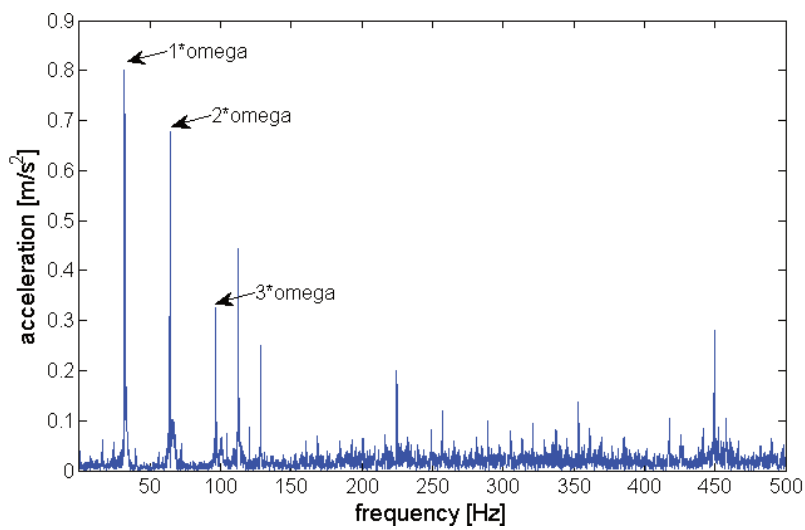

a) $X$ axis

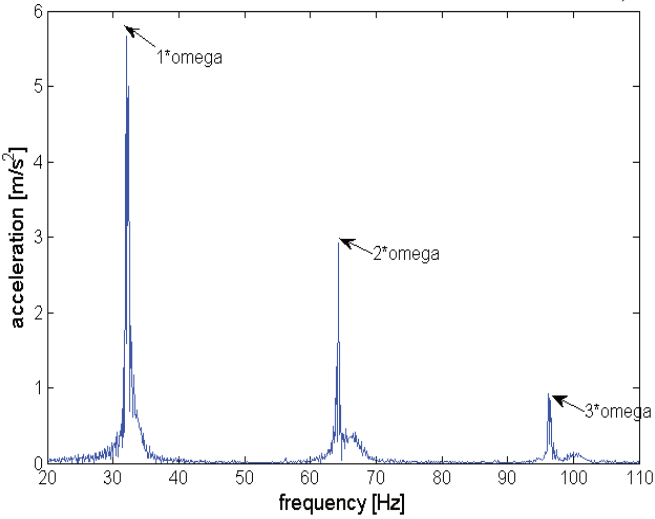

b) $Y$ axis

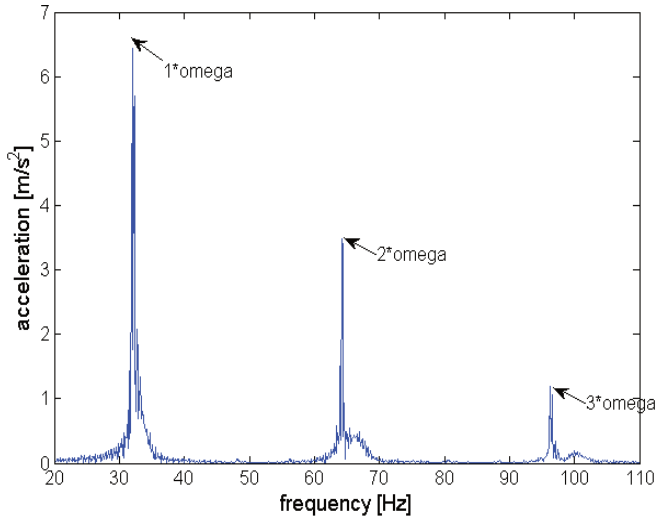

c) $Z$ axis

Fig. 11.4. Spectrums of vibration of the motor engine in $X, Y, Z$ axes, $750 \mathrm{rpm}$

Thus the frequency of unbalance disturbances is correlated to engine rotational speed (defined as rpm) and depends on the number of cylinders in the engine, the stroke number and the engine speed. For the four cylinders, four-stroke engine, which were investigated during research, the frequency of fundamental disturbances is at the second order of the engine speed. The frequency range is $20-200 \mathrm{~Hz}$ for an engine rotational speed range from 600 to $6000 \mathrm{rpm}$. Taking into consideration an eight-cylinder engine, the frequency of the disturbance torque is at the fourth orders of the engine rotational speed and the frequency range is $40-400 \mathrm{~Hz}$ for the same engine 
speeds. In general, at low engine speeds (near idle) the engine disturbance will result in an annoying shaking of the vehicle. At higher speeds, a booming sound is created inside the vehicle compartment when the engine disturbance force coincides with an acoustic resonance of the passenger compartment [210].

The main characteristics frequencies of the engine are marked on Fig. 11.4. Thus the analysis of different between amplitude of the harmonics vibration in axes $X, Y$ and $Z$ is possible.

In the spectrum of the signals the characteristics frequencies can be identified. In the presented results the ca. $32 \mathrm{~Hz}$ frequency is correlated with rotation velocity of the idle gear of the engine. The vertical vibrations have the largest amplitude but the signal is most stationary. The frequencies not correlated with rotation velocity of the idle gear of the engine (1-3 harmonics) are very low energy. The horizontal vibration have more frequencies, especially in the spectrum of the $X$ axis vibration can be identified many of different frequencies. The directional distribution of vibration can be resulted from the elements and systems of the suspension and the mounting of engine. It can be resulted of the unbalance of the rotating elements of the engine or other defects.

The received signal is strongly interfered by various vibration sources and that is why there is a necessity to use advanced methods of signal selection and observation in time-frequency domains. The TFR of the signals propagated in three orthogonal axes is presented in Fig. 11.5. It shows the complex structure of vibration of motor engine.

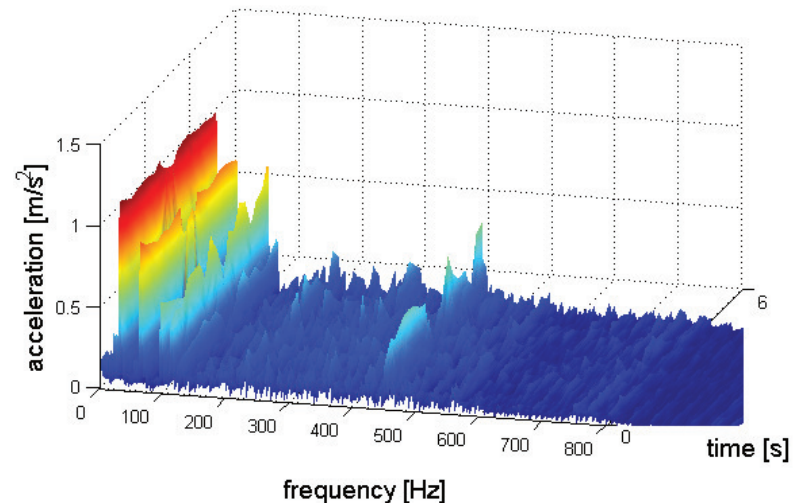

a) $X$ axis

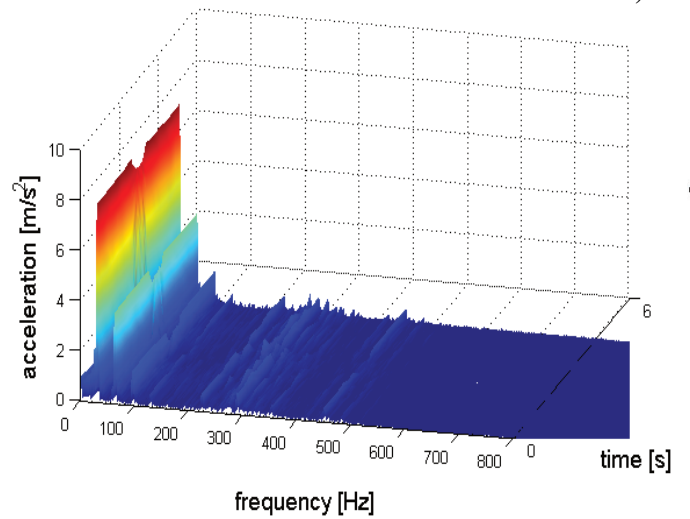

b) $Y$ axis

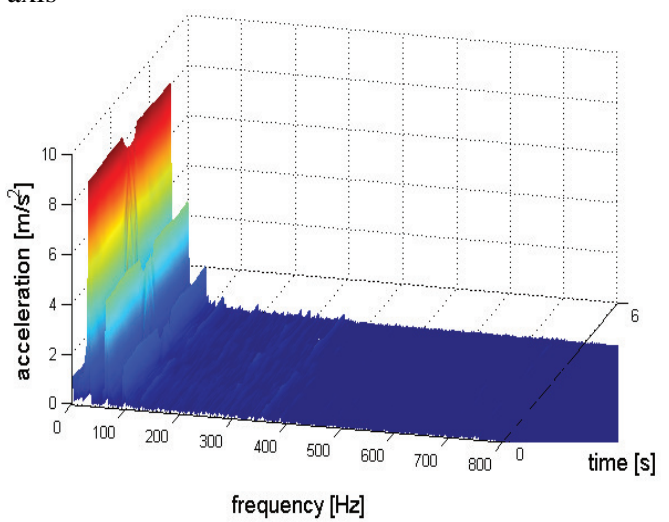

c) $Z$ axis

Fig. 11.5. Time-frequency structure of vibration of the motor engine in $X, Y, Z$ axes, $750 \mathrm{rpm}$ (time $0.25 \mathrm{~s}$, resolution $0.4884 \mathrm{~Hz}$ )

The transformations of the vibration signal into time-frequency representation enables analyzing of energy of vibration carrying by the defined frequency. Thus the periodic unstable of frequencies can be observed (Fig. 11.6). 


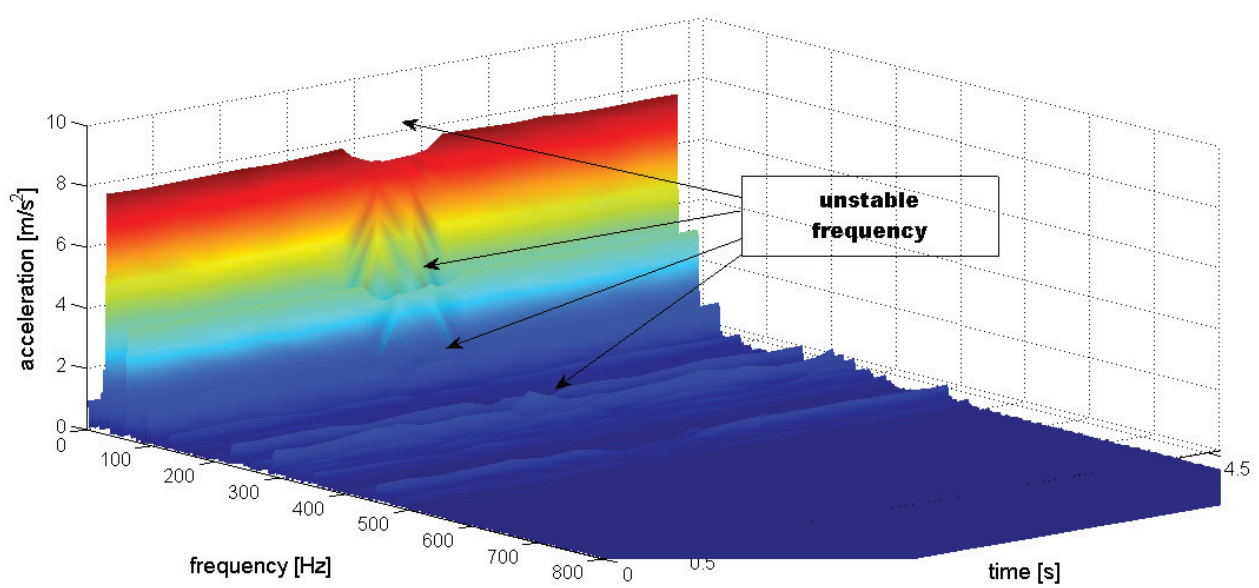

Fig. 11.6. Example of the unstable frequency (STFT of enfine block vibration, $Y$ axis - time window $0.25 \mathrm{~s}$, resolution $0.4884 \mathrm{~Hz}$ )

\subsection{Influence of engine rotational speed on the vibration generation}

To consider motor engine as vibration generator in vehicle investigation on influence of operating parameters and conditions of combustion engine were conducted. As most important condition of working engine the rotational speed expressed as revolutions per minute (rpm) was assumed.

The results of the investigation are presented in Fig. 11.7-11.9 as collection of charts presenting time and frequency functions of vibration generated for increasing rpm of engine.

For the purpose of analysis of rotational speed of engine influence on the vibration generation the RMS values were calculated. It shows changes in energy of vibration for different revolutions per minute of engine in orthogonal axes as direction of propagation. The distribution of RMS values were shown in Fig. 11.10.

Basing on the spectrums of the vibration the dominant dynamics component correlated with rotational speed of engine was identified. Those frequencies carry most of the energy of the vibration (a significant part of RMS). The Fig. 11.11 presents changes of the location of dominant frequency component for increasing of rotational speed of engine.

For the accurate identification of engine's rotational speed impact on generated vibration the observation in time-frequency domains is necessary. The TFR of the signals enables analyzing of structure of the vibration and time of exposure to the defined frequencies.

The changes of the structure of vibration generated in different rotational speed of engine are presented in Fig. 11.12-11.14. Apart of analyzing of constant in dominant frequencies realization, the periodic excitation of different frequencies can be observed. The periodic changes in distribution of defined frequency vibration can't be identified in regular spectrums obtained as Fourier transformation.

The higher values of vibration (RMS and amplitude of frequency component) can be a result of the variations in the gas pressure yield the principal force of disturbance at low engine rotational speeds, while the inertia forces may be considerable larger at higher speeds.

Spectrums of the vibration shows that the first order forces as identified from FFT are balanced and the second order forces carrying most of the vibration energy. These frequency components are result in an unbalance force. Apart from the magnitude of the unbalanced force, the frequency of dominant component of vibration depends on the engine rotational speed.

The transformations of the vibration signal into time-frequency representation show distribution and structure of vibration. This transformation enables identification of the periodic unstable of frequencies and evaluation of amplitude-time exposure to vibration in chosen frequencies bands. 


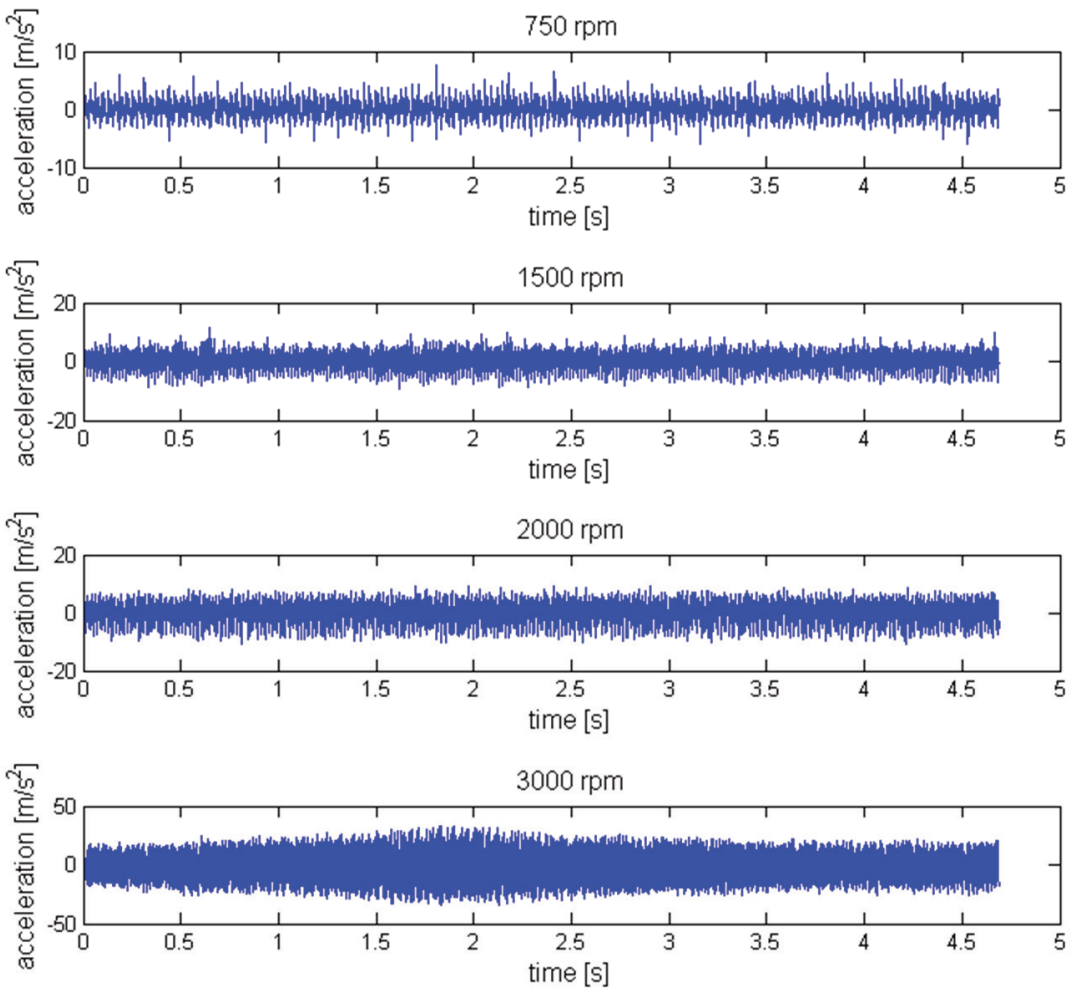

a) Time function
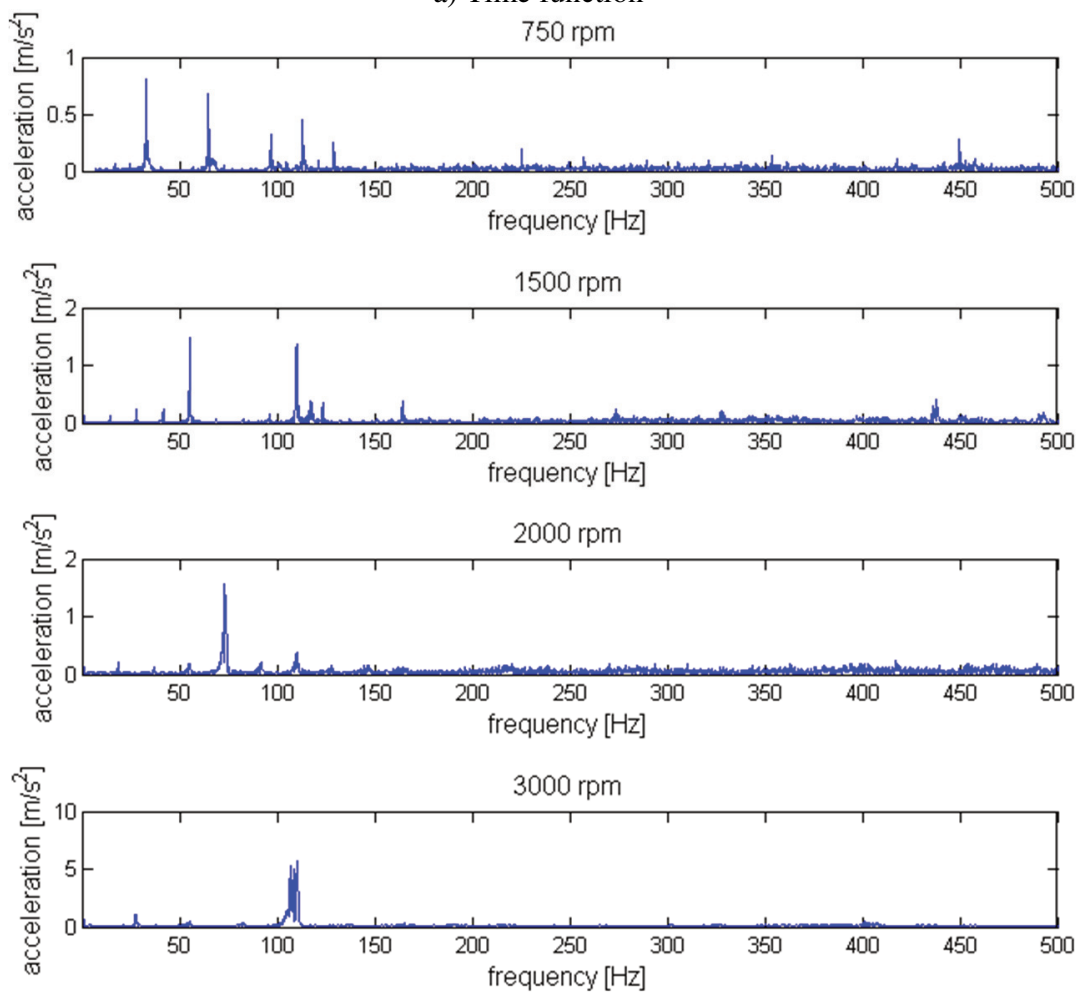

b) Frequency function

Fig. 11.7. The vibration of motor engine for increasing rpm value, $X$ axis - longitudinal, $750 \mathrm{rpm}$ 


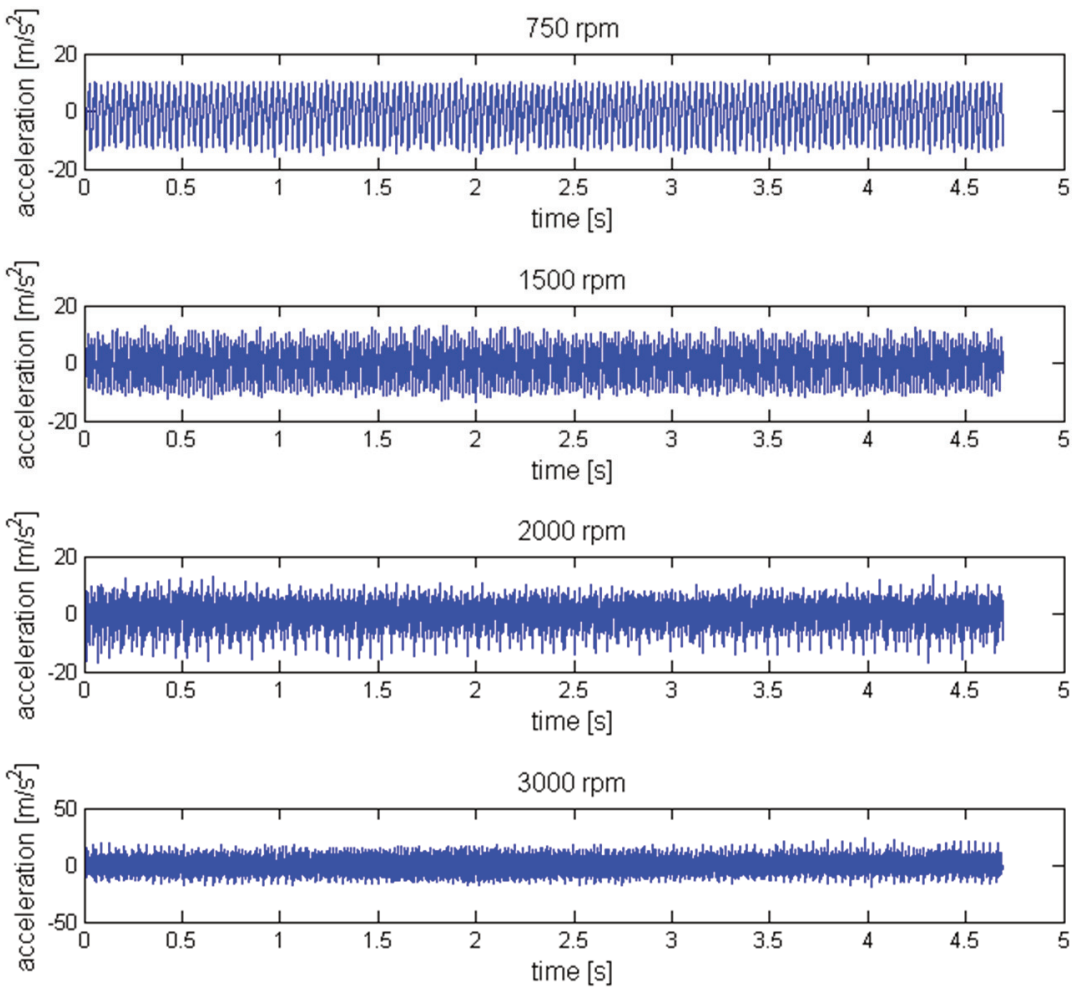

a) Time function
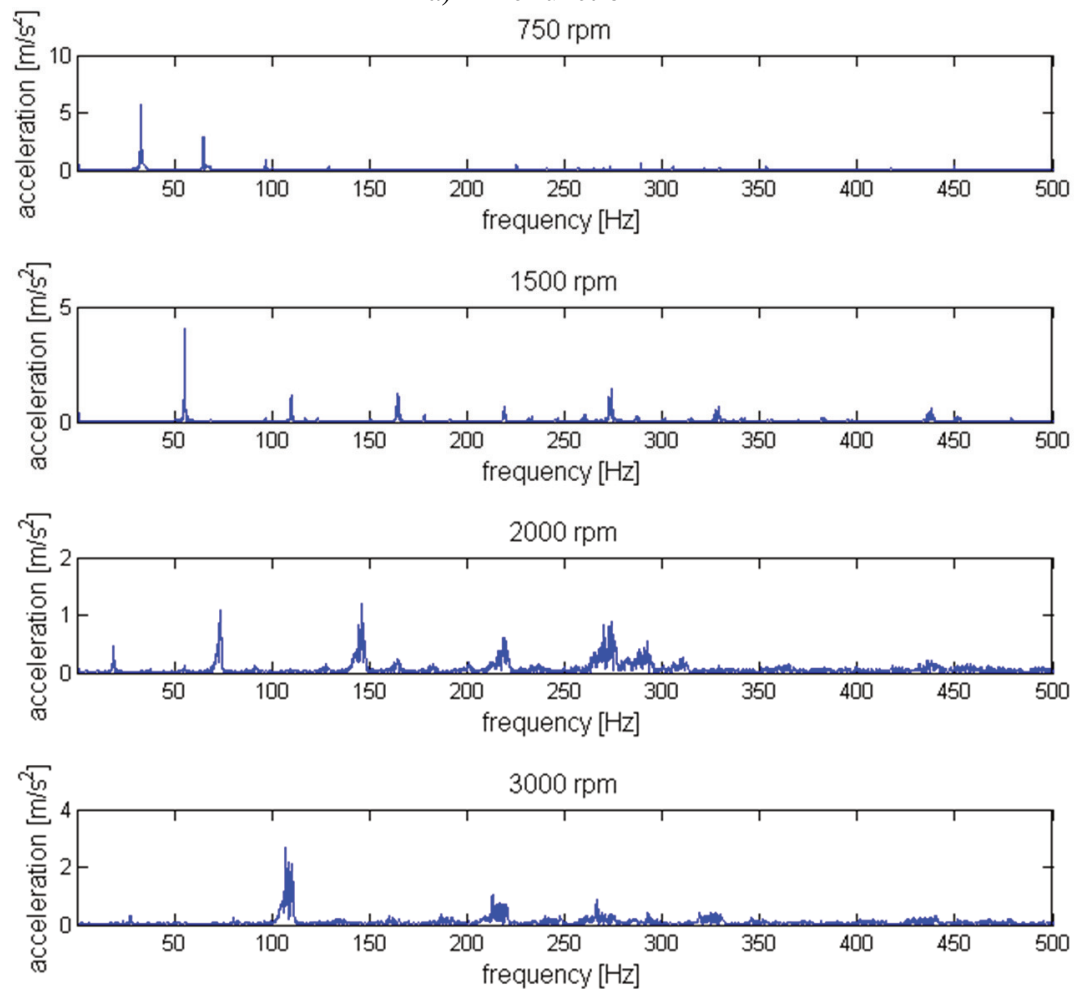

b) Frequency function

Fig. 11.8. The vibration of motor engine for increasing rpm value, $Y$ axis - lateral 


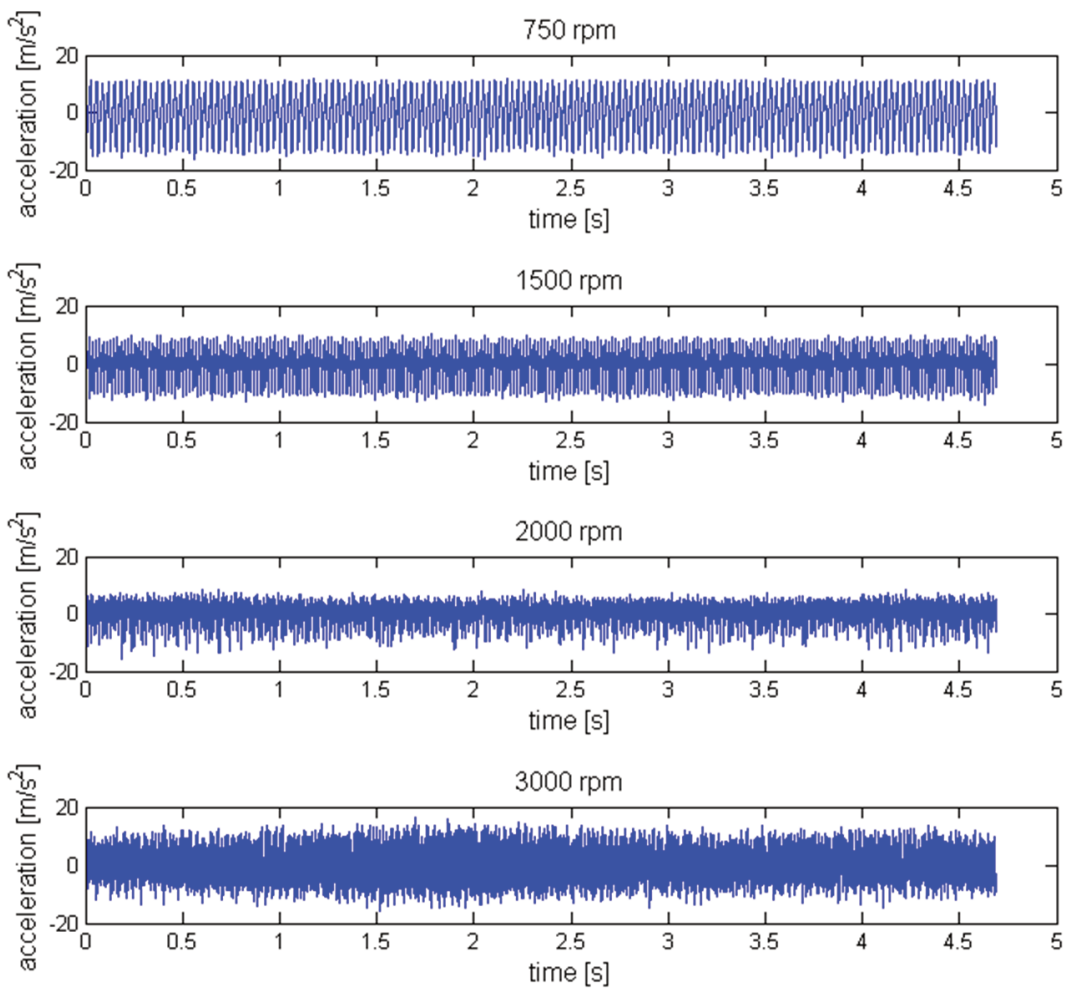

a) Time function
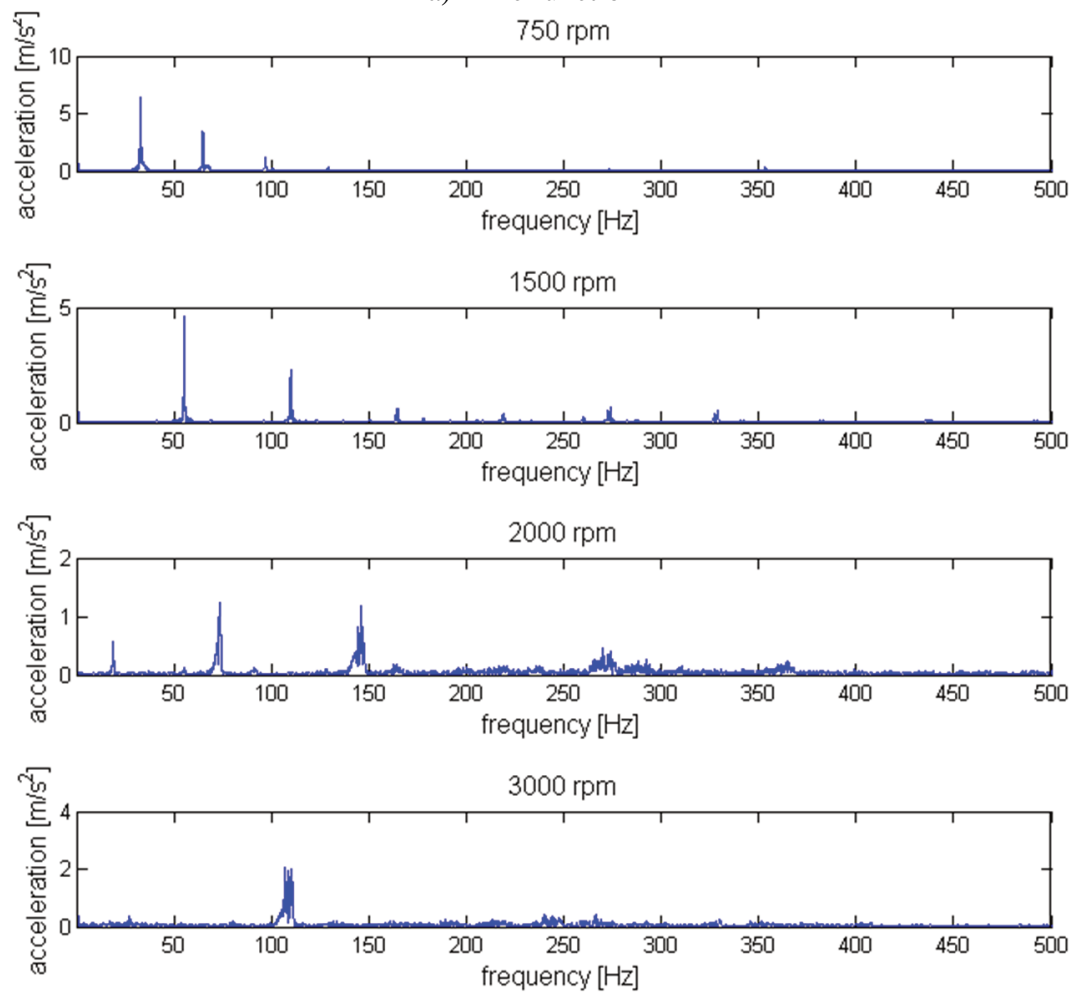

b) Frequency function

Fig. 11.9. The vibration of motor engine for increasing rpm value, $Z$ axis - vertical 


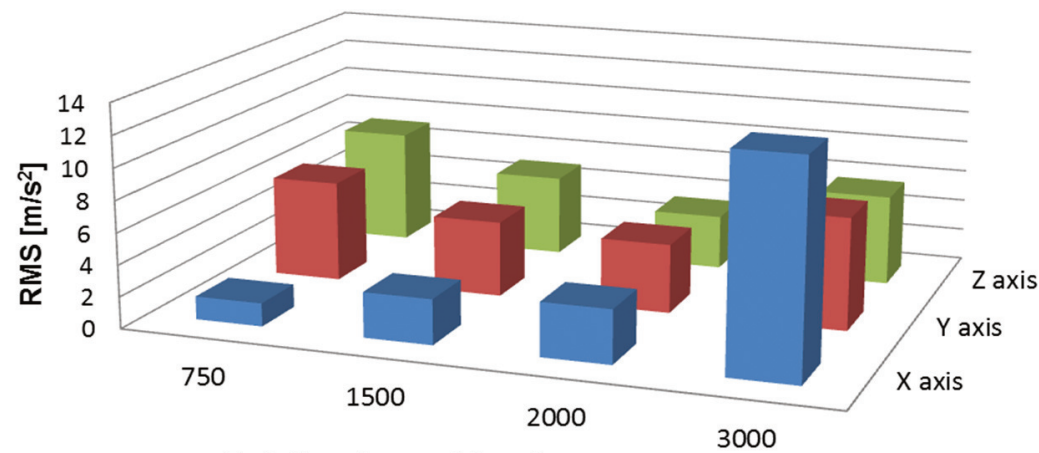

Rotational speed [rpm]

\begin{tabular}{|c|c|c|c|c|}
\hline & 750 & 1500 & 2000 & 3000 \\
\hline $\mathrm{X}$ axis & 1.4615031 & 2.851705344 & 3.37566313 & 13.38934669 \\
\hline $\mathrm{Y}$ axis & 6.391705216 & 4.788299865 & 4.368266727 & 7.073067795 \\
\hline $\mathrm{Z}$ axis & 7.215869997 & 5.100786875 & 3.432679143 & 5.709143522 \\
\hline
\end{tabular}

Fig. 11.10. The distribution of RMS of vibration for different rotational speed in 3 orthogonal axes

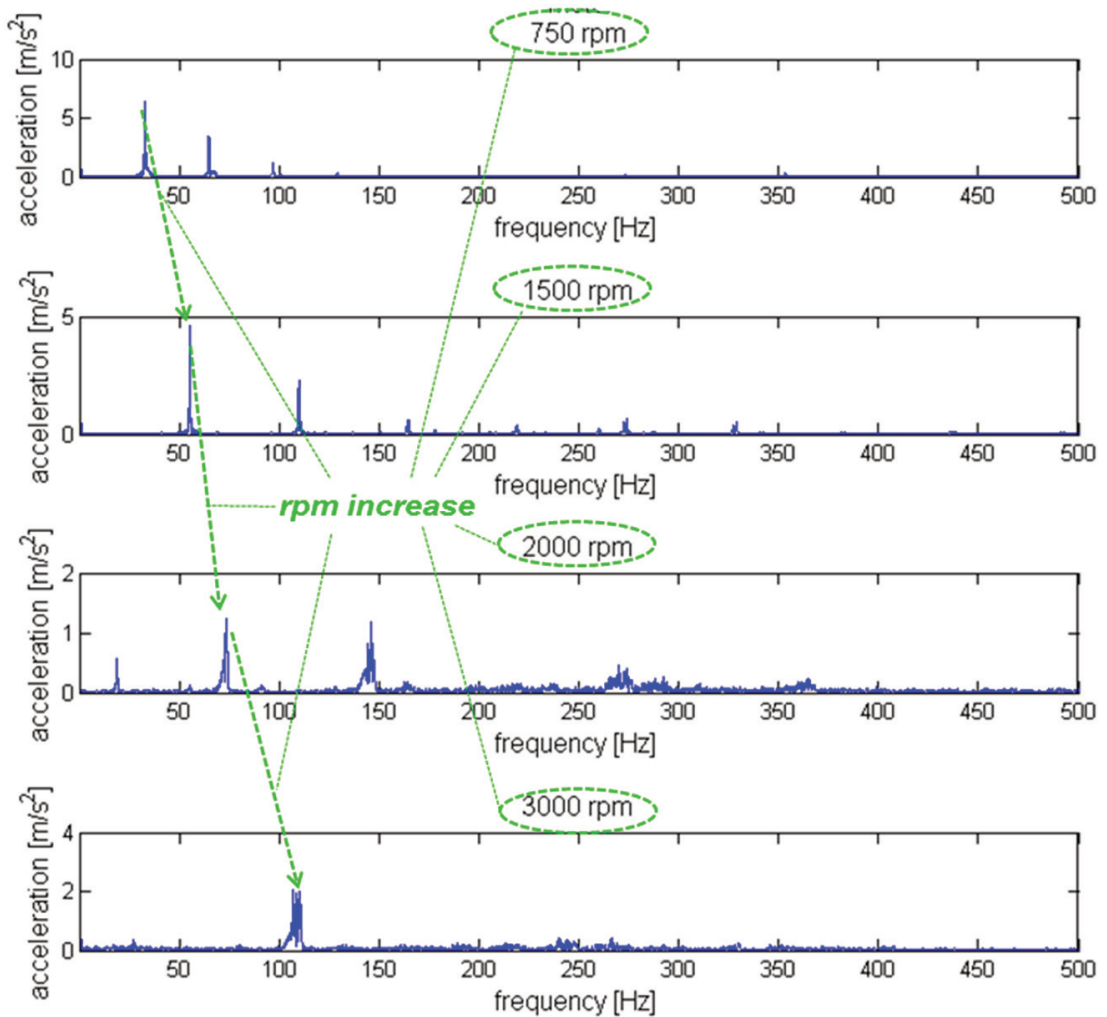

Fig. 11.11. Illustration of influence of rotational speed on dominant frequency component of vibration

Analysis of dynamics of vibration of 3000 [rpm] engine rotational speed shows concentration of vibration energy in narrow frequency band, close to frequency peak, especially for longitudinal and vertical directions of propagation. 


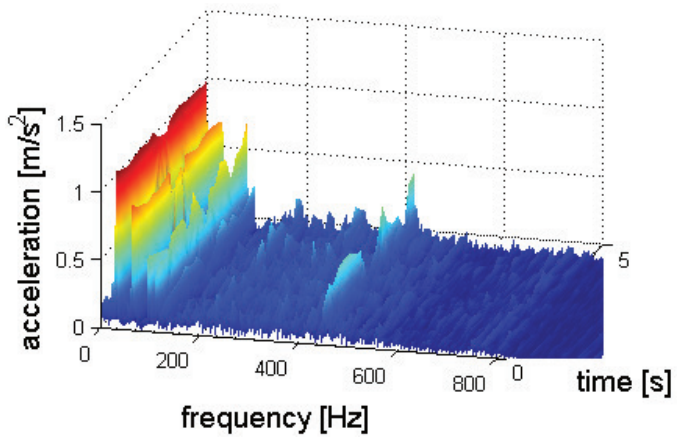

a) $750 \mathrm{rpm}$

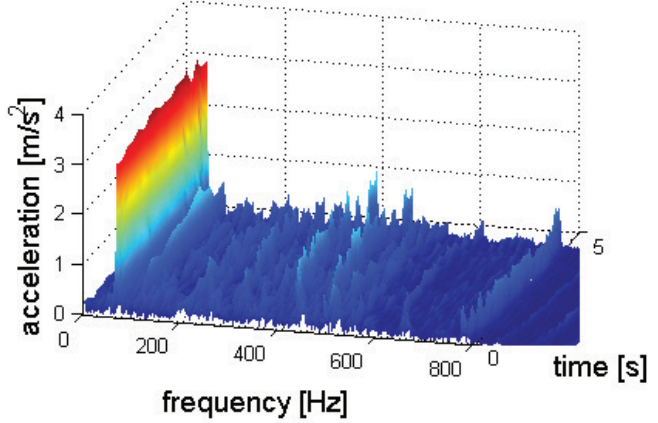

c) $2000 \mathrm{rpm}$

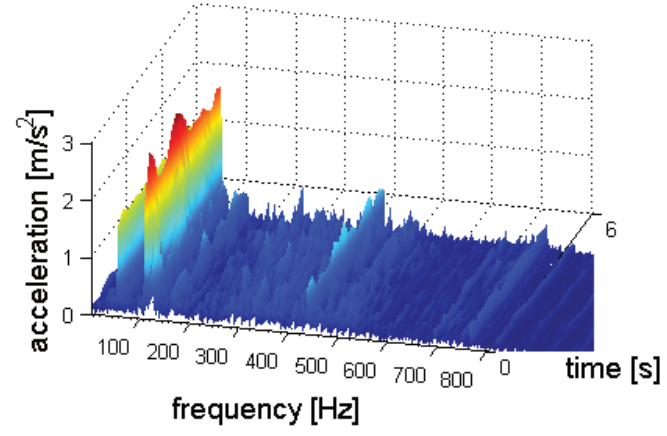

b) $1500 \mathrm{rpm}$

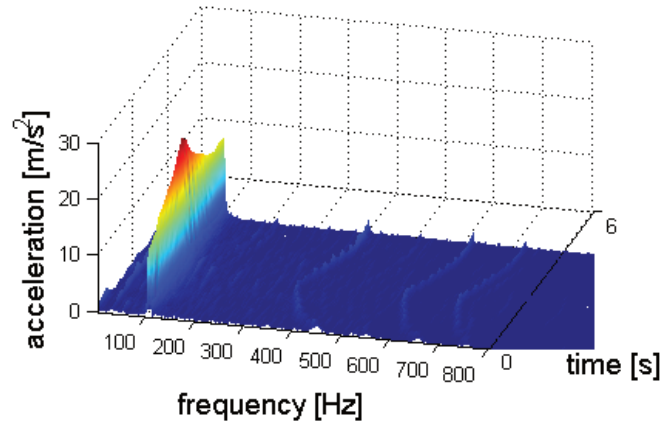

d) $3000 \mathrm{rpm}$

Fig. 11.12. The structures of vibration for different of rotational speed of engine, $X$ axis - longitudinal (time window $0.25 \mathrm{~s}$, resolution $0.4884 \mathrm{~Hz}$ )

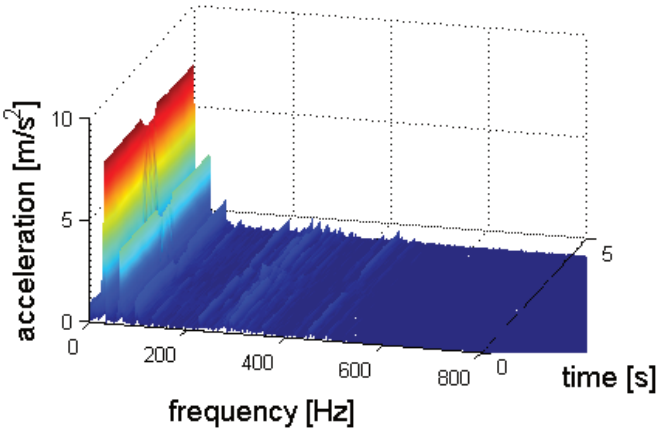

a) $750 \mathrm{rpm}$

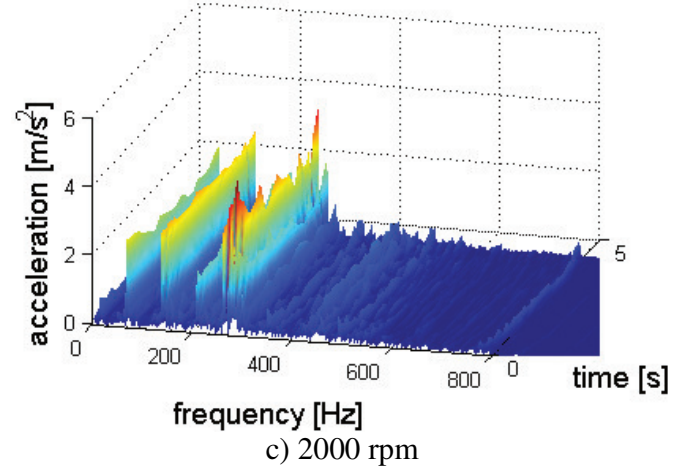

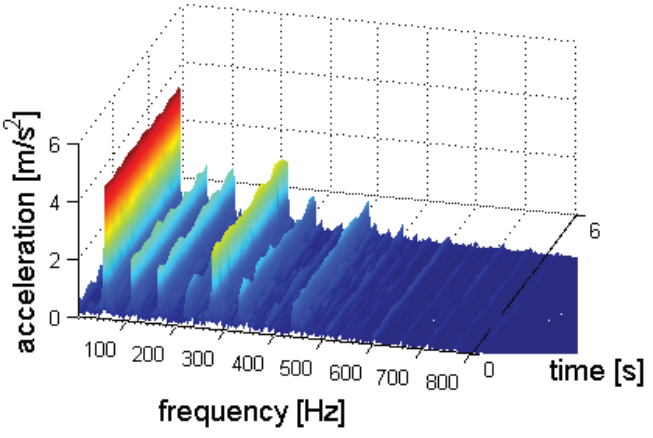

b) $1500 \mathrm{rpm}$

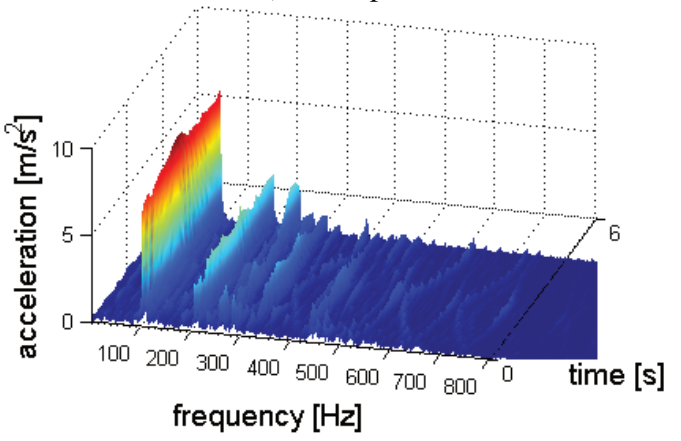

d) $3000 \mathrm{rpm}$

Fig. 11.13. The structures of vibration for different of rotational speed of engine, $Y$ axis - lateral (time window $0.25 \mathrm{~s}$, resolution $0.4884 \mathrm{~Hz}$ ) 


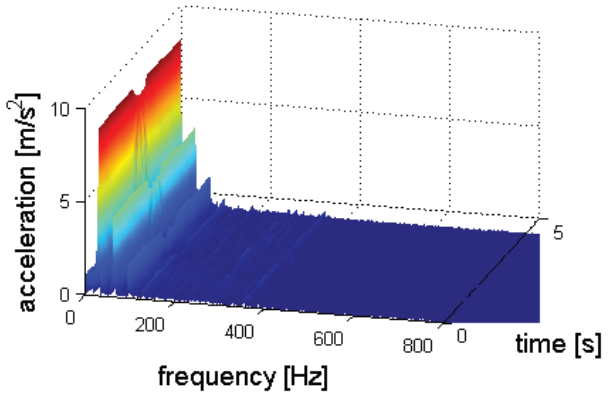

a) $750 \mathrm{rpm}$

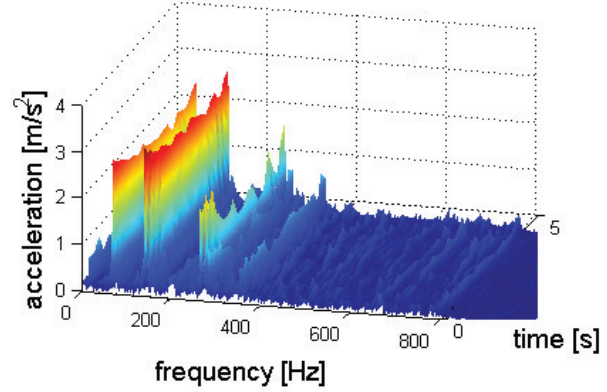

c) $2000 \mathrm{rpm}$

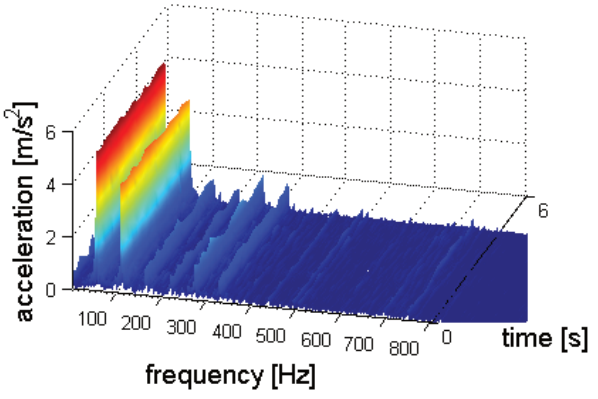

b) $1500 \mathrm{rpm}$

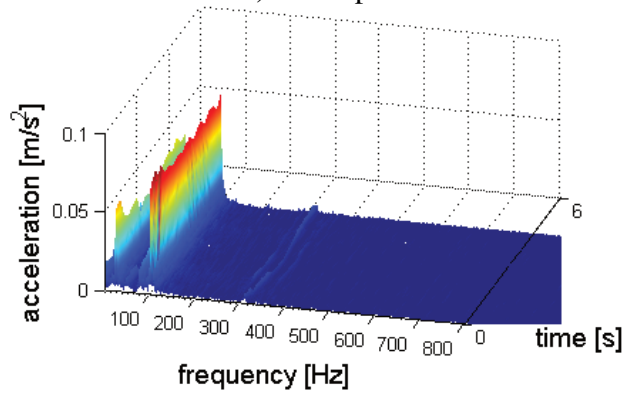

d) $3000 \mathrm{rpm}$

Fig. 11.14. The structures of vibration for different of rotational speed of engine, $Z$ axis - vertical (time window $0.25 \mathrm{~s}$, resolution $0.4884 \mathrm{~Hz}$ ) 


\section{Identification of vibration propagation paths from engine into the occupants}

Vibration propagation research in vehicles should primarily pertain to their effects on occupants. Consequences of the human exposure to vibrations include various harmful changes occurring in the organism. They have a direct or indirect influence on comfort and safety. The scope and the procedure of handling them depend to a considerable extent on the location where they penetrate the organism. The vibration energy affecting the human organism originates in the direct penetration area exclusively, therefore, what matters for the vibration perception is the energy affecting a unit of surface area in a unit of time. Thus the identification of propagation paths is significant for isolating or preventing to the vibration transfer from sources.

As it was elaborated in previous chapter one of the major vibration source, especially in the traffic jam, is engine. Fig. 12.1 illustrates propagation of the engine vibration to the driver body.

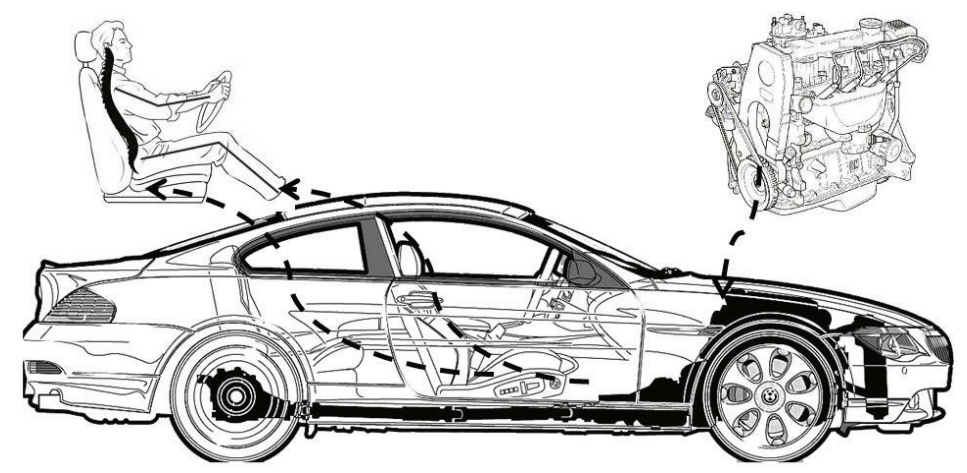

Fig. 12.1. Engine vibration transfer into human organism

\subsection{Research on vibration propagation paths from engine to car body in locations of penetrate into human body}

Active experiments were undertaken featuring measurements of vibration accelerations in a three directions in numerous selected points to analyse propagation of vibration generated by engine to human body via feet and lumbar spine. Vibration in three orthogonal axes ( $X$ - longitudinal, $Y$ - lateral, $Z$ - vertical) was recorded. The purpose of the research was to analyses the car body vibration generated from motor engine. The vehicles tested during research were passenger cars with most common used four cylinder, four-stroke engine. The identification of those kind of engine as vibration source was described in previous chapter. The experiments were conducted on the car vehicle which was placed on the special test racks. It allows to eliminate the road roughens impact on the suspension and in result to car body.

The vibration signals were measured in 10 points in vehicle (Fig. 12.2). It enables identifying many combinations of vibration propagation paths. The chapter presents result of vibration grouped in 4 dominants propagation paths:

I. Vibration source - engine, dash panel, floor panel under the driver's feet, driver's seat (Fig. 12.2 points 1-2-3-4); 
II. Vibration source - engine, floor panel under the rear left passenger's feet, left rear seat, right rear seat (Fig. 12.2 points 1-6-7-5);

III. Vibration source - engine, floor panel under the driver's feet, driver's seat, right front (front passenger) seat (Fig. 12.1 points 1-3-4-8);

IV. Vibration source - engine, floor panel under the front passenger's feet, right front seat, floor panel under the rear right passenger's feet (Fig. 12.2 points 1-9-8-10).

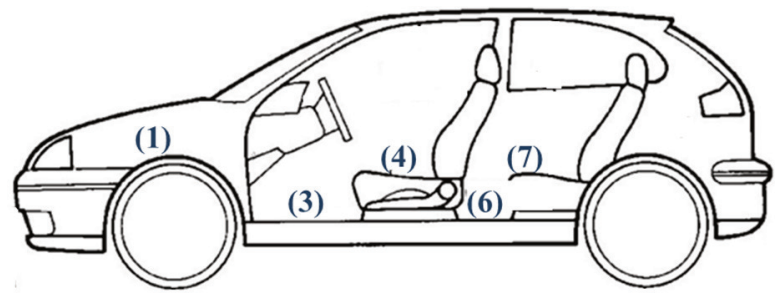

a)

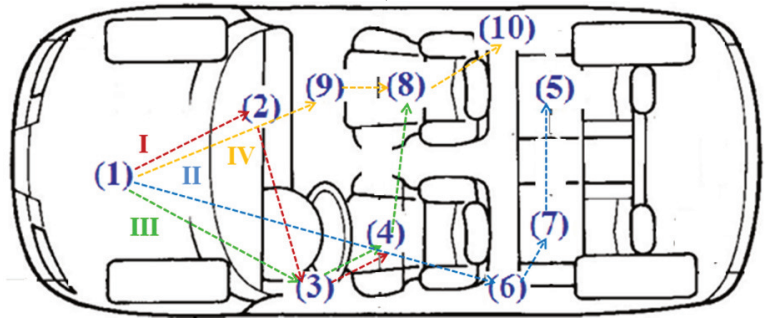

b)

Fig. 12.2. Locations of vibration measurement points in vehicle structure

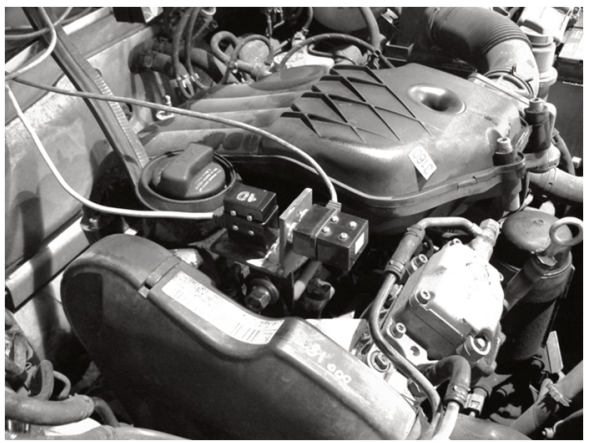

a) Propagation path: engine

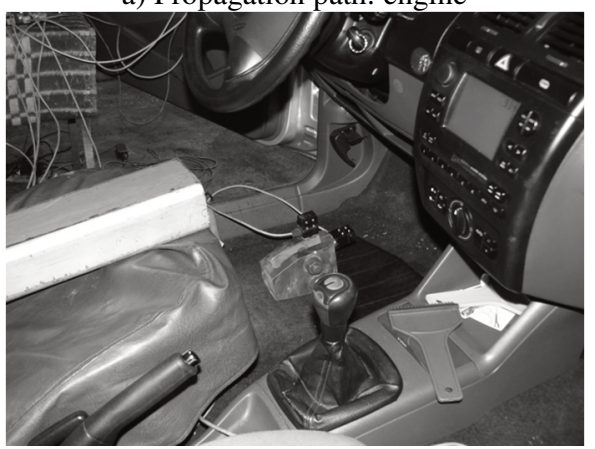

c) Propagation path: dash panel

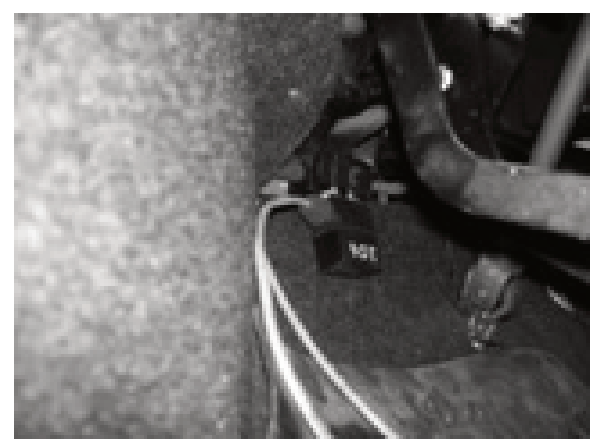

b) Propagation path: floor panel under driver feet

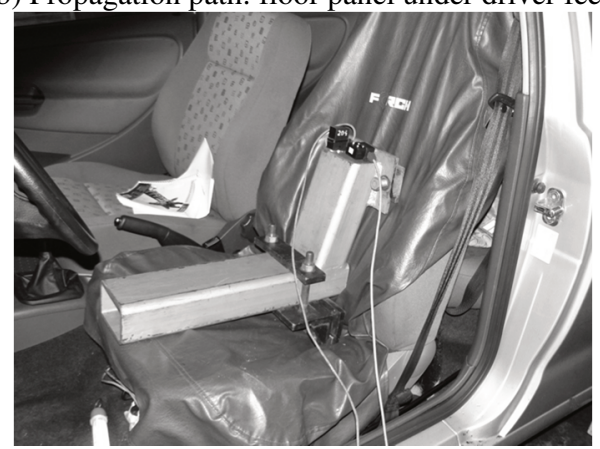

d) Propagation path: river seat

Fig. 12.3. Examples of vibration sensors mounting

The main reasons of choosing these 4 propagation paths were possibilities of structural waves 
propagation from engine via frame and car body into human body (feet and lumbar spine). It enables to analyse the way of vibration transfer from the source to occupants. Vehicle vibrations are transmitted to the occupant's lumbar spine and chest by the seat. The floor panel transmit additional vibrations to the feet. These vibrations cause a level of discomfort for driver and passengers. Examples of vibration sensors mounting for propagation path: engine, dash panel, floor panel under driver's feet and driver's seat are presented in Fig. 12.3.

The established scope of research enables to observe changes of the vibration for chosen points on the vibration propagation paths from engine. The three orthogonal axes were analysed separately. Thus the comparison of the acceleration of vibration signals allows determine which directions of the vibration propagation is parent.

\subsection{Propagation and structure of vibration transferred from engine to car body}

For the purpose of identifying of vibration propagation paths from engine to car body in terms of comfort and safety the transfer of the vibration to the locations of penetration into occupants have to be consider. Vibrations propagate into the human organism through vehicle seats via the pelvis, the back and lateral parts of the body as well as through the vehicle floor panel via feet.

The vibration signals were grouped in 4 dominants propagation paths included locations where they penetrate the organism. Thus the vibration transfer from engine to the floor panel and seats have been analysed. The complex analysis of vibrations due to the human exposure require observation of distribution on time, frequency and time-frequency representation.

\subsubsection{Vibration propagation into driver}

Observation of time distribution of vibration transferred from engine, via dash panel to the driver by the feet or seat enables evaluation of dissipation of energy. The Fourier transformation shows influence of propagation paths on dynamics of vibration (Figs. 12.4-12.6). The analysis of the vibration structure, as TFR, permit to identify the periods of temporary excited vibration. The collection of vibration distribution in the following measurement points on propagation path are presented in Figs. 12.7-12.9.

The lateral vibration measured on the floor panel under the driver's feet have constant low-frequency component which can be correspond to the rocking movement. Thus the energy of the frequency dominant peak is lower. It can be result of measurement process. Apart this the decrease of the vibration energy for following measurement points is significant. It can be assumed that lateral and vertical vibration transferred from engine to the driver are ca. one hundred times lower. 


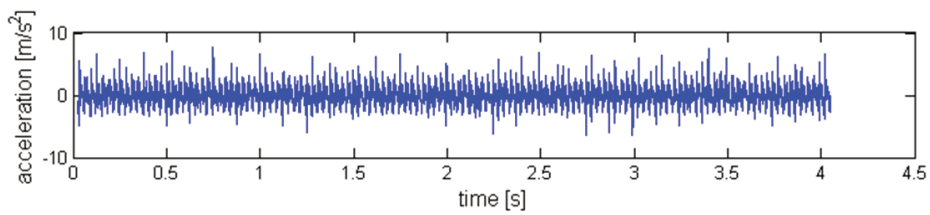

Motor-car engine

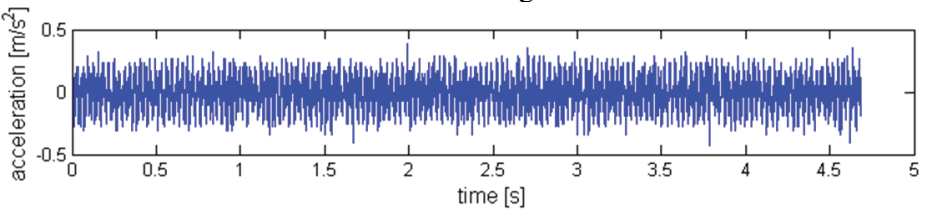

Dash panel

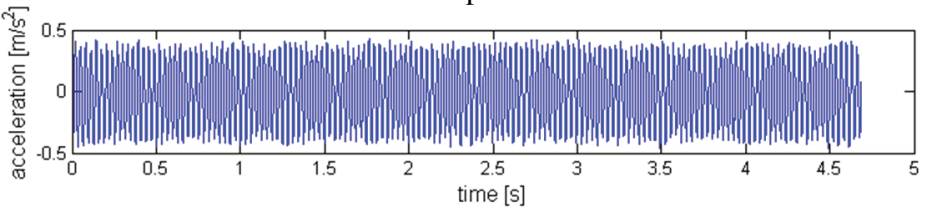

Driver floor

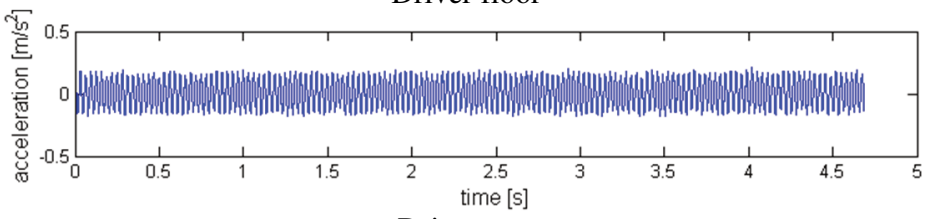

Driver seat

a) Time function

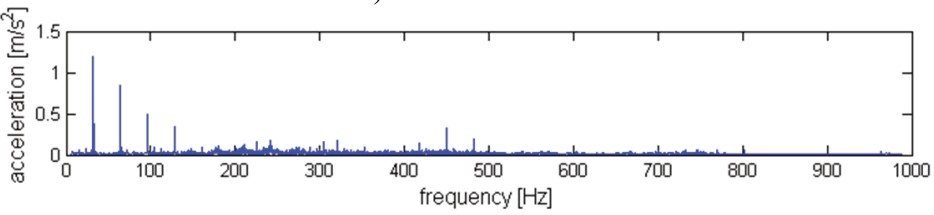

Motor-car engine

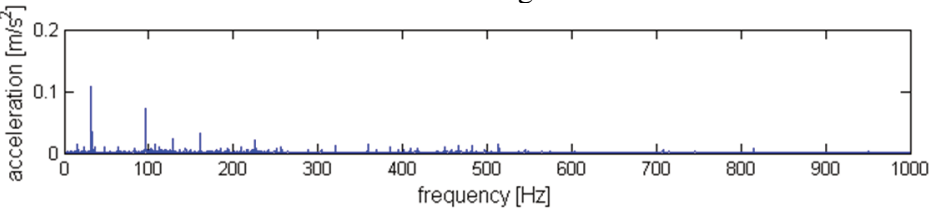

Dash panel

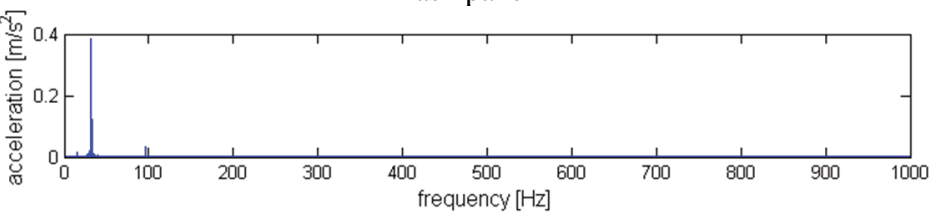

Driver floor

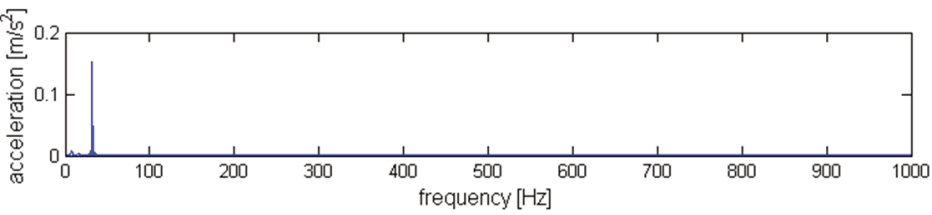

Driver seat

b) Frequency function

Fig. 12.4. Propagation of the vibration, path No. I., $X$ axis - longitudinal, $750 \mathrm{rpm}$ 


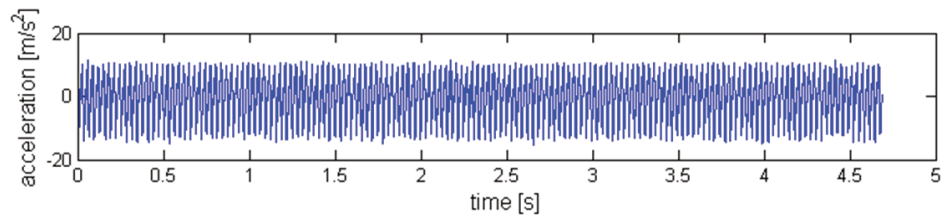

Motor-car engine

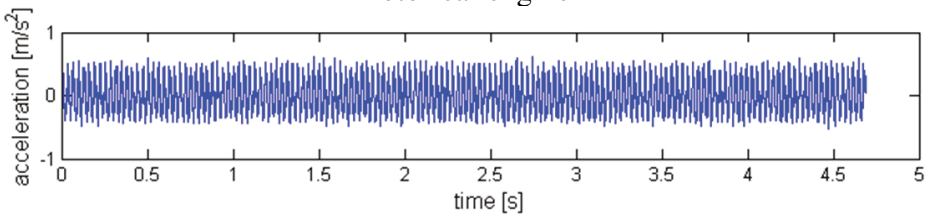

Dash panel

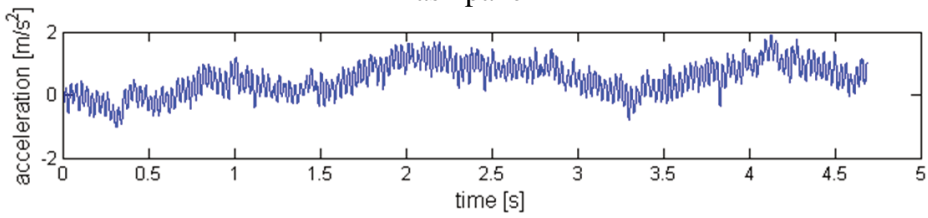

Driver floor

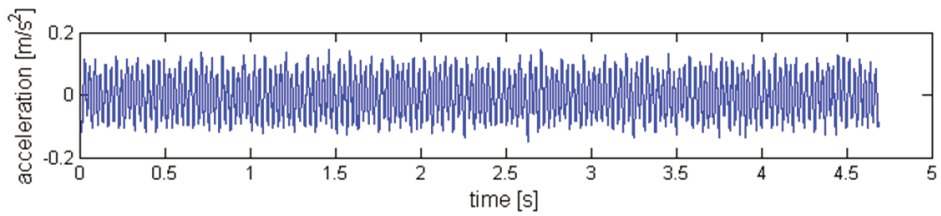

Driver seat

a) Time function

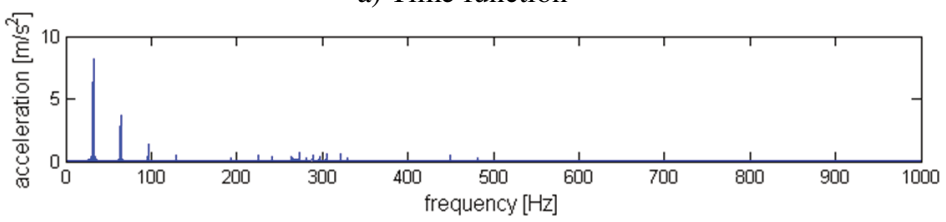

Motor-car engine

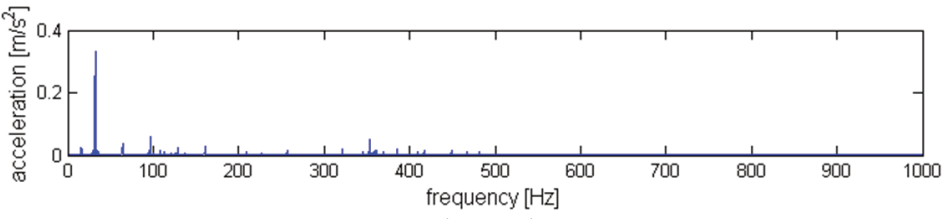

Dash panel

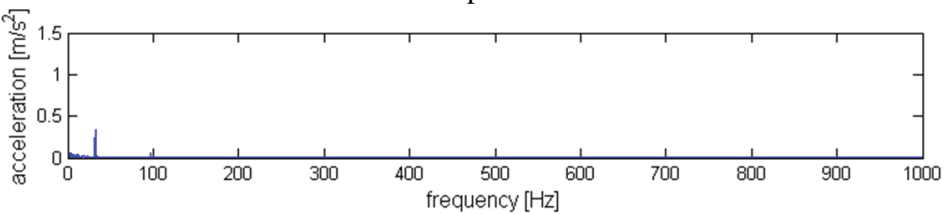

Driver floor

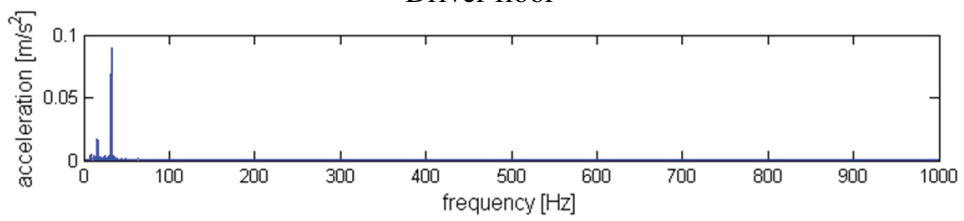

Driver seat

b) Frequency function

Fig. 12.5. Propagation of the vibration, path No. I., $Y$ axis - lateral, $750 \mathrm{rpm}$ 


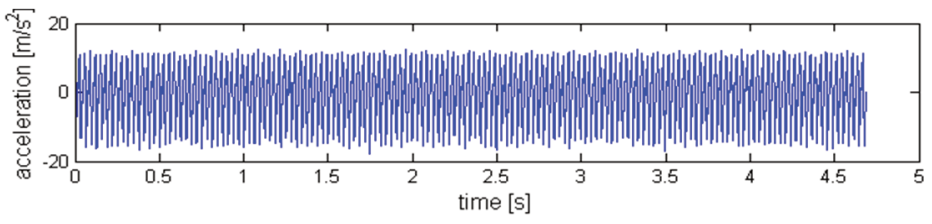

Motor-car engine

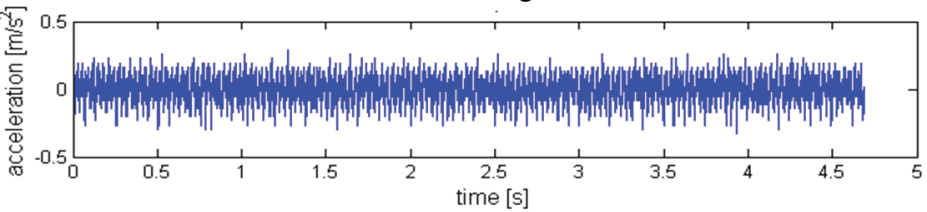

Dash panel

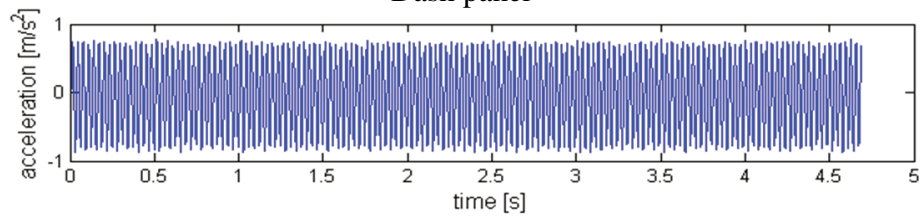

Driver floor

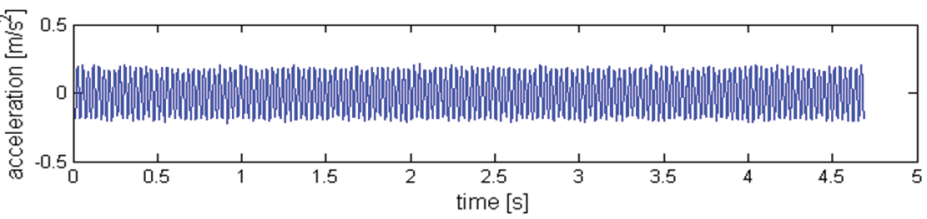

Driver seat

a) Time function

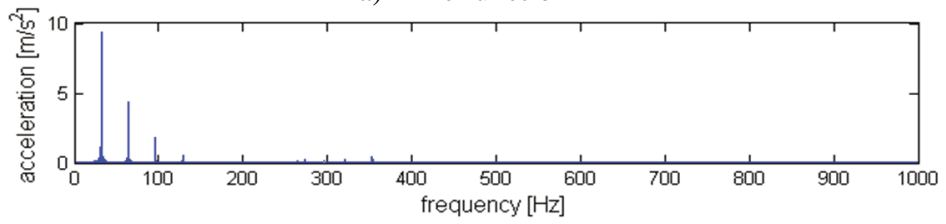

Motor-car engine

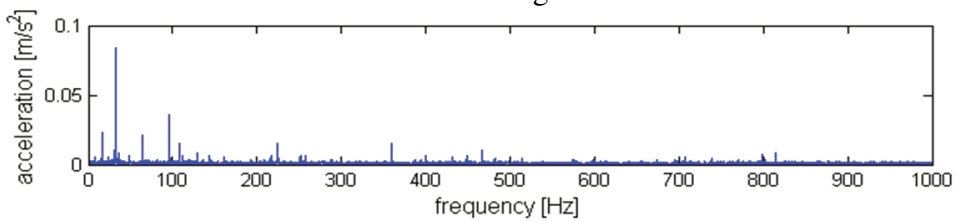

Dash panel

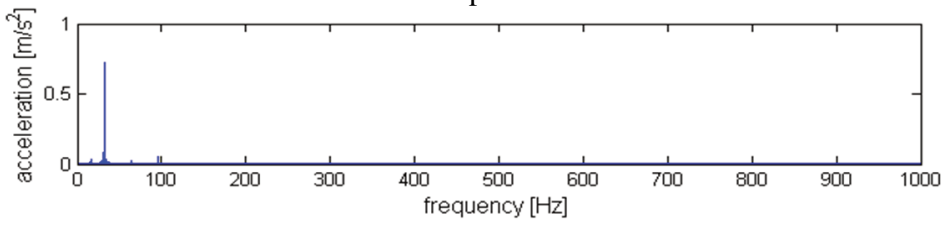

Driver floor

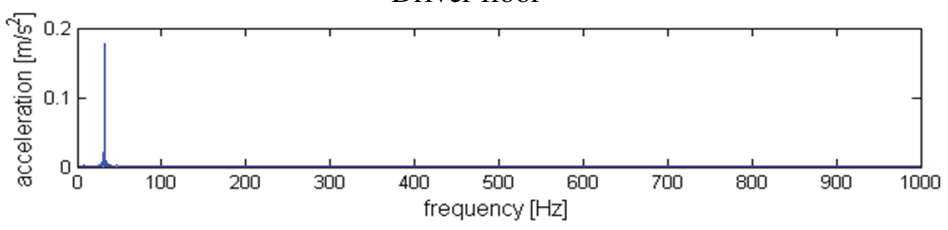

Driver seat

b) Frequency function

Fig. 12.6. Propagation of the vibration, path No. I., $Z$ axis - vertical, $750 \mathrm{rpm}$ 


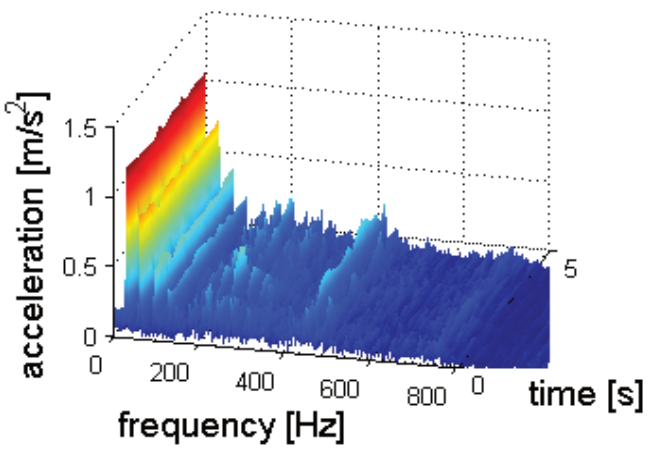

a) Motor car engine

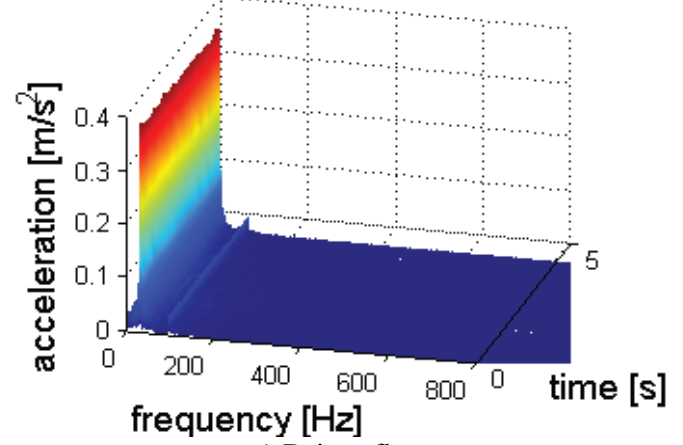

c) Driver floor

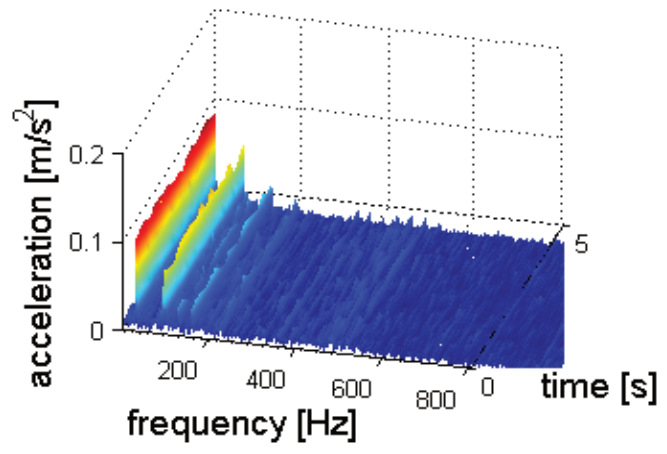

b) Dash panel

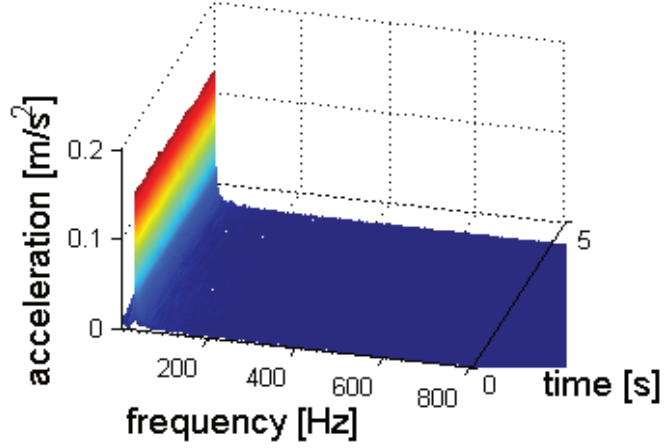

d) Driver seat

Fig. 12.7. Changes of the structure of vibration during propagation, path No. I., $X$ axis - longitudinal

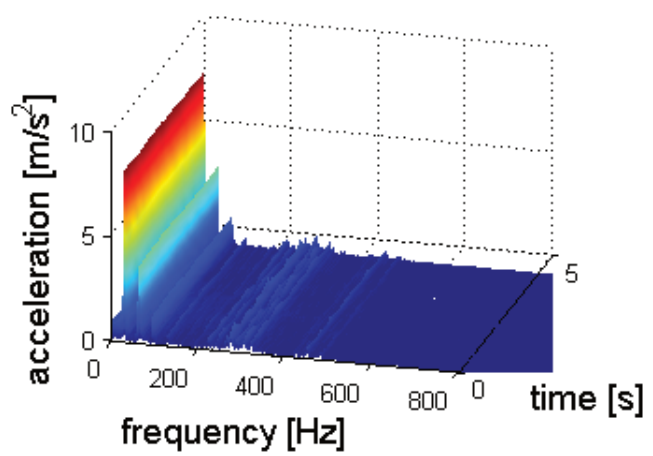

a) Motor car engine

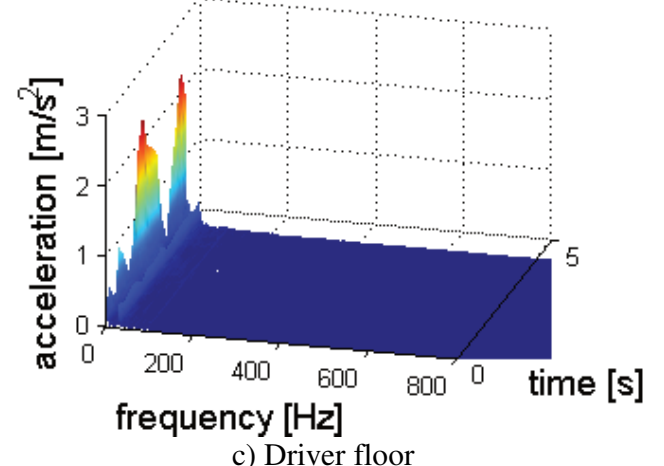

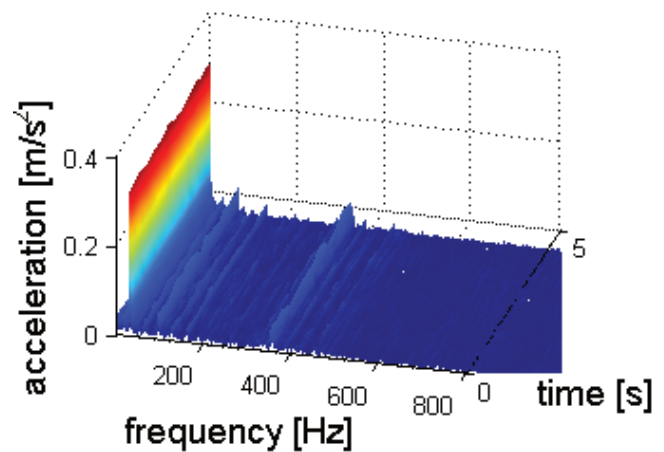

b) Dash panel

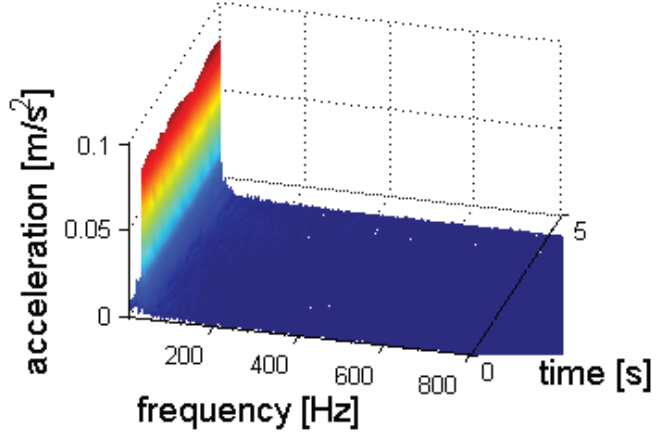

d) Driver seat

Fig. 12.8. Changes of the structure of vibration during propagation, path No. I., $Y$ axis - lateral 


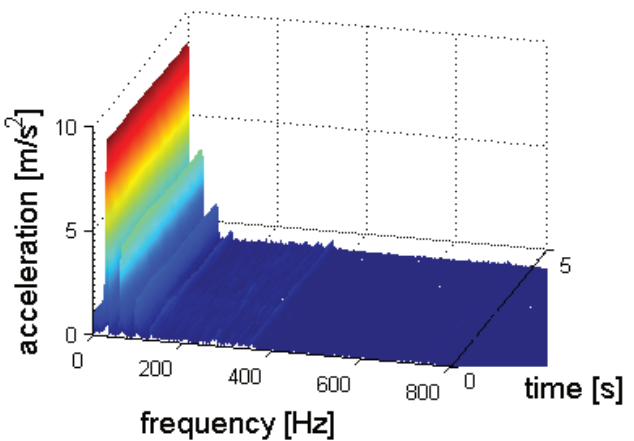

a) Motor car engine

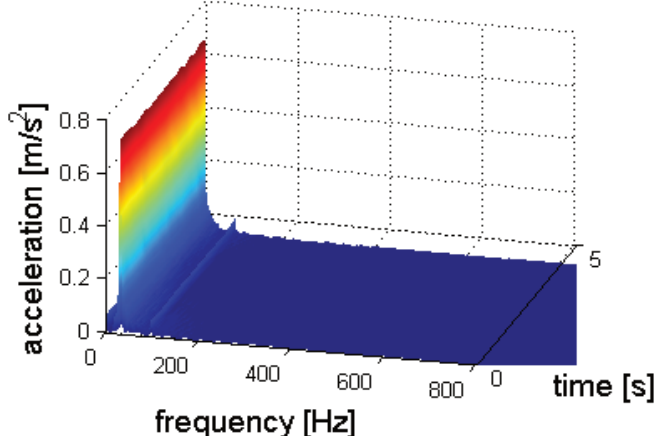

c) Driver floor

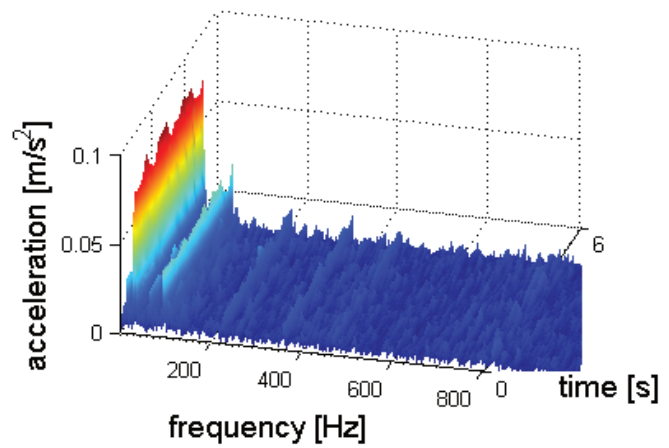

b) Dash panel

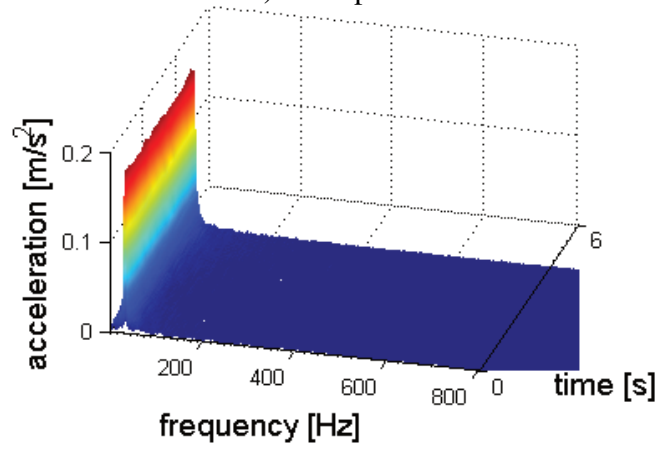

d) Driver seat

Fig. 12.9. Changes of the structure of vibration during propagation, path No. I., $Z$ axis - vertical

\subsubsection{Vibration propagation into rear passengers}

The propagation of the vibration generated by the engine has to be considered for all occupants. The scope of the research included observation of the vibration effected on passengers sitting on the rear seats. The main propagation path consist the location points on floor panel under left rear passenger's feet, left rear passenger's seat and right rear passenger's seat. These are location where whole body vibration penetrate into the human organism.

The collection of vibration distribution in the following measurement points on propagation path are shown in Figs. 12.10-12.12.

The analysis of structure of longitudinal and lateral vibration enable evaluation of exposure to whole body vibration in horizontal directions (Figs. 12.13-12.15). The dissipation of the vibration energy on propagation path is large between source (engine) and floor panel and seats. It shows the level of isolation or absorption of engine mounting system and suspension of the seats. The energy of the vibration affecting rear passengers in horizontal surface is similar.

The structure of vertical vibration affecting rear passengers via the floor and seats are similar as well, i.e. amplitude and frequency domains. For all result the vibration time distribution of dominant frequency band determined from the signals registered on floor panel (under the left rear passenger's feet) aren't constant. 


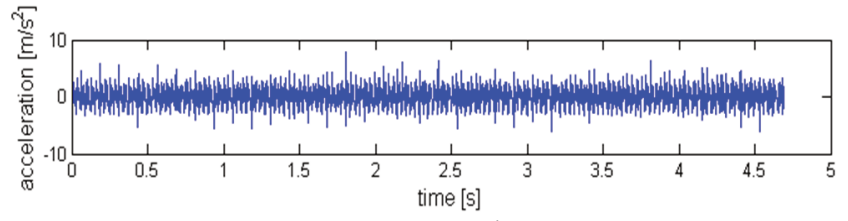

Motor-car engine

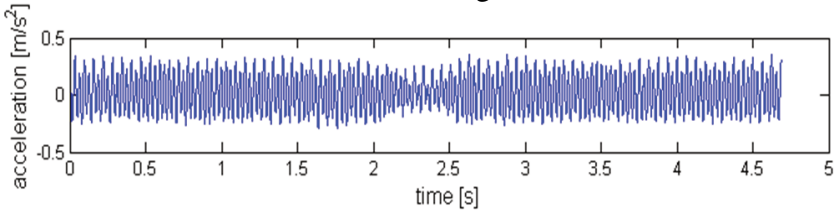

Left rear passenger floor

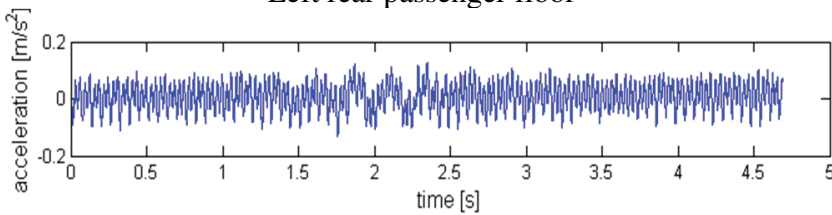

Left rear passenger seat

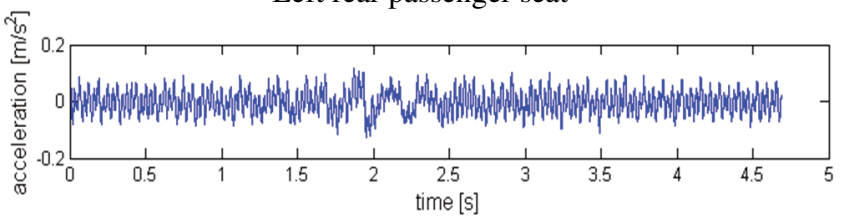

Right rear passenger seat

a) Time function

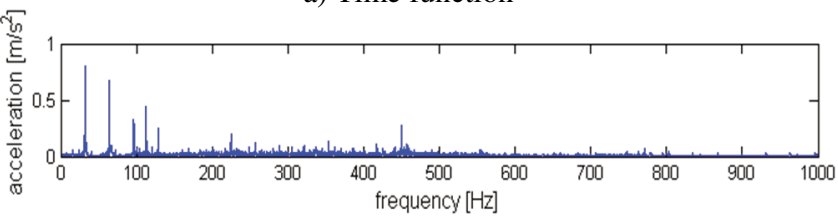

Motor-car engine

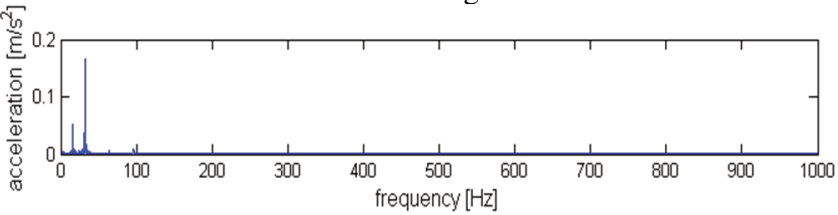

Left rear passenger floor

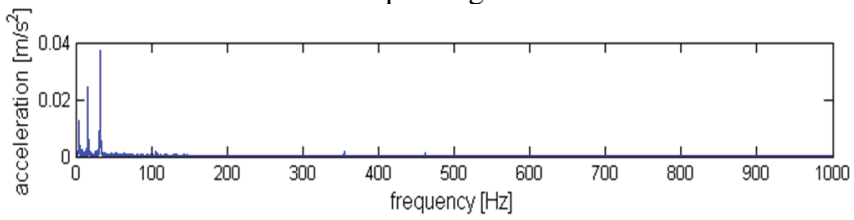

Left rear passenger seat

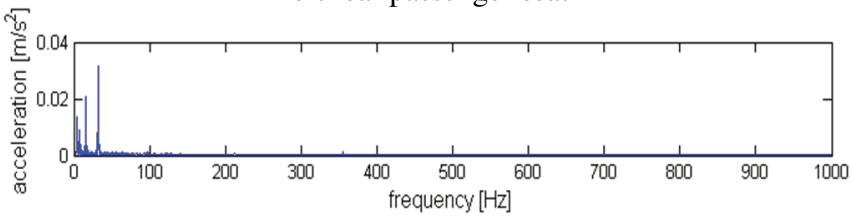

Right rear passenger seat

b) Frequency function

Fig. 12.10. Propagation of the vibration, path No. II., $X$ axis - longitudinal, $750 \mathrm{rpm}$ 


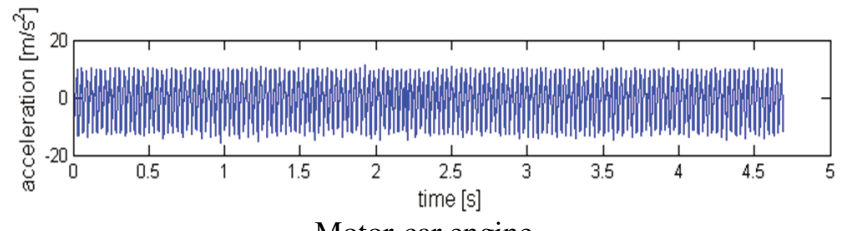

Motor-car engine

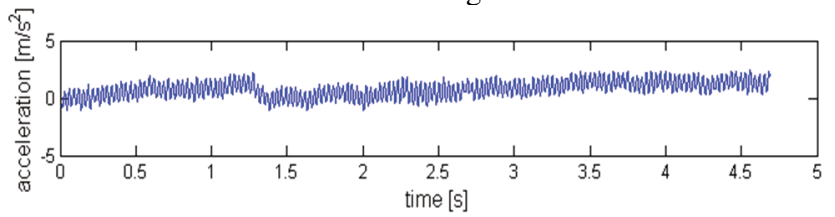

Left rear passenger floor

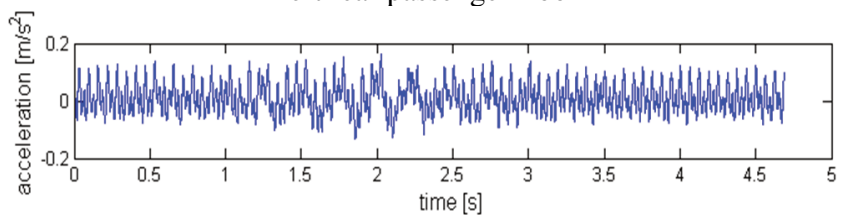

Left rear passenger seat

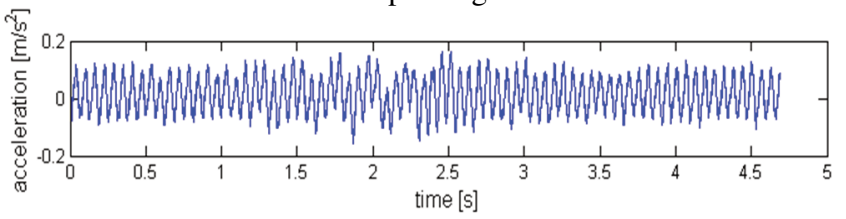

Right rear passenger seat

a) Time function

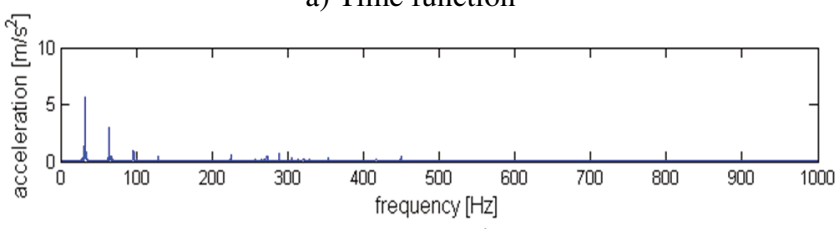

Motor-car engine

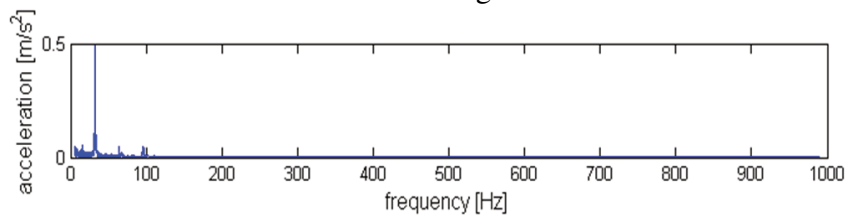

Left rear passenger floor

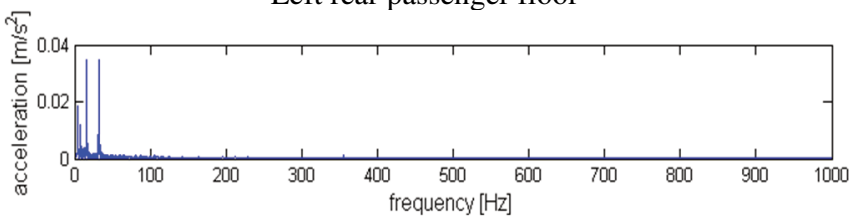

Left rear passenger seat

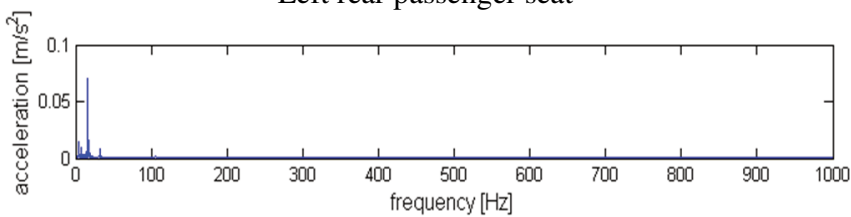

Right rear passenger seat

b) Frequency function

Fig. 12.11. Propagation of the vibration, path No. II., $Y$ axis - lateral 


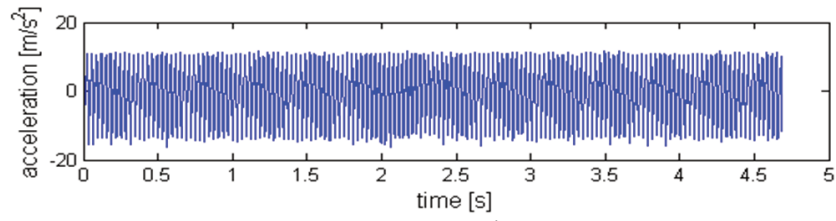

Motor-car engine

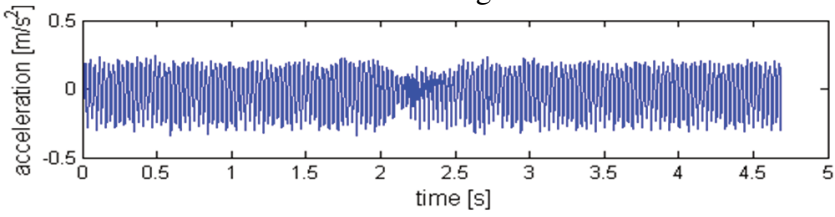

Left rear passenger floor

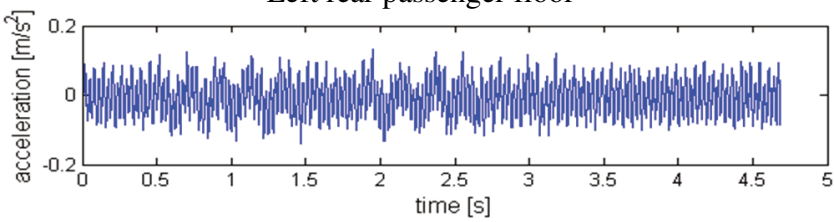

Left rear passenger seat

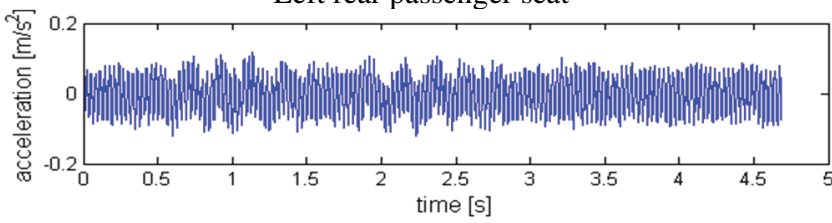

Right rear passenger seat

a) Time function

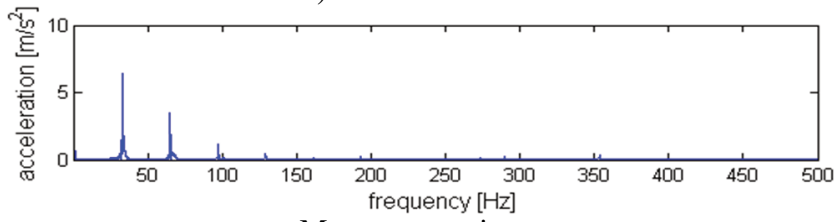

Motor-car engine

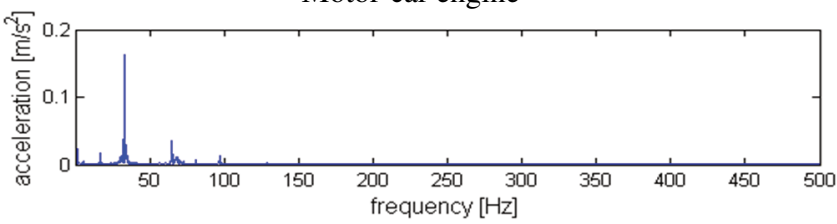

Left rear passenger floor

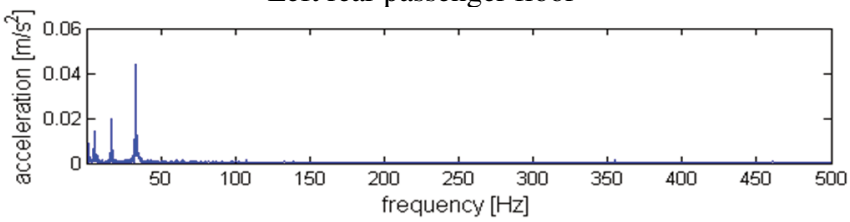

Left rear passenger seat

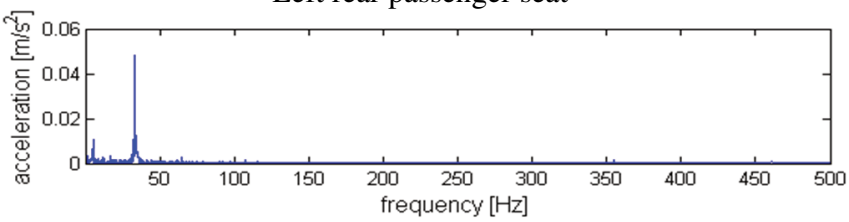

Right rear passenger seat

b) Frequency function

Fig. 12.12. Propagation of the vibration, path No. II., $Z$ axis -vertical, $750 \mathrm{rpm}$ 


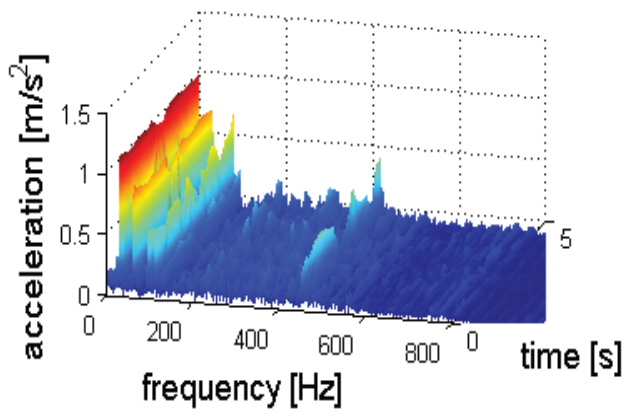

a) Motor car engine

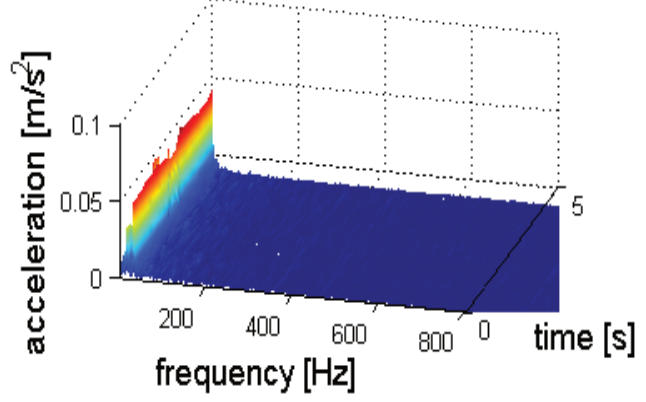

c) Left rear passenger seat

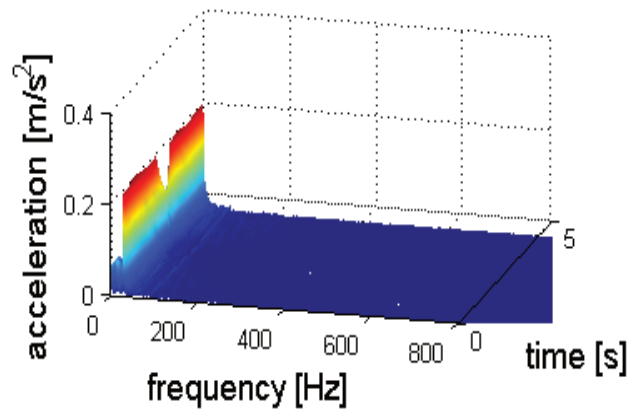

b) Left rear passenger floor

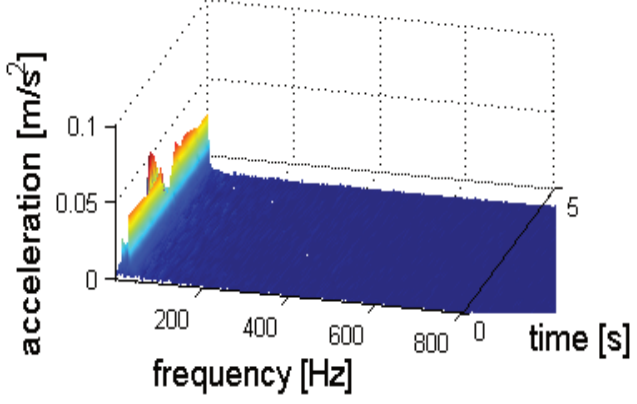

d) Right rear passenger seat

Fig. 12.13. Changes of the structure of vibration during propagation, path No. II., $X$ axis - longitudinal

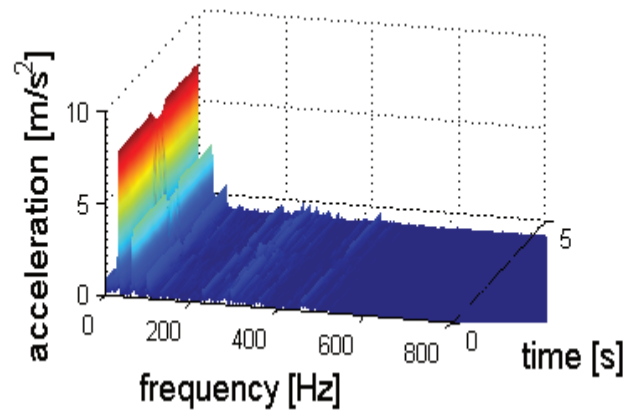

a) Motor car engine

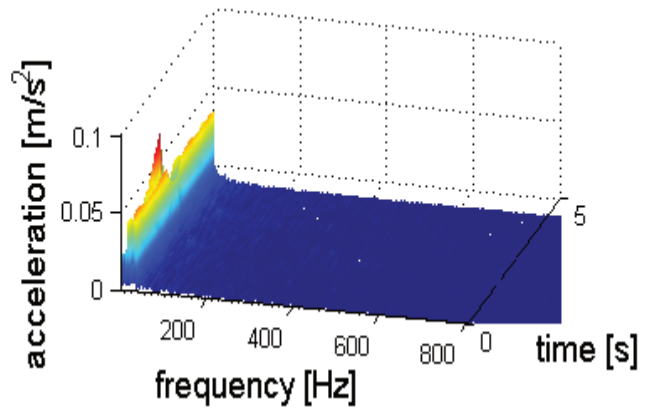

c) Left rear passenger seat

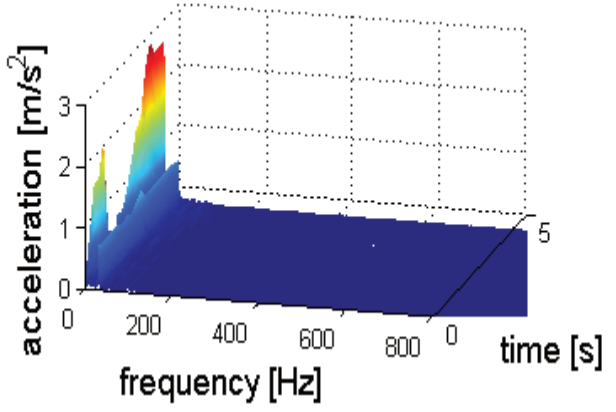

b) Left rear passenger floor

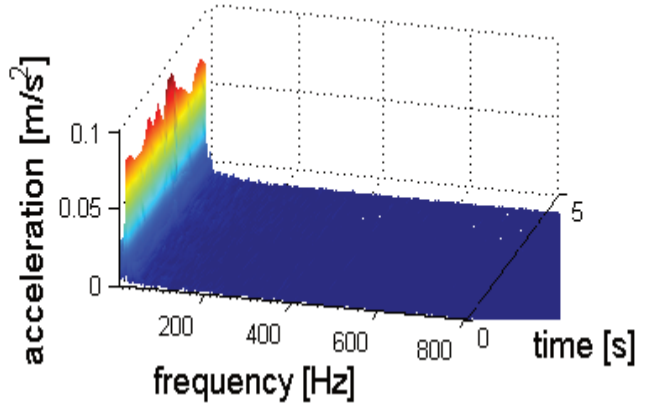

d) Right rear passenger seat

Fig. 12.14. Changes of the structure of vibration during propagation, path No. II., $Y$ axis - lateral 


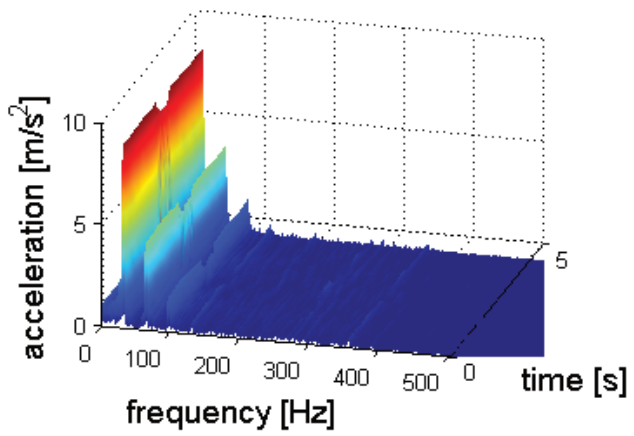

a) Motor car engine

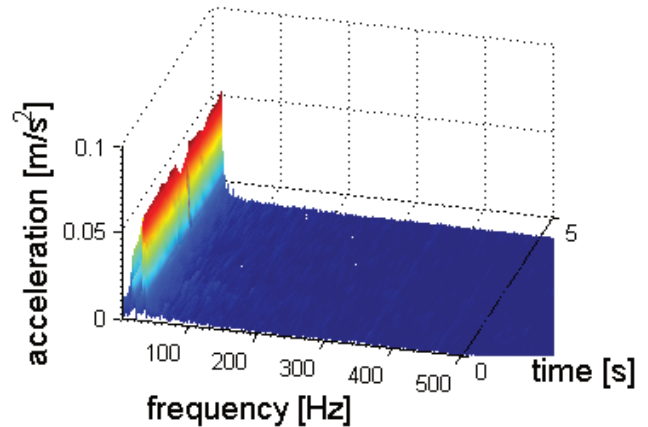

c) Left rear passenger seat

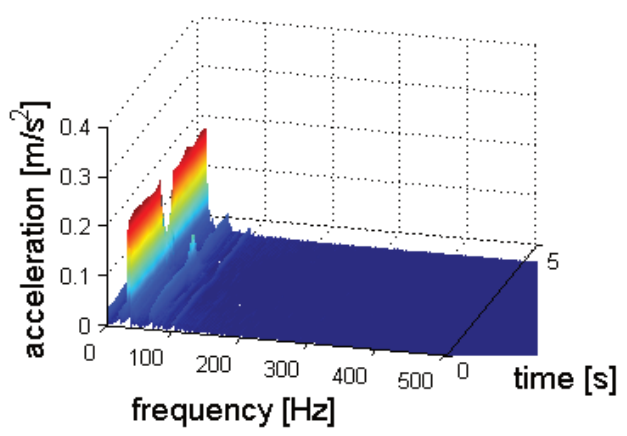

b) Left rear passenger floor

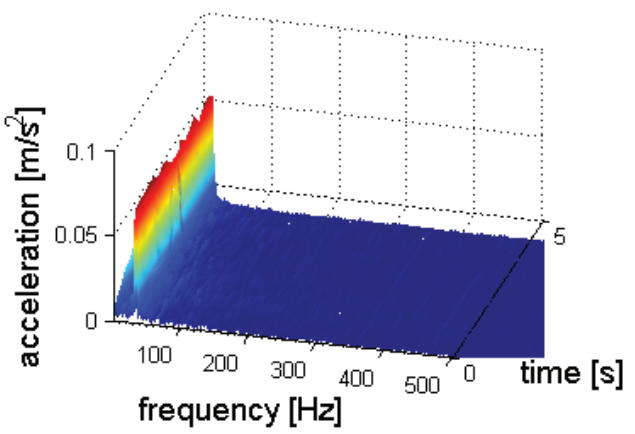

d) Right rear passenger seat

Fig. 12.15. Changes of the structure of vibration during propagation, path No. II., $Z$ axis -vertical

\subsubsection{Vibration propagation into front occupants}

For the purpose of comparison of vibration exposure to all occupants of vehicle the propagation of the vibration generated by the engine to the front occupants were analysed. The front occupants mean driver and front passenger.

The collection of vibration distribution in the following measurement points on propagation path are shown in Figs. 12.16-12.21.

Basing on the analysis of vertical vibration registered on front occupants seats it can be assumed that suspension systems of front seats have good vibroisolation properties. Apart of minimize of the amplitudes in dominant frequencies its filters the higher frequencies similar to lowpass filters. 


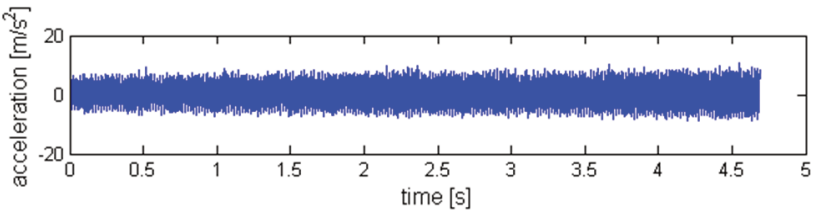

Motor-car engine

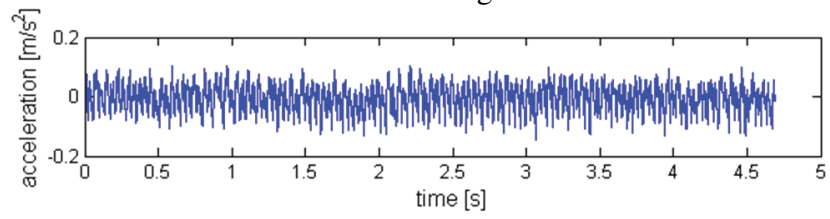

Driver floor

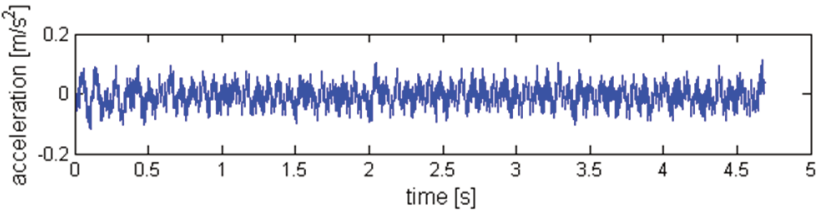

Driver seat

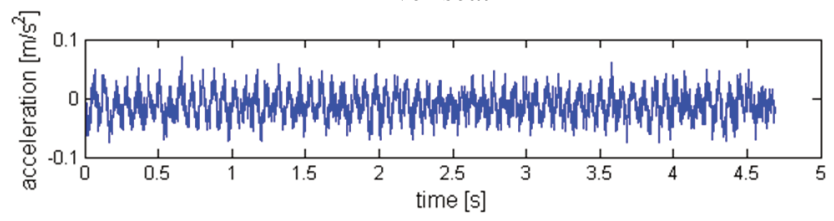

Front passenger seat

a) Time function

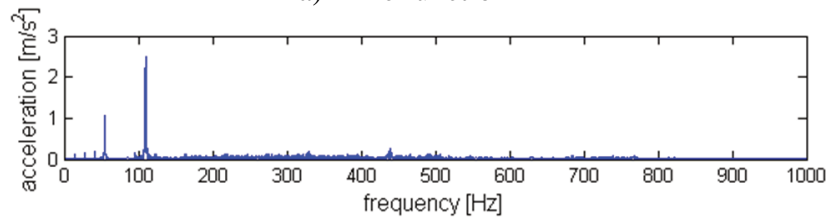

Motor-car engine

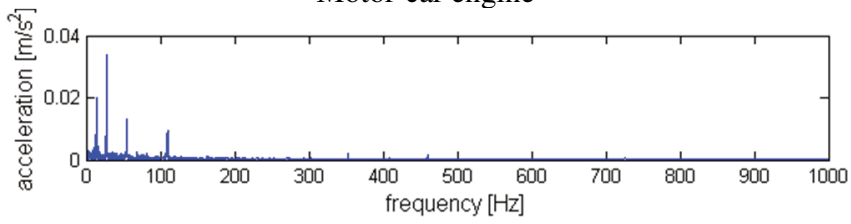

Driver floor

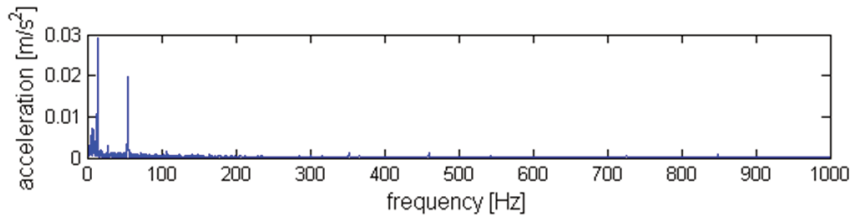

Driver seat

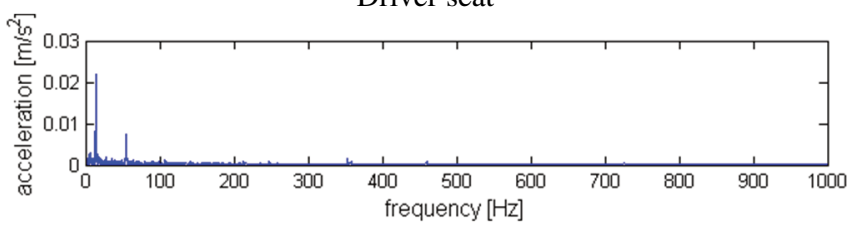

Front passenger seat

b) Frequency function

Fig. 12.16. Propagation of the vibration, path No. III, $X$ axis - longitudinal, $750 \mathrm{rpm}$ 


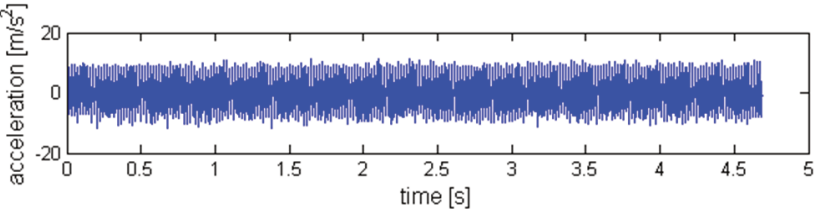

Motor-car engine

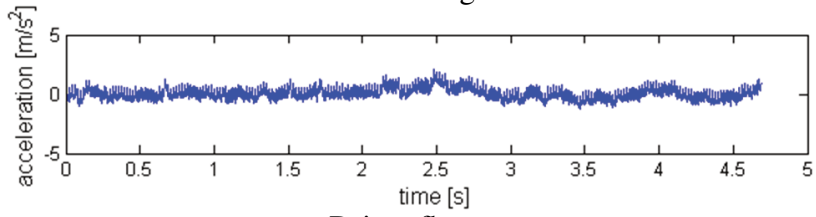

Driver floor

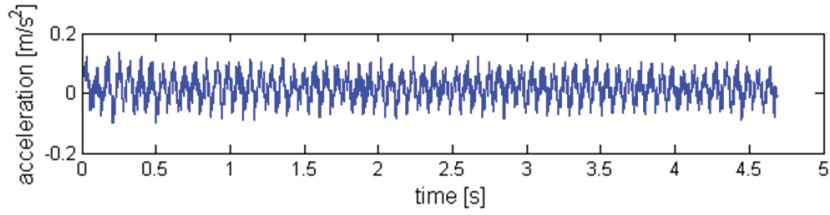

Driver seat

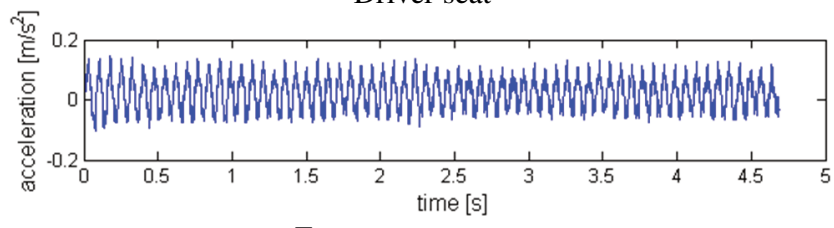

Front passenger seat

a) Time function

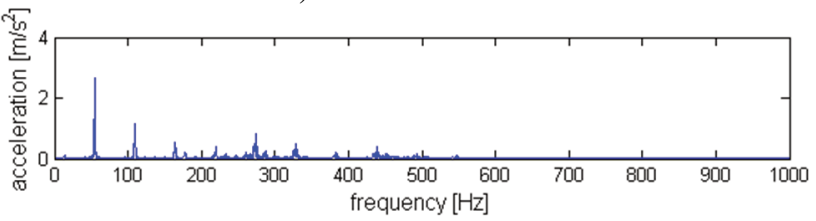

Motor-car engine

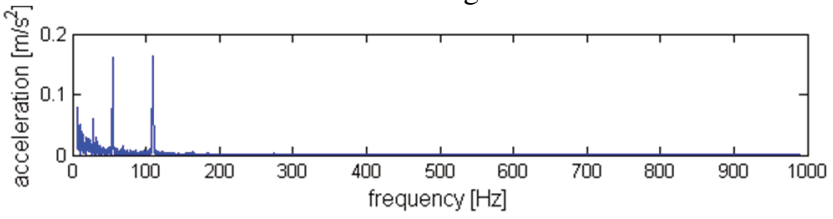

Driver floor

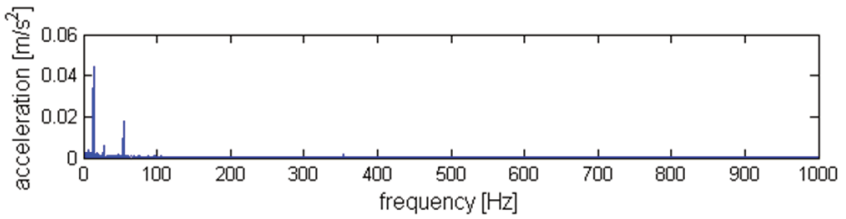

Driver seat

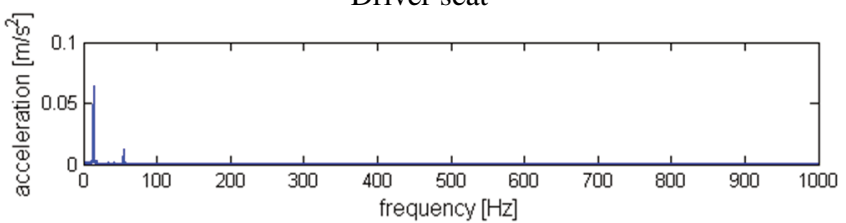

Front passenger seat

b) Frequency function

Fig. 12.17. Propagation of the vibration, path No. III, $Y$ axis - lateral, $750 \mathrm{rpm}$ 


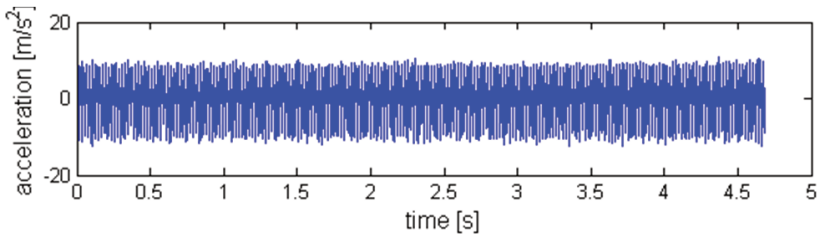

Motor-car engine

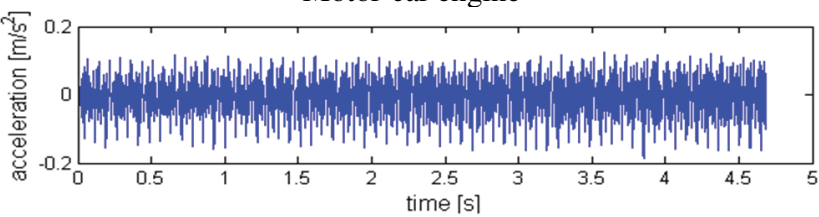

Driver floor

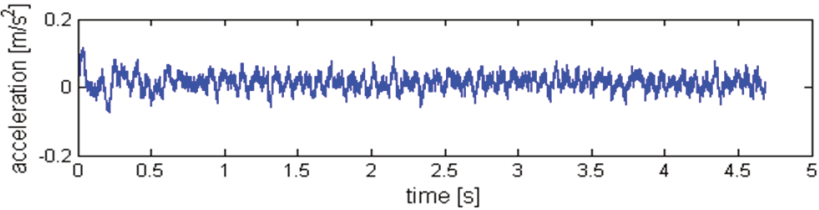

Driver seat

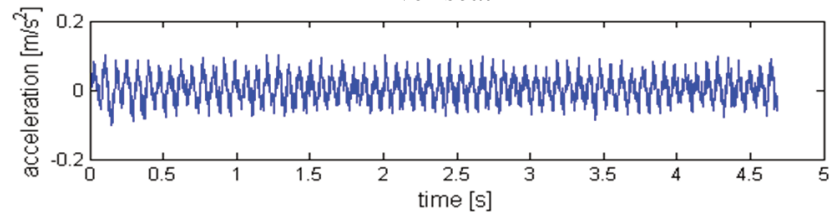

Front passenger seat

a) Time function

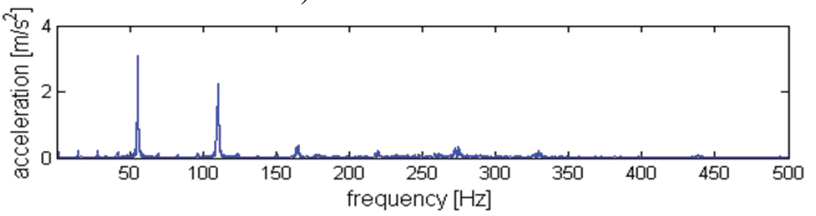

Motor-car engine

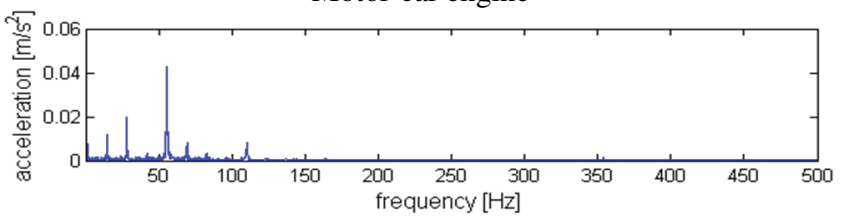

Driver floor

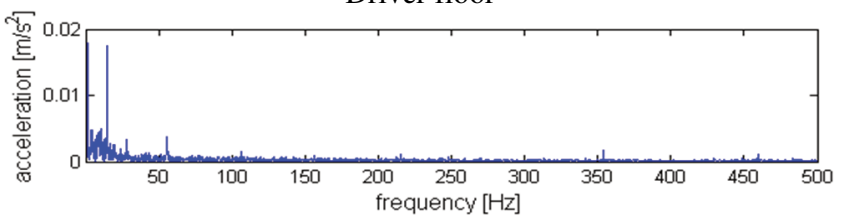

Driver seat

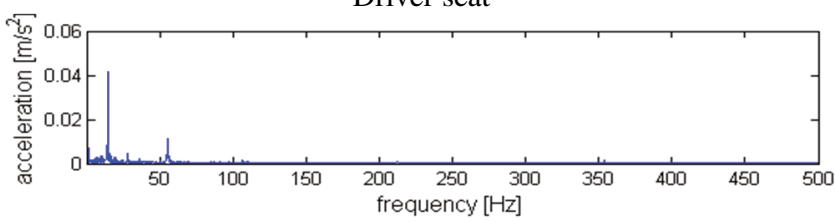

Front passenger seat

b) Frequency function

Fig. 12.18. Propagation of the vibration, path No. III, $Z$ axis - vertical 


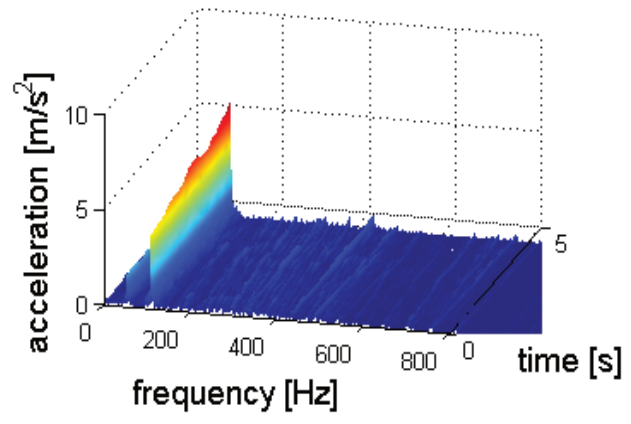

a) Motor car engine

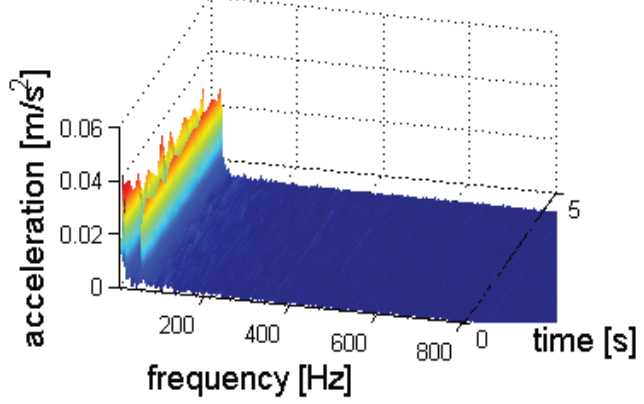

c) Driver seat

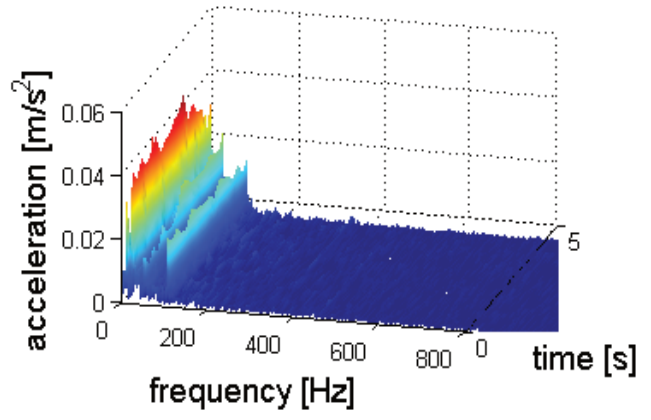

b) Driver floor

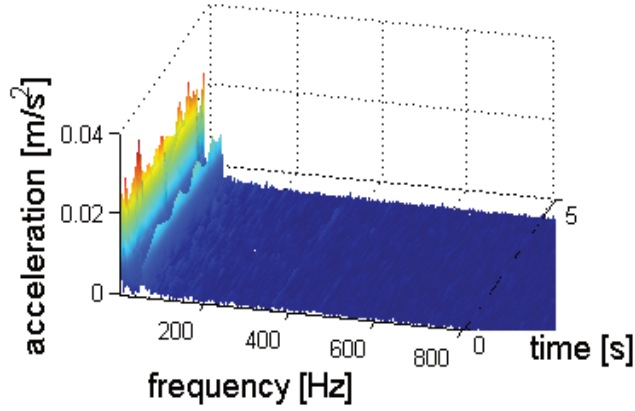

d) Front passenger seat

Fig. 12.19. Changes of the structure of vibration during propagation, path No. III, $X$ axis - longitudinal

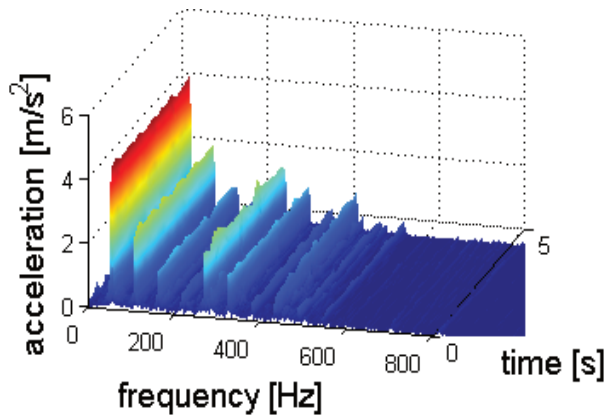

a) Motor car engine

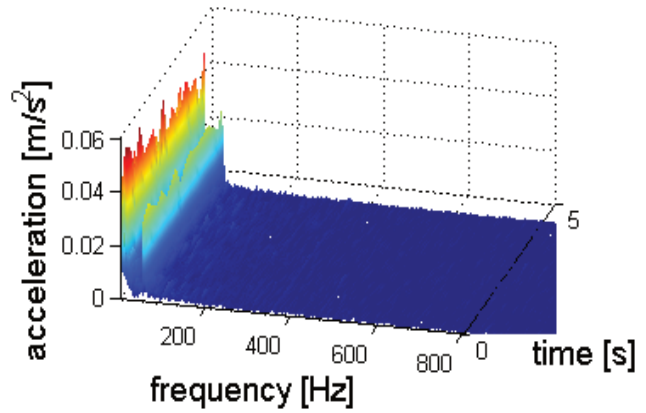

c) Driver seat

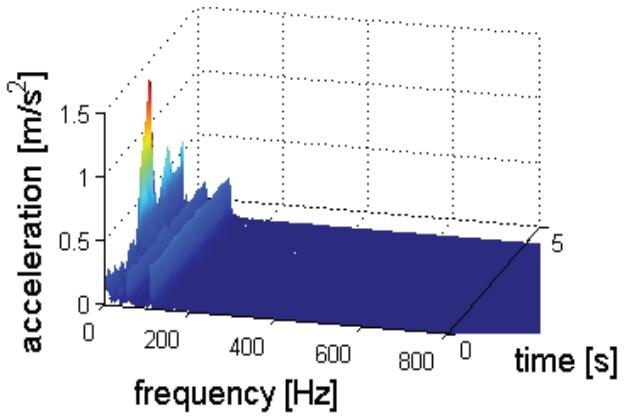

b) Driver floor

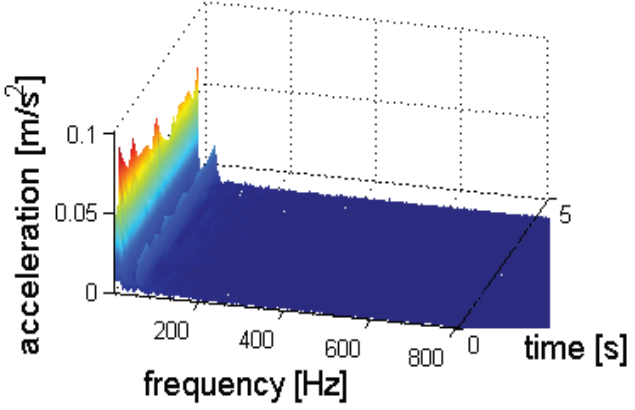

d) Front passenger seat

Fig. 12.20. Changes of the structure of vibration during propagation, path No. III, $Y$ axis - lateral 


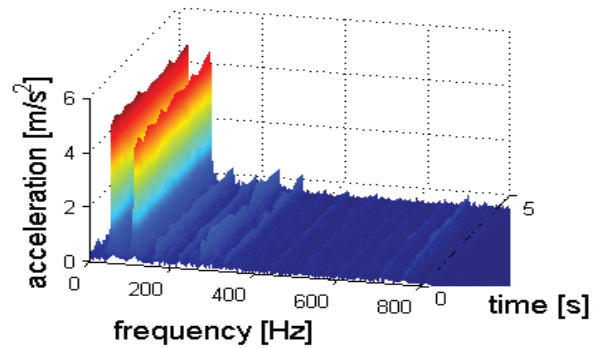

a) Motor car engine

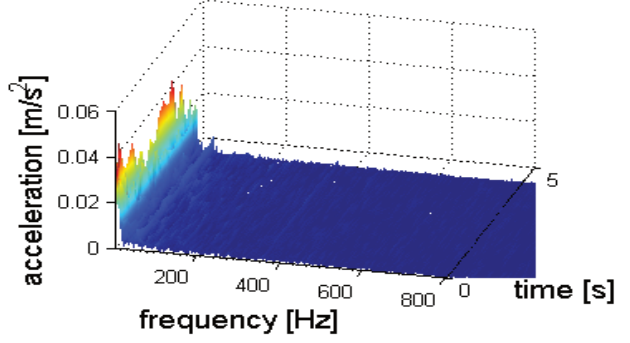

c) Driver seat

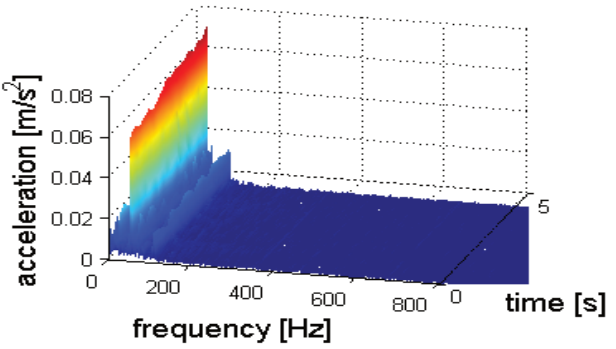

b) Driver floor

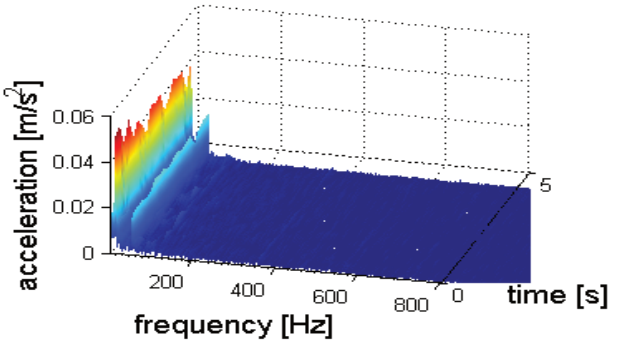

d) Front passenger seat

Fig. 12.21. Changes of the structure of vibration during propagation, path No. III, $Z$ axis - vertical

\subsubsection{Vibration propagation into front passenger}

For the purpose of identifying of vibration propagation paths into front passenger the engine vibration dynamic response of the vehicle structure on the right side was registered. The signals were measured in location of front passenger feet and seat and floor panel under rear right passenger feet.

The collection of vibration distribution in the following measurement points on propagation path are shown in Figs. 12.22-12.27. 


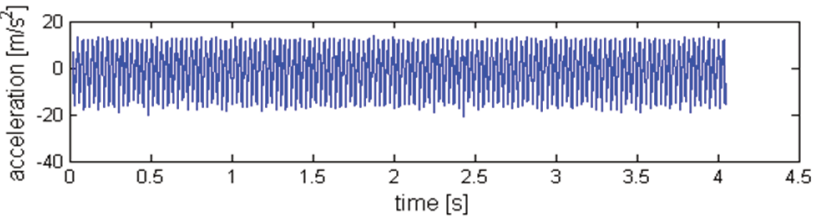

Motor-car engine

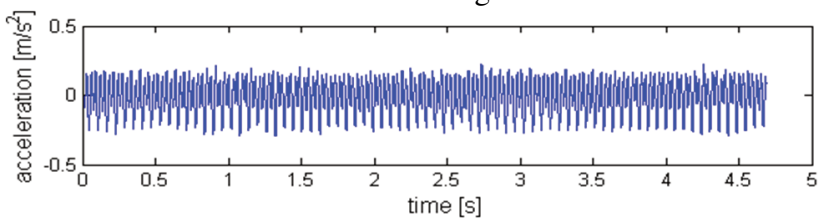

Front passenger floor

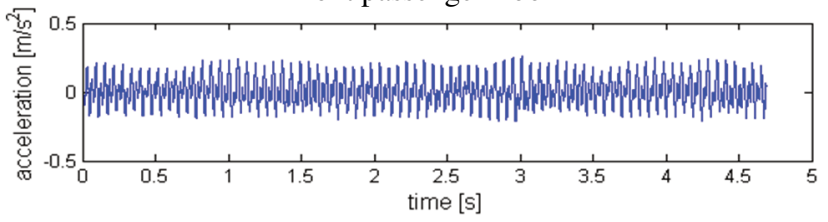

Front passenger seat

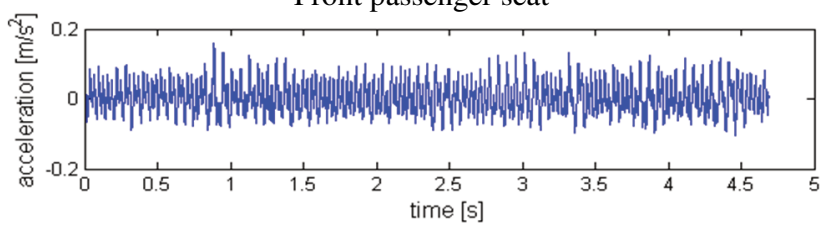

Right rear passenger floor

a) Time function

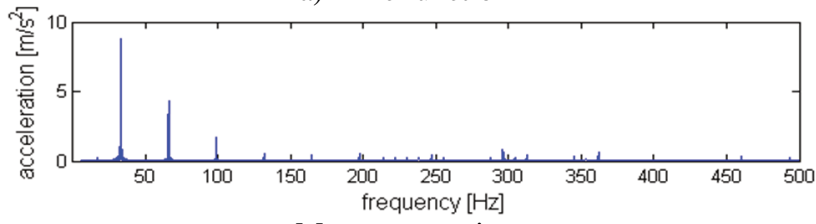

Motor-car engine

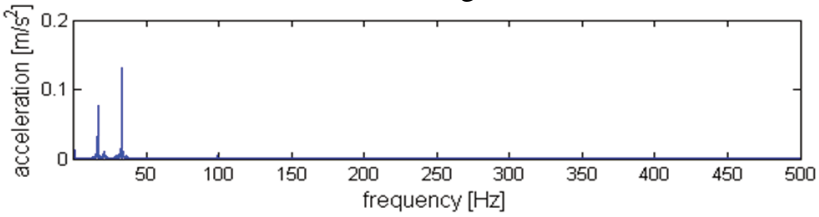

Front passenger floor

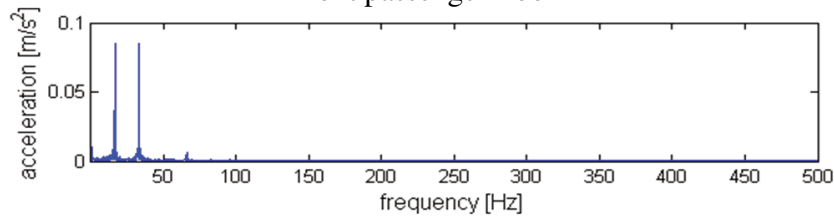

Front passenger seat

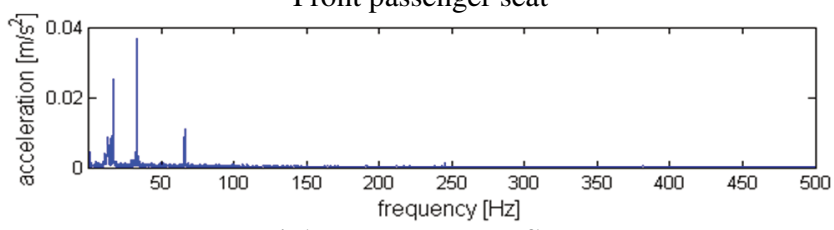

Right rear passenger floor

b) Frequency function

Fig. 12.22. Propagation of the vibration, path No. IV, $X$ axis - longitudinal, $750 \mathrm{rpm}$ 


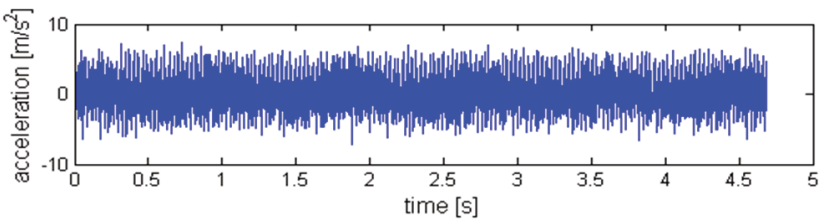

Motor-car engine

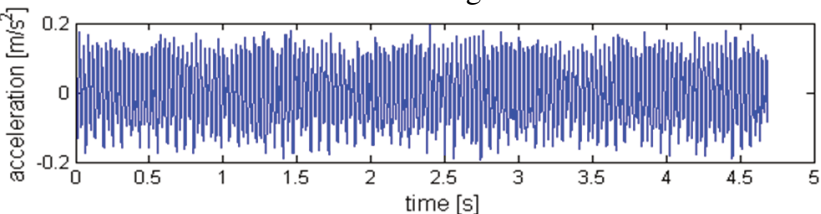

Front passenger floor

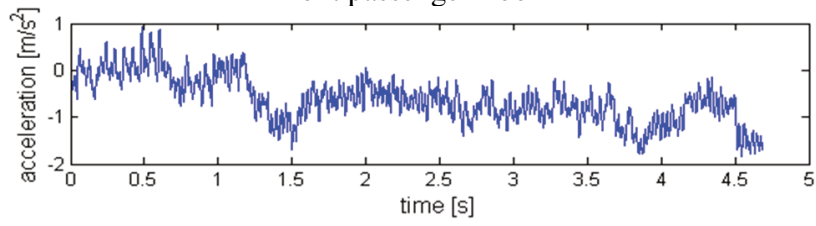

Front passenger seat

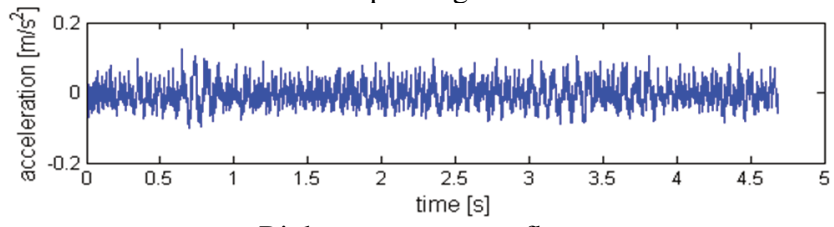

Right rear passenger floor

a) Time function

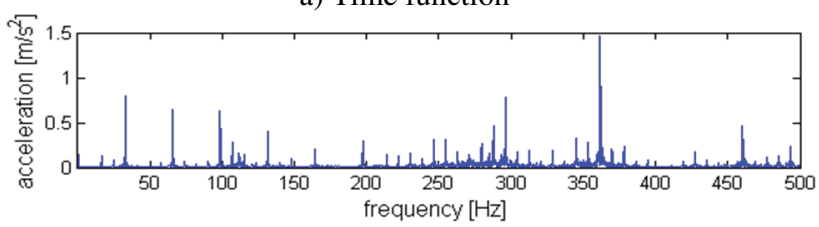

Motor-car engine

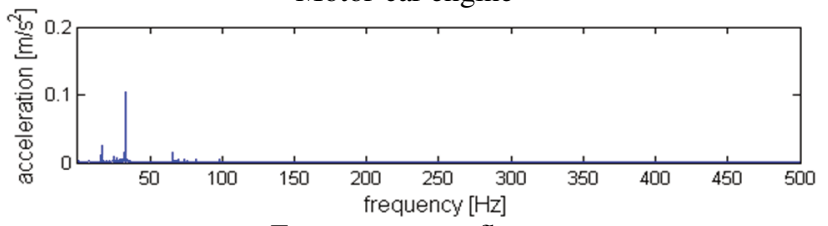

Front passenger floor

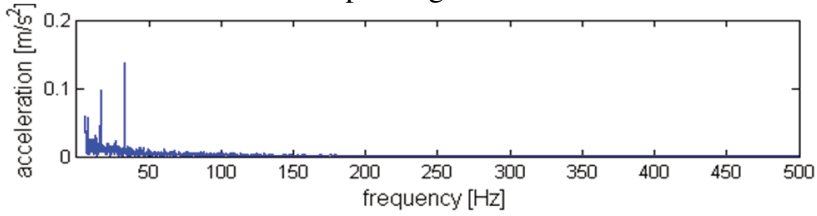

Front passenger seat

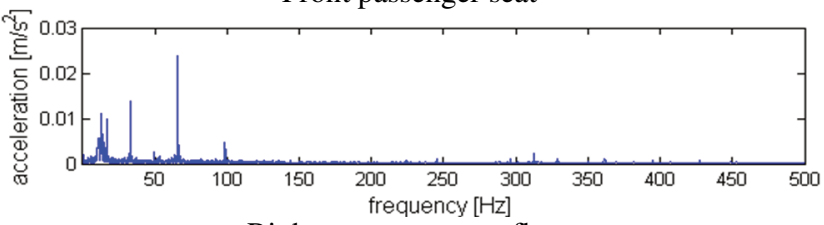

Right rear passenger floor

b) Frequency function

Fig. 12.23. Propagation of the vibration, path No. IV, $Y$ axis - lateral, $750 \mathrm{rpm}$ 

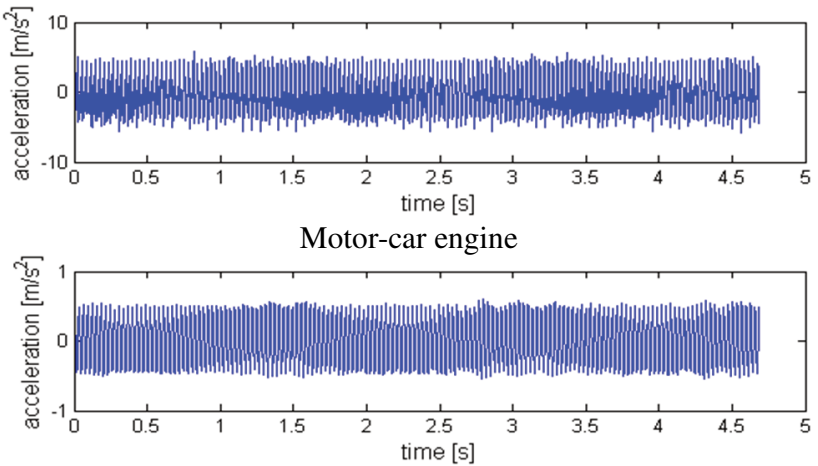

Front passenger floor

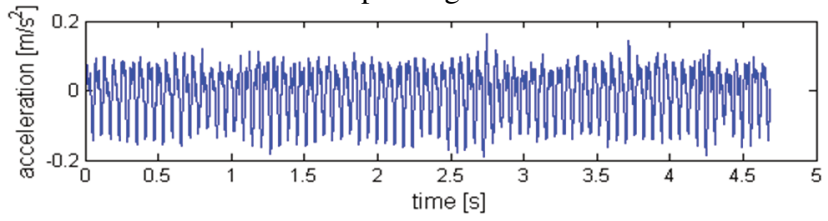

Front passenger seat

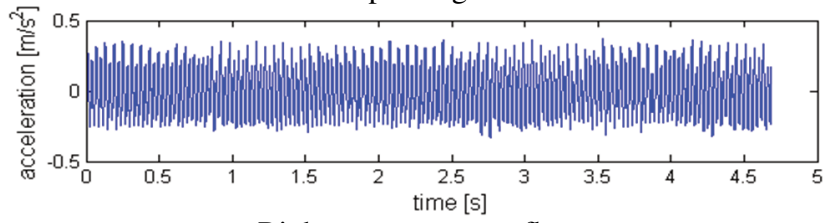

Right rear passenger floor

a) Time function

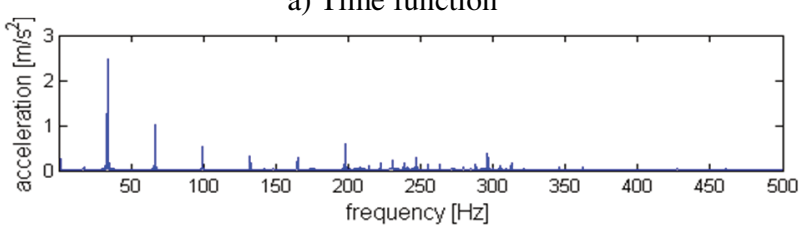

Motor-car engine

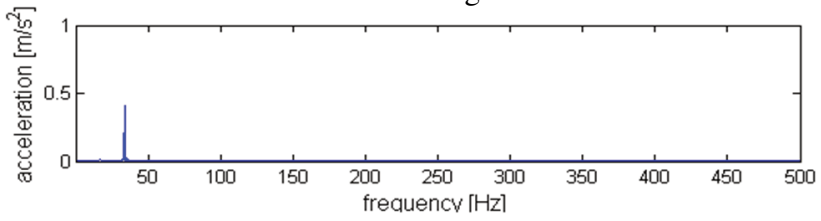

Front passenger floor

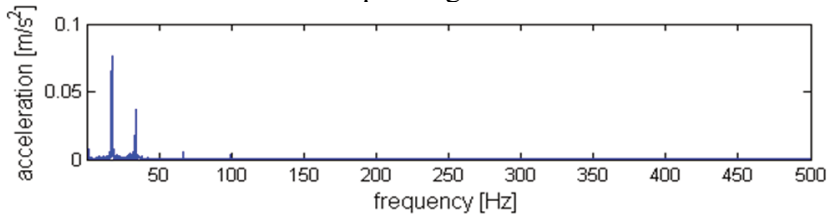

Front passenger seat

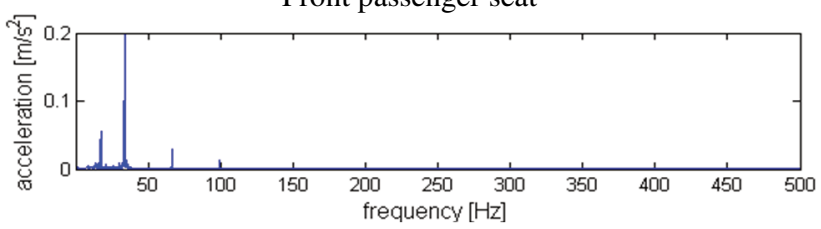

Right rear passenger floor

b) Frequency function

Fig. 12.24. Propagation of the vibration, path No. III, $Z$ axis -vertical, $750 \mathrm{rpm}$ 


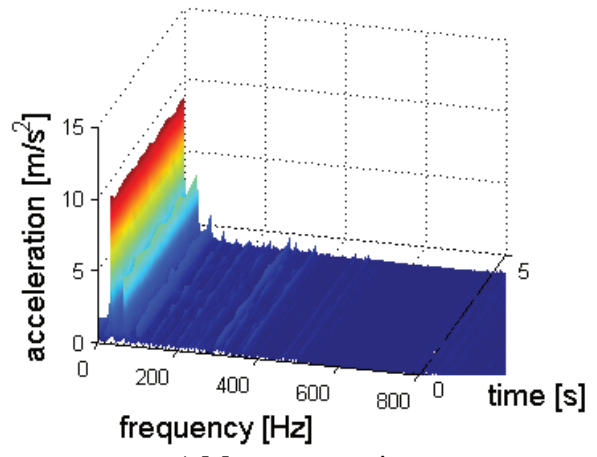

a) Motor car engine

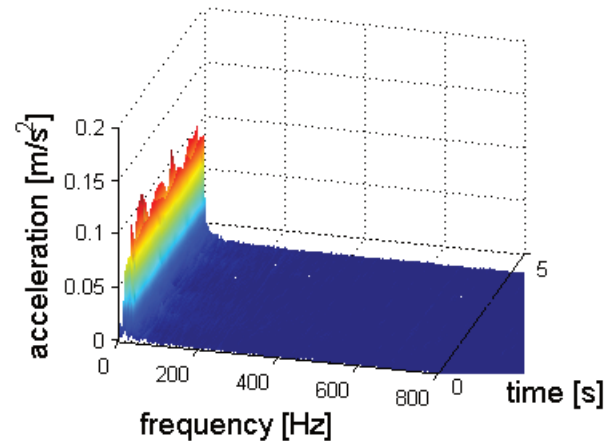

c) Front passenger seat

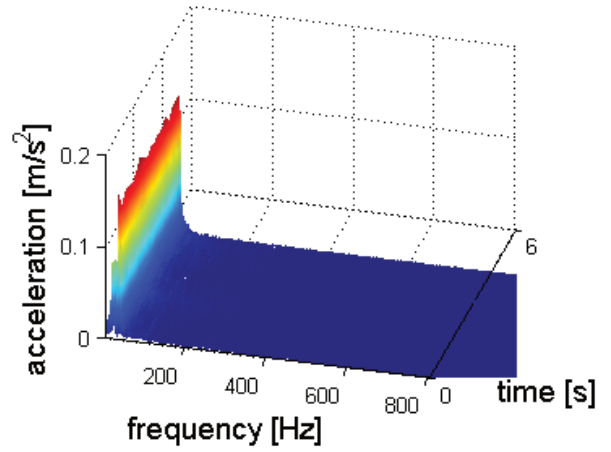

b) Front passenger floor

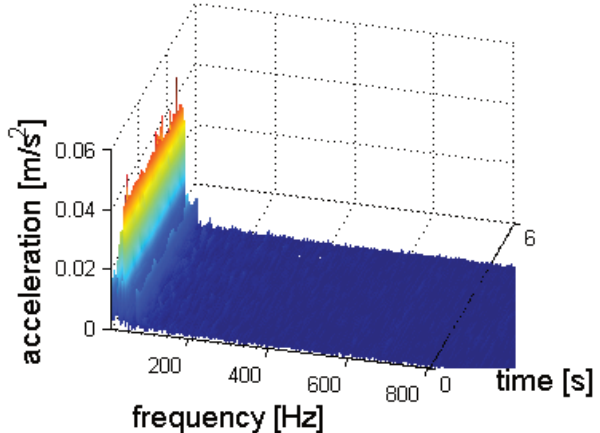

d) Right rear passenger floor

Fig. 12.25. Changes of the structure of vibration during propagation, path No. IV, $X$ axis - longitudinal

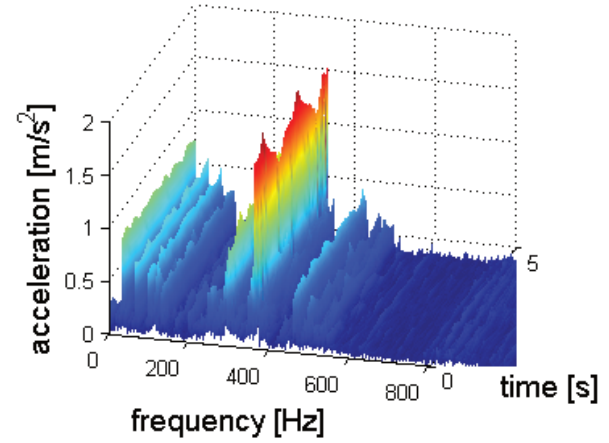

a) Motor car engine

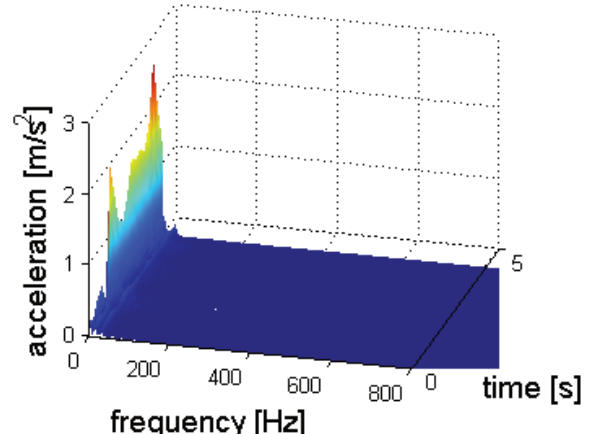

c) Front passenger seat

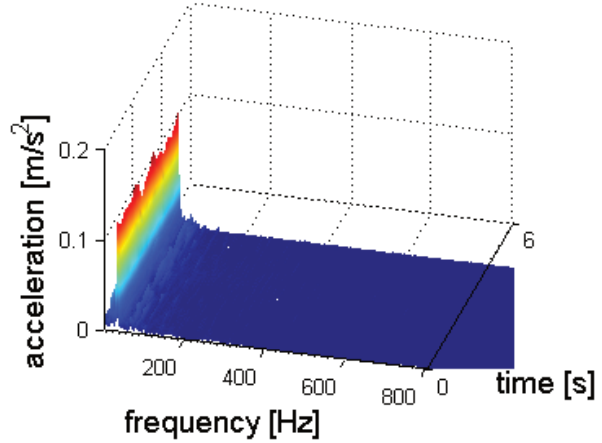

b) Front passenger floor

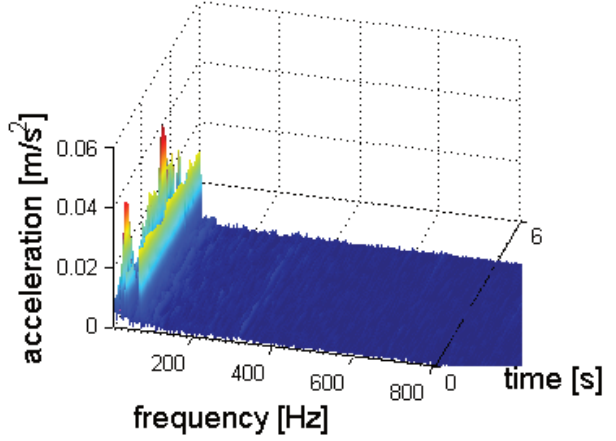

d) Right rear passenger floor

Fig. 12.26. Changes of the structure of vibration during propagation, path No. III, $Y$ axis - lateral 


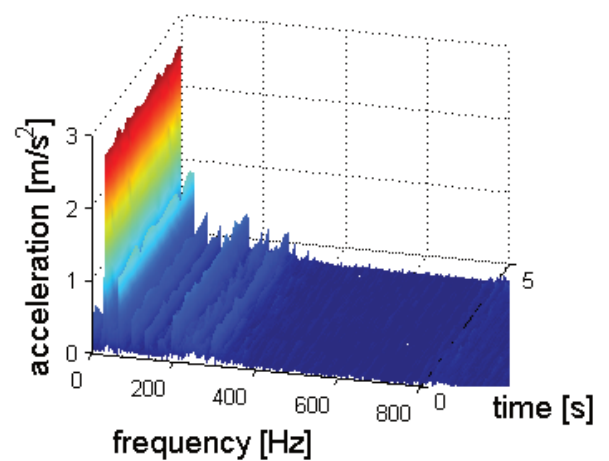

a) Motor car engine

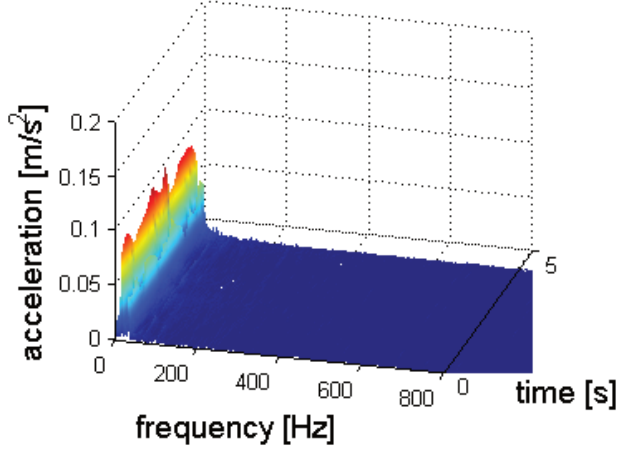

c) Front passenger seat

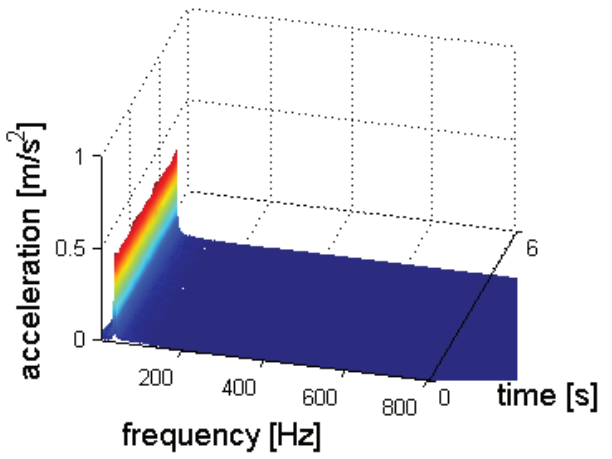

b) Front passenger floor

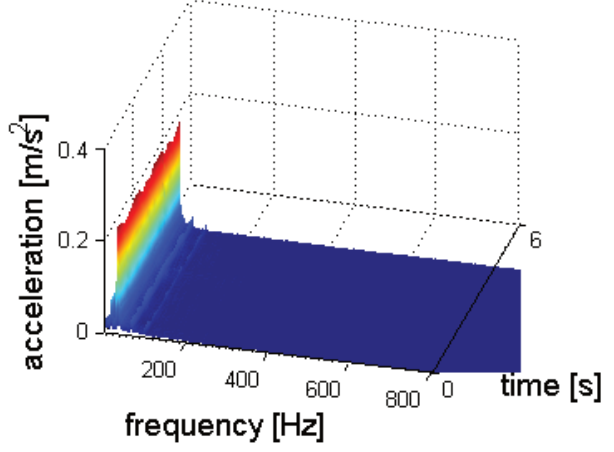

d) Right rear passenger floor

Fig. 12.27. Changes of the structure of vibration during propagation, path No. III, $Z$ axis - vertical

Propagation of the horizontal vibration shows relation between vibration and distance from the source. Thus the vibration of the floor panel in location of rear right passenger feet are much smaller than the vibration of the floor panel closer to the engine source in location of front passenger feet. The vibration registered on the front passenger seat are higher than long distance vibration of the floor panel. The vertical vibration are higher on the floor panel than on the seat. It can be assumed that suspension of the seat has better properties for absorbing the vertical vibration.

The results of the research enable analyzing the vibration propagation from engine to human body. The structures and directional distribution of the vibrations have been registered in the location where vibrations penetrate into the human organism. The vibration of floor panel and seats have got much more less higher frequencies components. Thus it can be assumed that vibration during propagation into the vehicle construction and equipment (seats) dissipate in the first step energy correlated to the higher than main harmonic frequencies. Also the whole-body vibration in location of penetration into the human organism transfer part of the vibration into the lower frequencies due to the decrease of the total energy volume. The values of the vibration of the motor engine are much higher than floor panel vibration. This proves good vibration isolation in a vehicle cab. Domination of lower frequencies in the signal registered on vehicle floor panel can be observed.

For the purpose of comparison of the vibration propagation paths into the occupants of the passenger car some estimators of the energy dissipation can be very helpful. Propositions of the vibration dissipation estimators for time and frequency domains are presented in Chapter 17. 



\section{Influence of engine rotational speed on floor panel vibration}

Considering the relation between idle gear rotational speed and engine vibration, which is described in Chapter 11, the investigation on influence of engine rotational speed on floor panel vibration should be conducted. Occupants of the means of transport are exposed on many kinds of vibration determined by different sources and driving conditions. During traffic jam when congestion increases in urban transport all passengers become more often exposed on vibration generated by the idle gear engine. During the drive there are more vibration sources which generate disruptions [43]. The chapter presents results of the research on the influence of engine rotational speed on floor panel vibration as disruptions for the passenger car occupants transferred to the human organism as whole-body vibration via feet.

\subsection{Research on vibration propagation paths from engine to car body in locations of penetrate into human body}

The scope of research included investigation featuring measurements of vibration acceleration in a three orthogonal directions in locations under the occupant's feet on the floor panel [45]. The vibration signals, being an effect of a force excitation, obtained by working process of engine, were recorded. The vibration in the vehicle structure were excited by engine working on idle gear. It enables to consider only the vibration generated by vehicle mechanism without vibration from road roughness during the drive. The experiment was conducted under laboratory conditions to ensure reproducibility of results.

The research was conducted on the car vehicle which was placed on the special test racks. It allows eliminate the road roughens impact on the suspension and in result to car-body.

\subsection{Research results}

The established scope of research enables to observe changes of the floor panel vibration for idle gear rotational speed increase. The idle gear rotational speed founded during research were: $750 \mathrm{rpm}, 1500 \mathrm{rpm}, 2000 \mathrm{rpm}$ and $3000 \mathrm{rpm}$.

The three orthogonal axes were analyzed separately. The comparison of the acceleration of vibration signals allows determine which directions of the vibration propagation is parent and how the vibration is changing under engine rotational speed increase.

\subsubsection{Influence of engine rotational speed on floor panel vibration}

The comparison of vibration registered under feet of vehicle occupants for different engine rotational speed are collected in Figs. 13.1-13.12. 

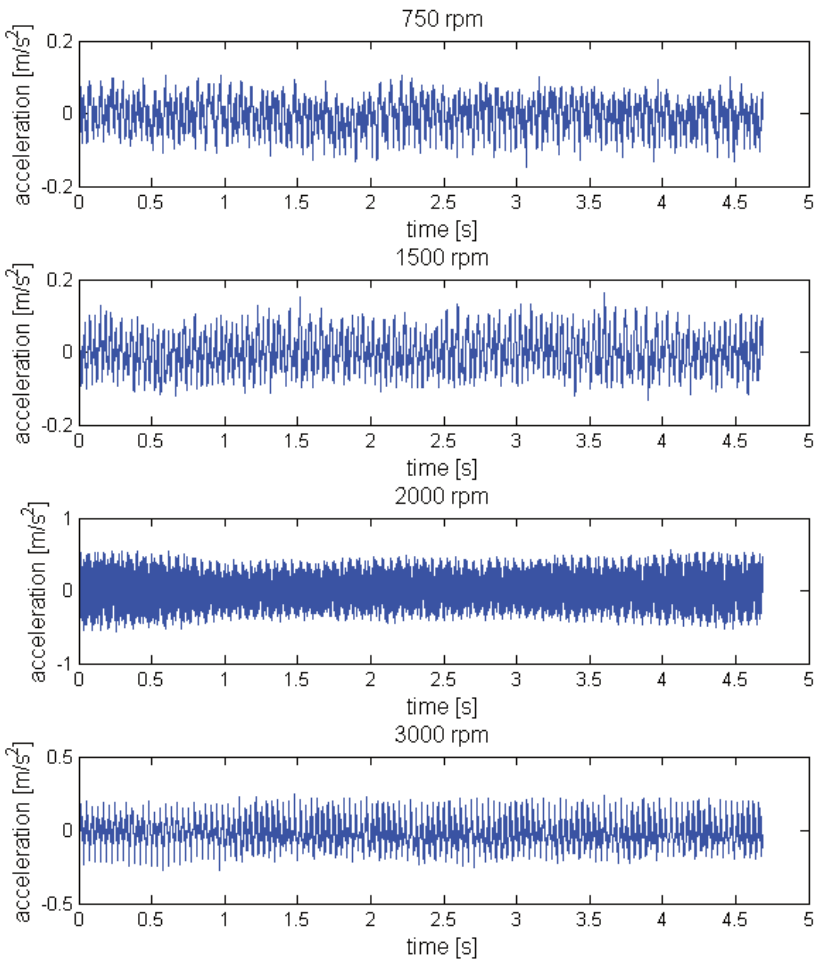

Fig. 13.1. Influence of engine rotational speed on longitudinal vibration of floor panel under driver's feet
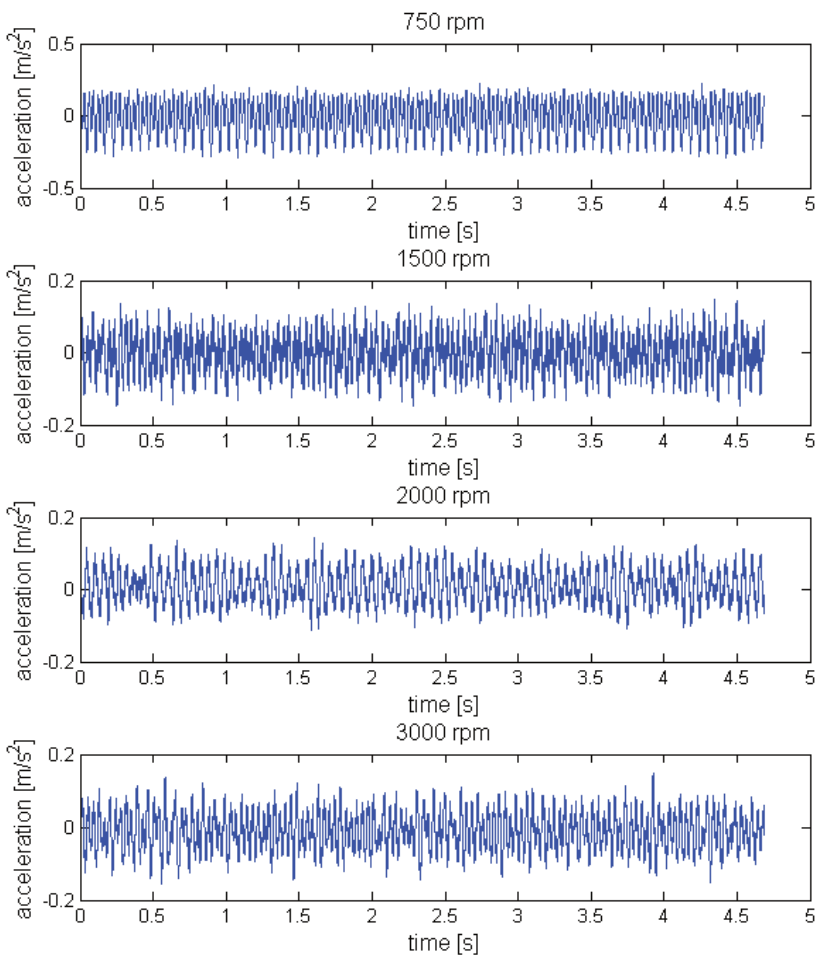

Fig. 13.2. Influence of engine rotational speed on longitudinal vibration of floor panel under front passenger's feet 

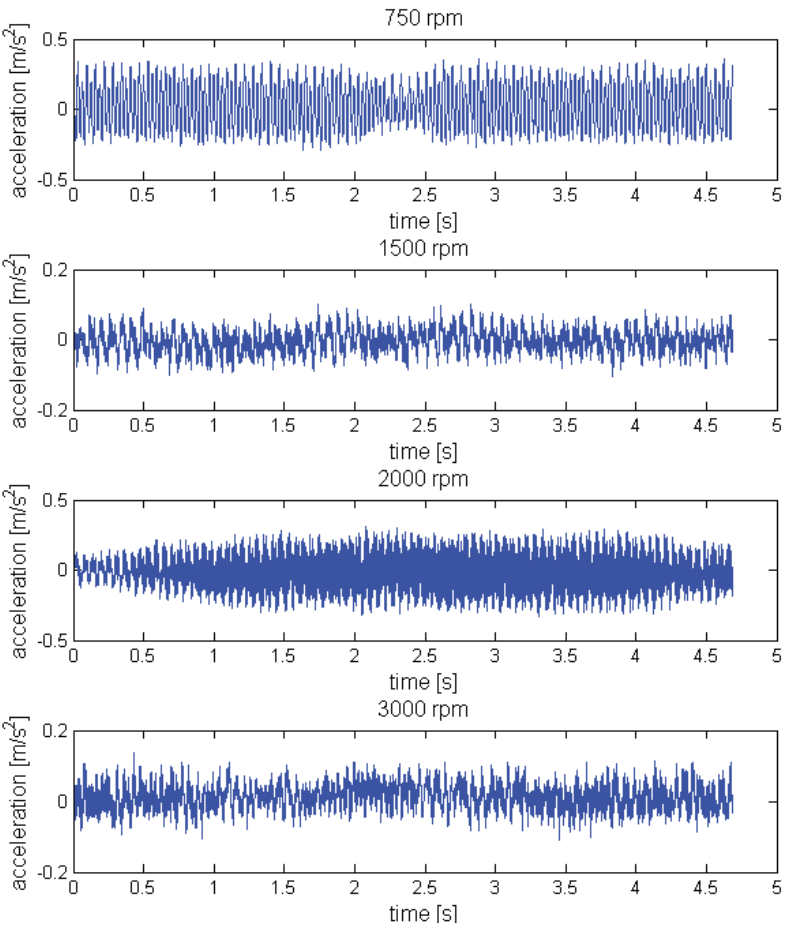

Fig. 13.3. Influence of engine rotational speed on longitudinal vibration of floor panel under rear left passenger's feet
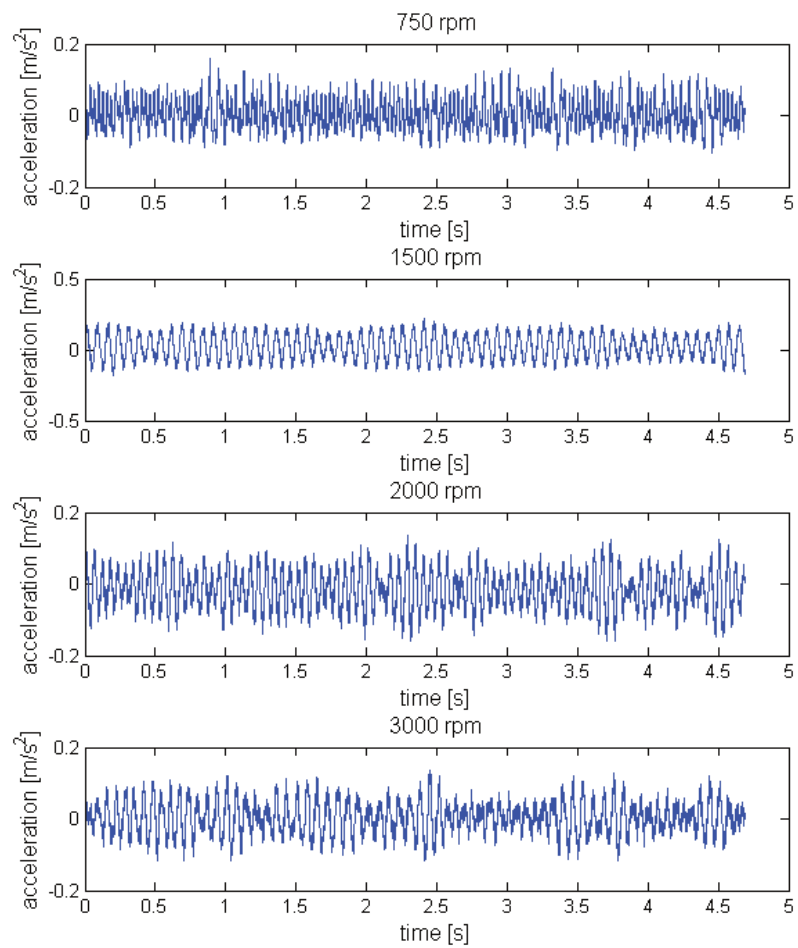

Fig. 13.4. Influence of engine rotational speed on longitudinal vibration of floor panel under rear right passenger's feet 

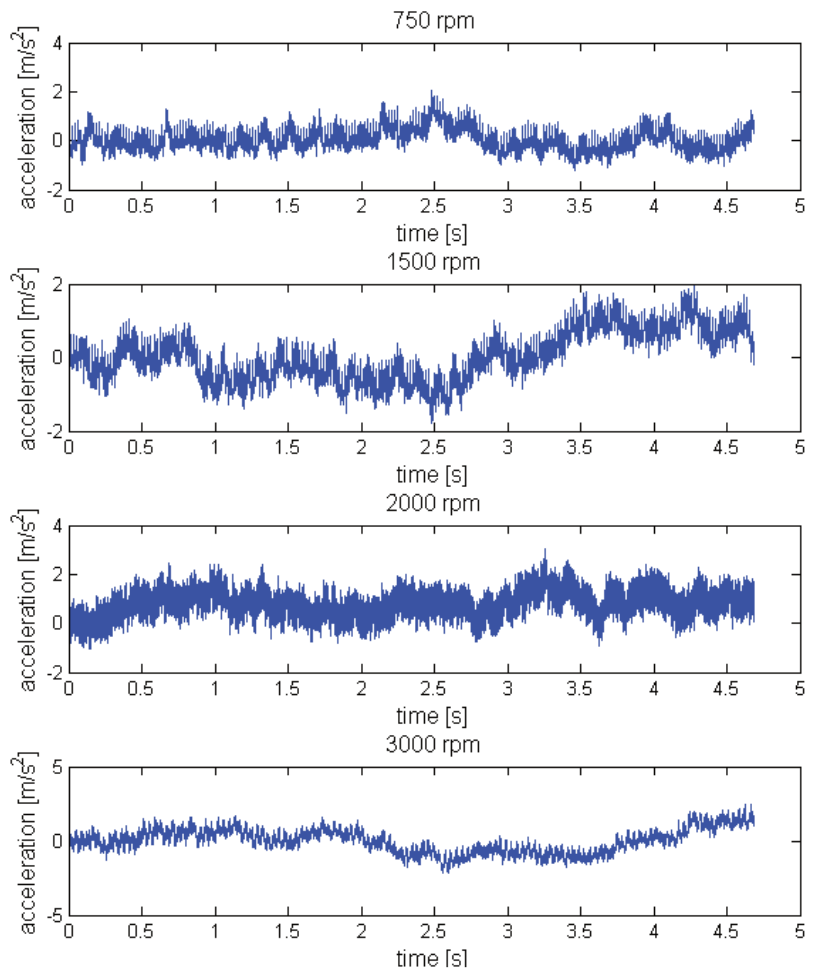

Fig. 13.5. Influence of engine rotational speed on lateral vibration of floor panel under driver's feet
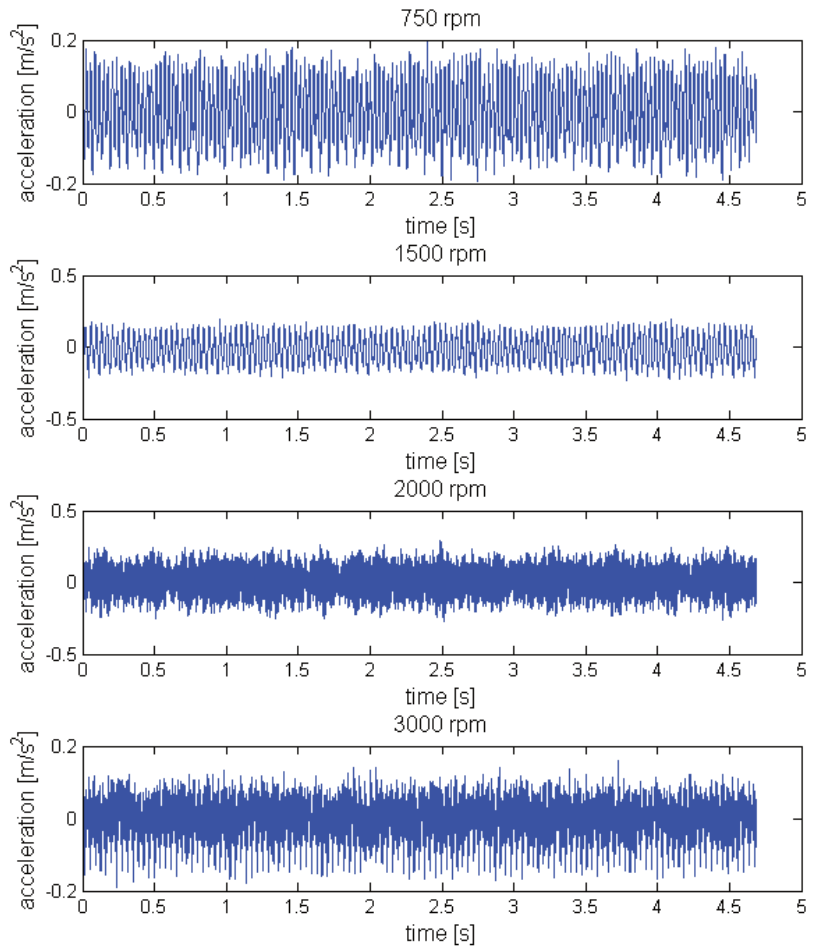

Fig. 13.6. Influence of engine rotational speed on lateral vibration of floor panel under front passenger's feet 

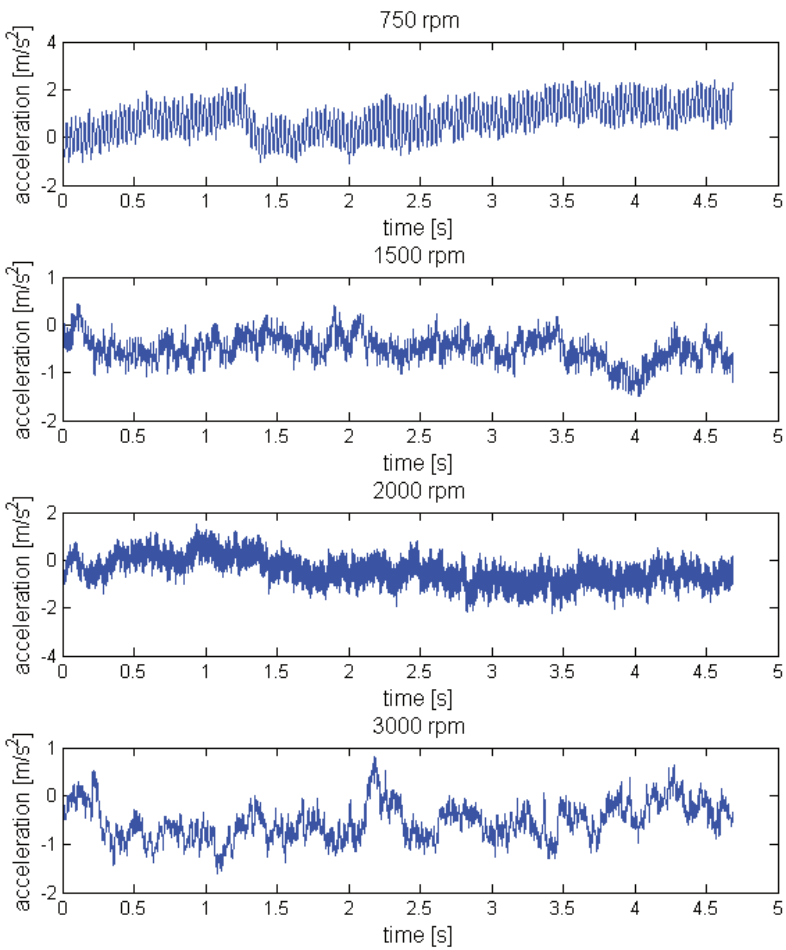

Fig. 13.7. Influence of engine rotational speed on lateral vibration of floor panel under rear left passenger's feet
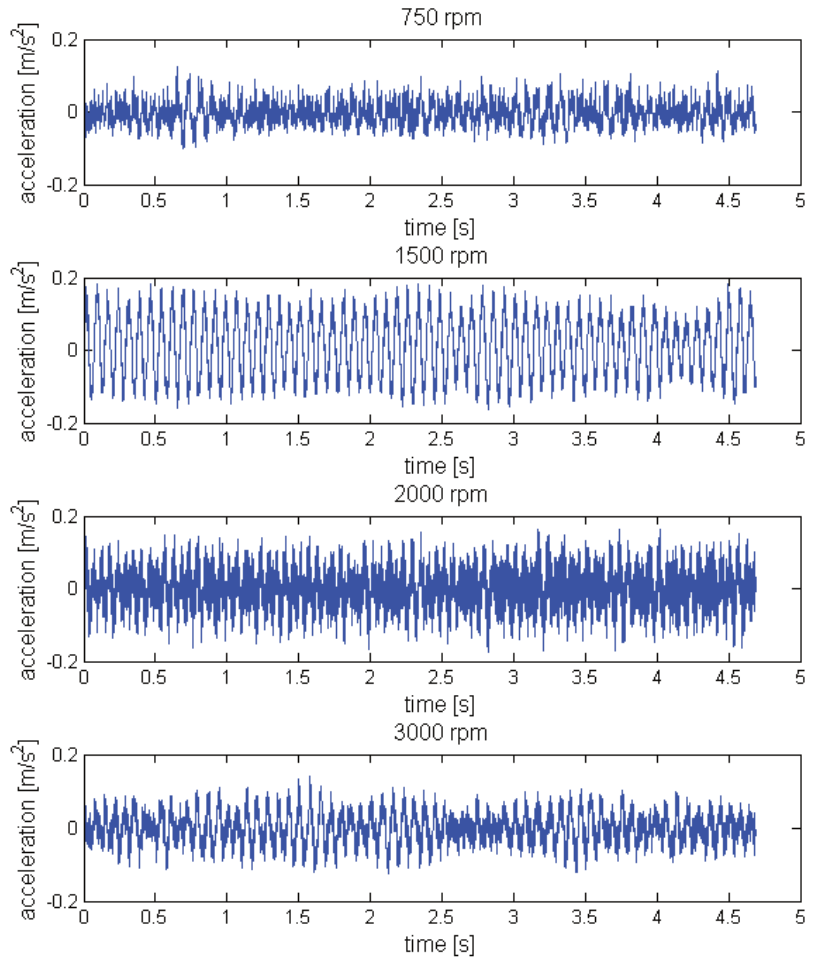

Fig. 13.8. Influence of engine rotational speed on lateral vibration of floor panel under rear right passenger's feet 

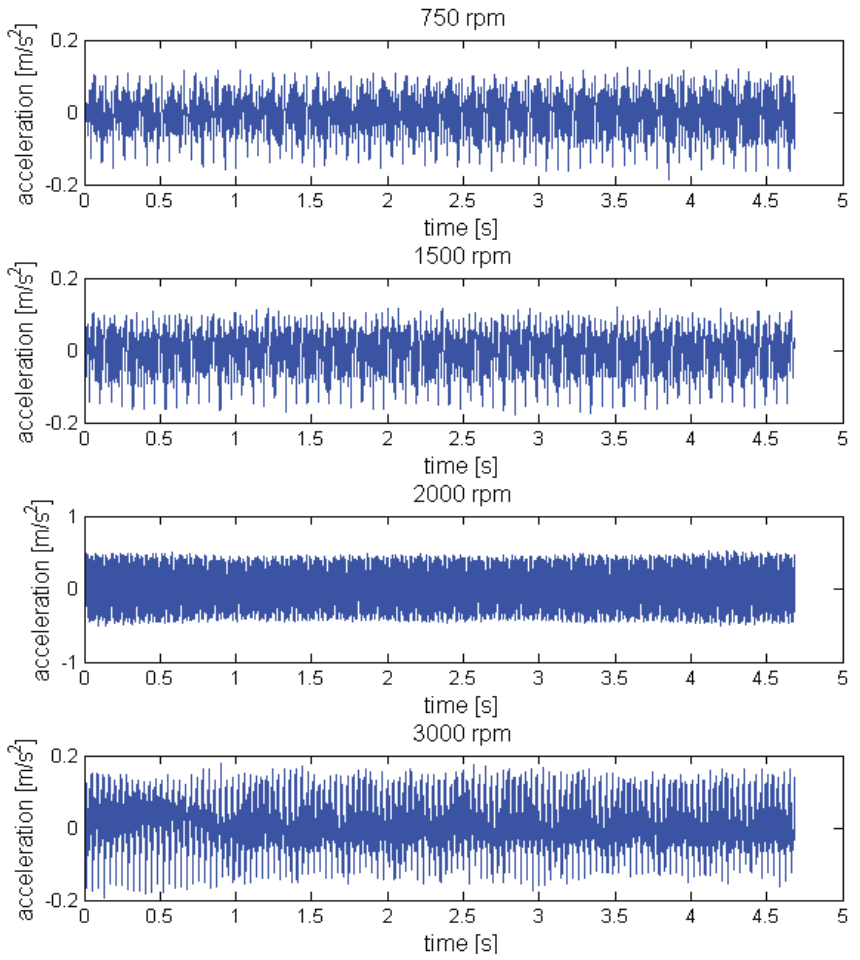

Fig. 13.9. Influence of engine rotational speed on vertical vibration of floor panel under driver's feet
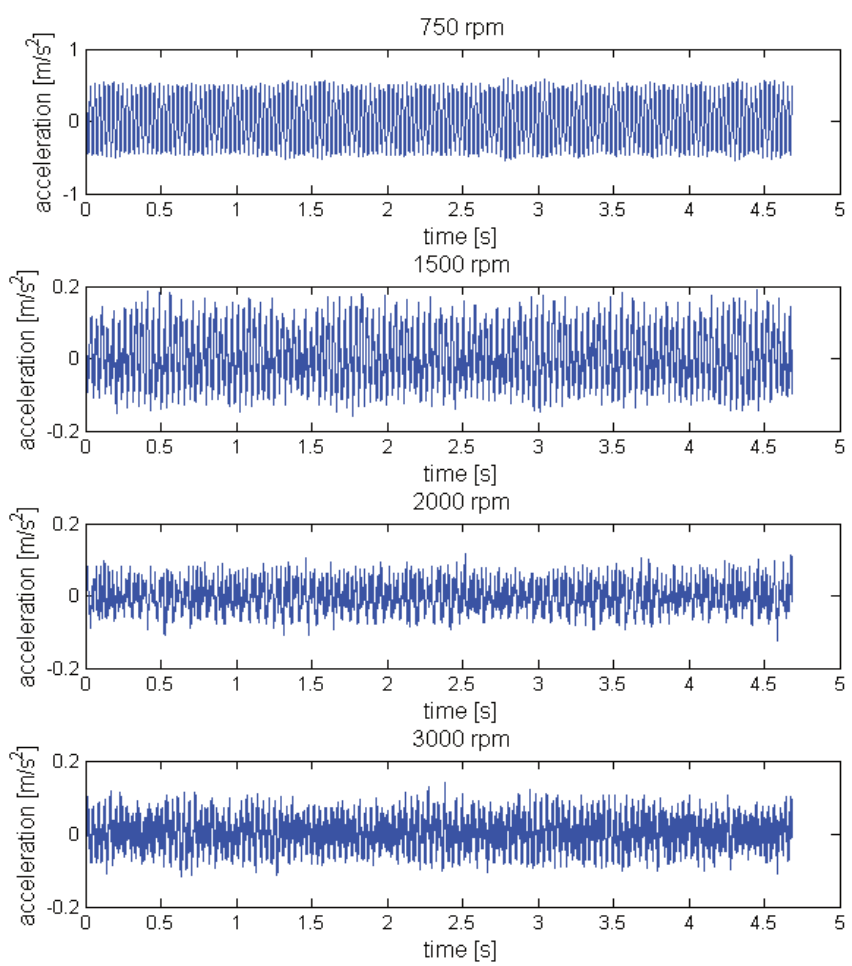

Fig. 13.10. Influence of engine rotational speed on vertical vibration of floor panel under front passenger's feet 

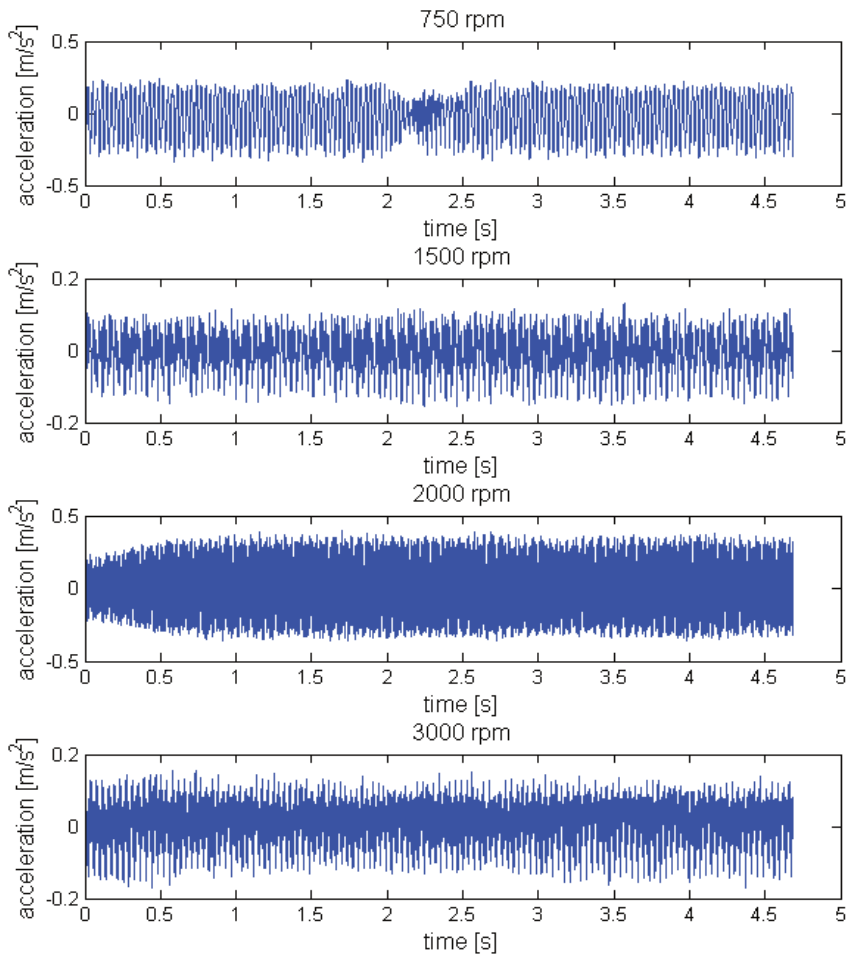

Fig. 13.11. Influence of engine rotational speed on vertical vibration of floor panel under rear left passenger's feet
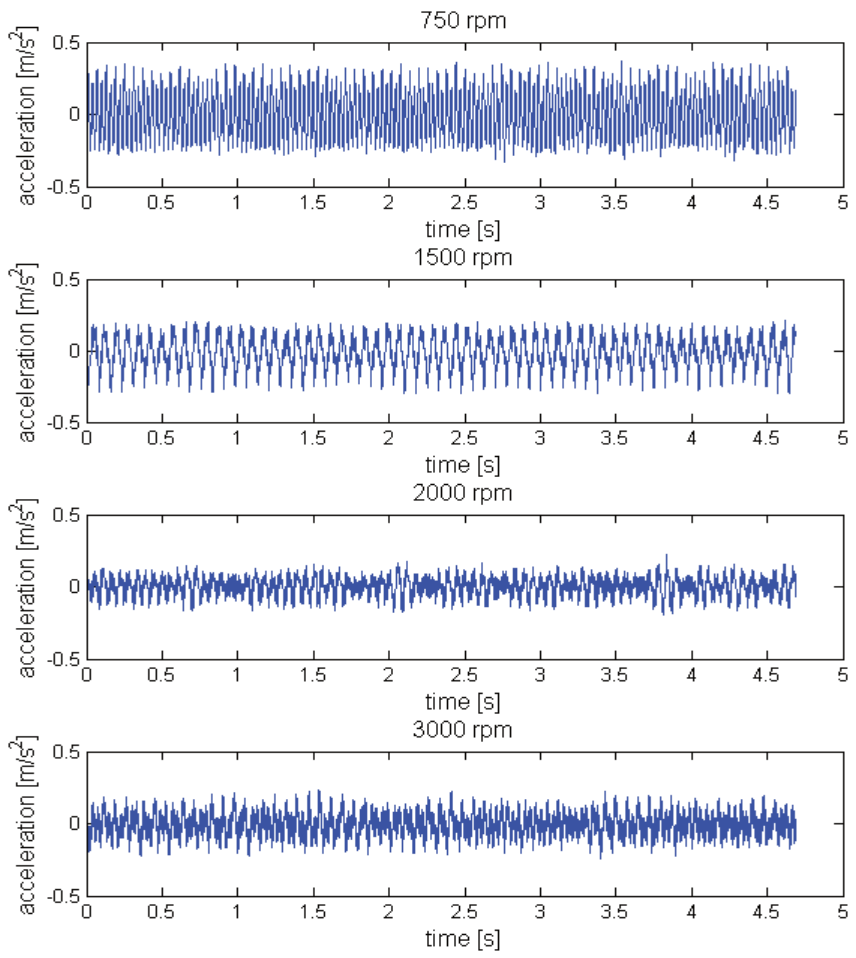

Fig. 13.12. Influence of engine rotational speed on vertical vibration of floor panel under rear right passenger's feet 

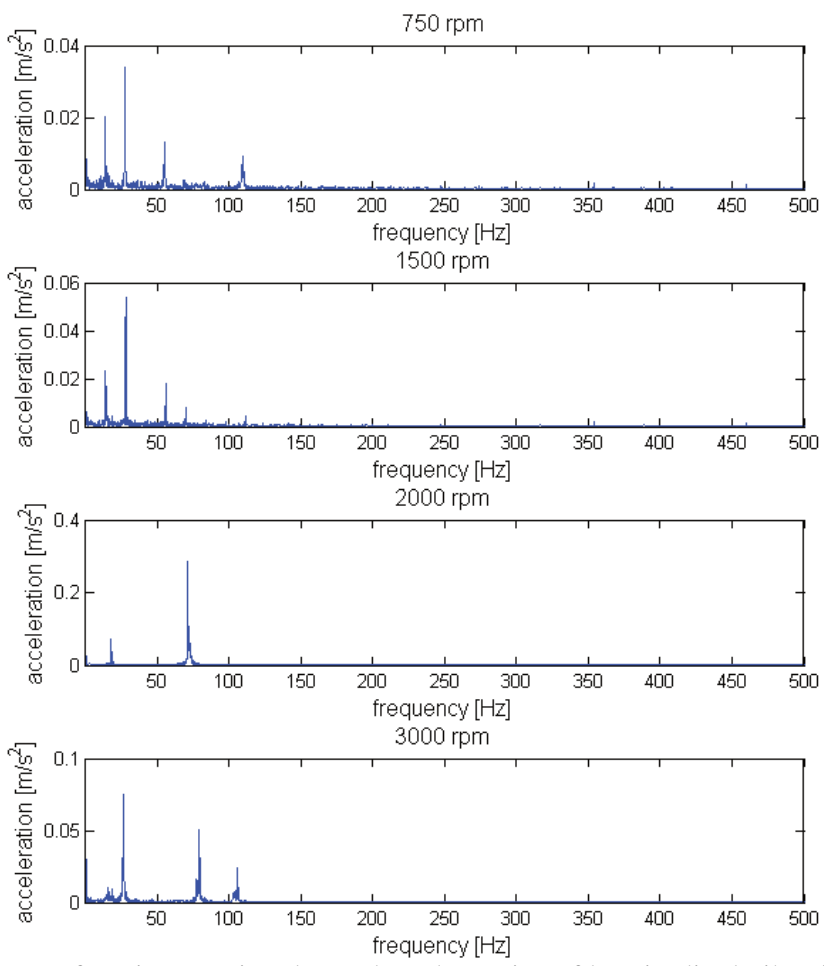

Fig. 13.13. Influence of engine rotational speed on dynamics of longitudinal vibration of floor panel under driver's feet
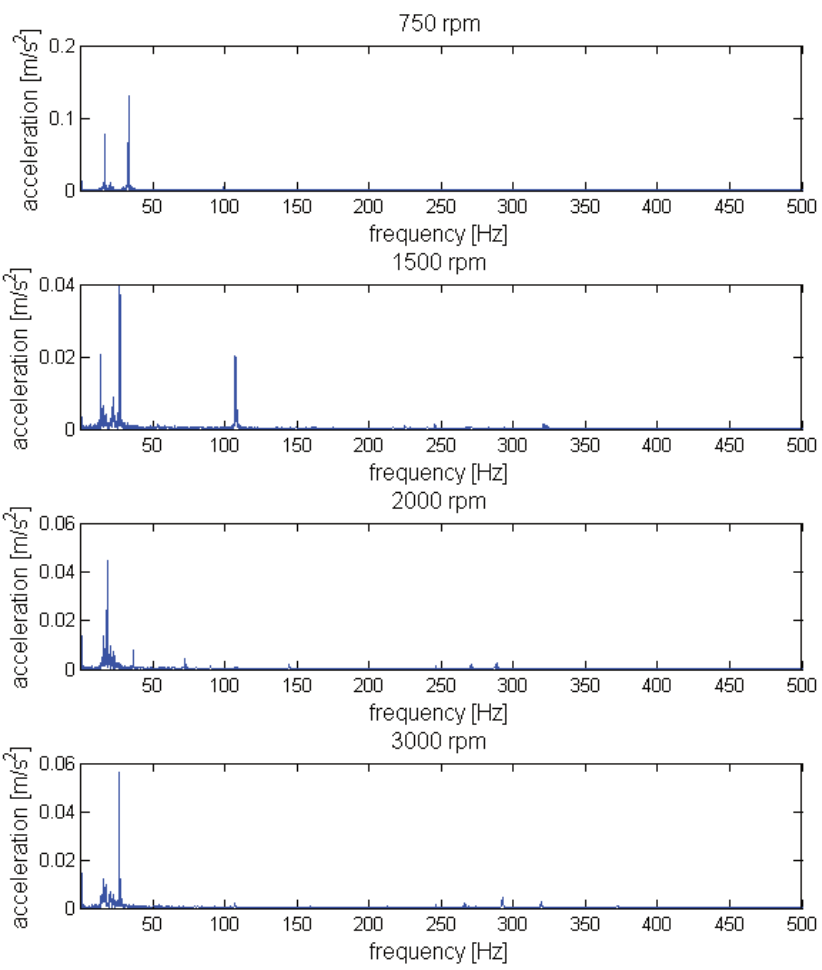

Fig. 13.14. Influence of engine rotational speed on dynamics of longitudinal vibration of floor panel under front passenger's feet 

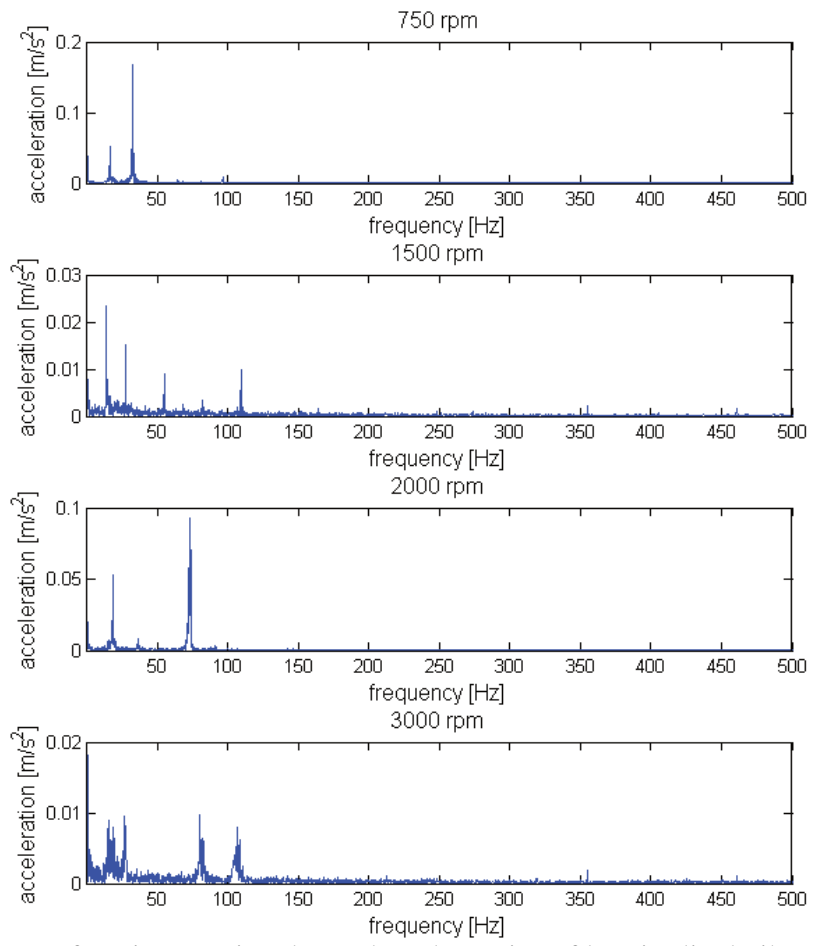

Fig. 13.15. Influence of engine rotational speed on dynamics of longitudinal vibration of floor panel under rear left passenger's feet
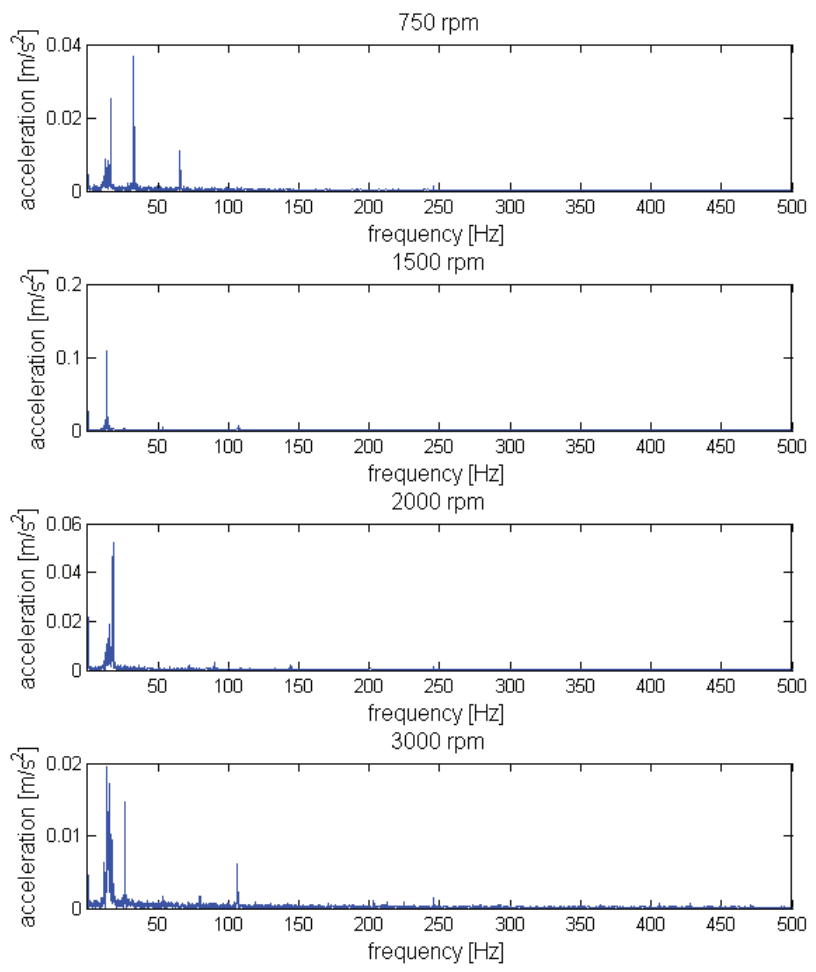

Fig. 13.16. Influence of engine rotational speed on dynamics of longitudinal vibration of floor panel under rear right passenger's feet 

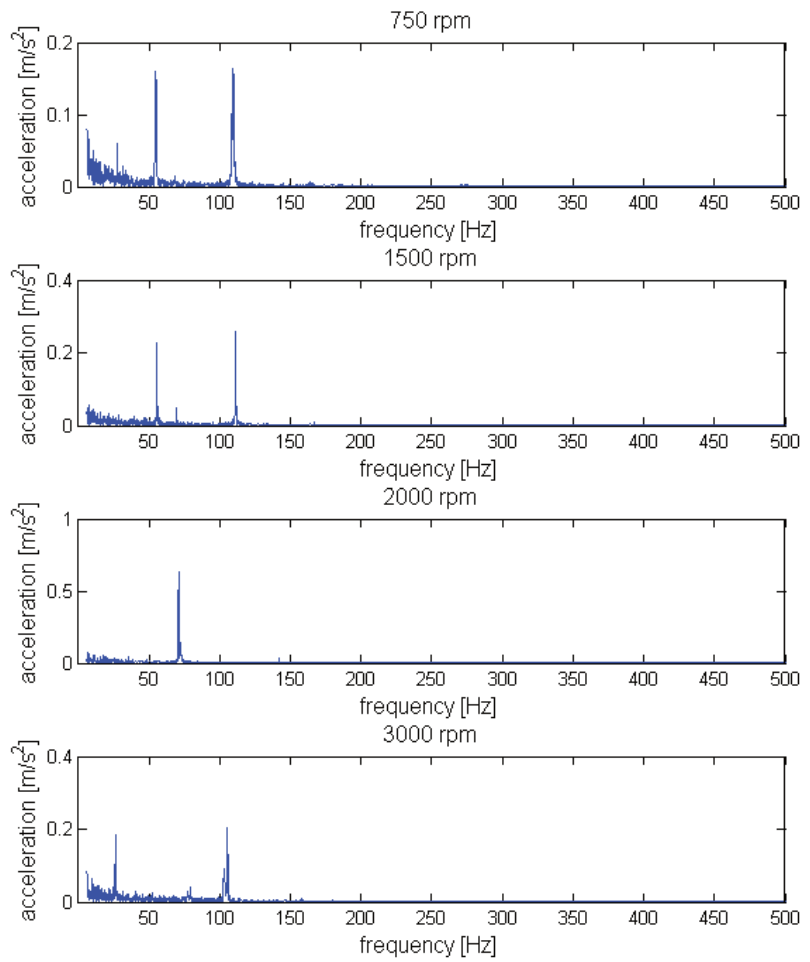

Fig. 13.17. Influence of engine rotational speed on dynamics of lateral vibration of floor panel under driver's feet
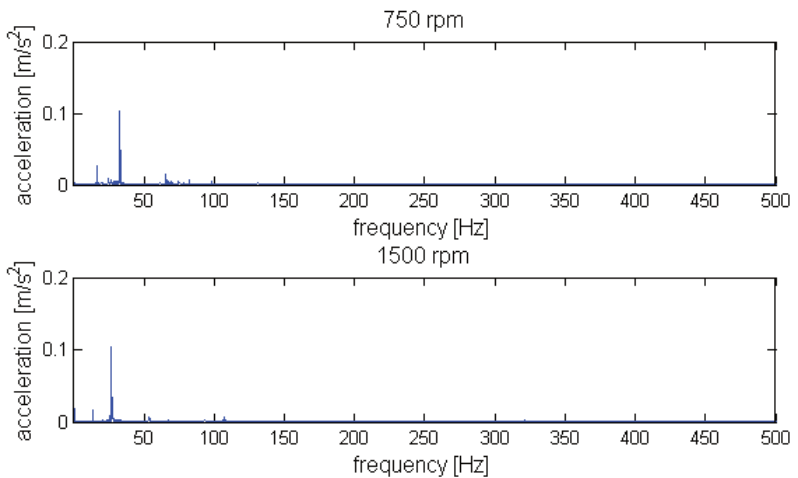

$2000 \mathrm{rpm}$
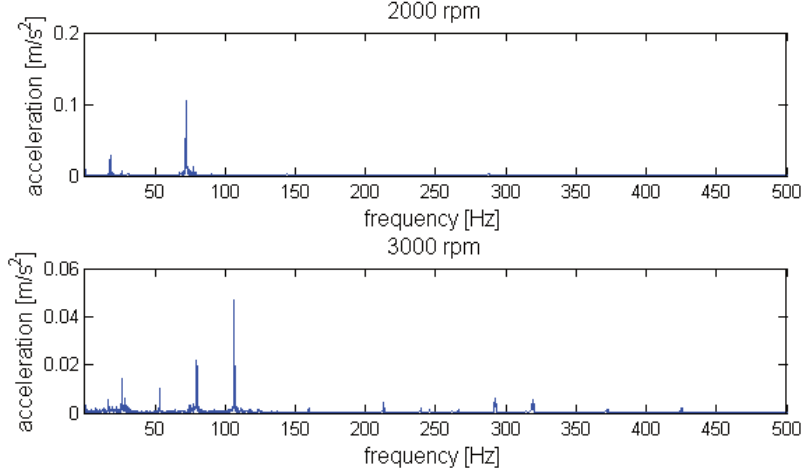

Fig. 13.18. Influence of engine rotational speed on dynamics of lateral vibration of floor panel under front passenger's feet 

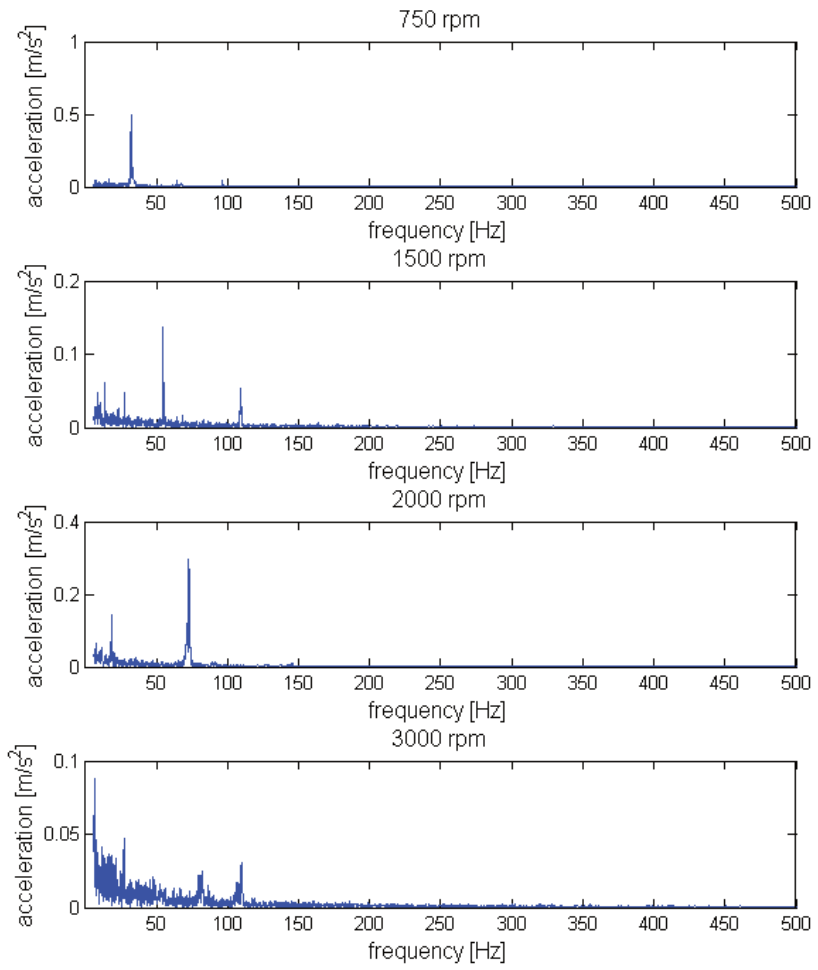

Fig. 13.19. Influence of engine rotational speed on dynamics of lateral vibration of floor panel under rear left passenger's feet
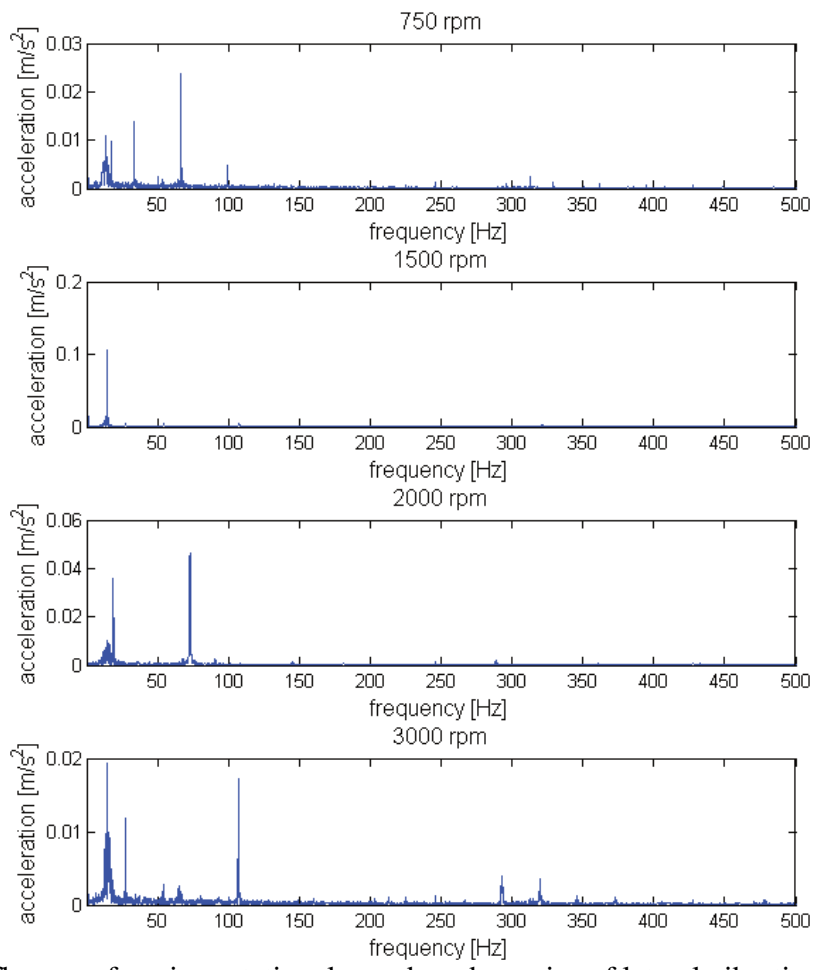

Fig. 13.20. Influence of engine rotational speed on dynamics of lateral vibration of floor panel under rear right passenger's feet 

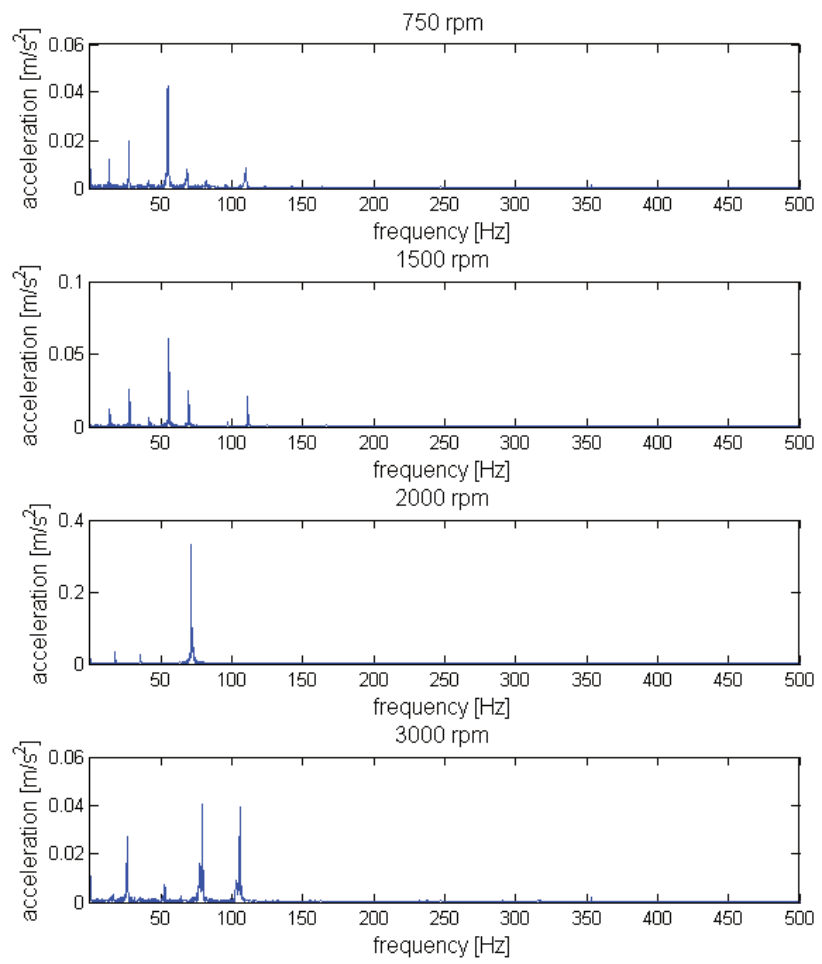

Fig. 13.21. Influence of engine rotational speed on dynamics of vertical vibration of floor panel under driver's feet
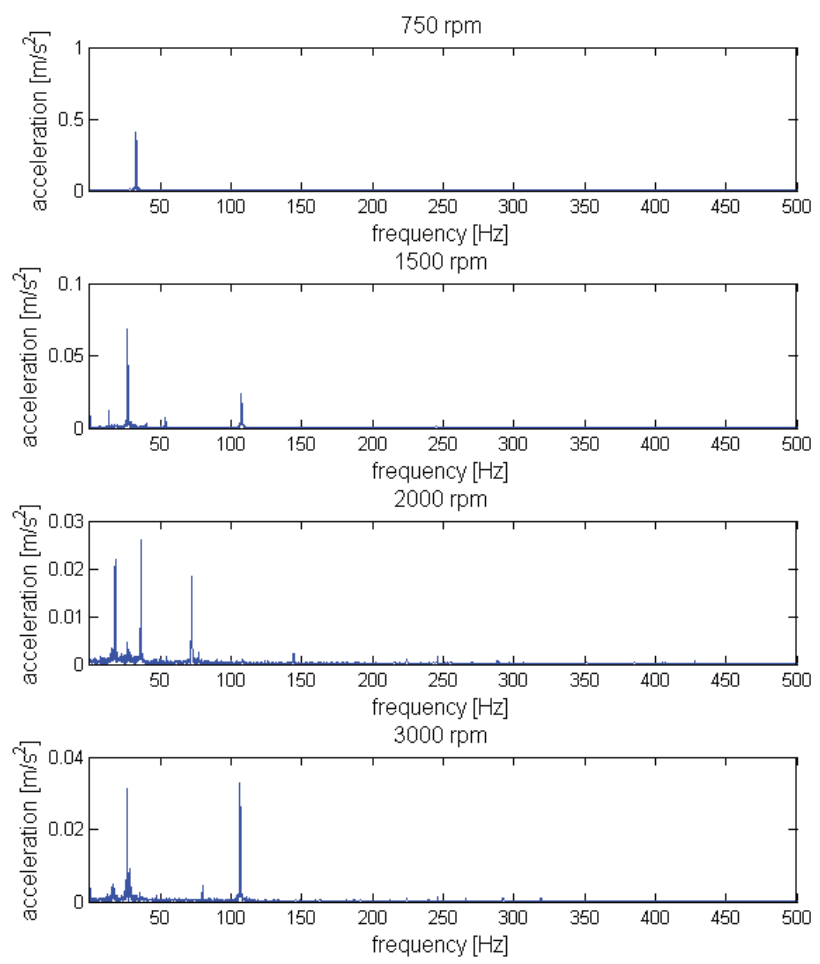

Fig. 13.22. Influence of engine rotational speed on dynamics of vertical vibration of floor panel under front passenger's feet 

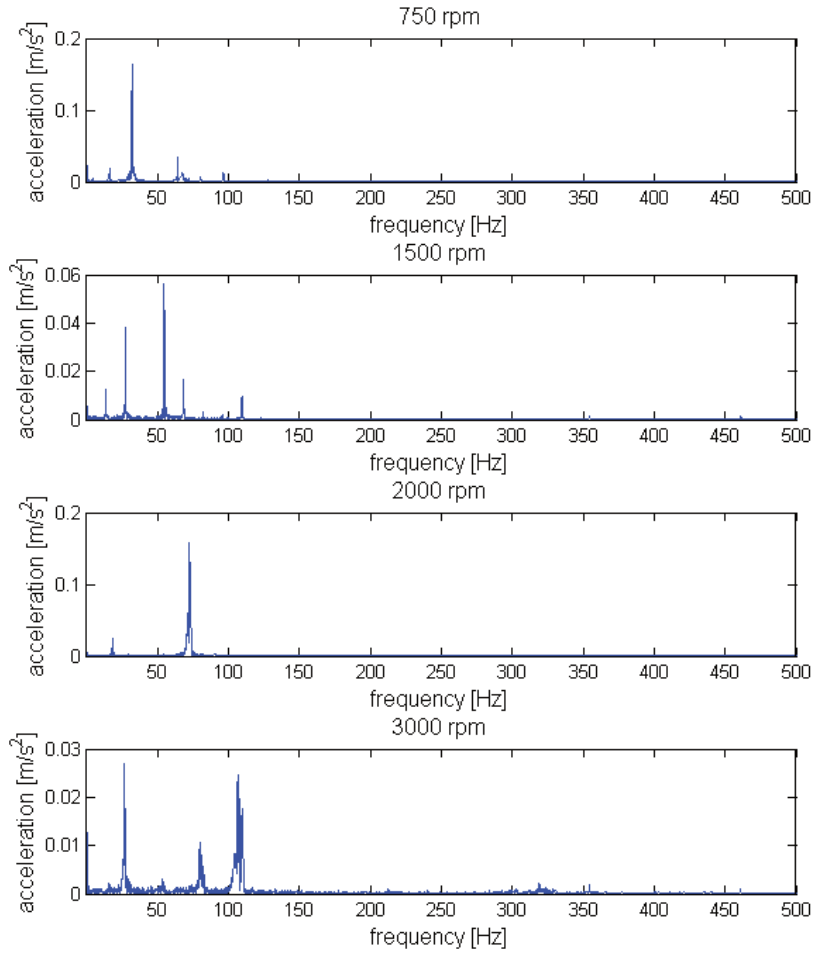

Fig. 13.23. Influence of engine rotational speed on dynamics of vertical vibration of floor panel under rear left passenger's feet
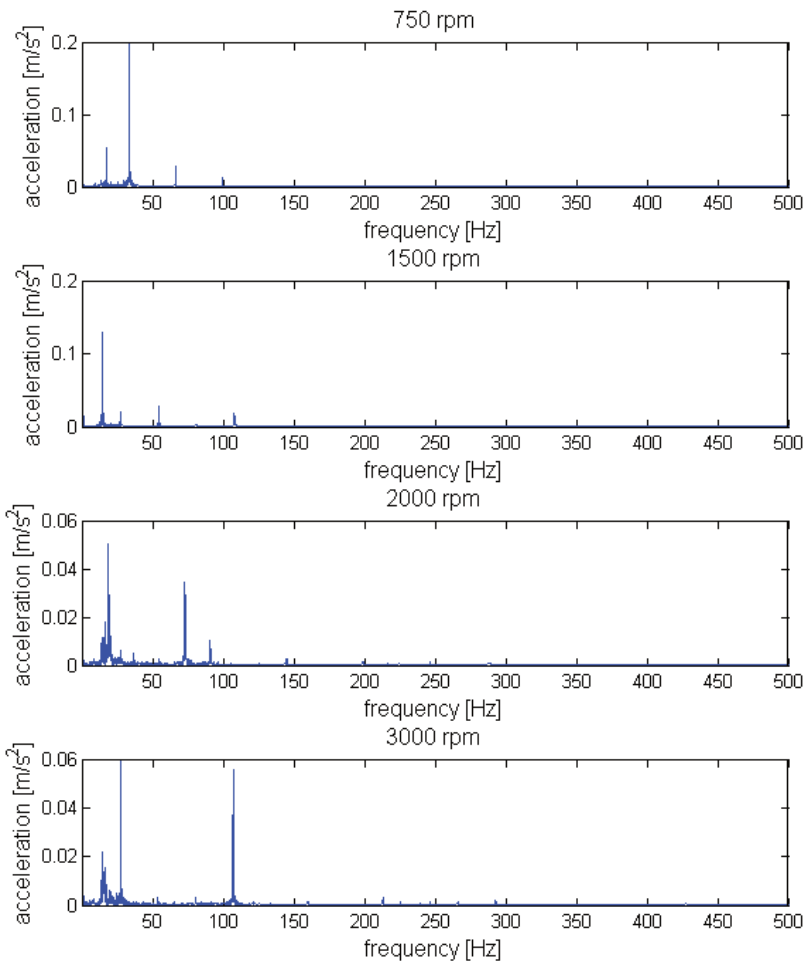

Fig. 13.24. Influence of engine rotational speed on dynamics of vertical vibration of floor panel under rear right passenger's feet 


\subsubsection{Influence of engine rotational speed on dynamics of floor panel vibration}

To investigate this phenomena the spectrum of the recorded signals were determined. It is possible to observe changes of the vibration's dynamics for the different rpm transferred in three orthogonal axes (Figs. 13.13-13.24). The frequency components correlated to rpm were indicated similar to results presented in Fig 11.11. For proper analysis of the vibration amplitude the scale of the $Y$ axis have to be taken into consideration.

\subsubsection{Influence of engine rotational speed on structure of floor panel vibration}

To analyze the influence of engine rotational speed on structure of vibration the timefrequency representation of the signal were determined. For this sake the STFT transformation was calculated. The differences in vibration structure were compared and collected in Figs. 13.25-13.36.

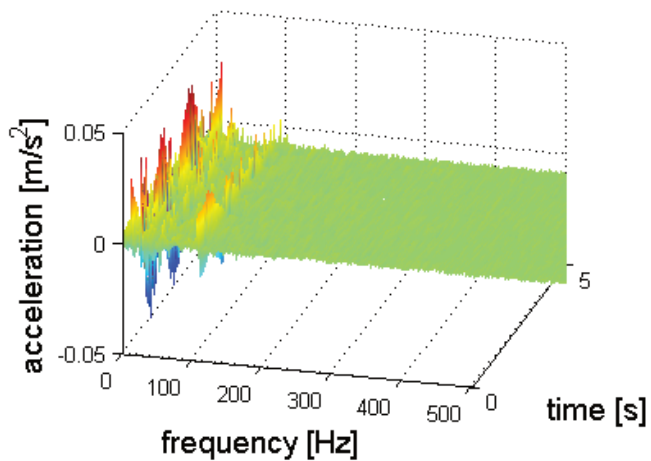

a) $750 \mathrm{rpm}$

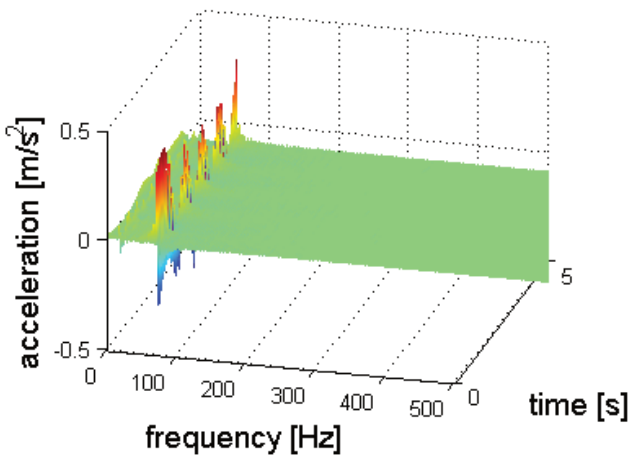

c) $2000 \mathrm{rpm}$

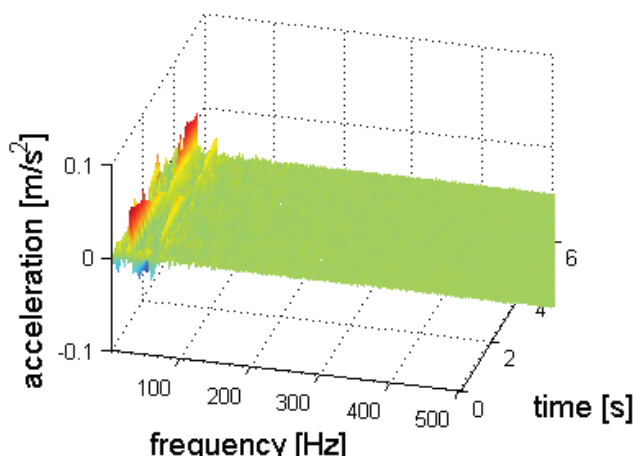

b) $1500 \mathrm{rpm}$

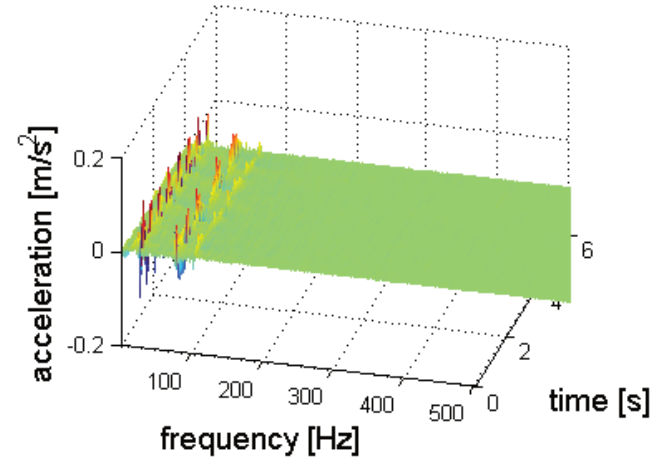

d) $3000 \mathrm{rpm}$

Fig. 13.25. Changesof structure of longitudinal vibration of floor panel under driver's feet for different engine rotational speed (time window $0.25 \mathrm{~s}$, resoliution $0.4884 \mathrm{~Hz}$ ) 


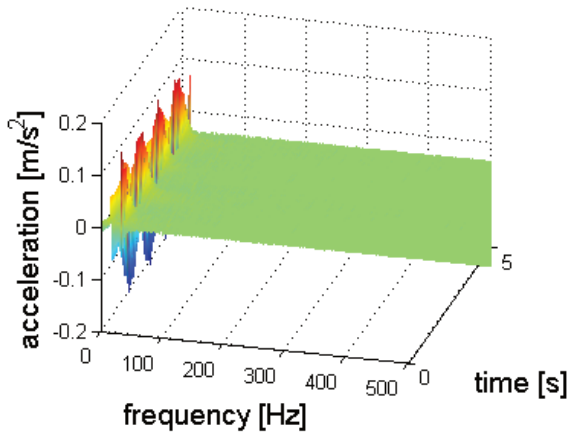

a) $750 \mathrm{rpm}$

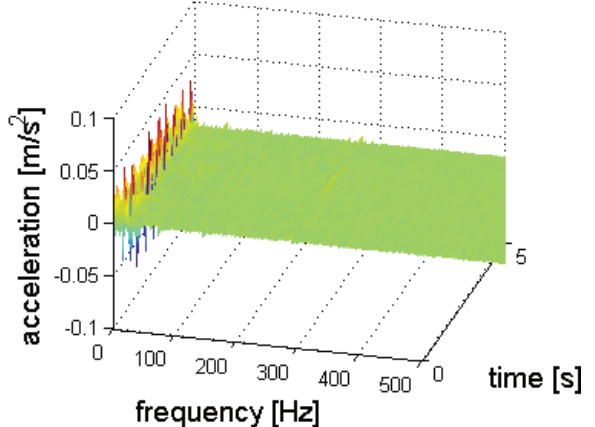

c) $2000 \mathrm{rpm}$

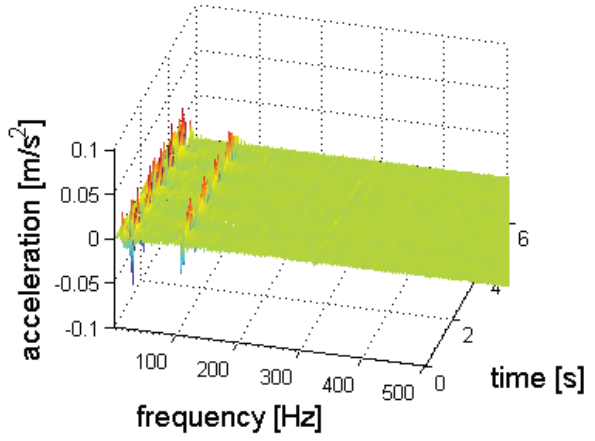

b) $1500 \mathrm{rpm}$

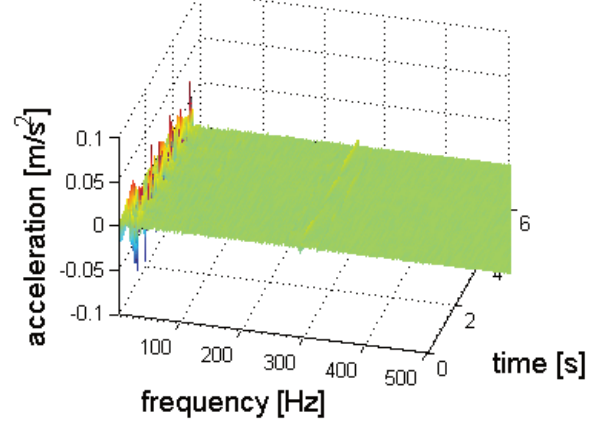

d) $3000 \mathrm{rpm}$

Fig. 13.26. Changesof structure of longitudinal vibration of floor panel under front passenger's feet for different engine rotational speed (time window $0.25 \mathrm{~s}$, resoliution $0.4884 \mathrm{~Hz}$ )

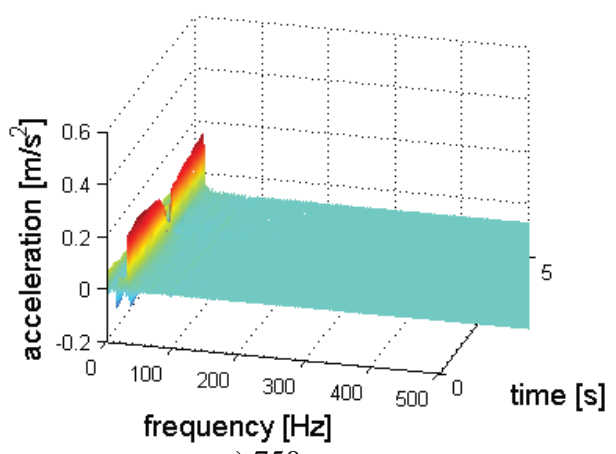

a) $750 \mathrm{rpm}$

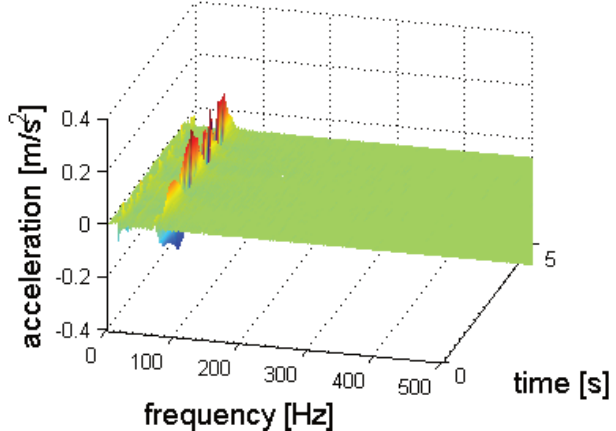

c) $2000 \mathrm{rpm}$

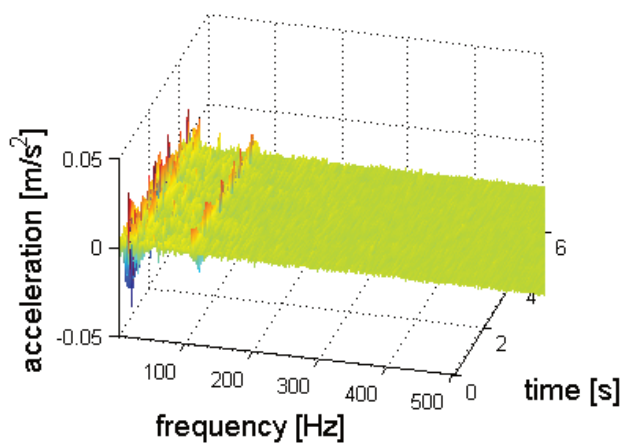

b) $1500 \mathrm{rpm}$

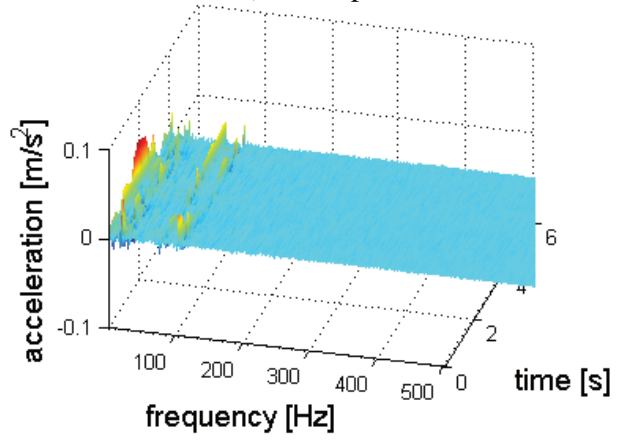

d) $3000 \mathrm{rpm}$

Fig. 13.27. Changesof structure of longitudinalvibration of floor panel under rear left passenger's feet for different engine rotational speed (time window $0.25 \mathrm{~s}$, resoliution $0.4884 \mathrm{~Hz}$ ) 


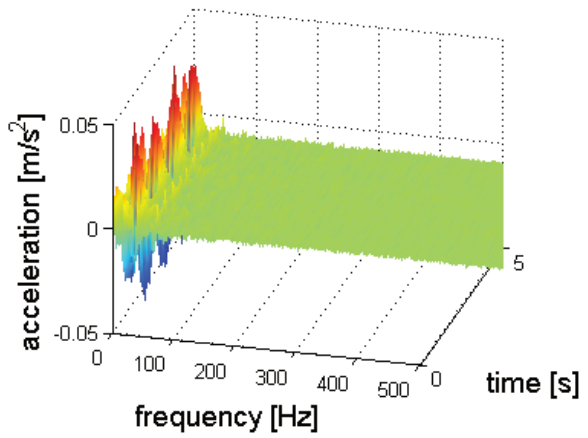

a) $750 \mathrm{rpm}$

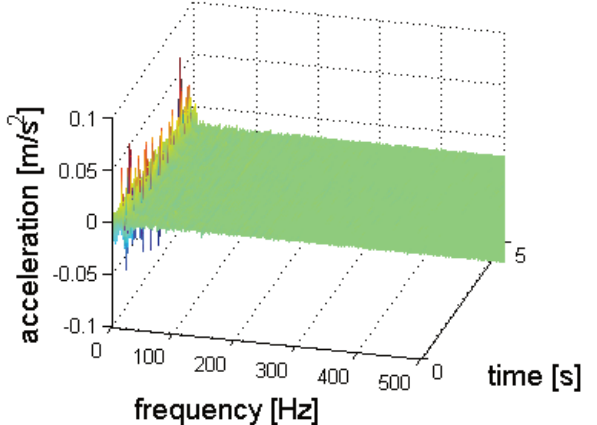

c) $2000 \mathrm{rpm}$

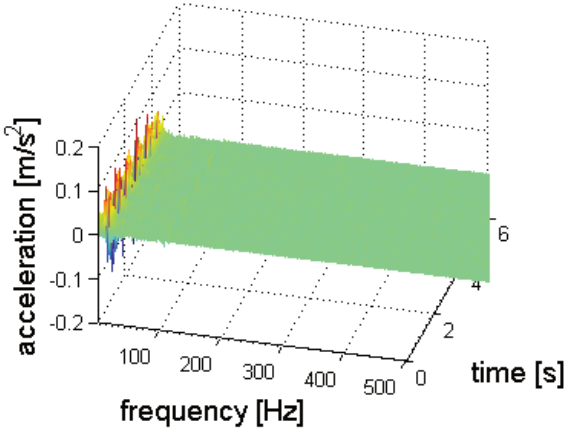

b) $1500 \mathrm{rpm}$

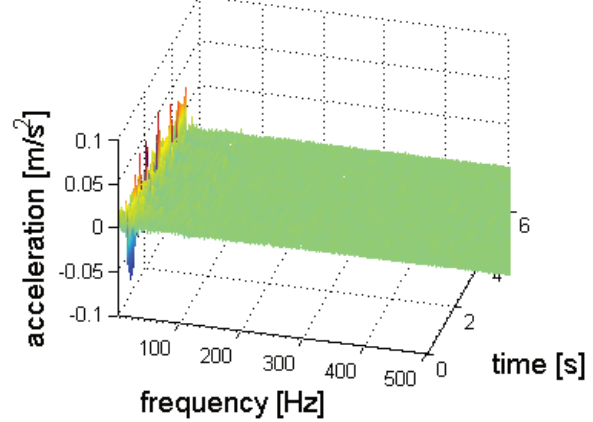

d) $3000 \mathrm{rpm}$

Fig. 13.28. Changesof structure of longitudinalvibration of floor panel under rear right passenger's feet for different engine rotational speed (time window $0.25 \mathrm{~s}$, resoliution $0.4884 \mathrm{~Hz}$ )

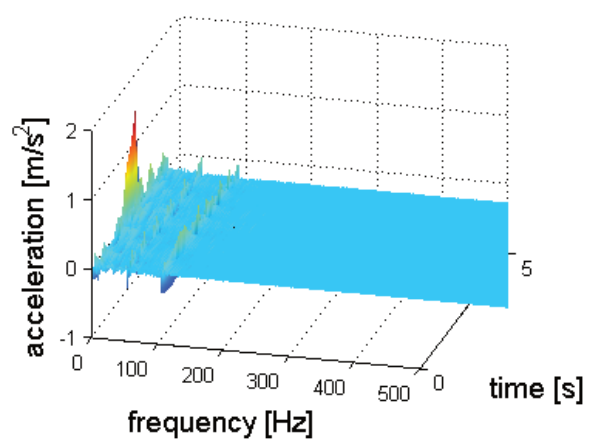

a) $750 \mathrm{rpm}$

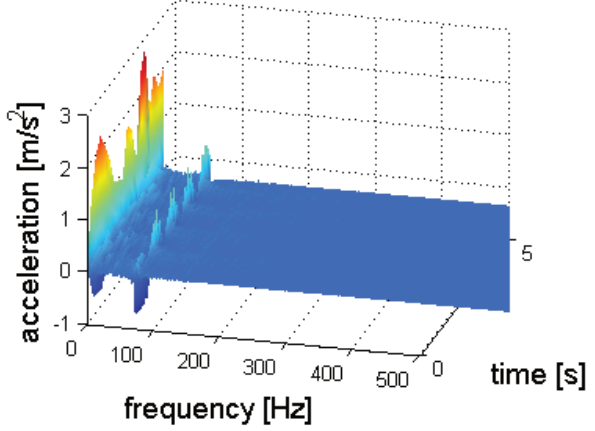

c) $2000 \mathrm{rpm}$

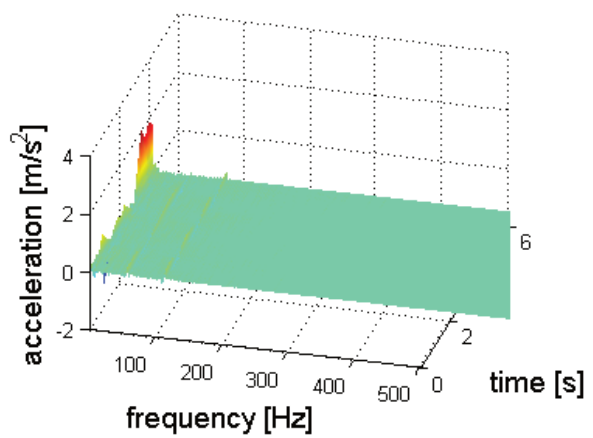

b) $1500 \mathrm{rpm}$

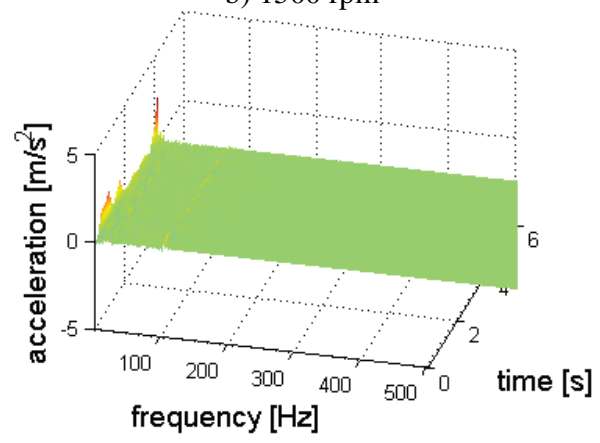

d) $3000 \mathrm{rpm}$

Fig. 13.29. Changesof structure of lateral vibration of floor panel under driver's feet for different engine rotational speed (time window $0.25 \mathrm{~s}$, resoliution $0.4884 \mathrm{~Hz}$ ) 


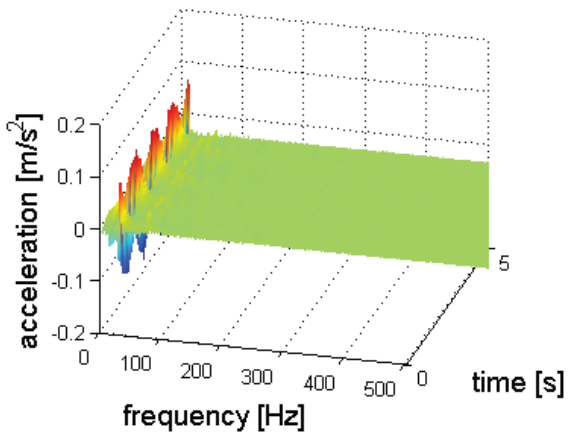

a) $750 \mathrm{rpm}$

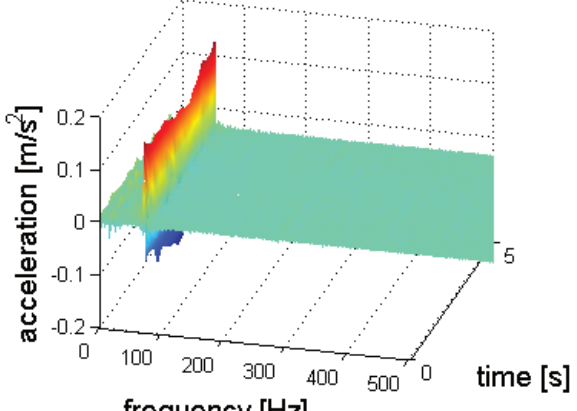

frequency $[\mathrm{Hz}]$

c) $2000 \mathrm{rpm}$

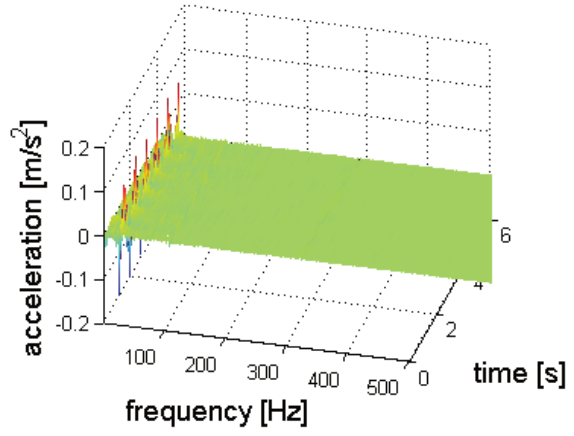

b) $1500 \mathrm{rpm}$

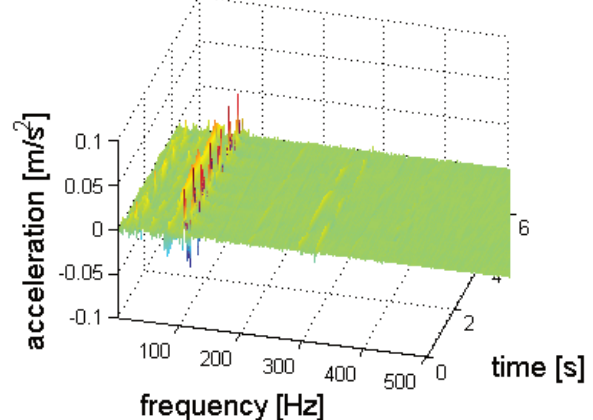

d) $3000 \mathrm{rpm}$

Fig. 13.30. Changesof structure of lateral vibration of floor panel under front passenger's feet for different engine rotational speed (time window $0.25 \mathrm{~s}$, resoliution $0.4884 \mathrm{~Hz}$ )

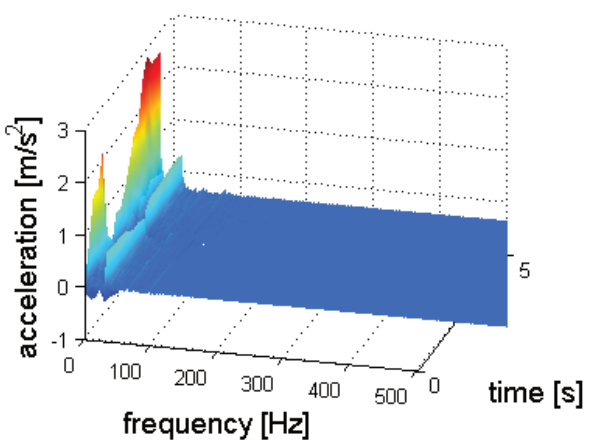

a) $750 \mathrm{rpm}$

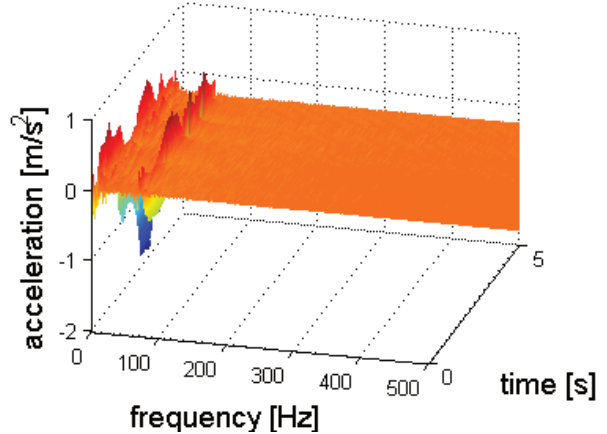

c) $2000 \mathrm{rpm}$

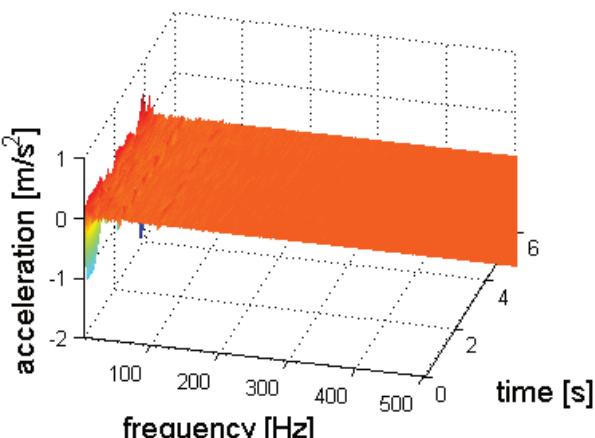

b) $1500 \mathrm{rpm}$

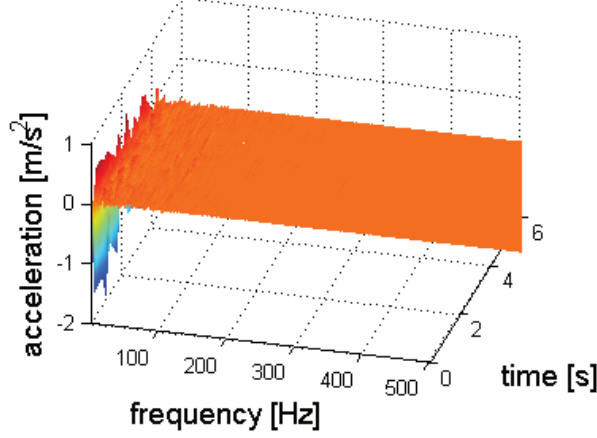

d) $3000 \mathrm{rpm}$

Fig. 13.31. Changesof structure of lateral vibration of floor panel under rear left passenger's feet for different engine rotational speed (time window $0.25 \mathrm{~s}$, resoliution $0.4884 \mathrm{~Hz}$ ) 


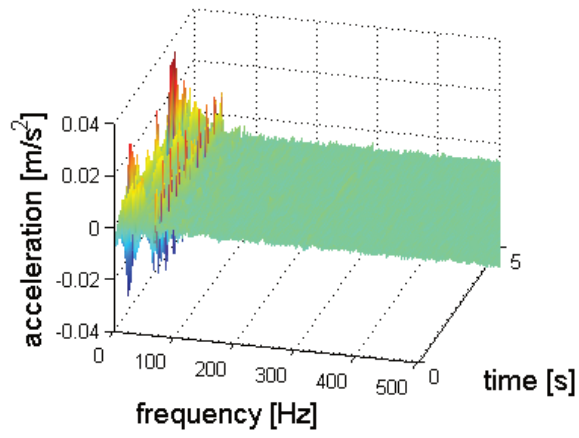

a) $750 \mathrm{rpm}$

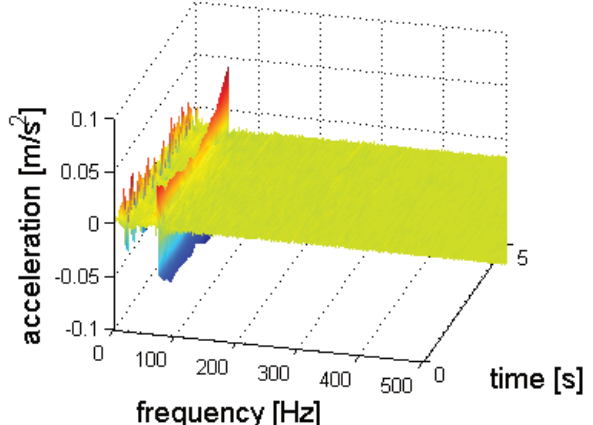

c) $2000 \mathrm{rpm}$

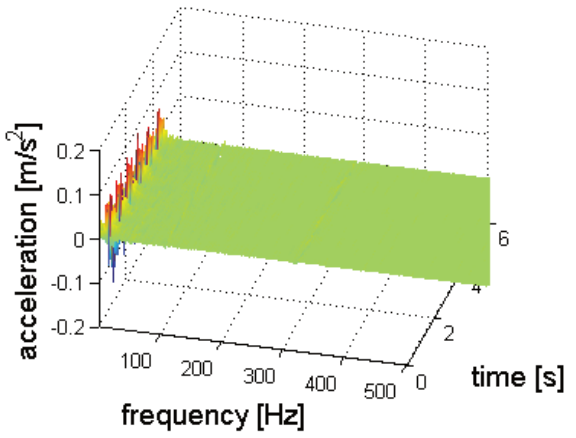

b) $1500 \mathrm{rpm}$

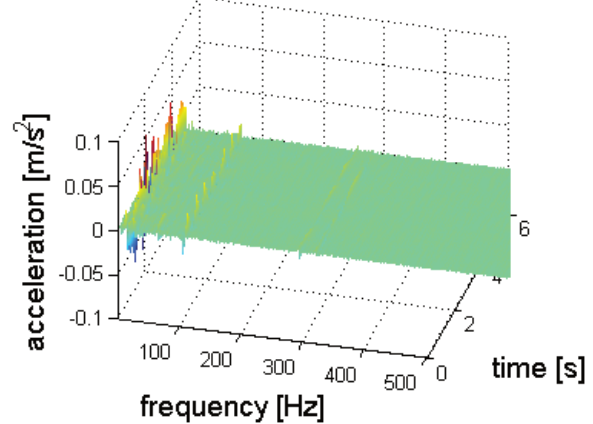

d) $3000 \mathrm{rpm}$

Fig. 13.32. Changesof structure of lateral vibration of floor panel under rear right passenger's feet for different engine rotational speed (time window $0.25 \mathrm{~s}$, resoliution $0.4884 \mathrm{~Hz}$ )

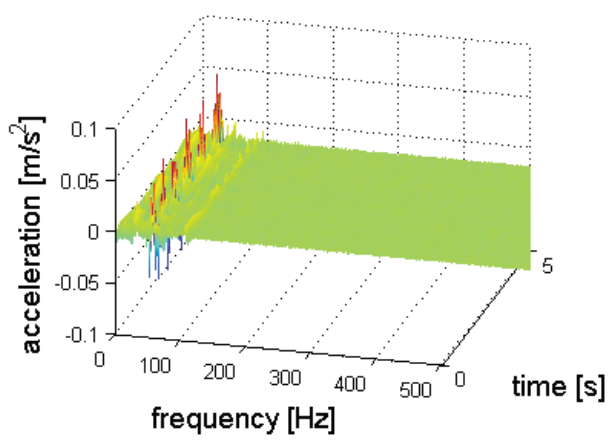

a) $750 \mathrm{rpm}$

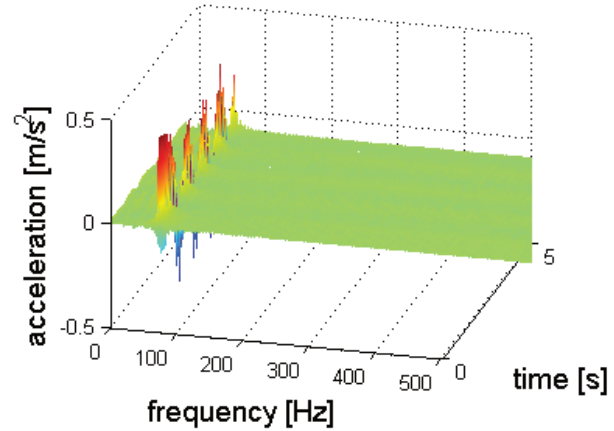

c) $2000 \mathrm{rpm}$

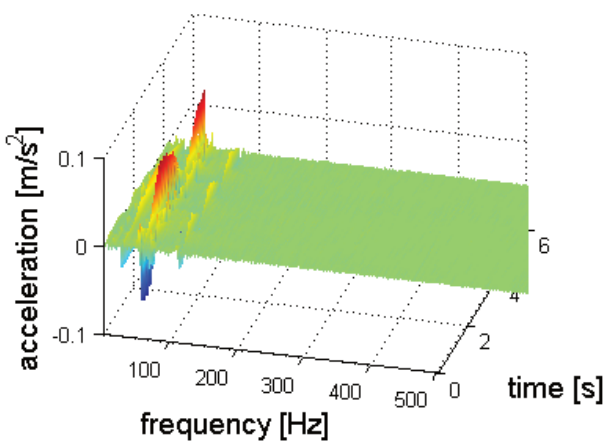

b) $1500 \mathrm{rpm}$

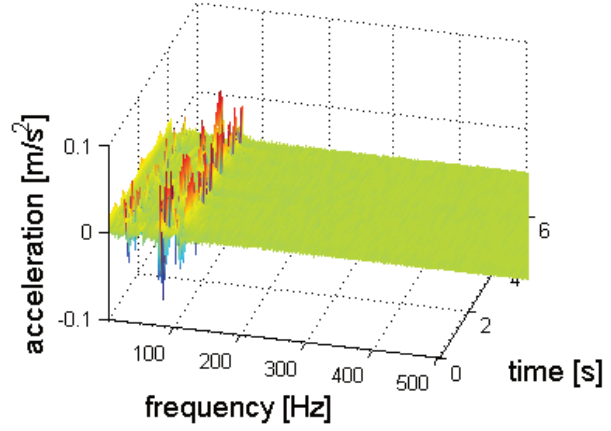

d) $3000 \mathrm{rpm}$

Fig. 13.33. Changesof structure of vertical vibration of floor panel under driver's feet for different engine rotational speed (time window $0.25 \mathrm{~s}$, resoliution $0.4884 \mathrm{~Hz}$ ) 


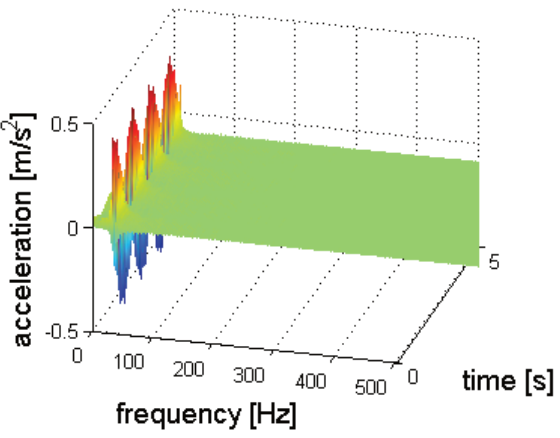

a) $750 \mathrm{rpm}$

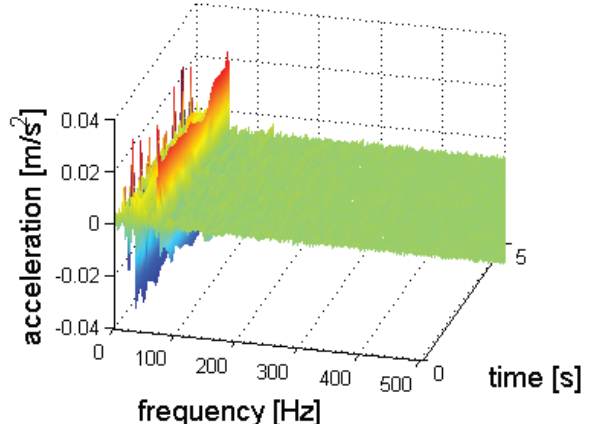

c) $2000 \mathrm{rpm}$

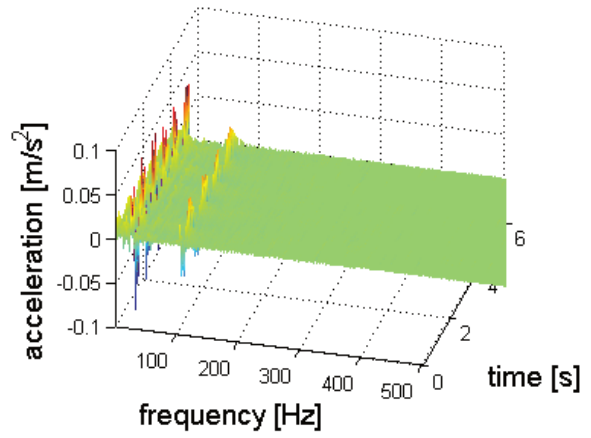

b) $1500 \mathrm{rpm}$

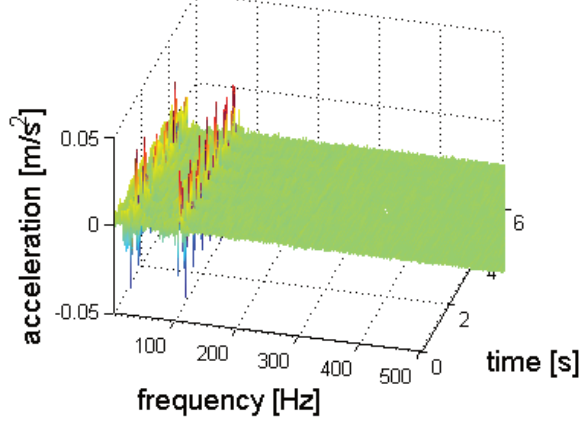

d) $3000 \mathrm{rpm}$

Fig. 13.34. Changesof structure of vertical vibration of floor panel under front passenger's feet for different engine rotational speed (time window $0.25 \mathrm{~s}$, resoliution $0.4884 \mathrm{~Hz}$ )

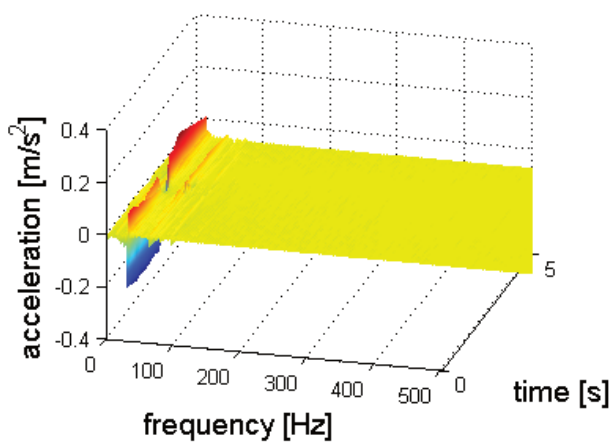

a) $750 \mathrm{rpm}$

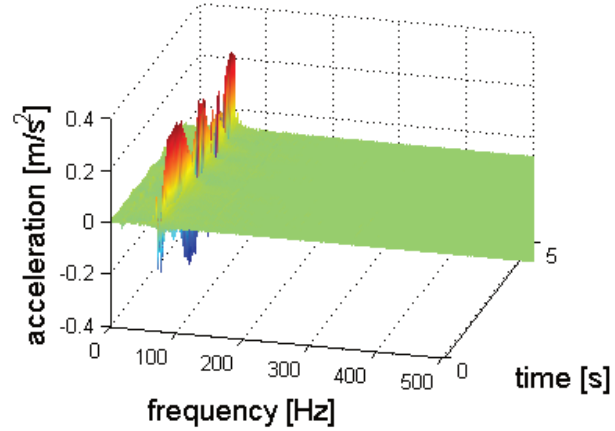

c) $2000 \mathrm{rpm}$

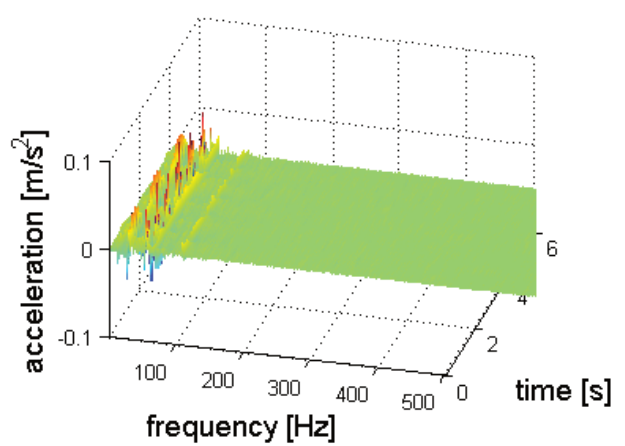

b) $1500 \mathrm{rpm}$

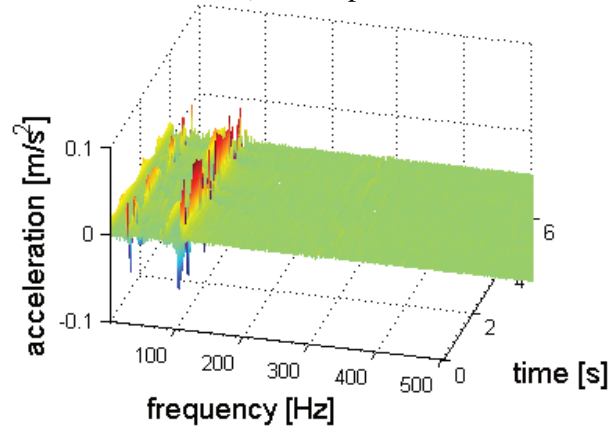

d) $3000 \mathrm{rpm}$

Fig. 13.35. Changesof structure of vertical vibration of floor panel under rear left passenger's feet for different engine rotational speed (time window $0.25 \mathrm{~s}$, resoliution $0.4884 \mathrm{~Hz}$ ) 


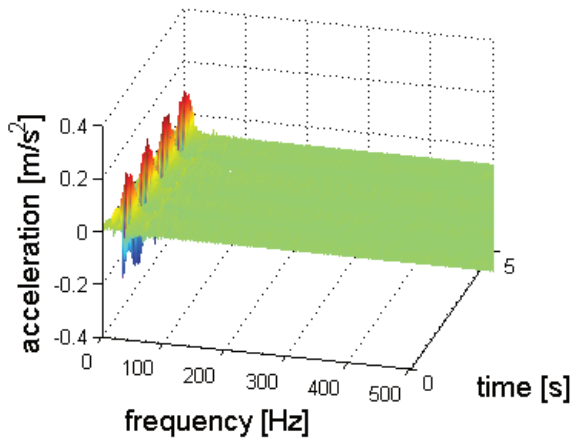

a) $750 \mathrm{rpm}$

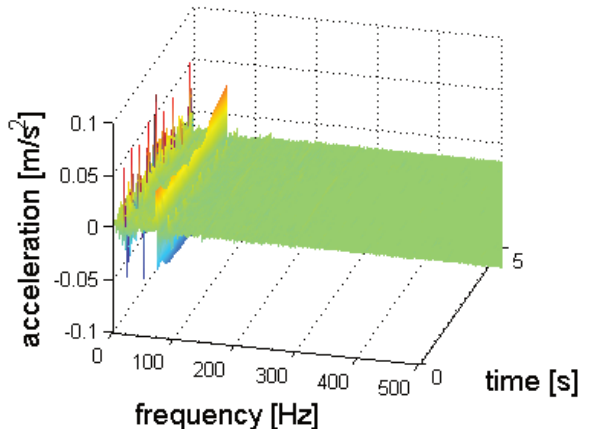

c) $2000 \mathrm{rpm}$

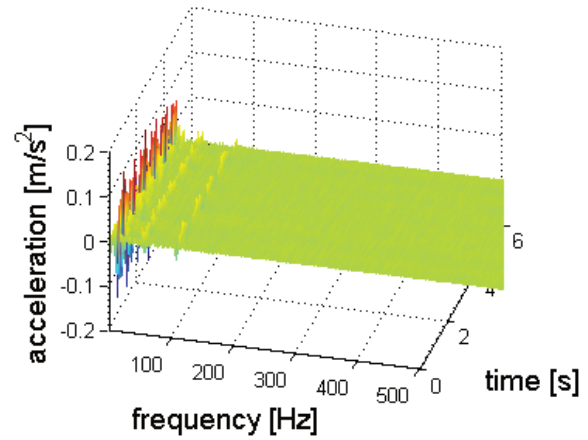

b) $1500 \mathrm{rpm}$

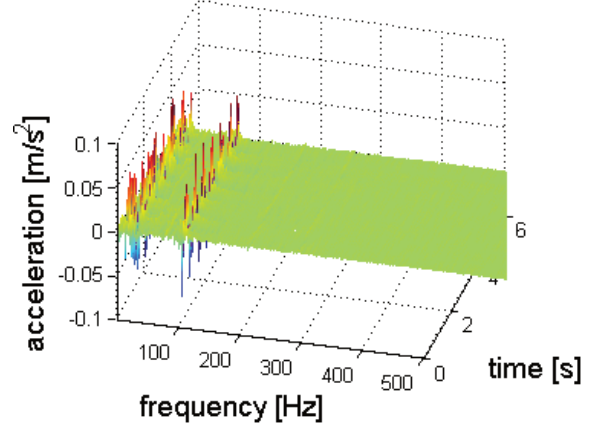

d) $3000 \mathrm{rpm}$

Fig. 13.36. Changesof structure of vertical vibration of floor panel under rear right passenger's feet for different engine rotational speed (time window $0.25 \mathrm{~s}$, resoliution $0.4884 \mathrm{~Hz}$ )

\subsection{Analysis of engine rotational speed impact on floor panel vibration}

To assess exposure to vibrations of the overall impact on the human body by the vibration of the floor panel of the vehicle stopped with working engine the total estimators were calculated. For the analysis of energy of directional distribution of vibrations the $R M S$ values were used. As the energy total estimators it was compared the $S_{R M S}$, which is expressed as:

$S_{R M S}=\sum_{i=1}^{n} X_{R M S d_{i}}$

where: $X_{R M S}$ - root mean square of vibration signal, $i$ - number of different exposure type, $d-$ direction ( $x$ - longitudinal, $y$ - lateral, $z$ - vertical), $n$ - total number of exposure type.

The distribution of these estimators are shown in Fig. 13.37.

What's most interesting in analysis results is that the energy estimators have larger values for the smallest idle gear rotational speed. The analysis of vibration signal energy value correlated to frequency distribution based on defined estimator can be more sensitive to exposure and perception on human vibration. Thus the estimator of energy of vibration spectrum for separate direction was proposed and determined by equation:

$T_{a b s(F F T)}=\frac{1}{D} \sum_{i}^{N F F T / 2}|F F T(i)|$,

where: NFFT - number of Fast Fourier Transform samples, $D$ - standardizing parameter (i.e. $D=100), F F T(i)-i$ th component of Fast Fourier Transform, $i$ - next sample of FFT. 


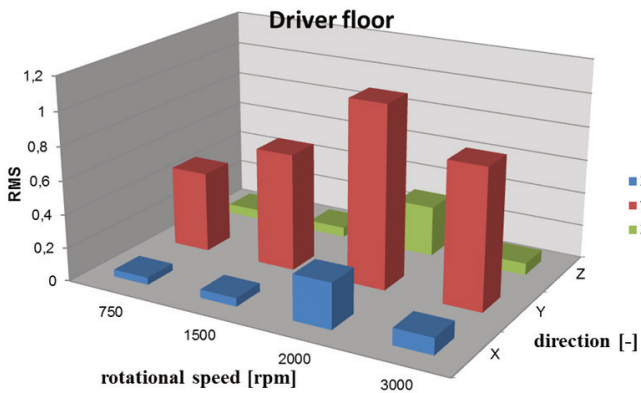

a) Driver floor $R M S$

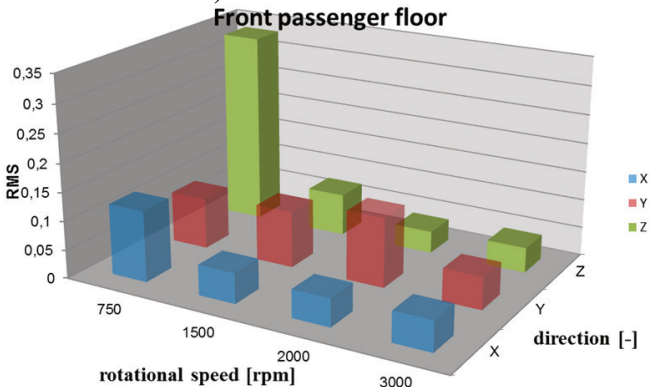

c) Front passenger floor $R M S$

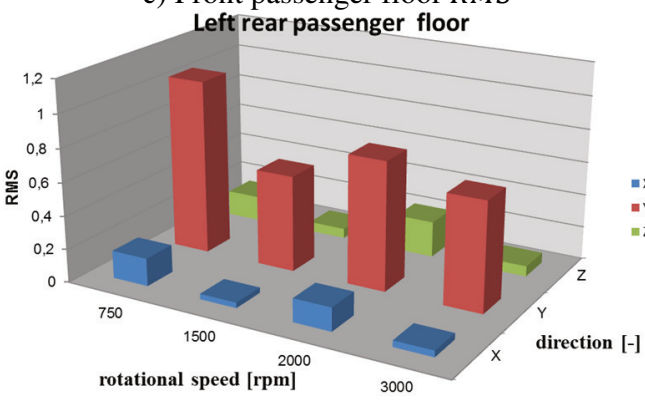

e) Left rear passenger floor $R M S$

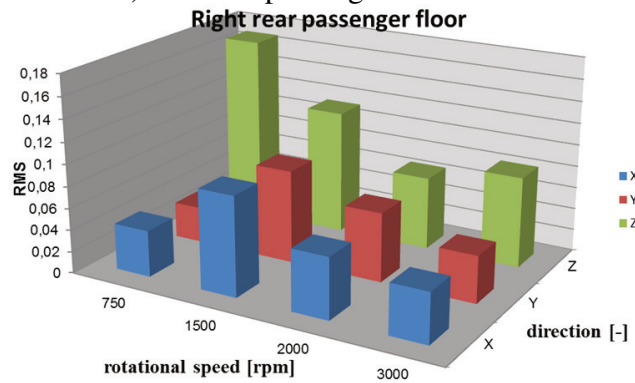

g) Right rear passenger floor $R M S$

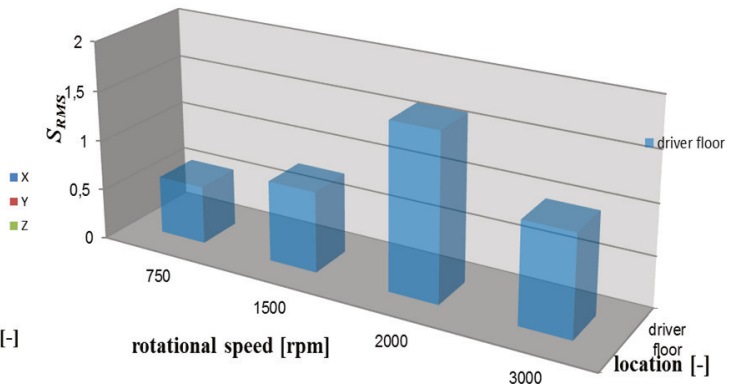

b) Driver floor $S_{R M S}$

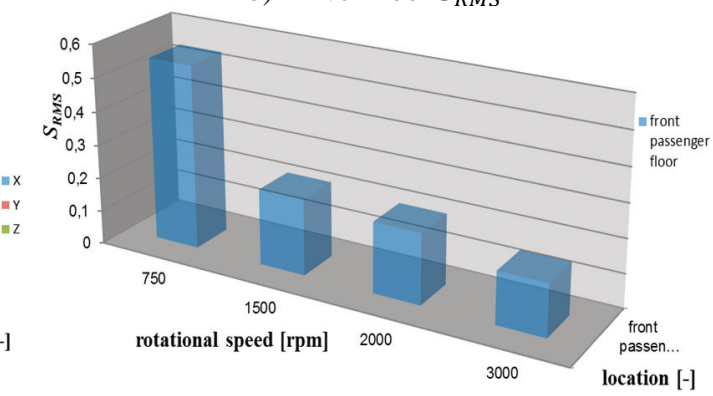

d) Front passenger floor $S_{R M S}$

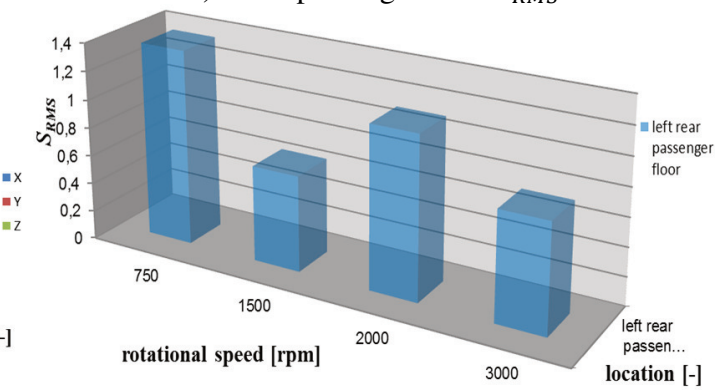

f) Left rear passenger floor $S_{R M S}$

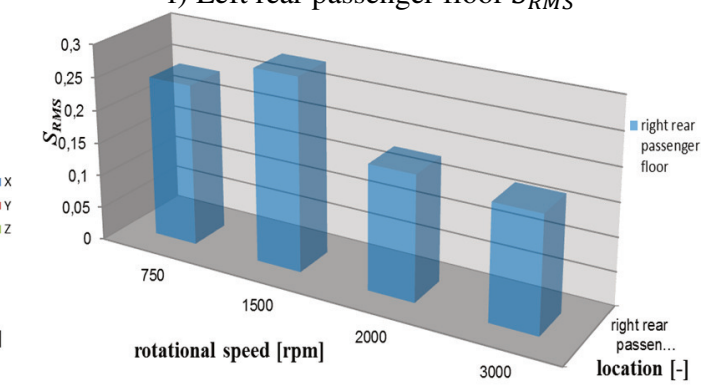

h) Right rear passenger floor $S_{R M S}$

Fig. 13.37. Distribution of time energy floor panel vibration estimators $R M S$ and $S_{R M S}$ for different engine rotational speed

As the total energy of vibration spectrum the $S_{\text {Tabs }}$ estimator was proposed and expressed as:

$$
S_{\text {Tabs }}=\sum_{i=1}^{n} T_{a b s(F F T) d_{i}} .
$$

Figs. 13.38-13.39 present the distribution of these frequency estimators. 


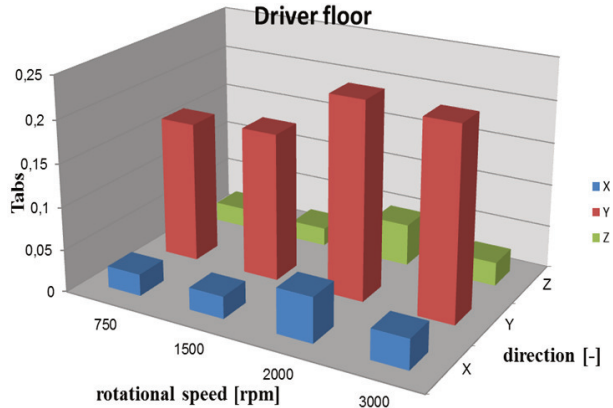

a) Driver floor $T_{a b s(F F T)}$

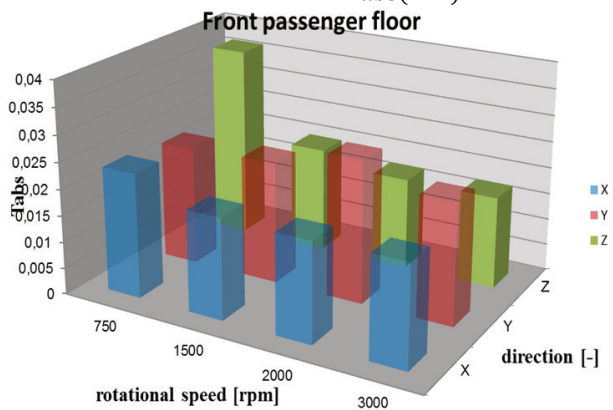

c) Front passenger floor $T_{a b s(F F T)}$

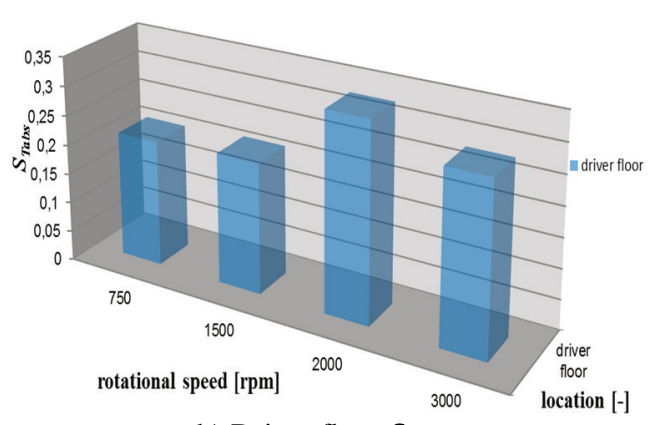

b) Driver floor $S_{\text {Tabs }}$

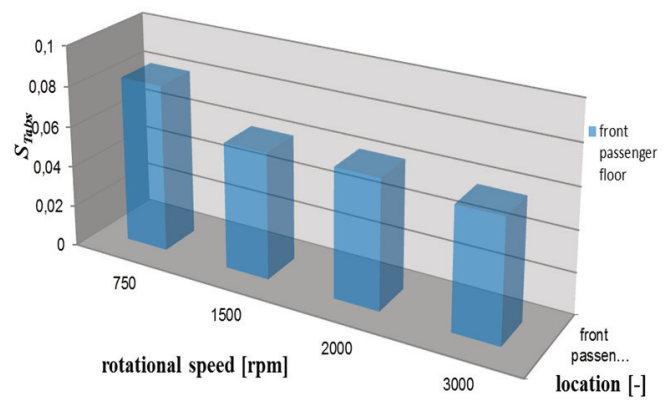

d) Front passenger floor $S_{T a b s}$

Fig. 13.38. Distribution of frequency energy front floor panel vibration estimators $T_{a b s(F F T)}$ and $S_{T a b s}$ for different engine rotational speed

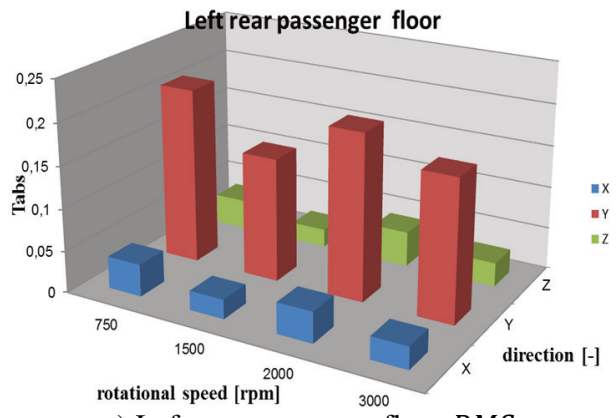

e) Left rear passenger floor $R M S$

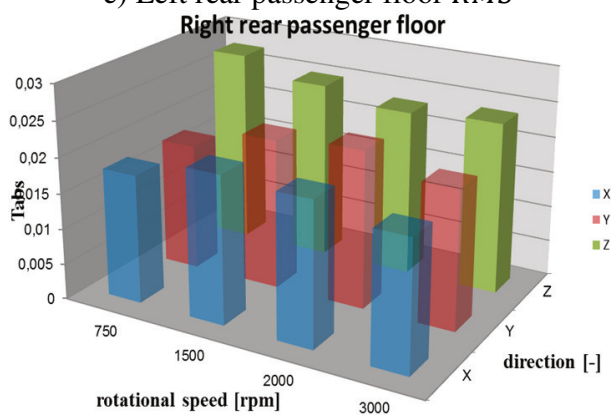

g) Right rear passenger floor $R M S$

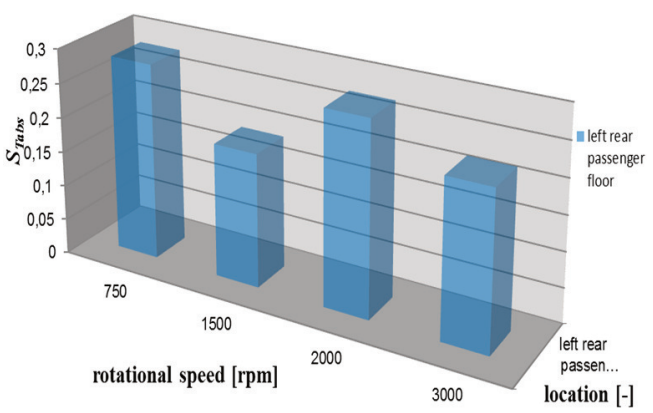

f) Left rear passenger floor $S_{R M S}$

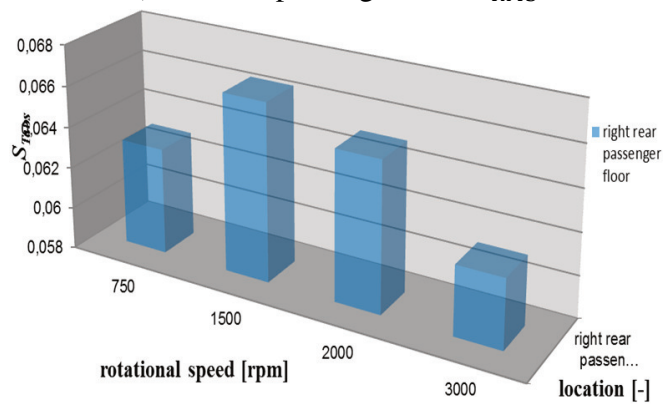

h) Right rear passenger floor $S_{R M S}$

Fig. 13.39. Distribution of frequency energy rearfloor panel vibration estimators $T_{a b s(F F T)}$ and $S_{T a b s}$ for different engine rotational speed

Analysing of engine rotational speed influence on structure of vibration presented as TFR the method of TFR energy estimator were developed. This method enables determining the energy of 
TFR of vibration estimator expressed as:

$T_{\text {absTFR }}=\frac{1}{D} \sum_{i}^{N F F T / 2} \mid F F T\left(X_{R M S}(T F R(i, t)) \mid\right.$,

where: $X_{R M S}(T F R(i, t))$ - root mean square of time distribution of $i$ th frequency component of TFR, $t$ - next sample of time (correlated with time window of TFR method).

The developed method is illustrated in Fig. 13.40. From TFR of the vibration signal next time distributions of the frequency component of the TFR are determined and the RMS of these distributions are calculated. As the result the frequency distribution of RMS of time components of TFR are obtained (Fig. 13.41). Thus the estimator of energy of vibration RMS spectrum can be determined.

As the total energy of TFR of vibration the $S_{\text {TabsTFR }}$ estimator is proposed and can be expressed as:

$S_{\text {TabsTFR }}=\sum_{i=1}^{n} T_{\text {absTFR }} d_{i}$.

Fig. 13.42 present the distribution of these TFR estimators.

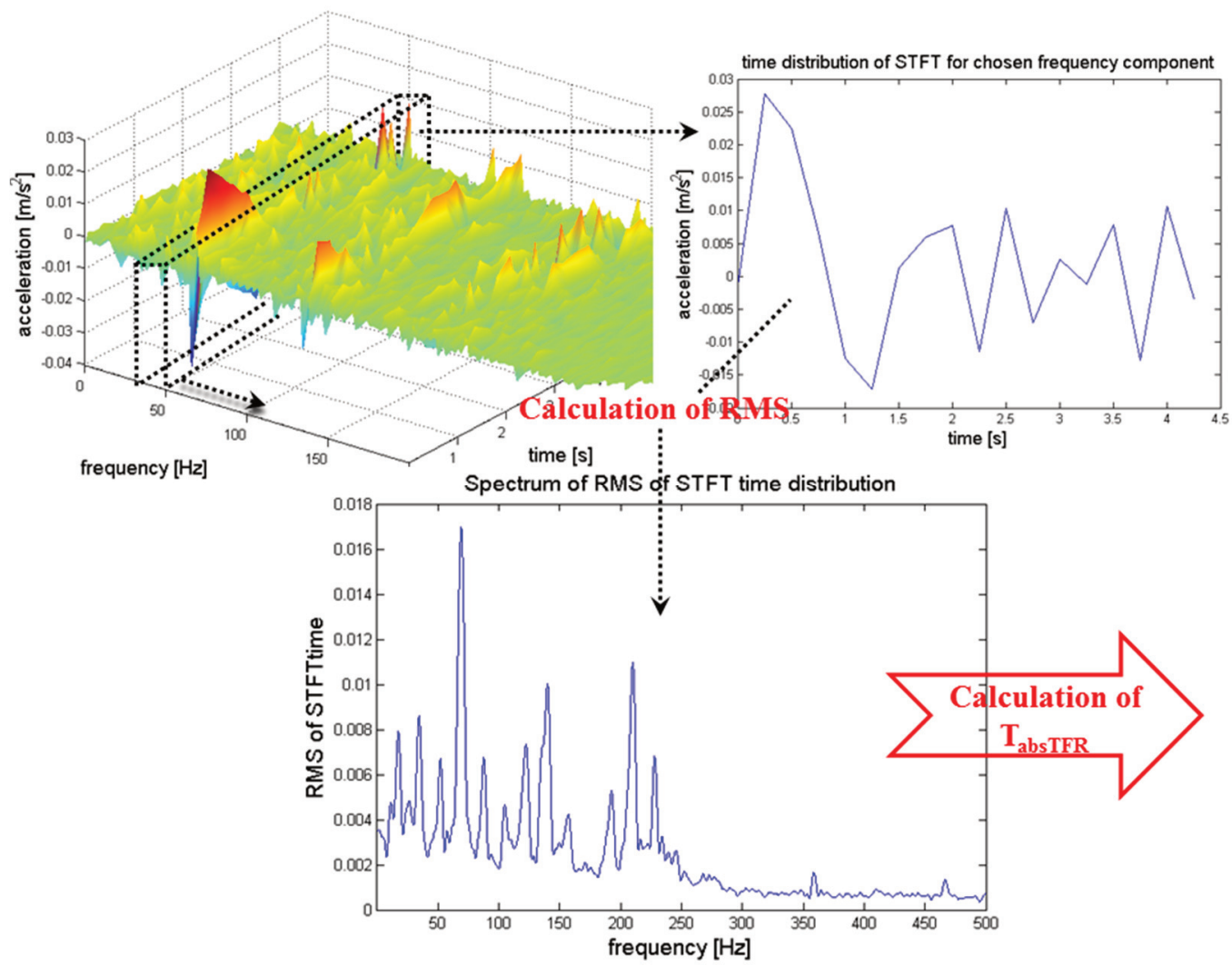

Fig. 13.40. Illustration of the method for the estimation of energy of TFR of vibration $\left(T_{\text {abSTFR }}\right)$ determination 

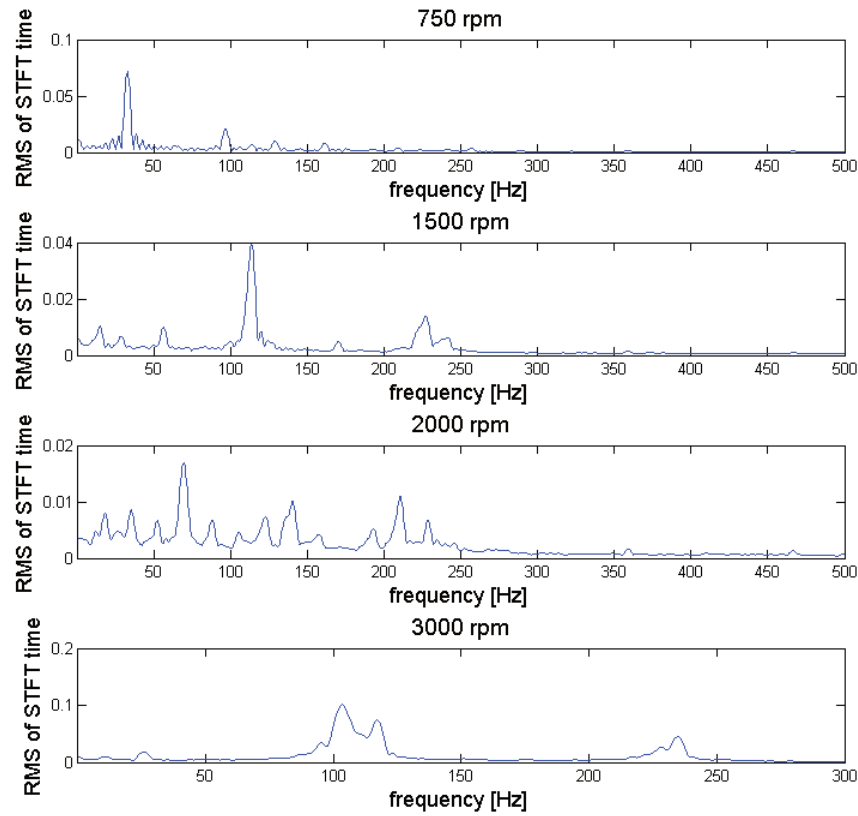

Fig. 13.41. Example of frequency distribution of RMS of time components of TFR of vibration on the floor panel under the driver's feet

\subsection{Result and functions analysis}

Results obtained show that increase of the engine rotational speed is not directly proportional to generated floor panel vibration. The analysis of the signal's spectrums allows to clearly identify the frequency components correlated to engine rotational speed.

In conclusion of results analysis some interesting observations were obtained. The vibration penetration into the driver via feet registered on the floor panel have unstable dynamic structure. Based on the spectrums analysis it can be observed that due to the increase of rotational speed the floor panel vibration have more frequency components. For the spectrum of the idle gear vibration the energy is focused around one major frequency component.

The TFR of vibration occurring due to increase of engine rotational speed shown some interesting phenomena in vibration structure. The time distribution for main frequency component become more inconstant for occurring and shifting in the time when main frequency vibration are lower to the other frequency components.

For the purpose of assessing exposure to vibrations of the overall impact on the human body by the vibration of the floor panel of the standing vehicle with working engine the total estimators were proposed and compared. Distribution of time energy floor panel vibration estimators $R M S$ and $S_{R M S}$ for different engine rotational speed shows that the energy estimators can have larger values for the smallest idle gear rotational speed. It is correlated to the feeling of vibration discomfort in the traffic jam, when engine is working on idle gear. To consider the influence on the dynamic of the vibration due to increase of the dynamic of the source the frequency estimators $T_{a b s(F F T)}$ and $S_{T a b s}$ were developed. The directional distribution of $T_{a b s(F F T)}$ is different than $R M S$ but the $S_{\text {Tabs }}$ distribution is much more similar to the $S_{R M S}$. Some differences in sensitivity on engine rotational speed of those estimators can be observed. The most complex analysis of influence of engine rotational speed on floor panel vibration can be conducted basing on TFR of the signals. Thus the method of TFR energy estimator was developed. It allows to calculate the estimator depends on time and frequency distribution of the vibration. Thus the estimator of energy of vibration RMS spectrum can be determined as $T_{a b s(F F T)}$ for directional propagation and $S_{\text {TabsTFR }}$ for total vibration energy. 


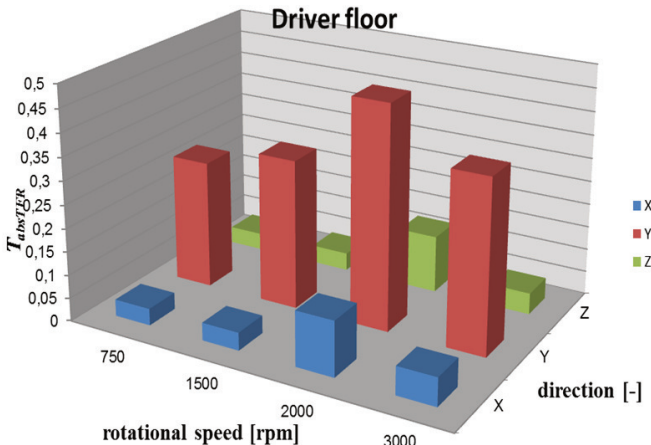

a) Driver floor $T_{a b S T F R}$

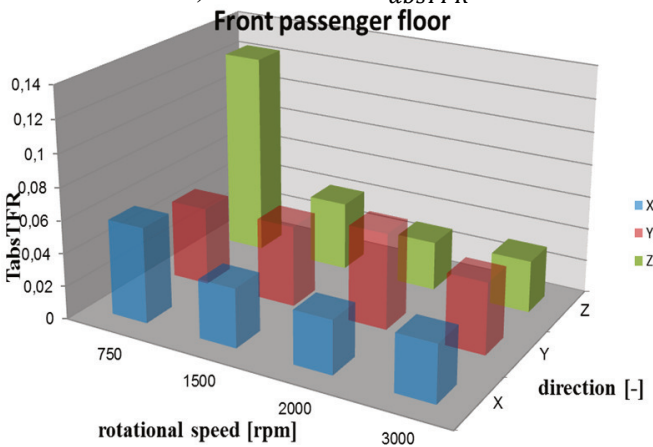

c) Front passenger floor $T_{a b S T F R}$

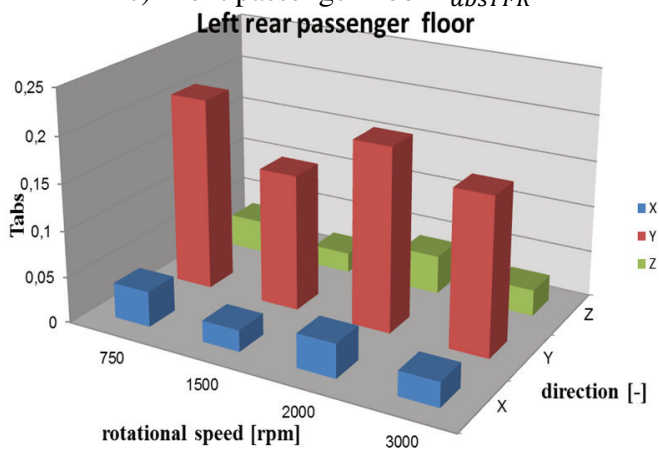

e) Left rear passenger floor $T_{a b s T F R}$

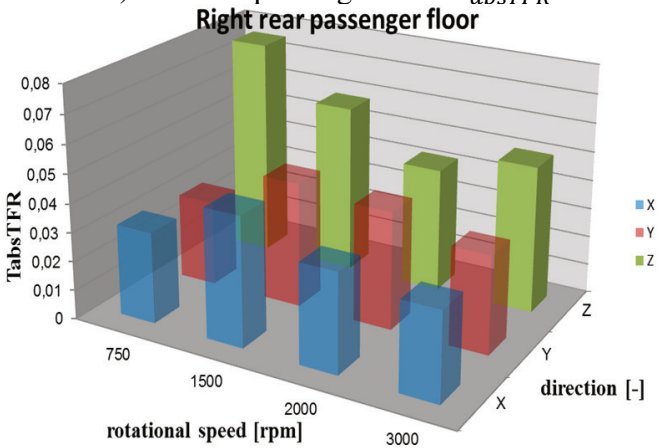

g) Right rear passenger floor $T_{a b s T F R}$

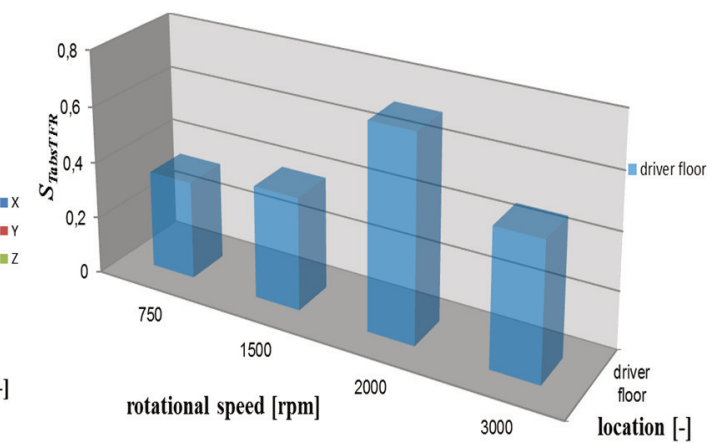

b) Driver floor $S_{\text {TabsTFR }}$

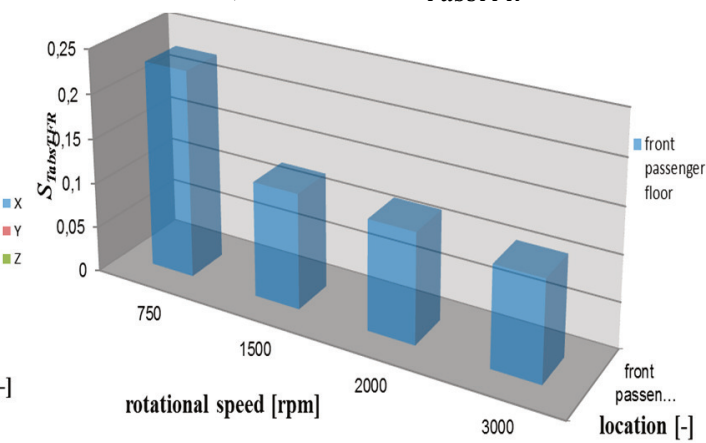

d) Front passenger floor $S_{\text {TabsTFR }}$

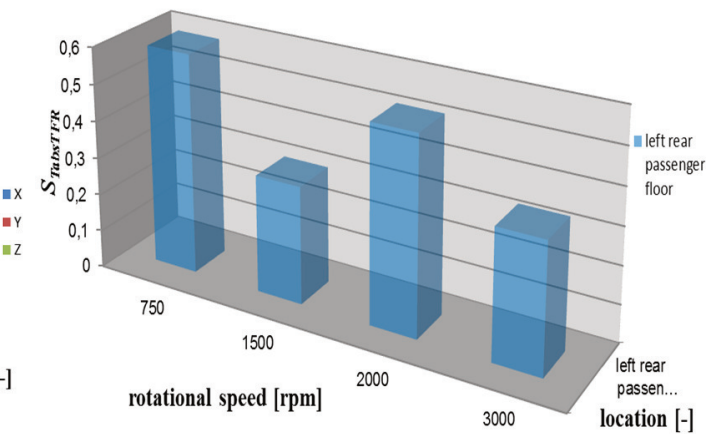

f) Left rear passenger floor $S_{\text {TabsTFR }}$

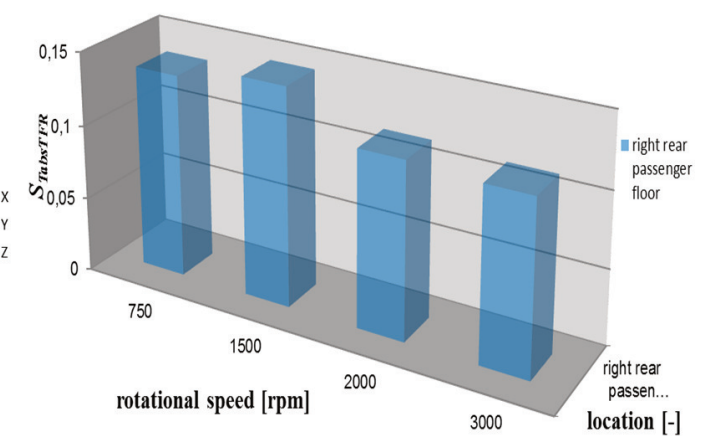

h) Right rear passenger floor $S_{\text {TabsTFR }}$

Fig. 13.42. Distribution of frequency energy floor panel vibration estimators $T_{a b S T F R}$ and $S_{\text {TabsTFR }}$ for different engine rotational speed 



\section{Influence of gear position in transmission gearbox on vibration}

What is required in order to consider the engine as a source of vibrations in a moving vehicle is an analysis of the impact exerted by other mechanisms of the power transmission system on the vibration propagation from the source. And bearing the vibroacoustic emission in mind, one of the most crucial mechanisms is the transmission. There is real abundance of publications discussing the problems of gear transmission vibrations [20, 82, 130, 159, 167, 185, 189, 201, 212]. Propagation paths of the vibrations generated by the engine and transmission operation may be convergent or divergent, all depending on the system of mounting and coupling of the power transmission system elements. In order to analyse the vehicle vibrations from the perspective of their impact on passengers, it is not required to distinguish between individual propagation paths. It was assumed that the propagation paths were convergent, whereas the vibrations recorded at locations where they penetrated the human organism were considered as a random dynamic response of the vehicle structure to numerous input functions of vibration sources.

\subsection{Gearbox vibration}

The gearbox is a source of vibration and, consequently, noise. Except for bearing fatal defects or extreme structure-resonance amplification, gears are the main sources of high frequency vibration and noise, even in newly built units. Gears, by their inherent nature, cause vibrations due to the large pressure which occurs between the meshing teeth when gears transmit power. Meshing of gears involves changes in the magnitude, the position and the direction of large concentrated loads acting on the contacting gear teeth, which as a result causes vibrations. The main parts of the gearbox are the gears, shafts, bearings, housing, and the outside of the gearbox clutches and couplings. Gears are machine elements that transmit motion by means of successively engaging teeth [185].

A simple gear train (a pair of meshing gears extended optionally by idler gears) is characterized by only one toothmeshing frequency. All the basic spectrum components are usually broken down into a combination of the following effects [188]:

- low harmonics of the shaft speed originating from unbalance, misalignments, a bent shaft, and resulting in low frequency vibration;

- harmonics of the base toothmeshing frequency and their sidebands due to the modulation effects that are well audible, the vibration of the geared axis systems originated from parametric, self-excitation due to the time variation of tooth-contact stiffness in the mesh cycle, the inaccuracy of gears in mesh, and non-uniform load and rotational speed;

- ghost components due to errors in the teeth of the index wheel of the gear cutting machine, especially gear grinding machines employing the continuous shift grinding method that results in high frequency due to the large number of index-wheel teeth, these ghost components disappear after running-in;

- components originating from faults in rolling-element bearings usually of the low level noise except for fatal bearing faults as the cracking or pitting of the inner or outer race or of the rolling element itself.

Except for the frequency spectrum components originating from the rolling bearings, the 
frequency of the components, which are associated with the meshing gears, is an integer multiple of the shaft rotational frequency. There are subharmonic components as well. These components are excited at the half of the teeth resonant frequency in high-speed units (thousands RPM) due to the non-linearity of tooth stiffness.

\subsection{Research method}

The aim of the investigation was to find the influence of the gear position (gear ratio) on exposure to whole-body vibration of driver. The scope of the research included measurements of vibration for constant engine rotational speed and different gear positions. The car was set on special laboratory stand to allow start the power transmission by the engine and gearbox into lifted drive wheels. The experiments were conducted for the engine rotational speed of $1500 \mathrm{rpm}$ and successive gear positions: neutral, first-speed gearwheel, second-speed gearwheel, third-speed gearwheel, fourth-speed gearwheel, fifth-speed gearwheel and reverse gear.

\subsection{Research result}

The vibration signals were transformed into frequency domain and TFR as STFT transformation. The collection of obtained results in three orthogonal axes are shown in Figs. 14.1-14.9.

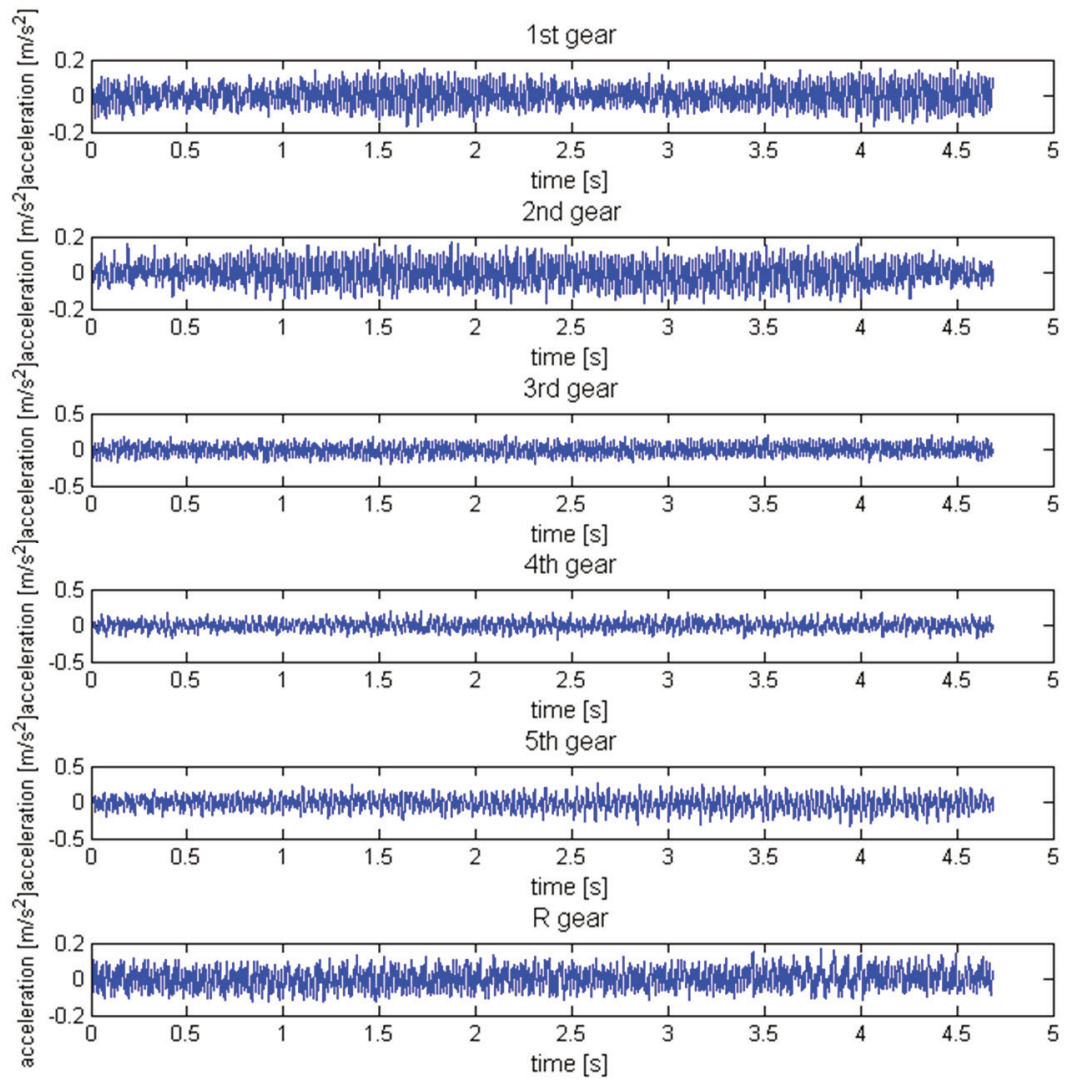

Fig. 14.1. The collection of longitudinal vibration signals measured on the floor panel in location of driver feet for different gear position 


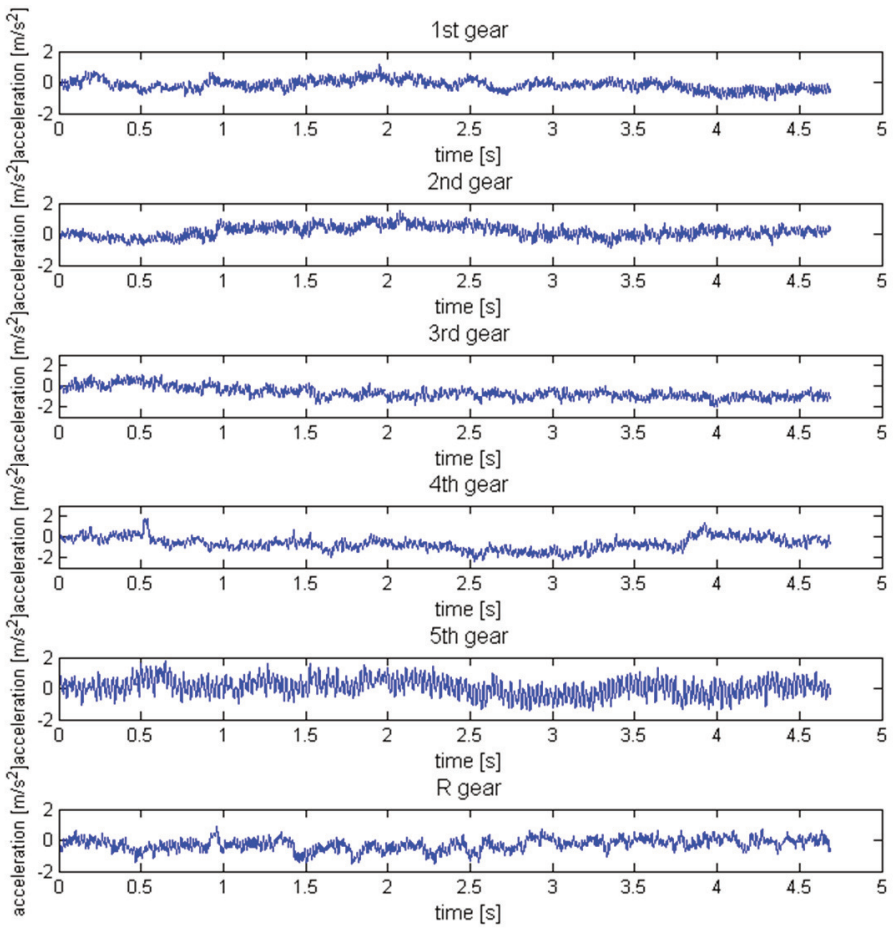

Fig. 14.2. The collection of lateral vibration signals measured on the floor panel in location of driver feet for different gear position

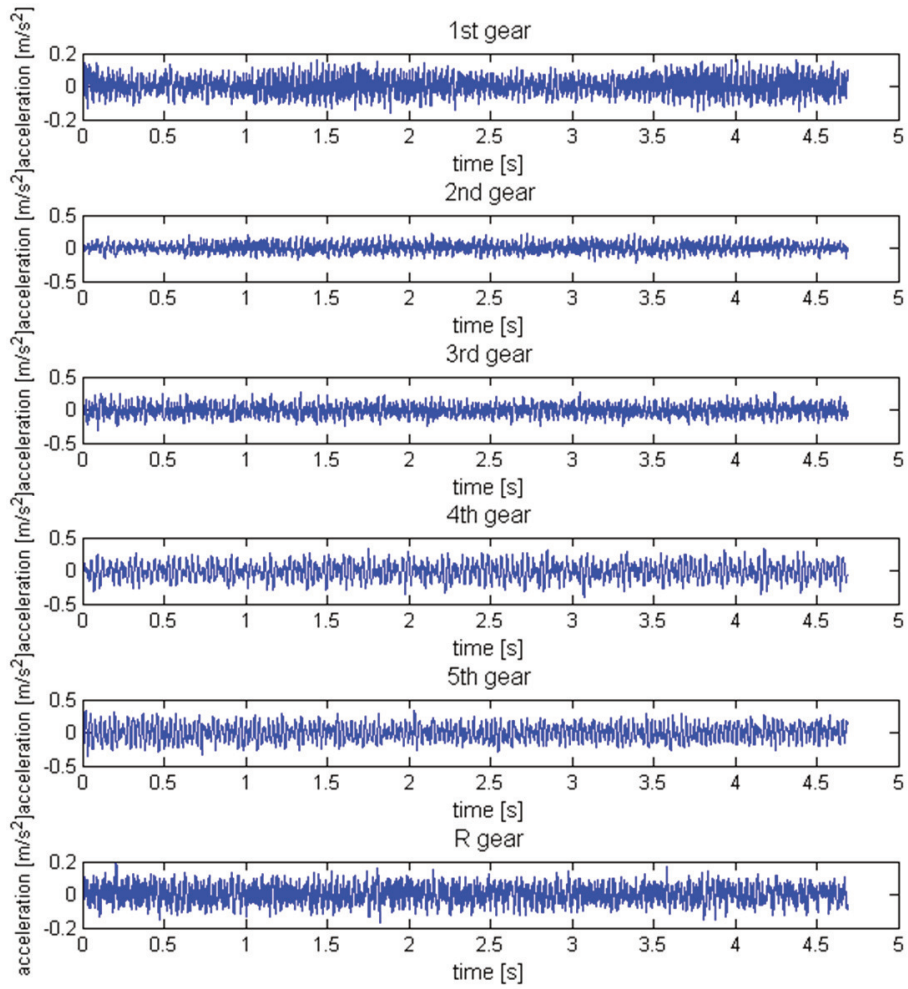

Fig. 14.3. The collection of vertical vibration signals measured on the floor panel in location of driver feet for different gear position 


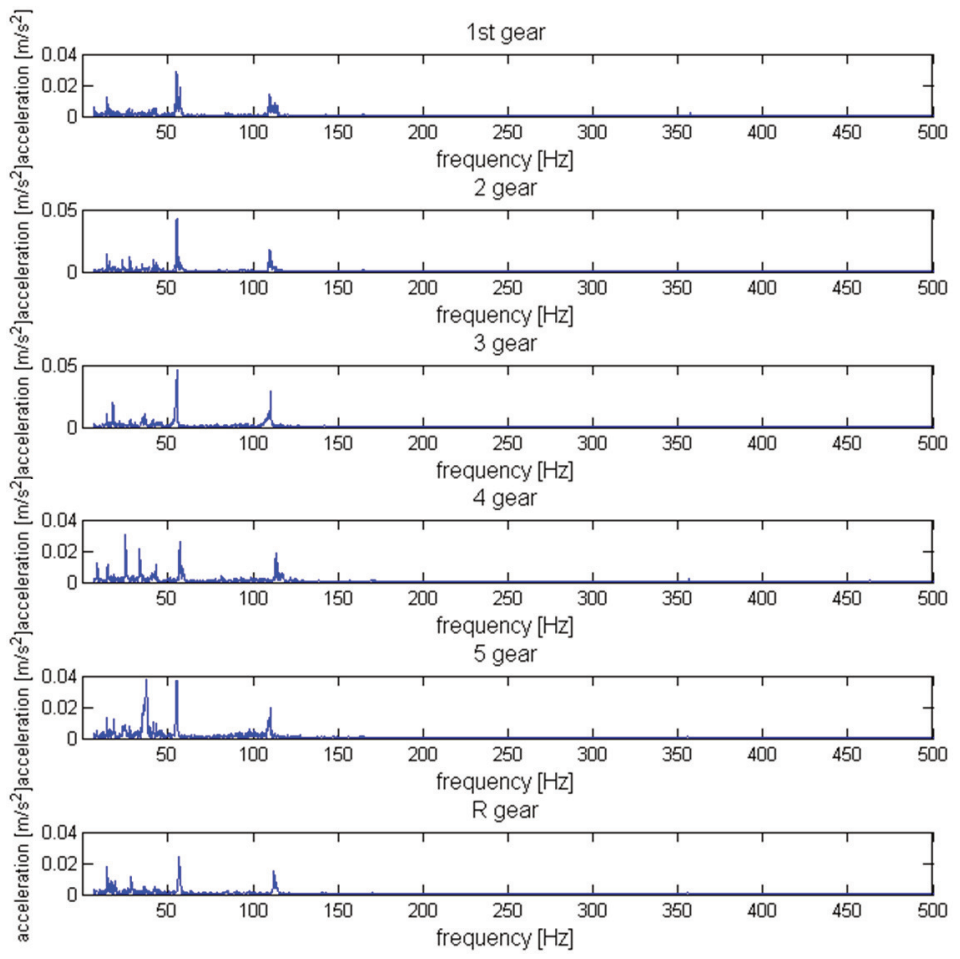

Fig. 14.4. The collection of spectrums of longitudinal vibration signals measured on the floor panel in location of driver feet for different gear position

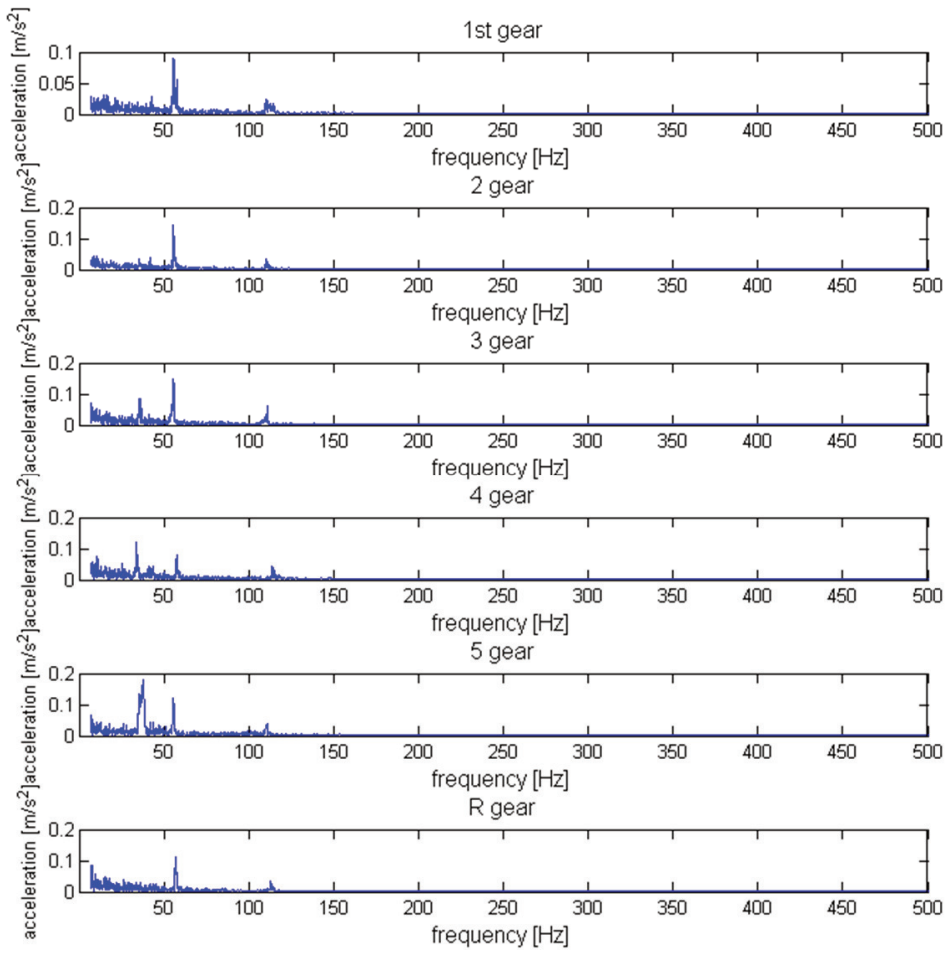

Fig. 14.5. The collection of spectrums of lateral vibration signals measured on the floor panel in location of driver feet for different gear position 


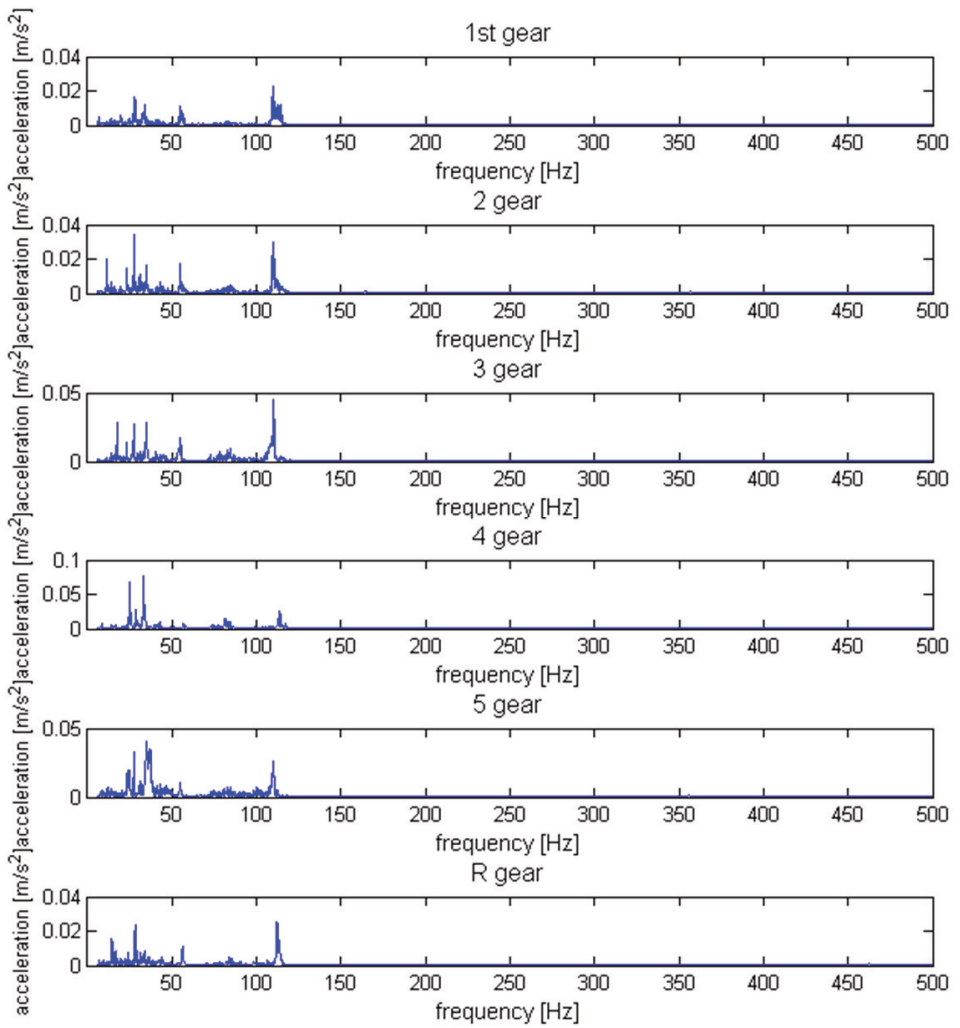

Fig. 14.6. The collection of spectrums of vertical vibration signals measured on the floor panel in location of driver feet for different gear position

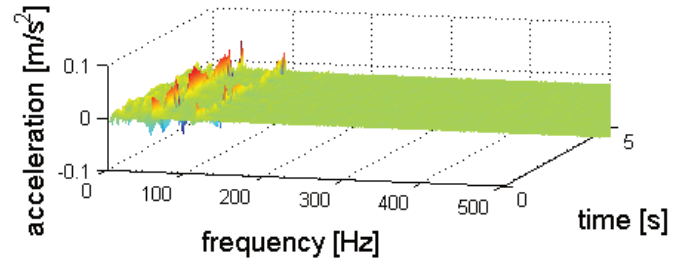

a) 1 st gear

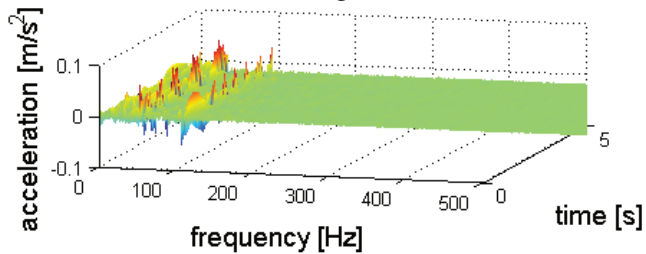

c) 3rd gear

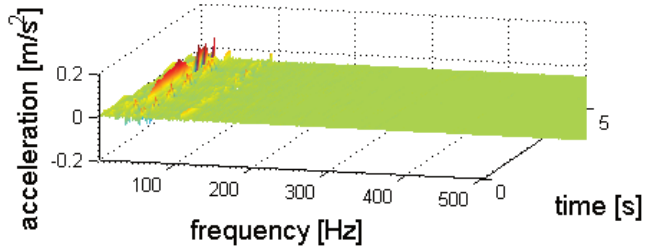

e) 5 th gear

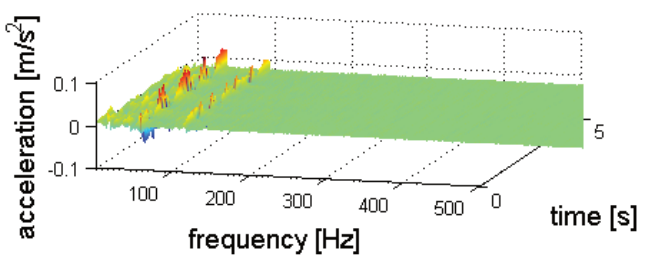

b) 2nd gear

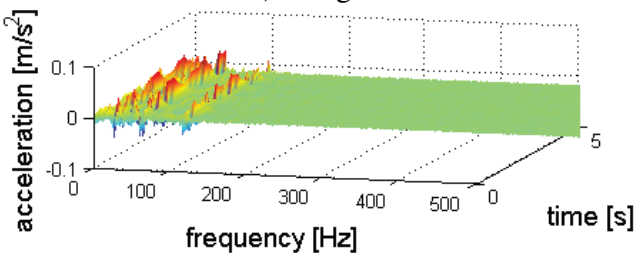

d) 4th gear

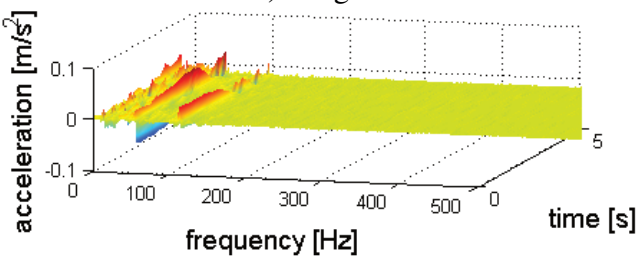

e) R gear

Fig. 14.7. The collection of TFR of longitudinal vibration signals measured on the floor panel in location of driver feet for different gear position (time window $0.25 \mathrm{~s}$, resolution $0.4884 \mathrm{~Hz}$ ) 


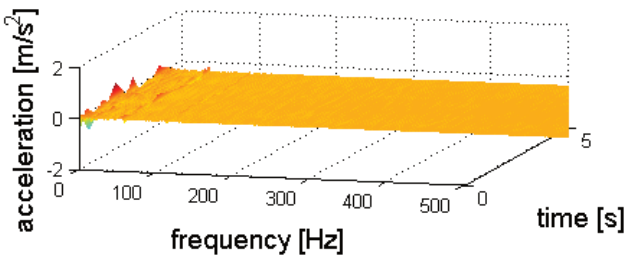

a) 1 st gear

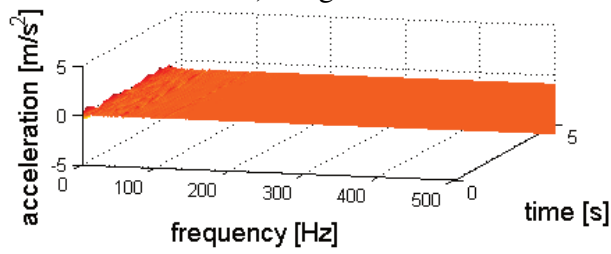

c) 3rd gear

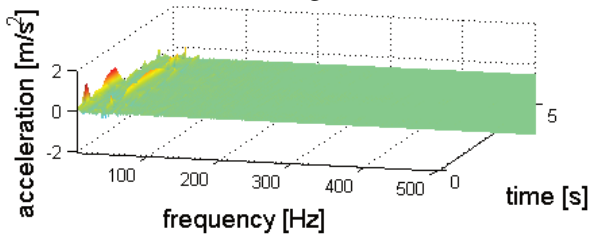

e) 5 th gear

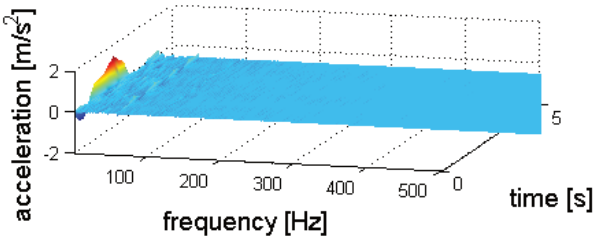

b) 2nd gear

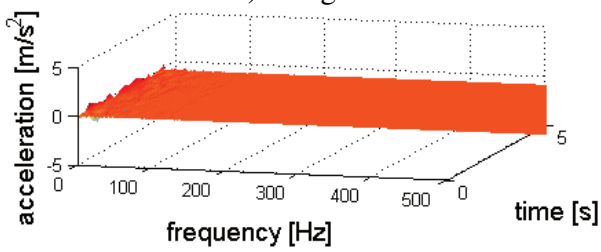

d) 4th gear

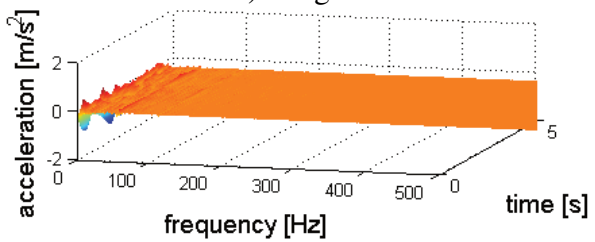

e) R gear

Fig. 14.8. The collection of TFR of lateral vibration signals measured on the floor panel in location of driver feet for different gear position (time window $0.25 \mathrm{~s}$, resolution $0.4884 \mathrm{~Hz}$ )

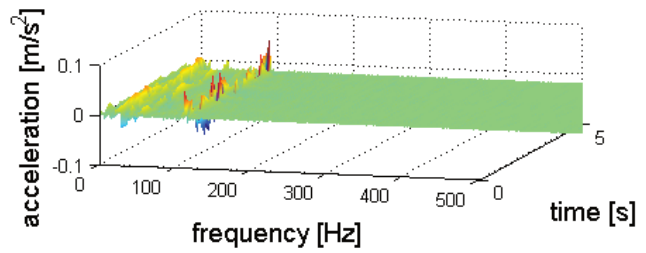

a) 1 st gear

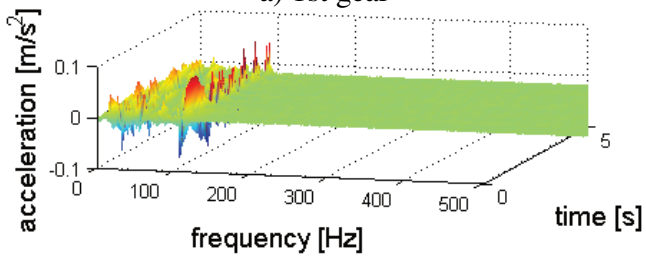

c) 3rd gear

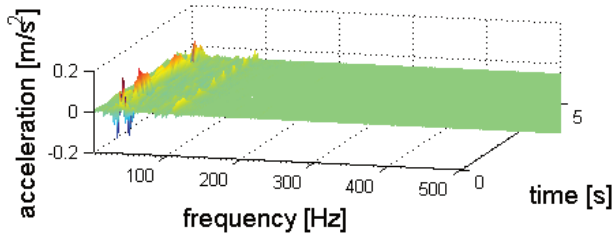

e) 5 th gear

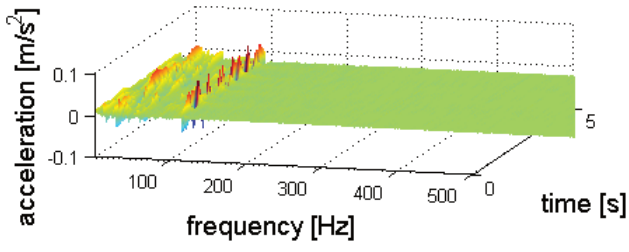

b) 2nd gear

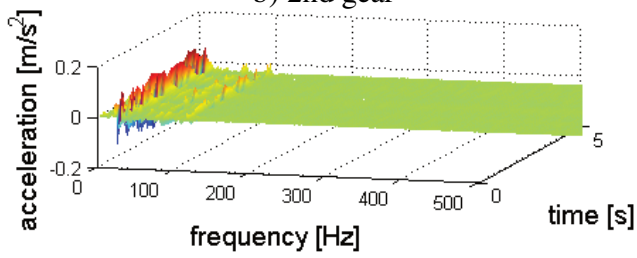

d) 4th gear

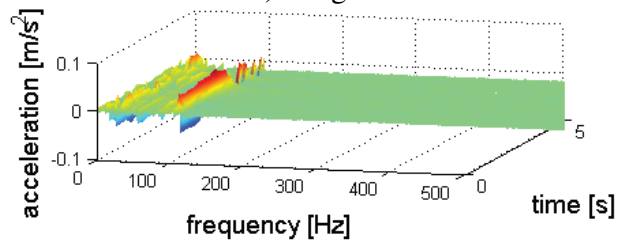

e) R gear

Fig. 14.9. The collection of TFR of vertical vibration signals measured on the floor panel in location of driver feet for different gear position (time window $0.25 \mathrm{~s}$, resolution $0.4884 \mathrm{~Hz}$ )

For the purpose of analysis of influence of gear position in transmission gearbox on vibration the estimators, defined in previous chapter, were compared. Fig. 14.10 present the distribution of these estimators with values for neutral gear. 


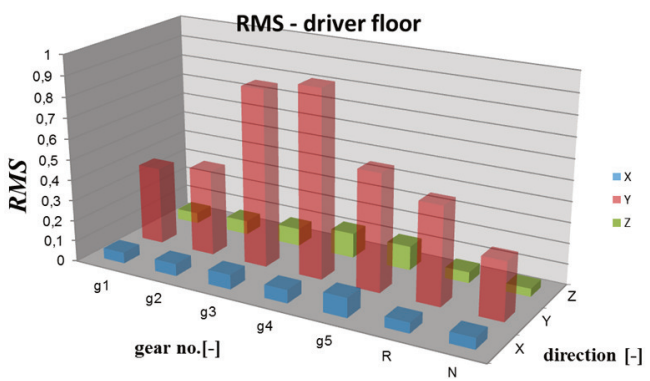

a)

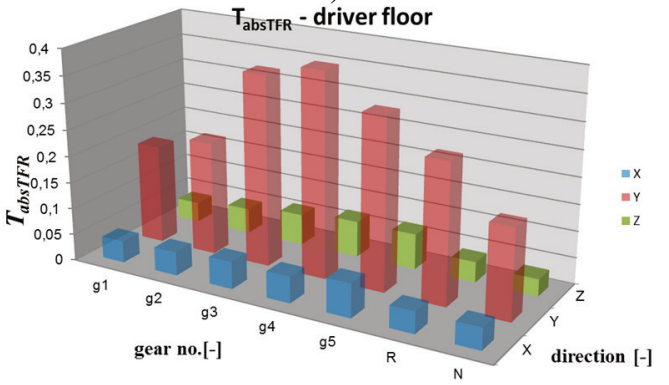

c)

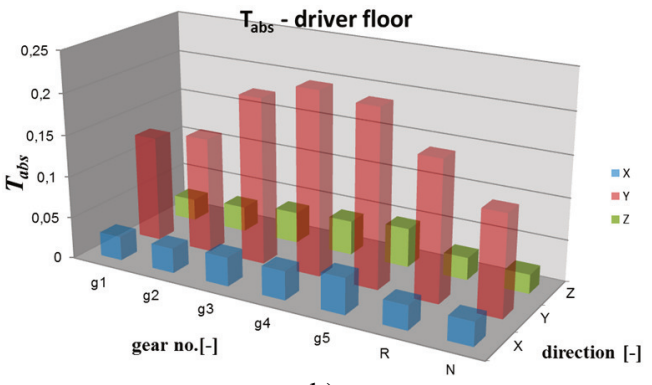

b)

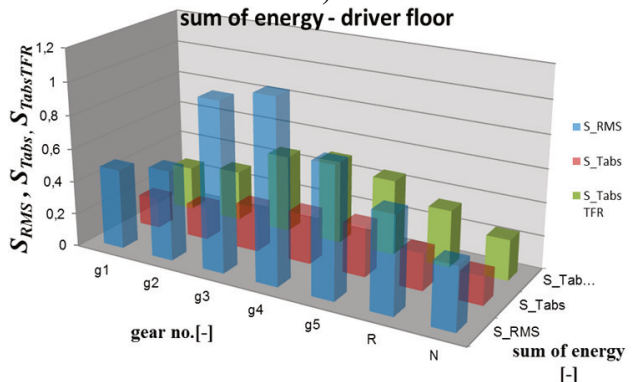

d)

Fig. 14.10. The distribution of directional estimators: a) $R M S$, b) $T_{a b s}$, c) $T_{a b s T F R}$ and

d) total energy estimators $S_{R M S}, S_{T a b s}$ and $S_{\text {TabsTFR }}$ for different gear position (vibration signals measured on the floor panel in location of driver feet)

\subsection{Identification of vibration components caused by the gearbox}

The purpose of the analysis was to identify vibration components caused due to the large pressure which occurs between the meshing teeth when gears transmit power. For these goal the time domain vibration was compared, which enables observation of vibration energy changes for the neutral idle gear and first-speed or fifth-speed gear position (Figs. 14.11-14.13).

The dynamic components can be identified by the comparison of spectrums and differential spectrum of neutral gear and running gear vibration (Figs. 14.14-14.16), expressed from equation:

$F_{d}(\omega)=\left|F_{g}(\omega)-F_{n}(\omega)\right|$

where: $F_{g}(\omega)$ - Fourier transform of running gear vibration, $F_{n}(\omega)$ - Fourier transform of neutral gear vibration.

For the analysis of structure of vibration the TFR of the vibration of first-speed and fifth-speed gear and neutral idle gear are presented in Figs. 14.17-14.19. 


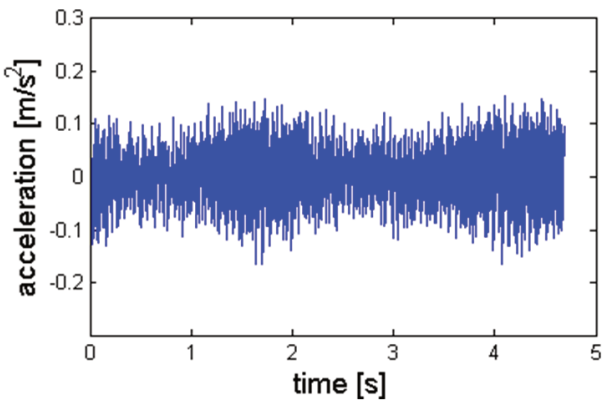

a) 1 st gear

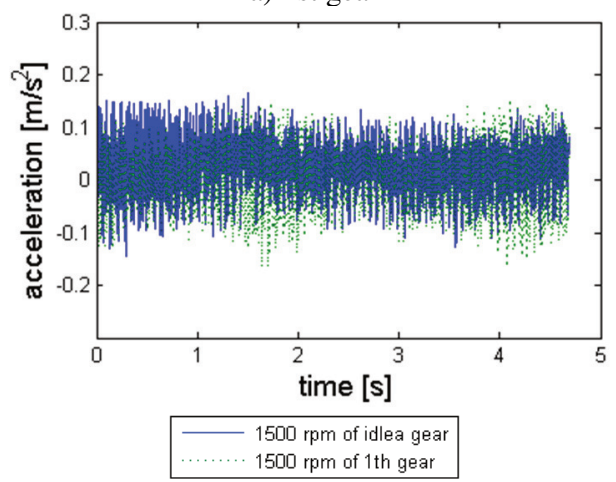

c) Idle gear vs 1 st gear

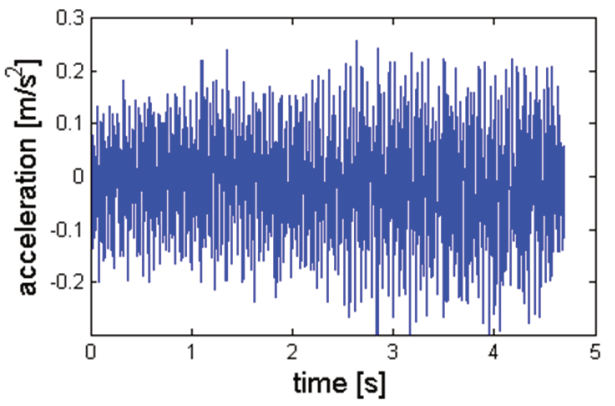

b) 5 th gear
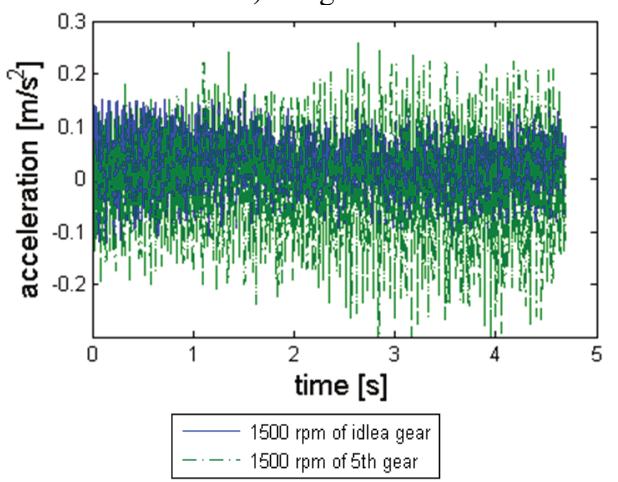

d) Idle gear vs 5 th gear

Fig. 14.11. The comparison of longitudinal vibration for neutral and first-speed and fifth-speed gear position (floor panel in location of driver feet, $1500 \mathrm{rpm}$ )

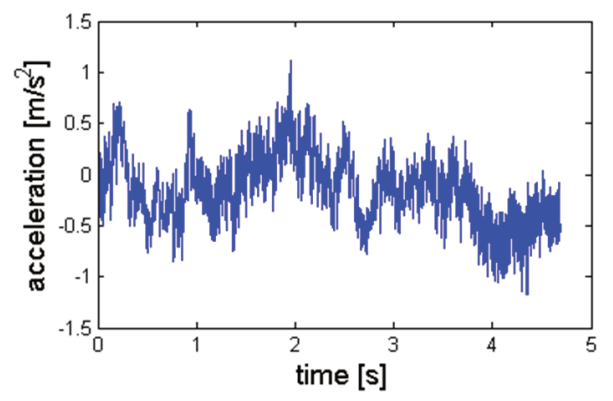

a) 1 st gear

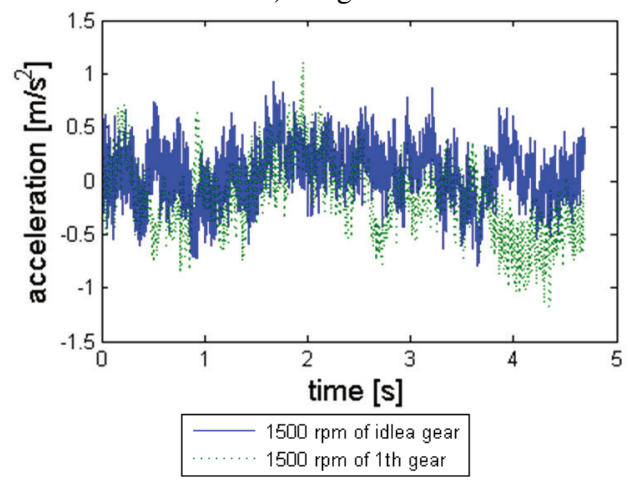

c) Idle gear vs 1 st gear

Fig. 14.12. The comparison of lateral vibration for neutral and first-speed and fifth-speed gear position (floor panel in location of driver feet, $1500 \mathrm{rpm}$ )

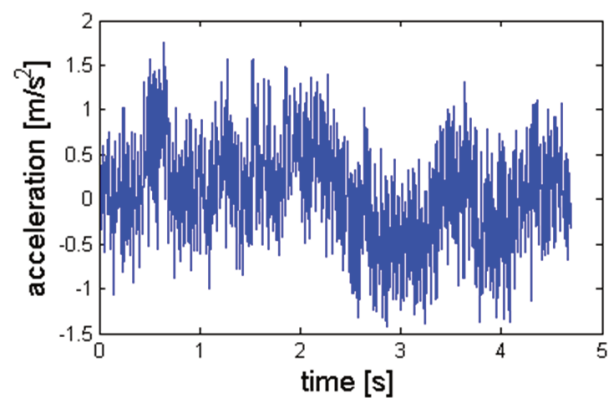

b) 5th gear

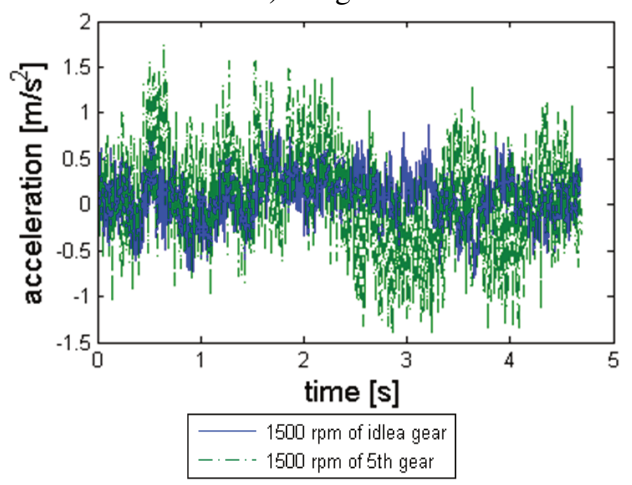

d) Idle gear vs 5 th gear 


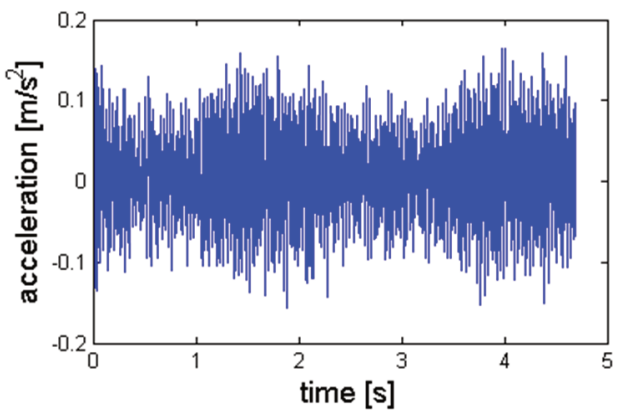

a) 1 st gear

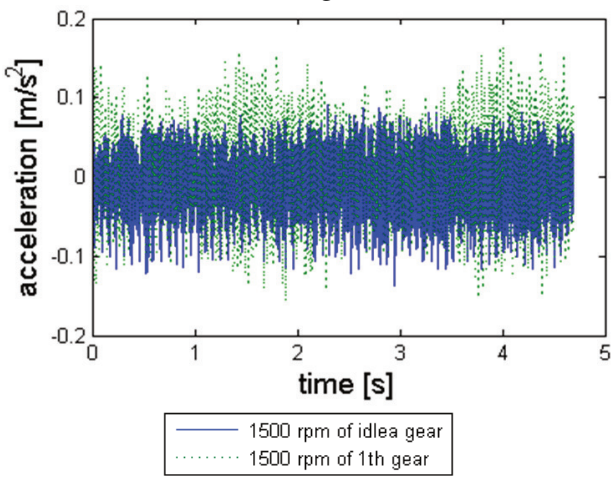

c) Idle gear vs 1 st gear

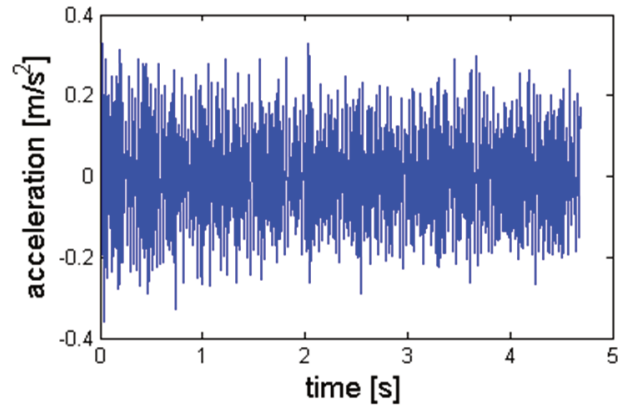

b) 5th gear

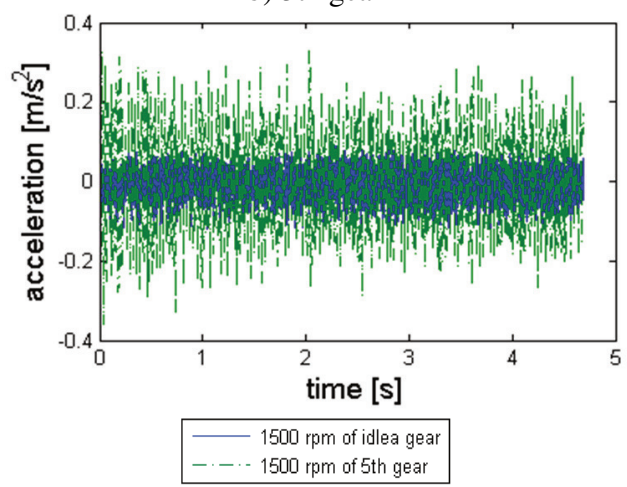

d) Idle gear vs 5 th gear

Fig. 14.13. The comparison of vertical vibration for neutral and first-speed and fifth-speed gear position (floor panel in location of driver feet, $1500 \mathrm{rpm}$ )

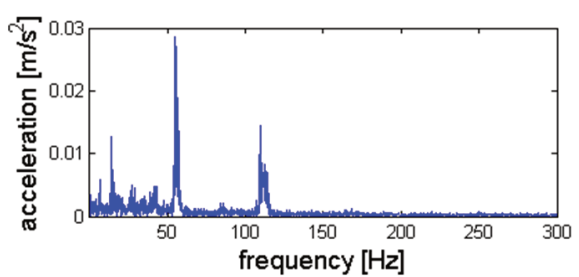

a) 1 st gear

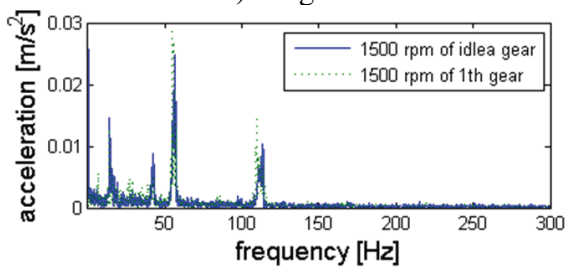

c) Idle gear vs. 1st gear

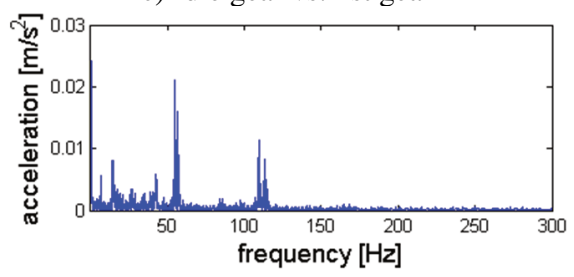

e) Differential spectrum of 1 st and idle gears

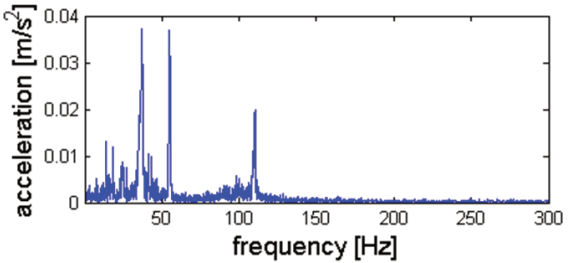

b) 5 th gear

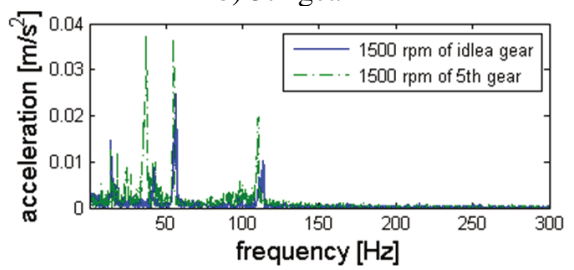

d) Idle gear vs. 5th gear

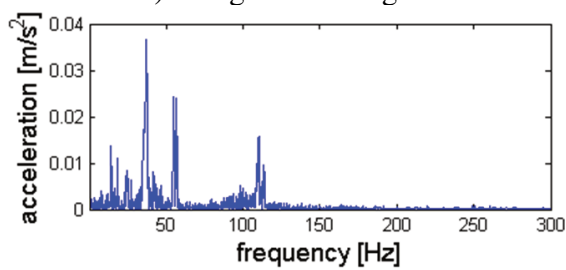

f) Differential spectrum of 5 th and idle gears

Fig. 14.14. The comparison of spectrums of longitudinal vibration for neutral and first-speed and fifth-speed gear position and differential spectrum of first and fifth-speed gears (floor panel in location of driver feet, $1500 \mathrm{rpm}$ ) 


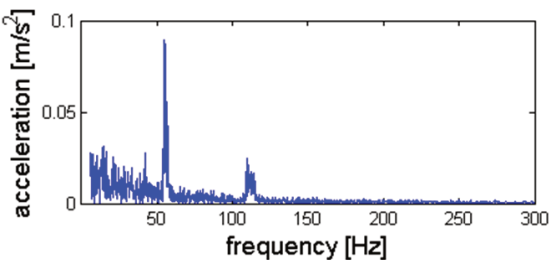

a) 1 st gear

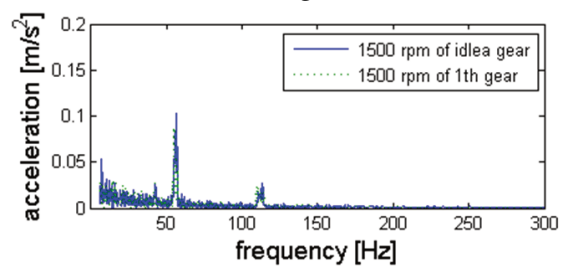

c) Idle gear vs. 1st gear

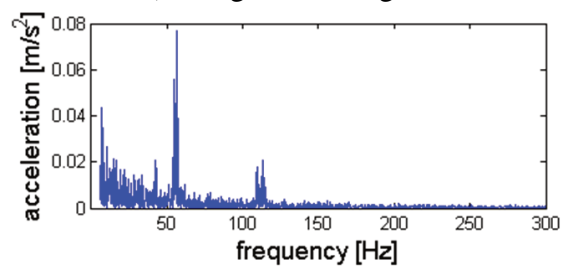

e) Differential spectrum of 1st and idle gears

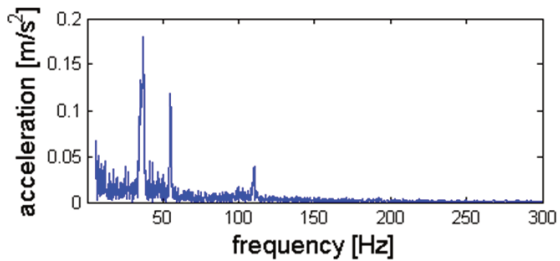

b) 5 th gear

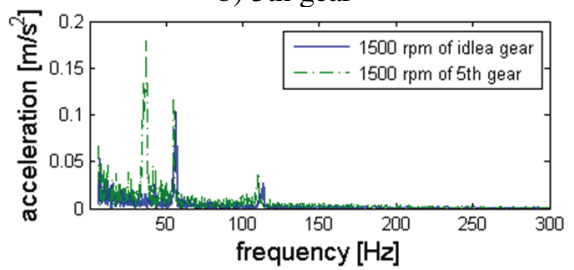

d) Idle gear vs. 5th gear

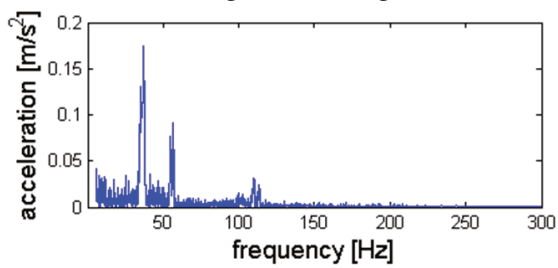

f) Differential spectrum of 5 th and idle gears

Fig. 14.15. The comparison of spectrums of lateral vibration for neutral and first-speed and fifth-speed gear position and differential spectrum of first and fifth-speed gears (floor panel in location of driver feet, $1500 \mathrm{rpm}$ )

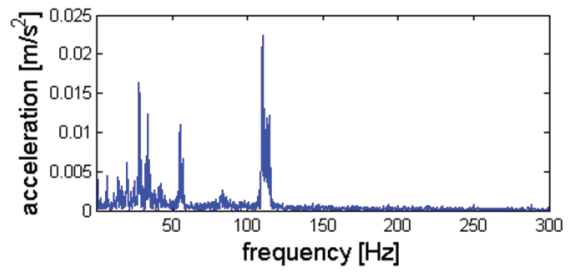

a) 1 st gear

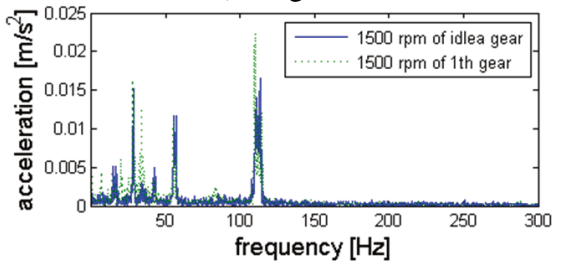

c) Idle gear vs. 1 st gear

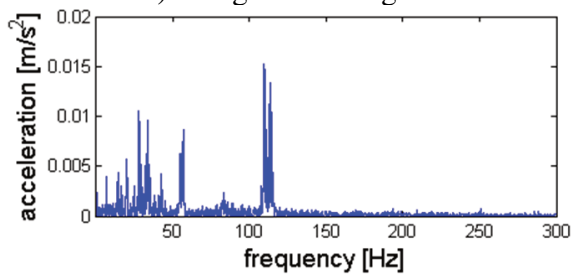

e) Differential spectrum of 1st and idle gears

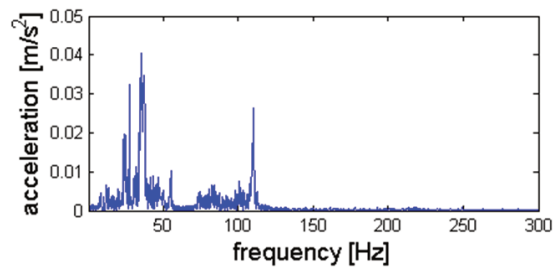

b) 5th gear

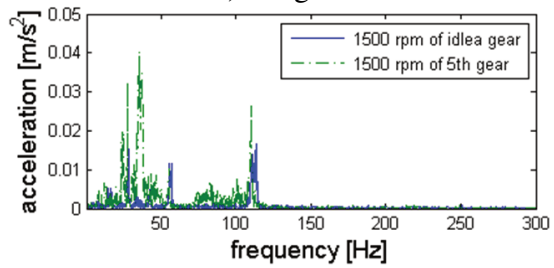

d) Idle gear vs. 5th gear

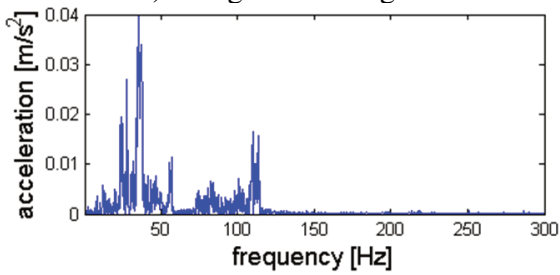

f) Differential spectrum of 5 th and idle gears

Fig. 14.16. The comparison of spectrums of vertical vibration for neutral and first-speed and fifth-speed gear position and differential spectrum of first and fifth-speed gears (floor panel in location of driver feet, $1500 \mathrm{rpm}$ ) 


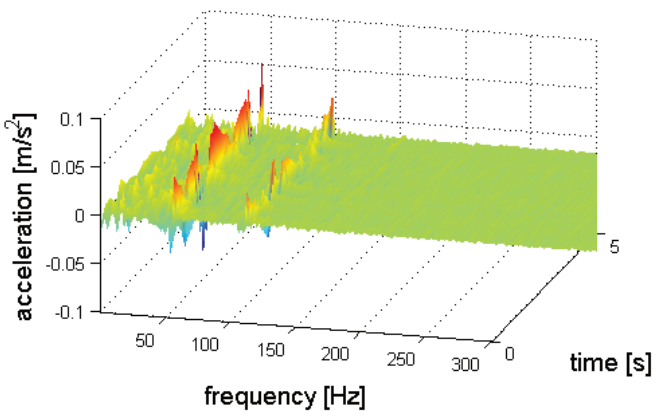

a) 1 st gear

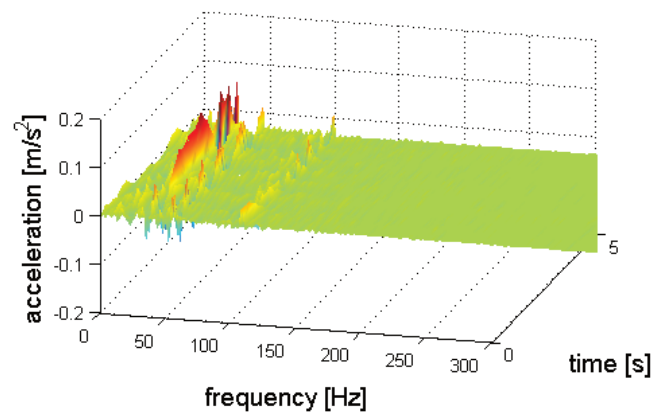

b) 5 th gear

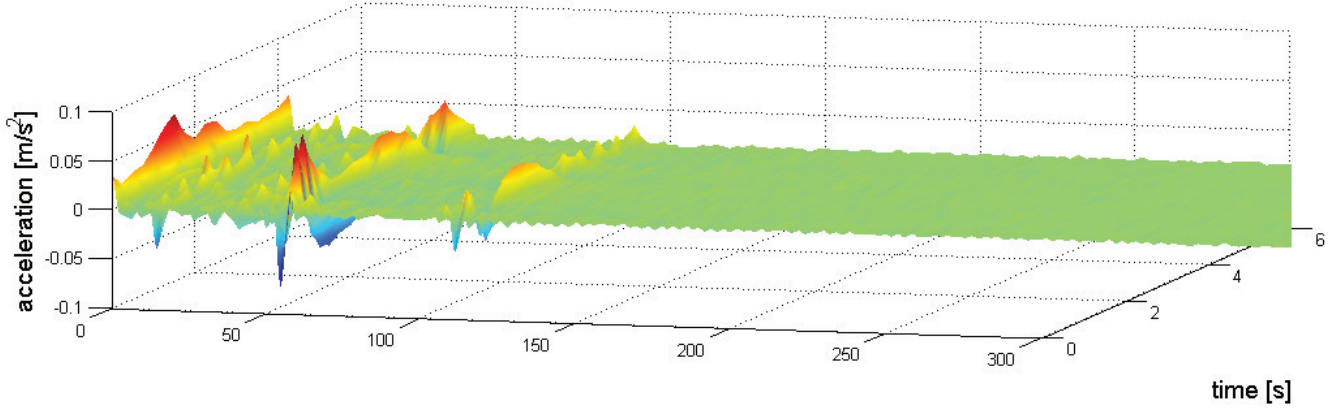

frequency $[\mathrm{Hz}]$

c) Idle gear

Fig. 14.17. The comparison of TFR of longitudinal vibration for neutral and first-speed and fifth-speed gear position (floor panel in location of driver feet, $1500 \mathrm{rpm}$ )

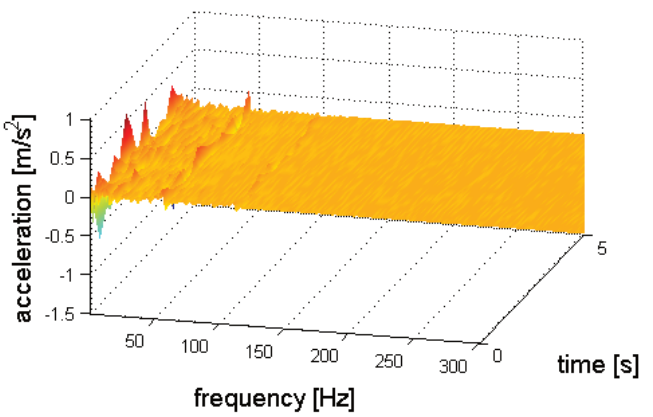

a) 1 st gear

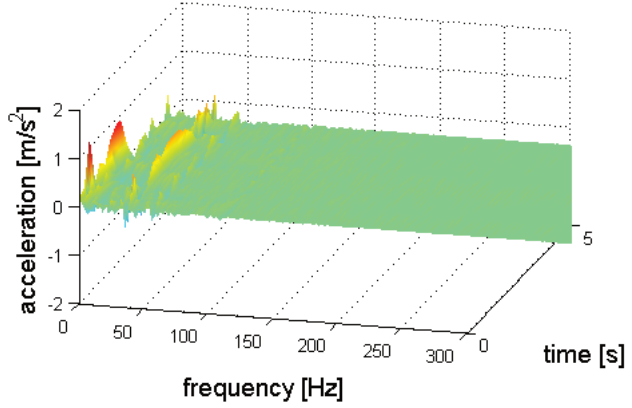

b) 5 th gear

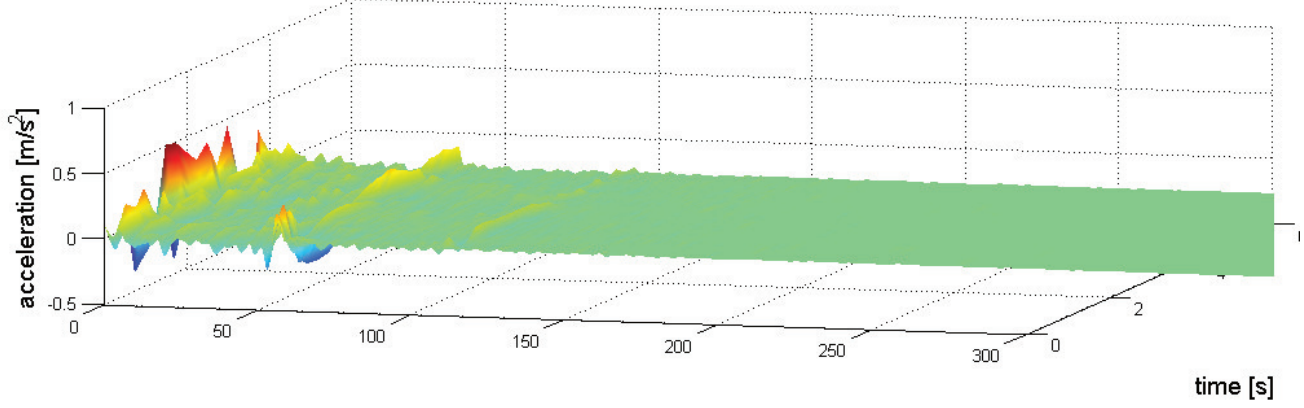

frequency $[\mathrm{Hz}]$

c) Idle gear

Fig. 14.18. The comparison of TFR of lateral vibration for neutral and first-speed and fifth-speed gear position (floor panel in location of driver feet, $1500 \mathrm{rpm}$ ) 


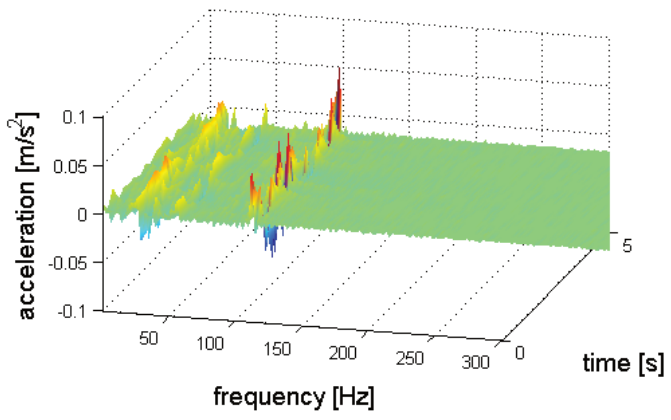

a) 1 st gear

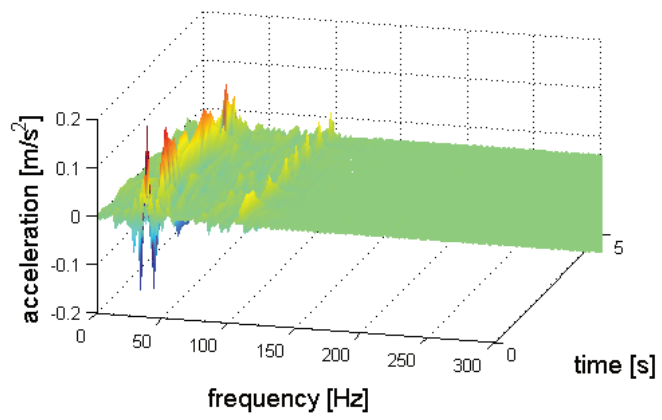

b) 5 th gear

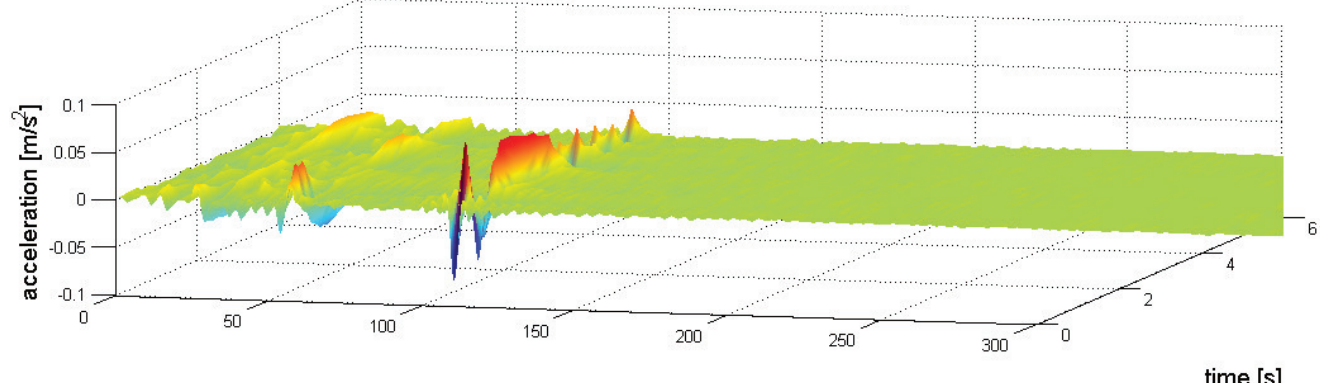

frequency $[\mathrm{Hz}]$

c) Idle gear

Fig. 14.19. The comparison of TFR of vertical vibration for neutral and first-speed and fifth-speed gear position (floor panel in location of driver feet, $1500 \mathrm{rpm}$ )

\subsection{Discussion on influence of gear position in transmission gearbox on vibration}

The directional distribution of vibration exposed on driver penetrate via feet for different gear position in gearbox, from first to fifth and reverse gear were presented in time, frequency and time-frequency domains. The successive gear ratio generate higher energy vibration with assumption that fifth and reverse gear vibration become a little lower. The values of estimators of energy of vibration considered in time, frequency and TFR representation of the signal were collected and compared to value reached for neutral gear, without gear ratio. All obtained results show an increase of energy of vibration compared to isolated engine vibration sources on the same rotational speed. The spectrums of the vibration allow to identify generation of lower to the main frequency components of the signal. Basing on the observation of TFR of vibration for different gear position some interesting phenomena can be found. Due to the increase of gear ratio the time distribution of the vibration of dominant frequency components become more constant.

For the purpose of identification of vibration components caused by the gearbox the result of vibration of neutral and first and fifth gears were summarized. The dynamic components can be identified by the comparison of spectrums and differential spectrum of neutral gear and running gear vibration. The results show that due to increase of gear ratio the correlation of spectrums of neutral and running gear become diminishes. The comparison of vibration spectrum of neutral and fifth gears shows many more different (low frequencies) components caused by the gear ratio. The differential spectrum allows to evaluate the influence of gear ratio on vibration dynamics. Due to the comfort and vibration exposure aspects it is important to analyse the energy, frequency but also the time of vibration components affecting on occupants. The TFR of the vibration collected as STFT of the vibration of gear ratio position and neutral idle gear position allow to observe simultaneous these exposure parameters. 


\section{Identification of vibration components of ride vehicle caused by road roughness}

The road unevenness determines the ride comfort, possible damage to goods, and vehicle wear and safety on one hand, and pavement damage and environmental noise and vibrations on the other hand. The study of traveling vehicle is the case of multiple sources of vibration interacting on vehicle and transfer into driver and passengers. In order to examine vibration related phenomena occurring in a moving vehicle or a stopped one with its engine in operation, identification of vibration sources should be done in the first place. The sources of vibration in a vehicle are dynamic forces but also free vibrations as well as forced, self-induced, parametrical, non-parametrical, random and stationary ones, all generated by the driving unit, the power transmission system and the road [41].

For the purpose of analysis of exposure to WHV and vibration absorption or isolation to the vehicle cabin the identification of different vibration sources should be performed. Thus the dedicated systems can be developed for vehicle vibration control and minimisation. The chapter presents simple method for identification vibration components caused by interior sources, i.e. engine and power transmission system and external sources as forces excited by the ground unevenness.

\subsection{Ride vehicle vibration generated by the ground unevenness}

Ride vehicle vibration results from interaction of a vehicle with: road roughness, aerodynamic forces, engine and driveline dynamics and tire/wheel assembly imbalance dynamics. All of these sources can induce vehicle body vibration and noise. The suspension system has conflicting demands, as isolation of the car-body from the forces generated by the ground unevenness and control of tire normal forces on the ground, thus the tires will have traction and lateral control (road holding).

The road roughness is the term used for deviations in a road surface compared to a real plane, which affect vehicle movement, ride quality, dynamic loads, drainage and winter maintenance. IRI is the most common metric to describe road roughness. It has become recognized as a general purpose roughness index and is strongly correlated to most kinds of vehicle responses that are of interest. The key importance of IRI is that road profiler users have shared experiences measuring IRI. Roughness is measured as the accumulated suspension stroke normalized by the total travelled distance. IRI is usually presented in engineering units such as $\mathrm{mm} / \mathrm{m}, \mathrm{m} / \mathrm{km}$ or inc/mile. It is highly correlated with acceleration of vehicle passengers (ride quality) and tyre load (vehicle controllability). Roads may have different names and visual characteristics around the world but researchers can compare vibration analyses results for roads with similar IRIs [146, 168].

Thus the ground excitation is very important. The description of road surface profile can be very helpful. The wave number of the road $\gamma$, is a measure of the rate of change with respect to distance or length. In space, we relate wave number $\gamma$, to wavelength, $\lambda$ :

$$
\lambda=\frac{2 \pi}{\gamma}
$$


The spatial cycle of wave length $\lambda$, is traversed by a vehicle with constant velocity $v$ during a period $T$, given by:

$T=\frac{\lambda}{v}$

If we use the relation between period $T$ and frequency $\omega$, we can write:

$\omega=\left[\frac{2 \pi}{\lambda}\right] v=\gamma v \Rightarrow v=f \lambda$

Thus the relation between frequency and forward vehicle velocity allows to calculate the frequency using the wavenumber, based description of a road profile, and velocity of the vehicle.

To consider the vehicle ride dynamics in terms of vibration the 3-directional movement has to be defined. Thus we can separate the roll (around the $X$ - longitudinal axis), pith (around the $Y$ - lateral axis) and bounce (in the $Z$ - vertical axis) motions of the car and especially car-body. Understanding pitch and bounce dynamics can provide insight into how the vehicle responds to a road profile. Bounce motion can be excited when the road has a wavelength equal to wheelbase (WB) and for much longer multiples and shorter with integer multiples. Pitch motion can be excited by wavelengths that are twice the WB, and by shorter wavelengths that are odd integer multiples of this value.

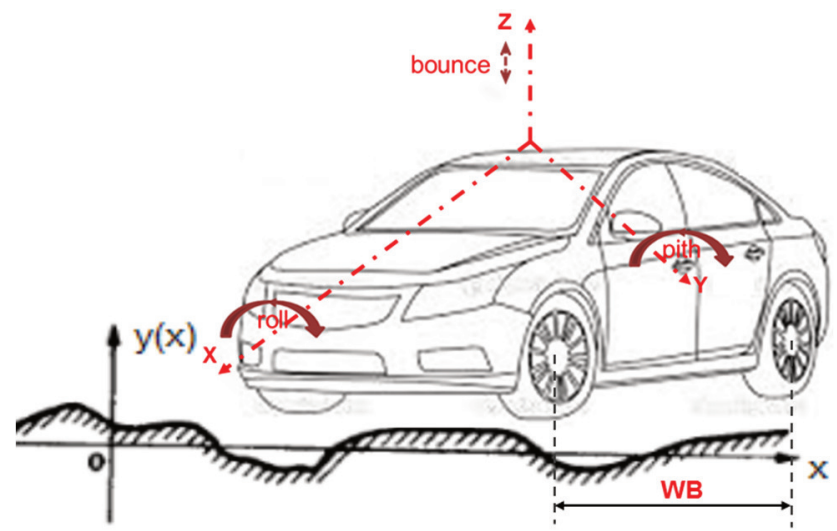

Fig. 15.1. Ride vehicle movement generated by the ground unevenness

\subsection{Method of identification of vibration components of riding vehicle caused by the road roughness}

The identification and separation of vibration components of riding vehicle caused by the powertrain system and road roughness requires large scope of the research included tests in laboratory and on the road. The same conditions of engine rotational speed and gear ratio of gearbox have to be conducted during each road test and laboratory research. For the elimination of the weather conditions the road tests were conducted in a tunnel. Thus allows to eliminate the wind forces from analysis. The section of the road was without any turns and equal rise level with angle between start and end of the road section ca. zero degree. Thus the lateral inertia forces and rolling resistance caused by hills have been minimalized.

For the purpose of proper identification of vibration transferred to car-body from road roughness it is necessary to provide measurement in multiple points located on vehicle construction. Human vibration perception depends on area and place of contact of human organism and vibrating machine. It depends also on dynamics of the vibration and exposure time. The identification of vibration transfer from road roughness to car-body in driving car was 
performed as the analysis of transformation of signals in time and frequency domains. This method of vibration signal processing allows to observe changes of the energy in selected frequency bands and correlate it with time.

\subsection{Identification of vibration components caused by the powertrain system and road roughness}

Due to the number of vibration sources affecting on motion of vehicle the method of comparison analysis have to be conducted. The scope of the research allows to assume that the dynamic responses of car-body are results of vibration sources i.e.: engine and powertrain system, road roughness impact on moving vehicle and aerodynamics resistances without wind forces.

First the comparison of time realization of vibration was collected in Figs. 15.2-15.4. It enables observation of vibration energy changes for the vehicle moving at $50 \mathrm{~km} / \mathrm{h}$ (Gear No. 3/2500 rpm) and $70 \mathrm{~km} / \mathrm{h}$ speed (Gear No. 5/2300 rpm) versus vehicle under laboratory test with the same gear number and engine rotational speed.

The chapter presents results of directional distribution of vibration of floor panel in location of driver feet as major WBV penetration surface into driver.

Basing on the analysis of directional distribution of vibration it was assumed that impact of aerodynamics resistances is much less important than road roughness in terms of generation of vibration in dynamic response. Thus it was assumed that it may be negligible. The dynamic components caused by the ride on the road roughness can be identified by the comparison of spectrums and differential spectrums of moving vehicle and laboratory tested vehicle at the same gear ratio and engine rotational speed, expressed from equation:

$F_{d}(\omega)=\left|F_{r}(\omega)-F_{g}(\omega)\right|$,

where: $F_{r}(\omega)$ - Fourier transform of vibration of riding vehicle, $F_{g}(\omega)$ - Fourier transform of running gear vibration.

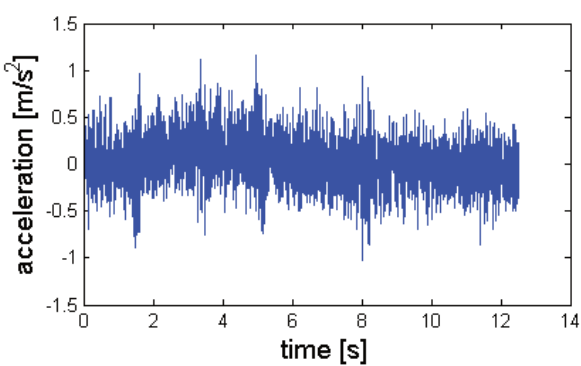

a) Road test - gear No. 3/2500 rpm

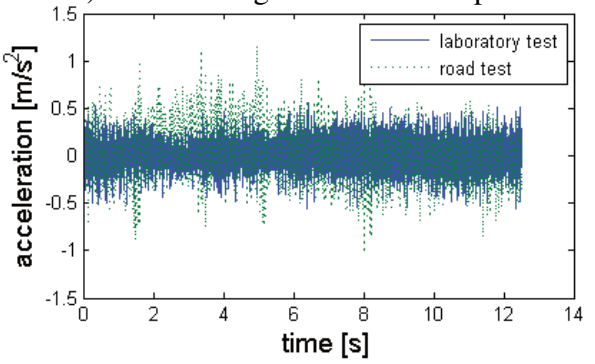

c) Laboratory test vs. road test gear No. 3/2500 rpm

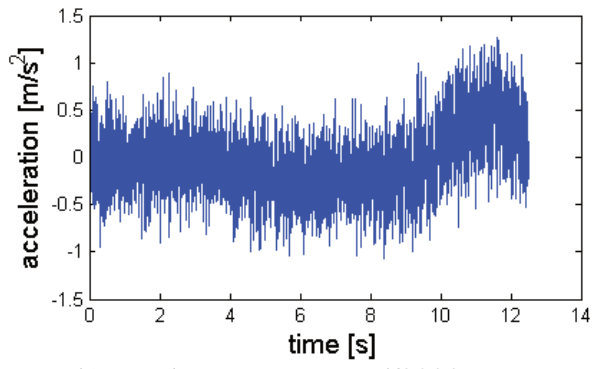

b) Road test - gear No. $5 / 2300 \mathrm{rpm}$

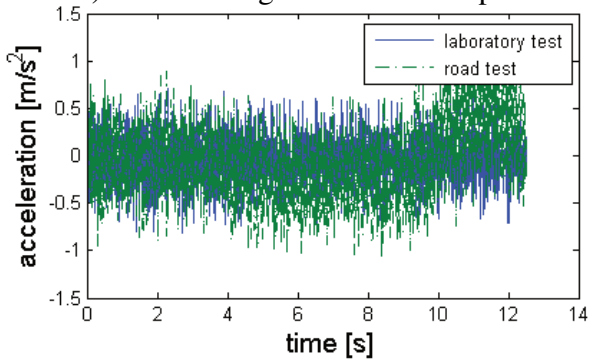

d) Laboratory test vs. road test gear No. 5/2300 rpm

Fig. 15.2. The comparison of longitudinal vibration of vehicle moving at $50 \mathrm{~km} / \mathrm{h}$ and $70 \mathrm{~km} / \mathrm{h}$ speed versus laboratory tested vehicle at the same gear ratio and engine rotational speed

(floor panel in location of driver feet) 


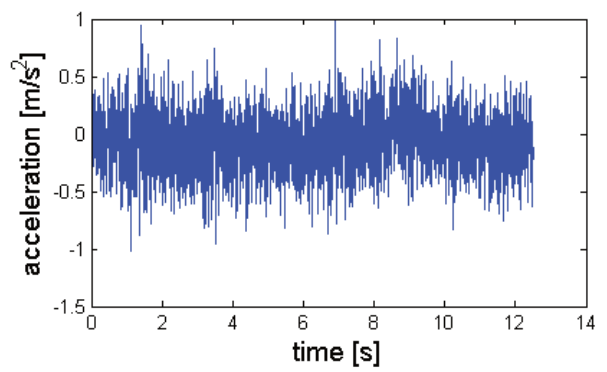

a) Road test - gear No. $3 / 2500 \mathrm{rpm}$

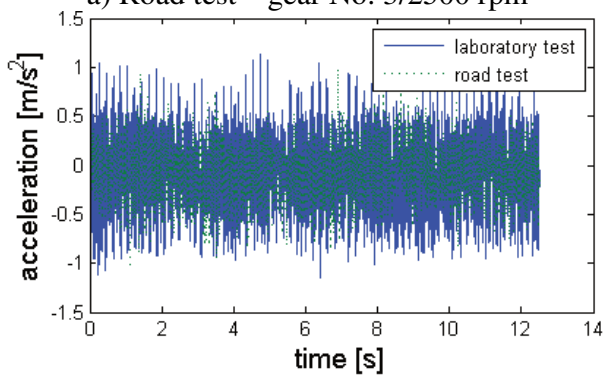

c) Laboratory test vs. road test gear No. 3/2500 rpm

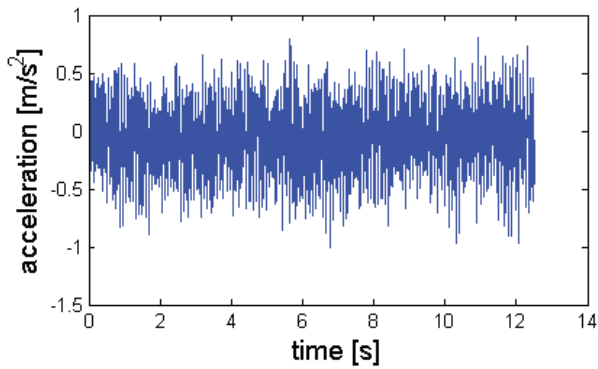

b) Road test - gear No. 5/2300 rpm

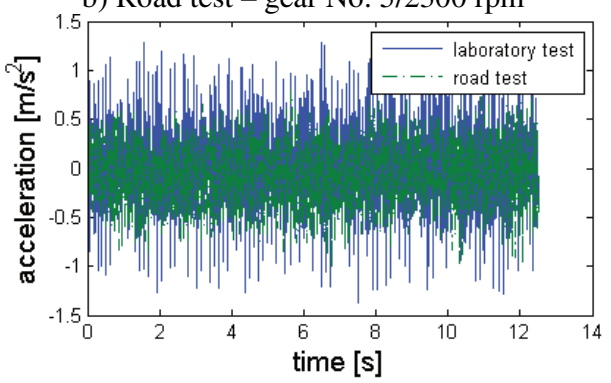

d) Laboratory test vs. road test gear No. $5 / 2300 \mathrm{rpm}$

Fig. 15.3. The comparison of lateral vibration of vehicle moving at $50 \mathrm{~km} / \mathrm{h}$ and $70 \mathrm{~km} / \mathrm{h}$ speed versus laboratory tested vehicle at the same gear ratio and engine rotational speed

(floor panel in location of driver feet)

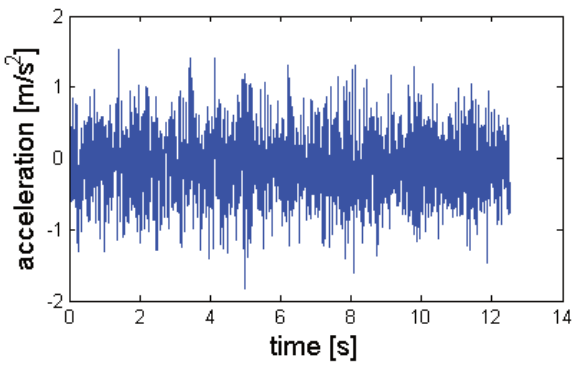

a) Road test - gear No. 3/2500 rpm

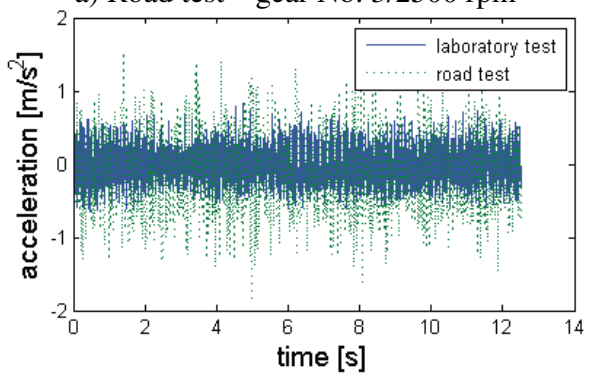

c) Laboratory test vs. road test gear No. 3/2500 rpm

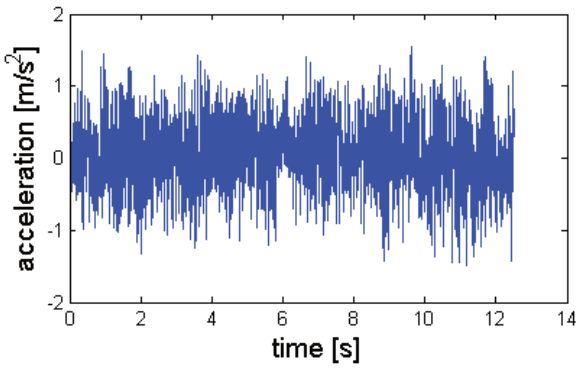

b) Road test - gear No. 5/2300 rpm

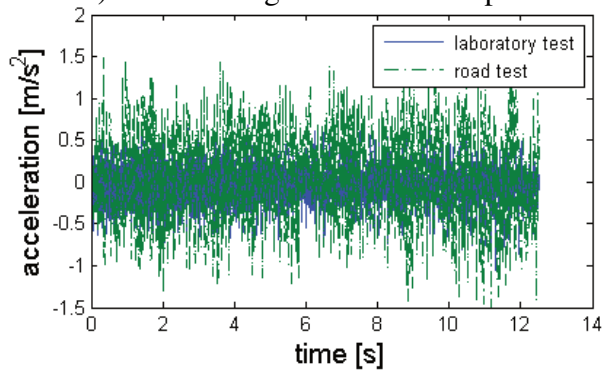

d) Laboratory test vs. road test gear No. 5/2300 rpm

Fig. 15.4. The comparison of vertical vibration of vehicle moving at $50 \mathrm{~km} / \mathrm{h}$ and $70 \mathrm{~km} / \mathrm{h}$ speed versus laboratory tested vehicle at the same gear ratio and engine rotational speed

(floor panel in location of driver feet)

For the analysis of structure of vibration the TFR of the signals of moving vehicle and laboratory tested vehicle at the same gear ratio and engine rotational speed are presented in Figs. 15.8-15.10. 


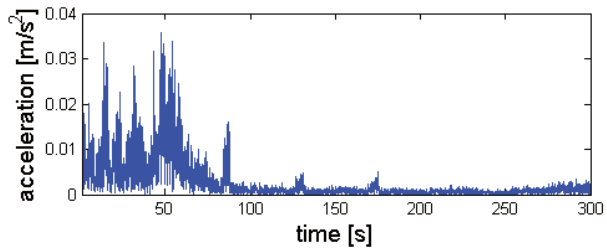

a) Road test - gear No. 3/2500 rpm

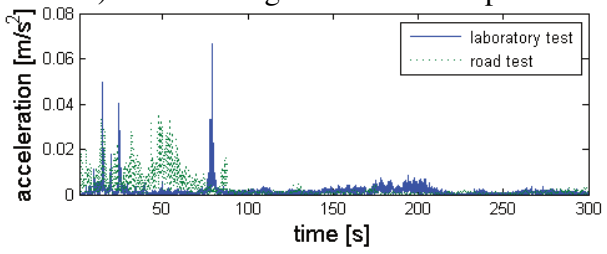

c) Laboratory test vs. road test gear No. 3/2500 rpm

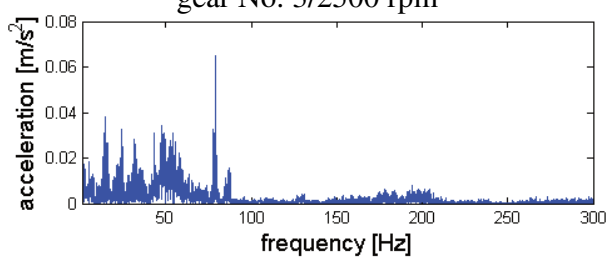

e) Differential spectrum of laboratory test and road test - gear No. 3/2500 rpm

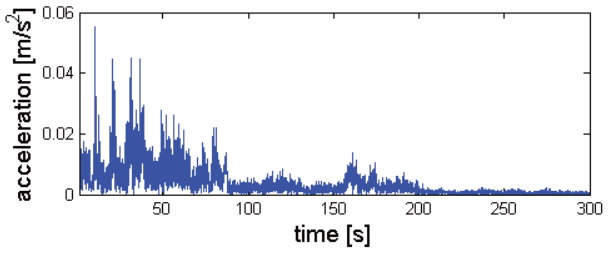

b) Road test - gear No. 5/2300 rpm

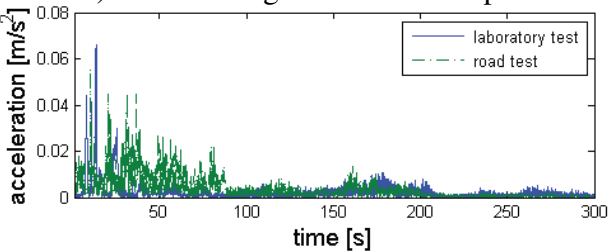

d) Laboratory test vs. road test gear No. 5/2300 rpm

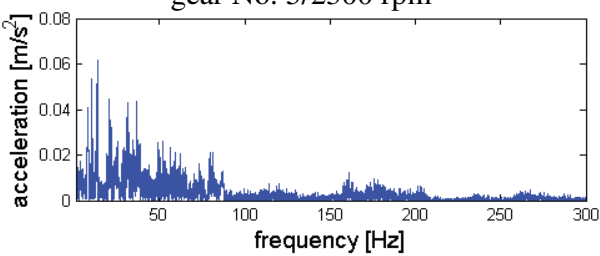

f) Differential spectrum of laboratory test and road test - gear No. 5/2300 rpm

Fig. 15.5. The comparison of spectrums and differential spectrums of longitudinal vibration of vehicle moving at $50 \mathrm{~km} / \mathrm{h}$ and $70 \mathrm{~km} / \mathrm{h}$ speed versus laboratory tested vehicle at the same gear ratio and engine rotational speed (floor panel in location of driver feet)

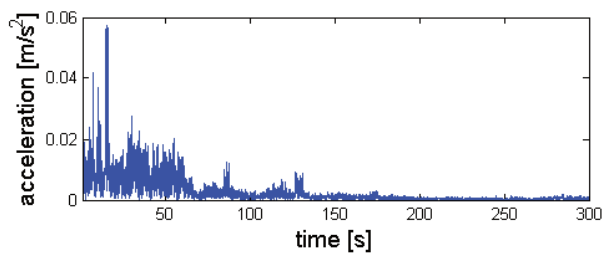

a) Road test - gear No. $3 / 2500 \mathrm{rpm}$

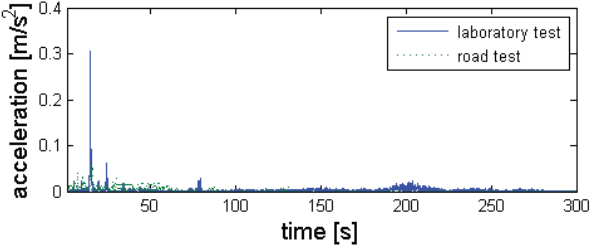

c) Laboratory test vs. road test gear No. 3/2500 rpm

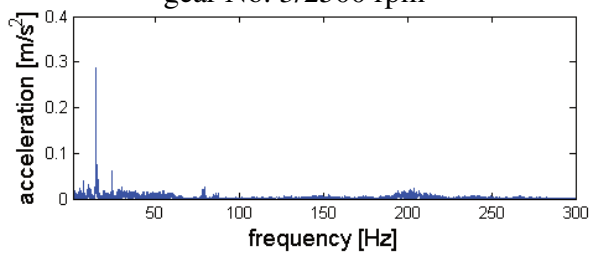

e) Differential spectrum of laboratory test and road test - gear No. 3/2500 rpm

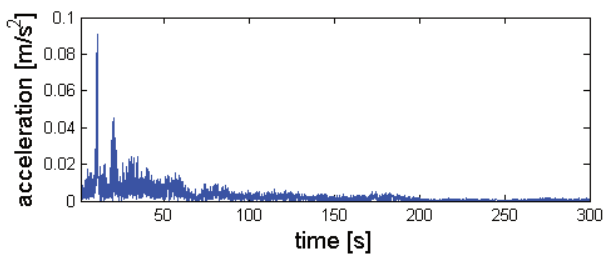

b) Road test - gear No. $5 / 2300 \mathrm{rpm}$

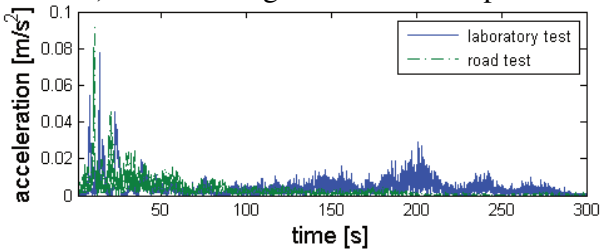

d) Laboratory test vs. road test gear No. 5/2300 rpm

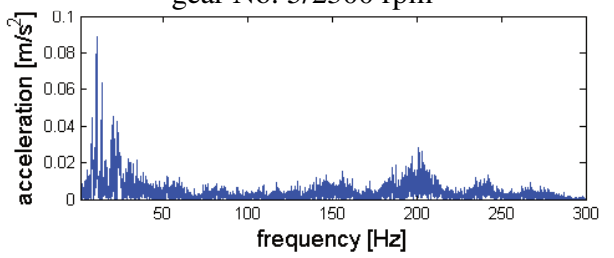

f) Differential spectrum of laboratory test and road test - gear No. 5/2300 rpm

Fig. 15.6. The comparison of spectrums and differential spectrums of lateral vibration of vehicle moving at $50 \mathrm{~km} / \mathrm{h}$ and $70 \mathrm{~km} / \mathrm{h}$ speed versus laboratory tested vehicle at the same gear ratio and engine rotational speed (floor panel in location of driver feet) 


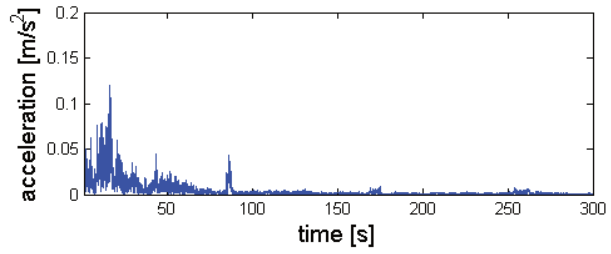

a) Road test - gear No. 3/2500 rpm

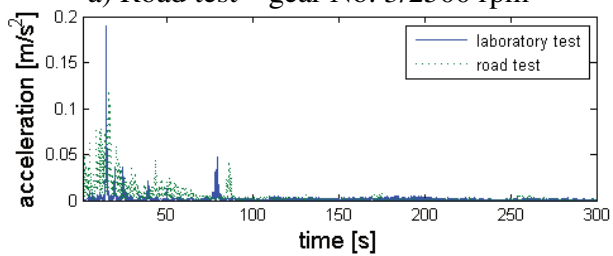

c) Laboratory test vs. road test gear No. 3/2500 rpm

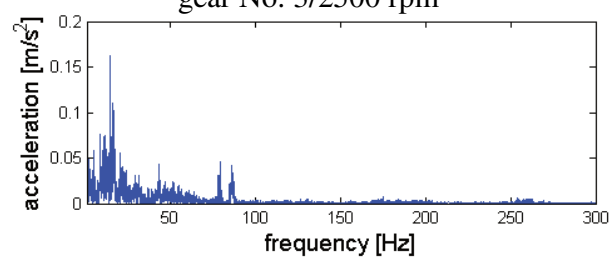

e) Differential spectrum of laboratory test and road test - gear No. 3/2500 rpm

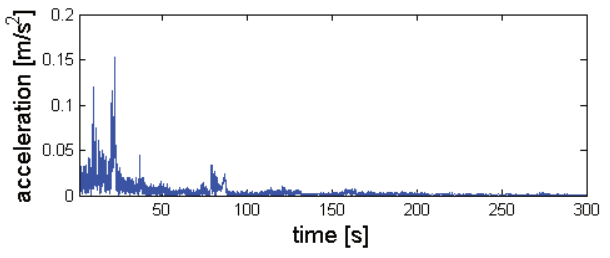

b) Road test - gear No. $5 / 2300 \mathrm{rpm}$

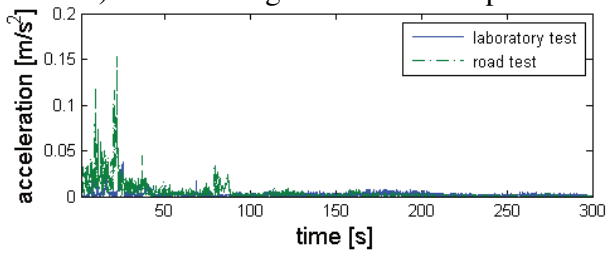

d) Laboratory test vs. road test gear No. 5/2300 rpm

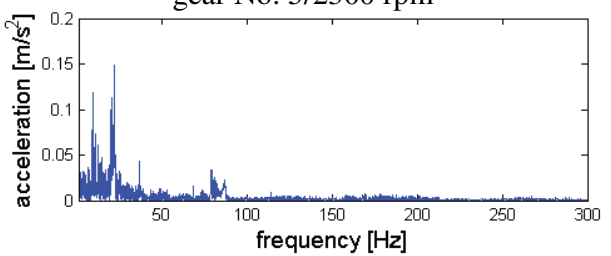

f) Differential spectrum of laboratory test and road test - gear No. 5/2300 rpm

Fig. 15.7. The comparison of spectrums and differential spectrums of vertical vibration of vehicle moving at $50 \mathrm{~km} / \mathrm{h}$ and $70 \mathrm{~km} / \mathrm{h}$ speed versus laboratory tested vehicle at the same gear ratio and engine rotational speed (floor panel in location of driver feet)

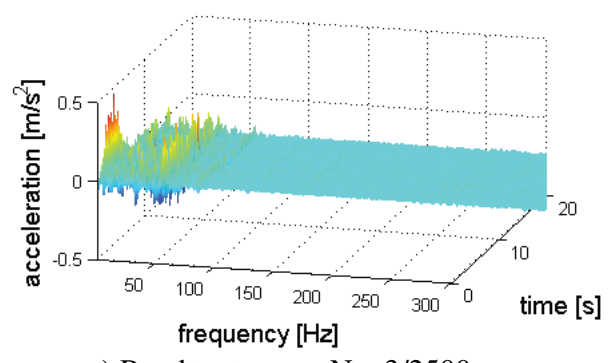

a) Road test - gear No. 3/2500 rpm

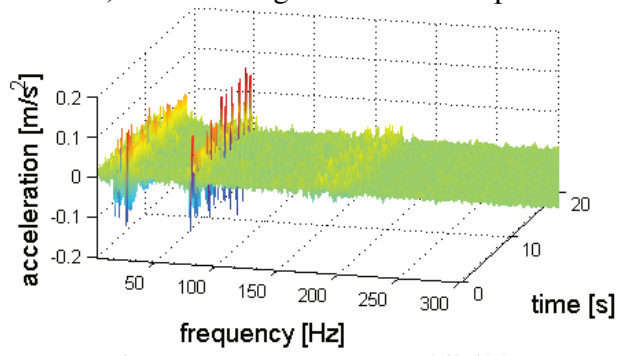

c) Laboratory test - gear No. 3/2500 rpm

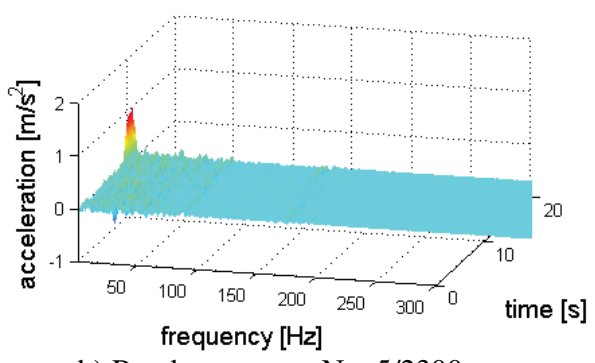

b) Road test - gear No. 5/2300 rpm

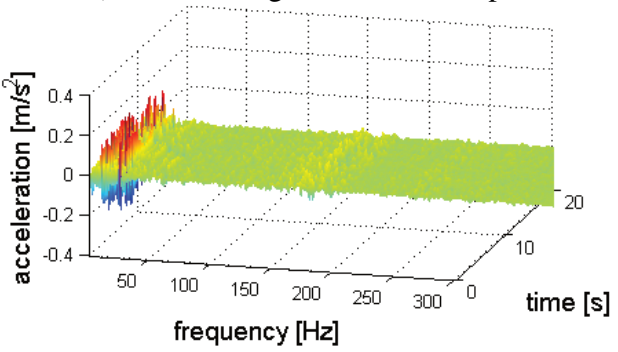

d) Laboratory test - gear No. 5/2300 rpm

Fig. 15.8. The comparison of TFR of longitudinal vibration of vehicle moving at $50 \mathrm{~km} / \mathrm{h}$ and $70 \mathrm{~km} / \mathrm{h}$ speed versus laboratory tested vehicle at the same gear ratio and engine rotational speed (floor panel in location of driver feet)

Analysis of distributions of vibration in time, frequency domains and its TFR allows to identify all components of the vibration caused by the road roughness and powertrain system. These presentations enable observing the time of excitation on defined frequencies. For the comparison 
of total energy of vibration transferred into the human organism the distribution of estimators defined in previous chapters are collected in Fig. 15.11.

The chapter presents some results of identification of structure and directional distribution of vibration transferred to car-body from road roughness.

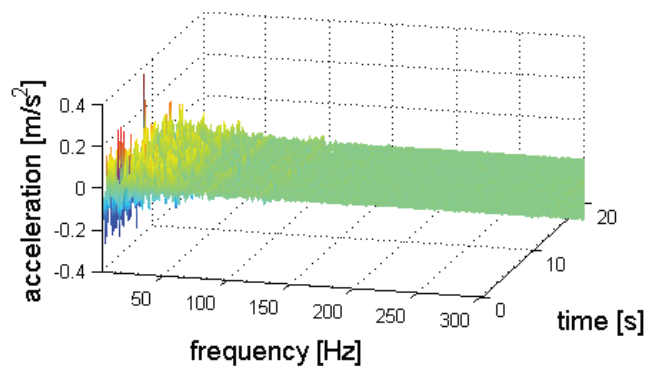

a) Road test - gear No. 3/2500 rpm

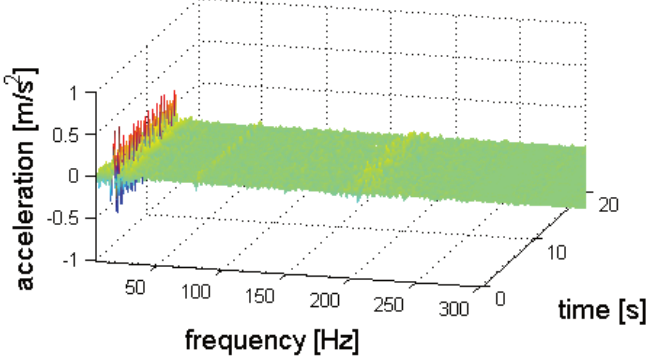

c) Laboratory test - gear No. 3/2500 rpm

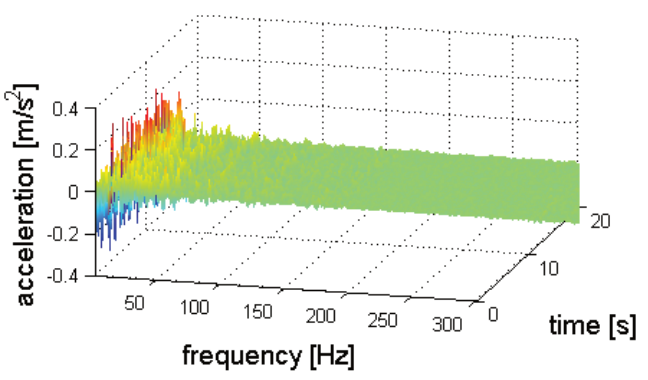

b) Road test - gear No. 5/2300 rpm

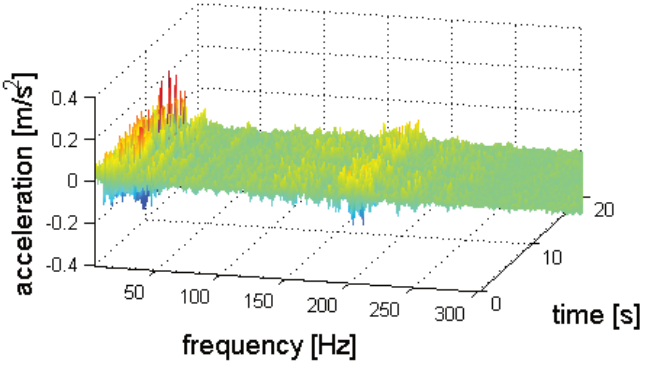

d) Laboratory test - gear No. 5/2300 rpm

Fig. 15.9. The comparison of TFR of lateral vibration of vehicle moving at $50 \mathrm{~km} / \mathrm{h}$ and $70 \mathrm{~km} / \mathrm{h}$ speed versus laboratory tested vehicle at the same gear ratio and engine rotational speed

(floor panel in location of driver feet)

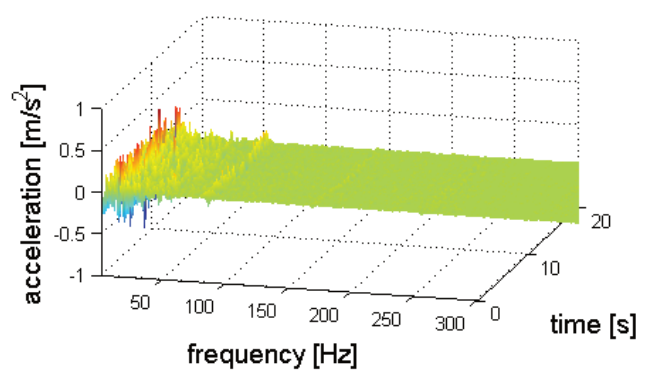

a) Road test - gear No. 3/2500 rpm

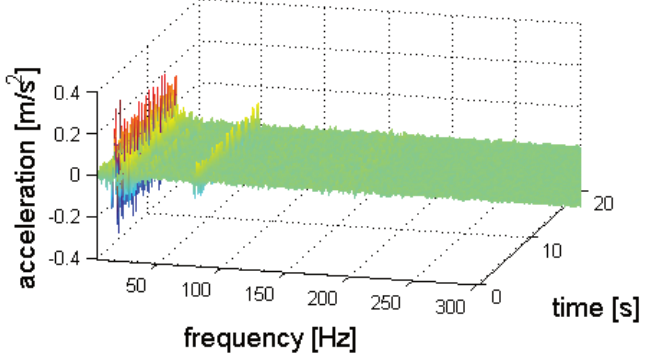

c) Laboratory test - gear No. 3/2500 rpm

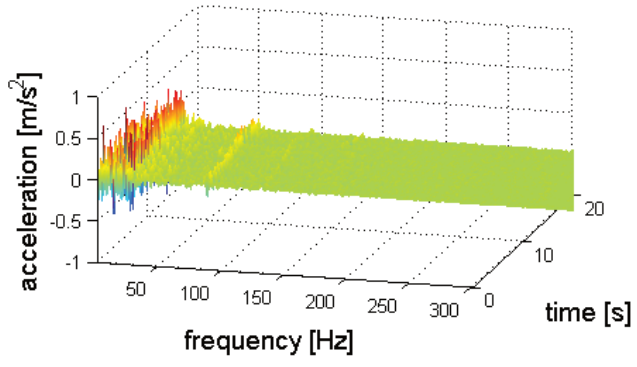

b) Road test - gear No. 5/2300 rpm

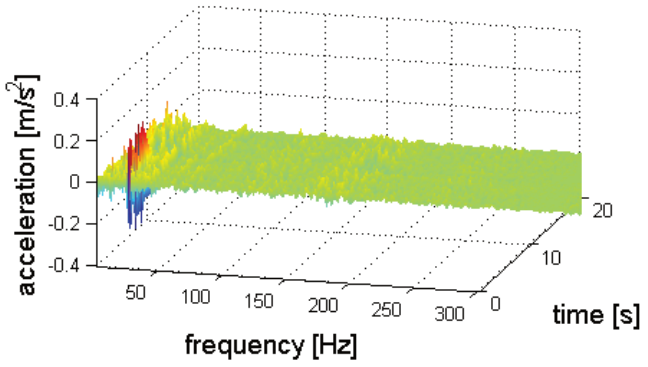

d) Laboratory test - gear No. 5/2300 rpm

Fig. 15.10. The comparison of TFR of vertical vibration of vehicle moving at $50 \mathrm{~km} / \mathrm{h}$ and $70 \mathrm{~km} / \mathrm{h}$ speed versus laboratory tested vehicle at the same gear ratio and engine rotational speed

(floor panel in location of driver feet) 


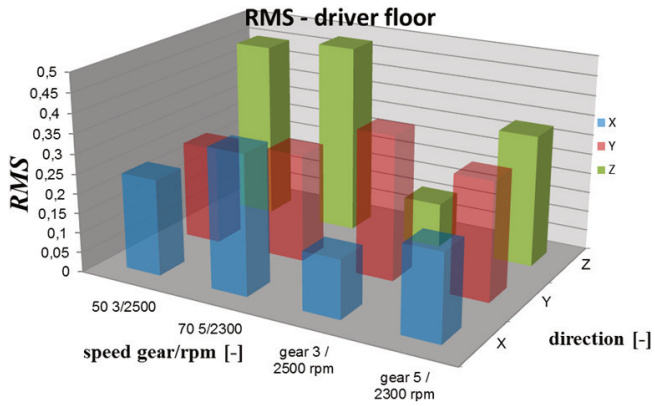

a) $R M S$

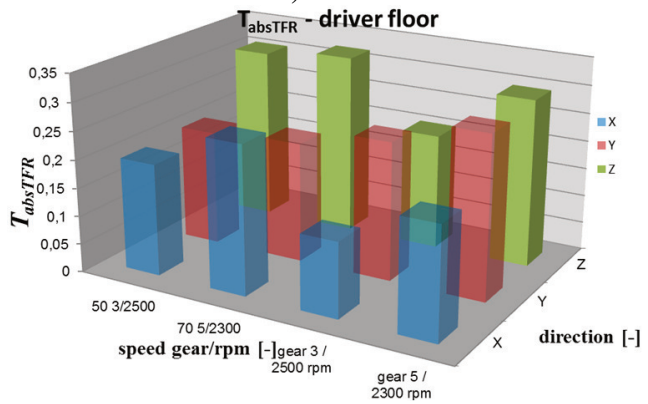

c) $T_{\text {absTFR }}$

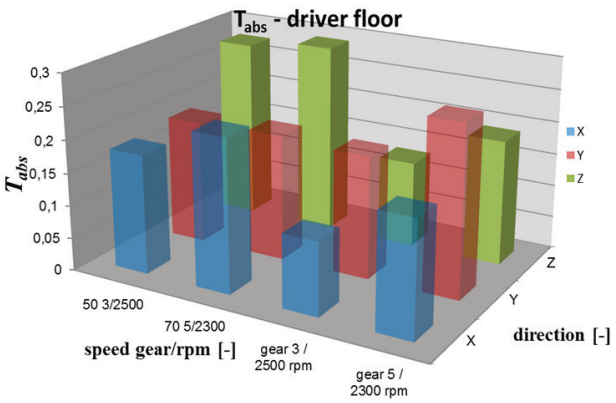

b) $T_{a b s}$

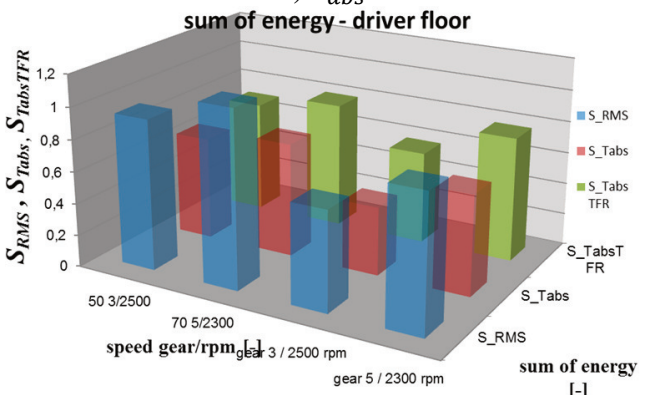

d) Sum of energy

Fig. 15.11. The comparison of energy estimators of vibration of vehicle moving at $50 \mathrm{~km} / \mathrm{h}$ and $70 \mathrm{~km} / \mathrm{h}$ speed versus laboratory tested vehicle at the same gear ratio and engine rotational speed

(floor panel in location of driver feet)

The method proposed for identification of components of road irregularity induced vibrations for a moving vehicle may be brought down to comprehensive laboratory and road tests of the same vehicle while maintaining identical engine and power transmission operating parameters. It can be achieved by isolating components correlated with the signals obtained in the course of laboratory tests of a motionless car, excited to vibrate by an engine operating at a constant rotational speed and with the given transmission ratio, from the recorded signals of directional vibration propagation of a moving vehicle's load bearing structure. The foregoing is most evident in the difference spectrum function defined, one which represents the frequency distribution of absolute values based on the difference of vibration spectra obtained in road and laboratory tests.

By application of the TFR analysis a conclusion of the inferior stability of time distributions for predominant frequency components can be drawn, and hence the superior non-stationary characteristic of vibration signals of a moving vehicle, which only confirms the need for the TFR method to be applied to assess the human exposure to vibrations in transport.

A comparison of estimator distributions reveals the predominant share of vertical vibration components for a moving car. As for the laboratory tests, which was explicitly laid down in the previous sections as well, a considerable share in the directional distribution of vibrations can also be attributed to transverse vibration components. Hence one can deduce that the difference in question results from the stability difference between a car placed on a test rig (with brackets lifting the car) and a car tested on the road. For the manner in which the car is set on a foundation in laboratory studies, it may display higher propensity for side tilts due to being excited to vibrate. Irrespective of the foregoing, in each case analysed, both for directional and total estimators, the vibration energy of a moving vehicle was larger. Having analysed the differences between these measures, the volume of energy of the vibrations caused by road irregularities and aerodynamic resistances of a moving vehicle can be estimated. 


\section{Influence of vehicle technical condition and power transmission system on evaluation of human exposure to whole body vibration penetrated via feet}

The statistics reported in western European countries imply that the number of people exposed to general vibrations come to 4-7\% of all working persons [79]. Studies [96] devoted to work-related risk of exposure to general mechanical vibrations in professional environments provide numbers of people exposed to vibrations broken into 6 NDN classes. They mention the following professional groups as the ones most endangered by general vibrations: drivers of locomotives and other traction vehicles - 10.211 persons, drivers of motor and delivery trucks -7.811 persons, bus drivers -5.740 persons, tractor drivers -4.053 persons, fork-lift truck operators -1.603 persons, loading machine operators - 1.607 persons, excavating machine operators -1.513 persons, bulldozer operators -1.325 persons, operator of other heavy gear -772 persons and operator of road machinery -728 persons. As regards vehicle drivers, they correspond to $15-30 \%$ of all persons employed. With reference to European data (4-7 \%) or results of national studies (ca. $0.5 \%$ ), the share of people exposed to vibrations in means of transport is far larger [96].

The occupants of means of transport assess the ride discomfort on the grounds of the accelerations to which they are exposed. The harmful effects of vibrations generated by any means of transport on the human body chiefly depend on the vibration amplitude and frequency. In most cases, a measure of the health risk and danger is based on the fact whether the vibration volume exceeds a specific limit that is determined experimentally. Important is also the duration of exposure, especially in the situation of increasing amount of time spent by people when riding vehicles [197].

All research described in previous chapters were focused on identification of vibration sources and paths of propagation in vehicle. The scope of the research included investigation on influence of chosen operating parameters of investigated sources on vibration propagation into the vehicle structure in locations where vibration penetrate into the human organism. Thus the influence of technical condition of suspension elements, vehicle operating parameters, engine rotational speed and gear ratio on evaluation of human exposure to whole body vibration can be performed.

There are many publication, documents, standards describing human exposure to vibration but the state of art on influence of mechanical systems conditions or operating parameters of machine on human exposure to vibration is much fewer in numbers of publications. Some interesting investigation on influence of chosen driving parameters on vibration comfort according to Human-Vehicle-Road (HVR) model and vibration exposure metric described in the ISO 2631 are presented in [127]. In a wide variety of transport environments the vibration transmitted through seats is associated with discomfort [197]. Seats can either reduce vibration discomfort or increase vibration discomfort [88]. The paper [88] presents results of the study on determine how factors, as age, gender, physical characteristics, backrest contact, and magnitude of vibration affect seat transmissibility.

The chapter presents some results of different methods evaluation of exposure to WBV for different suspension technical condition and power transmission system operating parameters. 


\subsection{Human exposure to Whole-Body vibration}

Mechanical vibrations are among the harmful physical factors occurring in working environments. Overloading an organism with vibrations contributes to development of non-specific pathological processes, also known as the head-arm vibration syndrome, which has been entered into the list of professional diseases. The nature and the location of health effects depend on the penetration point and propagation of vibrations in the organism.

The prediction of spinal stress is a prerequisite for a quantitative assessment of the health risk of the lumbar spine. Repetitive peak compressive forces are assumed to be responsible for fatigue failure of vertebral endplates [169-171]. The processing of predicted compressive forces in the time domain enables the calculation of a dose measure characterising the probability of fatigue failure. One procedure for the quantification of health risk described in ISO 2631-5.

Although the human body dampens most vibration frequencies transmitted through the operator-seat interface, WBV between $1 \mathrm{~Hz}$ and $20 \mathrm{~Hz}$ results in resonance of the spinal column, pelvis, internal organs and soft tissues. Health effects associated with short term WBV include muscle fatigue, discomfort, distorted motor performance, headache, loss of balance, motion sickness, increased heart rate, hyperventilation, decreased cognitive functions, as well as, diminished speech and vision. An even greater concern arises from chronic health effects associated with regular exposure to WBV, which include: spinal degeneration, spinal disc disease, disc failure, sciatic pain, herniated discs, low back pain, and gastrointestinal disorders.

\subsection{The standards of WBV for means of transport}

These standards comprise a very broad area of standardization with a small, but important, portion of it directly related to shock and vibration. ISO TC 108/SC 2 (Measurement and Evaluation of Mechanical Vibration and Shock as Applied to Machines, Vehicles, and Structures) is involved with the vibration of ships, and ISO 4867, 4868, and 6954 specifically address the measurement and reporting of vibration onboard ships. Much of the U.S. participation in this work is contributed by members of the Society of Naval Architects and Marine Engineers (SNAME). ANSI S2.16 covers the measurement and acceptance criteria for the vibratory noise of shipboard equipment, and ANSI S2.25 covers the evaluation and reporting of hull and superstructure vibration in ships. ISO TC 108/SC 2 is also involved with vibration of land-based vehicles, and ISO 8002, 8608, and 10326 are specifically related to the evaluation and reporting of the vibration associated with either land-based vehicles or road surface profiles. ISO TC 20 (Aircraft and Space Vehicles) is involved with standards related to aerospace vehicles in general, and a number of ISO technical committees exist that generally cover specific types of land-based vehicles, e.g., construction, agricultural, and off-road vehicles. The U.S.TAG for ISOTC 20 and the U.S.TAGs for many of the ISO technical committees on land-based vehicles in general are administered by the Society of Automotive Engineers (SAE). The CEN document CEN EN 1032 on testing mobile machinery has been published, and CEN TC 231 with respect to testing mobile machinery to determine whole-body vibration and vibration emission values. CENTC 231 maintains liaisons with CEN TC 144 and CEN TC 151 on tractors and agricultural machines, and construction equipment, respectively.

Human exposure to shock and vibration, as the program of work on human exposure to shock and vibration is assigned to ISO TC 108/SC 4 (Human Exposure to Mechanical Vibration and Shock). ISO TC 108/SC 4 maintains liaisons with about a dozen ISO technical committees and subcommittees including ISO TC 43 (Acoustics), as well as with other organizations such as the European Committee of Associations of Manufacturers of Agricultural Machinery (CEMA), the International Maritime Organization (IMO), and the International Union of Railways (UIC). There are a number of ISO and ANSI standards on exposure to whole-body and hand-arm vibration including standards covering occupants of fixed-structures, single shocks, guidance on safety aspects of tests and experiments, transmissibility of gloves and resilient materials, and terminology. 
According to technical report of European Commission Quality of Life and Management of Living Resources [142] most of these procedures ignores the fact that only one time series of compressive force exists as a result of the superposition of different components. Hence, any sufficient dose of peak values can be based on one time series only and not on peak values of several non-existing components. The origin of this error is the approach described in [142]. The models used in ISO 2631-5 has been previous described by Seidel in [6]. The existing methods for the quantification of health risk caused by WBV require time series of internal forces acting on lumbar vertebral endplates. The later research of Siedal presented in [170] assumed that this model cannot be considered as verified. Many aspects of a critical analysis and discussion of the very first results of research conducted under Morrison [142] underlying ISO 2631-5, were interpreted and published in [169]. The possible underestimation of health risk described in publication of Siedel deserves special attention. Variable postures, anthropometric characteristics, spinal geometry, and individual spinal tolerance were identified as important factors that codetermine the effects of occupational WBV on health. A re-analysis of the paper [140] led to the conclusion that the exponent derived from this study [6] should not be used for the dose calculation, because the pronounced non-linearity of the stress-strain relationship. It does not permit the calculation of a correct equivalent stress.

The European Directive (2002) states an extremely high limit value for whole-body vibration (WBV) in $z$-direction without any limitation of an energy-equivalent evaluation. The consequence is a very dubious assessment of health effects, especially for WBV containing high peak values and/or short daily exposure times [90].

Unlike ISO 2631-5 (2004), the FIOSH-approach considers additionally significant variables like posture, body mass and body height, body mass index (BMI), disc area, disc level. Combinations of body mass, body height and BMI were chosen in order to provide representative characteristics of European drivers. It offers the possibility to predict the health risk for different shares of the exposed population as a contribution to subsequent decisions on tolerated risks [11]. There are further differences between the FIOSH-approach and the method described by ISO 2631-5 (2004). A variable static stress is predicted instead of the "constant c representing the static stress due to gravitational force" in ISO 2631-5, $R$ factor equation. Another consequence is the variable dynamic stress predicted for different anthropometric characteristics. The FIOSH-approach also offers the possibility to use different exponents for the dose calculations. The procedure was developed to assess the health risk related to whole-body vibration (WBV) based on the results of model calculations and using the prediction of cumulative fatigue failure of vertebral endplates caused by compression as criterium. The procedure permits the consideration of individual exposure conditions, posture and personal characteristics which are reflected by different finite element models [76].

Since 2002 the implementation of the provisions of Directive 2002/44/EC of the European Parliament and of the Council on the minimum health and safety requirements regarding the exposure of workers to the risks arising from physical agents (vibration) were presented. In 2007 as the annex 19 to final technical report of European Commission Quality of Life and Management of Living Resources, programme Key Action 4 - Environment and Health Risks of Occupational Vibration Exposures, prediction of spinal stress in drivers from field measurements were published.

\subsection{The model of vibration spine response - ISO 2631}

Despite of discussion presented above the most popular standards for measurement and evaluation of human response to whole-body vibration are ISO 2631-1 (1997) and ISO2631-5 (2004). ISO 2631 - Mechanical vibration and shock - Evaluation of human exposure to whole-body vibration, was prepared by Technical Committee ISO/TC 108, Mechanical vibration and shock, subcommittee SC 4, Human exposure to mechanical vibration and shock. It is consists of the following parts: 
- Part 1: General requirements,

- Part 2: Vibration in buildings (1 Hz to $80 \mathrm{~Hz}$ ),

- Part 4: Guidelines for the evaluation of the effects of vibration and rotational motion on passenger and crew comfort in fixed-guideway transport systems,

- Part 5: Method for evaluation of vibration containing multiple shocks.

For the assessment of the effects of vibration on health, it is proposed to use the frequency-weighted RMS of ISO 2631-1 based on translational motion of the each axis on the seat surface. ISO 2631-1 also suggests that if the crest factor is less than 9, the weighted RMS is normally sufficient for evaluation of vibration. On the other hand, ISO 2631-5 provides the additional guidance on assessment of vibration containing multiple shocks. ISO 2631-5 focused on the lumbar response in humans exposed to whole-body vibration. Although the assessment point on humans differ depending on the ISO standard indices, it is necessary to compare effects of vibration on health by the current standard (ISO2631-1) with that by the multiple shocks standard (ISO2631-5).

In ISO2631-5 recommendations, the vibration effects to the human body are evaluated by compression stress in the lumbar spine. The frequency-weighted spinal stress is calculated.

In the $x$ - and $y$-axes, the spinal response is approximately linear and is represented by a single-degree-of-freedom (SDOF) lumped-parameter model. The lumbar response is calculated from equation:

$a_{l k}(t)=2 \varsigma \omega_{n}\left(v_{s k}-v_{l k}\right)+\omega_{n}^{2}\left(s_{s k}-s_{l k}\right)$,

where: $\varsigma$ - critical damping ration $(\varsigma=0.22), \omega_{n}$ - natural frequency, $k-X$ or $Y$ axes, $s_{s k}, s_{l k}-$ displacement time histories in the seat and in the spine, $v_{s k}, v_{l k}$ - velocity time histories in the seat and in the spine.

In the $z$-direction, the spinal response is non-linear and can be represented by a recurrent neural network (RNN) model. Lumbar spine $Z$-axis acceleration is predicted by using the following equations:

$a_{l z}=\sum_{i=1}^{7} W_{j} u_{j}(t)+W_{8}$

$u_{j}(t)=\tanh \left[\sum_{i=1}^{4} w_{j i} a_{l z}(t-i)+\sum_{i=5}^{12} w_{j i} a_{s z}(t-i+4)+w_{j 13}\right]$,

where: $w_{j i}$ - connection weights are given in Table 2 in the draft of ISO 2631-5, $W_{j}-z$-axis model coefficients are given in Tables 1 in the draft of ISO 2631-5, $a_{s}$-seat acceleration, $a_{l}-$ lumbar spine acceleration, $a_{l z}(t-i)$ - feed back delayed output, $a_{s z}-z$-axis seat acceleration.

The RNN model for the $\mathrm{z}$ axis presented in ISO 2631-5 was trained using vibration in the range of $-20 \mathrm{~m} / \mathrm{s}^{2}$ to $40 \mathrm{~m} / \mathrm{s}^{2}$ and $0.5 \mathrm{~Hz}$ to $40 \mathrm{~Hz}$ with frequency sampling $160 \mathrm{~Hz}$. As the model is non-linear, this constitutes the range of the applicability of this part of ISO 2631-5.

\subsection{WBV evaluation}

The vibration evaluation base on many methods and equations for assessment of health effects and risk. The section shows chosen equation of WBV evaluation.

The vibration evaluation according to ISO 2631 shall always include measurements of the weighted root mean square acceleration. This method evaluates the vibration transmitted to the body through the supporting surfaces. The standard defines the total vibration value of weighted $R M S$ acceleration for all directions in respective position.

The method of determining the vibration exposure and discomfort is the use of a measure defined as the vector sum of squared (or $R M S$ ) acceleration values for particular vibration axes 
(components), with extracting the root thereof. For the purpose of research goal the weighting factors for directions of floor panelvibration were used as 1.4 for horizontal directions. This approach is based on the running $R M S$ method (MTVV). However it takes into account dominant direction of the vibration, instead of occasional shocks and transient vibration by use of a short integration time constant. The vibration magnitude is defined as a maximum transient vibration value $\left(A_{\text {wmax }}\right)$, given as the maximum in time of root mean square acceleration $a_{d}(t)$. The $A_{w \max }$ is expresses as:

$$
A_{w \max }=\max \left\{1.4 R M S\left(a_{x}\right), 1.4 R M S\left(a_{y}\right), \operatorname{RMS}\left(a_{z}\right)\right\}
$$

where: $R M S$ - root mean square of vibration signal, $a_{x}$ - acceleration of vibration in $X$ axis, $a_{y}-$ acceleration of vibration in $Y$ axis, $a_{z}$ - acceleration of vibration in $Z$ axis.

For the long term exposure to vibration, i.e. work shift period as 8 hour, the daily exposure to vibration $A(8)$ is expressed as:

$A(8)_{l}=\sqrt{\frac{1}{T} \sum_{i=1}^{n} X_{R M S d_{i}} t_{i}}$,

where: $A(8)_{l}$-daily (8 hour) exposure to vibration, $X_{R M S d_{i}}$ - root mean square of vibration signal in $i$-direction, $t_{i}$ - time of exposure to vibration in separate direction (minutes), $T$ - total time of exposure to vibration $A(8)$ is calculated for 8 hours (480 min.).

The crest factor is defined as the modulus of the ratio of the maximum instantaneous peak value of the frequency - weighted acceleration signal to its $R M S$ value. The peak valueshall be determined over the duration of measurement. The crest factor may be used to investigate if the basic evaluation method is suitable for describing the severity of the vibration in relation to its effects on human beings. For vibration with crest factors below or equal to 9 , the basic evaluation method is normally sufficient.

The guidelines by BS 6841 [33] suggest for motions with high crest factors (greater than 6) the vibration dose value must be used. Vibration dose value is a fourth power relation between acceleration and exposure duration. Vibration dose value is used to evaluate the time-dependency, for the effects of vibration on health, for all durations up to $24 \mathrm{hrs}(T=86400 \mathrm{~s})$. The vibration dose value is defined as:

$V D V=\left[\int_{t=0}^{t=T} a^{4}(t) d t\right]^{1 / 4}$.

Alternatively, the vibration dose value might be calculated from the $R M S$ acceleration using the "estimated vibration dose value", expressed as:

$e V D V=\left[\left(1.4 X_{R M S}\right)^{4} t\right]^{1 / 4}$

The ISO 2631 [100-105] standard defines methods for calculation of acceleration dose and average daily acceleration dose. There are expressed as:

$$
\begin{aligned}
& D_{k}=\left[\sum_{i} A_{i k}^{6}\right]^{1 / 6}, \\
& D_{k d}=D_{k}\left[\frac{t_{d}}{t_{m}}\right]^{1 / 6},
\end{aligned}
$$


where: $D_{k}$ - acceleration dose, $D_{k d}$ - average daily acceleration dose, $A_{i k}$ - is the $i$ th peak of response acceleration $a_{l k}(t), k-x, y$ or $z$ axis, $t_{d}$ - is the duration of the daily exposure, $t_{m}-$ isthe period over which $D_{k}$ has been measured.

The peaks are picked up in both the positive and negative directions for $x$ - and $y$-directions. Otherwise, for $z$-direction, only positive peaks shall be counted. Eq. 16.6 can be used when the total daily exposureis represented from a single measurement period. Whenthe daily vibration exposure consists of two or more periodsof different magnitudes the average daily accelerationdose can be calculated by Eq. 16.7.

The ISO 2631 allows to assess the vibration health effects by use of a biomechanical model described in previous section. Thus the equivalent static compressive stress and its daily equivalent dose are calculated as follows:

$S_{e}=\left[\sum_{k=x, y, z}\left(m_{k} D_{k}\right)^{6}\right]^{1 / 6}$,
$S_{e d}=\left[\sum_{k=x, y, z}\left(m_{k} D_{d k}\right)^{6}\right]^{1 / 6}$,

where: $S_{e}$ - equivalent static compressive stress, $S_{e d}$ - daily equivalent static compressive stress dose, $k-x, y$ or $z$ axis, $D_{k}-$ is the acceleration dose in the $k$-direction, $D_{k d}-$ is the average daily acceleration dose in the $k$-direction, $m_{x}-$ is the stress weighting factor for $x$-direction $\left(m_{x}=0.015 \mathrm{MPa}\right), m_{y}-$ is the stress weighting factor for $x$-direction $\left(m_{x}=0.035 \mathrm{MPa}\right), m_{z}-$ is the stress weighting factor for $x$-direction $\left(m_{x}=0.032 \mathrm{MPa}\right)$.

The FIOSH-Approach of risk assessment requires another equations for calculating compressive stress dose in term of dynamic conditions. Based on transformation into dynamic compressive stress all peak-to-peak values are related to the disc area of the lumbar level. The dynamic peak-to-peak compressive stress is expressed as:

$P_{d y n}=\frac{C_{d y n}}{A \cdot 100}$

where: $C_{d y n}$ - is the dynamic peak-to-peak values of the compressive force (in Newton) due to vibration acting on the lumbar level, $A$ - is the area of the endplates at the lumbar level and for one of three categories (small, medium, large) (in $\mathrm{cm}^{2}$ ).

The dynamic compressive stress dose and daily compressive stress dose for disc level are calculated as follows:

$S_{j}=\left[\sum_{k=1}^{s} P_{d y n_{k}}^{\exp }\right]^{1 / \exp ,}$
$S_{e d}=\left[\sum_{j=1}^{r} S_{j}^{\exp } \frac{t_{d j}}{t_{m j} \cdot 2}\right]^{1 / \exp }$,

where: $S_{j}$ - the compressive stress dose for the exposure $j$ and disc level $l, S_{e d}-$ daily compressive stress dose for disc level, $j$ - is the exposure condition, $t_{d j}$ - is the duration of the daily exposure to condition $j, t_{m j}$ - is the period over which $S_{j}$ has been calculated based on the measurement.

This daily compression dose does not consider the extent of the simultaneously acting static peak-to-peak compressive stress that could be taken into account, e.g., by Goodman's law [171]. 
The static compressive stress is considered in the calculation of the risk factor $R$, expressed as:

$$
R=\left[\sum_{i=1}^{q}\left(\frac{S_{e d} n d^{1 / \exp }}{S_{u i}-P_{\text {stat }}}\right)^{\exp }\right]^{1 / \exp },
$$

where: $S_{u i}$ - is the ultimate (subscript $u$ ) strength of the lumbar spine for a person of age $(b+i)$ years that can be predicted by two different regression equations and considering the share of the population that shall be covered by the prediction (50 or 95 percent), $b$ is the age at which the exposure starts, $P_{\text {stat }}$ - is a constant representing the static compressive stress acting on disk level $l$ during acertain posture, $i-$ is the year counter, $n d-$ is the number of exposure days per year, $\exp -$ is the exponent chosen by the user, default is 6 .

$R$ factor is a quantity that predicts the risk of fatigue failure of the vertebral endplate due to repeated compressions, if it reaches or exceeds the value 1 . It can also be designated as 'equivalent static compressive stress', because it is equivalent to the static compressive stress that would cause failure, if the value 1 is reached.

\subsection{Investigation on influence of vehicle technical condition and power transmission system on evaluation of human exposure to whole body vibration via feet}

All of methods for assessment of vibration exposure are dedicated to the measurement of vibration signal on the seat surface. The second surface of whole-body vibration penetration into human organism are the area of floor panel contact to human's feet. Thus the research were conducted on implementation of chosen evaluation methods for assessment of exposure to vibration penetrated via feet of seating person.

The results described in the book show influence of chosen operating parameters of vibration sources on its propagation into the vehicle structure in locations where vibration penetrate into the human organism. Thus the experiments were performed on influence of technical condition of suspension elements, vehicle operating parameters, engine rotational speed and gear ratio on evaluation of human exposure to whole body vibration. For each case the vibration of floor panel in location of occupant's feet were registered. The chapter presents the results obtained for vibration penetrated to the feet of driver.

As the energy total estimators it were compared results of the parameter value of vibration dominant effective weighted vibration acceleration $A_{w \max }$ and daily exposure parameter $A(8)_{l}$. The influence of engine rotational speed and gear ratio on evaluation of human exposure to whole body vibration have been presented as the distribution of these exposure estimators and it is presented in Figs. 16.1-16.2.

For the complex analysis of WBV exposure of driver by areas of possible penetration of the vibration into driver body the estimators of exposure were calculated basing on the vibration signals measurement on the front dash panel and floor panel in location where driver's feet can be located during driving (Fig. 16.3). The third measurement point was located on driver seat, as it is suggested in ISO 2631.

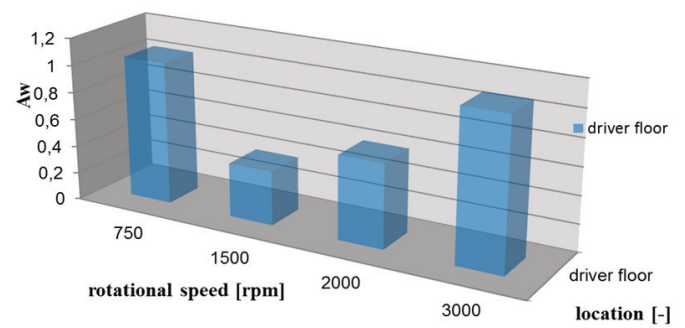

a)

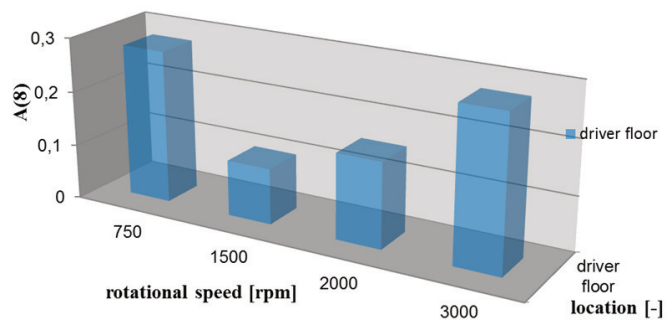

b)

Fig. 16.1. Influence of engine rotational speed on vibration exposure, expressed as a) $A_{w \max }$ and b) $A(8)$ 


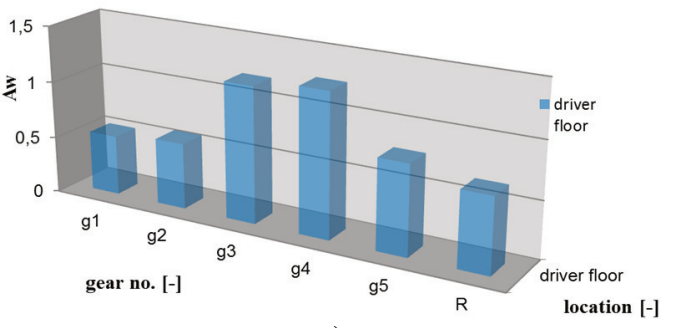

a)

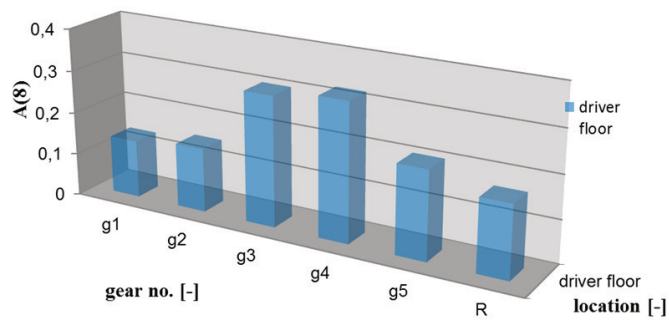

b)

Fig. 16.2. Influence of gear ratio on vibration exposure, expressed as a) $A_{w m a x}$ and b) $A(8)$

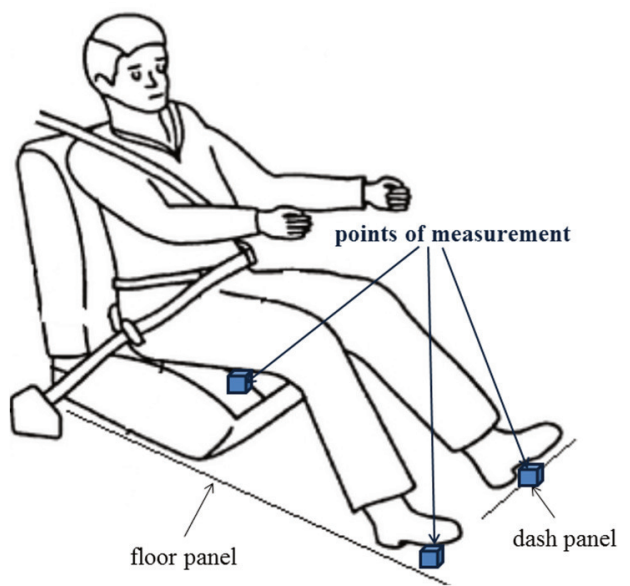

Fig. 16.3. The localization of points of measurement for evaluation of exposure to WBV

The comparison of estimators of exposure to WBV for vibration transferred through floor panel, dash panel and seat are shown in Figs. 16.4-16.5. It shows the influence of operating parameters of power transmission system on exposure to WBV.

When analysing a human exposure to whole-body vibration the methods of quantifying vibration containing multiple shocks in relation to human health is used. For the evaluation of vibration acceleration dose and average daily acceleration dose affecting to the driver the $D_{k}$ and $D_{k d}$ were compared for different engine rotational speed and gear ratio, as gear number position. Adverse effects on the lumbar spine are the dominating health risk. Therefore the analysis is concerned with the lumbar spine response. For the spine response calculating the presented in section 16.4 model was used. The calculation of acceleration dose and equivalent and daily static compressive stress $S_{e}$ and $S_{e d}$ for the assessment of health effects were done.

The example of results obtained for engine rotational speed $750 \mathrm{rpm}$ and $3000 \mathrm{rpm}$ or $1 \mathrm{st}$ and 5th gear with constant rotational speed is shown in Figs. 16.6-16.9.

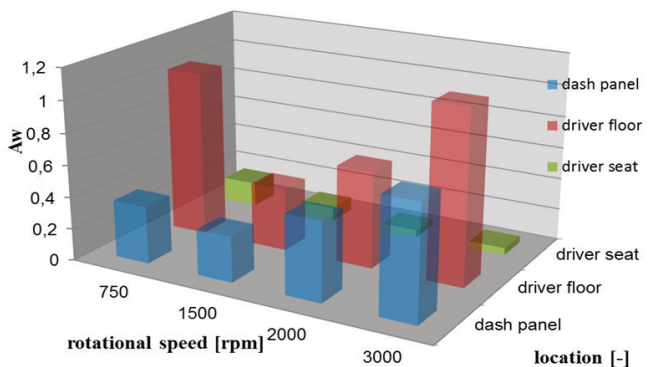

a)

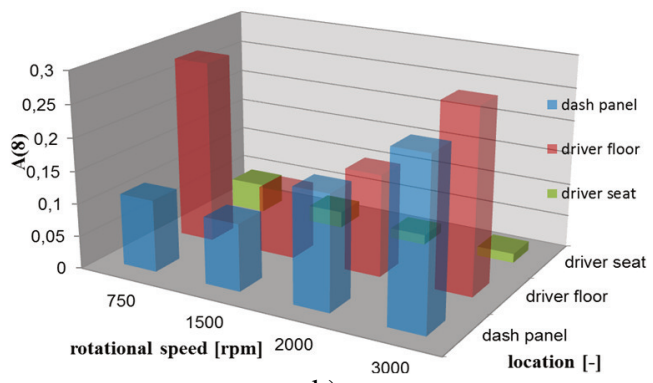

b)

Fig. 16.4. Comparison of influence of engine rotational speed on vibration exposure, expressed as a) $A_{\text {wmax }}$ and b) $A(8)$, calculated for vibration of dash panel, floor panel and driver seat 


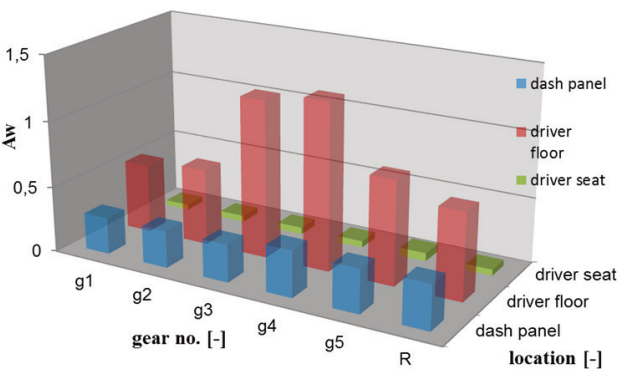

a)

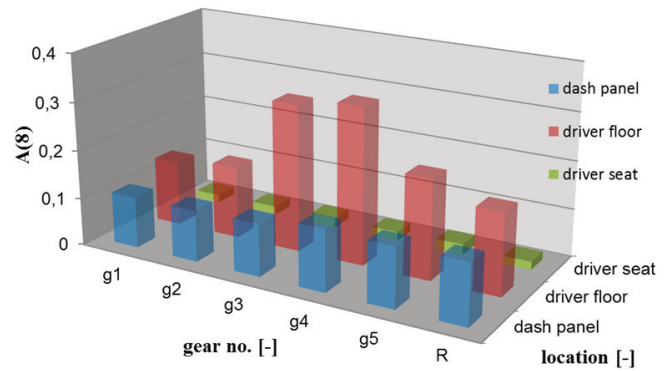

b)

Fig. 16.5. Comparison of influence of gear ratio on vibration exposure, expressed as a) $A_{w \max }$ and b) $A(8)$, calculated for vibration of dash panel, floor panel and driver seat
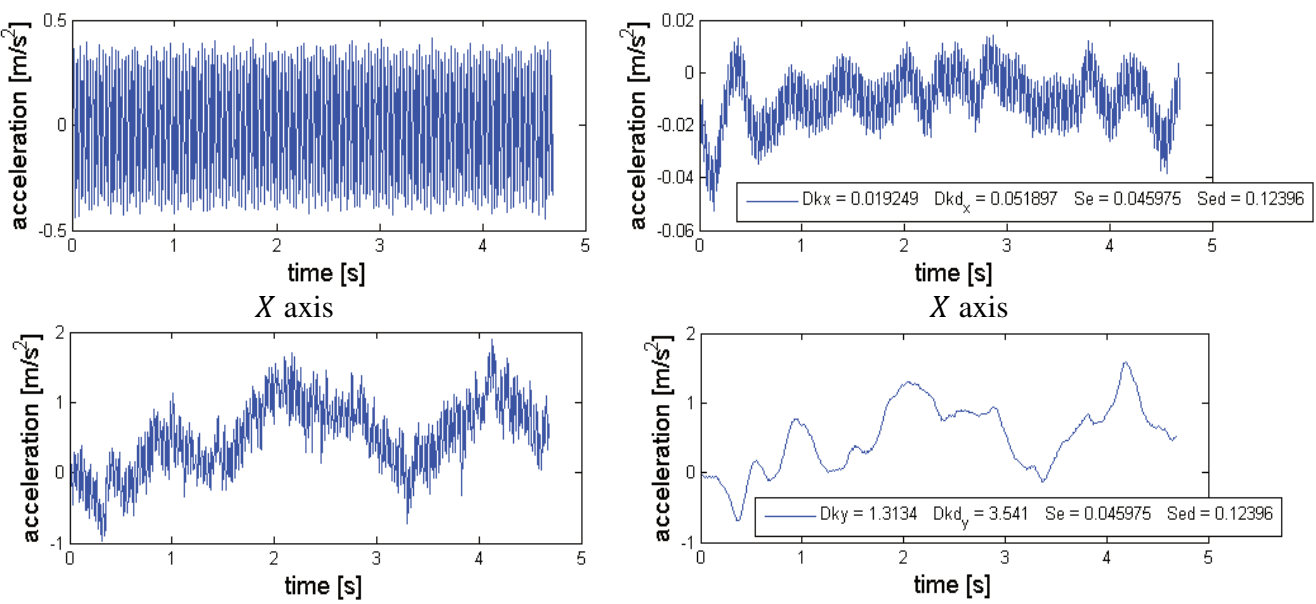

$Y$ axis

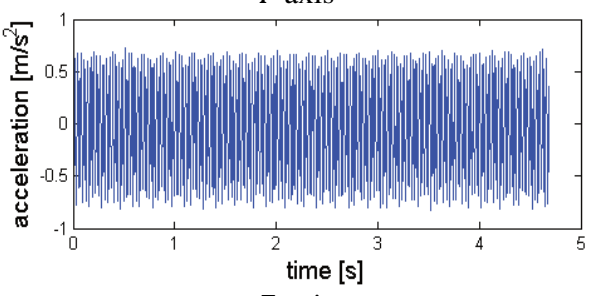

$Z$ axis

a) Vibration signal

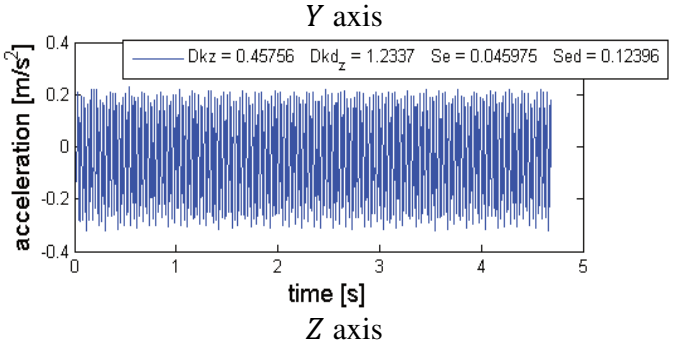

a) Response in the Spine

Fig. 16.6. a) Vibration of floor panel under driver's feet for 3 orthogonal axes and b) lumbar spine response for $750 \mathrm{rpm}$ engine rotational speed 

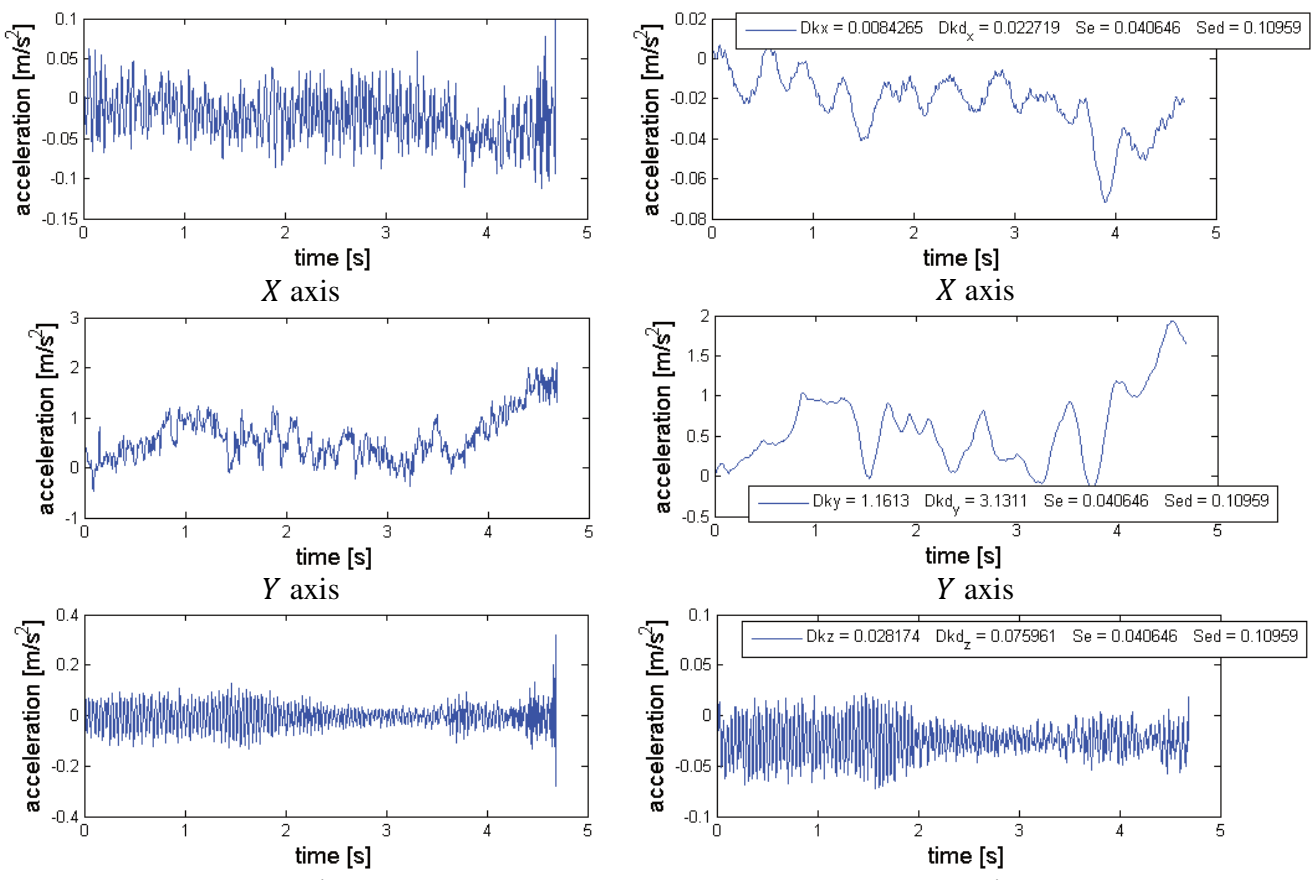

$Z$ axis

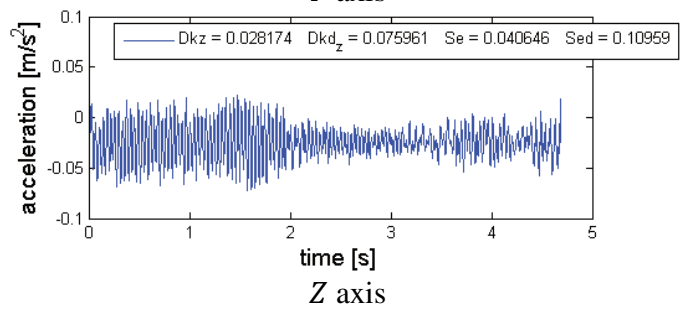

a) Vibration signal

a) Response in the Spine

Fig. 16.7. a) Vibration of floor panel under driver's feet for 3 orthogonal axes and

b) lumbar spine response for $3000 \mathrm{rpm}$ engine rotational speed
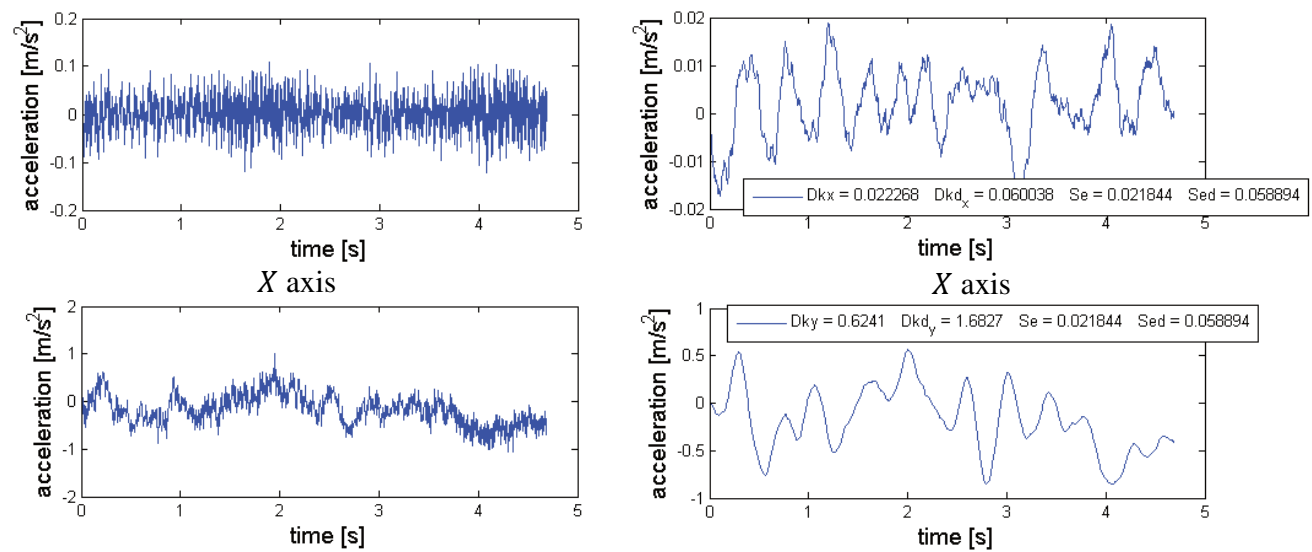

$Y$ axis

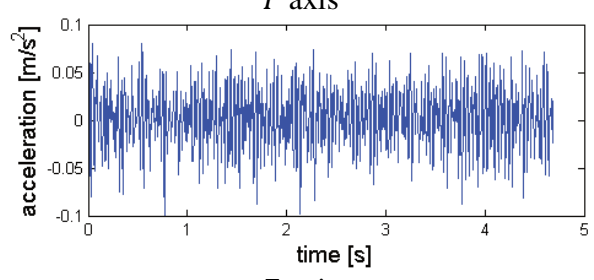

$Z$ axis

a) Vibration signal

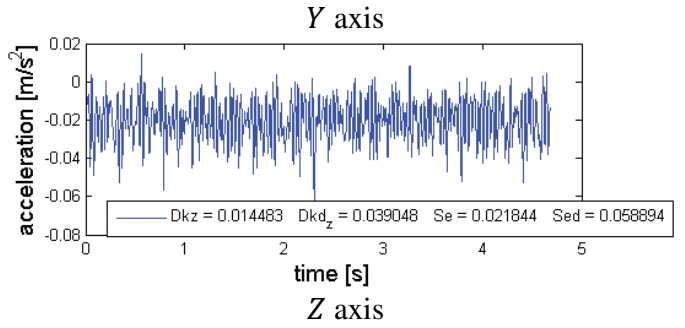

a) Response in the Spine

Fig. 16.8. a) Vibration of floor panel under driver's feet for 3 orthogonal axes and

b) lumbar spine response for 1th gear, $1500 \mathrm{rpm}$ 

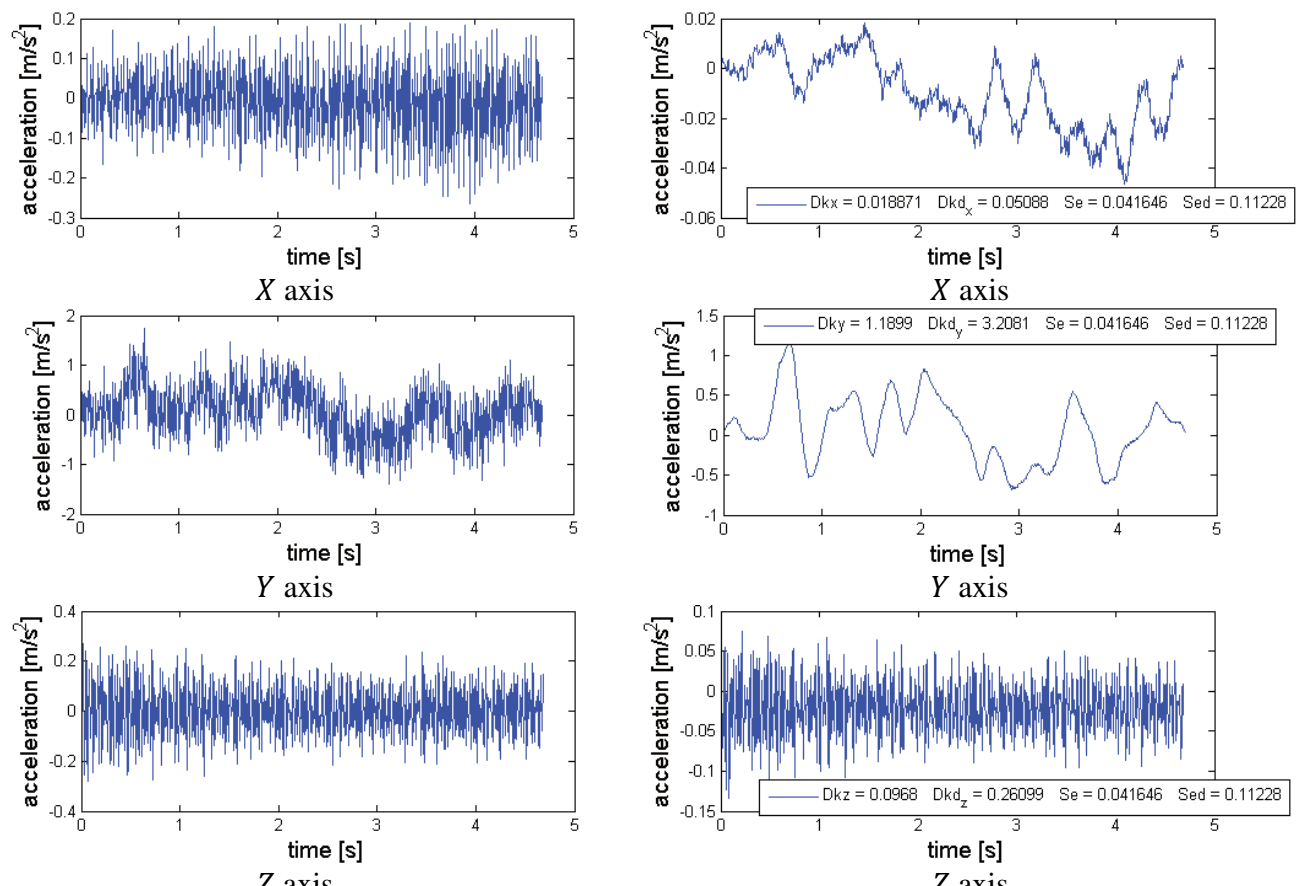

a) Vibration signal

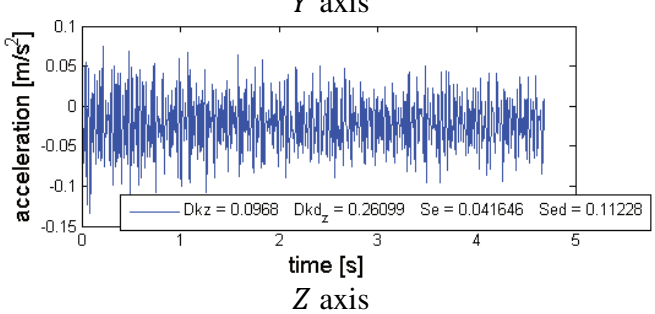

a) Response in the Spine

Fig. 16.9. a) Vibration of floor panel under driver's feet for 3 orthogonal axes and b) lumbar spine response for 5 th gear
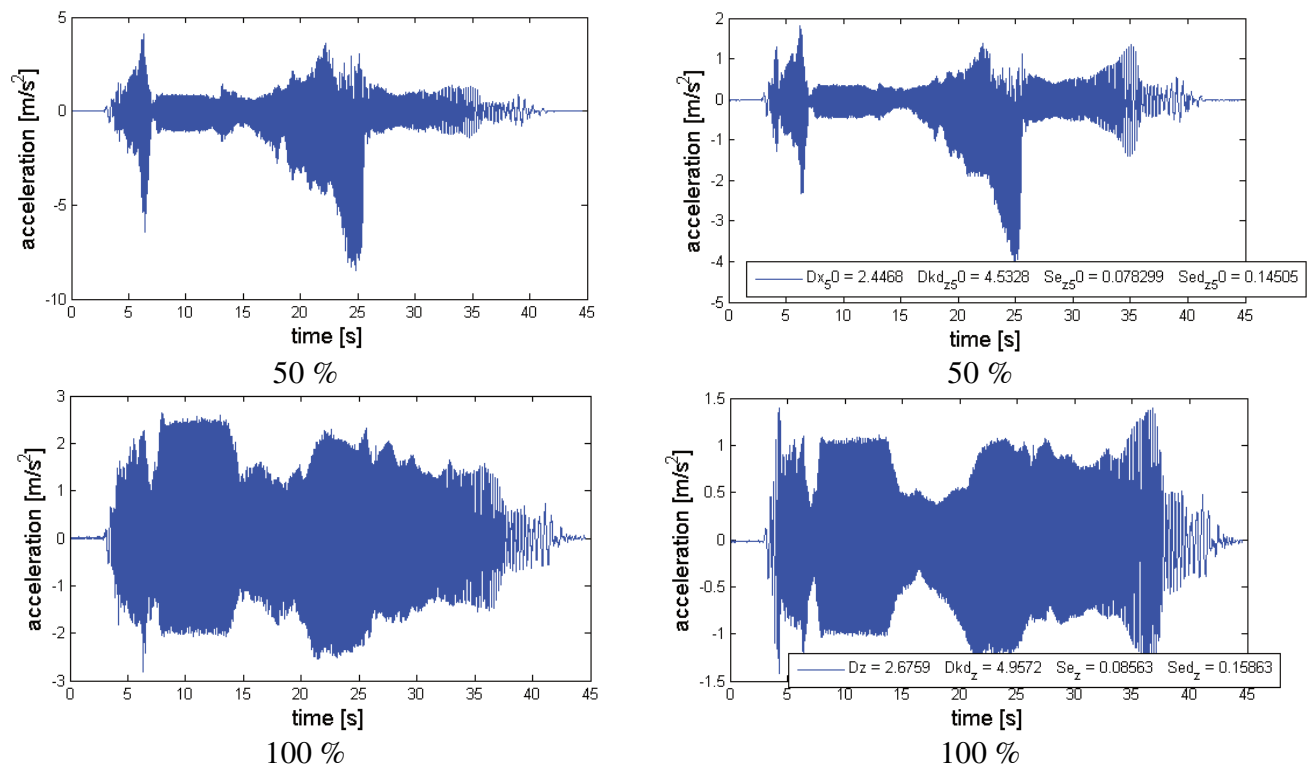

a) Vibration signal

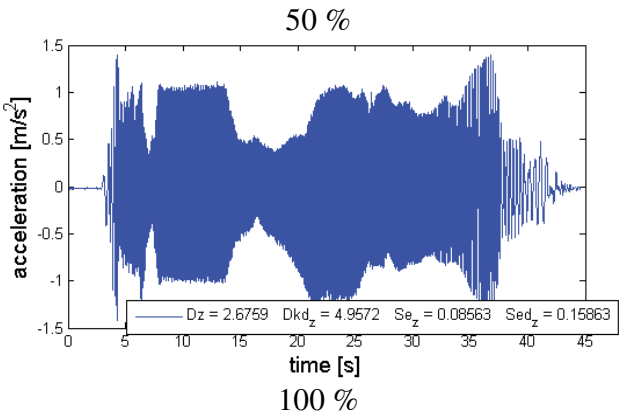

b) Response in the Spine

Fig. 16.10. a) Comparison of vibration of floor panel under driver's feet for $z$-axis and

b) lumbar spine response for shock absorber with $50 \%$ and $100 \%$ volume of fluid

Basing on the result of research on influence of technical condition of shock absorber and pressure in tires on vibration propagation, described in previous chapters, the analitical experiment on lumbar spine response modeling for these parameters were performed. For the ground excitation simulated in the laboratory research by use the vibration shaker the forces are acting to 
the wheel in vertical direction. Thus the result of floor panel vibration under driver's feet and lumbar spine response for $z$-direction was calculated.

The example of results obtained for shock absorber with $50 \%$ and $100 \%$ volume of fluid or $600 \mathrm{hPa}$ and $2600 \mathrm{hPa}$ of tire pressure level are shown in Fig. 16.10-16.11.

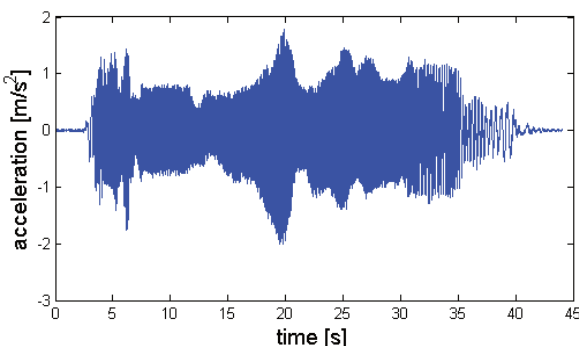

$600 \mathrm{hPa}$

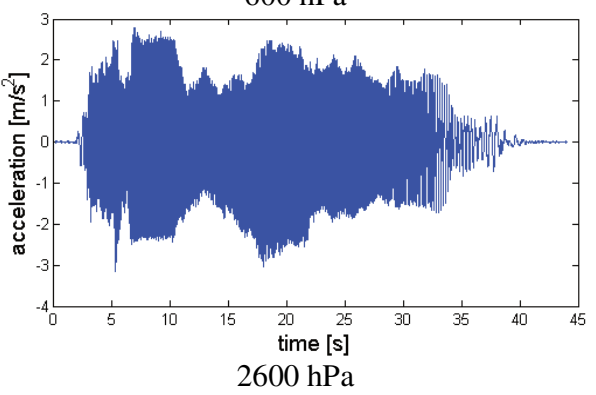

a) Vibration signal

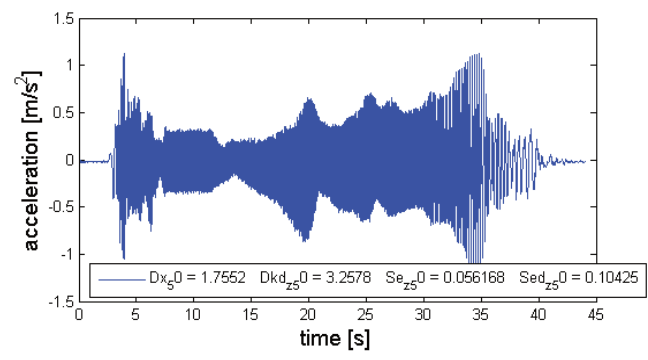

$600 \mathrm{hPa}$

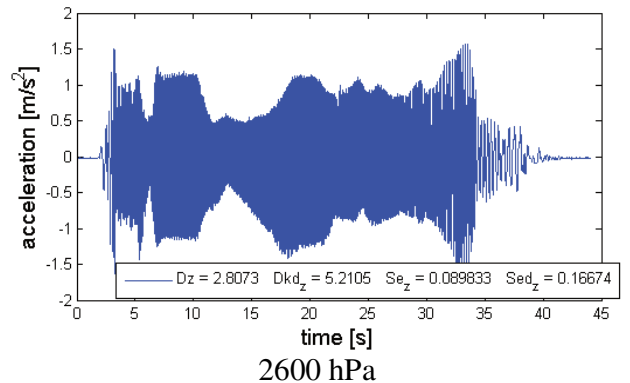

b) Response in the Spine

Fig. 16.11. a) Comparison of vibration of floor panel under driver's feet for $z$-axis and

b) lumbar spine response for tire pressure $600 \mathrm{hPa}$ and $2600 \mathrm{hPa}$

The values of vibration acceleration doses and equivalent static compressive stress for different operating parameters of vehicle were collected in Table 16.1.

Table 16.1. The values of vibration acceleration doses and equivalent static compressive stress for different operating parameters of vehicle

\begin{tabular}{|c|c|c|c|c|c|c|}
\hline Vehicle parameter & Value / as & & $D_{k}$ & $D_{k d}$ & $S_{e}$ & $S_{e d}$ \\
\hline \multirow{6}{*}{ Engine rotational speed } & \multirow{3}{*}{$750 \mathrm{rpm}$} & $X$ & 0.0192 & 0.0519 & \multirow{3}{*}{0.0460} & \multirow{3}{*}{0.1240} \\
\hline & & $Y$ & 1.3134 & 3.5410 & & \\
\hline & & $Z$ & 0.4576 & 1.2337 & & \\
\hline & \multirow{3}{*}{$3000 \mathrm{rpm}$} & $X$ & 0.0084 & 0.0227 & \multirow{3}{*}{0.0406} & \multirow{3}{*}{0.1096} \\
\hline & & $Y$ & 1.1613 & 3.1311 & & \\
\hline & & $Z$ & 0.0282 & 0.0760 & & \\
\hline \multirow{6}{*}{ Gear no. } & \multirow{3}{*}{ 1th } & $X$ & 0.0223 & 0.0600 & \multirow{3}{*}{0.0218} & \multirow{3}{*}{0.0589} \\
\hline & & $Y$ & 0.6241 & 1.6827 & & \\
\hline & & $Z$ & 0.0145 & 0.0390 & & \\
\hline & \multirow{3}{*}{5 th } & $X$ & 0.0189 & 0.0509 & \multirow{3}{*}{0.0416} & \multirow{3}{*}{0.1123} \\
\hline & & $Y$ & 1.1899 & 3.2081 & & \\
\hline & & $Z$ & 0.0968 & 0.2610 & & \\
\hline \multirow{2}{*}{ Volume of shock absorber fluid } & $50 \%$ & $Z$ & 2.4468 & 4.5328 & 0.0783 & 0.1450 \\
\hline & $100 \%$ & $Z$ & 2.6759 & 4.9572 & 0.0856 & 0.1586 \\
\hline \multirow{2}{*}{ Tire pressure level } & $600 \mathrm{hPa}$ & $Z$ & 1.7552 & 3.2578 & 0.0562 & 0.1043 \\
\hline & $2600 \mathrm{hPa}$ & $Z$ & 2.8073 & 5.2105 & 0.0898 & 0.1667 \\
\hline
\end{tabular}

The chapter presents experimental approach to exposure to WBV penetrated through floor panel via feet into human organism. The different methods for evaluation were compared for assessment influence of vehicle technical condition and power transmission system on human 
exposure to whole body vibration. For the purpose of comparison of total energy estimators it were collected results of the parameter value of vibration dominant effective weighted vibration acceleration $A_{w \max }$ and daily exposure parameter $A(8)_{l}$. The exposure to vibration occurring for idle gear rotational speed is higher than for $1500 \mathrm{rpm}$ and $2000 \mathrm{rpm}$. The increase of these estimators due to the idle gear were registered for the $3000 \mathrm{rpm}$ and more. For the successive gear number and gear ratio the maximum exposure can be observed for 3rd and 4th gear. The comparison of estimators of exposure to WBV for vibration transferred through floor panel, dash panel and seat show the level of the exposition to the vibration for feet located on dash or floor panel and for the lumbar spine on the seat.

The attempts to use the acceleration dose and equivalent static compressive stress for the assessment of health effects of WBV transferred from floor panel according to methods from ISO 2631-5 has been successful. The vibration measurement requirements included in ISO 2631-1 are measure in 3 orthogonal directions on driver seat. The presented calculation was for measurement on floor panel, but it is still corresponded with WBV and adverse effects on the lumbar spine. According to ISO 2631-5 assessment of adverse health effect at lifetime exposure are acceptable where $S_{e d}$ is below 0.5 and very danger when it is above 0.8 . The results obtained, with notification that the results of shock absorber and tire pressure are only for the vertical vibration, shows huge impact of damping parameters and tire stiffness on of human exposure to vibration in passenger car. If the horizontal vibration ( $x, y$ axis) is counted the probability of an adverse health effect will be very high. The influence of engine rotational speed and gear ratio are also significant and above 0.8 . The $S_{e d}$ difference between 1 st and 5 th gear is twice higher. 



\section{The measures of dispersion of vibration energy of vehicle construction}

Basing on an analysis of such a vast selection of study results, bearing in mind that the research purpose was to identify sources, propagation and structure of vibrations affecting men in means of transport based on the example of automotive vehicles, a decision was made to extend the studies with an analysis of the vibration energy dissipation across the vehicle structure. It will enable assessment of the damping properties of the vehicle structure and its equipment, and may prove to be a valid source of information to be applied while designing eliminators and dampers of vibrations propagating into the human organism.

When considering the vibration in human-structure relation system (i.e. building, platform, mean of transport) the identification of non-linear or active elements of human participation in structural vibrations has to be studied but the end result is usually seen in two forms: a moderate shift in structure natural frequency and a not so moderate increase in total damping capacity. Damping is energy dissipation and since the desirable end result of human participation is to help control vibration by energy dissipation it is natural to track energy flow in a vibrating human-structure system [32].

The state of art shows many approach to issue of dispersion of energy of vibration, especially in multi-body solid structure. One of the group of methods are based on the assumption that energy in the form of vibration in the structure behaves in the same way as energy in the form of heat. It diffuses from one substructure to another at a rate proportional to the difference in temperature of the substructures, and it is dissipated internally in each substructure at a rate proportional to the temperature of that substructure.

\subsection{Methods of analysis of dispersion of vibration energy}

Energy propagation and impact in mechanical systems are both closely linked with the phenomenon of energy dissipation and change to its emission forms. With regard to vibration related phenomena, the direct proportionality between the energy and the root mean square value of amplitude of the dynamic vibration course makes it possible to define global measures of propagation in the energy dimension [116]. The general vibration propagation form may be expressed in the following dependence:

$H=\frac{\int_{0}^{T}(x(t))^{2} d t}{\int_{0}^{T}(F(t))^{2} d t}$,

where: $x(t)$ - forced vibration, $F(t)$ - forcing vibration.

According to Parseval's theorem the result of the Fourier transform is unitary. Thus the integral of the square of a function is equal to the integral of the square of its transform. Therefore the propagation can be defined in the transform domain expression:

$H_{f}=\frac{\int_{-\infty}^{\infty}|X(f)|^{2} d f}{\int_{-\infty}^{\infty}|F(f)|^{2} d f}$, 
where: $X(f)$ - power spectral density of forced vibration, $F(f)$ - power spectral density of forcing vibration.

The different approach is presented as statistical-energy analysis. When applied appropriately it gives a very simple means of predicting the distribution of mean-square vibration amplitudes among the substructures in response to a known pattern of external driving. A major theoretical problem of statistical-energy analysis is to investigate the approximations implicit in these two fundamental assumptions, and the extent to which they are justified in various practical situations. The assumption that the rate of internal dissipation of energy is proportional to the kinetic energy of the substructure is a familiar idealization [68, 203]. In Rayleigh's language, it amounts to assuming that the dissipation function is simultaneously diagonalizable with the kinetic and potential energy functions. Rayleigh shows that this assumption does not lead to serious problems provided the damping is small [132].

The vibration energy dissipation may also be perceived as a specific case of the entropy phenomenon. Entropy is a measure of the system disorder degree. Following the second law of thermodynamics, if a thermodynamic system spontaneously evolves from one state of equilibrium towards another, its entropy always increases. With reference to the signal theory, entropy is defined as follows:

$E=-\sum_{i} x_{i}^{2}(t) \cdot \log \left(x_{i}^{2}(t)\right)$,

where: $x(t)$ - analyzed signal.

One of the energy entropy symptoms is dissipation which determines the energy form change into heat. This phenomenon clearly intensifies in systems or structures featuring friction forces and viscosity. As a result of the dissipation phenomenon, the oscillating motion energy declines and so vibration damping takes place. Consequently, the relative amount of thermal energy increases in the system and its entropy grows. An interesting engineering application of the said phenomenon can be observed in vibration damping systems based on magnetorheological fluids where, due to relative micro-motions, the oscillating motion power is dissipated through friction into heat [16-18].

\subsection{The measures of dispersion of vibration energy of vehicle construction}

The notion of vibration energy dissipation is inextricably linked with the wave propagation phenomenon, being most commonly described by means of wave propagation velocity derivatives $[110,113]$. What matters more in terms of the vibration related phenomena and their impact on men is the energy-oriented approach. Having established a correlation between energy measures and dynamic properties of vibrations or their TFR distribution, dedicated measures of propagation and dissipation of vibrations in a vehicle structure from the perspective of their impact on the vehicle passengers can be developed.

Basing on satisfactory results of the efficiency assessment for the measures proposed in Section 13.4 with reference to the studies of propagation of vibrations and how they affect the suspension system operating and technical parameters as well as the engine and transmission operating parameters, specific measures of the vibration energy dissipation at structural points of a vehicle were proposed.

The measures developed enable observation and assessment of the energy and amplitude dissipation of vibrations in the domains of time, frequency and TFR. The dissipation measures are correlated with vibration signals of the vibration sources envisaged as input signals. The foregoing measures are defined as follows:

$D_{R M S_{i}}=\frac{\left(X_{R M S s i}-X_{R M S d i}\right)}{X_{R M S s i}}$, 
$D_{S_{R M S}}=\frac{\left(S_{R M S S}-S_{R M S d}\right)}{S_{R M S S}}$

where: $D_{R M S_{i}}-R M S$ directional dispersion factor for $i$-direction, $D_{S_{R M S}}-$ total $R M S$ dispersion factor, $i$-direction $x, y$ or $z, X_{R M S s i}$ - root mean square of source vibration signal for $i$-direction, $X_{R M S d i}$ - root mean square of response vibration signal for $i$-direction, $S_{R M S s}$ - total root mean square of source vibration signal, $S_{R M S d}$ - total root mean square of response vibration signal.

$\begin{aligned} D_{T a b s_{i}} & =\frac{\left(T_{a b s(F F T) s i}-T_{a b s(F F T) d i}\right)}{T_{a b s(F F T) s i}} \\ D_{S_{T a b s}} & =\frac{\left(S_{T_{a b s_{S}}}-S_{T_{T a b s}}\right)}{S_{T a b s_{S}}}\end{aligned}$

where: $D_{T a b s}$ - frequency directional dispersion factor for $i$-direction, $D_{S_{T a b s}}-$ total frequency dispersion factor, $T_{a b s(F F T) s i}-T_{a b s(F F T)}$ of source vibration signal for $i$-direction, $T_{a b s(F F T) d i}-$ $T_{a b s(F F T)}$ of response vibration signal for $i$-direction, $S_{T a b s_{S}}$ - total $T_{a b s(F F T)}$ of source vibration signal, $S_{\text {Tabs }_{d}}$ - total $T_{a b s(F F T)}$ of response vibration signal.

$D_{\text {TabsTFR }}=\frac{\left(T_{\text {absTFRsi }}-T_{\text {absTFRdi }}\right)}{T_{\text {absTFRsi }}}$,
$D_{S_{\text {TabsTFR }}}=\frac{\left(S_{\text {TabsTFR }_{S}}-S_{\text {TabsTFR }_{d}}\right)}{S_{\text {TabsTFR }_{S}}}$,

where: $D_{\text {TabsTFR }}$ - TFR directional dispersion factor for $i$-direction, $D_{S_{\text {TabsTFR }}}-$ total TFR dispersion factor, $T_{a b s T F R s i}-T_{a b s T F R}$ of source vibration signal for $i$-direction, $T_{a b s T F R d i}-$ $T_{a b s T F R}$ of response vibration signal for $i$-direction, $S_{\text {TabsTFR }}$ - total $T_{a b s T F R}$ of source vibration signal, $S_{\text {TabsTFR }_{d}}$ - total $T_{a b s T F R}$ of response vibration signal.

The methodology of determining partial measures for the calculation of the vibration energy dissipation coefficients was already described in detail in Chapter 13.

\subsection{Dispersion of floor panel vibration generated by the engine and power transmission system}

In order to verify the efficiency of the measures proposed for the vibration dissipation in a vehicle structure, analytical experiments were conducted, comprising assessment of the susceptibility of the said measures to changes of the input function parameters. The source of vibrations was assumed to be the engine and the transmission. The studies included two experiments. In the course of the first one, the engine rotational speed was changed when running idle. In the second one, on fixed rotational speed, the gear ratio was changed by engaging successive gears. Time courses, spectra and TFR distributions of the vibration signals envisaged for the experiments were discussed in Chapters 13 and 14.

The results obtained in the course of the first experiment for the vibrations recorded on the vehicle floor panel were compared and collated in the bar graphs provided in the Figs. 17.1-17.4. 


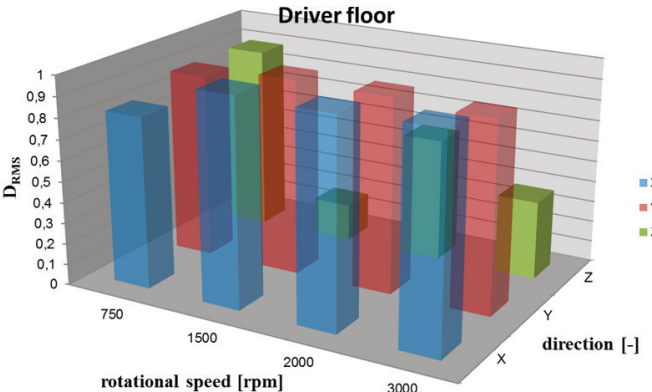

a) Driver floor

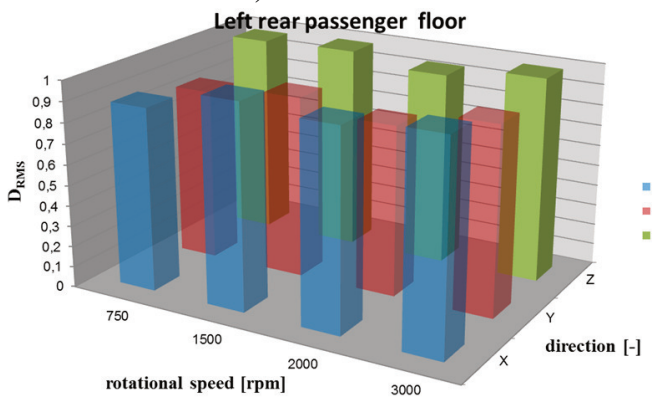

c) Left rear passenger floor

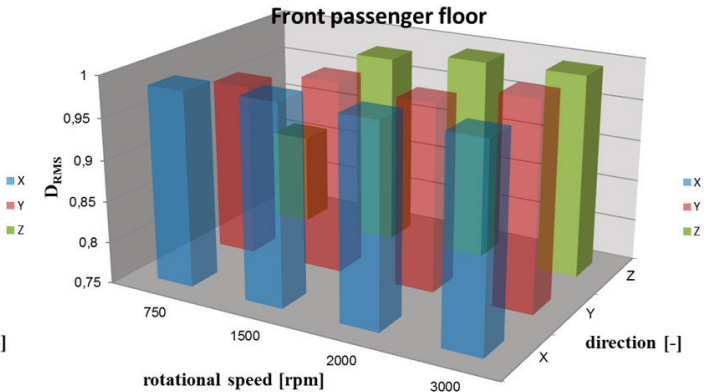

b) Front passenger floor

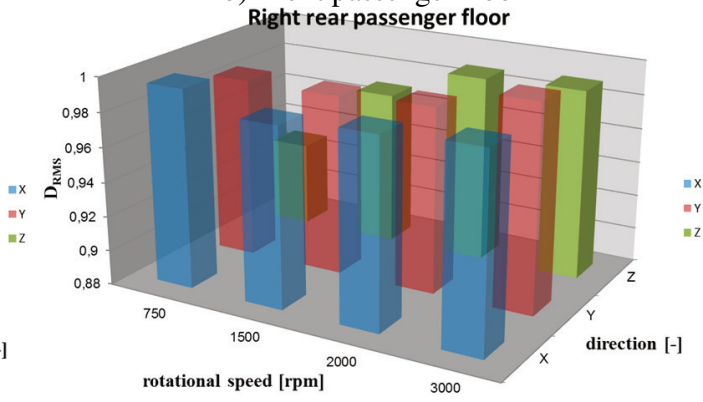

d) Right rear passenger floor

Fig. 17.1. Comparison of $R M S$ directional dispersion factor of floor panel vibration for different engine rotational speed

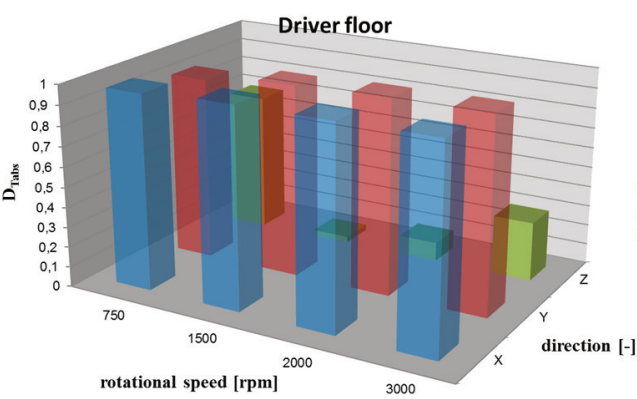

a) Driver floor

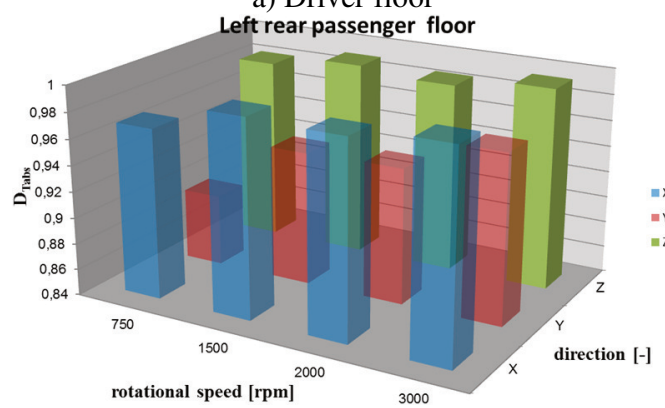

c) Left rear passenger floor

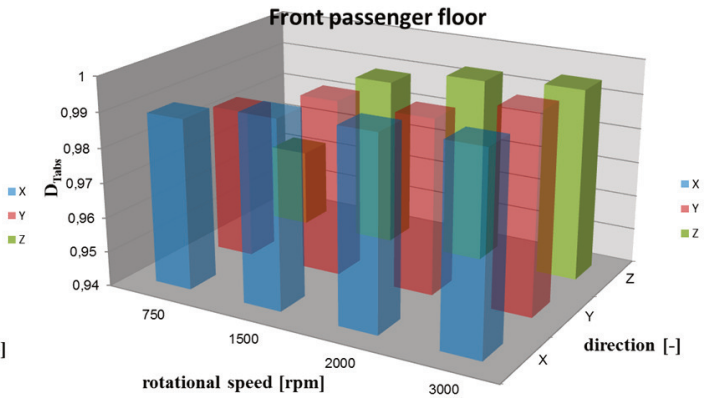

b) Front passenger floor

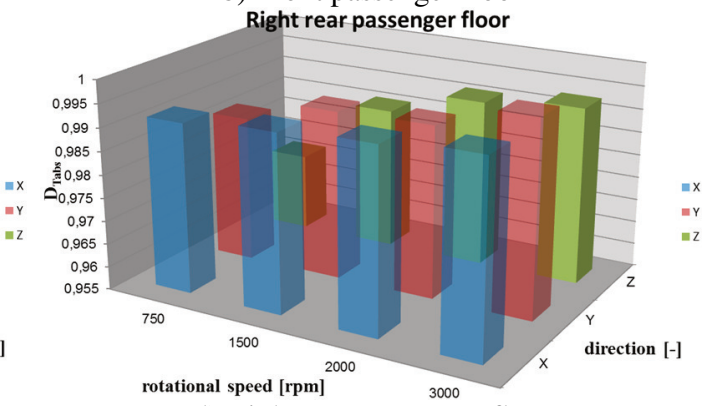

d) Right rear passenger floor

Fig. 17.2. Comparison of frequency directional dispersion factor of floor panel vibration for different engine rotational speed 


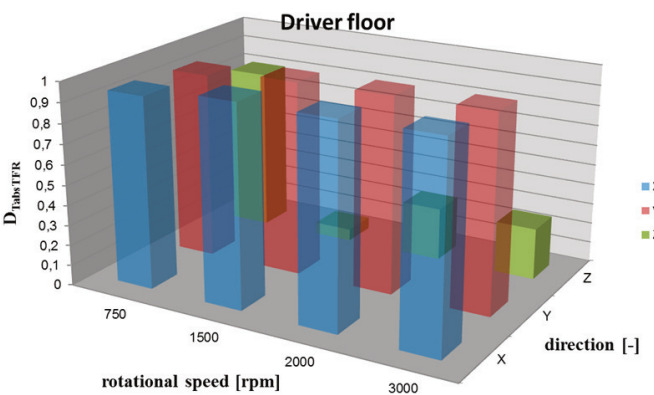

a) Driver floor

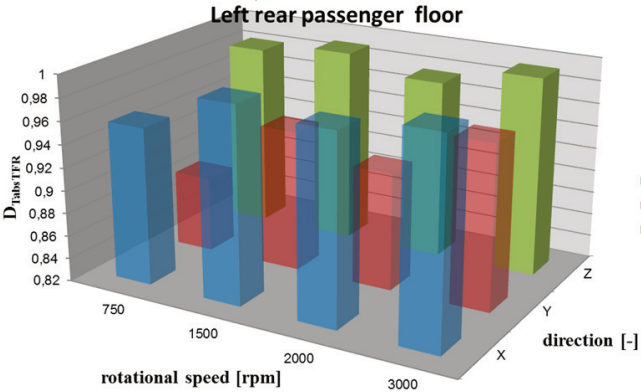

c) Left rear passenger floor

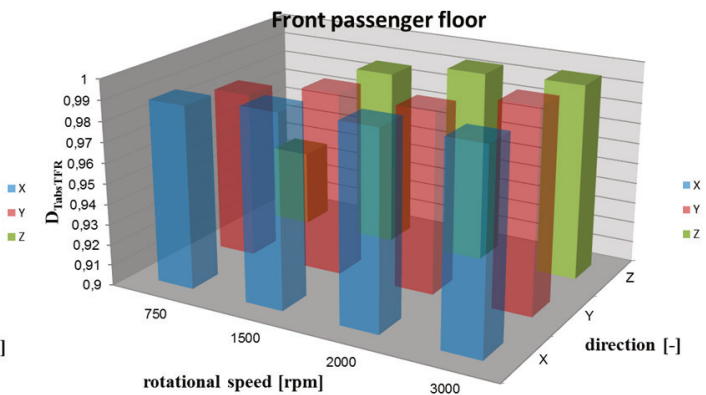

b) Front passenger floor

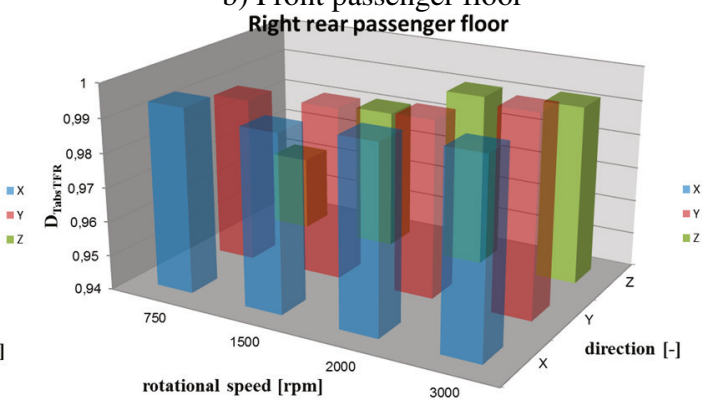

d) Right rear passenger floor

Fig. 17.3. Comparison of TFR directional dispersion factor of floor panel vibration for different engine rotational speed

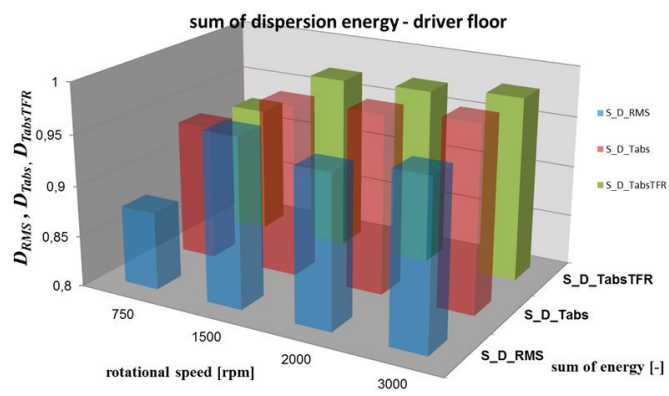

a) Driver floor

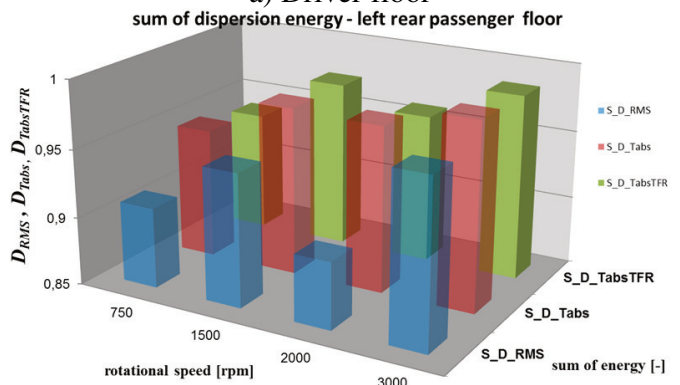

c) Left rear passenger floor

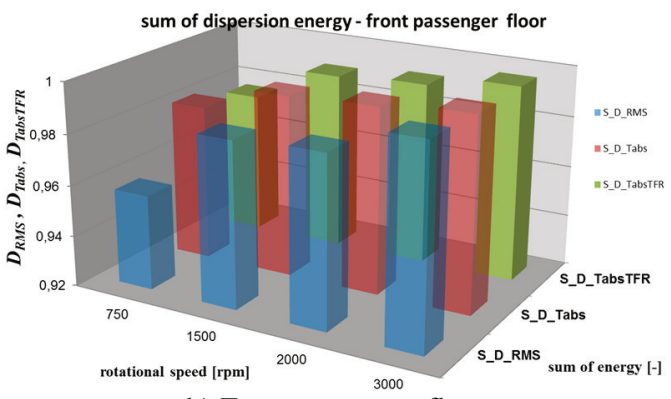

b) Front passenger floor

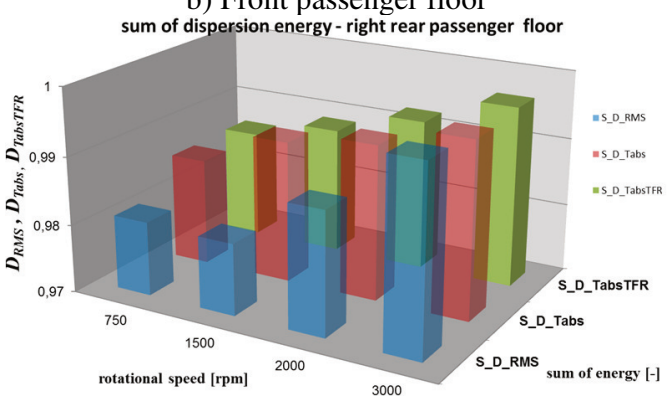

d) Right rear passenger floor

Fig. 17.4. Comparison of total energy directional dispersion factors of floor panel vibration for different engine rotational speed

For the purpose of analysis the vibration dispersion factors at the path of propagation into human body via dash panel, floor panel and seat the comparison was collected in Fig. 17.5. 


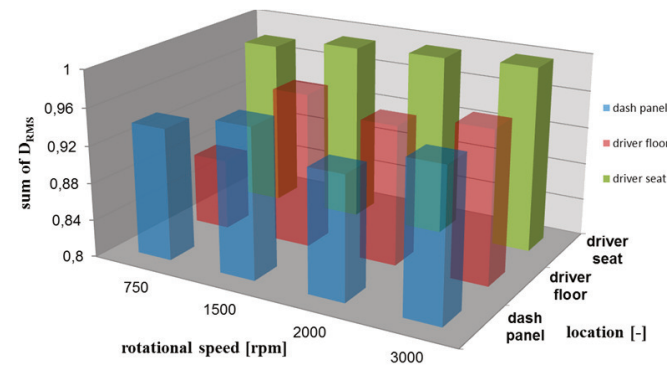

a)

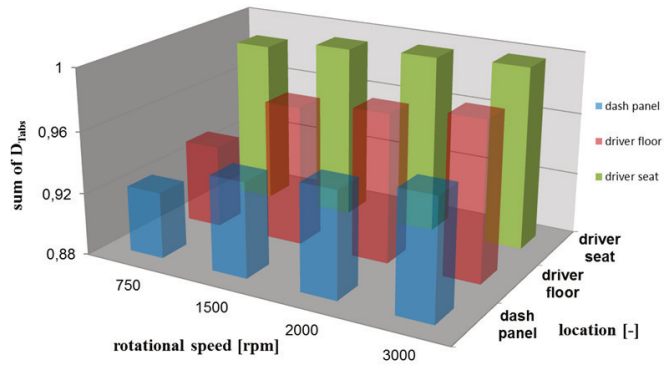

b)

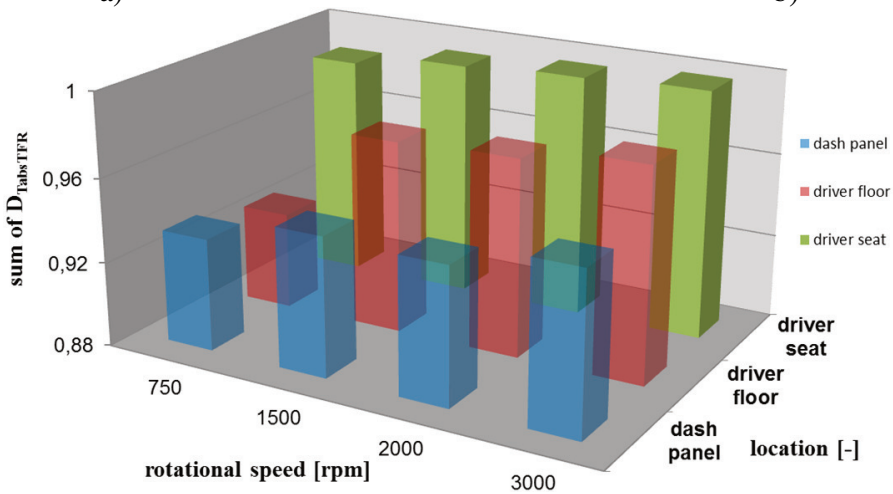

c)

Fig. 17.5. Comparison of total vibration dispersion factors at the path of propagation into human organism for different engine rotational speed

The results of second investigation on influence of gear ratio on dispersion of vibration on floor panel under the driver's feet are shown in Figs. 17.6 and 17.7.

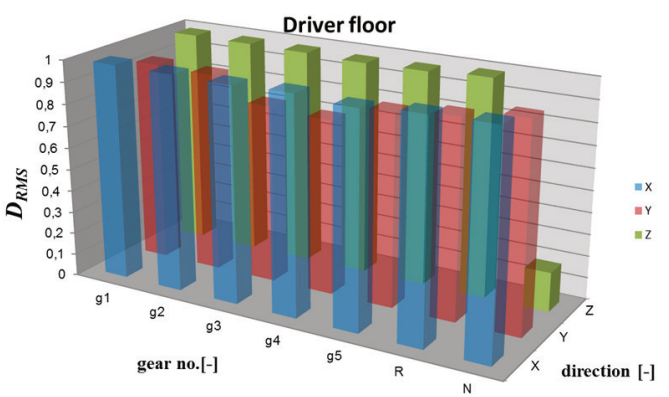

a)

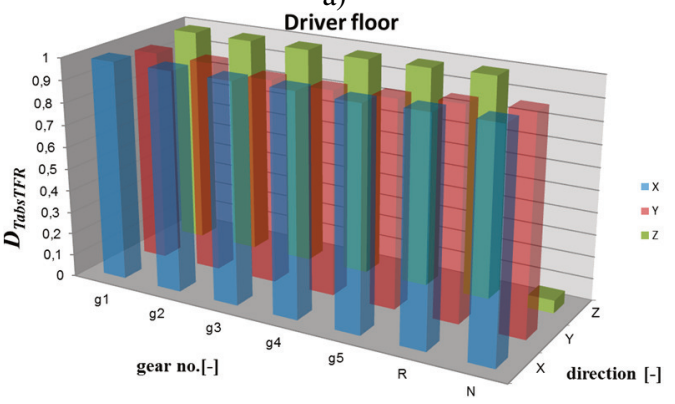

c)

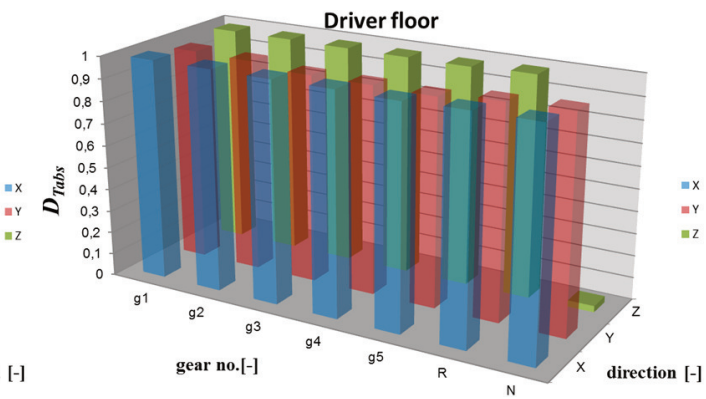

b)

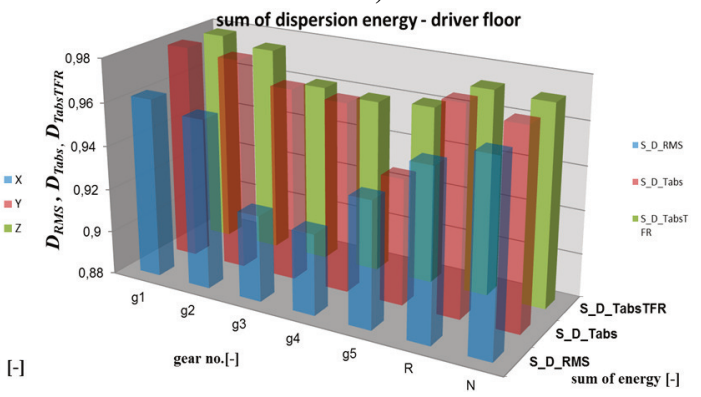

d)

Fig. 17.6. Comparison of directional and total vibration dispersion factors of floor panel under the driver's feet for different gear ratio (gear no.) 


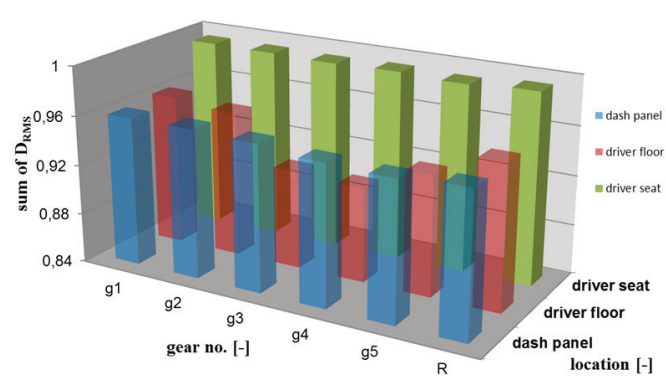

a)

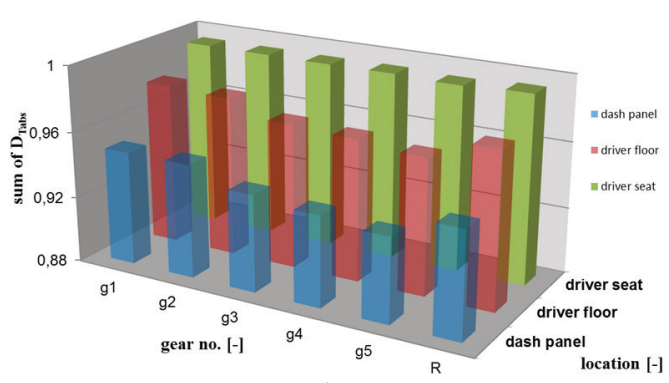

b)

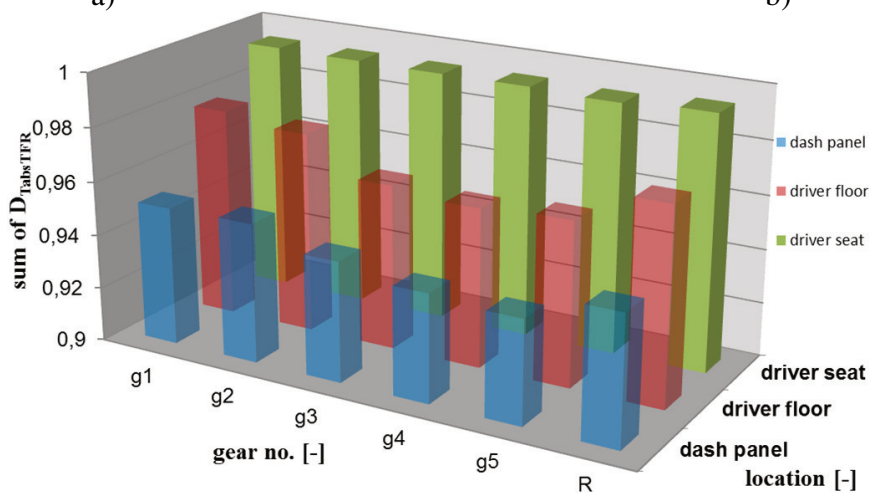

c)

Fig. 17.7. Comparison of total vibration dispersion factors at the path of propagation into human organism for different gear ratio (gear no.)

The measures of vibration dissipation in automotive vehicles were defined and verified. They enable the vibration propagation and damping to be analysed and assessed by application of simple energy measures in the domains of time and frequency. The measures envisaged can also be used in experimental studies, which were confirmed by the results of validation experiments. Directional distributions of the coefficients mentioned make it possible to determine the vibration damping characteristics and the directional propagation, whereas by comparing the values obtained at the selected structural points in the propagation path, the dynamics of vibration damping or suppression as well as determine the most exposed locations can be assessed.

All the vibration energy dissipation coefficients proposed may assume the maximum value of 1 , which implies that the entire vibration energy generated by the source dissipate, and that the vibration is completely suppressed at the given measurement point. Low values imply some small dissipation, whereas negative values prove that the vibration energy measured at the given point is larger than the one generated by the source. In this kind of case the vibration can be reinforced or interfered by other sources. 



\section{Application of vibroacoustic methods for monitoring of vibration propagation and comfort in vehicles}

\subsection{Impact of vibration on vehicle's comfort and safety}

The compromise between comfort and safety of the vehicle driving is very difficult to achieve. For the driving safety it is extremely important to provide constant contact of vehicle wheels to the road surface. It determines the high damping coefficient. For the comfort of the passengers it is important to minimize the vibration perception. It can be achieved by the gradual and smooth vibration absorbing $[124,125,175]$. The driver and passengers are exposed to whole-body vibration of the vehicle. It can affect from short-term body discomfort and inefficient performance to longterm physiological damage. The vibrations of the vehicle body are main problem in ride comfort. Ride comfort is extremely difficult to determine because of the variations in individual sensitivity to vibration.

The vibration exposure of the car depends on road roughness, speed, engine and powertrain parameters. To provide the best vibration isolation for the passengers the damping properties of the suspension have to be changeable to the drive condition. At the present the numerous automotive companies offer adaptive shock absorbers or active suspensions. It contains many of mechatronics systems and elements which are perfect to adjust the damping parameters of the suspension to the drive condition. The control system proper information on safety or comfort is required. The chapter presents the possibilities of application of vibroacoustic methods and signal processing algorithms for those purpose.

Analysis and evaluation of the vibration phenomena in car vehicles are very difficult and it requires using proper methods and mathematics algorithms. The number of physics and chemical phenomena occurring during working of many systems of vehicles which are affecting on propagation of energy in different forms. Thus research on this kind of phenomena has to be conducted and the results and developed methods should be analysed for different parameters of mechanical systems working. The chapter presents method verified for different exploitation parameters of the vehicle. As the results of observing and acquisition of vibration phenomena signals of displacement, velocity or acceleration of vibration are received. A vibration signal is a carrier of information on the state, the changes or the process to which the given physical or technical system is subject. Vibroacoustic signals are characterised by the largest information carrying capacity and they enable observation of changes occurring in a broad frequency band.

\subsection{Methods of vibration signal analysis}

Numerous measuring problems may be considered on a general level of a signal, perceiving the signal as an entirety in the course of observation. It may be examined in the domains of amplitudes, time and frequency. As far as random vibration phenomena are concerned, the signals recorded are of non-stationary nature which requires that the signal distribution is observed in the domains of time and frequency simultaneously.

One of the mathematical instruments enabling separation of non-stationary signal components is a wavelet transformation which consists in distinguishing a part of the $f(t)$ signal being similar to a present template, i.e. the part which corresponds to the determined component. The template 
role is performed by basic wavelet $\psi(t)$. The wavelet functions as a transformation kernel. A single wavelet is used in the given transformation, however, due to modification of scale coefficient $a$ and modification coefficient $b$, it forms what is referred to as a wavelet family. A continuous wavelet transform in the domain of time and frequency is defined as follows:

$\tilde{S}_{\Psi}(a, b)=\frac{1}{\sqrt{|a|}} \int_{-\infty}^{+\infty} s(t) \Psi\left(\frac{t-b}{a}\right) d t$,

where: $a$-scale coefficient, $b$ - modification coefficient, $s(t)$ - value of the signal examined in the function of time, $\tilde{S}_{\Psi}(a, b)$ - wavelet coefficient dependent on $a$ and $b, \psi$-wavelet function, $\Psi((t-b) / a)$ - transformation kernel.

The value of wavelet coefficient $\tilde{S}_{\Psi}(a, b)$ established by means of the above formula is generally understood as a measure of similarity between the signal examined and the chosen wavelet.

Furthermore, due to dimensional estimates' sensitivity to the stationary nature of operating conditions, in the process of identification of signal characteristics, besides dimensional estimates, one applies quotients of these measures being dimensionless amplitude discriminants. They are obtained by dividing moments of various ranks by one another.

\subsection{Method of multidimensional identification of signal characteristics in the analysis of vibration properties}

For the sake of identification of signal characteristics in the analysis of vibration properties of an automotive vehicle's floor panel, a complex mathematical algorithm was developed to be subsequently implemented in the MatLab environment, and a user interface was created named WIBROCAR. The programme developed was named Vibroacoustic Signals Analyzer (WSA), and it was then extended with several modules dedicated to analysis, monitoring and diagnostics of selected vehicle systems and structural assemblies. It is a self-designed program for the signal processing with friendly-user interface. The communication with the user is based on the dialog windows programmed in Graphical User Interface Tools in MATLAB environment. Procedure of testing starts with vehicle data and research parameters entry (Fig. 18.1).

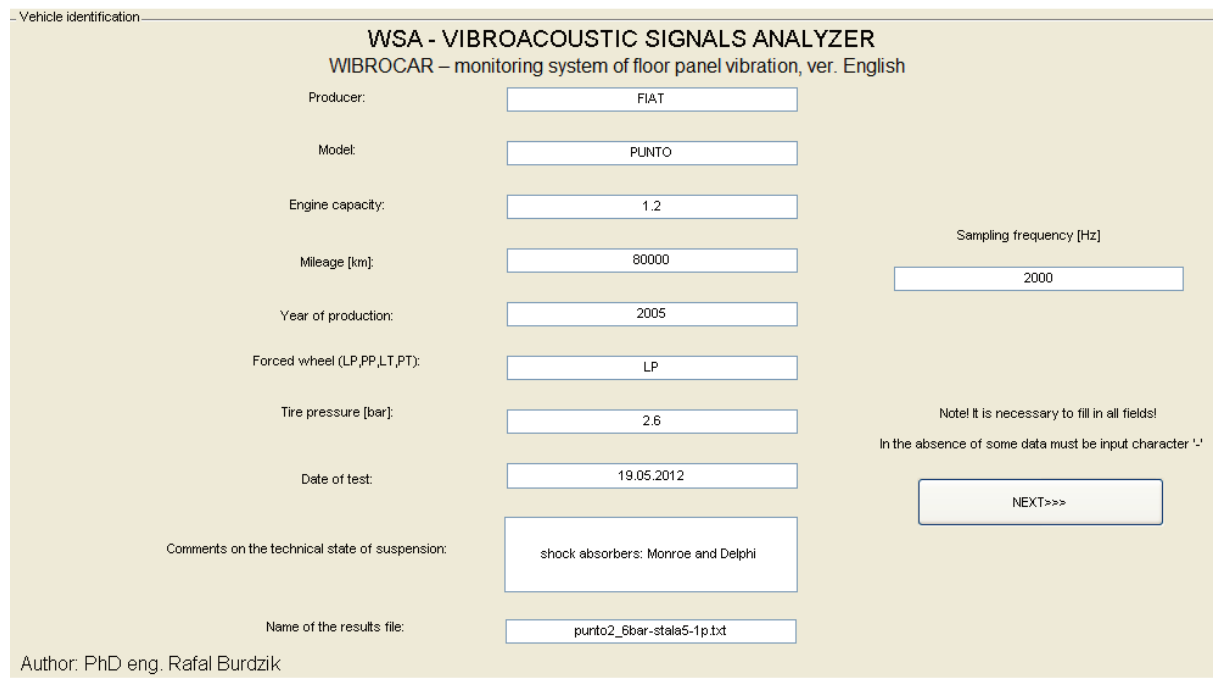

Fig. 18.1. First window of WSA program

It was assumed the utility properties of the software implementation of the WSA program. For 
this purpose it is very important to communicate with clear orders and information reports to user. The work in the WSA should be close to intuitive. Some examples of the communication windows are presented in Fig. 18.2.

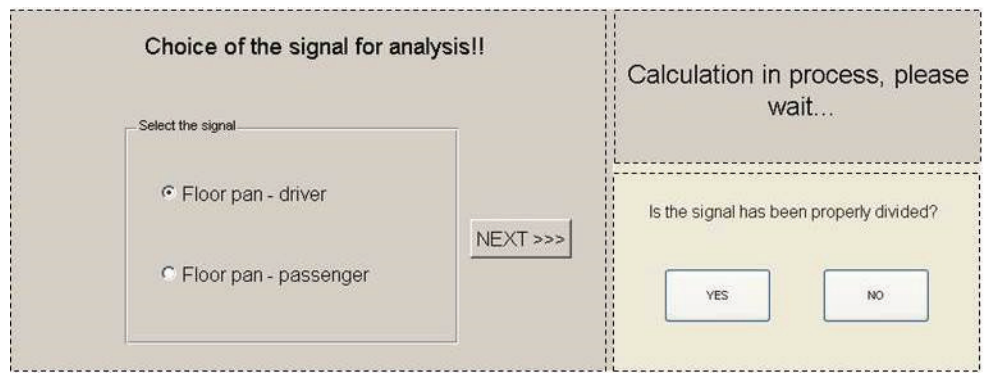

Fig. 18.2. User - WSA program communication windows

Due to the complexity resulting from nonlinear and random nature of vibration phenomena in automotive vehicles, the analysis in question is multidimensional. The property table being established consists of numerous measures and estimators, both dimensional and dimensionless ones, in the domains of amplitudes, time, frequency and time-frequency. In order to accurately identify signal characteristics, one needs appropriate analytical methods depending on the stationary and non-stationary nature of the signal. An automatic algorithm is developed for positioning of stationary and non-stationary signal cycles. For this purpose identification of next cycles of forced machine working there were next phases of vibration inductor working identification measure formulated. The markers of next cycles of forced machine working measures based on STFT (Short Time Fourier Transform) transformation is used. The main reason of choosing this transformation was short realization time. There was $21-22 \mathrm{~Hz}$ frequency band cut out from STFT spectrum for analysis. Based on time function of cut off frequency band identifying algorithm of end of stand run up and start of stand coasting time coordinates was created. Elaborated algorithm is based on comparing next value of analysed frequency band (,,analysis of edge") around set parameters. Locating of end of stand run up and start of stand coasting enables to divide signal on three time windows. First window for fragment of signal growing according to constant frequency increase of the forced system. Second window for signal with constant frequency and the third one for coasting stand - decrease of signal amplitudes according to constant frequency decrease of the forced system. This method and algorithm is presented in Fig. 18.3.
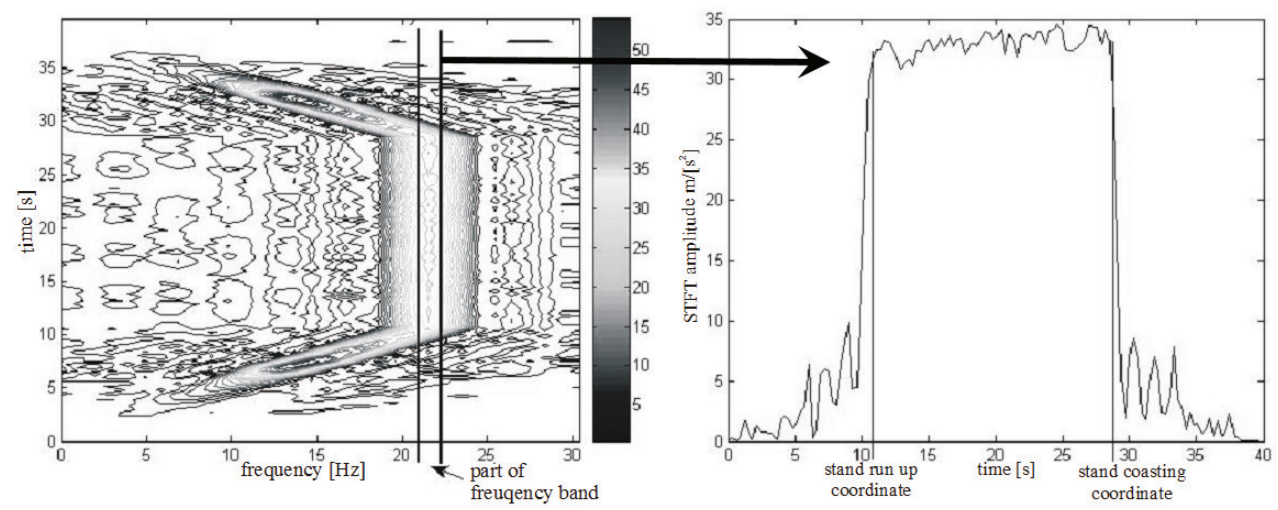

Fig. 18.3. Calculation and analysis of time function of STFT coefficients for identification of stationary and non-stationary parts of the signal

An example of such a division is presented in Fig. 18.4. It is the very first step towards 
identification of signal characteristics using dedicated methods in the analysis of stationary and non-stationary signals.

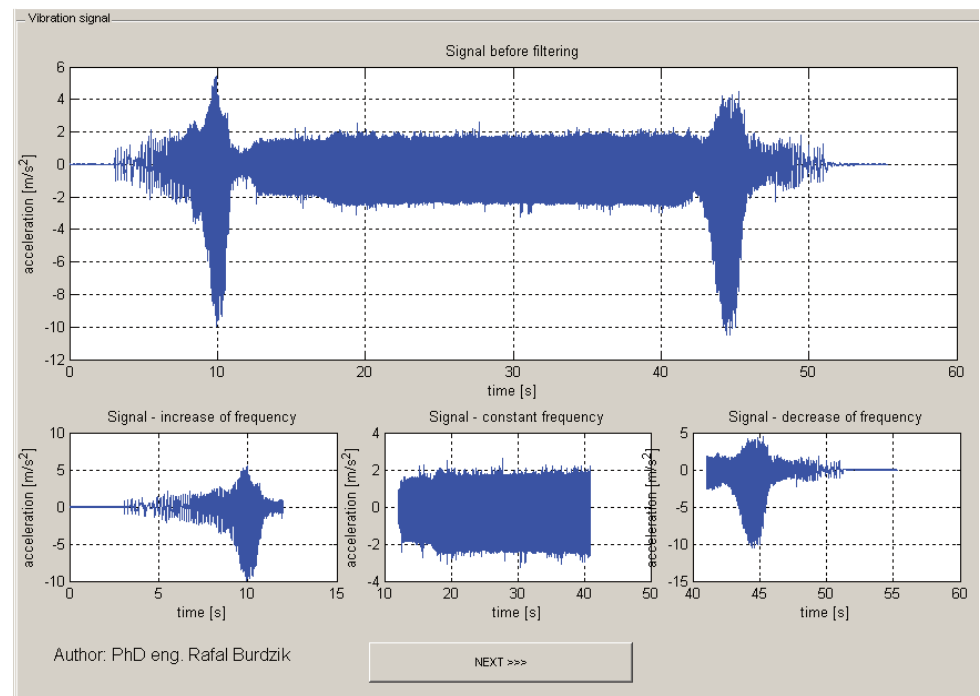

Fig. 18.4. Vibration of the floor panel - automatic algorithm for positioning of stationary and non-stationary signal cycles

For the purposes of analysis of the stationary signal part, an algorithm based on FFT was developed. The signal characteristics are then identified by amplitude based correlation of successive signal harmonics which were accurately separated from non-stationary signal components. Results of this algorithm are shown in Fig. 18.5. Preliminary tests of a car's floor panel proved various sensitivities to deviation of vibration damping parameters of successive harmonics from a constant input function.

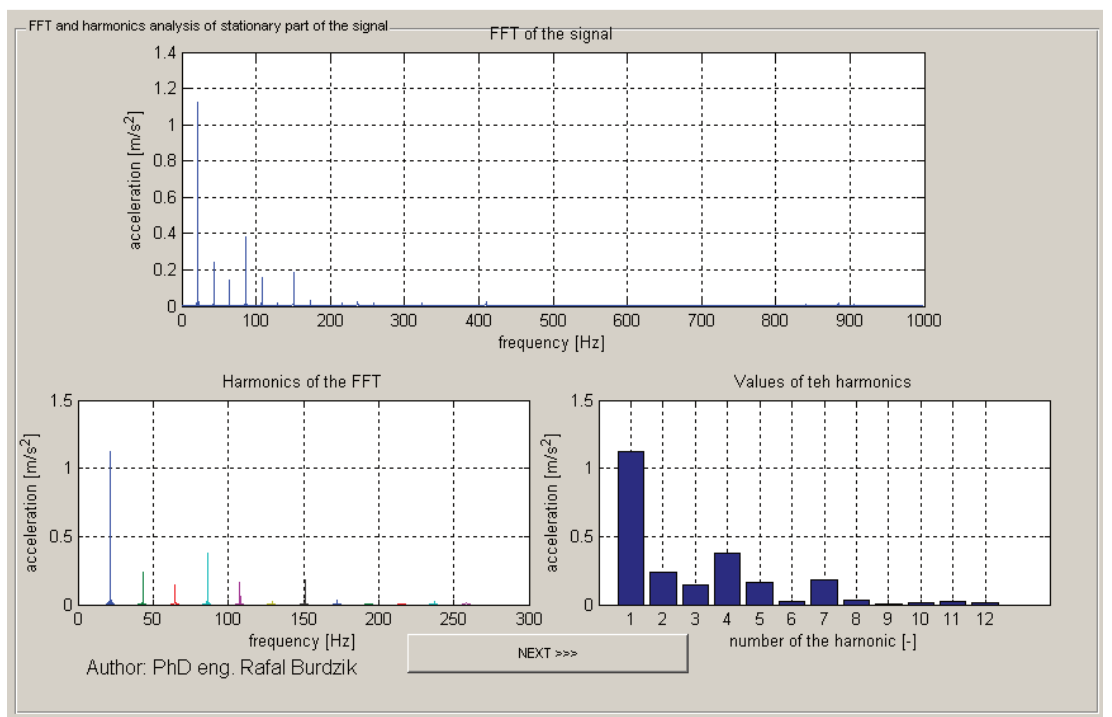

Fig. 18.5. Results of the FFT analysis for the stationary signal portion

In order to analyse predominant components of resonant frequencies of sprung and unsprung masses, a transformation algorithm was developed for the non-stationary signals recorded during a rundown of the vibration forcing station and once it was completely shut down. Finally, for the 
purposes of identification of the signal characteristics, a vehicle free vibration suppression window was chosen, where the vibrations of a system subject to free suppression were recorded. It enabled the system's free vibration frequency bands to be accurately observed and defined. The window used to analyse and define the range of resonant frequency bands for sprung and unsprung masses is presented in Fig. 18.6. The wavelet based time and frequency distribution of a signal enables accurate definition of resonant windows.

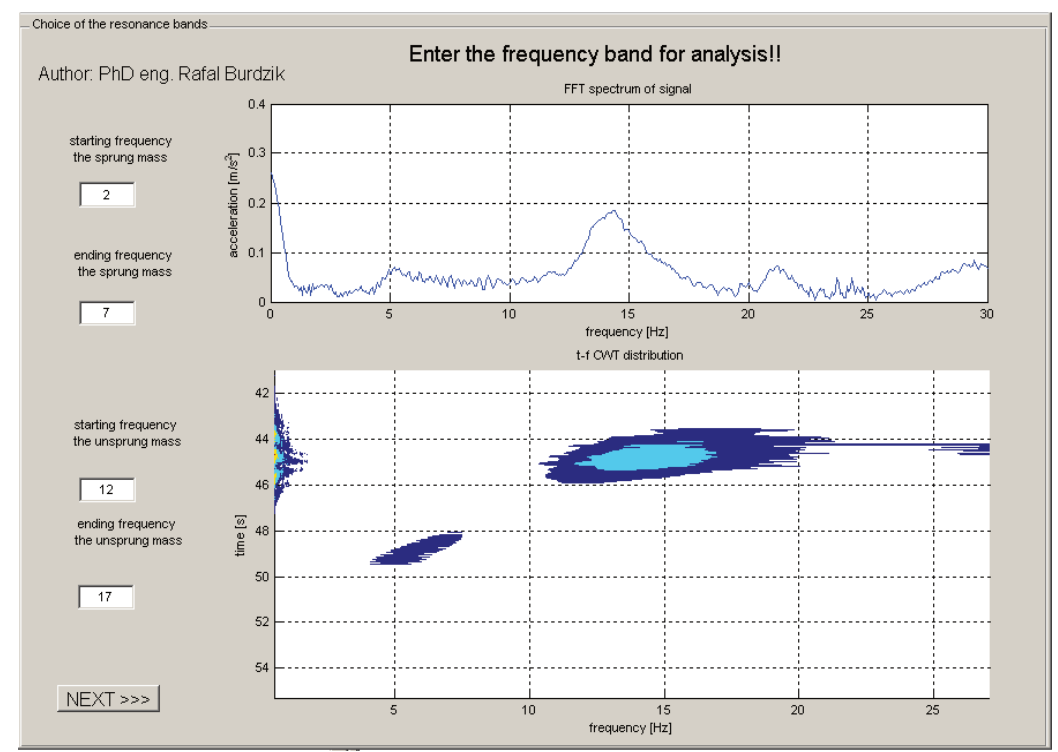

Fig. 18.6. Identification of resonance frequency bands - non-stationary signal portion

75-elemnents matrices of measures of signal characteristics were used as a multi-parameter measure of signal characteristics for an automotive vehicle's floor panel. They were established as estimators based on averaged time and frequency courses of resonant windows for sprung and unsprung masses (Fig. 18.7).

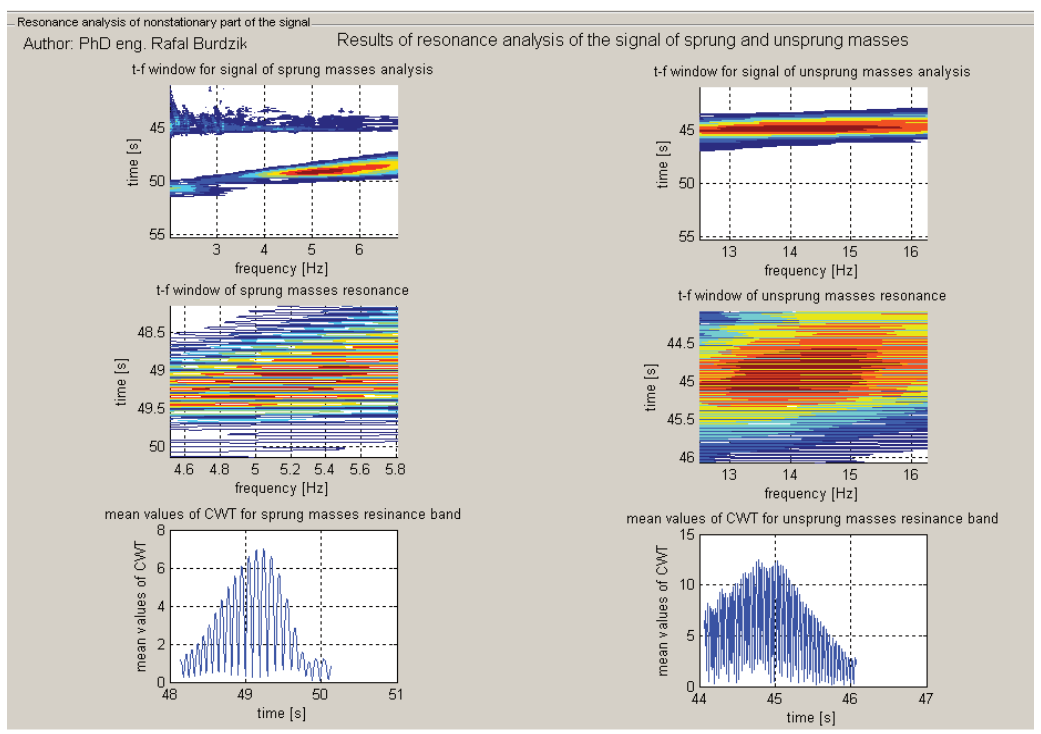

Fig. 18.7. Comparison of RMS directional dispersion factor of floor panel vibration for different engine rotational speed 
The method of multidimensional identification of vibration signal characteristics, described in previous chapter, allows to determine table of properties of an automotive vehicle's floor panel. The complicated vibration phenomena and random character of excitation forces acting on car vehicle determine to use many estimators to define vibration occurring in the car. The method described enables determining measures of signal distribution in time, frequency and timefrequency in terms of stationary and non-stationary parts of the signal.

The tables below contain a collation of the chosen estimators of vibration characteristics of an automotive vehicle's floor panel featuring built-in shock absorbers filled with working medium in $50 \%$. These form of 75-elements table is measures of signal characteristics. From the time realization of acceleration of vibration registered during slowing of excitation, when the mechanical system goes by resonance frequencies bands of sprung and unsprung masses of the vehicle the 16 global estimators were determined (Table 18.1).

Table 18.1. Global estimators of time realization of vibration

\begin{tabular}{|c|c|c|c|c|c|}
\hline \multicolumn{7}{|c|}{ Global estimators (amplitude, time) - resonance window } \\
\hline Max & Skewness & Kurtosis & Play factor & Root amplitude & Standard deviation \\
\hline 2.951 & -2.533 & 14.072 & -30.490 & 0.004 & 1.157 \\
\hline Shape factor & P2p & Peak factor & Impulsivity factor & Rms & Momentum 1 \\
\hline-10.206 & 5.663 & 4.229 & -43.166 & 1.339 & 0.000 \\
\hline Correlaction & Variance & covariance & Median & & \\
\hline 1.000 & 1.339 & 1.339 & 0.002 & & \\
\hline
\end{tabular}

Basing on the preliminary experimental research of stationary part of the vibration signal, during excitation force with constant frequency, the sensitivity on changes of technical condition of car suspension were specified. Thus for the vibration properties table were added estimators calculated on spectrum of vibration as 12 th next harmonics values. The values of those estimators for the same case study (shock absorbers filled with working medium in $50 \%$ ) are presented in Table 18.2.

Table 18.2. Spectrum of the vibration estimators (stationary signal)

\begin{tabular}{|c|c|c|c|c|c|}
\hline \multicolumn{7}{|c|}{ FFT estimators } \\
\hline 1st harm. & 2nd harm. & 3rd harm. & 4th harm. & 5th harm. & 6th harm. \\
\hline 1.121 & 0.242 & 0.142 & 0.378 & 0.159 & 0.019 \\
\hline 7th harm. & 8th harm. & 9th harm. & 10th harm. & 11th harm. & 12th harm. \\
\hline 0.186 & 0.034 & 0.007 & 0.017 & 0.027 & 0.016 \\
\hline
\end{tabular}

Some extra „control” estimators of identification of resonance occurring in time and frequency domains for sprung and unsprung masses of vehicle have been added to the Table 18.3. The values can change for different technical parameters of the suspension system (masses, stiffness).

Table 18.3. Estimators of resonances location

\begin{tabular}{|c|c|c|c|c|c|}
\hline \multicolumn{4}{|c|}{ Estimators of value and location of the resonances } \\
\hline \multicolumn{3}{|c|}{ Sprung masses } & \multicolumn{3}{c|}{ Unsprung masses } \\
\hline Max value & Time & Frequency & Max value & Time & Frequency \\
\hline 7.511 & 49.142 & 5.078 & 13.909 & 45.072 & 13.542 \\
\hline
\end{tabular}

For the precise time-frequency characteristics of the resonance windows, according to the methodology described in Chapter 3, the estimators of CWT (Continuous Wavelet Transform) have been determined. Time and value of the exposure on resonance vibration were determined separately for sprung and unsprung masses. The Tables 18.4 and 18.5 contain a collation of the chosen estimators of vibration determined from resonance distribution of CWT. Those estimators were added to the table of properties of floor panel vibration. 
Table 18.4. Collation of estimators of sprung masses resonance distribution of CWT

\begin{tabular}{|c|c|c|c|c|c|}
\hline \multicolumn{5}{|c|}{ Estimators of resonance distribution of CWT - sprung masses window } \\
\hline Max & Skewness & Kurtosis & Play factor & Root amplitude & Standard deviation \\
\hline 6.995 & 0.800 & 2.437 & 1.642 & 1.483 & 1.900 \\
\hline Shape factor & P2p & Peak factor & $\begin{array}{c}\text { Impulsivity } \\
\text { factor }\end{array}$ & Rms & Momentum 1 \\
\hline 1.479 & 3.457 & 0.960 & 1.420 & 3.601 & 0.000 \\
\hline Correlaction & Variance & Covariance & Median & $\begin{array}{c}\text { Integral of } \\
\text { average cwt }\end{array}$ & Mean/max \\
\hline 1.000 & 3.610 & 3.610 & 1.703 & 4.883 & 0.698 \\
\hline
\end{tabular}

Table 18.5. Collation of estimators of unsprung masses resonance distribution of CWT

\begin{tabular}{|c|c|c|c|c|c|}
\hline \multicolumn{5}{|c|}{ Estimators of resonance distribution of CWT - unsprung masses window } \\
\hline Max & Skewness & Kurtosis & Play factor & Root amplitude & Standard deviation \\
\hline 12.512 & 0.246 & 1.938 & 0.726 & 7.591 & 3.357 \\
\hline Shape factor & P2p & Peak factor & $\begin{array}{c}\text { Impulsivity } \\
\text { factor }\end{array}$ & Rms & Momentum 1 \\
\hline 2.040 & 6.160 & 0.548 & 1.118 & 11.239 & 0.000 \\
\hline Correlaction & Variance & Covariance & Median & $\begin{array}{c}\text { Integral of } \\
\text { average cwt }\end{array}$ & Mean/max \\
\hline 1.000 & 11.267 & 11.267 & 5.283 & 11.048 & 0.883 \\
\hline
\end{tabular}

Basing on the previous research some extra estimators were proposed to the table of properties of floor panel vibration. The relative (total) estimators of CWT distribution between resonances of sprung and unsprung masses are presented in Table 18.6. These are the relation representation's measurements of vibration characteristics of sprung and unsprung masses.

Table 18.6. Relative dimensionless estimators of the relation of CWT vibration characteristics of sprung and unsprung masses

\begin{tabular}{|c|c|c|c|c|}
\hline \multicolumn{5}{|c|}{ Dimensionless relative estimators (CWT) } \\
\hline$C_{w}$ & $L$ & $E_{s r}$ & $E_{\max }$ & $E_{w}$ \\
\hline 6.352 & 0.726 & 7.946 & 19.507 & 4.910 \\
\hline
\end{tabular}

Those estimators are defined as below.

$C_{w}$ - half of the sum of maximum values of amplitude of CWT of unsprung masses resonances (unsprung resonance $\mathrm{P} 2 \mathrm{P}$ - scope range measurement):

$C_{w}=\frac{W z_{\max }+W z_{\min }}{2}$

where: $W z_{\max }$ - maximum value of the average of CWT distribution for the unsprung masses resonance window, $W z_{\min }$ - minimum value of the average of CWT distribution for the unsprung masses resonance window.

$L$ - play factor of average of CWT distribution for the unsprung masses resonance window:

$$
L=\frac{\bar{w}}{\left(\frac{1}{n} \sum\left|w_{i}\right|^{\frac{1}{2}}\right)^{2}}
$$

where: $w_{i}$ - average of CWT distribution for the unsprung masses resonance window, $n$ - number of samples of CWT distribution average values.

$E_{s r}$ - sum of the average of CWT distribution for the sprung and unsprung masses resonance windows:

$$
E_{s r}=W z_{s r}+W n_{s r}
$$


where: $W z_{s r}-$ mean value of CWT distribution for the unsprung masses resonance window, $W n_{s r}$ - mean value of CWT distribution for the sprung masses resonance window.

$E_{\text {max }}$ - sum of maximum values of the average of CWT distribution for the sprung and unsprung masses resonance windows:

$E_{\max }=W z_{\max }+W n_{\max }$

where: $W z_{\max }$ - maximum value of average of CWT distribution for the unsprung masses resonance window, $W n_{\max }$ - maximum value of average of CWT distribution for the sprung masses resonance window.

$E_{w}$ - concentration coefficient of the average of CWT distribution for the resonance windows:

$E_{w}=\frac{E_{\max }}{\frac{E_{s r}}{2}}$.

To sum up the table of properties of floor panel vibration is collected from estimators determined from time realization of the vibration, spectrum and time-frequency distribution of the vibration. Exemplary structure of those table is presented in Table 18.7. It represents the vibration estimators calculated on the results of the research of the real object, as passenger car with shock absorbers filled with $50 \%$ of fluid volume. The color of the next values represents the estimators presented in Tables 18.1-18.6.

Table 18.7. Table of properties of floor panel vibration

\begin{tabular}{|c|c|c|c|c|}
\hline 2.951 & 0.002 & 5.078 & 0.000 & 0.548 \\
\hline-2.533 & 1.121 & 13.909 & 1.000 & 1.118 \\
\hline 14.072 & 0.242 & 45.072 & 3.610 & 11.239 \\
\hline-30.490 & 0.142 & 13.542 & 3.610 & 0.000 \\
\hline 0.004 & 0.378 & 6.995 & 1.703 & 1.000 \\
\hline 1.157 & 0.159 & 0.800 & 4.883 & 11.267 \\
\hline-10.206 & 0.019 & 2.437 & 0.698 & 11.267 \\
\hline 5.663 & 0.186 & 1.642 & 12.512 & 5.283 \\
\hline 4.229 & 0.034 & 1.483 & 0.246 & 11.048 \\
\hline-43.166 & 0.007 & 1.900 & 1.938 & 0.883 \\
\hline 1.339 & 0.017 & 1.479 & 0.726 & 6.352 \\
\hline 0.000 & 0.027 & 3.457 & 7.591 & 0.726 \\
\hline 1.000 & 0.016 & 0.960 & 3.357 & 7.946 \\
\hline 1.339 & 7.511 & 1.420 & 2.040 & 19.507 \\
\hline 1.339 & 49.142 & 3.601 & 6.160 & 4.910 \\
\hline
\end{tabular}

\subsection{Prototype of systems of monitoring of vibration properties and propagation in vehicles}

The prototype of the system for monitoring of vibration properties and propagation in vehicles is based on vibration signals analysis by use of the Vibroacoustic Signals Analyzer (WSA).

Application of the automatic developed algorithm for recognition of stationary and non-stationary states makes it possible to use the appropriate tools assuming the form of frequency as well as time and frequency transforms. Fig. 18.8 presents the programmable algorithm for ,analysis of edge". Application of the automatic developed algorithm for recognition of stationary and non-stationary states makes it possible to use the appropriate tools assuming the form of frequency as well as time and frequency transforms. 


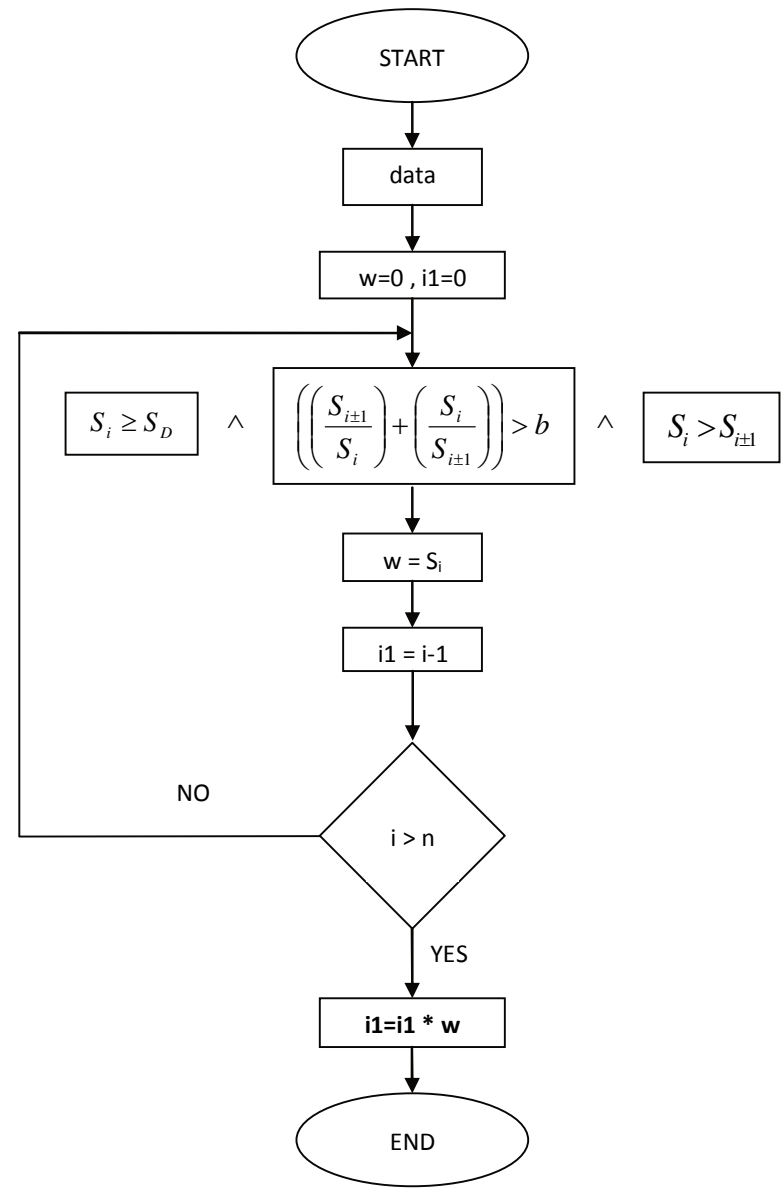

Fig. 18.8. The programmable ,analysis of edge” algorithm

The WSA program was extended with several modules dedicated to analysis, monitoring and diagnostics of selected vehicle systems and structural assemblies. For the control system of damping properties the AMOR module was developed. For the system of comfort monitoring the FLOOR module was developed.

The AMOR allows monitoring and determining the parameters of vibroisolation quantity of suspension system by comparison of vibration registered on suspension arm (as unsprung mass) and upper mounting of shock absorber (as sprung mass). Transformations of the signal in frequency and time-frequency domains enable observing the energy of the vibration in resonances and as the results evaluation of the damping properties. Basing on those information the control signal to adaptive suspension mechatronic systems can be formed to adjust the damping characteristics of shock absorbers.

The FLOOR module was designed for monitoring of exposure to whole-body vibration of passengers of car. The signal processing methodology is similar to AMOR but the possibility of determining by the user the frequency bands for analysis allows evaluating the exposure to vibration in free vibration bands of chosen human organs. It is very useful tool for analysing of driving comfort [36, 44, 47].

Both prototype systems have been tested during the experimental research. The results have already been presented in $[37,54]$. The scope of the experimental prototype researches included the tests of AMOR and FLOOR modules for the chosen damping properties parameters. There were researching on damping characteristics of shock absorbers, characteristics of suspension 
spring, pressure level in tires and value and location of extra load in the passenger cabin. The prototype of the system enables choosing of the vibration signal and frequency bands for the analysis. Some of the results are presented as the program's screens in Figs. 18.9 (AMOR) and 18.10 (FLOOR).

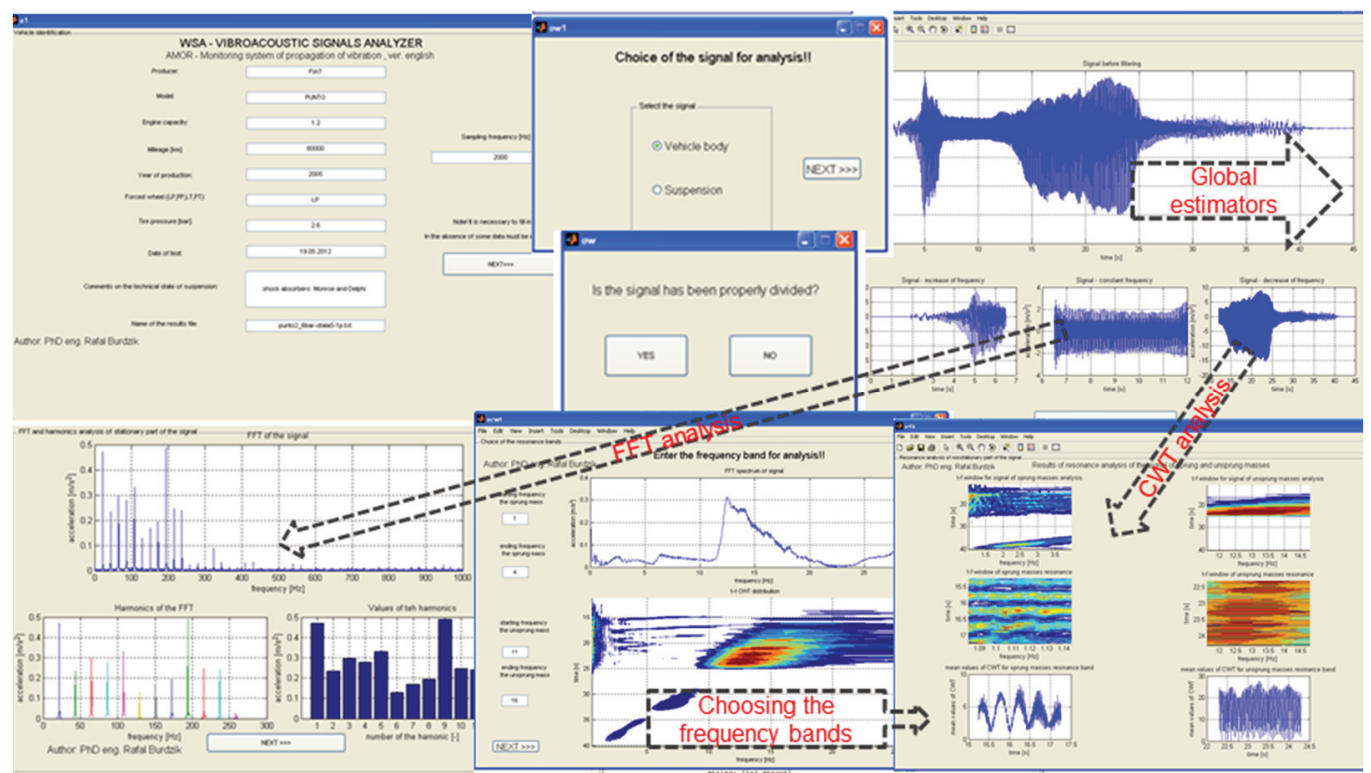

Fig. 18.9. Selected screens of the WSA program, module AMOR

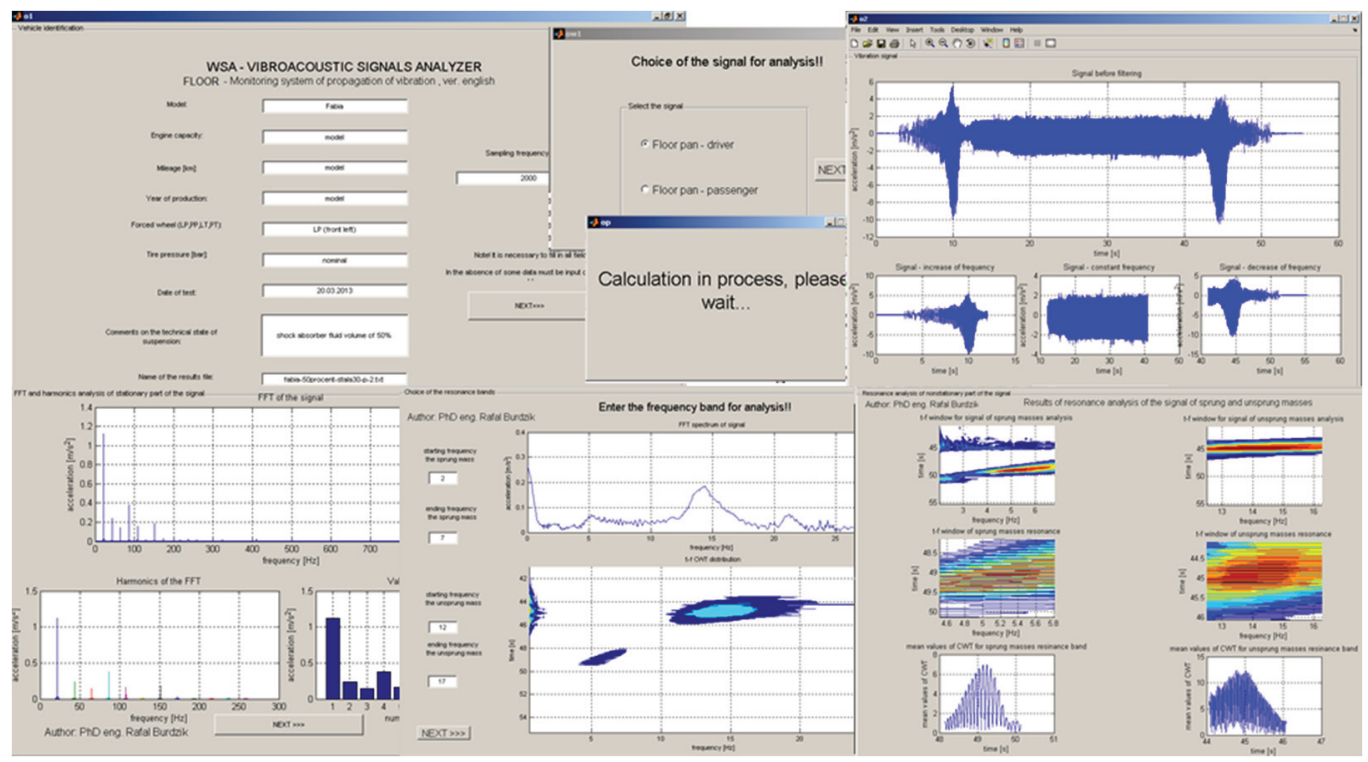

Fig. 18.10. Selected screens of the WSA program, module FLOOR

The results of WSA are vectors of estimators of resonances of unsprung and sprung masses or any others frequency bands defined by the user. Some of the example of the results are shown in Table 18.6. The proper conclusion basing on such large data collection is very difficult. Thus the papers $[37,59]$ present some application of neural networks as classifier or input module for the control system of vibration absorbing elements in vehicle structure. That choice was influenced by neural networks attributes such as: generalization of knowledge, approximation of functions 
and recognizing of patterns. The possibility of constant development of the basic knowledge by numerous examples and simple adaptation of a monitoring system is very significant as well. The neural classification system can be applied as a decision-making module in a control system [136].

The simulating test of application of neural network in abnormal tire pressure and shock absorber defect detection basing on vibration signals were conducted. The architectures of the network were designed and the transfer functions in the layers were programmed. The results obtained were satisfying. Fig. 18.11 presents the architectures of the example feed-forward network and training process.

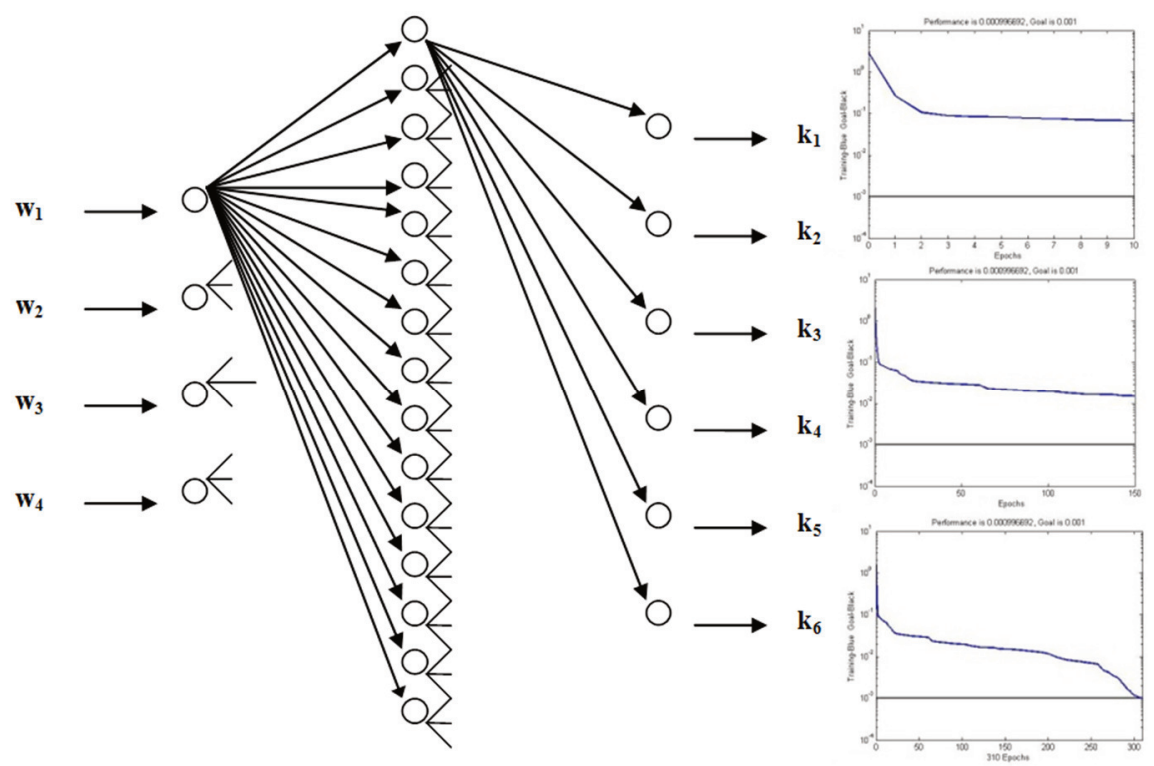

Fig. 18.11. Neural network classification module with training process graphs

The prototype of monitoring system is based on the assumption that vibrations can be recorded at any chosen structural points of a vehicle which enables the vibration propagation to be analysed. The monitoring system of vibration propagation in vehicles includes methods and algorithms for signals acquisition, pre-processing, transmission and data processing and storage. Consequently, the whole system consists of the following modules: data acquisition, stationary and non-stationary signal localization, WSA and neural classification system. It can be used as the input signal for the damping properties control system integrated with mechatronics system of vibration absorbing elements of suspension or engine and powertrain system mounting and springing elements.

\subsection{Concept of on-board comfort and propagation of vibration monitoring system for vehicles}

The conception of passenger cars comfort and safety monitoring and control system is based on prototype described in section above. The system has parameters for monitoring and control of the vibration. It is designed as 3-modular system integrated with WSA. First FLOOR module registers the floor panel vibration for surface and mapping of vibration transferred to human organism in multiple geometrical points distribution of vibrations. It can be consider as the comfort monitoring system. Second AMOR module monitors the damping properties of the suspension. It records the vibration of the suspension elements (sprung and unsprung). This module enables safety monitoring and controlling of the suspension elements. Basing on the previous research the system was expanded to third module. The last TIRES module is for the identification of pressure level in tires. It is very important exploitation parameter affecting on 
vibration transfer to car-body of the vehicle.

As the results of each module the defined estimators is reached. For the AMOR and TIRES some analytical experiment were conducted on application of neural network for the classification. For the comfort analysis the measures recommended in ISO 2631 and described in Chapter 16 for assessing exposure to vibrations of the overall impact on the human body are calculated. For the monitoring purpose those estimators are compared to some references values. Then system can generate the input function to the control unit of vibroisolation elements.

The architecture of the monitoring and control system of comfort and safety of passenger cars is shown in Fig. 18.12.

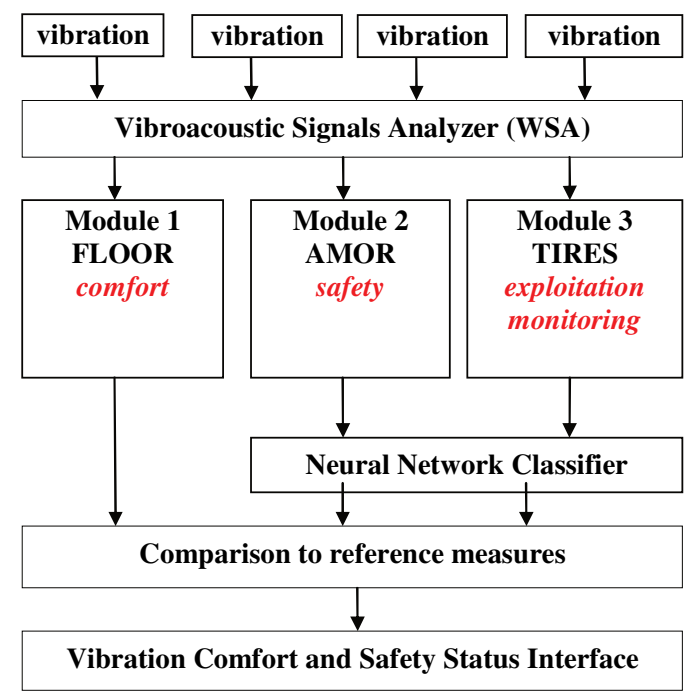

Fig. 18.12. Architectures of the monitoring and control system of comfort and safety of passenger cars

\subsection{The potential practical application of the WSA}

The method of multidimensional identification of signal characteristics in the analysis of vibration properties proposed and described in the chapter enables observation and separation of signal components in various domains. It also makes it possible to define signal measures depending on stationary and non-stationary characteristics as well as accurate time positioning of resonant frequencies. The measures applied in the table of signal characteristics determine a range of properties such a dynamics, amplification, scattering, concentration, attenuation, stability etc. The described software implementation of those method has the utilitarian character. WSA program is provided in friendly user interface. The results as table of properties of vehicle vibration could be adopted as mapping input signal to system of monitoring and control of vibration.

Vibroacoustic Signals Analyzer (WSA) was used for the signal processing. It is a self-designed program for the signal processing with friendly-user interface. Application of the automatic developed algorithm for recognition of stationary and nonstationary states makes it possible to use the appropriate tools assuming the form of frequency as well as time and frequency transforms. As the estimators of the vibration signals the measures calculated from time, frequency and time-frequency domains is used. The global measures are calculated from time realization of the signal. The stationary part of the signal allows calculating the measures of FFT harmonics. The nonstationary signal is transformed by Continuous Wavelet Transform so the time-frequency resonances windows can be determined. The WSA enables complex signal processing for the monitoring and comfort system application.

The preliminary prototype experimental research were successful. The scope of research 
presented in the chapter included the tests of AMOR and FLOOR modules for the chosen damping properties parameters. It enables developing the conception of the system for monitoring and control of comfort and safety of passenger cars is based on vibration signals analysis. The system based on Vibroacoustic Signals Analyzer (WSA). The WSA program is extended with several modules dedicated to analysis, monitoring and diagnostics of selected vehicle systems and structural assemblies. For the comfort monitoring system the module FLOOR was developed. For the safety control the AMOR can be used. The TIRE module enables monitoring of chosen exploitation parameters important for vibration transfer. As the results of each module are generated defined estimators. Monitoring of driving safety and comfort based on comparison to some references values. System can generate the input signal for the damping properties control system integrated with mechatronics system of vibration absorbing elements of suspension or engine and powertrain system mounting and springing elements. 



\section{Conclusions}

This book provides a discussion on vibration problems in means of transport. The issues addressed in the book include the identification of vibration sources and analysis of propagation and structure of vibrations affecting men in means of transport basing on the example of automotive vehicles. The author proposes specific methods dedicated for following characteristics identification: vibration structure in the function of time, frequency and time-frequency distributions vibration structure of wave propagation in three orthogonal axes as well as vibration distribution in a vehicle structure.

A majority of studies pertaining to vibrations in automotive vehicles focuses on vehicles in motion, subject to numerous dynamic impacts. The vibration waves being generated propagate into the vehicle structure and, via the body and equipment components, they are transferred to traffic participants. The book presents the experimental research analysis of natural vibrations of vehicle structures from the perspective of the most vital element in terms of the human exposure to vibration - the floor panel. The analysis of the results obtained and described in Chapter 5 enabled identification of characteristics of the vehicle floor panel vibrations.

The Chapters 6 to 10 present studies of the impact exerted by the technical condition of suspension components and the chosen operating parameters of a vehicle on the propagation of vibrations generated by a road wheel vertical motion to the vehicle. The purpose of those studies was to identify factors affecting the propagation of vibrations caused by the dynamic impact of road irregularities on wheels of a moving vehicle. Exposure to vibration in means of transport depends on many factors. For the perception properties as type of vibration the value, dynamics and exposure time of chosen frequency components of the vibration can be considered. The possibilities of simultaneous observation of signal in time and frequency domains distribution are reached by the time-frequency representation (TFR) of the signal. The Chapter 8 addresses analysis results of one of the TFR techniques application for the structure of vibration identification. The possibilities of characteristics components of vibration structure and sensitivity identification on the changes in mechanical system parameters were investigated on the research results concerning influence of technical condition of suspension and operating parameters of the vehicle.

The Chapters 9 and 10 describe proposition of function represents structure of vibration in terms of exposure. Technical condition of suspension elements analysis and vehicle's operating parameters influence on the vibration in terms of human perception and exposure require identification of frequency component carrying most of the vibration energy. The results of time function of average vibration in chosen frequency bands presented in Chapter 9 represents very sensitivity symptom estimator. Almost in every investigated case the functions were totally separable in whole time domain. This function of exposure time to the vibration of defined frequency bands is sensible for vibration of the floor panel in location of driver and passengers feet. The developed functions $S_{t}, S_{\text {Tavr }}$ represent dominant dynamics components of vibration excited during most vibro-activity time of exposure and function of average value of time period vibration in frequency domain allow identify frequency components.

Second part of the book is focused on engine as source of vehicle vibration. In Chapter 11 the engine as vibration source was analysed. The structures of vibration occurring on different conditions of working engine expressed as rotational speed were presented. 
Subsequently the identification of vibration propagation paths from engine into the occupants were presented. Basing on active experiments featuring measurements of vibration accelerations in a three directions in numerous selected points the analysis of propagation of vibration generated by engine to human body via feet and lumbar spine was conducted.

For the purpose of assessing exposure to vibrations of the overall impact on the human body by the vibration of the floor panel of the stopped vehicle with working engine the total estimators were proposed and compared in Chapter 13. Distribution of time energy floor panel vibration estimators $R M S$ and $S_{R M S}$ for different engine rotational speed shows that the energy estimators can have larger values for the smallest idle gear rotational speed. It is correlated to the feeling of vibration discomfort in the traffic jam, when engine is working on idle gear. To consider the influence on the dynamic of the vibration due to increase of the dynamic of the source the frequency estimators $T_{a b s(F F T)}$ and $S_{\text {Tabs }}$ were described. The most complex analysis of influence of engine rotational speed on floor panel vibration can be conducted basing on TFR of the signals. Thus the method of TFR energy estimator was developed. It allows to calculate the estimator depends on time and frequency distribution of the vibration.

The next chapter presents results of investigation on the directional distribution of vibration exposed on driver penetrate via feet for different gear position in gearbox. The values of estimators of energy of vibration considered in time, frequency and TFR representation of the signal were collected and compared to value reached for neutral gear, without gear ratio.

Chapter 15 presents some results of identification of structure and directional distribution of vibration transferred to car-body from road roughness. The method proposed for identification of components of road irregularity induced vibrations for a moving vehicle may be brought down to comprehensive laboratory and road tests of the same vehicle while maintaining identical engine and power transmission operating parameters.

The issues of exposure to vibration in means of transport were discussed in Chapter 16. The chapter presents experimental approach to exposure to WBV penetrated through floor panel via feet into human organism. The different methods for evaluation were compared for assessment influence of vehicle technical condition and power transmission system on human exposure to whole body vibration. The analysis is concerned with the lumbar spine response. The calculation of acceleration dose and equivalent and daily static compressive stress $S_{e}$ and $S_{e d}$ for the assessment of health effects were done.

The phenomena of vibration propagation is related to dispersion of energy. Thus Chapter 17 presents research on dispersion of vibration energy in vehicle construction. The measures of vibration dissipation in automotive vehicles were defined and verified. They enable the vibration propagation and damping to be analysed and assessed by application of simple energy measures in the domains of time and frequency.

The final chapter of the book describes possibilities of application presented methods. The preliminary prototype experimental research were successful. The scope of research presented in the Chapter 18 included the tests of 2 modules for the chosen damping properties parameters. It enables developing the conception of the system for monitoring and control of comfort and safety of passenger cars is based on vibration signals analysis. The system was based on Vibroacoustic Signals Analyzer (WSA). The WSA program is extended with several modules dedicated to analysis, monitoring and diagnostics of selected vehicle systems and structural assemblies. 


\section{References}

[1] Adamczyk J., Cioch W., Krzyworzeka P. Diagnosing of rotating machinery operating under variable conditions. The Naval Academy Scientific Papers, Vol. 48, Issue 169 K/1, 2007, p. 9-15.

[2] Adamczyk J., Kowal J., Wszołek W., Wszołek T. The impact of road traffic on a TV studio. Proceedings of the Tenth International Congress on Sound and Vibration, 2003, p. 4807-4814.

[3] Adamczyk J., Targosz J. Protection from Vibration Caused By the Road Transport. AGH, Cracow, 2000 , p. 68.

[4] Adamczyk J., Targosz J. Road Vibration. KRiDM AGH, Cracow, 2003, p. 181.

[5] Adams D. Health Monitoring of Structural Materials and Components. Willey, New York, 2007.

[6] Alleyne D. N. The nondestructive testing of plates using ultrasonic Lamb waves. PhD thesis Imperial College of Science, Technology and Medicine University of London, 1991.

[7] Amari M., Parizet E., Roussarie V. Multimodal approach to automobile driving comfort: The influence of visual setting on assessments of vibro-acoustic comfort in simulators. Applied Acoustic, Vol. 74, 2013, p. 1378-1387.

[8] Andrianov I. V., Awrejcewicz J., Danishevs'kyy V. V., et al. Wave propagation in periodic composites: higher-order asymptotic analysis versus plane-wave expansions method. Journal of Computational and Nonlinear Dynamics, Vol. 6, Issue 1, 2011.

[9] Au F. T. K., Cheng Y. S., Cheung Y. K. Vibration analysis of bridges under moving vehicles and train: an overview. Progress in Structural Engineering and Materials, Vol. 3, 2006, p. 299-304.

[10] Avramov K. V., Awrejcewicz J., Danishevs'kyy V. V., et al. Localization of vibrations in blade assemblies. Journal of Vibration and Control, Vol. 16, Issue 11, 2010, p. 1605-1622.

[11] Awrejcewicz J. Theoretical and numerical approaches to dynamics of selected engineering problems preface. International Journal of Structural Stability and Dynamics, Vol. 13, Issue 7, 2013.

[12] Awrejcewicz J., Krysko A. V., Kutepov I. E., et al. Analysis of chaotic vibrations of flexible plates using fast Fourier transforms and wavelets. International Journal of Structural Stability and Dynamics, Vol. 13, Issue 7, 2013.

[13] Awrejcewicz J., Krysko A. V., Soldatov V. On the wavelet transform application to a study of chaotic vibrations of the infinite length flexible panels driven longitudinally. International Journal of Bifurcation and Chaos, Vol. 19, Issue 10, 2009, p. 3347-3371.

[14] Awrejcewicz J., Krysko A. V., Yakovleva T. V., et al. Chaotic synchronization of vibrations of a coupled mechanical system consisting of a plate and beams. Latin American Journal of Solids and Structures, Vol. 10, Issue 7, 2013, p. 163-174.

[15] Awrejcewicz J., Papkova I. V., Krylova E. U., et al. Wavelet-based analysis of the regular and chaotic dynamics of rectangular flexible plates subjected to shear-harmonic loading. Shock and Vibration, Vol. 19, Issue 5, 2012, p. 979-994.

[16] Bajkowski J., Jasiński M., Mączak J., et al. The active magnetorheological support as an element of damping of vibrations transferred from the ground to large-scale structure supports. Structural Health Monitoring II: Key Engineering Materials, Vol. 518, 2012, p. 350-357.

[17] Bajkowski J., Jasiński M., Mączak J., Radkowski S., Zalewski R. The active magnetorheolo-gical support as an element of damping of vibrations transferred from the ground to large-scale structure supports. Key Engineering Materials, Vol. 518, 2012, p. 350-357.

[18] Bajkowski J., Nachman J., Shillor M., et al. A model for a magnetorheological damper. Mathematical and Computer Modelling, Vol. 48, Issue 1-2, 2008, p. 56-68.

[19] Balageas J., Fritzen C., Guemes A. Structural Health Monitoring Systems. ISTE, 2006.

[20] Bartelmus W. Mathematical modelling and computer simulations as an aid to gearbox diagnostics. Mechanical Systems and Signal Processing, Vol. 15, Issue 5, 2001, p. 855-871. 
[21] Batko W., Majkut L. Classification of phase trajectory portraits in the process of recognition the changes in technical condition of monitored machines and constructions. Archives of Metallurgy and Materials, Vol. 55, Issue 3, 2010, p. 757-762.

[22] Batko W., Majkut L. The phase trajectories as the new diagnostic discriminants of foundry machines and devices usability. Archives of Metallurgy and Materials, Vol. 52, Issue 3, 2007, p. 389-394.

[23] Beards C. F. Structural Vibration: Analysis and Damping. Halsted Press, New York, 1996.

[24] Bejger A., Gawdzinska K. Identification of structural defects of metal composite castings with the use of elastic waves. Archives of Metallurgy and Materials, Vol. 56, Issue 1, 2011, p. 129-133.

[25] Belgacem W., Berry A., Masson P. Active vibration control on a quarter-car for cancellation of road noise disturbance. Journal of Sound and Vibration, Vol. 331, 2012, p. 3240-3254.

[26] Bin Xu, Jian-Fei Lu, Jian-Hua Wang Numerical analysis of the isolation of the vibration due to Rayleigh waves by using pile rows in the poroelastic medium. Arch. Applied Mechanics, Vol. 80, 2010, p. 123-142.

[27] Blacha L., Burdzik R., Smalcerz A., Matuła T. Effects of pressure on the kinetics of manganese evaporation from the Ot4 Alloy. Archives of Metallurgy and Materials, Vol. 58, Issue 1, 2013, p. 197-201.

[28] Borowiec M., Hunicz J., Sen A., Litak G., Koszalka G., Niewczas A. Vibration of a vehicle excited by real road profiles. Forschung im Ingenieurwessen, Vol. 74, 2010, p. 99-109.

[29] Bouazara M., Richard M. J., Rakheja S. Safety and comfort analysis of a 3-D vehicle model with optimal non-linear active seat suspension. Journal of Terramechanics, Vol. 43, 2006, p. 97-118.

[30] Bradford S. C., Yang J., Heaton T. Variations in the dynamic properties of structures: the Wigner-Ville distribution. 1906 Earthquake Centennial Conference, San Francisco, 2006.

[31] Brauch R. Vibration analysis and standards, a review of vibration exposure regulations, standards, guidelines and current exposure assessment methods. AIHA Florida Conference, USA, 2009.

[32] Brownjohn James M. W. Energy dissipation from vibrating floor slabs due to human-structure interaction. Shock and Vibration, Vol. 8, 2001, p. 315-323.

[33] BS 6841. Guide to measurement and evaluation of human exposure to whole-body mechanical vibration and repeated shock, 1987.

[34] Bubulis A., Jūrẻnas V., Stepanenko D., Chigarev A., Minchenya V. Nonlinear effects related to vibrations of long elastic waveguides: formulation of nonlinear equations. Journal of Vibroengineering, Vol. 10, Issue 2, 2008, p. 222-224.

[35] Bubulis A., Reizina G., Korobko E., Bilyk V., Efremov V. Controllable vibro-protective system for the driver seat of a multi-axis vehicle. Journal of Vibroengineering, Vol. 13, Issue 3, 2011, p. 552-557.

[36] Burdzik R. Material vibration propagation in floor pan. Archives of Materials Science and Engineering, Vol. 59, Issue 1, 2013, p. 22-27.

[37] Burdzik R. Monitoring system of vibration propagation in vehicles and method of analysing vibration modes. 12th International Conference on Transport Systems Telematics, Vol. 329, p. 406-413.

[38] Burdzik R. Research on structure and directional distribution of vibration generated by engine in the location where vibrations penetrate the human organism. Diagnostics, Vol. 14, Issue 2, 2013, p. 57-61.

[39] Burdzik R. The influence of the rotational speed of engine on vibrations transferred on vehicle construction. Silesian University of Technology Scientific Papers, Transport, Vol. 72, 2011, p. 13-23.

[40] Burdzik R. The research of vibration of vehicle floor panel. Silesian University of Technology Scientific Papers, Transport, Vol. 67, 2010, p. 23-30.

[41] Burdzik R. Identification of structure and directional distribution of vibration transferred to car-body from road roughness. Journal of Vibroengineering, Vol. 16, Issue 1, 2014, p. 324-333.

[42] Burdzik R. Research on the influence of engine rotational speed to the vibration penetration into the driver via feet - multidimensional analysis. Journal of Vibroengineering, Vol. 15, Issue 4, 2013, p. 2114-2123.

[43] Burdzik R. Research of the vibration in 3 axes of car body for different idle gear rotational speed. Transactions on Transport Systems, Telematics and Safety, Chapter 5. Vehicles parameters, Gliwice, 2011, p. 203-214.

[44] Burdzik R. Multidimensional identification of signal's characteristics properties for the analysis of vibration properties of vehicle floor panel. Scientific Papers of the Warsaw University of Technology, s. Transport, Vol. 98, 2013, p. 29-36.

[45] Burdzik R., Czech P., Konieczny L., Folęga P. Exposure to vibrations generated by the motor vehicle. Journal of Polish Cimac Energetic Aspects, Vol. 8, Issue 1, 2013, p. 23-30. 
[46] Burdzik R., Czech P., Konieczny Ł., Wojnar G. Analysis of directional distribution of vibrations generated by the combustion engine. Journal of Polish Cimac Energetic Aspects, Vol. 7, Issue 1, 2012, p. 27-32.

[47] Burdzik R., Doleček R. Research of vibration distribution in vehicle constructive. Perner's Contacts, Vol. 7, Issue 4, 2012, p. 16-25.

[48] Burdzik R., Doleček R. Advanced signal processing methods for research on vibration propagation in vehicle construction. Perner's Contacts, Vol. 8, Issue 2, 2013, p. 15-26.

[49] Burdzik R., Folęga P., Lazarz B. Frequency based estimators of dynamics of exposure to vibration and technical condition od shock absorber with signals registered in location of vibration penetration into human organism. Scientific Papers of the Warsaw University of Technology, s. Transport, Vol. 98, 2013, p. 29-36.

[50] Burdzik R., Folęga P., Lazarz B. Research on influence of technical contition of spring element and vehicle operating parameters on vibration distribution in vehicle construction. Scientific Papers of the Warsaw University of Technology, s. Transport, Vol. 98, 2013, p. 29-36.

[51] Burdzik R., Folęga P., Lazarz B., Stanik Z., Warczek J. Analysis of the impact of surface layer parameters on wear intensity of frictional couples. Archives of Metallurgy and Materials, Vol. 57, Issue 4, 2012, p. 987-993.

[52] Burdzik R., Folęga P., Węgrzyn T., Silva Abílio P. Influence of exploitation tire stiffness on vehicle vibration. Conferencia Engenharia, Portugalia, 2009, p. 168-171.

[53] Burdzik R., Konieczny $\mathbf{t}$. Diagnosing of shock-absorbers of car vehicles at changeable pressure in tires. Diagnostics, Vol. 3, Issue 51, 2009, p. 27-32.

[54] Burdzik R., Konieczny $\mathbf{~}$. Application of vibroacoustic methods for monitoring and control of comfort and safety of passenger cars. Solid State Phenomena, Vol. 210, 2014, p. 20-25.

[55] Burdzik R., Konieczny $\mathbf{\ell}$. Research on structure, propagation and exposure to general vibration in passenger car for different damping parameters. Journal of Vibroengineering, Vol. 15, Issue 4, 2013, p. 1692-1700.

[56] Burdzik R., Konieczny Ł., Łazarz B. Influence of damping characteristics changes on vehicles vibration research. 19th International Congress on Sound and Vibration, 2012, p. 657.

[57] Burdzik R., Konieczny Ł., Figlus T. Concept of on-board comfort vibration monitoring system for vehicles. 13th International Conference on Transport Systems Telematics, 2013, p. 418-425.

[58] Burdzik R., Mikulski J. Analysis of vibration distribution in means of transport. Symposium Proceedings EURO-Zel 2013, Recent challenges for European railways, 2013, p. 14-21.

[59] Burdzik R., Peruń G, Warczek J. Possibilities of using vibration signals for the identification of pressure level in tires with application of neural networks classification. Key Engineering Materials, Vol. 588, 2014, p. 223-231.

[60] Burdzik R., Stanik Z., Warczek J. Method of assessing the impact of material properties on the propagation of vibrations excited with a single force impulse. Archives of Materials and Metallurgy, Vol. 57, Issue 2, 2012, p. 409-416.

[61] Cao M., Xu W., Ostachowicz W., Su Z. Damage identification for beams in noisy conditions based on Teager energy operator-wavelet transform modal curvature. Journal of Sound and Vibration, Vol. 333, Issue 6, 2014, p. 1543-1553.

[62] Cempel C. Singular values of symptom observation matrix of a system in operation as indicators of system damage. Diagnostics and Structural Health Monitoring, Vol. 4, Issue 60, 2011, p. 27-38.

[63] Cempel C., Tabaszewski M. Multidimensional vibration condition monitoring of non-stationary systems in operation. Mechanical Systems and Signal Processing, Vol. 21, Issue 3, 2007, p. 1233-1241.

[64] Cho-Chung L., Chi-Feng C. A study on biodynamic models of seated human subjects exposed to vertical vibration. International Journal of Industrial Ergonomics, Vol. 36, 2006, p. 869-890.

[65] Choromański W., Gągrowski A. Synthesis of mechatronic suspension seats of vehicles from the point of view of minimizing the impact of vibration on the human. Proceedings of The Transport of the 21st century, Vol. 3, 2004, p. 59-65.

[66] Clemente P., Rinaldis D. Protection of a monumental building against traffic-induced vibrations. Soil Dynamics and Earthquake Engineering, Vol. 17, 1998, p. 289-96.

[67] Corbridge C., Griffin M. J., Harborough P. Seat dynamics and passenger comfort. Institute of Mechanical Engineers, Part F, Journal of Rail and Rapid Transit, Vol. 203, 1989, p. 57-64.

[68] Crocker M. J., Battacharyaa M. C., Price A. J. Sound and vibration transmission through panels and tie beams using statistical energy analysis. Trans. ASME J., p. 775-782. 
[69] Cutini M., Romano E., Bisaglia C. Assessment of the influence of the eccentricity of tires on the whole-body vibration of tractor drivers during transport on asphalt roads. Journal of Terramechanics, Vol. 49, 2012, p. 197-206.

[70] Dąbrowski Z. Diagnostic inferring on the bases of nonlinear models. Acta Physica Polonica A, Vol. 118, Issue 1, 2010, p. 45-48.

[71] Dąbrowski Z. Modeling and identification of nonlinear system for technical diagnostics. Proceedings of the 8th International Congress on Sound and Vibration, China, 2001, p. 1083-1090.

[72] Dąbrowski Z. Use of non-linear symptoms in technical diagnosis. International Journal of Comadem, Vol. 8, Issue 2, 2005, p. 36-41.

[73] Dąbrowski Z., Dziurdź J., Klekot G. Studies on propagation of vibroacoustic energy and its influence on structure vibration in a large-size object. Archives of Acoustics, Vol. 32, Issue 2, 2007, p. 231-240.

[74] Dąbrowski Z., Zawisza M. Investigations of the vibroacoustic signals sensitivity to mechanical defects not recognised by the OBD system in diesel engines. Diffusion and Defect Data, Part B, Solid State Phenomena, Vol. 180, 2012, p. 194-199.

[75] Dạbrowski Z., Deuszkiewicz P. Designing of high-speed machine shafts of carbon composites with highly nonlinear characteristics. Key Engineering Materials, Vol. 490, 2011, p. 76-82.

[76] Official Journal of the European Communities. Directive 2002/44/EC of the European Parliament and of the Council of 25 June 2002 on the minimum health and safety requirements regarding the exposure of workers to the risks arising from physical agents (vibration). Sixteenth individual Directive within the meaning of Article 16(1) of Directive 89/391/EEC.

[77] Dobry M. W. The method of experimental verification of power distribution and energy flow in a human - machine system, structures - waves - human health. Acoustical Engineering, Polish Acoustical Society, Vol. 14, Issue 1, p. 45-48.

[78] Dobry M. W., Kolecka M. The energy impact of whole-body vibration on the human body. Scientific Papers of Cracow University of Technology, Vol. 83, 2001, p. 69-76.

[79] Dupuis H. Medical and ocupational preconditions for vibration-induced spinal disorders: occupational disease no 2110 in Germany. Int. Arch. Occup. Envir. Health, Vol. 6, 1994, p. 303-309.

[80] Dziurdź J. Transformation of nonstationary signals into pseudostationary signals for the needs of vehicle diagnostics. Acta Physica Polonica A, Vol. 118, Issue 1, 2010, p. 49-53.

[81] Dżygadło Z., Manerowski J. Vibration and stability analysis of rotors on orthotropic supports. Journal of Technical Physics, Vol. 23, Issue 3-4, 1982, p. 299-308.

[82] Endo H., Randall R. B., Gosselin C. Differential diagnosis of spall vs. cracks in the gear tooth fillet region: experimental validation. Journal of Mechanical System and Signal Processing, Vol. 23, Issue 3, 2008.

[83] Engel Z. W., Kowalski P. Investigation of the influence of simultaneous vibroacoustic exposures on the operator. Journal of the Theoretical and Applied Mechanics, Vol. 46, Issue 4, 2008, p. 799-811.

[84] Engel Z. W., Kowalski P. Investigation of the influence of simultaneous vibroacoustic exposures on the operator. Journal of the Theoretical and Applied Mechanics, Vol. 46, Issue 4, 2008, p. 799-811.

[85] Figlus T., Wilk A. Application of analysis of envelope's spectrum for gearbox diagnosing. International Review of Mechanical Engineering, Vol. 6, Issue 6, 2012, p. 1350-1355.

[86] Figlus T., Wilk A. Comparison of the sound pressure measurement and the speed measurement of the gearbox vibrating surface. Journal of Transport Problems, Vol. 7, Issue 1, 2012, p. 37-42.

[87] Gabor D. Theory of communication, Part 2. The analysis of hearing, Part 3. Frequency compression and expansion, Electrical Engineers - Part 3: Radio and Communication Engineering. Journal of the Institution of Electrical Engineers, Vol. 93, Issue 26, 1946, p. 429-457.

[88] Griffin M. Discomfort from feeling vehicle vibration. Vehicle System Dynamics, Vol. 45, Vol. 7-8, 2007, p. 679-698.

[89] Griffin M. Handbook of Human Vibration. New York: Academic Press Ltd, 1990.

[90] Griffin M. Minimum health and safety requirements for workers exposed to handtransmitted vibration and whole-body vibration in the European Union, a review. Occup Environ Med., Vol. 61, 2004, p. 387-397.

[91] Griffin M. Biodynamic response to whole-body vibration. The Shock and Vibration Digest, Vol. 13, Issue 3, 1981.

[92] Griffin M. The evaluation of vehicle vibration and seats. Applied Ergonomics, Vol. 9, Issue 1, 1978, p. 15-21.

[93] Gronostajski Z., Polak S. Quasi-static and dynamic deformation of double-hat thinwalled elements of vehicle controlled body crushing zones joined by clinching. Archives of Civil and Mechanical Engineering, Vol.8, Issue 2, 2008, p. 57-66. 
[94] Grządziela A. Modelling of propeller shaft dynamics at pulse load. Polish Maritime Research, Vol. 15, Issue 4, 2008, p. 52-58.

[95] Halverson W. G., Brown D. L. Impulse technique for structural frequency response testing, University of Cincinnati. Reprinted from Sound and Vibration, 1977.

[96] Harazin B. Occupational exposure to whole-body vibration in Poland. Occupational Medicine, Vol. 53, Issue 6, 2002, p. 465-472.

[97] Hodges C. H., Woodhouse J. Theories of noise and vibration transmission in complex structures. Rep. Prog. Phys., Vol. 49, 1986, p. 107-170.

[98] Hunaidi O., Rainer J. H., Pernica G. Measurement and analysis of traffic-induced vibrations. Proceedings of the 2nd International Symposium on Transport Noise and Vibrations, Russia, 1994, p. 103-108.

[99] Hung H. H., Yang Y. B. A review of researches on ground-borne vibrations with emphasis on those induced by trains. Proc. Natl. Sci. Counc., Vol. 25, 2001, p. 1-16.

[100] International Organization for Standardization. ISO 1978, guide for the evaluation of human exposure to whole-body vibration. 2nd Edition, International Standard 2631-1978(E).

[101] International Organization for Standardization. ISO 1997, mechanical vibration and shock - evaluation of human exposure to whole-body vibration, International Standard 2631-1:1997(E).

[102] International Organization for Standardization. ISO 2631, mechanical vibration and shock-evaluation of human exposure to whole-body vibration, 1997.

[103] International Organization for Standardization. ISO 2631-1, mechanical vibration and shock. Evaluation of human exposure to whole - body vibration. Part 1: General requirements, 1997.

[104] International Organization for Standardization. ISO 2631-4, mechanical vibration and shock. Evaluation of human exposure to whole - body vibration. Part 4: Guidelines for the evaluation of the effects of vibration and rotational motion on passenger and crew comfort in fixed-guide way transport system, 2001.

[105] International Organization for Standardization. ISO 2631-5, mechanical vibration and shock. Evaluation of human exposure to whole - body vibration. Part 5: Method for evaluation of vibration containing multiple shock, 2004.

[106] International Organization for Standardization. ISO 5982, mechanical vibration and shock - Range of idealized values to characterize seated-body biodynamic response under vertical vibration, 2001.

[107] International Organization for Standardization. ISO 7962, mechanical vibration and shock Mechanical transmissibility of the human body in the direction, 1987.

[108] Jasiński M., Radkowski S. Use of bispectral-based fault detection method in the vibroacoustic diagnosis of the gearbox. Engineering Asset Lifecycle Management, 2010, p. 651-660.

[109] Jia-Yi Yeh, Jiun-Yeu Chen, Chen-Yang Liu, Chih-Chieh Chang Control of wave propagation in periodic structures having defects. Journal of Science and Engineering Technology, Vol. 3, Issue 3, 2007, p. 45-50.

[110] Kacalak W., Krzyżyński T., Lewkowicz R., Balasz B. Specific energy minimization in processes of abrasive machining. Journal of Applied Mathematics and Mechan, Vol. 80, Issue 3, 2000, p. 587-588.

[111] Kardas-Cinal E., Droździel J., Sowiński B. Simulation study of a relation between derailment coefficient and track condition. Archives of Transport, Vol. 21, Issue 1-2, 2009, p. 85-98.

[112] Khoshravan M. R., Paykani A., Akbarzadeh A. Design and modal analysis of composite drive shaft for automotive application. International Journal of Engineering Science and Technology, Vol. 3, Issue 4, 2011, p. 2543-2549.

[113] Kiciński J. Microturbines in dispersed cogeneration - vibroacoustic threats. Archives of Acoustics, Vol. 32, Issue 2, 2007, p. 263-277.

[114] Kiciński J. New method of analysis of non-linear stochastic and random vibrations. Vibrations in Physical Systems, Vol. 25, 2012, p. 13-22.

[115] Kiciński J., Drozdowski R., Materny P. Nonlinear model of vibrations in a rotor-bearings system. Journal of Vibration and Control, Vol. 4, Issue 5, 1998, p. 519-540.

[116] Klekot G. Application of structural vibration propagation for evaluation of technical objects. Mechanical Review, Vol. 10, 2010, p. 38-42.

[117] Kocańda A., Sadłowska H. Automotive component development by means of hydroforming. Archives of Civil and Mechanical Engineering, Vol.8, Issue 3, 2008, p. 55-72.

[118] Kollek W., Kudzma Z., Stosiak M., Mackiewicz J. Possibilities of diagnosing cavitation in hydraulic systems. Archives of Civil and Mechanical Engineering, Vol. 7, Issue 1, 2007, p. 61-74. 
[119] Konieczny Ł., Burdzik R., Lazarz B. Analysis of properties of automotive vehicle suspension arm depending on different materials used in the MSC.Adams environment. Archives of Materials Science and Engineering, Vol. 58, Issue 2, 2012, p. 171-176.

[120] Konieczny Ł., Burdzik R., Lazarz B. Application of the vibration test in the evaluation of the technical condition of shock absorbers built into the vehicle. Journal of Vibroengineering, Vol. 15, Issue 4, 2013, p. 2068-2074.

[121] Korzeb J., Nader M., Rózowicz J. Review and estimation of traffic generated vibration developed in proximity of Warsaw subway line. 12th International Congress on Sound and Vibration, 2005, p. 5048-5055.

[122] Korzeb J., Różowicz J. Analysis of dynamic influences on buildings and the people in them staying in the impact zone of transport. Logistics, Vol. 4, 2010, p. 9.

[123] Korzeb J. Prediction of dynamic responses in area of influence of transport infrastructure. Scientific Papers of the Warsaw University of Technology, Transport, Vol. 90, 2013, p. 182.

[124] Kowal J., Konieczny J. Active control of vibration with eigenvalue placement controller. International Congress on Noise Control Engineering, Internoise, Vol. 1, 2005, p. 149-158.

[125] Kowal J., Pluta J., Konieczny J., Kot A. Energy recovering in active vibration isolation system results of experimental research. Journal of Vibration and Control, Vol. 14, Issue 7, 2008, p. 1075-1088.

[126] Kudela P., Krawczuk M., Ostachowicz W., Palacz M., Zak A. Wave propagation modelling in composite plates with damage. Proceedings of the 3rd European Workshop, Structural Health Monitoring, 2006, p. 1214-1221.

[127] Kuznetsov A., Mammadov M., Sultan I., Hajilarov E. Optimization of a quarter-car suspension model coupled with the driver biomechanical effects. Journal of Sound and Vibration, Vol. 330, 2011, p. 2937-2946.

[128] Kuznetsov A., Mammadov M., Sultan I.A., Hajilarov E. Vibration analysis optimization of parameters of the two mass model based on Kelvin elements. Proceedings of the Eighth IEEE International Conference on Control and Automation, China, 2010, p. 1326-1332.

[129] Lak M. A., Degrande G., Lombaert G. The effect of road unevenness on the dynamic vehicle response and ground-borne vibrations due to road traffic. Soil Dynamics and Earthquake Engineering, Vol. 31, 2011, p. 1357-1377.

[130] Lazarz B., Wojnar G., Czech P. Early fault detection of toothed gear in exploitation conditions. Maintenance and Reliability, Vol. 1, Issue 49, 2011, p. 68-77.

[131] Lee T. K., Kim B. S. Vibration analysis of automobile tire due to bump impact. Applied Acoustics, Vol. 69, 2008, p. 473-478.

[132] Lord Rayleigh The Theory of Sound. 2nd ed. MacMillan, London, 1894, 1st ed. Dover, New York, 1945.

[133] Lozia Z. A two-dimensional model of the interaction between a pneumatic tire and an even and uneven road surface. Vehicle System Dynamics, Vol. 17, Supplement S, 1988, p. 227-238.

[134] Lozia Z. Truck front wheels and axle beam vibrations. 5th Mini Conference on Vehicle System Dynamics, Identification and Anomalies, Budapest, 1996.

[135] Magala Schoeller M., Tautzb J., Casasc J. The role of leaf structure in vibration propagation. Journal of the Acoustical Society of America, Vol. 108, Issue 5, 2000, p. 2412-2418.

[136] Manerowski J., Zgrzywa F., Sibilski K. A neural model of coefficients of forces and moments of aerodynamic forces for a turboprop aircraft. Collection of Technical Papers, Atmospheric Flight Mechanics Conference, Vol. 1, 2006, p. 682-691.

[137] Mazur K., Pawełczyk M. Active noise control with a single nonlinear control filter for a vibrating plate with multiple actuators. Archives of Acoustics, Vol. 38, Issue 4, 2013, p. 537-545.

[138] Mead D. J. Wave propagation in continuous periodic structures: research contributions from Southampton 1964-1995. Journal of Sound and Vibration, Vol. 190, Issue 3, 1996, p. 495-524.

[139] Michalski R., Wierzbicki S. An analysis of degradation of vehicles in operation. Maintenance and Reliability, Vol. 1, Issue 3, 2008, p. 30-32.

[140] Michel M., Guo X., Gibson L., McMahon T., Hayes W. Compressive fatigue behavior of bovine trabecular bone. J. Biomech., Vol. 26, 1993, p. 453-463.

[141] Mityurich G. S., Aleksiejuk M., Ranachowski P., Pelivanov I. M., Serdyukov A. N. Photoacoustic diagnostics of inhomogeneous gyrotropic materials with internal stress using bessel light beams. Archives of Metallurgy and Materials, Vol. 56, Issue 4, 2011, p. 1235-1242. 
[142] Morrison J., Robinson D., Roddan G., Nicol J., Springer M., Martin S., Cameron B. Development of a standard for the health hazard assessment of mechanical shock and repeated impact in army vehicles. Phase 5. B.C. Research Inc., Vancouver, 1997, Project No: 6-06-600.

[143] Nader M. Influence of mechanical vibrationon the human body in the means of transport and its modeling. Archives of Transport, Vol. 12, Issue 2, 2000, p. 33-53.

[144] Nader M., Chudzikiewicz A. The energy flow method for estimation of the vibrations effect on man-vehicle systems. Machine Dynamic Problems, Vol. 28, Issue 2, Warsaw, 2004, p. 7-22.

[145] Nader M., Korzeb J. Analysis of the dynamic influence of urban transport on the environment. Proceedings of 5th IC-SCCE, Greece, 2012, p. 67-74.

[146] Nahvi H., Fouladi M. H., Mohd Nor M. J. Evaluation of whole - body vibration and ride comfort in a passenger car. Proceedings of ICSV16, Cracow, Poland, 2009.

[147] Nguyen V. N., Inaba S. Effects of tire inflation pressure and tractor velocity on dynamic wheel load and rear axle vibrations. J. Terrramech, Vol. 48, 2011, p. 3-16.

[148] Ostachowicz W., Krawczuk M., Zak A., Kudela P. Damage detection in elements of structures by the elastic wave propagation method. Computer Assisted Mechanics and Engineering Sciences, Vol. 12, Issue 1, 2006, p. 109-124.

[149] Ostachowicz W., Kudela P., Krawczuk M., Zak A. Guided waves in structures for SHM: the timedomain spectral element method. Polish Academy of Sciences, Institute of Fluid Flow Machinery, Poland, 2012, p. 337.

[150] Ostachowicz W., Kudela P., Radzieński M. Guided wavefield images filtering for damage localization. Key Engineering Materials, Vol. 558, 2013, p. 92-98.

[151] Ostachowicz W., Radzieński M. Structural health monitoring by means of elastic wave propagation. Journal of Physics, Conference Series, Vol. 382, Issue 1, 2012.

[152] Paddan G., Griffin M. Evaluation of whole-body vibration in vehicles. Journal of Sound and Vibration, Vol. 253, Issue 1, 2002, p. 195-213.

[153] Pai P., Nguyen B., Sundaresan M. Nonlinearity identification by time-domain only signal processing. International Journal of Non-Linear Mechanics, Vol. 54, 2013, p. 85-98.

[154] Papalukopoulos C., Giadopulos D., Natsiavas S. Dynamics of large scale vehicle models coupled with driver biodynamic models. Proceedings of the fifth GRACM International Congresson Computational Mechanics, Limassol, 2005.

[155] Pawełczyk M. Acoustics and vibration as fascinating challenges to specialists in automatic control and signal processing. International Journal of Acoustics and Vibrations, Vol. 11, Issue 3, 2006, p. 118-119.

[156] Popescu Th. D. Analysis of traffic-induced vibrations by blind source separation with application in building monitoring. Mathematics and Computers in Simulation, Vol. 80, 2010, p. 2374-2385.

[157] Prasad Ravi, Seshu D. R. A study on dynamic characteristics of structural materials using modal analysis. Asian Journal of Civil Engineering, Vol. 9, Issue 2, 2008, p. 141-152.

[158] Qiu Y., Griffin M. J. Transmission of vibration to the backrest of a car seat evaluated with multiinput models. Journal of Sound and Vibration, Vol. 274, 2004, p. 297-321.

[159] Radkowski S., Smalko Z., Pietak A., Woropay M. Use of bispectral analysis in condition monitoring of machinery. Structural Health Monitoring, 2006, p. 627-634.

[160] Radkowski S., Szczurowski K. Use of vibroacoustic signals for diagnosis of pre-stressed structures. Maintenance and Reliability, Vol. 14, Issue 1, 2012, p. 84-91.

[161] Raghavan A., Cesnik C. E. S. Review of guided - waves structural health monitoring. The Shock and Vibration Digest, Vol. 39, 2007, p. 91-114.

[162] Ragulskis K., Kanapeckas K., Jonušas R., Juzėnas K. Vibrations generator with a motion converter based on permanent magnet interaction. Journal of Vibroengineering, Vol. 12, Issue 1, 2010, p. 124-132.

[163] Ragulskis L., Palevicius R., Ragulskis M., Rubliauskas D., Palevicius A. Analytical, numerical and experimental investigation of self resonance in vibration excitation systems. Proceedings of SPIE, The International Society for Optical Engineering, Vol. 5764, 2005, p. 641-649.

[164] Ragulskis M., Maskeliunas R. Measurement of transverse vibrations of piezoelectric ceramics by atomic force microscopy. Experimental Techniques, Vol. 30, Issue 2, 2006, p. 37-41.

[165] Ragulskis M., Palevicius A., Ragulskis L., Bubulis A. Generalized Abel transform for the analysis of fluid vibration in a tube. Optical Engineering, Vol. 46, Issue 6, 2007.

[166] Rustighi E., Elliott S. J., Finnveden S., Gulyas K., Mocsai T., Dantid M. Linear stochastic evaluation of tire vibration due to tire/road excitation. Journal of Sound and Vibration, Vol. 310, 2008, p. 1112-1127. 
[167] Sawalhi N., Randall R. B. Simulating gear and bearing interaction in the presence of faults, Part I The combined gear bearing dynamic model and simulation of localized bearing faults. Journal of Mechanical System and Signal Processing, Vol. 22, 2008, p. 1924-1951.

[168] Sayers M. W., Karamihas S. M. The Little Book of Profiling. Regent of the University of Michigan, 1998.

[169] Seidel H. Effects and Evalution of Whole-Body Vibration - Biological Aspects. Humanschwingungen, 2004, p. 1-24.

[170] Seidel H. On the relationship between whole-body vibration exposure and spinal health risk. Industrial Health, Vol. 43, 2005, p. 361-377.

[171] Seidel H., Blüthner R., Hinz B., Schust M. On the health risk of the lumbar spine due to whole-body vibration - theoretical approach, experimental data and evaluation of whole-body vibration. J. Sound and Vibration, Vol. 215, Issue 4, 1998, p. 723-741.

[172] Seidel H., Blüthner R., Menzel G., Hofmann J., Gericke L., Schust M. in cooperation with H. Kaiser and Ch. Mischke B. Hinz. Prediction of spinal stress in drivers from field measurements. European Commission Quality of Life and Management of Living Resources Programme Key Action 4, Environment and Health Risks of Occupational Vibration Exposures, Annex 19 to Final Technical Report, 2007.

[173] Sekulic D., Dedovic V., Rusov S., Šalinic S., Obradovic A. Analysis of vibration effects on the comfort of intercity bus users by oscillatory model with ten degrees of freedom. Applied Mathematical Modelling, Vol. 37, 2013, p. 8629-8644.

[174] Sherwin L. M., Owende P. M. O., Kanali C. L., Lyons J., Ward S. M. Influence of tire inflation pressure on whole-body vibrations transmitted to the operator in a cut-to-length timber. Appl Ergon, Vol. 35, Issue 3, 2004, p. 235-261.

[175] Snamina J., Kowal J., Orkisz P. Active suspension based on low dynamic stiffness. Acta Physica Polonica A, Vol. 123, Issue 6, 2013, p. 1118-1122.

[176] Sobczyk K. Methods of Stochastic Mechanics. PWN, Warsaw, 1973.

[177] Sobczyk K. Stochastic Mechanics: Genesis, Models, Applications. Polish and World Achievements of Science, Science Technical, p. 430-462.

[178] Sobczyk K. Stochastic Wave Propagation. Elsevier, Amsterdam, 1985, p. 1-248.

[179] Strzyżakowski Z. Changing the intensity of the surface wave in the ground from a means of transport. International Scientific Conference on Transport Twenty-First Century, Bialowieza, 2010.

[180] Stypuła K. Ground vibrations due to underground train influence on the buildings. Scientific Papers of Cracow University of Technology, Civil Engineering, Vol. 72, 2001.

[181] Su Z., Ye L. Identification of Damage Using Lamb Waves. Springer, Berlin Heidelberg, 2009.

[182] Szemplinska-Stupnicka W., Bajkowski J. Multi-harmonic response in the regions of instability of harmonic solution in multi-degree-of-freedom non-linear systems. International Journal of Non-Linear Mechanics, Vol. 15, Issue 1, 1980, p. 1-11.

[183] Szemplinska-Stupnicka W., Bajkowski J. The 1/2 subharmonic resonance and its transition to chaotic motion in a nonlinear oscillator. International Journal of Non-Linear Mechanics, Vol. 21, Issue 5, 1986, p. 401-419.

[184] Takemiya H. Environmental vibrations: prediction monitoring and evaluation. Proceedings of the 2nd International Symposium on Environmental Vibrations, Prediction, Monitoring and Evaluation, Proceedings and Monographs in Engineering, Water and Earth Sciences, Taylor \& Francis, 2005.

[185] Taylor J. I. The Gear Analysis Handbook. Vibration Consultants, Inc., USA, 2000.

[186] Thompson A. G., Pearce C. E. M. RMS values for force, stroke and tyre deflection in a quarter-car model active suspension. Vehicle System Dynamics, Vol. 39, 2002, p. 57-75.

[187] Toward M., Griffin M. J. The transmission of vertical vibration through seats: influence of the characteristics of the human body. Journal of Sound and Vibration, Vol. 330, 2011, p. 6526-6543.

[188] Tuma J. Transmission and gearbox noise and vibration prediction and control. Handbook of Noise and Vibration Control, Chapter 88, Wiley, New York, 2007, p. 1080-1089.

[189] Tuma J., Šimek J., Škuta J., Los J. Active vibrations control of journal bearings with the use of piezoactuators. Mechanical Systems and Signal Processing, Vol. 36, Issue 2, 2013, p. 618-629.

[190] Uhl T. Modern methods of monitoring and diagnosing the structure. Polish and World Achievements of Science, Science Technical, p. 194-254.

[191] Uhl T. The use and challenge of modal analysis in diagnostics. Diagnostics, Vol. 30, Issue 2, 2004, p. 151-160.

[192] Uhl T., Chudzikiewicz A., Karpiński J. Dynamic problems in rail vehicle design. Archives of Transport, Vol. 12, Issue 1, 2000, p. 57-71. 
[193] Verver M. M., Hoof J., Oomens C. W. J., Wouw N., Wismans J. S. H. M. Estimation of spinal loading in vertical vibrations by numerical simulation. Clinical Biomechanics, Vol. 18, 2003, p. 800-811.

[194] Ville J. Theory and applications of the notion of complex signal, Rand Corporation Technical Report T-92, Santa Monica, CA, 1958.

[195] Watts G. R. Traffic induced vibrations in buildings. Research Report 246, Transport and Road Research Laboratory, 1990.

[196] Węgrzyn T., Piwnik J., Burdzik R., Wojnar G., Hadryś A. New welding technologies for car body frame welding. Archives of Materials Science and Engineering, Vol. 58, Issue 2, 2012, p. 245-249.

[197] Wicher J., Więckowski D. Influence of vibrations of the child seat on the comfort of child's ride in a car. Maintenance and Reliability, Vol. 77, Issue 1, 2010, p. 77-93.

[198] Więckowski D., Wicher J. Safety and comfort of the of child's ride in child seat in a car. Scientific Papers of Warsaw University of Technology Institute of Vehicle, Vol. 4, 2010, p. 102-110.

[199] Wigner E. On the quantum correction for thermodynamic equilibrium. Physical Review, Vol. 40, 1932, p. 749-759.

[200] Wilder D. G., Aleksiev A. R., Magnusson M. L., Pope M. H., Spratt K. F., Goel V. K. Muscular response to sudden load. A tool to evaluate fatigue and rehabilitation. Spine, Vol. 21, Issue 22, 1996, p. 2628-39.

[201] Wilk A., Madej H., Figlus T. Analysis of the possibility to reduce vibroactivity of the gearbox housing. Maintenance and Reliability, 2011, p. 42-49.

[202] Wong J. Y. Theory of Ground Vehicles. John Wiley and Sons, Inc., New York, 2001.

[203] Woodhouse J. An approach to the theoretical background of statistical energy analysis applied to structural vibration. J. Acoust. Soc. A., Vol. 69, Issue 6, 1981, p. 1965-1709.

[204] Xian-feng Du, Li Zhi-jun, BI Feng-rong, Zhang Jun-hong, Wang Xia, Shao Kang Vibration-based feature extraction of determining dynamic characteristic for engine block low vibration design. J. Cent. South Univ., Vol. 19, 2012, p. 2238-2246.

[205] Xua Y. L., Guo W. H. Effects of bridge motion and crosswind on ride comfort of road vehicles. Journal of Wind Engineering and Industrial Aerodynamics, Vol. 92, 2004.

[206] Yang J., Suematsu Y., Kang Z. Twodegree-of-freedom controller to reduce the vibration of vehicle engine-body system. IEEE Trans. on Control Systems Technology, Vol. 9, Issue 2, 2001, p. 295-304.

[207] Yang Y. B., Hung H. H. Soil vibrations caused by underground moving trains using the 2.5D finite/infinite element approach. J. Geotech. Geoenviron. Eng. ASCE, Vol. 134, 2008, p. 1633-1644.

[208] Yang Y. B., Hung H. H., Hsu L. C. Ground vibrations due to underground train considering soil-tunnel interactions. Interact. Multiscale Mech. Int. J., Vol. 1, 2005, p. 157-175.

[209] Yoshida J. Idle shake simulation with engine exciting force. JSAE Review, Vol. 17, 1996, p. 65-77.

[210] Yunhe Yu, Naganathan Nagi G., Dukkipati Rao V. A literature review of automotive vehicle engine mounting systems. Mechanism and Machine Theory, Vol. 36, 2001, p. 123-142.

[211] Zheng J., Suzuki K., Fujita M. Car-following behavior with instantaneous driver-vehicle reaction delay: a neural-network-based methodology. Transportation Research Part C, Vol. 36, 2013, p. 339-351.

[212] Zimroz R., Urbanek J., Barszcz T., Bartelmus W. Milioz F. Martin N. Measurement of instantaneous shaft speed by advanced vibtation signal processing - application to wind turbine gearbox. Metrology and Measurement Systems, Vol. 18, Issue 4, 2011, p. 701-711. 


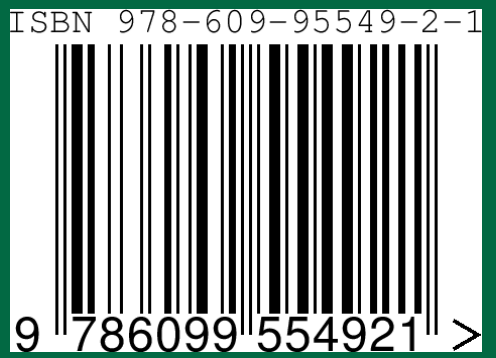

